\title{
Large-Eddy Simulation of Turbulent Flow Using the Finite Element Method
}

R. C. McCallen

(Ph.D. Thesis)

February 15, 1995 


\section{DISCLAIMER}

This document was prepared as an account of work sponsored by an agency of the United States Government. Neither the United States Government nor the University of California nor any of their employees, makes any warranty, express or implied, or assumes any legal liability or responsibility for the accuracy, completeness, or usefulness of any information, apparatus, product, or process disclosed, or represents that its use would not infringe privately owned rights. Reference herein to any specific commercial product, process, or service by trade name, trademark, manufacturer, or otherwise, does not necessarily constitute or imply its endorsement, recommendation, or favoring by the United States Government or the University of California. The views and opinions of authors expressed herein do not necessarily state or reflect those of the United States Government or the University of California, and shall not be used for advertising or product endorsement purposes.

This report has been reproduced directly from the best available copy.

Available to DOE and DOE contractors from the Office of Scientific and Technical Information

P.O. Box 62, Oak Ridge, TN 37831

Prices available from (615) 576-8401, FTS 626-8401

Available to the public from the National Technical Information Service

U.S. Department of Commerce 5285 Port Royal Rd. Springfield, VA 22161 


\section{DISCLAIMER}

Portions of this document may be illegible in electronic image products. Images are produced from the best available original document. 
UCRL-LR-114962

Distribution Category UC-700

\title{
Large-Eddy Simulation of Turbulent Flow Using the Finite Element Method
}

\author{
R. C. McCallen \\ (Ph.D. Thesis)
}

Manuscript date: February 15, 1995

\section{LAWRENCE LIVERMORE NATIONAL LABORATORY}

University of California • Livermore, California • 94551

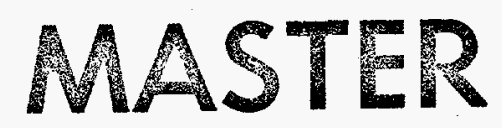




\title{
Large-Eddy Simulation of Turbulent Flow \\ Using the Finite Element Method
}

\author{
By
}

ROSE CLARA MCCALLEN

B.S. (St. Mary's College, Moraga) 1977

B.S. (California State University, Chico) 1980

M.S. (University of California, Davis) 1984

DISSERTATION

Submitted in partial satisfaction of the requirement for the degree of

DOCTOR OF PHILOSOPHY

in

Mechanical Engineering

in the

GRADUATE DIVISION

of the

UNIVERSITY OF CALIFORNIA

DAVIS

APPROVED:

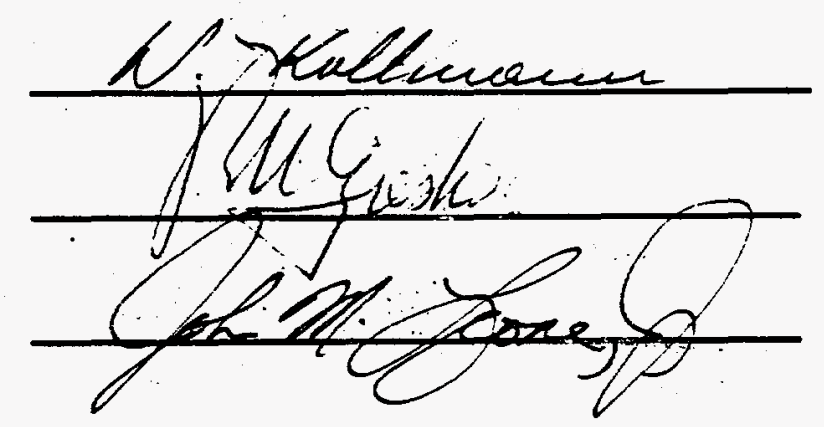

Committee in Charge 


\begin{abstract}
The motivation for this research is the development of a finite element turbulence modeling method which will ultimately be used to predict the wind flow around buildings. The turbulence model is a large-eddy simulation (LES) in which the large-eddy motion is directly simulated and the small-eddy motion is modeled (i.e., approximated by semi-empirical/analytical relations). The LES model is incorporated into an existing time-dependent, laminar, incompressible flow code that uses the finite element numerical approach. The objective of this research is to show that the finite element method (FEM) is a viable numerical approach for the solution of the LES equations and that there are clear advantages to using the FEM approach over the more commonly used finite difference and spectral method approaches.
\end{abstract}

The backward-facing step problem is used as the benchmark problem. The backward-facing step is a simplification of the building flow problem.

Included in this report is a brief discussion of turbulent flow characteristics and the difficulties in numerically modeling turbulent flow. The direct simulation approach is described along with an explanation of why current results are limited to simple flows at low Reynolds number. A description of the Reynolds-average method is also included, highlighting its major deficiencies regarding complex flows.

A detailed description of the LES method and its history of development and use is given. The discretized LES equations are derived and the solution methodology is described. The method and code are first verified for the laminar case.by comparing the numerical results from this study to the numerical and experimental results of other researchers. The results for turbulent flow over a backward-facing step for various filter widths and grid sizes with the LES method are compared to an accurate solution (i.e., the Navier-Stokes equations without a turbulence model) generated with the same code used to generate the LES results. The LES approach using the finite element method is found to accurately predict the shedding vortices off the backward-facing step and the time-averaged turbulent statistics. 


\section{ACKNOWLEDGMENTS}

The author wishes to express her gratitude to Dr. Wolfgang Kollmann, Professor of Mechanical Engineering at U.C. Davis, to Dr. Phil Gresho, Staff Scientist at Lawrence Livermore National Laboratory (LLNL) and Adjunct Professor at U.C. Davis, and to Dr. John Leone, Jr., Staff Scientist at LLNL, for their guidance and valuable assistance in this research and in the preparation of this report, and to Dr. William Comfort, the author's supervisor at LLNL, for his support, and encouragement.

The financial support from LLNL's Mechanical Engineering Department and Environmental Protection Department and the use of computer and office facilities by the Atmospheric and Geophysical Sciences Division, computer time allocations from the National Supercomputing Center for Energy and the Environment at the University of Nevada, Las Vegas, and computer time allocations from the National Energy Research Supercomputer at LLNL are gratefully acknowledged. The author is also thankful to Katie Tyree and Elaine Conners of LLNL for their skillful typing of the manuscript. 


\section{TABLE OF CONTENTS}

\section{PAGE}

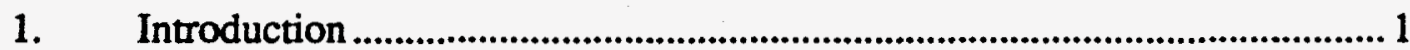

$1.1 \quad$ Background .............................................................................................. 1

1.2 Objective and Scope of Work ……..............................................2

1.3 General Flow Characteristics ............................................................ 6

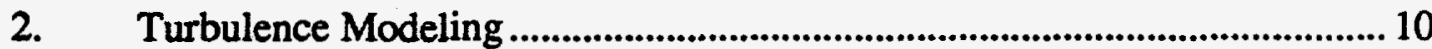

2.1 General Background and Theory ....................................................... 10

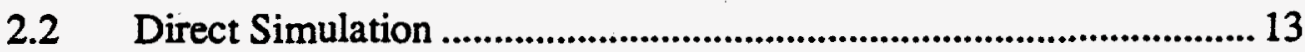

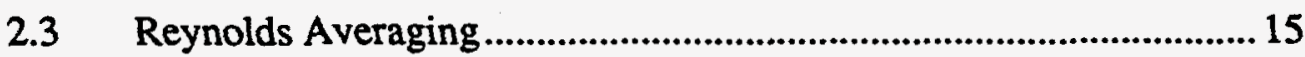

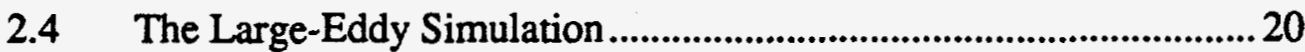

2.4.1 Method Description................................................................... 20

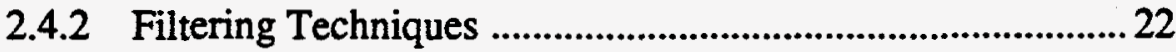

2.4.3 Formulation of the Continuum Equations ..................................31

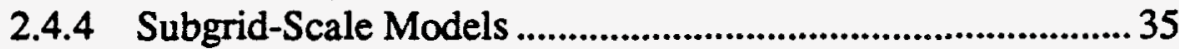

2.4.5 Wall Models..........................................................................44

2.4.6 Inflow and Outflow Conditions ............................................... 47

2.5 Two-Dimensional Simulations of Three-Dimensional

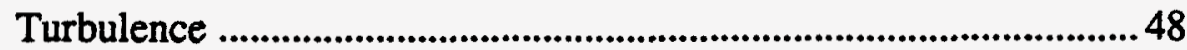

2.5.1 Three-Dimensional Theory ..........................................................49

2.5.2 Two-Dimensional Simulation of the Large-Scale

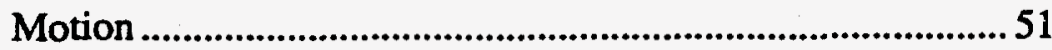

2.5.3 Two-Dimensional Simulation of the Small-Scale

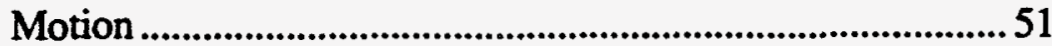

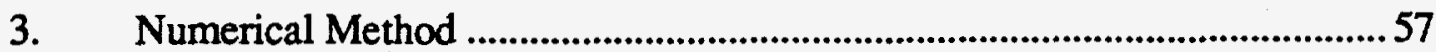

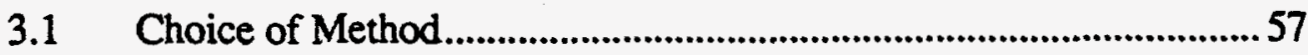


3.2 The Finite Element Method in Fluid Mechanics ............................... 59

3.2.1 Outline of Methodology ......................................................5

3.2.2 Choice of Test and Basis Functions and Order of

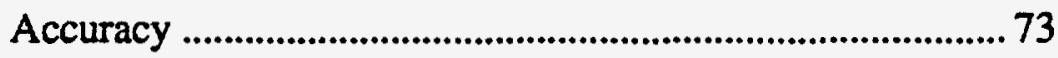

3.2.3. Spatial Integration of Terms in the Coefficient

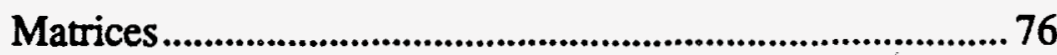

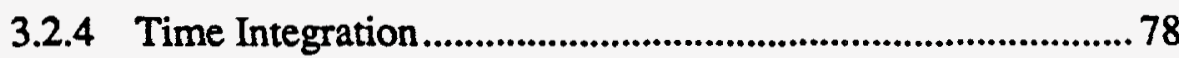

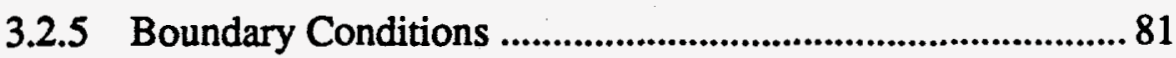

3.2.6 Hour-Glass Matrix and Balancing Tensor

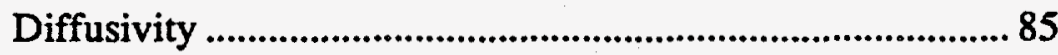

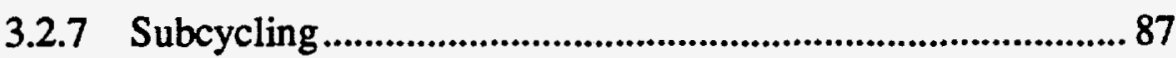

3.3 Finite Element Solution Approach.................................................... 89

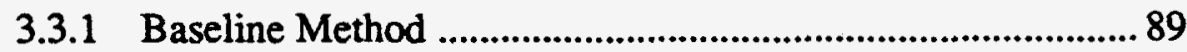

3.3.2 Explicit Calculation of the Advection Term ..........................89

4. Method and Code Validation ...................................................................99

4.1 The Laminar Case ........................................................................ 100

4.2 Comparison with Experimental Data or Reynolds-

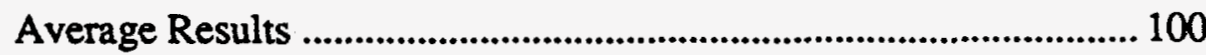

4.3 Comparison with Direct Simulation .................................................. 102

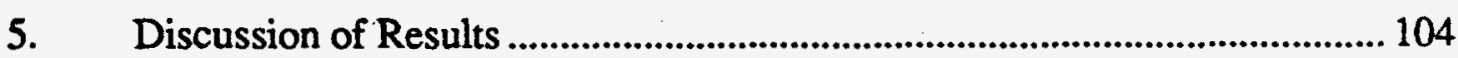

5.1 The Laminar Case ........................................................................ 104

5.2 An Accurate Solution for the 'Turbulent' Case ................................ 120

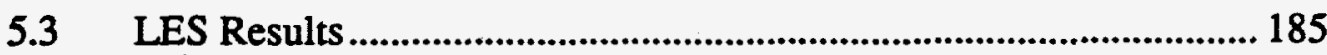

5.3.1 LES Without Explicit Filtering of the Advection

Term

5.3.2 LES With Explicit Filtering of the Advection

Term 


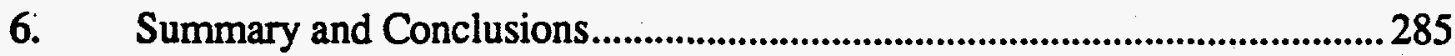

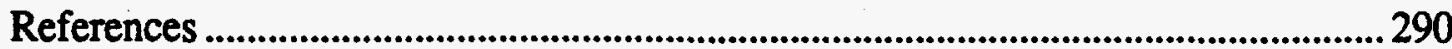

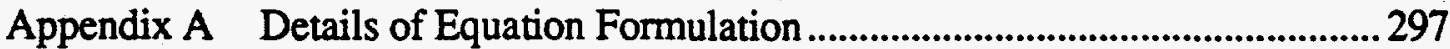

Appendix B Time Averaging of Instantaneous Quantities......................................... 301

Appendix C Methodology for Developing the Element Coefficient

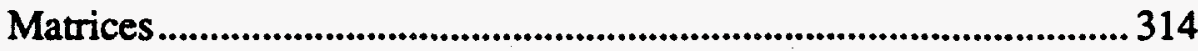

Appendix D Description of The Existing Software ..................................................328

Appendix E Details of How the Vorticity Model is Implemented in the

Existing Finite Element Code

Appendix F Estimating a Steady State from the Velocity Time History at a Point Location ……........................................................................ 338

Appendix G Spactial Filtering of the Direct Simulation ............................................340

Appendix $\mathrm{H}$ Power Spectrum Calculaitons Using the Fast Fourier

Transform 


\section{Introduction}

\subsection{Background}

The equations of motion describing turbulent flows (in both the low and high Reynolds-number regimes) are well established. However, present day computers cannot meet the enormous computational requirement for numerically solving the governing equations for common engineering flows in the high Reynolds number turbulent regime. The characteristics that make turbulent, high Reynolds number flows difficult to simulate is the extreme range of time and space scales of motion. Most current engineering calculations are performed using semi-empirical equations, developed in terms of the flow mean (average) properties. These turbulence 'models' (semi-empirical/analytical approximations) do not explicitly account for the eddy structures and thus, the temporal and spatial flow fluctuations are not resolved. In these averaging approaches, it is necessary to approximate all the turbulent structures using semi-empirical relations, and as a result, the turbulence models must be tailored for specific flow conditions and geometries with parameters obtained (usually) from physical experiments.

The motivation for this research is the development of a finite element turbulence modeling approach which will ultimately be used to predict the wind flow around buildings. Accurate turbulence models of building flow are needed to predict the dispersion of airborne pollutants. The building flow turbulence models used today are not capable of predicting the three-dimensional separating and reattaching flows without the manipulation of many empirical parameters. These empirical parameters must be set by experimental data and they may vary unpredictably with building geometry, building orientation, and upstream flow conditions. 
The turbulence modeling approach proposed in this study is a large-eddy simulation (LES) in which the large eddies are directly simulated and the small eddies are modeled. Some experts believe that the LES concept is the preferred approach for developing a more general application model (e.g., Ferziger 1990 and Wyngaard 1984). It provides a high degree of accuracy with a minimum of empiricism. The LES method should be capable of accurately predicting the flow for all building geometries and orientation and upstream flow conditions without the manipulation of parameters. The building flow calculations made with an LES model should show the time dependence of the mean flow (e.g., flapping of the building wake) along with local velocity fluctuations.

The backward-facing step (Figure 1.1) is a simple geometry with a separating and reattaching flow. A good deal of experimental and numerical results for the turbulent, backward-facing step problem are available, and thus, it is commonly used for testing and verifying a modeling approach. It will be used as the benchmark problem for this study. The backward-facing step problem is a simplification of the building flow problem. The geometry is two-dimensional (even though the turbulent flow field is still three-dimensional), only one corner separation needs to be considered (where building flows have multiple corner separations and stagnation flow on sides that face into the wind), and the upper part of the field is wall bounded (instead of free as for atmospheric flows).

\subsection{Objective and Scope of Work}

The purpose of this study is to develop a universal, accurate flow model of timedependent, incompressible, turbulent separated flow. The flow geometry to be considered is a two-dimensional backward-facing step. After the completion of this 
study, the model will be expanded to three-dimensions and used to calculate the flow around buildings to predict the dispersion of airborne pollutants. Thus, the scope of the research is the model development, validation, and testing for the case of a backwardfacing step, but the ultimate motivation is to model the flow around buildings.

The LES model is incorporated into an existing laminar, incompressible flow code that uses a finite element numerical approach. Use of LES to model the turbulent flow in conjunction with a finite element numerical scheme is uncommon. Most LES modeling has been performed with finite difference or spectral schemes. Thus, a large part of this research will require the development of the finite element approach. The objective of this research is to show that the finite element method (FEM) is a viable numerical approach for the solution of the LES equations and that there are clear advantages to using the FEM approach over the more commonly used finite difference and spectral method approaches.

To reduce computer system demand, run time, postprocessing, and the actual turnaround time in obtaining results and to reduce the complexity of evaluating the finite element solution approach and results, the calculations are performed in twodimensions (i.e., two-dimensional planar backward-facing step with out-of-plane symmetry assumed). Two-dimensional turbulence theory is used in the formulation of the continuous and discrete equations and in the formulation of the LES turbulent model so that all of the code development is consistent with the two-dimensional assumption. Two-dimensional flow has different turbulent spectral characteristics than threedimensional flow, however, both exhibit a continuous range of scales and transfer of energy between scales (see Section 2.5 for a detailed discussion). Thus, the twodimensional simulations should be adequate to achieve our goal of demonstrating the advantages in using the finite element method to solve the LES equations. 


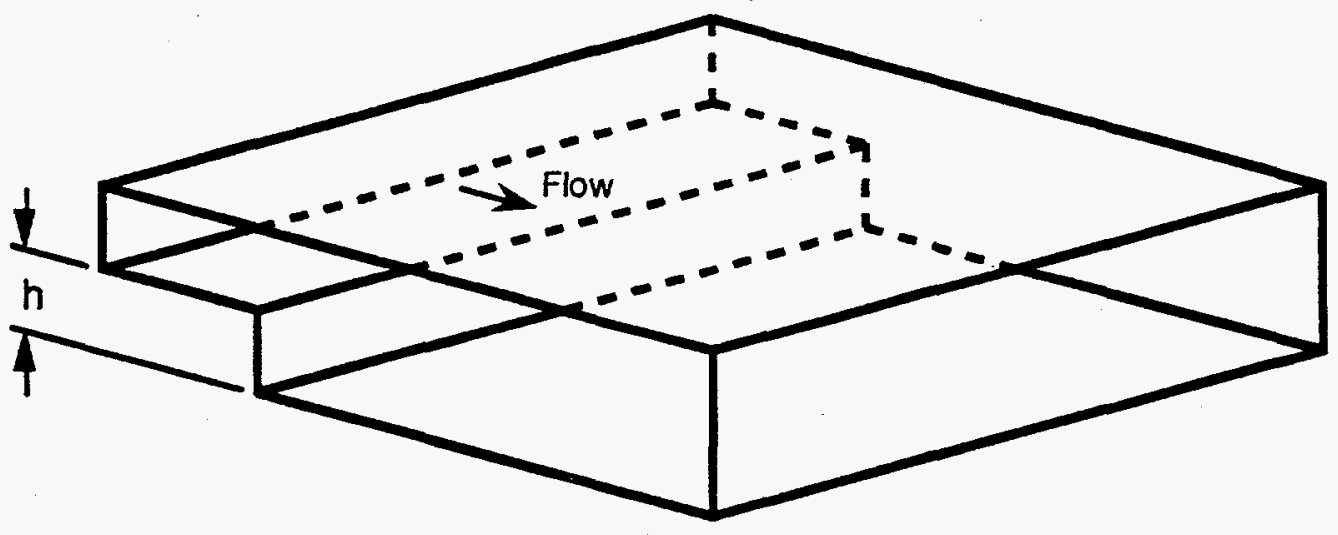

(a)

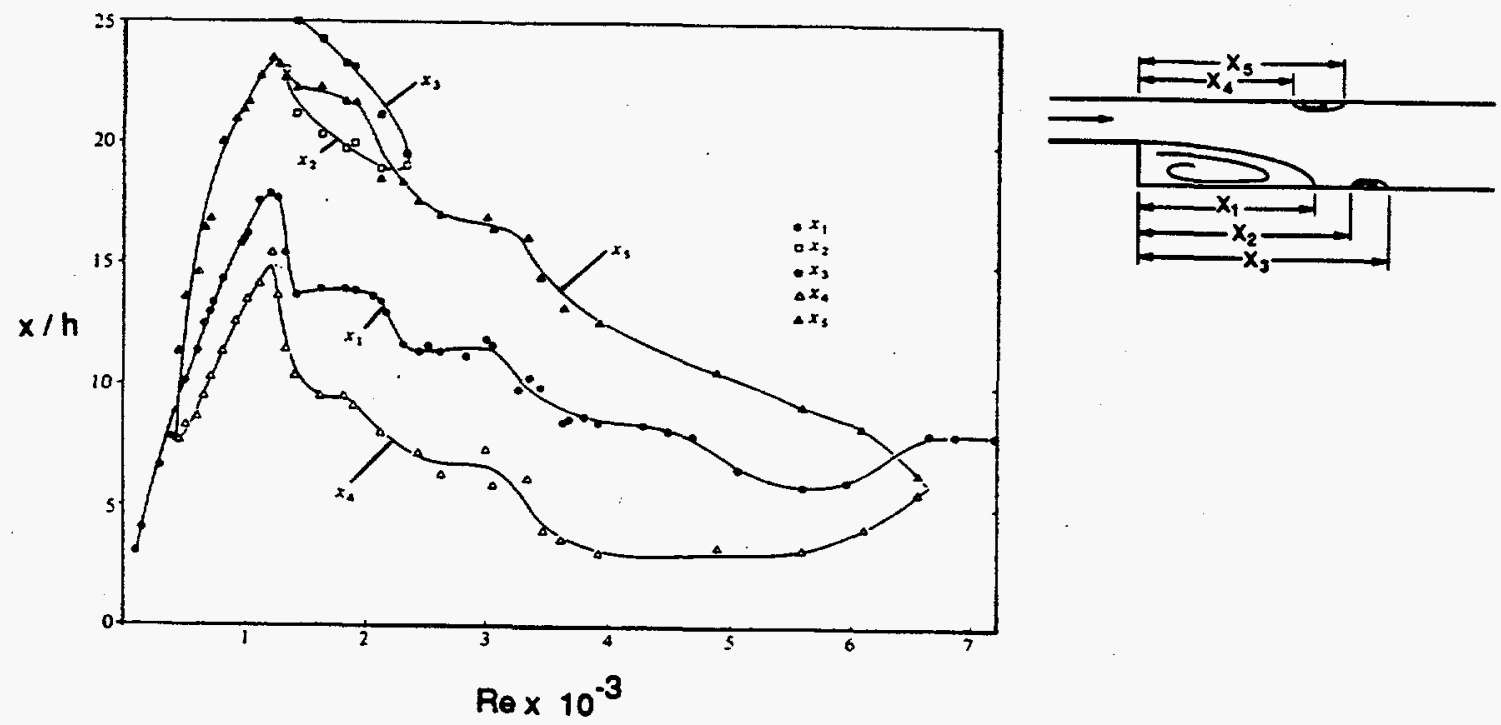

(b)

Figure 1.1 Backward-facing step (a) geometry and (b) experimental data of Armaly et al. (1983). 
The method and code are first verified for the laminar case by comparing the numerical results from this study to the numerical and experimental results of other researchers. The results for 'turbulent' flow (i.e., two-dimensional without input random pertubation) over a backward-facing step with the LES method are compared to an accurate solution or 'direct' simulation (i.e., the Navier-Stokes equation without a turbulence model on a very fine grid) generated with the same code used to generate the LES results.

The items to be investigated in this research are:

development of the discrete LES equations using the finite element method (described in Section 3) and implementation into an existing laminar finite element code,

- development and implementation of a scheme for explicitly solving the LES advection term with the finite element method (described in Section 3.3.2),

- $\quad$ application of current subgrid-scale models (described in Section 2.4.4) in $2 \mathrm{D}$ with the finite element method,

- investigation of wall models for separating flow for a backward-facing step and use of the LES subgrid-scale model to the wall (described in Section 2.4.5),

- generation of an accurate solution for comparison to the LES results, and 
- investigation of methods for validating the numerical approach and calculated code results for the backward-facing step.

The modeling scheme will be validated as described in Section 4.0.

\subsection{General Flow Characteristics}

The backward-facing step is a two-dimensional channel expansion in the streamwise direction (Figure 1.1a). The step expansion causes the flow to separate at the step corner and reattach at the base of the expanded channel, forming a recirculation zone. If the flow is laminar and steady, the recirculation zone traps the fluid inside of a dividing streamline. Armaly et al. (1983) found that in the laminar regime, the flow exhibits an additional recirculation zone at the top of the channel downstream of the step (for approximately $500<R e<1200$ in Figure 1.1b). During transition, an additional small recirculation zone forms on the channel bottom, which is not present in the fully turbulent regime.

The backward-facing step calculations in the laminar regime by Kim and Moin (1985) and Durst and Pereira (1988) predicted the recirculation zone at the top of the channel. The author is unaware of computational results in the transition regime that show the small recirculation zone at the bottom of the channel. The computational results for the reattachment points in the laminar regime by Kim and Moin (1985) and Durst and Pereira (1988) are in good agreement with each other and experiment for $\operatorname{Re}_{h}$ (Reynolds number based on step height and the average velocity upstream of the step) up to 600 . However, at higher $\mathrm{Re}_{h}$, the predictions and experimental data are not in good agreement.

In the turbulent regime, the average or mean data from different experimental 
investigations exhibits a 20-50\% scatter for the major reattachment length, $x_{1} / h$. Adams and Johnston (1988a) were able to explain the variation by performing a detailed parametric study and by obtaining their own carefully controlled experimental results for $\operatorname{Re}_{h}$ ranging from 8,000 to 40,000 and upstream boundary layer thickness, $\delta / h$, from 0 to 2. Their choice of dimensionless parameters were: i) $\operatorname{Re}_{h}$ to specify the role of viscous effects, ii) outlet to inlet expansion ratio, $E R$, as a geometric parameter, and iii) $\delta / h$ and a statement concerning the state of the upstream boundary layer (laminar or turbulent) to specify upstream conditions (Figure 1.2). Their results indicate that at high $\mathrm{Re}_{h}, x_{1} / h$ for a laminar upstream boundary layer is $30 \%$ smaller than for a turbulent upstream boundary layer. They conclude that this is due to increased entrainment of the free shear layer for the laminar case. For the turbulent upstream boundary layer, $x_{1} / h$ is approximately constant with $\mathrm{Re}_{h}$, except for a slight decrease with increasing $\delta / h$.

In most of the recent publications on the numerical results for turbulent flow over a backward-facing step, the numerical results are validated (i.e., checked for validity of numerical or modeling approach) and verified (i.e., checked for accuracy) by comparison to the experimental results of Kim et al. (1980) or those of Eaton and Johnston (1980). In the experiments of Kim et al. (1980), tufts and a mixture of oils were used to visualize the flow field and to determine the average or mean reattachment zone. They report that 'most of the recirculating zone contained unsteady flow' and that 'the tufts moved forward and backward continuously'. By taking still photographs of the tufts, they found that the instantaneous reattachment length was not a straight line across the span (i.e., the instantaneous flow field is three-dimensional). The oil-flow visualization gave a mean flow result which indicated a straight line in the spanwise direction. This mean distance to reattachment was found to be $x_{1} / h=7 \pm 1$ for $\operatorname{Re}=$ 132,000 (based on the upstream centerline mean velocity and downstream channel 
width) and a ratio of channel height to step height of 3:2 (i.e., expansion ratio of 1.5). There is no explanation given for the large uncertainty band of \pm 1 (or 29\%) placed on the reattachment position.

The published three-dimensional LES or direct simulation results for a backward facing step show a highly unsteady flow with vortices that shed from the step and top channel wall and travel downstream (Kondoh and Nagano 1989, Neto et al., 1991a, b, Kobayashi et al. 1992). These researchers validate their results by time averaging their transient solution to obtain a mean velocity field from which they determine the mean reattachment zone length. Kobayashi et al., (1992) state that their LES results had to be averaged over 10,000 time steps $(\Delta t=0.01)$ to obtain a steady time-averaged (i.e., stationary) solution. Since their time averaging procedure gives a reattachment length of $x_{1} / h=9$ for a Smagorinsky constant (i.e., the single parameter in the LES model) $c=0.15$ and $x_{1} / h=8$ for $c=0.10$, they conclude that their LES results overpredict the reattachment length. Neto et. al. (1991b) compare their time-averaged LES results $\left(x_{1} / h=8.1\right.$ for $\mathrm{Re}=38,000$ based on upstream bulk velocity and step height for an expansion ratio of 2.5 with $c=0.23$ ) to the experimental results of Eaton and Johnston $(1980)\left(x_{1} / h=7.8\right)$, but they make an additional comparison to what they call a 'direct' simulation using the same code as used to generate the LES result. However, they report on only the time averaged quantities. (Further discussion on the Smagorinsky constant is in Section 2.4.4.) 


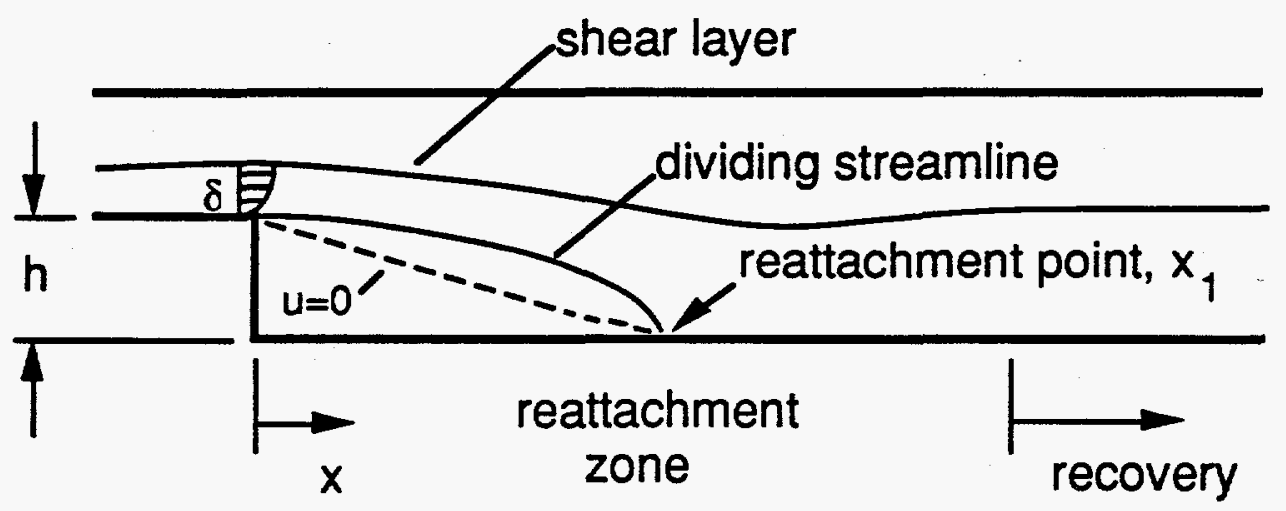

Figure 1.2 Backward-facing step showing important parameters as defined by Adams and Johnston (1988a). 


\section{Turbulence Modeling}

The following includes a brief discussion of turbulent flow characteristics and the difficulties in numerically modeling turbulent flow. The direct-simulation approach is described along with an explanation of why current results are limited to simple flows at low Reynolds number. A description of the Reynolds-average approach is also included, highlighting its major deficiencies regarding complex flows. A detailed description of the LES approach is then given along with a historical perspective. In the final subsection, the three-dimensional theory of turbulence is described and compared to a description of the two-dimensional theory. The derivation of the two- and threedimensional LES models for the small-scale motion are outlined.

\subsection{General Background and Theory}

Turbulent flow is stochastic (i.e., nondeterministic), three-dimensional, timedependent, and contains structures called eddies with a wide range of scales (eddy sizes). The eddy sizes can range from the size of the flow domain to many orders of magnitude smaller and the energy is a continuous function of the wave number (Figure 2.1). From laboratory experiments, it has been observed that the large eddies are responsible for most transport. They are inhomogeneous, anisotropic, and geometry dependent. The small eddies are responsible for energy dissipation. They are approximately homogeneous and isotropic, and may be only weekly geometry dependent. The eddies become more homogeneous and isotropic and less geometry dependent with higher wave number, and the range of wave numbers increases with Reynolds number. As discussed in Section 2.4.4, not all researchers agree on the turbulent scale size that defines the cutoff between large and small eddies. The term small eddies used here will imply all the eddies below a cutoff eddy size that display the characteristics of being homogeneous, isotropic, and not geometry dependent. 
The Navier-Stokes equations (conservation of mass and momentum equations) describe the motion of many fluids whether the flow is laminar or turbulent. However, because turbulent motion exhibits a much larger range of length and time scales than laminar flow, its computation requirements are much greater (see discussion in Section 2.2 on direct simulations). As a result of these computational requirements, direct simulations (wherein all scales are resolved) for high Reynolds number complex turbulent flows are not possible in the foreseeable future. Most complex turbulent flow analyses are done by laboratory experiments.

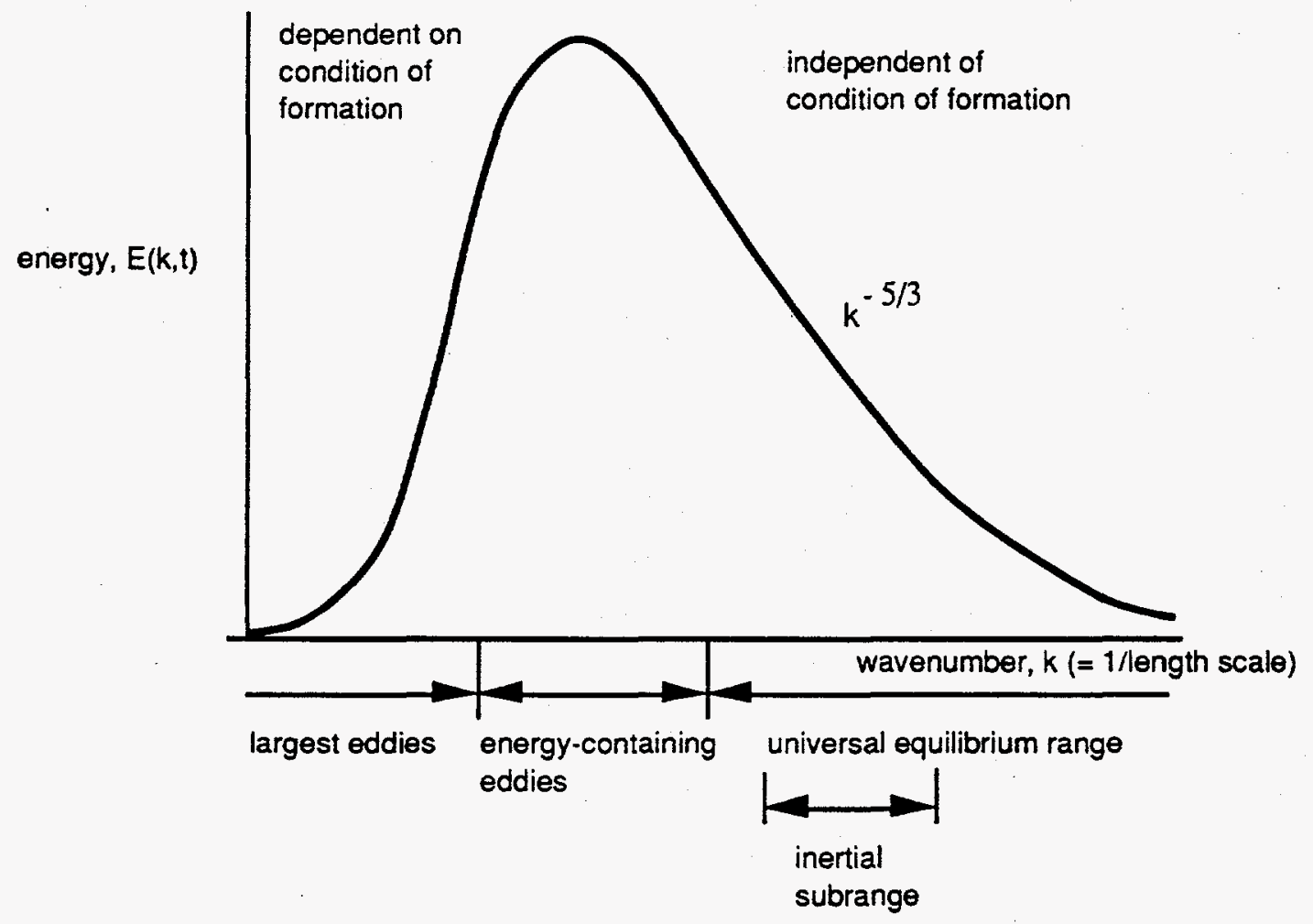

Figure 2.1 Plot of turbulent energy spectrum showing a continuous range of eddy sizes. 
In practice, computations usually take the traditional approaches of $O$. Reynolds where the Navier-Stokes equations are averaged over an ensemble of flows or over an interval of time or space, called one-point-average methods (see Section 2.3 for discussion on Reynolds averaging). The information on the turbulent fluctuations is necessarily discarded by averaging and as a result, the equations no longer form a closed system. The information on all the eddy scales has been removed and the effects of the wide range of scales must be modeled by using semi-empirical relations for the nonclosed terms. It is common practice to use one-point-average methods with first- or second-order closure models. The order of the closure model depends on how many differential equations are used for the nonclosed terms.

As mentioned above, large eddies are geometry or flow field dependent and the small eddies are more universal. The LES approach is designed to take advantage of this turbulent flow characteristic (see Section 2.4). In an LES, the large eddies are resolved and the small ones are modeled. The models for the small eddies are called subgrid-scale (SGS) models. By restricting the averaging to small spatial regions, only the information on the small-scale fluctuations is removed. Thus, instantaneous results along with averaged results can be generated. (The averaged results are computed from the LES instantaneous results.)

Over the past 20 years, the LES approach has been successfully applied to simple engineering and meteorological flows. Most experts agree that both Reynoldsaverage and LES approaches will be used in the future for practical applications, with LES having the best predictive capability. 


\subsection{Direct Simulation}

Direct simulations of turbulent flow involve the numerical solution of the threedimensional, time-dependent Navier-Stokes equations. The computational domain must be large enough to capture the large-scale motion, and the discretization must be fine enough such that even the smallest scales are resolved. Thus, in a direct simulation, the system of equations is closed and a turbulence model is not needed.

The results from a direct simulation can be used to study the turbulent physics of particular flow fields or they can be used to develop and assess turbulent models for a Reynolds-average or an LES approach. The information obtained from a direct simulation far exceeds that from experimental data in both accuracy and quantity. The instantaneous velocity (mean plus fluctuation) is available at every grid point in the computational domain (and everywhere in the domain when using FEM).

Clark et al. (1979) developed an approach they called 'a priori testing' where the data from a direct simulation is used to evaluate the LES SGS models. The approach is to filter the direct simulation velocity field and compare the resulting SGS stresses to those calculated with the LES SGS models. The measure of comparison is usually expressed as a statistical correlation coefficient between the modeled and direct simulation SGS stresses. Piomelli et al. (1988) and Bardina et al. (1983) usually found correlation coefficients between 0.5 and 0.2 depending on filter and SGS model used. In spite of an apparently poorly performing SGS model, the LES still produced statistical predictions (e.g., total Reynolds stress equal to resolved plus SGS) in good agreement with the direct simulation data. Piomelli et al. (1988) explain that the LES is insensitive to the choice of SGS model because the resolved eddies adjust themselves dynamically to the SGS model being used. The total Reynolds stress is not as sensitive to the SGS model. 
The only, but severe, difficulty in applying a direct-simulation approach is with its computational requirements. The direct-simulation approach requires accurate numerical methods and detailed resolution in both space and time. Present directsimulation results are limited to low Reynolds number turbulent flows because of computer storage and run time requirements. Since both the largest and smallest scales in the flow must be resolved (Reynolds 1989), there is a required overall domain size and there are domain discretization requirements. To resolve the smallest scales, the mesh size must be less than the viscous dissipation length scale which is on the order of the Kolmogorov scale $\eta=\left(v^{3} / \varepsilon\right)^{1 / 4}$ (where $v$ is the kinematic viscosity and $\varepsilon$ is the dissipation rate of turbulent kinetic energy per unit mass). The dissipation rate $\varepsilon \approx q^{3} / L$ where the velocity scale $q$ is chosen to be the root-mean-square amplitude of the velocity fluctuations $\left(q=\left[\left\langle u_{1}^{-2}\right\rangle+\left\langle u_{2}^{2}\right\rangle+\left\langle u_{3}^{-2}\right\rangle\right]^{1 / 2}\right.$ where $u_{\alpha}^{*}$ is the $\alpha$-component of the fluctuating velocity and the brackets, $\langle>$, signify a time or ensemble average) and $L$ is the length scale of the large eddies (see Section 2.3 on Tennekes and Lumley 1972). Thus, the required number of mesh points to capture the large and small scales of motion can be estimated as

$$
N=\left(\frac{L}{\eta}\right)^{3}=\left(\frac{q L}{v}\right)^{9 / 4} \equiv \operatorname{Re}_{T}^{9 / 4}
$$

where $\mathrm{Re}_{T}$ is the Reynolds number for the large-scale turbulence. For example, using 128 mesh points in each direction will limit the direct simulation to $\operatorname{Re}_{T} \approx 645$ (a more careful estimate by Reynolds (1989) gives $\operatorname{Re}_{T}=500$ for a $128^{3}$ mesh). It is clear that with our present computer capabilities, direct simulations can only be performed for low Reynolds number flows of simple geometry. 


\subsection{Reynolds Averaging}

Most numerical solutions of complex turbulent flows (i.e., typical engineering and atmospheric flows) involve averaged equations. It is assumed that the velocity field can be decomposed into a mean velocity $\left\langle u_{i}\right\rangle$ (time, space, or ensemble averaged) and a fluctuating velocity $u_{i}^{\prime \prime}$ such that the instantaneous velocity $u_{i}$ is defined as

$$
u_{i}=<u_{i}>+u_{i}^{*}
$$

The $<>$ signifies the Reynolds-averaging operator. Hinze (1975), for example, describes the three methods of averaging, which are time, space, and ensemble averaging. He also outlines the flow conditions that must be satisfied for each averaging method. In each case, the averaging scheme is defined such that $\left\langle u_{i}^{*}\right\rangle=0$ (i.e., the mean or average of the fluctuating velocity is zero). It is also true by definition that $\left\langle\left\langle u_{i}\right\rangle\right\rangle=\left\langle u_{i}\right\rangle$.

Substituting this relation into the incompressible Navier-Stokes equations (see Appendix $\mathrm{A}$ for equations) for a constant viscosity and averaging the equation, we arrive at the so-called Reynolds-averaged equations

$$
\begin{gathered}
\frac{\left.\partial<u_{j}\right\rangle}{\partial x_{j}}=0 \\
\frac{\left.\partial<u_{i}\right\rangle}{\partial t}+<u_{j}>\frac{\partial<u_{i}>}{\partial x_{j}}=-\frac{1}{\rho} \frac{\partial<P>}{\partial x_{i}}+v \frac{\partial^{2}<u_{i}>}{\partial x_{j}^{2}}-\frac{\partial}{\partial x_{j}}\left\langle u_{i} u_{j}^{*}\right\rangle .
\end{gathered}
$$

Averaging of the nonlinear momentum equation for the decomposed mean plus fluctuating velocity field, as above, leads to the so-called closure problem. Unknown terms called Reynolds flux or stress terms, $\left\langle\overline{u_{i}} u_{j}^{*}\right\rangle$, are present in the resulting 
equation. We could derive a transport equation for the unknown stress terms (a secondorder model), but it would in turn include third-order nonclosed terms involving $\left\langle\dot{u_{i}} u_{j} u_{k}\right\rangle$. It is more common, in engineering practice, to approximate the nonclosed terms in the momentum equation with a semi-empirical algebraic relation which involves the closed variable mean velocity field, $\left\langle u_{i}\right\rangle$. These algebraic (zero-equation) models are called eddy-viscosity models and are of the form

$$
\left.<u_{i}^{\prime \prime} u_{j}^{\prime \prime}\right\rangle=-v_{T}\left(\frac{\partial<u_{i}>}{\partial x_{j}}+\frac{\partial<u_{j}>}{\partial x_{i}}\right)+\frac{2}{3} k \delta_{i j} \text {, where } v_{T} \equiv C L q
$$

and the turbulent kinetic energy is

$$
k=\frac{<u_{i}^{\prime \prime} u_{i}^{n}>}{2}
$$

The coefficient $v_{T}$ is a so-called eddy viscosity which is a function of an eddy length scale, $L$, eddy velocity scale, $q$, and a constant $C$ which is a model parameter. Additional transport equations may also be included to define these length and velocity scales. Most commonly used in engineering is the $k-\varepsilon$ model (a two-equation model) with model parameters as defined by Jones \& Launder (1972) where

$$
v_{T}=C_{\mu} \frac{k^{2}}{\varepsilon}
$$

where $\varepsilon$ is the dissipation rate of turbulent kinetic energy, and $C_{\mu}$ is a model parameter usually set to a value of 0.09 (where they chose $L=k^{3 / 2} / \varepsilon, q=k^{1 / 2}$ ). The transport equations for $k$ and $\varepsilon$ include 4 more parameters which are determined from experimental data. 
Unfortunately, commonly used Reynolds-average approaches, with the above referenced (linear; see below) $k-\varepsilon$ model of turbulence, inaccurately predict the reattachment point for the backward-facing step problem. For example, the experimental results of Kim et al. (1980) for $\mathrm{Re}=132,000$ (based on the upstream channel velocity and downstream channel width) and an expansion ratio of 3:2 give a reattachment length $x_{1} / h=7.0 \pm 1$ (where $x_{1}$ is the distance from the step to the reattachment point and $h$ is the step height). The computed reattachment length by Speziale and Ngo (1988) with the linear $k-\varepsilon$ model is $x_{1} / h=5.5$. However, they predict $x_{1} / h=6.4$ when using their nonlinear $k-\varepsilon$ model, which is within the experimental uncertainty band. This nonlinear $k-\varepsilon$ model introduces two new empirical constants, which along with the other 5 constants, must be determined by experimental data and may vary (unpredictably) with geometry and flow conditions. Thus, one should be cautious when applying the model outside the specific range of flows for which it has been calibrated.

Second-order closure models (or sometimes referred to as Reynolds-stress models) include the transport equations for the unknown stress terms, $\left\langle u_{i}^{\prime} u_{j}^{*}\right\rangle$. In general, the six partial differential equations for the Reynolds-stress components and one for the dissipation must be solved. As already mentioned, the equations for the Reynolds stress include higher-order moment terms that are modeled with semiempirical relations. By including the transport equations for the various Reynolds stresses, various velocity scales are represented and their transport can be accounted for (Rodi 1980). These models have shown some improvement over $k-\varepsilon$ models, but they are more complicated and much more costly to run. They also include more model parameters than a two-equation model which must be determined from experiments and that may vary unpredictably with changing flow conditions. 
The major deficiency of the Reynolds average approach is that all of the turbulence (all the eddy structures) must be modeled. As a result, the parameters must be tailored to specific flows and may vary as the flow field varies. In general, the assumption that the higher-order moments (e.g., $\left\langle u_{i} u_{j}\right\rangle$ ) are a unique function of lower-order moments (e.g., $\left\langle u_{i}\right\rangle$ ) which is the basis for the modeling approach is not supported by experiment. As stated by Ferziger (1990), "It is probably wise to regard models as engineering approximations rather than the consequences of a predictive theory".

In terms of building flow problems, the published literature for the Reynoldsaverage approach gives an average solution of the spatial- and time-varying problem. It is not clear from the theory whether the Reynolds-averaged solution should predict the flapping wake behind a building and some of the local spatial and temporal velocity fluctuations. To clarify this statement, the following is a brief discussion of the theory and review of recent calculations of flow past two-dimensional and three-dimensional square cornered objects using the Reynolds-average approach.

As explained in Appendix B, if the flow field is stationary (i.e., statistically steady), averaging with respect to time can be used for the averaging operator $<>$ and thus, $\partial<u>/ \partial t$ is zero (or negligibly small). If in the equation formulation for a Reynolds-average approach, we set the time-derivative term to zero and solve the steady state equations, we are assuming that the flow is stationary and we will generate a steady mean flow solution. If instead the time-derivative is included in the formulation of the Reynolds-average equations, we are keeping open the possibility of time-vary statistics (i.e., $\left\langle u_{i}\right\rangle,\left\langle\dot{u_{i}} u_{j}^{\prime}\right\rangle, k$, etc.), and the implied averaging scheme is then an ensemble average (see Cardot and Pironneau 1988). Even though, in theory, including the time-derivative in the equation formulation should allow the prediction of 
time- and spatially-varying flow fields (like a building wake), as discussed below, the computational results of most researchers show a steady solution.

Considering first the backward-facing step, the author is unaware of any published numerical results for the backward-facing step using a Reynolds-average approach that predicts a time-varying flow (i.e., vortices shedding from the step and top wall). For example, the calculations of Speziale and Ngo (1988) with a $k-\varepsilon$ model and the calculations of Lasher and Taulbee (1989) with a Reynolds-stress model both predict a steady flow field, even though the time-dependent equations are solved.

For the two-dimensional calculations of flow past a square-cylinder, some researchers have predicted the vortex shedding off the cylinder corners using a Reynolds average approach. For example, Franke and Rodi (1991), predict the vortexshedding flow past a square cylinder with both a $k-\varepsilon$ model and a Reynolds-stress model. They found that if the $k-\varepsilon$ model is combined with wall functions at the solid boundaries of the cylinder, too much damping is introduced so that no vortex shedding is predicted. With a two-layer approach (i.e., use of a one-equation model at the wall), the $k$ - $\varepsilon$ model does predict vortex shedding. The Reynolds-stress model predicts vortex shedding with both wall functions or the two-layer approach.

For the three-dimensional calculations of flow past a surface mounted cube, the author is unaware of a time-varying solution using the Reynolds-average approach. The published literature for the cube shows a steady solution even though the timedependent equations are solved. For example, Murakami and Mochida (1988) predict a steady solution for the flow past a surface mounted cube using a $k$ - $\varepsilon$ model with the solution of the time-dependent equations. They report steady solutions when using either a logarithmic-law or a power-law wall function. 
Some obvious questions that arise from the above review are:

- Is turbulent flow over a backward-facing step truly stationary?

- Does the introduction of wall models for the three-dimensional surface mounted cube solution and the two-dimensional backward-facing step introduce too much damping so that vortex shedding is not predicted?

The answers to the above questions are beyond the scope of this research. However, it is the author's opinion that the limitations of the Reynolds-average approach in predicting flow around a building need to be known before the full advantages of the LES approach over the Reynolds-average approach can be stated.

The 'assumption' that the flow over a backward-facing step is stationary seems to be universally accepted as a 'truth' by all of the above cited researchers. It is this author's opinion that if you average any transient flow field over a long enough time period, you can usually arrive at what appears to be a statistically steady result. However, doesn't this type of averaging give you a nonphysical steady solution of a transient problem? Again, the answer (e.g., probably ensemble averaging of an accurate solution) is beyond the scope of this research.

\subsection{The Large-Eddy Simulation}

\subsubsection{Method Description}

In an LES, the large-scale motion is calculated explicitly and the small-scale 
motion is modeled. The large-scale motion is defined by applying an averaging operator to the equations to filter out the small-scale motions. The LES averaging is different than that used for Reynolds averaging. The LES averaging is done spatially over a small region of the flow. The size of the region is on the order of magnitude of the small turbulent scales (usually) in the inertial subrange. Whereas for Reynolds averaging the space scale is on the order of magnitude of the flow field (i.e., the largest scale) and the time scale is on the order of magnitude of the ratio between the size of the flow field and mean-flow velocity. The LES averaging of the instantaneous velocity for all the turbulent motions, $u_{i}$, filters out the small-scale motions, $u_{i}{ }^{\prime}$, and results in the instantaneous velocity for only the large-scale motions, $\bar{u}_{i}$. Thus, the LES average can be regarded as a short-range filter, whereas the Reynolds average is a long-range filter. The large-scale motions which are solved for explicitly are called the resolved field and the small-scale motions which are modeled are called the subgrid-scale motion or residual field. The term subgrid scale comes from the grid-based method approach where the modeled small-scale motion is smaller than the grid size. In this report, as in most LES publications, the term subgrid scale (SGS) will be used even when the modeled small-scale size is not the grid size.

Deardorff (1970) credits O. Reynolds (1895) as the individual who first came up with the idea of applying an averaging operator to the governing equation to filter out the SGS motions. The approach was successfully employed by several meteorologists (Smagorinsky et al. 1965 and Leith 1965) to atmospheric models that were somewhat two dimensional (i.e., vertical accelerations and velocities were small compared to those in the horizontal direction). The research of Smagorinsky et al. (1965) and Lilly (1967) was used to define the first SGS models and is still the basis for current models. Deardorff $(1970 a, b)$ applied the LES approach to the three-dimensional flow in a plane channel and to a planetary boundary layer. Schumann (1975) also used the LES 
approach to calculate flow in plane channels and annuli. One difference in these two researchers' approaches was that Schumann's averaging was defined over a cell surface so as to model the effects of anisotropic grids, where Deardorff's averaging was over a cell volume. More details on Deardorff's and Schumann's work, and the work of other researchers, is given in the following sections.

\subsubsection{Filtering Techniques}

An averaging or filtering process separates the resolvable from the subgrid scales. The following includes definitions of the averaging and filtering approaches, descriptions of various filters, and a discussion of applications.

The purpose of filtering or averaging (as defined below) is the same; however, filtering approaches have only been applied to infinite domains (i.e., without walls), whereas averaging has been used for wall-bounded domains. Also, filtering is the method used by researchers using a spectral method of solution where the filter width (i.e., the size of the smallest resolved eddies) is chosen. The averaging approach is that used by the researchers using a finite difference method where the filter (or average) width is the same as the grid size (i.e., the so-called subgrid scale). However, those using the finite difference method do not explicitly apply an averaging operator; rather, there is an implied operator resulting from the differencing scheme. For instance, Rogallo and Moin (1984) show how the use of a second-order central-difference formula on a variable $v(x)$ gives exactly the derivative of an average of the variable:

$$
\frac{v(x+\Delta)-v(x-\Delta)}{2 \Delta}=\frac{d}{d x}\left[\frac{1}{2 \Delta} \int_{x-\Delta}^{x+\Delta} v(\xi) d \xi\right] .
$$


The difference formula filters out scales less than the mesh size $\Delta$. Thus, the use of finite difference operators on the terms in the Navier-Stokes equations introduces an averaged variable for each term.

If instead, the Navier-Stokes equations are explicitly filtered, the choice of filter width $\Delta_{f}$ can be chosen independently of the grid size $\Delta$. The filter width then sets the scale of the smallest resolved eddy. Some researchers claim that $\Delta_{f}$ should be considerably larger than the grid size $\Delta$ to assure independence of the LES results from the numerical method (Rogallo and Moin 1984 and Piomelli et al. 1987).

As will be shown in Section 3, explicit averaging in a finite element scheme allows the choice of a filter (or average) width independent of the grid size. However, as discussed briefly below, there has been almost no attempt by LES researchers to develop filtering schemes for wall-bounded domains, and thus, cell volume-averaging will be used in this study.

Cell Volume-Averaging (Deardorff 1970, following the original definition by O. Reynolds 1895)

With cell volume-averaging, the resolved field is defined by

$$
\bar{f}(x, y, z, t)=\frac{1}{\Delta x_{f} \Delta y_{f} \Delta z_{f}} \int_{x-\frac{1}{2} \Delta x_{f}}^{x+\frac{1}{2} \Delta x_{f}} \int_{y-\frac{1}{2} \Delta y_{f}}^{y+\frac{1}{2} \Delta y_{f}} \int_{z-\frac{1}{2} \Delta z_{f}}^{z+\frac{1}{2} \Delta z_{f}} f(\xi, \eta, \zeta, t) d \zeta d \eta d \xi
$$

where $\Delta x_{f}, \Delta y_{f}$, and $\Delta z_{f}$ defines the size of the volume averaging over. Using Leibnitz' rule, it can be shown that the averaging operator commutes with differentiation (i.e., $\overline{\partial f / \partial x_{\beta}}=\partial \bar{f} / \partial x_{\beta}$ ) for a constant filter width (i.e., $\Delta x_{f}, \Delta y_{f}$, and $\Delta z_{f}$ all constant). (See 
Appendix $\mathrm{C}$ for details.)

Filtering (Leonard 1974, Kwak et al. 1975)

With filtering, the resolved field is defined by

$$
\bar{f}(\underline{x}, t)=\int_{-\infty}^{\infty} G\left(\underline{x}-\underline{x}^{\prime}, t\right) f\left(\underline{x}^{\prime}, t\right) d \underline{x}^{\prime}
$$

where $G$ is a selected filter function, such that when $f$ is constant, $\bar{f}=f$, which requires that $G$ be normalized such that

$$
\int_{-\infty}^{\infty} G(\underline{x}, t) d \underline{x}=1
$$

If the filter function, $G$, is piecewise continuously differentiable and $G(r) \rightarrow 0$ as $r \rightarrow \infty$, then using integration by parts ${ }^{1}$

${ }^{1}$ If we let $\underline{x}-\underline{x}=\underline{s}$, then

$$
\begin{aligned}
& \frac{\partial G}{\partial \underline{x}^{\prime}}(\underline{s}, t)=\frac{\partial G}{\partial \underline{s}} \frac{\partial \underline{s}}{\partial \underline{x}^{\prime}}=\frac{-\partial}{\partial \underline{s}} G(\underline{s}, t) \\
& \frac{\partial G}{\partial \underline{x}}(\underline{s}, t)=\frac{\partial G}{\partial \underline{s}} \frac{\partial \underline{s}}{\partial \underline{x}}=\frac{\partial}{\partial \underline{s}} G(\underline{s}, t)
\end{aligned}
$$

Thus, $\frac{\partial G}{\partial \underline{x}^{\prime}}=\frac{-\partial G}{\partial \underline{x}}$. Also, $f\left(\underline{x}^{\prime}, t\right)$ is not a function of $\underline{x}$. 


$$
\begin{aligned}
\frac{\overline{\partial f}}{\partial \underline{x}}=\int_{-\infty}^{-} G\left(\underline{x}-\underline{x}^{\prime}, t\right) \frac{\partial f\left(\underline{x}^{\prime}, t\right)}{\partial \underline{x}^{\prime}} d \underline{x}^{\prime} \\
= \\
=\left.G\left(\underline{x}-\underline{x}^{\prime}, t\right) f\left(\underline{x}^{\prime}, t\right)\right|_{\underline{x}^{\prime}=-\infty} ^{\infty}-\int_{-\infty}^{\infty} f\left(\underline{x}^{\prime}, t\right) \frac{\partial}{\partial \underline{x}^{\prime}} G\left(\underline{x}-\underline{x}^{\prime}, t\right) d \underline{x}^{\prime} \\
=\frac{\partial}{\partial \underline{x}} \int_{-\infty}^{\infty} G\left(\underline{x}-\underline{x}^{\prime}, t\right) f\left(\underline{x}^{\prime}, t\right) d \underline{x}^{\prime} \\
=\frac{\partial \bar{f}}{\partial \underline{x}} .
\end{aligned}
$$

Thus, the filter commutes with differentiation. Similarly, if $G$ is not a function of time (and as shown below, it usually isn't), then

$$
\frac{\overline{\partial f}}{\partial t}=\frac{\partial \bar{f}}{\partial t}
$$

Then, using either cell volume-averaging or filter functions, the variable $f$ can be decomposed into a resolved field, $\bar{f}$, and an SGS field, $f^{\prime}$;

$$
f=\bar{f}+f^{\prime}
$$

However, in general $\overline{\bar{f}} \neq \bar{f}$ unless $f=$ constant, and $\overline{f g} \neq \bar{f} \bar{g}$. It should also be noted that in general, $\overline{f^{\prime}} \neq 0$. For the case of cell volume-averaging or a top-hat filter (described below), $\overline{\bar{f}}=\bar{f}$ for a constant $\Delta_{f}$ (i.e., each bar operator is for the same $\Delta_{f}$ ) such that $\bar{f}$ is constant over $\Delta_{f}$. It then follows that $\bar{f}^{\prime}=0.1$ However, if $\bar{f}$ varies over $\Delta_{f}$, then $\bar{f} \neq \bar{f}$ and also $\overline{f^{\prime}} \neq 0$. These two situations are shown graphically in Figure 2.2 (Leonard 1974). In Figure 2.2a, $\bar{f}$ is constant over $\Delta_{f}$ such that $\overline{\bar{f}}=\bar{f}$ and $\overline{f^{\prime}}=0$. In Figure 2.2b, $\bar{f}$ varies over $\Delta_{f}$ such that $\overline{\bar{f}} \neq \bar{f}$ and $\overline{\bar{f}}^{\prime} \neq 0$. Understanding

$1 \overline{f^{\prime}}=\overline{f-\bar{f}}=\bar{f}-\bar{f}=0$. 
these two situations is important in the formulation of the LES advection term. As explained in Section 2.4.4, for the situation shown in Figure 2.2a, the bar operator is equivalent to a Reynolds-average operator applied over a small spatial domain.

\section{Various Filters}

- Top-Hat or Box Filter (equivalent to cell volume-averaging for $\Delta_{f}=\Delta$ )

$$
G\left(\underline{x}-\underline{x}^{\prime}\right)= \begin{cases}1 / \Delta_{f}^{3} & \text { for }\left|\underline{x}-\underline{x}^{\prime}\right|<\frac{\Delta_{f}}{2} \\ 0 & \text { otherwise }\end{cases}
$$

where $\Delta_{f}$ is the filter size (i.e., all scales smaller than $\Delta_{f}$ are modeled) or for a finite difference method $\Delta_{f}$ is the grid size $\Delta$ (e.g., $\Delta^{3}=\Delta x \Delta y \Delta z$ ). For finite difference methods, the top-hat filter (or cell volume-averaging) is actually implicit; i.e., as discussed above, applying the finite difference operators results in an averaged variable.

- Gaussian Filter

$$
G\left(\underline{x}-\underline{x}^{\prime}\right)=\left(\frac{\gamma}{\pi \Delta_{f}^{2}}\right)^{\frac{3}{2}} \exp \left(\frac{-\gamma\left(\underline{x}-\underline{x}^{\prime}\right)^{2}}{\Delta_{f}^{2}}\right)
$$

Most researchers choose $\gamma=6$ so that if the Leonard term (the advection term, $\overline{\bar{u}_{\alpha} \bar{u}_{\beta}}$, discussed in Section 2.4.3) is modeled with a Taylor series expansion, the expansion with the Gaussian filter is the same as that for a top-hat filter (Kwak et al. 1975, p. 11). Rogallo and Moin (1984) state that the Gaussian filter is usually the filter choice for 'homogeneous' (i.e., not normal to walls) dimensions because it provides a smooth transition between resolved and subgrid scales and is positive definite in both physical and wave space. 


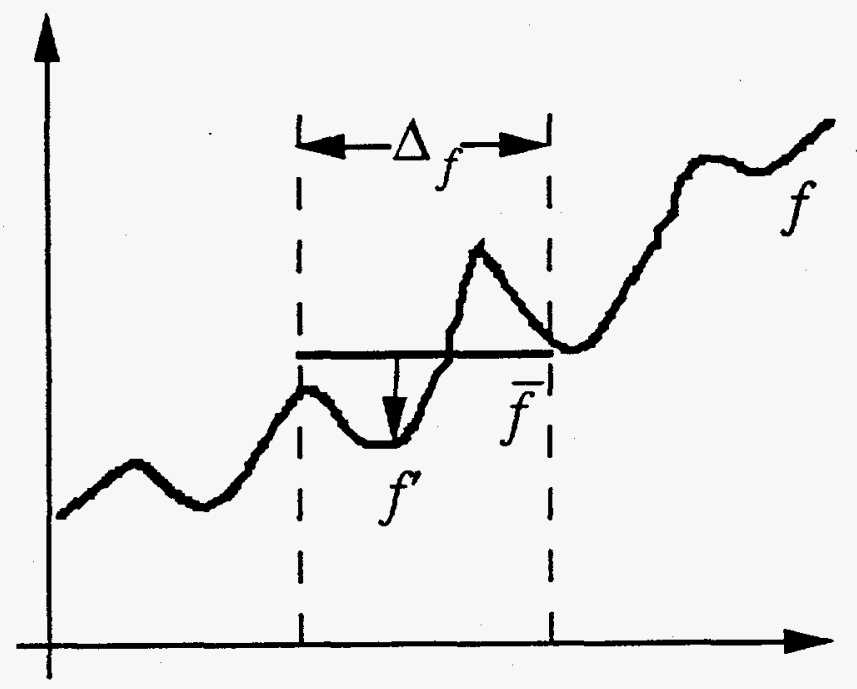

(a)

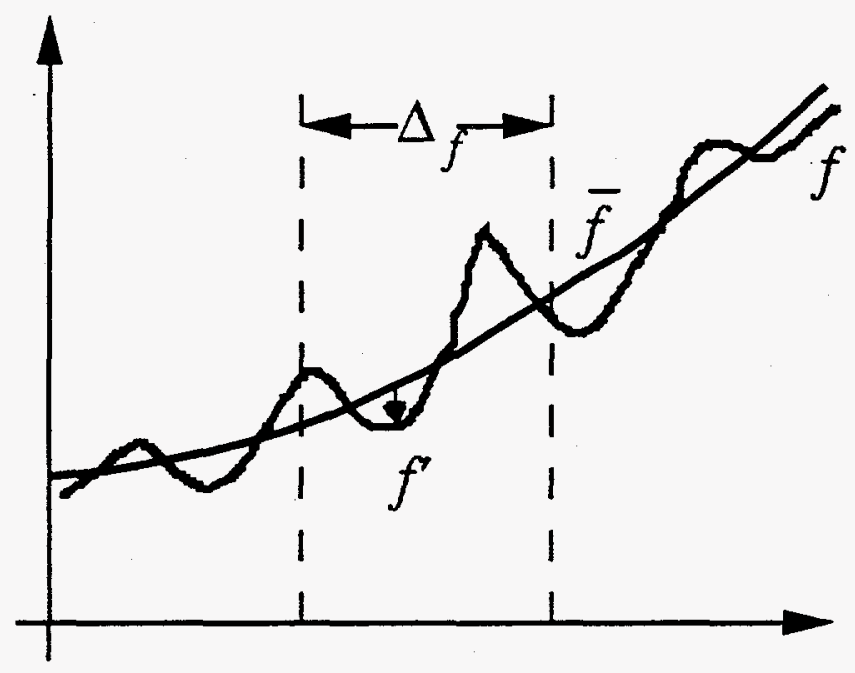

(b)

Figure 2.2 Two possible definitions of the bar operator for cell volume-averaging were a) $\bar{f}$ is constant over $\Delta_{f}$ and b) $\bar{f}$ varies over $\Delta_{f}$. 
- Sharp-Cutoff Filter

$$
G\left(\underline{x}-\underline{x}^{\prime}\right)=\prod_{i=1}^{3} \frac{\sin \left(\frac{\pi\left(x_{i}-x_{i}^{\prime}\right)}{\Delta_{f}}\right)}{\pi\left(x_{i}-x_{i}^{\prime}\right)}
$$

For small scales $\left(x^{\prime} \rightarrow \underline{x}\right)$ the sharp-cutoff filter approximates a top-hat filter $(G(\underline{x}-\underline{x})$ $\left.\rightarrow 1 / \Delta_{f}^{3}\right)$, and at $\underline{x}-\underline{x}^{\prime}=\Delta_{f}, G\left(\underline{x}-\underline{x}^{\prime}\right)=0$. Thus, this filter removes modes with wavenumbers $\left|k_{f}\right|>\pi / \Delta_{f}$. For large scales $\left(\underline{x}^{\prime}>>x\right), G\left(\underline{x}-\underline{x}^{\prime}\right) \equiv 0$.

For homogeneous flows, with filtering, the integration extends over all space (i.e., from plus infinity to minus infinity). There are no restrictions that the filters be isotropic or homogeneous. However, the domain of integration may be restricted for the inhomogeneous case of a wall-bounded flow. Germano (1986a) states that his 'differential' filters for the LES approach can be extended to anisotropic, inhomogeneous turbulence and he derives the resulting consistent filter and SGS model. To my knowledge, testing of this filter and SGS model has not yet been done, and the current published literature does not include the application of a filter normal to the walls of a bounded domain.

In an LES, the calculated total energy is less than the experimental (actual) total energy, because the energy in the subgrid scales is filtered out and usually the largest scales are not captured (i.e., the computational domain is usually smaller than the largest scales). Thus, the energy spectra can not always be accurately calculated from the filtered field (Kwak et al. 1975, Bardina et al. 1983). However, Bardina et al. (1983) found that with the Gaussian filter, the filtered energy spectra are similar to the real spectra at least for low Reynolds number flows. Kwak et al. (1975) derive the 
expression

$$
\bar{E}(k)=E(k) \exp \left(\frac{-\Delta_{f}^{2}}{2 \gamma} k^{2}\right)
$$

for converting the three-dimensional energy spectra of a filtered field, $\bar{E}(k)$, to the spectra of the actual field, $E(k)$, when a Gaussian filter is used.

Kwak et al. (1975) show that with the top-hat filter, the formula for calculating the actual spectrum can be singular. ${ }^{1}$ Thus, total energy spectral features can not be computed using the top-hat filter with finite difference methods. Kwak et al. (1975) also show that the optimum filter scale is twice the grid mesh scale $\left(\Delta_{f}=2 \Delta\right)$. This choice of optimum filter scale is based on a comparison of the calculated energy spectra with the filtered experimental energy spectra for the decay of homogeneous isotropic turbulence (grid generated). As will be discussed in Section 2.4.4, the optimum choice of filter is dependent on the choice of subgrid-scale model.

As already stated, the numerical approach in this research is the finite element method. Very few LES researchers have chosen the finite element method of solution and thus, the choice of filter or averaging with respect to a finite element approach is

1From Kwak et al. (1975), the formula for calculating the actual spectrum, when using a top-hat filter, is

$$
\hat{\bar{u}}(\underline{k})=\left\{\prod_{i=1}^{3} \frac{\sin \left(k_{i} \frac{\Delta_{f}}{2}\right)}{k_{i} \frac{\Delta_{f}}{2}}\right\} \hat{u}(\underline{k})
$$

where $\hat{u}(\underline{k})$ is the Fourier transform of the actual spectrum, $u(\underline{k})$, and $\bar{u}(\underline{k})$ is the filtered spectrum. The coefficient is zero for some wavenumbers and thus, the inverse formula is what Kwak et al. (1975) called singular. 
not described in detail in the literature. Like the finite difference method, the finite element method is usually considered a grid-based method. Based on this interpretation, one would probably conclude that a top-hat filter would be implicit with the finite element method, as it is with the finite difference method. However, as will be shown in Section 3.2.1 and in Appendix C, with the finite element method, the equations can be filtered over a width $\Delta_{f}>\Delta$ and the numerical scheme does not necessarily imply the filter width. The key to this flexibility is in the fact that even though the method is grid based, the flow variables are defined continuously at all points in the flow (as with spectral methods) and not just at discrete points.

As mentioned above, the domain of integration for filtering should probably be restricted for an inhomogeneous wall-bounded flow (i.e., we can not integrate over an infinite domain). How this should be done has not yet been determined and will not be addressed in this study. Thus, for the backward-facing step the cell volume-average approach will be used (which implies a top-hat filter) for a chosen averaging width. The averaging operator and its implications with respect to the finite element approach are further discussed in Appendix C. In summary, it is shown (in Appendix C) that a cell volume-averaging operator commutes with differentiation (which is assumed in the development of the LES equations) only if the filter width is constant over the flow domain. The error introduced for a variable filter width is small as long as the spatial variation of the filter width is small (i.e., $\partial \Delta_{f} / \partial x_{\alpha}$ is small where $\Delta_{f}$ is the filter width). It is shown (in Appendix C) that applying the cell volume-averaging operator to one-dimensional piecewise-linear basis functions (described in Section 3.0) results in higher order piecewise-continuous functions that span over a greater number of grid lengths than the number averaging over. It is also shown that for a constant filter width, the cell volume-averaging operator commutes with differentiation for the product of piecewise-linear basis functions. 
As described in Section 3.3, the analytical approach for choosing a filter width in the solution of the advection term is simplified in this study, because of the 'centroid advection velocity' simplification made in the existing code. (For the explicit calculation of the advection term,

$$
\overline{\bar{u}_{\beta} \frac{\partial \bar{u}_{\alpha}}{\partial x_{\beta}}}
$$

(summation over $\beta$ implied) the resolved velocity, $\bar{u}_{\beta}$, varies over the element according to the velocity basis functions, $\bar{u}_{\beta}=\bar{u}_{\beta}^{j} \phi_{j}$ (summation over $j$ implied), and the explicit bar operator over the entire term can be and is chosen for $\Delta_{f} \geq \Delta$.) It should be emphasized that the simplifications herein are not the result of the finite element method, but are instead made to reduce computational effort so that we can solve computationally intensive problems with limited computer resources.

\subsubsection{Formulation of the Continuum Equations}

The details of the LES equation formulation are given in Appendix A and are summarized here. For an incompressible flow with constant molecular viscosity the LES governing equations are

$$
\begin{gathered}
\frac{\partial \bar{u}_{\alpha}}{\partial x_{\alpha}}=0 \\
\frac{\partial \bar{u}_{\alpha}}{\partial t}+\frac{\partial}{\partial x_{\beta}} \overline{u_{\alpha} u_{\beta}}=-\frac{1}{\rho} \frac{\partial \bar{p}}{\partial x_{\alpha}}+v \frac{\partial^{2}}{\partial x_{\beta}^{2}} \bar{u}_{\alpha}
\end{gathered}
$$

where $u_{\alpha}$ is the instantaneous velocity field that has been decomposed into a resolved 
field, $\bar{u}_{\alpha}$, and an SGS field, $\dot{u}_{\alpha}\left(u_{\alpha}=\bar{u}_{\alpha}+\dot{u}_{\alpha}\right)$, and similarly for $p$, which also includes the gravitational body force potential. (For an incompressible flow it is of course assumed that density changes in time and space are negligible. This assumption implies that the fluid speed of sound is infinite and that any flow perturbation is instantaneously felt over the entire flow field.)

For the velocity product we have, in expanded form,

$$
\overline{u_{\alpha} u_{\beta}}=\overline{\bar{u}_{\alpha} \bar{u}_{\beta}}+\overline{u_{\alpha} \bar{u}_{\beta}}+\overline{\bar{u}_{\alpha} u_{\beta}}+\overline{u_{\alpha} u_{\beta}}
$$

The first term on the right can actually be explicitly computed (by using the solution from the previous time step; see Piomelli et al. 1987), but it is commonly modeled. Some researchers claim that a second order approximation for $\overline{\bar{u}_{\alpha} \bar{u}_{\beta}}$, developed by Leonard (1974), can be used to model this term when second-order accurate numerical schemes are used (Rogallo and Moin 1984). Since most researchers use second-order accurate schemes, they commonly model $\overline{\bar{u}}_{\alpha} \bar{u}_{\beta}$. The last three terms in the velocity product involve the residual field and must be modeled. Various ways of modeling these terms are discussed in the next section.

Comparing the above LES filtered velocity product with that for Reynolds averaging $\left.\left.\left.\left(<u_{\alpha} u_{\beta}\right\rangle=<u_{\alpha}><u_{\beta}\right\rangle+<u_{\alpha} u_{\beta}\right\rangle\right)$, we see that there are two extra (nonclosed) terms with LES filtering. These terms do not vanish because, in general, with LES filtering, $\overline{u_{\alpha}} \neq 0$ and $\overline{\bar{u}}_{\alpha} \neq \bar{u}_{\alpha}$.

It is helpful to break up the advection term into three parts, to help clarify the SGS modeling. We substitute the above expanded form of the velocity product into the 
momentum equation and subtract and add

$$
\delta_{\alpha \beta} \frac{\overline{u_{\gamma} \dot{u_{\gamma}}}}{3}
$$

to the right side (for reasons discussed in Section 2.4.4) and add $\partial\left(\bar{u}_{\alpha} \bar{u}_{\beta}\right) / \partial x_{\beta}$ to both sides of the equation. The equations of motion for the large-scale eddies are then

$$
\begin{gathered}
\frac{\partial \bar{u}_{\alpha}}{\partial x_{\alpha}}=0 \\
\frac{\partial \bar{u}_{\alpha}}{\partial t}+\frac{\partial}{\partial x_{\beta}} \bar{u}_{\alpha} \bar{u}_{\beta}=-\frac{1}{\rho} \frac{\partial \bar{P}}{\partial x_{\alpha}}-\frac{\partial}{\partial x_{\beta}}\left(L_{\alpha \beta}+C_{\alpha \beta}+R_{\alpha \beta}\right)+v \frac{\partial^{2} \bar{u}_{\alpha}}{\partial x_{\beta}^{2}}
\end{gathered}
$$

where

$$
\begin{aligned}
& \bar{P}=\bar{p}+\frac{1}{3} \rho \delta_{\alpha \beta} \overline{u_{\gamma} \dot{u_{\gamma}}} \\
& L_{\alpha \beta}=\overline{\bar{u}_{\alpha} \bar{u}_{\beta}}-\bar{u}_{\alpha} \bar{u}_{\beta} \\
& C_{\alpha \beta}=\overline{\dot{u}_{\alpha} \bar{u}_{\beta}}+\overline{\bar{u}_{\alpha} \dot{u_{\beta}}} \\
& R_{\alpha \beta}=\overline{u_{\alpha} \dot{u_{\beta}}}-\frac{1}{3} \delta_{\alpha \beta} \overline{u_{\gamma} u_{\gamma}}
\end{aligned}
$$

The modeling of nonclosed terms, $C_{\alpha \beta}$ and $R_{\alpha \beta}$, will be discussed (along with the sometimes modeled term $L_{\alpha \beta}$ ) in the following section. This form for the LES momentum equations is quite common and will be referred to in the following discussions of past and present LES research. This form will be used in calculations with the Smagorinsky model (described in Section 2.4.4).

An alternate form of the momentum equations which will be used in discussions 
of Bardina's Model (described in Section 2.4.4) is

$$
\frac{\partial \bar{u}_{\alpha}}{\partial t}+\frac{\partial}{\partial x_{\beta}} \overline{\overline{u_{\alpha}} \bar{u}_{\beta}}=-\frac{1}{\rho} \frac{\partial \bar{p}}{\partial x_{\alpha}}-\frac{\partial}{\partial x_{\beta}}\left(\overline{u_{\alpha} \bar{u}_{\beta}}+\overline{\overline{u_{\alpha} \bar{u}_{\beta}}}+\overline{u_{\alpha} u_{\beta}}\right)+v \frac{\partial^{2} \bar{u}_{\alpha}}{\partial x_{\beta}^{2}}
$$

where the Leonard term $\overline{\bar{u}_{\alpha} \bar{u}_{\beta}}$, has been brought over to the left side and has the form of a filtered advection term, which can, using equation 2.1 , also be written as

$$
\frac{\partial}{\partial x_{\beta}} \overline{\bar{u}_{\alpha} \bar{u}_{\beta}}=\overline{\bar{u}_{\beta} \frac{\partial \bar{u}_{\alpha}}{\partial x_{\beta}}}
$$

This term is called the Leonard term because Leonard (1974) developed an improved approximation for the term. Previous to this, the approximation

$$
\frac{\partial}{\partial x_{\beta}} \overline{\bar{u}_{\alpha} \bar{u}_{\beta}} \cong \frac{\partial}{\partial x_{\beta}} \bar{u}_{\alpha} \bar{u}_{\beta}
$$

had been used. Leonard's approximation uses a truncated Taylor series expansion (truncated after the second derivative term) that results in a model of order $\Delta_{f}^{2}$. Leonard (1974) also showed that the advection term removes significant energy from the resolved scales and thus, it should not be lumped with the SGS terms. The assumption that $\overline{\bar{u}_{\alpha} \bar{u}_{\beta}}=\bar{u}_{\alpha} \bar{u}_{\beta}$ is satisfied if $\bar{u}_{\alpha}$ remains constant over the averaging or filtering domain (i.e., the Reynolds-average assumption). This situation is graphically shown in Figure 2.2a and discussed in Section 2.4.2.

The trace of the SGS Reynolds-stress tensor is not included in the SGS terms in equation 2.3 and thus, not included in the pressure term, because of the choice of SGS model used with this form of the equation. The SGS model is called Bardina's model 
and is described in the following Section 2.4.4. An explanation of why the trace of the SGS Reynolds stress is not needed is also given in the following section. The discretized form of this formulation will be described in detail in Section 3.2 along with the discretized form of equation 2.2 .

\subsubsection{Subgrid-Scale Models}

By filtering the Navier-Stokes equation, we are discarding information and as a result, the filtered equations are not closed. The filtered advection term includes the SGS motion, $u_{\alpha}$, for which we have no closed equation. Thus, the SGS motion must be modeled.

Even though twenty years have passed since Deardorff's first LES modeling of a channel flow, very few advances in SGS modeling have occurred. The following is a brief summary of past development of SGS modeling, present usage, and new research approaches.

Deardorff (1970) and Schumann (1975) applied the Reynolds-averaging assumption

$$
\overline{\bar{u}_{\alpha} \bar{u}_{\beta}}-\bar{u}_{\alpha} \bar{u}_{\beta}+\overline{\bar{u}_{\alpha} u_{\beta}}+\overline{u_{\alpha} \bar{u}_{\beta}}=0
$$

which effectively reduces the velocity product to ${ }^{1}$

$$
\overline{u_{\alpha} u_{\beta}}=\bar{u}_{\alpha} \bar{u}_{\beta}+\overline{u_{\alpha} u_{\beta}}
$$

${ }^{1}$ This is equivalent to setting $L_{\alpha \beta}+C_{\alpha \beta}=0$ in equation 2.2 in Section 2.4.3. 
Both Deardorff (1970) and Schumann (1975) state that primed terms represent the deviation from the local grid mean (which is not the general representation implied in this study). They both go on to label $\overline{u_{\alpha} u_{\beta}}$ as the SGS Reynolds-stress term. This statement, which labels $\dot{u_{\alpha}}$ as a deviation from the mean, $\bar{u}_{\alpha}$, is the only rationale offered by Deardorff (1970) and Schumann (1975) for applying the Reynolds-averaging assumption. Rogallo and Moin (1984) criticize this approach for the channel flow problem because the Reynolds-averaging assumption is applicable only to cell volumeaveraging that is uniform over an unbounded homogeneous space. The assumption should not be applied to averages over bounded domains (Monin and Yaglom 1971, Leonard 1974). (See discussion in Section 2.4.2 and 2.4.3 describing when the LES operator reduces to a Reynolds-average operator.) However, the resulting approximation is still used in some current LES calculations (e.g., Murakami et al. 1987). As described below, this approximation is not only a simplified approach (and easy to implement into a laminar code), but it has been shown to give results that compare well with experiment.

In using the Reynolds-averaging assumption, the only term that requires modeling is the SGS term, $\overline{u_{\alpha} u_{\beta}}$. This term can be interpreted as a stress (Hinze 1975, p.23), even though it stems from the turbulence advection. To arrive at a consistent model, the so called 'SGS turbulent pressure' (or also sometimes called the energy of the residual turbulence), $\delta_{\alpha \beta} \overline{u_{\gamma} u_{\gamma} / 3}$ is subtracted from the stresses and, as required, added to the pressure term in the equation,

$$
\bar{p}+\frac{\rho \delta_{\alpha \beta} \overline{u_{\gamma} \dot{u}_{\gamma}}}{3}
$$

Deardorff (1970), Schumann (1975), and many present day researchers model the 
resulting so called 'SGS Reynolds stress' term using the model of Smagorinsky et al. (1963)

$$
R_{\alpha \beta}=\overline{\overline{u_{\alpha} u_{\beta}}}-\frac{1}{3} \delta_{\alpha \beta} \overline{u_{\gamma} \dot{u_{\gamma}}}=-2 v_{T} S_{\alpha \beta}
$$

where

$$
S_{\alpha \beta} \equiv \frac{1}{2}\left(\frac{\partial \bar{u}_{\alpha}}{\partial x_{\beta}}+\frac{\partial \bar{u}_{\beta}}{\partial x_{\alpha}}\right) \text {, }
$$

$v_{T} \equiv(c \Delta)^{2}\left(2 S_{\alpha \beta} S_{\alpha \beta}\right)^{1 / 2}, \Delta \equiv(\Delta x \Delta y \Delta z)^{1 / 3}$, and $c$ is a constant. ${ }^{1}$ It can be seen that extracting the $\delta_{\alpha \beta} \overline{u_{\gamma} u_{\gamma} / 3}$ term causes the left and right sides of the relation to be zero when the indices are contracted (i.e., when $\alpha=\beta$ and a summation is performed). This form is invariant with respect to a Galilean transformation (i.e., translation of coordinate system) (Hinze 1975, p. 24, Speziale 1985). The Galilean invariance was important in Deardorff's channel flow calculation because the coordinate system was moved at a constant speed (the mean-flow velocity) in the downstream direction as a means of increasing the time step limit. As will be shown below, some current researchers who do not invoke the Reynolds-averaging assumption have governing equations which are not Galilean invariant.

There is no standard value for $c$ and researchers have chosen different values with various degrees of success. Deardorff (1970a) chose $c=0.1$ for his channel flow

${ }^{1}$ There is some variation in the literature on the definition of the grid scale $\Delta$. For example, Kobayashi et al. (1989) use $\Delta^{2}=\left(\Delta x^{2}+\Delta y^{2}+\Delta z^{2}\right) / 3$. Ferziger (1981) states that Bardina et al. (1980) show $\Delta=\left(\Delta x^{2}+\Delta y^{2}+\Delta z^{2}\right)^{1 / 2}$ is a better choice over $\Delta=(\Delta x$ $\Delta y \Delta z)^{1 / 3}$. However, in the new SGS model of Germano et al. (1990) (discussed at end of this section), the original definition is used, $\Delta=(\Delta x \Delta y \Delta z)^{1 / 3}$. 
problem and reported good comparison to experiment. Mason and Callen (1986) suggest $c=0.2$, however, Piomelli et al. (1987) use $c=0.1$ for the channel flow problem because they found that $c=0.2$ was too diffusive. For the results shown herein (Section 5.0) we use $c=0.1$ (or more precisely $c \omega=(c)^{2 / 3}=(0.1)^{2 / 3}=0.2154$ for $c \omega$ described in Section 2.5.3).

The Smagorinsky model is an eddy-viscosity model which implicitly assumes that the SGS turbulence is in equilibrium with the large eddies and that it adjusts itself instantaneously to changes in the large-scale velocity gradients (Rogallo and Moin 1984). The name eddy viscosity comes from the analogy that the SGS Reynolds stress behaves in the same manner as the viscous stress and is dependent on the gradient of the resolved field. The use of an eddy-viscosity model for the SGS terms usually produces the correct average energy removal from large eddies but poorly represents the effects of small eddies on large eddies. This random nonlinear action of the small eddies on the large eddies is called stochastic backscatter. Recently, Leith (1990) has developed a supplement to the Smagorinsky subgrid-scale viscosity to account for stochastic backscatter. Piomelli et al. (1990) investigated the energy transfer between the large scales and small scales using direct-simulation data bases for turbulent and transitional channel flows and compressible isotropic flow. They concluded that the mean transfer is indeed from large to small scales but it is caused by the small difference between large forward and backward components. When sharp-cutoff filters were used, it was found that at any instant about $50 \%$ of the grid points indicated transfer of energy from small to large scales. The fraction was less with a Gaussian filter (30\%), and in between the two for a top-hat filter (between $30 \%$ to $50 \%$ ).

If both the mesh size and the filter width are too large (e.g., greater than the integral scale), we no longer have an LES. In this case, an eddy-viscosity model may 
not be adequate for an SGS model. A length scale may need to be computed as with Reynolds-averaged methods. The required mesh size or filter width is still debatable. Some authors claim that an inertial subrange must exist and that the mesh size or filter width should be at or below the largest eddies in the inertial subrange. (High Reynoldsnumber flows exhibit an inertial subrange.) Ferziger (1977), however, claims that his calculations have shown that this is an unnecessarily strict restriction.

Schumann's (1975) SGS-stress model differed from the simple Smagorinsky model in that he splits the SGS stresses into two parts. One part accounts for locally isotropic turbulence (as in the bulk of the flow) and the other for the inhomogeneous turbulence (as in the near-wall region). The isotropic part is defined by an SGS eddyviscosity model where the velocity scale in the eddy viscosity relation is calculated by means of a separate transport equation for the SGS kinetic energy. The inhomogeneous part is also defined by an eddy-viscosity model, however with the restrictions that 1 ) it have negligible effect if the grid size is small enough so that the SGS stress is isotropic and homogeneous, and 2) when the grid size is larger than all scales in the inertial subrange, the eddy-viscosity model is equal to common models for the time-mean Reynolds stress (as one would have with a Reynolds-average code). Thus, the model is meaningful for the entire flow field, and even if the size of the grid volumes is very large. Schumann (1975) found that including the SGS kinetic energy equation did not result in much improvement over the simple Smagorinsky model. As a whole, Schumann's results were in better agreement with experiment than the results of Deardorff (1970).

Most of the LES research work from 1975 to approximately 1985 did not invoke the Reynolds-averaging assumption and instead the SGS Reynolds stress term, $\overline{u_{\alpha} \overline{u_{\beta}}}$, plus cross terms, $\overline{u_{\alpha} \bar{u}_{\beta}}+\overline{\bar{u}_{\alpha} u_{\beta}}$ were modeled with the Smagorinsky model; letting 


$$
Q_{\alpha \beta}=\overline{u_{\alpha} u_{\beta}}-\bar{u}_{\alpha} \bar{u}_{\beta}=\overline{u_{\alpha} \bar{u}_{\beta}}+\overline{\bar{u}_{\alpha} \bar{u}_{\beta}}+\overline{u_{\alpha} \bar{u}_{\beta}}
$$

and then

$$
Q_{\alpha \beta}-\frac{\delta_{\alpha \beta}}{3} Q_{n}=-2 v_{T} S_{\alpha \beta}
$$

Speziale (1985) showed that this SGS model results in equations of motion which are not Galilean invariant (the model does not retain the same form in all inertial frames of reference). However, most of the researchers during this same time period did see model improvement by explicitly computing the so-called Leonard term, $\overline{\bar{u}_{\alpha} \bar{u}_{\beta}}$.

One group of researchers, Bardina, Ferziger, and Reynolds (1983), included a separate model for the cross terms, $C_{\alpha \beta}=C_{r}\left(\bar{u}_{\alpha} \bar{u}_{\beta}-\overline{\bar{u}}_{\alpha} \overline{\bar{u}}_{\beta}\right)$, along with the Smagorinsky model for $R_{\alpha \beta}$. The model for $C_{\alpha \beta}$ is based on the assumption that the modeled scales are structurally similar to the smallest resolved scales. They assumed that the interaction between the subgrid scales $\left(\dot{u}_{\alpha}\right)$ and the resolved scales $\left(\bar{u}_{\alpha}\right)$ takes place between the smallest resolved eddies and the largest subgrid-scale eddies. They used the idea that filtering $u_{\alpha}$ once $\left(\bar{u}_{\alpha}\right)$ removes the small scales, and filtering it twice $\left(\bar{u}_{\alpha}\right)$ results in a term which contains only the largest scales. Thus, the difference $\bar{u}_{\alpha}-\overline{\bar{u}}_{\alpha}$ gives only the smallest resolved scales.

The actual final form of the model used by Bardina et al. (1983) is not clear in this reference, because of apparent inconsistencies in the text. However, Piomelli et al. (1987) clearly states what will here be called Bardina's Model; 


$$
\overline{u_{\alpha} \bar{u}_{\beta}}+\overline{u_{\alpha} \bar{u}_{\beta}}+\overline{\bar{u}_{\alpha} \dot{u_{\beta}}}=C_{r}\left(\bar{u}_{\alpha} \bar{u}_{\beta}-\overline{\bar{u}_{\alpha}} \overline{\bar{u}}_{\beta}\right)-2 v_{T} S_{\alpha \beta}
$$

With the model in this form, the trace of the SGS terms, $\frac{1}{3} \delta_{\alpha \beta}\left(\overline{u_{\gamma} \dot{u}_{\gamma}}+2 \overline{u_{\gamma} \bar{u}_{\gamma}}\right)$, does not need to be subtracted from the left side of the above expression. Then, the pressure also does not have these added terms (i.e., we have $\bar{P}=\bar{p}$ ). The trace of the SGS terms is needed when just the Smagorinsky model is used, because the strain tensor, $S_{\alpha \beta}$, is trace free (i.e., so that the nonclosed terms and their model are both trace free).

This model, used by Bardina et al. (1983), would have satisfied the Galileaninvariance constraint had their model constant $C_{r}$ been set to 1.0 instead of 1.1 (again, as verified by Speziale 1985). The model of Clark et al. (1979) was shown by Speziale to be Galilean invariant; however, they modeled the Leonard term and the cross terms by a Taylor series expansion, thus introducing unnecessary errors.

Bardina et al. (1983) and Piomelli et al. (1988) found that consistency between the SGS model and filter is essential to ensure accurate results. By comparing LES results for various combinations of filters and SGS models to the results from direct simulations, Piomelli et al. (1988) conclude that consistency is satisfied if the filter and SGS model carry the same length information (i.e., length scale implied in model agrees with filter width). Germano (1986a) develops a purely mathematical approach (rather than the empirical or trial and error approach of other researchers) for deriving an SGS model that is consistent with the choice of filter. He was also able to ensure Galilean invariance of the SGS model (Germano 1986b).

Recently, during the 1990 Summer Program at the NASA Ames/Stanford University Center for Turbulence Research, Germano et al. (1990) developed a new 
SGS eddy-viscosity model. The model is based on the Smagorinsky model and an algebraic identity between the SGS and the resolved turbulent stresses (Germano 1990). In the referenced literature, the model is developed for the combined nonclosed terms $\left(Q_{\alpha \beta}-\delta_{\alpha \beta} Q_{\gamma} \beta\right)$. With this new model, the SGS stresses vanish in laminar regions of the flow and asymptotically go to zero at walls. The Smagorinsky 'constant' is a function of space and time and can even be negative and thus, possibly, accounting for backscatter. The model has only one adjustable parameter. The parameter represents the ratio of test filter width to grid filter width and the ratio is defined such that its value is always greater than one. The test filter width is the turbulent scale size that is being filtered out. The grid filter represents the numerical scheme accuracy. Germano et al. (1990) states that if the chosen parameter value is too small, the results can be contaminated by numerical errors, and if, on the other hand, too large a value is chosen, it implies that the large energy carrying structures determine the contribution of the subgrid scales. It is hoped that the optimum value of this parameter will be approximately the same for a wide range of flow conditions. For a transitional and fully developed turbulent channel flow, when using a sharp-cutoff filter, Germano et al. (1990) found that setting the model parameter to a value of 2.0 yielded the best results. No other flow configurations have yet been attempted.

The space and time varying coefficient in this new SGS model also includes averaged terms. For the turbulent channel-flow problem (two-dimensional geometry), Germano et al. (1990) averaged these terms over a plane parallel to the channel wall. In general, the plane average would be replaced with some appropriate local space or time average.

Based on the above discussion, we can conclude that the current state-of-the-art seems to suggest that $\overline{\bar{u}_{\alpha} \bar{u}_{\beta}}$ should be computed explicitly (using values from a previous 
time step).

There are several options available for modeling the nonclosed terms, $\overline{u_{\alpha} \bar{u}_{\beta}}+\overline{u_{\alpha} \bar{u}_{\beta}}+\overline{\bar{u}_{\alpha} \dot{u}_{\beta}}$ (on $C_{\alpha \beta}$ and $R_{\alpha \beta}$ in equation 2.2 ):

- the model of Smagorinsky (1963) (as used by Deardorf 1970 and Schumann 1975),

- the model of Bardina et al. (1983) (labeled here as Bardina's model),

- the model of Germano et al. (1990) (from hereon to be referred to as Germano's model), and

- the model of Leith (1990).

The SGS turbulence model to be used in this study will be the Smagorinsky model (actually, it is called a vorticity model in two-dimensional problems; see Section 2.5); however, the finite element equations will also be developed for Bardina's model. The form of the continuum equations to be used in this study are equations 2.1 and 2.2 with the Smagorinsky model (given in the previous Section 2.4 .3 ). The primary reason for choosing the Smagorinsky model is because the model parameters and form are well defined (at least in comparison to Bardina's and Germano's model). Eliminating the need for turbulence model development should help simplify efforts and allow for more time to be spent on developing the numerical approach. Incorporating an improved model, such as Germano's model or Leith's model, should be less difficult once the numerical scheme has been established. However, to show the flexibility of the finite element method, the equations are also developed with Bardina's model. 
Future efforts should probably be the incorporation of Germano's model. This model has been shown to properly simulate the SGS motion near walls and thus, does not require ad hoc near-wall corrections if the wall region is resolved. However, implementation of Germano's model will require further investigation of how to compute the averaged terms and the adjustable parameter in the space and time varying coefficient for the backward-facing step problem and with a finite element solution approach.

\subsubsection{Wall Models}

To simulate the wall region 'exactly' requires the specification of a no-slip boundary condition, very fine discretization of the region, and the equations of motion must account for the low Reynolds-number flow close to the wall (e.g., the SGS stresses should asymptotically go to zero at the wall). The Stanford group (Moin et al. 1978, Moin and Kim 1981, Spalart 1988) has simulated wall regions as accurately as possible for simple (nonseparating) flows. They generated three-dimensional movies of their simulations (Robinson 1989 and Robinson et al. 1989) which display streaks, bursts, and ejections in the flow. However, most researchers applying LES to more complicated geometries do use wall models (artificial boundary conditions) to represent the physics in the regions close to the wall (Deardorff 1970, Schumann 1975, Mason and Callen 1986). This is usually done by setting the instantaneous stress at the wall proportional to the tangential velocity at the first grid point away from the wall. The coefficient of proportionality can be described by either a logarithmic, power, or linear law-of-the-wall relation depending on the type of flow simulation and location of the first grid point relative to the wall. The normal velocity component at the wall is set to zero. With LES, the approximate boundary conditions are applied everywhere on a 
plane surface parallel to the wall and are time dependent. For the Reynolds-average equations only a single average value is specified.

Piomelli et al. (1987) give a detailed review of wall models used in an LES by past researchers and propose wall model improvements. Piomelli et al. (1988) considered in their wall model for nonseparating channel flows that the turbulent structures at the wall are inclined away from the wall and in the downstream direction. The instantaneous wall shear stress was set proportional to the tangential velocity at the first grid point away from the wall, but at a distance (on the order of a cell length) downstream from the wall point.

For high Reynolds-number flows in three dimensions with separation, no one is really sure what to use. The commonly used law-of-the-wall approximations are accurately known only for two-dimensional geometries and then only for steady attached flows. For example, Ruderich and Fernholz (1986) have shown that the logarithmic law-of-the-wall is not valid in recirculating flow regions. Reynolds (1989) also states that assuming that the law-of-the-wall is satisfied instantaneously is unlikely to be valid.

Werner and Wengle (1989) computed the flow over a square rib in a channel with an LES approach using wall models. If the first grid point from the wall was located in the viscous sublayer, a linear law-of-the-wall was used to evaluate the coefficient. Otherwise, an integrated power-law of the local mean velocity was used.

One option that seems plausible to this author, but was not found in the literature, is the use of the LES approach all the way to the wall without resolution of the wall region. In other words, an SGS model is used for the elements in the wall 
region. This implies that the small-scale motion at the wall (that is smaller than the resolved scale) is the same as the small-scale motion away from the wall. This seems to be a reasonable assumption, especially for the shedding vortices of a backward-facing step, where the wall region and the core flow are similar (i.e., all swirling eddies).

It is clear from the above review that the use of wall models for separated flows and especially in conjunction with the LES approach is still under development. Preferably, in this study, it would be ideal to resolve the wall region, but this would require excessive computer resources. Resolution of the wall region is also not feasible with the code used in this study (see Appendix D for code description), because it is limited to structured grids (i.e., transition elements at wall regions are not available). Instead, the above described new idea of using the SGS model in the wall region is the approach used in this study.

In cases where the wall regions are resolved (which was not done here) and the Smagorinsky model is used, the length scale $(\ell=c \Delta)$ in the Smagorinsky model is usually adjusted so that $v_{T} \rightarrow 0$ as the wall is approached. For example, Piomelli et al. (1987) suggest that

$$
\ell=c\left[1-\exp \left(-y^{+3} / A^{+3}\right)\right]^{1 / 2}\left(\Delta_{1} \Delta_{2} \Delta_{3}\right)^{1 / 3}
$$

for $A^{+}=25$ and $c=0.065$ and where $y^{+}=y_{n} u_{\tau} / v, u_{\tau}=\left(\tau_{w} / \rho\right)^{1 / 2}, \tau_{w}=\mu\left(d u / d y_{n}\right)_{w}$ and $y_{n}$ is the direction normal to the wall. Some authors use variations of the above form for $\ell$ and choose different values for $c$ (e.g., Mason and Callen 1986). Piomelli et al. (1987) found that their form for $\ell$ with $c=0.065$ gives velocity profiles in good agreement with the exact profile for a channel flow. However, this approach has not been attempted for a separating flow problem. In fact, it also does not appear to be 
valid or might at least need some modification for application to separating flow simulations, because $\tau_{w}=0$ at separation points.

Another important point (which this author has not found mentioned in the literature) is the difference in wall boundary between a direct simulation and LES when the wall region is resolved and no slip conditions are specified for the LES. This situation is described in Figure 2.3 where it is shown that the boundary for the LES should be a time- and spatial-dependent boundary condition at a location one-half the filter width from the direct simulation boundary, but in practice this condition would not be known.

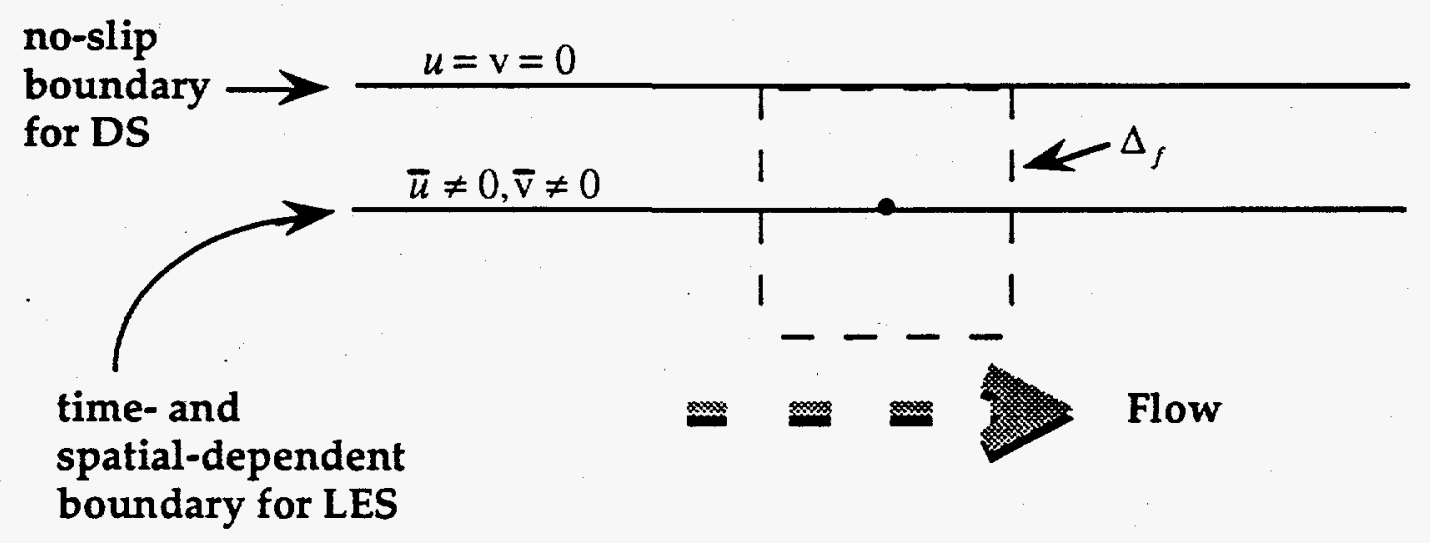

Figure 2.3 Description of no-slip boundary condition for a direct simulation versus the corresponding LES time- and spatial-dependent boundary.

\subsubsection{Inflow and Outflow Conditions}

Inflow conditions for the backward-facing step can be specified as the time dependent outflow calculated from the direct simulation or LES of a corresponding 
channel flow. One could also generate 'artificial' inflow conditions (e.g., randomly perturbed mean flow quantities) with the same mean flow and turbulence intensity characteristics as the experimental or computational results being considered for comparison. Sandham and Reynolds (1987) suggest that for the case of artificial inflow conditions, some randomizing of the inflow conditions may be necessary to represent turbulent flows. In this study, the inflow conditions will not be randomized so as to simplify the comparison of the transient LES calculations to the 'direct' simulation (generated with the same code) for the purpose of code validation. It is possible that the flow will exhibit random-like behavior due to insufficient field discretization (i.e., numerical pertubation). It is expected that with adequate resolution of the flow field, the results will be periodic. As shown in Section 5, the results reported herein display some random-like behavior that is reduced with finer discretization of the flow field.

For the laminar backward-facing step problem with a finite element code, a zero natural boundary condition (see Section 3.2.5) at the outflow gives accurate results. In the discretized LES formulation (Section 3.2.1 and 3.2.5), the natural boundary conditions include the subgrid-scale motion. Zero natural boundary conditions for the LES outflow condition will be used in this study.

\subsection{Two-Dimensional Simulations of Three-Dimensional Turbulence}

In this Section, the three-dimensional theory of turbulence is described in terms of its energy spectrum and energy transfer mechanisms. The eddy viscosity coefficient, $v_{T}$, derived from the three-dimensional theory is that used in the Smagorinsky SGS model. The eddy viscosity coefficient for the SGS model which follows from the twodimensional theory is also described along with a description of the two-dimensional equations for the large-scale motion. 
From the discussion that follows, it can be concluded that a two-dimensional LES of a backward-facing step is not a 'real' (i.e., occurring in nature) turbulent flow. However, the flow does have the spectral characteristics of cascading energy in a flow with a continuous range of scales. Thus, the demonstration of filtering with a finite element approach, which is one of the objectives of this research, can be accomplished with two-dimensional simulations. Therefore, while the two-dimensional LES for a backward-facing step presented herein are not true simulations of the naturally occurring backward-facing step flow, they do demonstrate a filtering scheme with a finite element method.

\subsubsection{Three-Dimensional Theory}

As described in Section 2.1, turbulent flow is three-dimensional and contains structures called eddies with a wide range of scales. Energy is extracted from the mean flow at large scales and the energy gain is approximately balanced by viscous dissipation of energy at small scales. The net transfer of energy is from large scales to small scales and is controlled by three-dimensional vortex motions. As explained, for example, by Tennekes and Lumley (1972), turbulent flows exhibit high levels of fluctuating vorticity that are maintained by a mechanism known as vortex stretching which is absent in two-dimensional flow. In particular, it is believed that for shear flows, vortex stretching in the direction of the mean strain rate is the mechanism for energy transfer from large to small scales. Therefore, two-dimensional simulations of turbulent shear flows lack the basic mechanism for energy transfer from large to small 
scales, and thus, 'real' turbulent flows cannot be modeled in two-dimensions. ${ }^{1}$

The cutoff length scale between the resolved large-scale motion and the unresolved small-scale motion for an LES is usually chosen to be in the inertial subrange (see Figure 2.1). (As discussed in Sections 2.1 and 2.4.4, most researchers agree that the eddies below this cutoff eddy size display the characteristics of being homogeneous, isotropic, and not geometry dependent.) In the inertial subrange, the three-dimensional energy spectrum exhibits a constant energy cascade rate $\tilde{\varepsilon}$ to higher wave numbers (or smaller scales), and by dimensional analysis (see Tennekes and Lumley 1972 , Section 8.3 ).

$$
E(k)=\alpha \tilde{\varepsilon}^{2 / 3} k^{-5 / 3}
$$

where $k$ is the wave number ( $k$ is proportional to $1 /$ length scale) and $\alpha$ is the dimensionless Kolmogorov constant (from experimental data, $\alpha \cong 1.5$ ). Thus, in the inertial subrange for three-dimensional turbulence, the energy cascades from large to small scales with a $-5 / 3$ power spectrum. Using dimensional analysis and Smagorinsky's (1963) procedure (in which a local estimate of $\tilde{\varepsilon}$ is made from the resolved large-scale motion), Leith (1969) shows that the eddy viscosity coefficient for three-dimensional turbulence is proportional to $k_{*}^{-2}$ (or $\Delta^{2}$ ) where $k_{*}$ is the cutoff wave number $\left(k_{*} \equiv 2 \pi / \Delta\right)$. Leith's derived eddy viscosity coefficient is that in Smagorinsky's model, $v_{T}=(c \Delta)^{2}\left(2 S_{\alpha \beta} S_{\alpha \beta}\right)^{1 / 2}$ (see Section 2.4.4).

\footnotetext{
${ }^{1}$ An exception to this may be atmospheric motion. The scales of planetary atmospheric motion have been observed to have a $k^{3}$ spectrum (described in Section 2.5.3) as is also predicted with two-dimensional simulations. Thus, two-dimensional simulations may be acceptable for this case.
} 


\subsubsection{Two-Dimensional Simulation of the Large-Scale Motion}

For two-dimensional large-scale motion, the out-of-plane velocity component is zero (i.e., $\bar{u}_{3}=0$ ), and the gradient of the in-plane components in the out-of-plane direction are zero (i.e., $\partial \bar{u}_{1} / \partial x_{3}=\partial \bar{u}_{2} / \partial x_{3}=0$ ). In equation 2.2 , the terms involving the SGS motion $u_{3}$ are modeled in terms of the gradient of the large scale motion (i.e., involve terms with $\bar{u}_{3}$ or $\partial / \partial x_{3}$ ), and thus, these terms are also zero. Thus, in two dimensions, equations 2.1 and 2.2 reduce to

$$
\begin{gathered}
\frac{\partial \bar{u}_{1}}{\partial x_{1}}+\frac{\partial \bar{u}_{2}}{\partial x_{2}}=0 \\
\frac{\partial \bar{u}_{1}}{\partial t}+\frac{\partial}{\partial x_{1}} \bar{u}_{1} \bar{u}_{1}+\frac{\partial}{\partial x_{2}} \bar{u}_{1} \bar{u}_{2}=-\frac{1}{\rho} \frac{\partial \bar{P}}{\partial x_{1}}-\frac{\partial}{\partial x_{1}}\left(L_{11}+C_{11}+R_{11}\right) \\
-\frac{\partial}{\partial x_{2}}\left(L_{12}+C_{12}+R_{12}\right)+v\left(\frac{\partial^{2} \overline{u_{1}}}{\partial x_{1}{ }^{2}}+\frac{\partial^{2} \bar{u}_{1}}{\partial x_{2}{ }^{2}}\right) \\
\frac{\partial \bar{u}_{2}}{\partial t}+\frac{\partial}{\partial x_{1}} \bar{u}_{2} \bar{u}_{1}+\frac{\partial}{\partial x_{2}} \bar{u}_{2} \bar{u}_{2}=-\frac{1}{\rho} \frac{\partial \bar{P}}{\partial x_{2}}-\frac{\partial}{\partial x_{1}}\left(L_{21}+C_{21}+R_{21}\right) \\
-\frac{\partial}{\partial x_{2}}\left(L_{22}+C_{22}+R_{22}\right)+v\left(\frac{\partial^{2} \bar{u}_{2}}{\partial x_{1}^{2}}+\frac{\partial^{2} \bar{u}_{2}}{\partial x_{2}{ }^{2}}\right)
\end{gathered}
$$

These assumptions might only be justified if the 'real' problem being simulated had large structures which were predominantly two-dimensional. (A justification we shall not attempt.)

\subsubsection{Two-Dimensional Simulation of the Small-Scale Motion}

In Section 2.5.1, three-dimensional turbulence was described as having an inertial subrange with a $-5 / 3$ power energy spectrum. Two-dimensional turbulence has two inertial subranges because it is not only characterized by an energy conservation 
constraint, but also by an enstrophy conservation constraint (Kraichnan 1967). Enstrophy is defined as one-half the square of the vorticity $\left(\omega^{2} / 2\right)$.

Due to the energy constraint, one of the inertial subranges has a $-5 / 3$ power spectrum (same as that given for three-dimensional theory, Section 2.5.1). However, the constant energy cascade rate $\varepsilon$ is from small to large scales, which is the opposite direction of that for three-dimensional turbulence. To show that the energy cascade rate is towards larger scales, we use the energy flux function (Leith 1968)

$$
F \equiv-\beta k^{7 / 2-m} \frac{\partial}{\partial k}\left(k^{m} E^{3 / 2}\right)
$$

where $\beta$ is a dimensionless positive coefficient. For three-dimensional turbulence $m=$ -3 and for two-dimensional turbulence $m=9 / 2$ (Leith 1968). Thus, for two-dimensional turbulence

$$
F=-\beta k^{-1} \frac{\partial}{\partial k}\left(k^{9 / 2} E^{3 / 2}\right) .
$$

Substituting the $-5 / 3$ power spectrum, $E=A k^{-5 / 3}$ where $A$ is a positive constant, we obtain

$$
F=-2 \beta A^{3 / 2}=-\tilde{\varepsilon}
$$

which is a negative constant. It follows that $A=\alpha \tilde{\varepsilon}^{2 / \beta}$ for a positive constant $\alpha$. Thus, energy is cascaded towards larger scales (lower wave numbers) at the constant rate $\tilde{\varepsilon}$ in the two-dimensional inertial subrange with a $-5 / 3$ power spectrum. 
For the enstrophy conservation constraint we have the enstrophy flux function (Leith 1968)

$$
H \equiv-\beta k^{3} \frac{\partial}{\partial k}\left(k^{5 / 2} E^{3 / 2}\right)
$$

Substituting $E(k)=\alpha \tilde{\varepsilon}^{2 / 3} k^{-5 / 3}$, we obtain $H=0$. Thus, for the $-5 / 3$ power spectrum the enstrophy cascade is zero.

The other inertial subrange has a -3 power spectrum (Kraichnan 1967)

$$
E(k)=\alpha_{2} \eta^{2 / 3} k^{-3}
$$

where $\eta$ is a constant enstrophy cascade rate and $\alpha_{2}$ is a positive dimensionless coefficient. (Lilly 1968 estimates $\alpha_{2}=4.5 \pm 0.5$ ). For the -3 power spectrum, $E(k)=$ $A \boldsymbol{k}^{-3}$,

$$
F=0
$$

and

$$
H=2 \beta A^{3 / 2}=\eta
$$

which is a positive constant. It follows that $A=\alpha_{2} \eta^{2 / 3}$ for a positive constant $\alpha_{2}$. Thus, enstrophy is cascaded to smaller scales (or higher wave numbers) at the constant rate $\eta$ and the energy cascade rate is zero in this two-dimensional inertial subrange with a -3 power spectrum. 
In summary, for two-dimensional turbulence in the inertial subrange, energy is cascaded with a $-5 / 3$ power spectrum at a constant rate $\tilde{\varepsilon}$ from small to large scales, and enstrophy is cascaded with a -3 power spectrum at a constant rate $\eta$ from large to small scales (see Figure 2.3). Thus, energy is trapped in the large scales. Since the energy is locked in the large scales for two-dimensional turbulence, the SGS model should have less effect on the large-scale motion than for three-dimensional turbulence. The Smagorinsky model for the eddy viscosity coefficient is not the appropriate model to use for the SGS motion in two-dimensions because it is based on a $-5 / 3$ power spectrum with the cascade of energy to smaller scales. For consistency and to avoid possible nonlinear instability, it is the enstrophy cascade that should be modeled in twodimensional simulations.
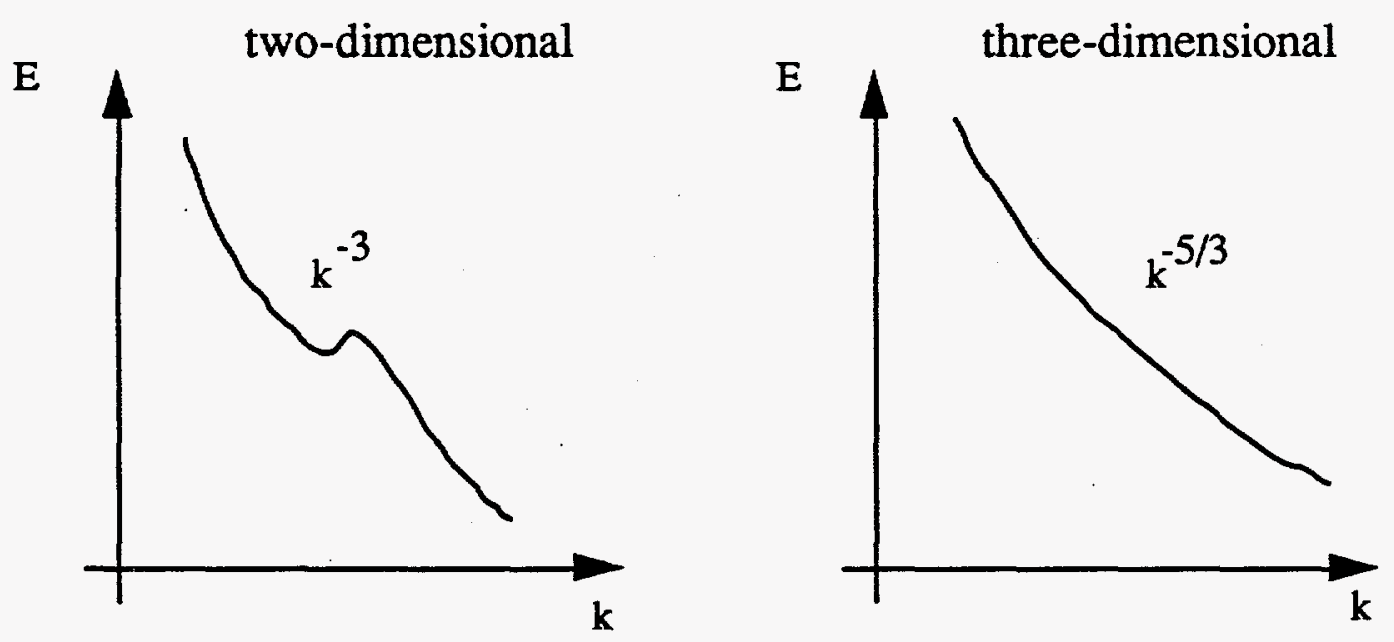

Figure 2.3 Comparison of two-dimensional and three-dimensional energy spectra.

Leith (1969) developed a vorticity relation for the eddy viscosity coefficient in two dimensions which accounts for the -3 power spectrum and the enstrophy cascade to small scales. The following is a description of Leith's SGS model-called a vorticity 
model.

Leith (1969) shows by dimensional analysis and with Smagorinsky's procedure that if the truncation wave number $k$ lies within a -3 power spectrum, the eddy viscosity coefficient is proportional to $k_{*}^{-3}$ (or $\Delta^{3}$ ). The derived vorticity relation for the eddy viscosity coefficient is

$$
v_{T}=\left(c_{\omega} \Delta\right)^{3}|\nabla \cdot \omega|
$$

where $\nabla . \omega$ is defined by Leith (1969) as 'the finite difference approximation to the vorticity gradient' and $c_{\omega}$ is a constant. The term $|\nabla . \omega|$ is analogous to the rate of strain term, $\left(2 S_{\alpha \beta} S_{\alpha \beta}\right)^{1 / 2}$ in the Smagorinsky model. ${ }^{1}$ Thus, the continuous form of $|\nabla . \omega|$ is

$$
|\nabla \omega|=\left(\frac{\partial \bar{\omega}}{\partial x_{\beta}} \frac{\partial \bar{\omega}}{\partial x_{\beta}}\right)^{1 / 2}
$$

where $\bar{\omega}$ is the resolved or large-scale vorticity.

1The factor 2 in this term allows simplification of the expanded expression:

$$
\begin{aligned}
2 S_{\alpha \beta} S_{\alpha \beta} & =2\left[\frac{1}{2}\left(\frac{\partial \bar{u}_{\alpha}}{\partial x_{\beta}}+\frac{\partial \bar{u}_{\beta}}{\partial x_{\alpha}}\right) \frac{1}{2}\left(\frac{\partial \bar{u}_{\alpha}}{\partial x_{\beta}}+\frac{\partial \bar{u}_{\beta}}{\partial x_{\alpha}}\right)\right] \\
& =\frac{1}{2}\left[\left(\frac{\partial \bar{u}_{\alpha}}{\partial x_{\beta}}\right)^{2}+2 \frac{\partial \bar{u}_{\alpha}}{\partial x_{\beta}} \frac{\partial \bar{u}_{\beta}}{\partial x_{\alpha}}+\left(\frac{\partial \bar{u}_{\beta}}{\partial x_{\alpha}}\right)^{2}\right]
\end{aligned}
$$

and since summation is implied over $\alpha$ and $\beta$,

$$
2 S_{\alpha \beta} S_{\alpha \beta}=\frac{1}{2}\left[2\left(\frac{\partial \bar{u}_{\alpha}}{\partial x_{\beta}}\right)^{2}+2 \frac{\partial \bar{u}_{\alpha}}{\partial x_{\beta}} \frac{\partial \bar{u}_{\beta}}{\partial x_{\alpha}}\right]=\frac{\partial \bar{u}_{\alpha}}{\partial x_{\beta}}\left(\frac{\partial \bar{u}_{\alpha}}{\partial x_{\beta}}+\frac{\partial \bar{u}_{\beta}}{\partial x_{\alpha}}\right)
$$


In two dimensions, where $\bar{\omega}=\frac{\partial \bar{u}_{2}}{\partial x_{1}}-\frac{\partial \bar{u}_{1}}{\partial x_{2}}$,

$$
\begin{aligned}
|\nabla \omega| & =\left[\left(\frac{\partial \bar{\omega}}{\partial x_{1}}\right)^{2}+\left(\frac{\partial \bar{\omega}}{\partial x_{2}}\right)^{2}\right]^{1 / 2} \\
& =\left[\left(\frac{\partial^{2} \bar{u}_{2}}{\partial x_{1}^{2}}-\frac{\partial^{2} \bar{u}_{1}}{\partial x_{1} \partial x_{2}}\right)^{2}+\left(\frac{\partial^{2} \bar{u}_{2}}{\partial x_{1} \partial x_{2}}-\frac{\partial^{2} \bar{u}_{1}}{\partial x_{2}^{2}}\right)^{2}\right]^{1 / 2} .
\end{aligned}
$$

With the LES approach, Findikakis (1980) calculated two-dimensional flow in a square cavity driven by a specified randomly varying shear at the surface. He performed the calculation with the eddy viscosity coefficient vr defined by the vorticity gradient and he repeated the calculation with $v_{T}$ defined by the rate of strain. He found that the spectrum (i.e., the spectral density function, $E(f) /<\bar{u}^{2}>$ versus frequency $f$ (in $\mathrm{rad} / \mathrm{sec}$ )) of the simulated flow were similar for both definitions of $v_{T}$. The calculated spectrum had a -3 slope for both $v_{T}$ definitions and were in good quantitative agreement. The choice of model constant (i.e., $c$ in the Smagorinsky model, $v_{T}=(c \Delta)^{2}$ $\left(2 S_{\alpha \beta} S_{\alpha \beta}\right)^{1 / 2}$ and $c_{\omega}$ in the vorticity model, $\left.v_{T}=\left(c_{\omega} \Delta\right)^{3}\left|\nabla_{*} \omega\right|\right)$ caused more variation in the predicted streamline plots than the relation chosen for $v_{T}$.

Since plans are to extend this research work to three-dimensions and since the Smagorinsky and vorticity model give approximately the same estimates for $v_{T}$ in two dimensions, the Smagorinsky model could probably be used here for all simulations (two- and three-dimensional). However, for the sake of consistency, the vorticity model was implemented in the code for the two-dimensional case. Appendix $\mathrm{E}$ gives the details of how the vorticity model is incorporated into the existing finite element code. The final modeled equations are equations $2.4,2.5$, and 2.6 with $C_{\alpha \beta}=0, L_{\alpha \beta}=0$ or solved explicitly, and $R_{\alpha \beta}$ modeled using the vorticity model. 


\section{Numerical Method}

The following includes a brief discussion of several numerical methods and gives a summary description of the finite element method, to be used for this study. Also, the discretized LES equations are derived and the solution approach is described.

\subsection{Choice of Method}

Numerical techniques available for LES computations include finite difference methods, spectral and pseudospectral methods, and finite element methods. Deardorff (1970) and Schumann (1975) used the finite difference method and most of the Stanford researchers have used spectral and pseudospectral methods. The use of finite element schemes has been far less prominent (scarce, in fact) than the other methods, but several researchers believe it to be the preferred numerical approach for complex geometries of technological interest (e.g., Hutton et al. 1987).

The following briefly summarizes the numerical approaches in relation to LES computations.

\section{Finite Difference Method}

The finite difference approach involves the approximation of time and spatial derivatives in the differential (strong) form of the equations of motion by the terms in a Taylor-series expansion. The more terms included, the higher the order of accuracy of the approximation. Transformation to generalized coordinates is required for the computation of complex geometries, a procedure that could reduce the accuracy of socalled higher-order methods. The finite difference method is a grid-based method in that the flow variables are calculated at discrete points in the field. In relation to an 
LES computation, the turbulent scales of motion larger than the grid size are said to be resolved and those smaller than the grid size are said to be modeled.

\section{Spectral and Pseudospectral Methods}

The spectral method involves the transformation of the governing equations from real space to spectral space. The transformation is done globally by the use of such functions as a Fourier series or spherical harmonics. The pseudospectral method involves performing some operations in physical space and then transforming to wave number space (Orzag 1971). For example, Piomelli et al. (1987) computed the LES convective terms, $\partial \overline{\overline{u_{i}}} \bar{u}_{j} / \partial x_{j}$, pseudospectrally by performing the velocity product in physical space and then using the Fourier transform to compute the derivative of the product.

An advantage of spectral methods over finite difference methods in an LES (which should also apply to finite element methods) is that the choice of filtering scheme is optional (e.g., Gaussian or sharp cutoff). The filter width, $\Delta_{f}$, may also be chosen independently of the numerical discretization. With finite difference methods, the filter width is the grid size and top-hat filtering is implicit (built-in).

A disadvantage of spectral methods is its difficulty in dealing with complex geometries. Also, for domains with solid boundaries, functions other than standard Fourier series must be used because periodic boundary conditions are then inappropriate. In these cases, the fast-Fourier-transform algorithm can not be used and the computational cost increases significantly (Ferziger 1987 and Gottlieb and Orzag 1977). A disadvantage of the pseudospectral method is that it may be susceptible to numerical instability due to aliasing terms (Orzag 1972). 


\section{Finite Element Method}

The finite element method is an expansion of the dependent variables using piecewise (local) polynomials rather than the global polynomials of the spectral method. Also, the finite element method is much more readily adaptable to complex geometry than the spectral method and the finite difference method. As with the finite difference method, the finite element method can be construed to be grid-based and from the discussion in the previous section, one would presume that the filter width is implicitly grid-controlled. However, as will be shown in the following sections, a choice of both averaging operator and averaging width is possible with the finite element method, just as for spectral methods. The key to this flexibility is the fact that even though the method is ostensibly grid based, the flow variables are defined continuously at all points in the flow field and not just at discrete points, just as for spectral methods.

In a literature search for LES results computed using the finite element method, only three references were found (Kondo et al. 1986, Findikakis et al. 1978, Findikakis and Street 1980). Even though very few LES researchers have chosen the finite element approach, past LES developments via the spectral and finite difference approach are adaptable to the finite element method.

\subsection{The Finite Element Method in Fluid Mechanics}

\subsubsection{Outline of Methodology}

The finite element method for solving a system of nonlinear partial differential equations (PDEs) can be outlined in the following steps (e.g., Carey and Oden 1986): 
- Derive the weak form of the PDEs, using what is effectively the method of weighted residuals.

- Expand solutions (variables solving for) into linearly independent piecewise polynomial basis functions.

- Insert expansions into the weak form of the equations to obtain a system of differential-algebraic equations.

- Solve system of differential-algebraic equations.

In this section, we will derive the 'general' (with some variation dependent on the SGS model) discretized form of the LES equations of motion given in Section 2.4.3. One additional step to be included that is not listed above is the replacement of the continuity equation with a Poisson equation. The benefits of solving the Poisson equation and a discussion of proposed solution techniques will be presented in the following sections.

\section{Weak Form of the Partial Differential Equations}

First, the strong form of the equations (Section 2.4.3, equations 2.1 and 2.3) are written in the slightly abbreviated form

$$
\begin{gathered}
\frac{\partial \bar{u}_{\alpha}}{\partial x_{\alpha}}=0 \\
\frac{\partial \bar{u}_{\alpha}}{\partial t}+\frac{\partial}{\partial x_{\beta}} \overline{\bar{u}_{\alpha} \bar{u}_{\beta}}=\frac{\partial}{\partial x_{\beta}} \bar{\tau}_{\alpha \beta}
\end{gathered}
$$


where

$$
\bar{\tau}_{\alpha \beta}=-\frac{\bar{p}}{\rho} \delta_{\alpha \beta}+v \frac{\partial \bar{u}_{\alpha}}{\partial x_{\beta}}-\left(\overline{u_{\alpha} \bar{u}_{\beta}}+\overline{\bar{u}_{\alpha} \dot{u}_{\beta}}+\overline{u_{\alpha} \dot{u}_{\beta}}\right)
$$

We then derive the weak form of the PDEs by

- forming the residual function, $r(x)$, (i.e., moving everything to one side of the equation), e.g., for the momentum equation

$$
\mathrm{r}_{\alpha}(\underline{x})=\frac{\partial \overline{u_{\alpha}}}{\partial t}+\frac{\partial}{\partial x_{\beta}} \overline{\bar{u}}_{\alpha} \bar{u}_{\beta}-\frac{\partial}{\partial x_{\beta}} \overline{\tau_{\alpha \beta}}
$$

- choosing appropriate test functions, $v(x)$, which multiply the residual,

- expanding the dependent variables, $\bar{u}_{\alpha}$ and $\bar{p}$, into appropriate basis functions, and

- $\quad$ integrating over the domain, $\Omega$.

In more mathematical terms, we determine appropriate test functions, $v$, in an appropriate function space, $H^{R}(\Omega)$, called a Hilbert space (where all $\mathrm{v} \in H^{R}(\Omega)$ ) containing functions whose derivatives up to and including order $R$ are square integrable over domain $\Omega$. The weak formulation is

$$
\int_{\Omega} \mathrm{r}(\underline{x}) \mathrm{v}(\underline{x}) d \underline{x}=0
$$

Thus, the residual is forced to be orthogonal to a linearly independent set of test functions (i.e., given $v, r$ is made orthogonal to $v$ ). If $r(x)=0$, then $u(x)$ is a strong solution. Thus, the residual function is the 'error' incurred by not satisfying the 
differential equation. It can be shown that if a strong solution exists, a weak solution also exists, and the solutions are identical. However, a weak solution need not be a strong solution. A very important advantage of the weak form of the equation is that the boundary conditions (Neumann, Dirichlet, or mixed) are built into the equations.

If the test functions are chosen to be the same as the basis functions used to express the appropriate solution, we have Galerkin's weighted residual method. If, further, these functions are the piecewise polynomials associated with the nodes of the finite element method, we have the Galerkin finite element method (GFEM). The Galerkin finite element method is used in the following formulation of the approximate solution.

Substituting for $r(\underline{x})$, we get the following weak forms of the PDEs

$$
\begin{gathered}
\int_{\Omega} \mathrm{w} \frac{\partial \bar{u}_{\alpha}}{\partial x_{\alpha}}=0 \\
\int_{\Omega} \mathrm{v}\left(\frac{\partial \bar{u}_{\alpha}}{\partial t}+\frac{\partial}{\partial x_{\beta}} \overline{\bar{u}_{\alpha} \bar{u}_{\beta}}\right)-\int_{\Omega} \mathrm{v} \frac{\partial \bar{\tau}_{\alpha \beta}}{\partial x_{\beta}}=0 .
\end{gathered}
$$

where the appropriate sets of test functions are chosen such that all $w \in H^{R_{1}}(\Omega)$ and all $\mathrm{v} \in H^{R_{2}}(\Omega)$ where $H^{R_{1}}$ and $H^{R_{2}}$ are not necessarily (and usually aren't) the same function space. (Specific function choices are discussed in Section 3.2.2.) Integrating the stress term by parts,

$$
\int_{\Omega} v \frac{\partial \bar{\tau}_{\alpha \beta}}{\partial x_{\beta}}=\int_{\Omega}\left(\frac{\partial}{\partial x_{\beta}}\left(v \bar{\tau}_{\alpha \beta}\right)-\bar{\tau}_{\alpha \beta} \frac{\partial \mathrm{v}}{\partial x_{\beta}}\right)
$$


and using the divergence theorem,

$$
\int_{\Omega} \frac{\partial}{\partial x_{\beta}}\left(\mathrm{v} \bar{\tau}_{\alpha \beta}\right)=\int_{\partial \Omega} \mathrm{v} n_{\beta} \bar{\tau}_{\alpha \beta},
$$

where $n_{\beta}$ is the $\beta$-component of the outward unit normal to $\partial \Omega$ (domain boundary), the weak form of the momentum equation becomes

$$
\int_{\Omega} v\left(\frac{\partial \bar{u}_{\alpha}}{\partial t}+\frac{\partial}{\partial x_{\beta}} \overline{\bar{u}_{\alpha} \bar{u}_{\beta}}\right)+\int_{\Omega} \bar{\tau}_{\alpha \beta} \frac{\partial v}{\partial x_{\beta}}=\int_{\partial \Omega} \mathrm{v} n_{\beta} \bar{\tau}_{\alpha \beta} .
$$

The differentiability requirements on $\bar{\tau}_{\alpha \beta}$ (and thus, on $\bar{p}$ and $\bar{u}_{\alpha}$ ) have been relaxed by shifting derivatives to the test functions (replaced the second derivative term with the product of two first derivative terms). This (weaker) form requires that the solution $\bar{u}_{\alpha}$ and the test function $v$ need only have 'square-integrable' first derivatives (i.e., be piecewise-continuous),

$$
\int_{\Omega}\left(\frac{\partial \bar{u}_{\alpha}}{\partial x_{\beta}}\right)^{2}<\infty
$$

This condition allows us to consider $\bar{u}_{\alpha}$ and $v$ as members of the same 'family' of functions. The pressure term $\bar{p}$ need only be square-integrable (piecewisediscontinuous). Note also that a boundary integral term has been introduced by use of the divergence theorem, a feature that leads to the natural boundary conditions.

The combined boundary conditions (essential and natural) are 


$$
-\frac{\bar{p}}{\rho} n_{\beta} \delta_{\alpha \beta}+v n_{\beta} \frac{\partial \bar{u}_{\alpha}}{\partial x_{\beta}}-n_{\beta}\left(\overline{\overline{u_{\alpha} \bar{u}_{\beta}}}+\overline{\overline{u_{\alpha}} \overline{u_{\beta}}}+\overline{u_{\alpha} \bar{u}_{\beta}}\right)+\gamma_{(\alpha)} \bar{u}_{\alpha}=f_{\alpha}
$$

on $\partial \Omega$ where $\gamma_{(\alpha)}$ is a given scalar and the subscript $(\alpha)$ indicates dependence of $\gamma$ on $\alpha$ and does not imply summation. (The natural boundary condition is $n_{\beta} \bar{\tau}_{\alpha \beta}$ (discussed in Section 3.2.5) and the essential boundary condition is a specified value for $\bar{u}_{\alpha}$.) The vector $f_{\alpha}$ is a vector of functions specified on the boundary $\partial \Omega$. From the definition of $\bar{\tau}_{\alpha \beta}$, this is equivalent to

$$
f_{\alpha}=n_{\beta} \bar{\tau}_{\alpha \beta}+\gamma_{(\alpha)} \bar{u}_{\alpha}
$$

on $\partial \Omega$.

As discussed in Gresho (1991), the natural boundary condition involves a surface force balance (or traction condition) where $\bar{\tau}_{\alpha \beta}$ is the stress tensor and $f_{\alpha}$ is the surface traction vector. However, $\bar{\tau}_{\alpha \beta}$ and $f_{\alpha}$ are true physical forces only when the viscous term in the momentum equation is left in its stress divergence form,

$$
\frac{\partial}{\partial x_{\beta}} v\left(\frac{\partial \bar{u}_{\alpha}}{\partial x_{\beta}}+\frac{\partial \bar{u}_{\beta}}{\partial x_{\alpha}}\right),
$$

and not simplified, as done here, to

$$
v\left(\frac{\partial^{2} \bar{u}_{\alpha}}{\partial x_{\beta}^{2}}\right) .
$$

This distinction is more important in theory than in practice; i.e., the form is expected to 
work quite well.

To set essential boundary conditions, we set $\bar{u}_{\alpha}=\bar{u}_{\alpha}^{s}$ (the specified value) on $\partial \Omega^{s}$ (a portion of the boundary) and let $\gamma_{(\alpha)} \rightarrow \infty$. (See Appendix D for explanation of how this is actually implemented in the code.) Then in the above boundary condition relation, $\bar{u}_{\alpha} \cong \bar{u}_{\alpha}^{s}$. To set natural boundary conditions, $\gamma_{(\alpha)}$ is set to zero and $f_{\alpha}$ can be set to a constant or a function of space and/or time. Boundary conditions are further discussed in Section 3.2.5 (which includes a discussion of pressure boundary conditions).

Substituting (3.1b) into the momentum equation (3.1a) and expanding the $\bar{\tau}_{\alpha \beta}$ term (i.e., $n_{\beta} \bar{\tau}_{\alpha \beta}=f_{\alpha}-\gamma_{(\alpha)} \bar{u}_{\alpha}$ ), we arrive at the final weak form of the momentum equation

$$
\begin{aligned}
\int_{\Omega}\left[\mathrm { v } \left(\frac{\partial \bar{u}_{\alpha}}{\partial t}+\right.\right. & \left.\frac{\partial}{\partial x_{\beta}} \overline{\bar{u}_{\alpha} \bar{u}_{\beta}}\right)+v \frac{\partial \bar{u}_{\alpha}}{\partial x_{\beta}} \frac{\partial \mathrm{v}}{\partial x_{\beta}}-\frac{\bar{p}}{\rho} \frac{\partial \mathrm{v}}{\partial x_{\alpha}} \\
& \left.-\left(\overline{u_{\alpha} \bar{u}_{\beta}}+\overline{\bar{u}_{\alpha} \dot{u}_{\beta}}+\overline{u_{\alpha} \dot{u_{\beta}}}\right) \frac{\partial \mathrm{v}}{\partial x_{\beta}}\right]+\int_{\partial \Omega} \gamma_{(\alpha)} \bar{u}_{\alpha} \mathrm{v}=\int_{\partial \Omega} \mathrm{v} f_{\alpha} .
\end{aligned}
$$

\section{Expanding the Equation Variables}

The first step in expanding the solutions is to discretize the domain $\Omega$, forming a union $\Omega_{h}$ of elements $\Omega_{e}, e=1,2, \ldots, M$, where $M$ is the total number of elements (and herein $M$ is the total number of pressures which are piecewise constant). Associated with these $M$ elements are $N$ velocity nodes. We then piece together local basis functions on each element constructing a global basis for the approximation subspace, $H^{h}$. For $H^{h}$ to be a subspace of $H^{R}(\Omega)$ (with $R=1$ here for velocity; $R=0$ for pressure) we need the global basis functions to be continuous across interelement interfaces. The 
basis function $\phi_{j}$ is the basis function associated with node $j$ having value unity at node $j$ and value zero at every other node. Thus, the velocity components $\bar{u}_{\alpha}$ and pressure $\bar{p}$ are expanded into the appropriate basis functions $\phi_{j}$ and $\psi_{j}$, respectively, as follows:

$$
\begin{aligned}
& \bar{u}_{\alpha}^{h}(\underline{x}, t)=\sum_{j=1}^{N} \bar{u}_{\alpha}^{j}(t) \phi_{j}(\underline{x}) \\
& \bar{p}^{h}(\underline{x}, t)=\sum_{j=1}^{M} \bar{p}_{j}(t) \psi_{j}(\underline{x})
\end{aligned}
$$

where $N$ is the total number of velocity basis functions, $\phi_{j}(\underline{x})$, and $\bar{u}_{\alpha}^{j}(t)$ is the nodal value of the resolved velocity, $\bar{u}_{\alpha}^{k}$, and $M$ is the total number of pressure basis functions $\psi_{j}(\underline{x})$, with $\bar{p}_{j}(t)$ being the value of $\bar{p}^{h}$ on element $j$. The superscript $h$ signifies that $\bar{u}_{\alpha}^{k}$ and $\bar{p}^{k}$ are the approximate weak solutions on a mesh of the computational domain (with characteristic element size $h$ ). The functions $\phi_{j}$ and $\psi_{j}$ are linearly independent piecewise polynomial basis functions. This expansion is consistent with the weak form derivation above where all boundary conditions are treated as natural boundary conditions.

The expansions will also be substituted for $\bar{u}_{\alpha}$ in the SGS terms and the resulting terms will depend on the chosen SGS model. These terms will be defined for the Smagorinsky model and Bardina's model below.

\section{Insert Expansions into Equations}

Substituting the expansions into the weak form of the equations is fairly straightforward for most of the terms except for the convection term and SGS terms. For example, setting $v=\phi_{i}$ (i.e., GFEM) we have for the time derivative term 


$$
\int_{\Omega} v\left(\frac{\partial \bar{u}_{\alpha}}{\partial t}\right) \rightarrow\left(\int_{\Omega} \phi_{i} \phi_{j}\right) \frac{\partial \bar{u}_{\alpha}^{j}}{\partial t}
$$

where a summation over $j$ is implied (i.e., $\bar{u}_{\alpha}^{h}=\bar{u}_{\alpha}^{j} \phi_{j}$ ).

Substitution of the expansions into the convection term and the SGS terms requires averaging (or filtering) of the velocity products. The substitution of the expansions and the averaging of these terms is shown below and averaging of the basis functions is discussed in Appendix C. For the convection term we have

$$
\frac{\partial}{\partial x_{\beta}} \overline{\bar{u}_{\alpha} \bar{u}_{\beta}}=\frac{\partial}{\partial x_{\beta}} \overline{\bar{u}_{\alpha}^{j} \phi_{j} \bar{u}_{\beta}^{k} \phi_{k}}=\bar{u}_{\alpha}^{j} \bar{u}_{\beta}^{k} \frac{\partial}{\partial x_{\beta}} \overline{\phi_{j} \phi_{k}} .
$$

The averaging operator is an integral over space, and $\bar{u}_{\alpha}^{j}$ and $\bar{u}_{\beta}^{k}$ are dependent on time only, and thus can be taken outside the averaging operator and spatial derivative. The form of the convection term at the element level and the transformation to local coordinates is shown in Appendix C.

For the SGS cross terms and Reynolds-stress terms, using Bardina's model, we have

$$
\overline{u_{\alpha} u_{\beta}}+\overline{u_{\alpha} \bar{u}_{\beta}}+\overline{\overline{u_{\alpha} u_{\beta}}}=C_{r}\left(\bar{u}_{\alpha} \bar{u}_{\beta}-\overline{\bar{u}}_{\alpha} \bar{u}_{\beta}\right)-2 v_{T} S_{\alpha \beta}
$$

where 


$$
\begin{aligned}
& v_{T}=(c \Delta)^{2}\left(2 S_{\alpha \beta} S_{\alpha \beta}\right)^{1 / 2}=(c \Delta)^{2}\left(\frac{\partial \bar{u}_{\alpha}}{\partial x_{\beta}}\left(\frac{\partial \bar{u}_{\alpha}}{\partial x_{\beta}}+\frac{\partial \bar{u}_{\beta}}{\partial x_{\alpha}}\right)\right)^{1 / 2}, \\
& S_{\alpha \beta}=\frac{1}{2}\left(\frac{\partial \bar{u}_{\alpha}}{\partial x_{\beta}}+\frac{\partial \bar{u}_{\beta}}{\partial x_{\alpha}}\right), \text { and } \Delta=(\Delta x \Delta y \Delta z)^{1 / 3} \text { or } \Delta=\left(\Delta x^{2}+\Delta y^{2}+\Delta z^{2}\right)^{1 / 2}
\end{aligned}
$$

(Bardina et al. (1983) recommend the latter choice for $\Delta$. .) For Galilean invariance we must set $C_{r}=1$ (see Speziale 1985). Substituting the expansions, we have

$$
\begin{aligned}
\overline{u_{\alpha} \bar{u}_{\beta}}+\overline{\bar{u}_{\alpha} \dot{u_{\beta}}}+\overline{u_{\alpha} u_{\beta}} & =\bar{u}_{\alpha}^{j} \bar{u}_{\beta}^{k}\left(\phi_{i} \phi_{k}-\bar{\phi}_{j} \bar{\phi}_{k}\right) \\
& -(c \Delta)^{2}\left(\bar{u}_{\alpha}^{m} \frac{\partial \phi_{m}}{\partial x_{\beta}}\left(\bar{u}_{\alpha}^{k} \frac{\partial \phi_{k}}{\partial x_{\beta}}+\bar{u}_{\beta}^{k} \frac{\partial \phi_{k}}{\partial x_{\alpha}}\right)\right)^{1 / 2}\left(\bar{u}_{\alpha}^{j} \frac{\partial \phi_{j}}{\partial x_{\beta}}+\bar{u}_{\beta}^{j} \frac{\partial \phi_{j}}{\partial x_{\alpha}}\right)
\end{aligned}
$$

The form of the average basis function, $\bar{\phi}_{j}$, and the product $\bar{\phi}_{j} \bar{\phi}_{k}$, at the element level and the transformation to local coordinates is described in Appendix C.

Inserting the expansions into the remaining terms in the weak formulation (equations 3.1 and 3.2), using $w=\psi_{i}$ and $v=\phi_{i}$, results in the GFEM equations

$$
\left(\int_{\Omega} \psi_{n} \frac{\partial \phi_{j}}{\partial x_{\alpha}}\right) \bar{\mu}_{\alpha}^{j}=0
$$




$$
\begin{aligned}
& \left(\int_{\Omega} \phi_{i} \phi_{j}\right) \frac{\partial \bar{u}_{\alpha}^{j}}{\partial t}+\left(\bar{u}_{\beta}^{k} \int_{\Omega} \phi_{i} \frac{\partial}{\partial x_{\beta}} \overline{\phi_{j} \phi_{k}}\right) \bar{u}_{\alpha}^{j}+\left(\int_{\Omega} v \frac{\partial \phi_{i}}{\partial x_{\beta}} \frac{\partial \phi_{j}}{\partial x_{\beta}}\right) \bar{u}_{\alpha}^{j}-\left(\int_{\Omega} \frac{\partial \phi_{i}}{\partial x_{\alpha}} \psi_{n}\right) \frac{1}{\rho} \bar{p}_{n} \\
& -\left(\bar{u}_{\beta}^{k} \int_{\Omega}\left(\phi_{j} \phi_{k}-\bar{\phi}_{j} \bar{\phi}_{k}\right) \frac{\partial \phi_{i}}{\partial x_{\beta}}\right) \bar{u}_{\alpha}^{j} \\
& +\left(\int_{\Omega} v_{T}^{h} \frac{\partial \phi_{j}}{\partial x_{\beta}} \frac{\partial \phi_{i}}{\partial x_{\beta}}\right) \bar{u}_{\alpha}^{j}+\left(\int_{\Omega} v_{T}^{h} \frac{\partial \phi_{j}}{\partial x_{\alpha}} \frac{\partial \phi_{i}}{\partial x_{\beta}}\right) \bar{u}_{\beta}^{j} \\
& +\left(\int_{\partial \Omega} \gamma_{(\alpha)} \phi_{i} \phi_{j}\right) \bar{u}_{\alpha}^{j}=\int_{\partial \Omega} \phi_{i} f_{\alpha}
\end{aligned}
$$

where

$$
v_{T}^{h}=(c \Delta)^{2}\left(\bar{u}_{\alpha}^{m} \frac{\partial \phi_{m}}{\partial x_{\beta}}\left(\bar{u}_{\alpha}^{k} \frac{\partial \phi_{k}}{\partial x_{\beta}}+\bar{u}_{\beta}^{k} \frac{\partial \phi_{k}}{\partial x_{\alpha}}\right)\right)^{1 / 2}
$$

and

$$
\begin{gathered}
i ; j ; k ; m=1,2,3, \ldots, N \\
n=1,2,3, \ldots, M .
\end{gathered}
$$

These are the global system of differential-algebraic equations. There are $N d+M$ equations where $N$ is the number of velocity basis functions, $\phi_{j}(\underline{x}), M$ is the number of pressure basis functions, $\psi_{j}(\underline{x})$, and $d$ is the space dimension (i.e., 2 or 3 ). The terms $\bar{u}_{\beta}^{j}$ represent the dependence on the other velocity components in the $\alpha$-momentum equation (e.g., the $\bar{u}_{1}$ momentum equation also has terms involving $\bar{u}_{2}$ and $\bar{u}_{3}$ ). If the equations are solved explicitly (as in this study), these other velocity components are treated as 'knowns' (i.e., the values at the previous time step are used). 
The above momentum equation $3.4^{\circ}$ is for the 'exact' solution of the advection term (i.e., it is not lumped with the modeled terms) and includes Bardina's model for the SGS terms. If the Smagorinsky model is used with the 'exact' solution of the advection term, the fifth term on the left of equation 3.4 is not considered and the pressure $\bar{P}_{n}$ includes the trace of the SGS Reynolds-stress term (see equation 2.2 with $C_{\alpha \beta}=0$ and $L_{\alpha \beta}$ solved for 'exactly'). The resulting equation for the Smagorinsky model is

$$
\begin{aligned}
& \left(\int_{\Omega} \phi_{i} \phi_{j}\right) \frac{\partial \bar{u}_{\alpha}^{j}}{\partial t}+\left(\bar{u}_{\beta}^{k} \int_{\Omega} \phi_{i} \frac{\partial}{\partial x_{\beta}} \overline{\phi_{j} \phi_{k}}\right) \bar{u}_{\alpha}^{j}+\left(\int_{\Omega} v \frac{\partial \phi_{i}}{\partial x_{\beta}} \frac{\partial \phi_{j}}{\partial x_{\beta}}\right) \bar{u}_{\alpha}^{j} \\
& -\left(\int_{\Omega} \frac{\partial \phi_{i}}{\partial x_{\alpha}} \psi_{n}\right) \frac{1}{\rho} \bar{P}_{n}+\left(\int_{\Omega} v_{T}^{k} \frac{\partial \phi_{j}}{\partial x_{\beta}} \frac{\partial \phi_{i}}{\partial x_{\beta}}\right) \bar{u}_{\alpha}^{j}+\left(\int_{\Omega} v_{T}^{h} \frac{\partial \phi_{j}}{\partial x_{\alpha}} \frac{\partial \phi_{i}}{\partial x_{\alpha}}\right) \bar{u}_{\beta}^{j} \\
& \quad+\left(\int_{\partial \Omega} \gamma_{(\alpha)} \phi_{i} \phi_{j}\right) \bar{u}_{\alpha}^{j}=\int_{\partial \Omega} \phi_{i} f_{\alpha} .
\end{aligned}
$$

The above equation 3.5 is solved in this study, but with some modification of the advection term (as described in Section 3.3). The finite element method in general allows for the 'exact' solution of the advection term by calculation of the derivative of the filtered product of the basis functions

$$
\frac{\partial}{\partial x_{\beta}} \overline{\phi_{j} \phi_{k}}
$$

or equivalently (i.e., considering continuity)

$$
\overline{\phi_{k} \frac{\partial}{\partial x_{\beta}} \phi_{j}}
$$

In theory, the filtering method and filter width can be chosen just as done with the spectral method approach. Appendix $C$ includes a detailed description of the 
characteristics of a cell-volume averaged basic function $\bar{\phi}_{j}$ and the product of basis functions $\overline{\phi_{j} \phi_{k}}$ for constant and variable filter widths.

\section{Putting Equations in Matrix Form}

The discretized continuity and momentum equations (equations 3.3 and 3.4) can now be written in matrix form

$$
\begin{gathered}
C^{T} \bar{u}=0 \\
M \dot{\bar{u}}+[K+N(\bar{u})] \bar{u}-C \bar{P}=F
\end{gathered}
$$

where $\bar{u}$ is the velocity vector, $\bar{P}$ is the pressure vector for $\bar{p}_{j} / \rho$, and in threedimensional space for equation 3.4 ,

$$
M=\left[\begin{array}{ccc}
m_{i j} & 0 & 0 \\
0 & m_{i j} & 0 \\
0 & 0 & m_{i j}
\end{array}\right], m_{i j}=\int_{\Omega} \phi_{i} \phi_{j}
$$$$
\boldsymbol{K}=\left[\begin{array}{lll}
k_{i j_{(1)}} & k_{i j_{(2)}} & k_{i j_{(3)}} \\
k_{i j_{(2)}} & k_{i j(2)} & k_{i j_{(2)}} \\
k_{i j_{(3)}} & k_{i j(z)} & k_{i j_{(3)}}
\end{array}\right], k_{i j_{(\infty)}}=\int_{\Omega} \delta_{\alpha \beta}\left(v+v_{T}^{h}\right)\left(\frac{\partial \phi_{j}}{\partial x_{s}} \frac{\partial \phi_{i}}{\partial x_{s}}\right)+\int_{\Omega} v_{T}^{h} \frac{\partial \phi_{j}}{\partial x_{\alpha}} \frac{\partial \phi_{i}}{\partial x_{\beta}}+\delta_{\alpha \beta} \int_{\partial \Omega} \delta_{i j} \gamma_{(\alpha)} \phi_{i} \phi_{j}
$$

(note: summation over $\varsigma$ implied)

$$
N=\left[\begin{array}{ccc}
n_{i j}(\bar{u}) & 0 & 0 \\
0 & n_{i j}(\bar{u}) & 0 \\
0 & 0 & n_{i j}(\bar{u})
\end{array}\right], n_{i j}(\bar{u})=\bar{u}_{\beta}^{k}\left[\int_{\Omega} \phi_{i} \frac{\partial}{\partial x_{\beta}} \overline{\phi_{j} \phi_{k}}-\int_{\Omega}\left(\phi_{j} \phi_{k}-\bar{\phi}_{j} \bar{\phi}_{k}\right) \frac{\partial \phi_{i}}{\partial x_{\beta}}\right]
$$




$$
\begin{aligned}
& C=\left[\begin{array}{l}
c_{i m_{(a)}} \\
c_{i m_{(2)}} \\
c_{i m_{(3)}}
\end{array}\right], c_{i m_{(\alpha)}}=\int_{\Omega} \frac{\partial \phi_{i}}{\partial x_{\alpha}} \psi_{n} \\
& C^{T}=\left[c_{n i_{(\Omega)}} c_{n i_{(\Omega)}} c_{n i_{(\Omega)}}\right], \quad c_{n i_{(\Omega)}}=\int_{\Omega} \frac{\partial \phi_{\Lambda}}{\partial x_{\alpha}} \psi_{i} \\
& F=\left[\begin{array}{l}
\mathbf{f}_{i_{(\sigma)}} \\
\mathrm{f}_{i_{(\partial)}} \\
f_{i_{(s)}}
\end{array}\right], \quad \mathbf{f}_{i_{(\alpha)}}=\int_{\partial \Omega} \phi_{i} f_{\alpha}
\end{aligned}
$$

If the Smagorinsky model is used (equation 3.5), $n_{i j}(\bar{u})$ reduces to

$$
n_{i j}(\bar{u})=\bar{u}_{\beta}^{k} \int_{\Omega} \phi_{i} \frac{\partial}{\partial x_{\beta}} \overline{\phi_{j} \phi_{k}}
$$

The coefficient calculations are performed at the element level. The element contributions are then added to form the global matrices described above using standard finite element method assembly techniques. A detailed description of how the element coefficient matrices are developed in terms of local (element level) coordinates is given in Appendix C. The integrals in the coefficient matrices are usually computed using a numerical quadrature technique.

\section{Replace Continuity Equation with a Poisson Equation}

The discretized Poisson equation for pressure can be solved in place of the continuity equation so that continuity and momentum are decoupled and an explicit time-integration scheme is used. 
The discrete Poisson equation for pressure is an approximation of the continuous Poisson equation. The continuous Poisson equation is derived by taking the divergence of the momentum equation and using the continuity equation, $\nabla \cdot \bar{u}=0$. The analogous discrete Poisson equation is derived by multiplying the matrix form of the momentum equation by $C^{T} M^{-1}$ and using $C^{T} \bar{u}=0$ :

$$
C^{T} M^{-1}(M \dot{\bar{u}}+[K+N(\bar{u})] \bar{u}-C \bar{P})=C^{T} M^{-1} F
$$

Since $M^{-1} M=I$ and $d C^{T} \bar{u} / d t=0$, we have

$$
C^{T} M^{-1} C \bar{P}=C^{T} M^{-1}[(K+N(\bar{u})) \bar{u}-F]
$$

where the coefficient matrix $C^{T} M^{-1} C$ is a discretized approximation of the Laplacian operator. Thus, the final spatially discretized equations in matrix form are

$$
C^{T} M^{-1} C \bar{P}=C^{T} M^{-1}[(K+N(\bar{u})) \bar{u}-F]
$$

and

$$
M \dot{\bar{u}}+[K+N(\bar{u})] \bar{u}-C \bar{P}=F .
$$

\subsubsection{Choice of Test and Basis Functions and Order of Accuracy}

The main idea behind the finite element method is that the finite element basis functions $\phi_{i}$ can be defined piecewise over subregions of the domain called elements (see e.g., Becker et al. 1981). We partition the domain into a finite number of elements. The fundamental criteria for defining the basis functions are (Becker et al. 1981):

- Basis functions are generated by simple functions defined piecewise 
(element-by-element) over the finite element mesh.

- Basis functions are smooth enough to be members of the class $H^{l} 0$ of test functions ${ }^{1}$.

- Basis functions are linearly independent (i.e., complete).

- Basis functions are chosen such that the approximate solution

$$
u^{h}=\sum_{i=1}^{N} u_{i} \phi_{i}(\underline{x})
$$

where $u^{h}=u_{i}$ at nodal points because $\phi_{i}(\underline{x})$ is interpolatory; i.e.,

$$
\phi_{i}\left(\underline{x}_{j}\right)=\left\{\begin{array}{l}
1 \text { if } i=j \\
0 \text { if } i \neq j
\end{array} .\right.
$$

The equation coefficients are calculated for each element and then the element contributions are assembled to form the global matricies. The parts of $\phi_{i}\left(\underline{x}_{j}\right)$ on the element are given in terms of local coordinates ${ }^{2}(\xi, \eta, \zeta)$ for the calculation of the element contributions. We construct a mapping for transforming from global to local coordinates. If the finite element basis functions are used to construct the mapping from global coordinates to local coordinates such that

${ }^{1} H^{l} O_{0}$ is the set of all functions that satisfy the homogeneous boundary conditions and whose first derivatives are square-integrable (see Becker et al. 1981).

${ }^{2}$ Local coordinates are defined with reference to the element (rather than with reference to the global coordinate system in which the element resides) and the local coordinate range is from -1 to 1 by convention. 


$$
\underline{x}=\sum_{j=1}^{n} \underline{x}_{j} \phi_{i}^{e}(\xi, \eta, \zeta)
$$

where $n$ is the number of nodes per element, the element is labeled an 'isoparametric' element.

If the space of test functions is chosen to be the same as that for the basis functions, the approach is called the Galerkin method. When the test functions are not the same as the basis functions, the approach is called the Petrov-Galerkin method. For example, upwind finite element schemes [sometimes used for convection dominated flows (Hughes, et al. 1982, 1988)] are called Petrov-Galerkin methods. For these upwind schemes the test functions are upwind biased (or weighted).

The basis functions, $\psi_{i}$, to be used here in the pressure expansion, have a value of one on element $i$ and zero on all other elements. The functions, $\phi_{i}$, were used as test functions in the momentum equation, and since we used the Galerkin method approach, $\phi_{i}$ is the basis function used in the velocity expansion. If $\psi_{i}$ and $\phi_{i}$ are from the same space of functions (i.e., the polynomial degree for $\psi_{i}$ is the same as that for $\phi_{i}$ ), the velocity solution is good but the pressure solution is usually poor (Hood and Taylor 1974 and Gresho 1985, pp. 13-14). If the polynomial degree for $\psi_{i}$ is one order lower than that for $\phi_{i}$, both the velocity and pressure solution are usually good. Sani et al. (1981) show that spurious pressure modes occur because of matrix singularities when $\psi_{i}$ and $\phi_{i}$ are from the same space of functions. The pressure modes are claimed to be the result of equation discretization (choice of basis functions) and the choice of grid point selection relative to variable location.

Gresho et al. (1984) performed a 'Taylor-series-like' analysis to determine how 
well $\nabla \cdot u$ (laminar case) is approximated with piecewise-constant basis functions for the pressure expansion. In summary, they found for two-dimensional problems that the error in the $\nabla P$ approximation is $O\left(h^{2}\right)$ on a uniform mesh of equal-sized rectangles, $O(h)$ on a general rectangular mesh, and $O(1)$ on a mesh of distorted elements. Even though these results imply that piecewise-constant basis functions are inadequate for distorted meshes, in practice, Gresho et al. (1984) find that with mesh refinement, their pressure solutions do converge. This indicates that a Taylor-series analysis is often an inappropriate error analysis approach for finite element schemes.

In the existing Galerkin finite element code to be used in this study, isoparametric elements with piecewise continuous multilinear basis functions are used for velocity (bilinear in two dimensions, trilinear in three dimensions) with piecewiseconstant basis functions for the pressure. This choice corresponds most closely to the staggered-grid approach that is so popular and successful in finite difference and finite volume methods.

\subsubsection{Spatial Integration of Terms in the Coefficient Matrices}

The integrals in the coefficient matrices are usually computed using a numerical quadrature technique (see Appendix C). The 1-point quadrature technique involves first, the exact computation of the element area in two dimensions (volume in three dimensions), and second, multiplying this element size by the value of the integrand at the element centroid.

Most of the integrals in the coefficient matrices are independent of time, with the exception of those involving $v_{T}^{h}$. These integrals could be generated once and input as needed. However, Gresho et al. (1984), found that input/output costs are high with 
this scheme, and that if a 1-point quadrature technique is used, it is more cost effective to calculate the coefficient matrices every time they are needed.

With 1-point quadrature, for two dimensional quadrilaterals and nondistorted three dimensional elements, element level and global mass balance is achieved (Gresho et al. 1984). However, if the three-dimensional elements are distorted, element level mass balance may not be achieved and, in turn, global mass conservation cannot be assured. For highly distorted three-dimensional meshes it may be necessary to use a 2point quadrature C-matrix. With 2-point quadrature, it may be more advantageous to calculate the coefficient matrices once and store them instead of calculating them at each time step. The 2-point quadrature integration will then result in added storage requirements and input/output cost.

A comparison of 1- and 2-point quadrature schemes for both the lumped and consistent mass matrix (see Section 3.2.4 for definition of lumped mass matrix) and centered finite difference schemes was performed by Gresho et al. (1984). A Fourier analysis of the constant-velocity, pure advection equation was performed to determine the effect of quadrature level and mass lumping on the advection matrix $(N)$. A Fourier analysis of the transient heat equation was performed to determine the effect on the diffusion matrix $(K)$. In summary, it was found that:

- A consistent mass matrix with 2-point quadrature is the most accurate scheme,

- The lumped mass matrix gives inferior results even compared to the centered finite difference scheme, 
- Mass lumping induces much larger errors than those caused by reduced quadrature (however, careful choice of a time integration scheme can reduce this error in the advection problem), and

- The diffusion matrix can be singular with respect to $2 \Delta x$ waves ('zero energy modes') with 1-point quadrature. (The use of hour-glass correction to deal with this problem is discussed in Section 3.2.6).

In the existing code to be used in this study, the integrals in the coefficient matrices are computed using 1-point quadrature with a lumped mass matrix (described in Section 3.2.4).

\subsubsection{Time Integration}

An implicit integration scheme is commonly used with a consistent 'mass' matrix, $\boldsymbol{M}$ (Gresho 1985). The $\boldsymbol{M}$-matrix is symmetric, positive-definitive, sparse, and banded. To avoid solving the linear system of equations and to keep the pressure matrix sparse, $\boldsymbol{M}$ can be diagonalized by 'mass lumping'. Mass lumping decouples the time derivative and makes easy the use of explicit time integration. A lumped mass matrix will be used for this study. The mass lumping is done by summing rows at the element level. For quadrilateral elements

$$
m_{i i}^{e}=\sum_{j} \int_{\Omega_{d}} \phi_{i} \phi_{j}=\int_{\Omega_{c}} \phi_{i} \sum_{j} \phi_{j}=\int_{\Omega_{c}} \phi_{i}, \text { since } \sum_{j} \phi_{j}=1
$$

In general, 


$$
m_{i j}^{e}=\delta_{i j} \int_{\Omega_{e}} \phi_{i}
$$

With $M$ diagonalized, the calculation of its inverse, $M^{-1}$, is trivial. Mass lumping is frequently used in practice. Mass lumping may reduce accuracy, especially for highly transient advection-dominated flow, but it also reduces run time cost. It requires less work per element and per time step and one can usually maintain desired accuracy by finer discretization (Gresho et al. 1984).

To solve the system of matrix equations 3.6 and 3.7 we first compute

$$
\begin{aligned}
& A^{n} \equiv M^{-1}\left[\left(K^{n}+N\left(\bar{u}^{n}\right)\right) \bar{u}^{n}-F\left(\bar{u}^{n}\right)\right] \\
& \left(C^{T} M^{-1} C\right) \bar{P}^{n}=C^{T} A^{n}
\end{aligned}
$$

and then solve for $\overline{\boldsymbol{P}}^{n}$ using a skyline solver.

An explicit time integration scheme was used for this study. Rearranging the momentum equation 3.7 to the form

$$
M \dot{\bar{u}}=-(K+N(\bar{u})) \bar{u}+F+C \bar{P}
$$

and multiplying through by $M^{-1}$,

$$
\dot{\bar{u}}=M^{-1}[-(K+N(\bar{u})) \bar{u}+F+C \bar{P}]
$$

and using a forward (explicit) Euler scheme gives 


$$
\bar{u}^{n+1}=\bar{u}^{n}+\delta t M^{-1}\left[-\left(K^{n}+N\left(\bar{u}^{n}\right)\right) \bar{u}^{n}+F^{n}+C \bar{P}^{n}\right],
$$

or

$$
\bar{u}^{n+1}=\bar{u}^{n}-\delta t\left[A^{n}-M^{-1} C \bar{P}^{n}\right]
$$

The discrete pressure Poisson equation ensures that if $C^{T} \overline{\boldsymbol{u}}^{n}=0$, then $C^{T} \bar{u}^{n+1}=0$ or more precisely, $C^{T} \bar{u}^{n}=$ constant for all $n$. This can be shown by substituting

$$
\bar{P}^{n}=\left[C^{T} M^{-1} C\right]^{-1} C^{T} A^{n}
$$

from the pressure Poisson equation 3.6, into the equation for $\bar{u}^{n+1}$ and multiplying by $C^{T}$ :

$$
\begin{aligned}
C^{T} \bar{u}^{n+1} & =C^{T} \bar{u}^{n}+C^{T} \delta t\left(A^{n}-M^{-1} C\left[C^{T} M^{-1} C\right]^{-1} C^{T} A^{n}\right) \\
& =0+\delta t\left(C^{T}-C^{T} M^{-1} C\left[C^{T} M^{-1} C\right]^{-1} C^{T}\right) A^{n} \\
& =0+\delta t\left(C^{T}-C^{T}\right) A^{n}=0
\end{aligned}
$$

Therefore, the initial velocity field must be divergence free so that $C^{T} \overline{u\left(t_{0}\right)}=0$ (i.e., it must satisfy the discrete divergence free condition).

Part of the discussion in Section 3.2.7 on subcycling describes a scheme for generating a mass-consistent velocity field. This same scheme is used in the existing code to generate a valid initial velocity condition that is discretely divergence-free (i.e., 
$\dot{C}^{\top} \bar{u}^{\circ}=0$ ). The scheme is called a 'mass adjustment' and in Section 5.2, it is shown that this mass adjustment gives approximately a potential flow solution for the initial condition, however, it does preserve vorticity.

With the above time integration scheme, the time step for stability is determined by either the Courant limit $(\Delta t<\Delta / V$ where $\Delta$ is the mesh size and $V$ is the characteristic velocity) or the diffusion limit $\left(\Delta t<\Delta^{2} / 2 v\right.$ where $v$ the kinematic viscosity).

It can be shown that the explicit forward Euler scheme effectively reduces the diffusivity. Gresho et al. (1984), show that for the laminar, transient advectiondiffusion equation, the diffusion matrix element $k_{i j}$ is reduced to $k_{i j}-u_{i} u_{j} \Delta t / 2$ (which is independent of mesh size). This negative diffusion term results from the discretization of the advection term. The reduction in diffusivity can be counterbalanced by the implementation of the so-called 'balancing tensor diffusivity', which is discussed in Section 3.2.6 (or see Gresho et al. 1984).

\subsubsection{Boundary Conditions}

Specification of a natural boundary condition for velocity corresponds to specifying a value or function for $f_{\alpha}$ where (as given in Section 3.2.1)

$$
f_{\alpha}=\bar{\tau}_{\alpha \beta} n_{\beta}+\gamma_{(\alpha)} \overline{u_{\alpha}}=-\frac{\bar{p}}{\rho} n_{\beta} \delta_{\alpha \beta}+v n_{\beta} \frac{\partial \bar{u}_{\alpha}}{\partial x_{\beta}}-n_{\beta}\left(\overline{u_{\alpha} \bar{u}_{\beta}}+\overline{\bar{u}_{\alpha} \bar{u}_{\beta}}+\overline{u_{\alpha} \bar{u}_{\beta}}\right)+\gamma_{(\alpha)} \bar{u}_{\alpha}
$$

and $n_{\beta}$ is the unit surface normal. The last term in $f_{\alpha}$ is included so that essential boundary conditions (i.e., specified value for variable $\bar{u}_{\alpha}$ or as sometimes called, 
Dirichlet boundary conditions) can be conveniently implemented using the natural boundary condition technique. Thus, input to the code for the $\bar{u}_{\alpha}$ natural boundary condition is a value or function for $\bar{\tau}_{\alpha \beta} n_{\beta}$ for the boundary with the unit normal $n_{\beta}$.

To clarify the above description, let us look at the natural boundary conditions for a vertical two-dimensional boundary (planar problem). For example,

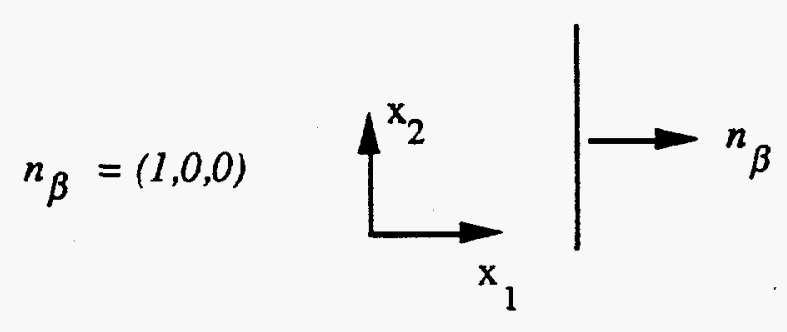

The $\bar{u}_{1}$ natural boundary condition is

$$
\begin{aligned}
f_{1} & =\bar{\tau}_{1 \beta} n_{\beta}+\gamma_{1} \bar{u}_{1}=\left(\bar{\tau}_{11}, \bar{\tau}_{12}, \bar{\tau}_{13}\right) \cdot(1,0,0)+\gamma_{1} \bar{u}_{1}=\tau_{11}+\gamma_{1} \bar{u}_{1} \\
& =-\frac{\bar{p}}{\rho}+v \frac{\partial \bar{u}_{1}}{\partial x_{1}}-\left(2 \overline{u_{1} \bar{u}_{1}}+\overline{u_{1} u_{1}}\right)+\gamma_{1} \bar{u}_{1} .
\end{aligned}
$$

The $\bar{u}_{2}$ natural boundary condition is

$$
f_{2}=\overline{\tau_{2 \beta}} n_{\beta}+\gamma_{2} \overline{u_{2}}=\overline{\tau_{21}}+\gamma_{2} \overline{u_{2}}=v \frac{\partial \bar{u}_{2}}{\partial x_{1}}-\left(\overline{u_{2} \bar{u}_{1}}+\overline{\bar{u}_{2} \bar{u}_{1}}+\overline{u_{2} u_{1}}\right)+\gamma_{2} \bar{u}_{2} .
$$

These boundary conditions can either be set to a constant value or a function of $x_{1}$ and $x_{2}$. If an essential boundary condition is desired, $\gamma_{(\alpha)}$ is set to a very large number (e.g., $10^{20}$ ), which sets $\bar{u}_{\alpha}$ to a specified value $\bar{u}_{\alpha}{ }^{s}$ (see Section 3.2.1). Otherwise, $\gamma_{(\alpha)}$ is set to zero. 
Although no pressure boundary conditions are required as input data to the code, it may be helpful to discuss the implied boundary conditions for pressure. With natural boundary conditions on velocity, the implied boundary condition for the pressure Poisson equation is defined by the normal component of the surface traction vector, $n$ $f$, (Gresho (1991)). The normal component for $f_{\alpha}$ is

$$
f_{n} \equiv n \cdot f=-\frac{\bar{p}}{\rho}+v \frac{\partial \bar{u}_{n}}{\partial n}-\left(2 \overline{u_{n} \bar{u}_{n}}+\overline{u_{n} u_{n}}\right)
$$

and solving for $\bar{p} / \rho$ we have

$$
\frac{\bar{p}}{\rho}=v \frac{\partial \bar{u}_{n}}{\partial n}-f_{n}-\left(2 \overline{u_{n} \bar{u}_{n}}+\overline{u_{n} \bar{u}_{n}}\right) .
$$

With Dirichlet boundary conditions on velocity, the implied (or 'built-in') boundary conditions for the pressure Poisson equation is a Neumann condition which is the normal component of the momentum equation at the boundary. (There are no boundary conditions on pressure to be specified, and the velocity solution determines the pressure only up to an arbitrary additive constant.) Gresho and Sani (1987) show that this is the correct boundary condition for all time, $t \geq 0$, (i.e., it provides a unique solution to the continuum Poisson equation for pressure). They also show that the Dirichlet boundary condition for the pressure Poisson equation, which is the tangential component of the momentum equation, is also satisfied and is essentially equivalent to the Neumann condition, but in general, is only appropriate for $t>0$.

The normal projection of the momentum equation (Navies-Stokes equations) onto the boundary $\partial \Omega$ is 


$$
n \cdot \nabla P \equiv \frac{\partial P}{\partial n}=v \nabla^{2} u_{n}-\left(\frac{\partial u_{n}}{\partial t}+u \cdot \nabla u_{n}\right) \text { on } \partial \Omega \text { for } t \geq 0
$$

where the density $\rho$ has been absorbed into the pressure $(P=p / \rho), n$ is the normal to $\partial \Omega$, and $u_{n}$ is the velocity component normal to $\partial \Omega$. For the LES formulation (equation 2.3) we have additional terms due to the SGS motion,

$$
\frac{\partial}{\partial x_{\beta}}\left(\overline{u_{\alpha} \bar{u}_{\beta}}+\overline{\bar{u}_{\alpha} \bar{u}_{\beta}}+\overrightarrow{u_{\alpha} \overline{u_{\beta}}}\right)
$$

Expanding these terms, before putting them in vector form, we have

$$
\overline{u_{\alpha} \frac{\partial \bar{u}_{\beta}}{\partial x_{\beta}}}+\overline{u_{\beta} \frac{\partial u_{\alpha}}{\partial x_{\beta}}}+\overline{u_{\alpha} \frac{\partial \dot{u_{\beta}}}{\partial x_{\beta}}}+\overline{\dot{u_{\beta} \frac{\partial \bar{u}_{\alpha}}{\partial x_{\beta}}}}+\overline{u_{\alpha} \frac{\partial \dot{u_{\beta}}}{\partial x_{\beta}}}+\overline{\dot{u_{\beta} \frac{\partial u_{\alpha}^{\prime}}{\partial x_{\beta}}}} .
$$

From mass continuity, the first, third, and fifth terms are zero, and thus, in vector form, we have

$$
\overline{(\bar{u} \cdot \nabla) \boldsymbol{u}^{\prime}}+\overline{\left(\boldsymbol{u}^{\prime} \cdot \nabla\right) \bar{u}}+\overline{\left(\boldsymbol{u}^{\prime} \cdot \nabla\right) \boldsymbol{u}^{\prime}}
$$

The normal projection of the LES momentum equation can then be written as

$$
n \cdot \nabla \bar{P} \equiv \frac{\partial \bar{P}}{\partial n}=v \nabla^{2} \bar{u}_{n}-\left(\frac{\partial \bar{u}_{n}}{\partial t}+\overline{\bar{u} \cdot \nabla \bar{u}_{n}}\right)-\left(\overline{\bar{u} \cdot \nabla \bar{u}_{n}}+\overline{u^{\prime} \cdot \nabla \bar{u}_{n}}+\overline{u^{\prime} \cdot \nabla u_{n}}\right)
$$

This is the implied pressure boundary condition for the LES formulation (equations 2.1 and 2.3) when Dirichlet boundary conditions are specified for velocity. Note that most of the right hand side terms are zero for solid walls because $\bar{u}$ and $\bar{u}$ are then zero. 


\subsubsection{Hour-Glass Matrix and Balancing Tensor Diffusivity}

When 1-point quadrature is used, so-called 'zero energy modes' may be present (Gresho et al. 1984). These zero energy modes are undiffused 'waves' that are null vectors of the diffusion matrix. In two dimensions, there is one $2 \Delta x$ by $2 \Delta y$ wave, which has alternating nodal values of \pm 1 . In three dimensions, there are four null vectors, one $2 \Delta x$ by $2 \Delta y$ by $2 \Delta z$ wave and three two-dimensional waves, all with nodal values of \pm 1 .

To insure diffusion of these waves, a so-called hour-glass matrix can be added to the 1-point quadrature diffusion matrix (Goudreau and Hallquist 1982 and Gresho et al. 1984). Gresho et al. (1984), showed that additional diffusion provided by the hourglass matrix compensates for the negative diffusion resulting from the spatial discretization scheme with 1-point quadrature. Gresho et al. (1984) comment that the current method may have difficulty with problems involving strong anisotropic diffusion and distorted meshes with large element aspect ratios.

Balancing tensor diffusivity (BTD) is a technique for applying an additive diffusivity (correction) to the diffusion matrix which balances the negative diffusion caused by the explicit Euler time integration. The forward-Euler scheme is unconditionally unstable if the diffusion matrix is zero $\left(k_{i j}=0\right)$, because its effective diffusivity is negative $\left(-u_{i} u_{j} \Delta t / 2\right)$. For laminar problems, the diffusion matrix $k_{i j}$ is replaced by $k_{i j}+u_{i} u_{j} \Delta t / 2$ (Gresho et al. 1984) and the added diffusivity is only in the streamline direction. The correction is applied at the element level at each time step.

Gresho et al. (1984) show for the advection-diffusion problem that the explicit 
Euler stability time step limits are relaxed with the use of BTD. The allowance of larger time steps with the use of BTD for multi-dimensional Navier-Stokes solutions is also seen in practice.

For the one-dimensional advection-diffusion equation with BTD (integrated in time with explicit Euler) Gresho et al. (1984) state that the local error is $O\left[\Delta x^{2}+k \Delta t+\Delta t^{2}\right]$ and the global error is $O\left[\Delta x^{2}+\Delta t^{2}\right]$. For the one- and twodimensional pure advection problem, the scheme is spatially fourth-order dissipative and second-order accurate in phase speed. Phase speed is the projection of the velocity vector onto the wave number vector. Overall, the time integration error increases both damping and phase speed. However, the phase speed is reduced by the spatial integration error, which helps to compensate for the time integration increase (the error compensation is better in one-dimensional solutions than in multi-dimensional solutions).

Gresho et al. (1984) also investigated the effect of applying both hour-glass correction and BTD to the diffusion term with the two-dimensional pure advection problem. The form of the diffusion coefficient for pure advection was $\sigma \Delta x \Delta y\left(c_{1}+c_{2}\right)^{2} / \Delta t$ with an 'optimal' value of $\sigma=1 / 32$ where $c_{1}=u_{1} \Delta t / \Delta x$ and $c_{2}=u_{2} \Delta t / \Delta x$ are the courant numbers. In summary, the hour-glass correction successfully damped the $2 \Delta x$ wave and the stability limit and phase speed were improved over that obtained with only BTD.

The steady state (or slow time-varying) simulation was also considered by Gresho et al. (1984). In summary, they found that with BTD, the added diffusivity is only in the streamline direction (as is also true for the transient case), the effective Reynolds number in the streamline direction is less than the desired one (which worsens 
with increase in characteristic velocity $(u$, where $R e=u L / v)$ and ratio of grid size to characteristic length $(\Delta x / L)$ ), and the added diffusivity suppresses wiggles (signals that warn user that too coarse a mesh is being used). These effects are only in the streamline direction, where in comparison, the effects of upwind differencing in a typical finite difference scheme are in all directions. Overall, it is recommended that BTD be used even when steady state solutions are sought.

\subsubsection{Subcycling}

To reduce run time, one can sometimes update the pressure field only when it has changed significantly, instead of at every time step. This is called subcycling. One method of subcycling, outlined by Gresho et al. (1984) (and in the code used for this study) involves the following:

1) Compute advection and diffusion only at minor time steps which are based on stability limits, with an extrapolated pressure gradient (the pressure at the minor steps are the linear extrapolation of the pressures from the previous two major time steps).

2) Reinforce continuity (i.e., adjust velocity field so it is divergence free) at each major time step, whose size is based on temporal accuracy.

3) Update the pressure field using the discrete pressure Poisson equation (see section 3.2.3).

4) Calculate the next major step size and return to step 1.

Mass consistency (the discrete continuity equation) is enforced only at major 
time steps. The velocity adjustment to a discretely divergence free field is made with minimum variation in the velocity field that was calculated during subcycling. Gresho et al. (1984), outline two equivalent techniques for adjusting the velocity. They are the following:

Method 1: Solve first for the vector of Lagrangian multipliers $\lambda$ with the velocity at the last minor time step at $t_{m+1}, \bar{u}_{s}^{m+1}$, using

$$
\left(C^{T} M^{-1} C\right) \lambda=C^{T} \bar{u}_{s}^{m+1}
$$

and then the mass-consistent velocity field is calculated from

$$
\bar{u}^{n+1}=\bar{u}_{s}^{m+1}-M^{-1} C \lambda
$$

Method 2: Perform an orthogonal projection of the velocity field at the last minor time step at $t_{m+1}, \bar{u}_{s}^{m+1}$, onto the subspace of vectors which is discretely divergence free. (The realization of this projection is, in fact, Method 1.)

Gresho et al. (1984) show that the two techniques are equivalent because the velocity adjustment of Method 1 and projection of Method 2 are both performed using the gradient of a scalar. The next major time step can be calculated based on the desired temporal accuracy (a specified error tolerance) and an estimate of the local error based on the last two major time step results.

Subcycling can prove to be very cost effective when the time step size for stability is much less than that for the required accuracy. 


\subsection{Finite Element Solution Approach}

The following sections briefly summarize the baseline numerical scheme that will be used to solve the turbulent backward-facing step problem with an LES approach. Also presented is the method that will be used for explicitly calculating the advection term.

\subsubsection{Baseline Method}

A modified Galerkin finite element method will be used with isoparametric quadrilateral (or in 3D, hexahedral) elements for modeling of the LES formulated equations 2.1 and 2.2. To reduce run time cost, a lumped mass matrix will be used and the coefficient matrices will be generated using 1-point quadrature. The hour-glass correction term and BTD terms used for the laminar flow case will be used for the LES formulation. The pressure Poisson equation will be solved directly with a skyline solver and an explicit forward Euler time integration scheme will be used for the velocity solution. Subcycling on the pressure field solution will be used when needed to reduce run time cost.

\subsubsection{Explicit Calculation of the Advection Term}

The advection term in the LES equations (equation 2.3) is

$$
\frac{\partial}{\partial x_{\beta}} \overline{\bar{u}_{\alpha} \bar{u}_{\beta}}
$$

or since we have a divergence free flow, we can write the advection term as 


$$
\overline{\bar{u}_{\beta} \frac{\partial \bar{u}_{\alpha}}{\partial x_{\beta}}}
$$

As discussed in Sections 2.4.3 and 2.4.4, the Leonard term, $\overline{\bar{u}}_{\alpha} \bar{u}_{\beta}$, has commonly been approximated as $\bar{u}_{\alpha} \bar{u}_{\beta}$, or with a truncated Taylor series expansion model of order $\Delta_{f}^{2}$ (i.e., Leonard's approximation). (As explained in section 2.4.3, $\bar{u}_{\alpha} \bar{u}_{\beta}=\bar{u}_{\alpha} \bar{u}_{\beta}$ is satisfied only if $\bar{u}_{\alpha}$ remains constant over the averaging or filtering domain.) Leonard (1974) showed that the advection term removes significant energy from the resolved scales. The Stanford group (e.g., Piomelli et al. 1987) believe that the advection term should not be lumped and modeled with the SGS terms (as in equation 2.2) but should be explicitly calculated. Using a pseudospectral method, the Stanford group have seen model improvement by explicitly computing for the advection term. For example, Piomelli et al. (1987) evaluated the advection term by first performing the velocity product, $\bar{u}_{\alpha} \bar{u}_{\beta}$, in physical space, and then used the Fourier transform to compute the derivative of the product, and finally, filtered the derivative in Fourier space.

One of the objectives of this research is to show that the advection term can be explicitly calculated with a finite element approach, so that it is possible to choose a filter width independently of the grid size, as is done with spectral methods. In Section 3.2.1, it was shown that the finite element method, in general, allows for the 'exact' solution of the advection term and the filtering method and the filter width may be chosen. However, because of constraints imposed by the existing code and limited computer resources, some simplification in the solution of the advection term is made here. The following describes the form of the explicitly calculated advection term and how it is solved in the finite element approach used in this research. 
In the weak form of the momentum equation (equation 3.2), the advection term is

$$
\int_{\Omega} v \frac{\partial}{\partial x_{\beta}} \overline{\bar{u}_{\alpha} \bar{u}_{\beta}}
$$

which, for the divergence free flow field, can be written as

$$
\int_{\Omega} v \overline{\bar{u}_{\beta} \frac{\partial \bar{u}_{\alpha}}{\partial x_{\beta}}}
$$

As shown in Section 3.2.1, using the finite element approach, the advection term has the form

$$
\left(\bar{u}_{\beta}^{k} \int_{\Omega} \phi_{i} \frac{\partial}{\partial x_{\beta}} \overline{\phi_{j} \phi_{k}}\right) \bar{u}_{\alpha}^{j}
$$

(see equation 3.4 or 3.5 ) or we could write, for the divergence free flow field,

$$
\left(\bar{u}_{\beta}^{k} \int_{\Omega} \phi_{i} \overline{\phi_{k} \frac{\partial \phi_{j}}{\partial x_{\beta}}}\right) \bar{u}_{\alpha}^{j}
$$

Thus, we can evaluate the advection term by calculating

$$
\frac{\partial}{\partial x_{\beta}} \overline{\phi_{j} \phi_{k}} \text { or } \overline{\phi_{k} \frac{\partial}{\partial x_{\beta}} \phi_{j}}
$$

which is dependent only on the geometry (i.e., basis functions) and the filter width. Thus, these terms need only be calculated once. However, the solution of the advection 
term in the above form can be expensive because of the integration of a triple product (i.e., the summation over $\beta$ where $\beta=1,2,3$ for three dimensions). The explicit solution of the momentum equation using the values of the velocity components from the previous time step decouples the equations. To reduce the triple product to a double product, Gresho et al (1984) suggest a simplification using what they call a 'centroid advection velocity', in which, at the element level

$$
\bar{u}_{\beta}^{e} \equiv\left[\sum_{k=1}^{N} \bar{u}_{\beta_{k}}^{e} \phi_{k}\right]_{\text {centroid of } \Omega}
$$

For piecewise linear basis functions, $\phi_{i}^{e}$ at the element centroid is $1 / 2^{n}$, and thus,

$$
\bar{u}_{\beta}^{e} \equiv \frac{1}{2^{n}} \sum_{k=1}^{2^{n}} \bar{u}_{\beta_{k}}^{e}
$$

where $n$ is the number of dimensions and $2^{n}$ is the number of nodes per element. We can then bring $\bar{u}_{\beta}^{e}$ outside the integral. Note that $\bar{u}_{\beta}^{e}$ is simply the average of the nodal values. Gresho et al. (1984) point out that when one-point Gaussian quadrature integration is used, the simplified and nonsimplified advection terms give equivalent results. (One-point quadrature is used herein.)

Using the centroid advection velocity simplification with the LES approach, $\bar{u}_{\beta}^{e}$ is constant over the element and thus, it would appear that $\overline{\bar{u}_{\beta}^{e}}=\bar{u}_{\beta}^{e}$ and $\overline{\bar{u}_{\alpha}^{e} \bar{u}_{\beta}^{e}}=\bar{u}_{\alpha}^{e} \bar{u}_{\beta}^{e}$ for a filter width equivalent to the grid size. The advection term is developed below for the case of the filter width chosen equivalent to the grid size with the centroid advection velocity simplification. It is shown that $\overline{\bar{u}}_{\alpha} \bar{u}_{\beta}=\bar{u}_{\alpha} \bar{u}_{\beta}$ if one-point quadrature is used in conjunction with the centroid advection velocity simplification. It should be 
emphasized that $\overline{\bar{u}_{\alpha} \bar{u}_{\beta}}=\bar{u}_{\alpha} \bar{u}_{\beta}$ is not true in general for the finite element method, but is due to the centroid advection velocity simplification along with one-point quadrature integration.

In using the centroid advection velocity simplification and one-point quadrature, if the filter width is chosen to be greater than the grid size, $\overline{\bar{u}_{\alpha} \bar{u}_{\beta}}=\bar{u}_{\alpha} \bar{u}_{\beta}$ is no longer true. The advection term is developed below for the finite element method for piecewise linear basis functions, one-point quadrature, and with the centroid advection velocity simplification, but for the general case of a chosen filter width. However, it is assumed that the filter width encompasses whole elements (i.e., fractions of elements are not considered). For comparison, the advection term is also developed with the initial assumption that $\overline{\bar{u}}_{\alpha} \bar{u}_{\beta}=\bar{u}_{\alpha} \bar{u}_{\beta}$ before putting the term in the discretized finite element form.

We consider first the approximation for the advection term (i.e., $\overline{\bar{u}_{\alpha} \bar{u}_{\beta}}=\bar{u}_{\alpha} \bar{u}_{\beta}$ )

$$
\int_{\Omega} v \bar{u}_{\beta} \frac{\partial \bar{u}_{\alpha}}{\partial x_{\beta}}
$$

We first substitute for $\bar{u}_{\alpha}$ the expansion $\bar{u}_{\alpha}=\bar{u}_{\alpha}^{j} \phi_{j}$, ignoring $\bar{u}_{\beta}$ for now, setting $\mathrm{v}=\phi_{i}$ (i.e., GFEM), and considering the element assembly, we have

$$
\left(\int_{\Omega} \phi_{i} \bar{u}_{\beta} \frac{\partial \phi_{j}}{\partial x_{\beta}}\right) \bar{u}_{\alpha}^{j}=\left[\sum_{e} \int_{\Omega_{e}} \phi_{i}^{e} \bar{u}_{\beta}^{e} \frac{\partial \phi_{j}^{e}}{\partial x_{\beta}}\right] \bar{u}_{\alpha}^{j}
$$

where $\Omega$, is the domain of element $e$ and $\sum_{e}$ symbolizes the element assembly to form the global matrix. Using the centroid advection velocity simplification for piecewise 
linear basis functions, we can then pull $\bar{u}_{\beta}^{e}$ outside the integral arriving at

$$
\int_{\Omega_{e}} \phi_{i}^{e} \bar{u}_{\beta}^{e} \frac{\partial \phi_{j}^{e}}{\partial x_{\beta}} \cong \frac{1}{2^{n}} \sum_{k} \bar{u}_{\beta_{k}}^{e} \int_{\Omega_{e}} \phi_{i}^{e} \frac{\partial \phi_{j}^{e}}{\partial x_{\beta}}
$$

Going one step further away from GFEM by using one-point Gaussian quadrature, the integral is reduced to the value of the integrand at the centroid times the area of the domain of integration. The result is

$$
\int_{\Omega_{e}} \phi_{i}^{e} \bar{u}_{\beta}^{e} \frac{\partial \phi_{j}}{\partial x_{\beta}} \cong \frac{1}{2^{n}} \sum_{k} \bar{u}_{\beta_{k}}^{e}\left[\phi_{i}^{e} \frac{\partial \phi_{j}^{e}}{\partial x_{\beta}}\right]_{\text {centroid of } \Omega_{e}}^{\Omega_{e}}
$$

Thus, the final form of the approximated advection term is

$$
\left(\int_{\Omega} \phi_{i} \bar{u}_{\beta} \frac{\partial \phi_{j}}{\partial x_{\beta}}\right) \bar{u}_{\alpha}^{j}=\left[\sum_{e}\left(\frac{1}{2^{n}}\right)^{2} \sum_{k} \bar{u}_{\beta_{k}}^{e}\left[\frac{\partial \phi_{j}^{e}}{\partial x_{\beta}}\right]_{\text {centroid of } \Omega_{e}}\right]_{\alpha} \bar{u}_{\alpha}^{j}
$$

Now considering the 'exact' advection term (i.e., the general situation where $\overline{\bar{u}}_{\alpha} \bar{u}_{\beta} \neq \bar{u}_{\alpha} \bar{u}_{\beta}$ ), we again substitute for $\bar{u}_{\alpha}$ the expansion $\bar{u}_{\alpha}=\bar{u}_{\alpha}^{\mathrm{j}} \phi_{\mathrm{j}}$, set $v=\phi_{i}$, and consider the element assembly, arriving at

$$
\left.\left(\int_{\Omega} \phi_{i} \overline{\bar{u}_{\beta} \frac{\partial \phi_{j}}{\partial x_{\beta}}}\right) \bar{u}_{\alpha}^{j}=\left[\sum_{e} \int_{\Omega_{e}} \phi_{i}^{e} \overline{\bar{u}_{\beta} \frac{\partial \phi_{j}}{\partial x_{\beta}}}\right]_{e}\right] \bar{u}_{\alpha}^{j} .
$$

Expanding the filtered part of the integrand using cell-volume averaging, we have 


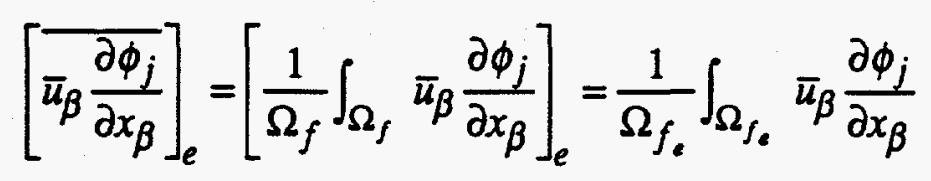

where $\Omega_{f_{e}}$ is the filtered domain size around element $e^{1}$ The domain $\Omega_{f_{e}}$ can be chosen to be any size greater than or equal to $\Omega_{e}$, but for ease of application we assume here that $\Omega_{f_{e}}$ encompasses whole elements. Thus,

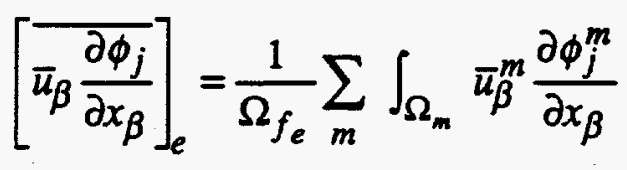

where the elements $m$ are the elements around and including element $e$ that are part of the domain $\Omega_{f_{e}}$. Using the centroid advection velocity simplification for the integral over each of the $m$ elements, we have

$$
\bar{u}_{\beta}^{m} \cong\left[\sum_{k} \bar{u}_{\beta_{k}}^{m} \phi_{k}^{m}\right]_{\text {centroid of } \Omega_{m}}=\frac{1}{2^{n}} \sum_{k}^{2^{n}} \bar{u}_{\beta_{k}}^{m}
$$

and substituting the above into the 'exact' advection term, the result is

$$
\left(\int_{\Omega} \phi_{i} \overline{\bar{u}_{\beta}} \overline{\partial \phi_{j}}\right) \bar{u}_{\alpha}^{j}=\left[\sum_{e} \int_{\Omega_{e}} \phi_{i}^{e}\left(\frac{1}{2^{n}}\right) \frac{1}{\Omega_{f_{e}}} \sum_{m}\left(\sum_{k} \bar{u}_{\beta_{k}}^{m}\right) \int_{\Omega_{m}} \frac{\partial \phi_{j}^{m}}{\partial x_{\beta}}\right] \bar{u}_{\alpha}^{j}
$$

If we perform one-point Gaussian quadrature on each of the $\Omega_{m}$ integrals and the $\Omega_{e}$ integral, we finally arrive at

${ }^{1}$ It should be noted that the bar operator in general can be any filtering scheme (e.g., those defined in Section 2.4.2). However, the cell-volume averaging scheme is used here, because it is the only one that has been developed for finite domains. 


$$
\left(\int_{\Omega} \phi_{i} \overline{u_{\beta} \frac{\partial \phi_{j}}{\partial x_{\beta}}}\right) \bar{u}_{\alpha}^{j}=\left[\sum\left(\frac{1}{2^{n}}\right)^{2} \frac{\Omega_{e}}{\Omega_{f_{e}}} \sum_{m}\left(\sum_{k} \bar{u}_{\beta_{k}}^{m}\right)\left[\frac{\partial \phi_{j}^{m}}{\partial x_{\beta}}\right]_{\text {cenoroid of } \Omega_{m}} \Omega_{\bar{u}_{\alpha}^{j}}\right.
$$

In comparison to the approximated advection term (equation 3.9), the 'exact' advection term (equation 3.11) includes coupling of the elements over the domain $\Omega_{f_{e}}$. These results can be shown to be consistent with the approximated advection term (equation 3.9) by setting $\Omega_{f_{e}}=\Omega_{e}$ in equation 3.11. In summary, for $\Omega_{f_{e}}=\Omega_{e}$ the 'exact' advection term with this finite element approach reduces to that for the approximated advection term. However, this is true only because of the combination of the use of the centroid advection velocity simplification along with one-point quadrature integration. This can be shown by backing up to the step where the centroid advection velocity simplification has been made, but before one-point quadrature is first applied (equation 3.10). Letting $\Omega_{f_{e}}=\Omega_{e}$ such that the summation over $m$ is over one element, $\Omega_{e}$, the integral in equation 3.10 reduces to

$$
\int_{\Omega_{e}} \phi_{i}^{e}\left[\overline{u_{\beta} \frac{\partial \phi_{j}}{\partial x_{\beta}}}\right]_{e} \equiv\left(\frac{1}{2^{n}}\right) \sum_{k} \bar{u}_{\beta_{k}}^{m} \int_{\Omega_{e}} \phi_{i}^{e}\left(\frac{1}{\Omega_{e}} \int_{\Omega_{e}} \frac{\partial \phi_{j}^{e}}{\partial x_{\beta}}\right)
$$

In comparison to equation 3.8 , where we approximate $\overline{\bar{u}_{\alpha} \bar{u}_{\beta}}=\bar{u}_{\alpha} \bar{u}_{\beta}$ before applying one-point quadrature, equation 3.12 has an integral of the derivative of the basis function over $\Omega_{e}$. Equations 3.8 and 3.12 are equivalent if

$$
\frac{1}{\Omega_{e}} \int_{\Omega_{e}} \frac{\partial \phi_{j}^{e}}{\partial x_{\beta}}=\frac{\partial \phi_{j}^{e}}{\partial x_{\beta}}
$$

which is true if $\partial \phi_{j}^{*} / \partial x_{\beta}$ is constant. However, if one-point quadrature is used, equations 3.8 and 3.12 reduce to the same form. For instance, using one-point 
quadrature, equation 3.8 reduces to

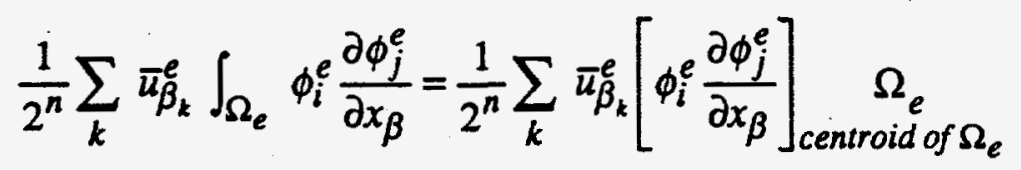

$$
\begin{aligned}
& =\left(\frac{1}{2^{n}}\right)^{2} \sum_{k} \bar{u}_{\beta_{k}}^{e}\left[\frac{\partial \phi_{j}^{e}}{\partial x_{\beta}}\right]_{\text {centroid of } \Omega_{e}},{ }_{e},
\end{aligned}
$$

and using one-point quadrature on equation 3.12 , we have

$$
\begin{aligned}
\frac{1}{2^{n}} \sum_{k} \bar{u}_{\beta_{k}}^{e} \int_{\Omega_{e}} \phi_{i}^{e}\left(\frac{1}{\Omega_{e}} \int_{\Omega_{e}} \frac{\partial \phi_{j}^{e}}{\partial x_{\beta}}\right) & =\frac{1}{2^{n}} \sum_{k} \bar{u}_{\beta_{k}}^{e} \int_{\Omega_{e}} \phi_{i}^{e}\left[\frac{\partial \phi_{j}^{e}}{\partial x_{\beta}}\right]_{\text {centroid of } \Omega_{e}} \\
& =\left(\frac{1}{2^{n}}\right)^{2} \sum_{k} \bar{u}_{\beta_{k}}^{e}\left[\frac{\partial \phi_{j}^{e}}{\partial x_{\beta}}\right]_{\text {cencoid of } \Omega_{e}} \Omega_{e}
\end{aligned}
$$

which is the same result for both equations. Thus, for the case of the centroid advection velocity used in conjunction with one-point Gaussian quadrature and a chosen filter width at the grid size, the resulting advection term (equation 3.11 ) is equivalent to the assumption that $\overline{\bar{u}_{\alpha} \bar{u}_{\beta}}=\bar{u}_{\alpha} \bar{u}_{\beta}$. However, if the filter width is greater than the grid size, then $\overline{\bar{u}_{\alpha} \bar{u}_{\beta}}=\bar{u}_{\alpha} \bar{u}_{\beta}$ is not implied by equation 3.11 .

In this research, the centroid advection velocity simplification is used with onepoint Gaussian quadrature (equation 3.11), and filter widths at the grid size and greater than the grid size are chosen. To separate the turbulence model from the numerics, the Stanford group recommends that for the case of homogeneous isotropic flow the filter width $\Delta_{f}$ should be at least two times the grid size $\Delta$. Since $v_{T}$ is an element centered quantity, choosing $\Delta_{f}=2 \Delta$ would require that we include contributions from parts of 
elements (see Figure 3.1). Thus, for ease in calculation, we choose $\Delta_{f}=3 \Delta$. More specifically, for the two-dimensional results presented here we use $\Omega_{f_{e}}=3 \Delta x 3 \Delta y$ such that $\Delta_{f}=\sqrt{3 \Delta x 3 \Delta y}=3 \sqrt{\Delta x \Delta y}=3 \Delta$. (In three or one dimensions, we would also have $\Delta_{f}=3 \Delta$.)

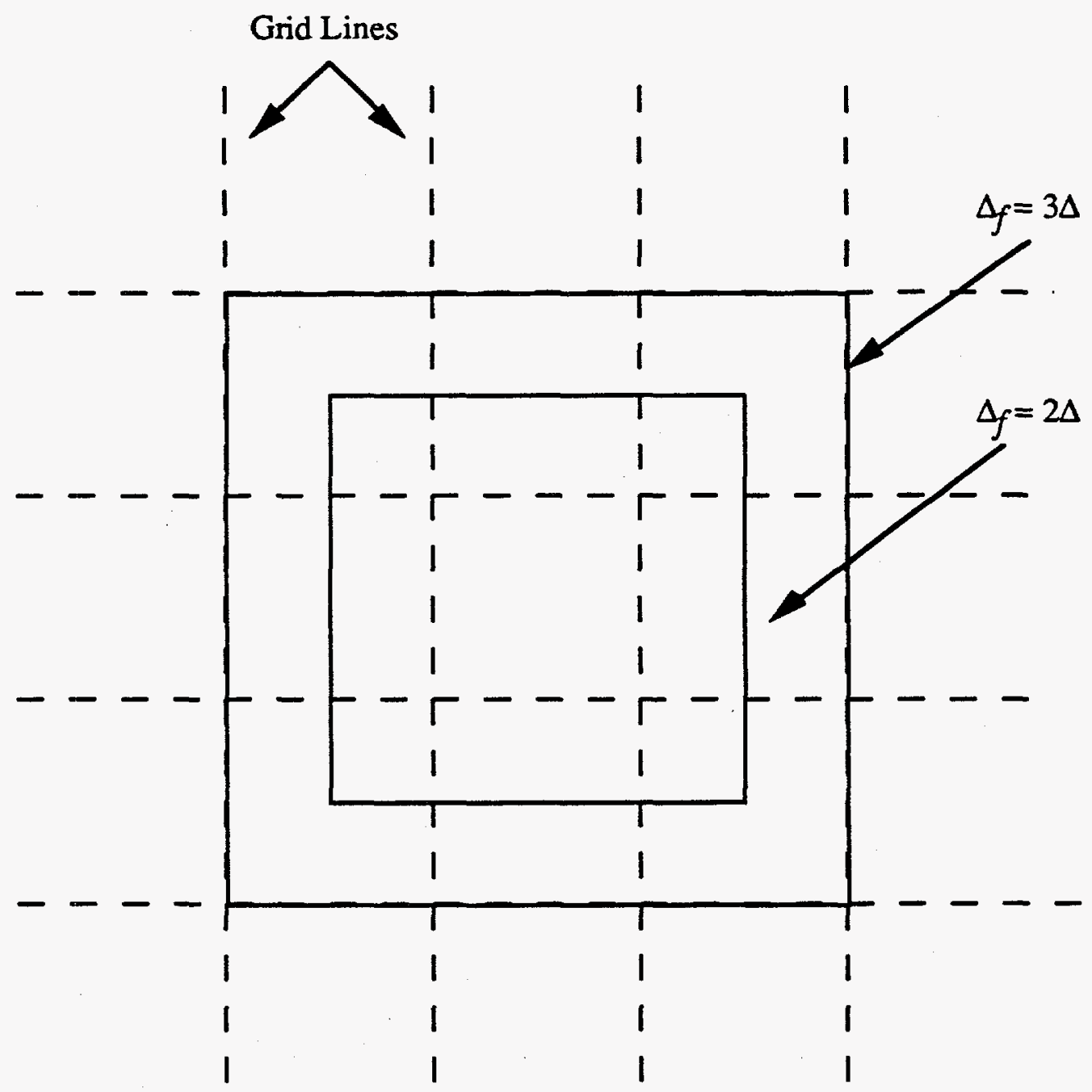

Figure 3.1 Sketch of filter width choices $\Delta_{f}=2 \Delta$ and $\Delta_{f}=3 \Delta$. 


\section{Method and Code Validation}

The following is a description of the approach used for method and code validation. The goal is to evaluate both the numerical and turbulence modeling approaches used in this study by comparison to equivalent or more accurate data.

Before discussing the specifics of the validation approach, some general comments regarding LES results should be noted.

(i) The statistical information that can be extracted from the LES are mean field quantities, resolved and SGS turbulent stresses, one-dimensional spectra of resolved and SGS energy, and turbulence intensities (rms of the velocity fluctuations). Geometric characteristics may also be determined from LES results (e.g., the point of reattachment for recirculation regions). However, these quantities must be obtained through time, space, or ensemble averaging which can require excessive computer usage.

(ii) The filtered (resolved) LES results should be compared with the filtered results from direct simulations or experimental data.

(iii) The SGS turbulent stresses may be large (especially when the computational grid is coarse). Thus, if total turbulent stresses are desired from the LES results, it is necessary to add the SGS turbulent stresses to the resolved-field turbulent stresses to obtain the total turbulent stress (Reynolds 1989). Thus, 


$$
\left.<u_{\alpha} u_{\beta}\right\rangle=\left\langle\overline{u_{\alpha}} \overline{u_{\beta}}\right\rangle+\left\langle\overline{\bar{u}_{\alpha} \bar{u}_{\beta}}+\overline{u_{\alpha} \bar{u}_{\beta}}+\overline{u_{\alpha} \bar{u}_{\beta}}\right\rangle
$$

where $<>$ denotes an average (e.g., volume averages for homogeneous turbulence).

(iv) Comparison of the LES total energy to experimental data may not be possible with grid-based methods (as discussed in Section 2.4.2).

\subsection{The Laminar Case}

The first step in method and code validation is to exercise the existing code for the laminar backward-facing step. The effects of mesh refinement are evaluated for the laminar case for two-dimensional calculations. The results are compared to the experimental results of Armaly et al. (1983) and the numerical results of Kim and Moin (1985), Durst and Pereira (1988), and the recent benchmark solution of Gartling (1990) (see Section 5.0). This establishes a baseline case (i.e., code accuracy in absence of turbulence models).

\subsection{Comparison with Experimental Data or Reynolds-Average Results}

In Section 1.3, the experimental results of Kim et al. (1980) and Eaton and Johnston (1980) for a backward-facing step are described. Also discussed in Section 1.3 are the methods used by LES researchers to validate and verify their codes by comparison to this experimental data. These experimental results and others (e.g., Adams and Johnston 1988b and Berbee and Ellzey 1989) found in the literature give only qualitative results for the instantaneous flow field and the methods used to obtain 
the mean quantities result in large error bands. It is this author's opinion that the experimental mean flow data is not adequate by itself for validation and verification of the instantaneous flow field predicted by an LES code. Time averaging of the LES instantaneous result is also very computationally expensive. More information on the instantaneous flow field is needed.

As described in Section 2.5, the calculations in this study.are performed for a two-dimensional backward-facing step which can not be validated by comparison to three-dimensional experimental results. In developing the two-dimensional equations in Section 2.5.2, we made the assumptions that $\bar{u}_{3}=0$ and $\partial \bar{u}_{1} / \partial x_{3}=\partial \bar{u}_{2} / \partial x_{3}=0$. In reality, these terms are not negligible, and can even be quite significant. Even the timeaveraged LES results should not be expected to compare to the time-averaged experimental results or the results from a Reynolds-average solution. With twodimensional Reynolds-average solutions of the backward-facing step, we obtain $\left\langle u_{1}\right\rangle$ and $\left\langle u_{2}\right\rangle$ assuming the $\left.\left.<u_{3}\right\rangle=0, \partial<u_{1}\right\rangle / \partial x_{3}=0$, and $\left.\partial<u_{2}\right\rangle / \partial x_{3}=0$. These terms have been shown by experiment to be small. Therefore, the two-dimensional Reynoldsaverage results can be compared to experiment. The two-dimensional assumption for the backward-facing with the LES approach is not consistent with reality because $\bar{u}_{3}, \partial \bar{u}_{1} / \partial x_{3}$ and $\partial \bar{u}_{2} / \partial x_{3}$ are not negligible.

In this study, for one Reynolds number case $\left(\operatorname{Re}_{k}=10,000\right.$ based on the average velocity at the step and the downstream channel height) the 'direct' simulation result (described in Section 4.4) and the LES result that most closely compares to the 'direct' simulation are time averaged to arrive at statistically steady results. $\operatorname{Re}_{h}=10,000$ was chosen because the flow should be well within the turbulent regime (see Armaly et al. 1983) and the Reynolds number should be low enough that an adequate DS can be obtained with the available computure resources. The predicted 
recirculation zone length from these time averaged results will be reported and compared (as discussed in Section 4.3), but because these calculations are twodimensional, they can not be verified by comparison to three-dimensional experimental results.

\subsection{Comparison with Direct Simulation}

For validation of LES results, more information on the instantaneous flow field is needed than that provided by experimental data or calculations with a Reynoldsaverage model. An 'established' direct simulation for comparison to LES results would be ideal. The LES results could then be compared to the direct simulation results by filtering the latter. However, the available direct simulation results are limited to low Reynolds number flows without flow separation. Kondoh and Nagano (1989) have published what they call a direct simulation for the backward-facing step. They used third-order upwind finite difference schemes in their calculations, which introduce fourth-derivative error terms that may play the role of an SGS model. Ferziger (1990) states that such results using third-order schemes should be interpreted as large-eddy simulations. Results from the Stanford/NASA Ames Center for Turbulence Research for $\operatorname{Re}>2000$ based on step height and a mean inlet velocity have been reported in an abstract (Le et al. 1990), but the results have not been published.

As mentioned in Section 1.3, Neto et al. (1991b) compare their LES results to what they call a 'direct' simulation. Their direct simulation is generated using the same code as that used to generate the LES results but without any model or approximation for the small-scale motion (i.e., the Navier-Stokes equations without averaging or filtering). This approach will be used here for validation of the LES results generated in this study. The direct simulation results generated with the same code used to generate 
the LES results are described in Section 5.2. The direct simulation results are filtered before comparison to the LES results. Specifically, velocity time histories at chosen points in the flow and a time series of streamline plots (i.e., snapshots of the instantaneous results) for the unfiltered and filtered direct simulation and LES results for grid-size and greater-than-grid-size filter widths are compared. Also included are power spectrum calculations for the velocity time histories. 


\section{Discussion of Results}

The following sections include discussions of the computational results for flow over a backward-facing step. The goal is to evaluate both the numerical and turbulence modeling approaches used in this study.

Section 5.1 is a discussion of the numerical results for the case of laminar flow over a backward-facing step. The method and code are verified for the laminar case by comparing the numerical results from this study to the numerical and experimental results of other researchers.

Sections 5.2 and 5.3 discuss the results for turbulent flow over a backwardfacing step. All calculations were performed in two dimensions. The 'direct' simulation generated with the same code used to generate the LES results is described in Section 5.2. This accurate solution was obtained by using a very fine computational mesh and by not including a turbulence model. The LES results are presented and compared to the 'direct' simulation in Section 5.3.

\subsection{The Laminar Case}

The first step was to exercise the existing code for laminar flow over a backward-facing step for the purpose of method and code verification without the presence of a turbulence model. Figure 5.1.1 shows our two-dimensional results for Reynolds number $(\mathrm{Re})$ of 648 and 1000 , based on the inlet channel hydraulic diameter 
and the average inlet velocity. ${ }^{1}$ Plotted are the distances from the step to the point of reattachment of the step recirculation zone $\left(x_{1}\right)$ and the points of separation $\left(x_{2}\right)$ and reattachment $\left(x_{3}\right)$ of the top-wall recirculation zone. Also shown on the plots are the numerical and experimental results of other researchers (Durst and Pereira (1988), Ghia et al. (1987), Mansour et al. (1983), and Armaly et al. (1983)).

The calculations at $\mathrm{Re}=1000$ were performed for the $60 \times 200$ mesh and geometry shown in Figure 5.1.2. Calculations for $R e=1000$ were also performed for a $30 \times 100$ mesh with the same geometry (i.e., same grading but with a quarter of the nodes). A parabolic velocity profile was specified for the inlet conditions (Figure 5.1.3) and zero natural boundary conditions were specified at the outlet. A streamline plot (Figure 5.1.4) clearly shows the two recirculation zones. A closer look reveals another small recirculation zone at the base of the step (Figure 5.1.5). The point of separation and reattachment of the recirculation zones was determined by calculating the vorticity along the upper and lower walls of the channel (Figure 5.1.6). The separation or reattachment point is the location where the vorticity is zero (i.e., zero shear stress). ${ }^{2}$ By refining the mesh from $30 \times 100$ to $60 \times 200$, the calculated separation and

${ }^{1}$ From Armaly et al. (1983), $\operatorname{Re}=V D / v$ where $V=2 U_{\max } / 3$ and $U_{\max }$ is the measured velocity at the center of the inlet channel $1 \mathrm{~cm}$ upstream of the step, $D=2 h$ where $h$ is the inlet channel height (i.e., $D$ is the inlet channel hydraulic diameter), and $v$ is the kinematic viscosity. The experimental results at the channel center plane show a parabolic velocity profile.

${ }^{2}$ The vorticity $\omega=\nabla \times \mathbf{v}$, and in two dimensions, $\omega=\left(\frac{\partial u_{2}}{\partial x_{1}}-\frac{\partial u_{1}}{\partial x_{2}}\right) i_{3}$ where $i_{3}$ is the unit vector in the $x_{3}$ direction. In two dimensions, the shear stress $\tau_{12}=\tau_{21}=v\left(\frac{\partial u_{2}}{\partial x_{1}}+\frac{\partial u_{1}}{\partial x_{2}}\right)$. At the wall, $\frac{\partial u_{2}}{\partial x_{1}}=0$ so that $\omega=\frac{\partial u_{1}}{\partial x_{2}}$ and $\tau_{12}=\tau_{21}=v \frac{\partial u_{1}}{\partial x_{2}}$. Separation is defined as the point where the shear stress is zero or in two-dimensions, when $\partial u_{1} / \partial x_{2}=0$, which is true for $\omega=0$ and only at steadystate. 
reattachment positions moved to close agreement with the numerical results of Durst and Pereira (1988) (Figure 5.1.1); however, the numerical results do not quantitatively agree with experiment. Kim and Moin (1985) suggest that the differences between the numerical and measured results are due to three-dimensional effects (i.e., the vertical side walls). To the author's knowledge, this hypothesis of three-dimensional effects has not been numerically verified (i.e., three-dimensional, laminar calculations for a backward-facing step have not been found in the published literature).

The calculations at $\operatorname{Re}=648$ were performed with two different mesh geometries. The first geometry was the same as that for the $\operatorname{Re}=1000$ case (Figure 5.1.2) with a $30 \times 100$ mesh. The second mesh geometry was a $58 \times 100$ mesh with a shorter inlet channel length and a shorter expanded channel length, but with no change in height (Figure 5.1.7). The geometry and inlet conditions (Figure 5.1.8) for the $58 \mathrm{x}$ 100 mesh geometry more closely match that used by Durst and Pereira (1988) for their numerical calculations. A streamline plot (Figure 5.1.9) shows the two recirculation zones, and the plots of vorticity along the upper and lower walls (Figure 5.1.10) show the separation and reattachment locations (i.e., where the vorticity or shear stress is zero). The results for the $58 \times 100$ mesh are in closer agreement with the numerical results of Durst and Pereira (1988) than the results for the $30 \times 100$ mesh. The results with the $58 \times 100$ mesh geometry are in good agreement with the numerical results of Durst and Pereira (1988); however, the numerical results do not quantitatively agree with the experiment (Figure 5.1.1).

A set of calculations were also performed for $\mathrm{Re}=800$ for comparison to the 'mesh converged' results of Gartling (1990). Two mesh geometries, 8,421 nodes (20 x 400 elements) and 32,841 nodes (40 x 800 elements), were used. (Gartling used 9 node elements, where as the code used in this study uses 4 node elements.) A description of 
the geometry and mesh is given in Figure 5.1.11. The boundary conditions used by Gartling and used here are the no-slip velocity condition for all solid surfaces. The inlet condition is a specified parallel flow condition with a parabolic horizontal component $u_{1}\left(x_{2}\right)=-12\left(2 x_{2}^{2}-3 x_{2}+1\right)$. A zero natural boundary condition was used for the outflow condition. A comparison of the calculated results is given in Table 5.1.1 and they are in good agreement with Gartling's benchmark results.

All of the above described solutions were obtained using the direct solver for the pressure and the forward (explicit) Euler time integration scheme (as described in Section 3.2.4). The calculations were initiated with a divergence free velocity field (i.e., the code performs a 'mass adjustment' ${ }^{1}$ before the first time step), and the calculations were advanced in time until a steady solution was achieved. Steadiness was determined from velocity time histories at several flow locations (see Appendix F) and from time histories of the separation and reattachment positions (i.e., the zero vorticity or shear stress positions). Subcycling on the pressure solution was used at early times in the solution to decrease computer usage. However, subcycling was not used during the final approach to the steady solution. Turning off the subcycling option eliminated small oscillations (see Gresho et al. 1984) in the final solution.

${ }^{1}$ The mass adjustment is performed the same way it is done for subcycling (see Section 3.2.7). The velocity adjustment to a discretely divergence free field is made with minimum variation in the specified initial velocity field. For details see Tuerpe et al. (1978). 


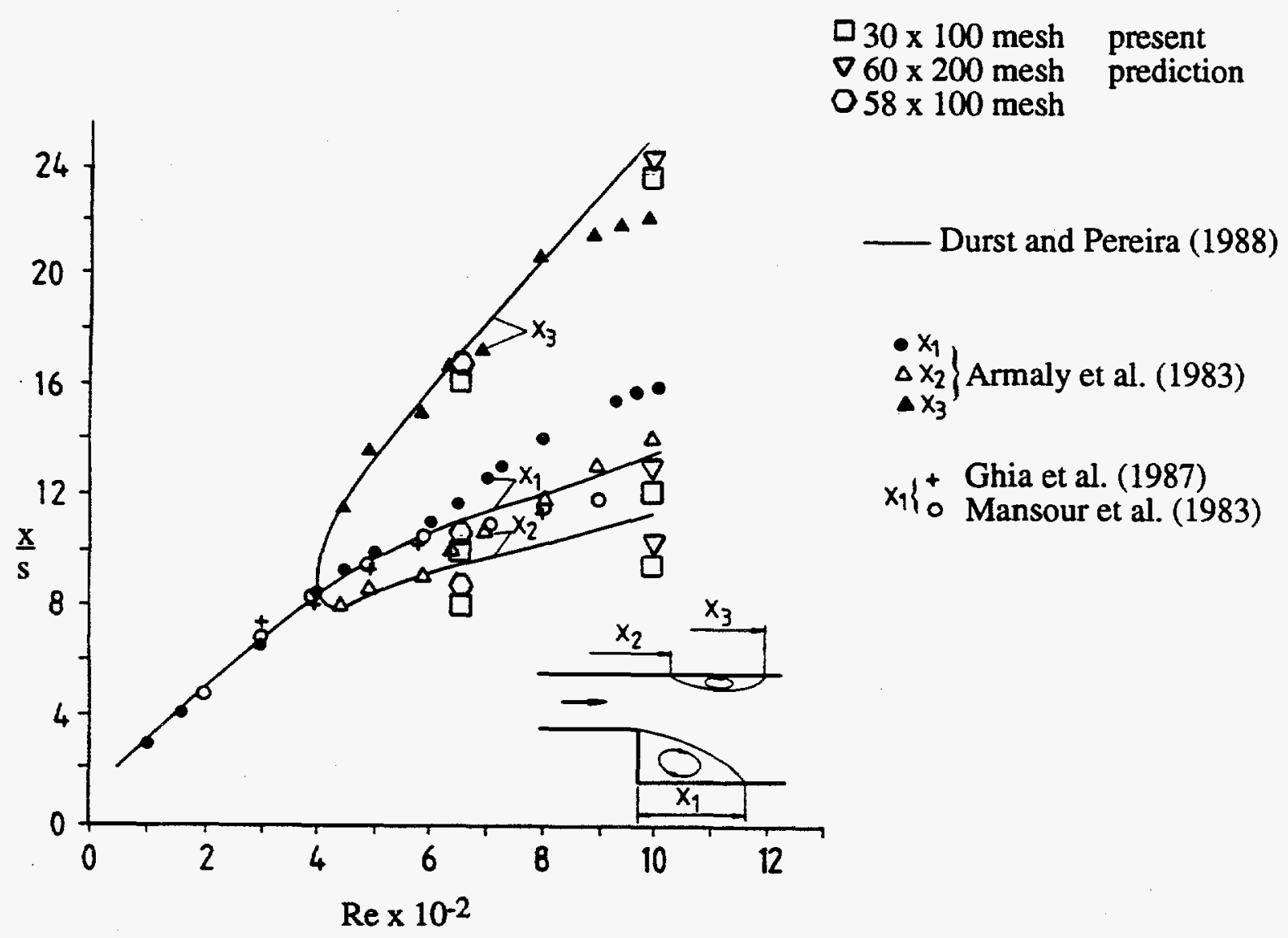

Figure 5.1.1 Comparison of experimental and predicted separation and reattachment points downstream of a backward-facing step. (Distances $x_{1}, x_{2}$, and $\mathrm{x}_{3}$ are measured from the step and $\mathrm{s}$ is the step height.) 


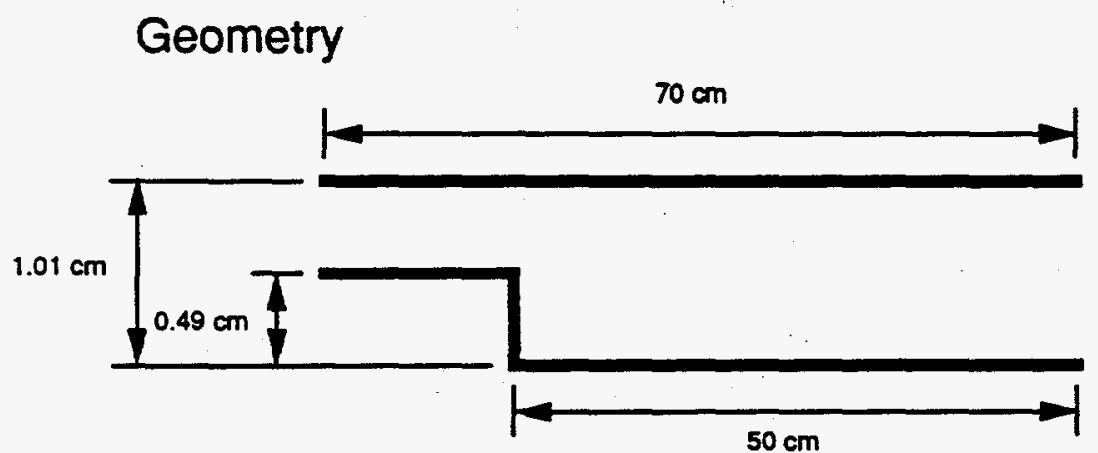

Logical Grid

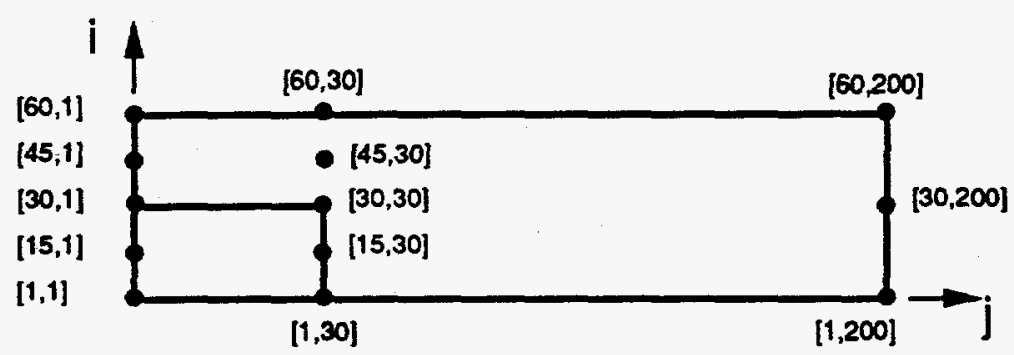

Details of Mesh Near Step

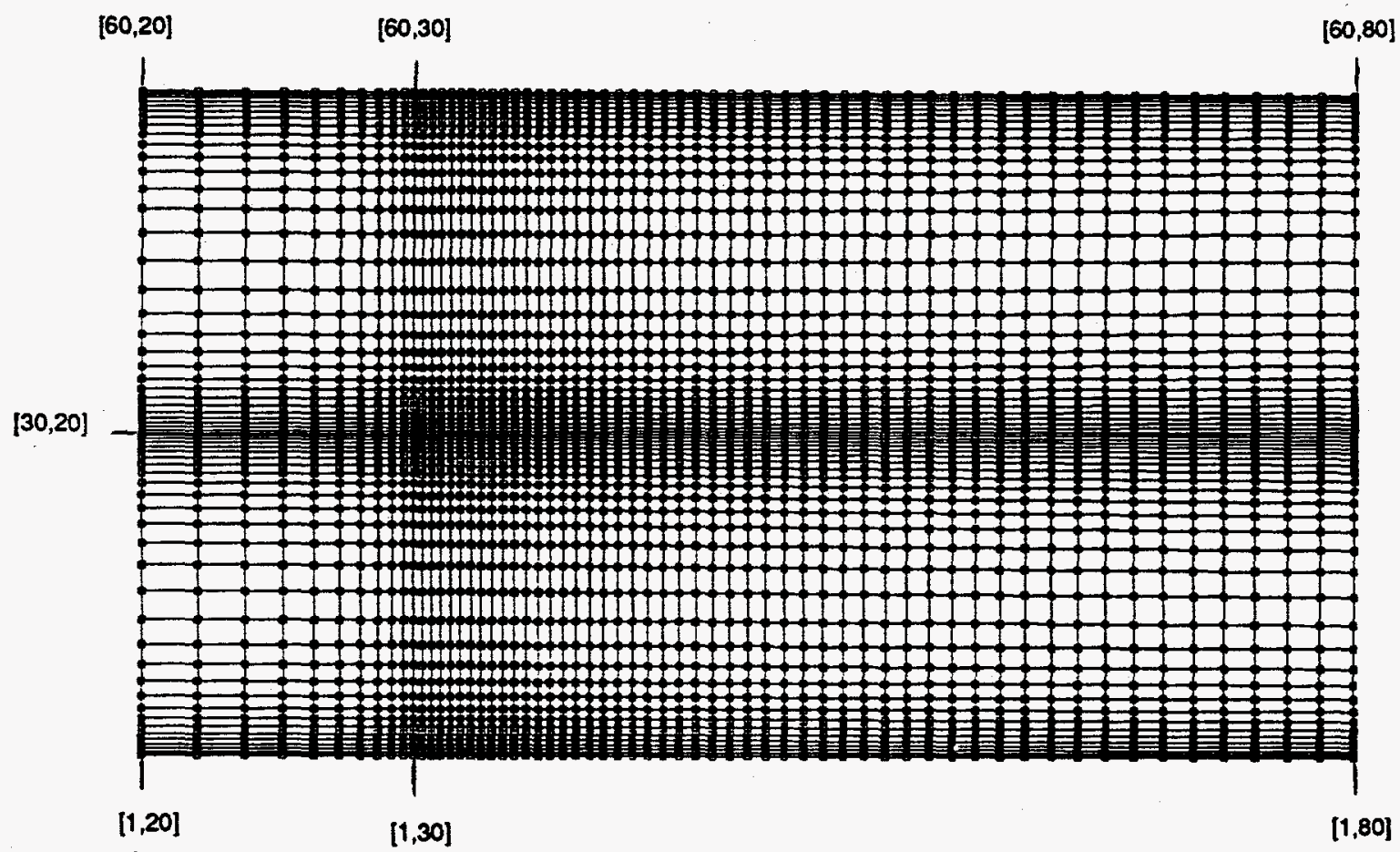

Figure 5.1.2 The $60 \times 200$ mesh and geometry. 


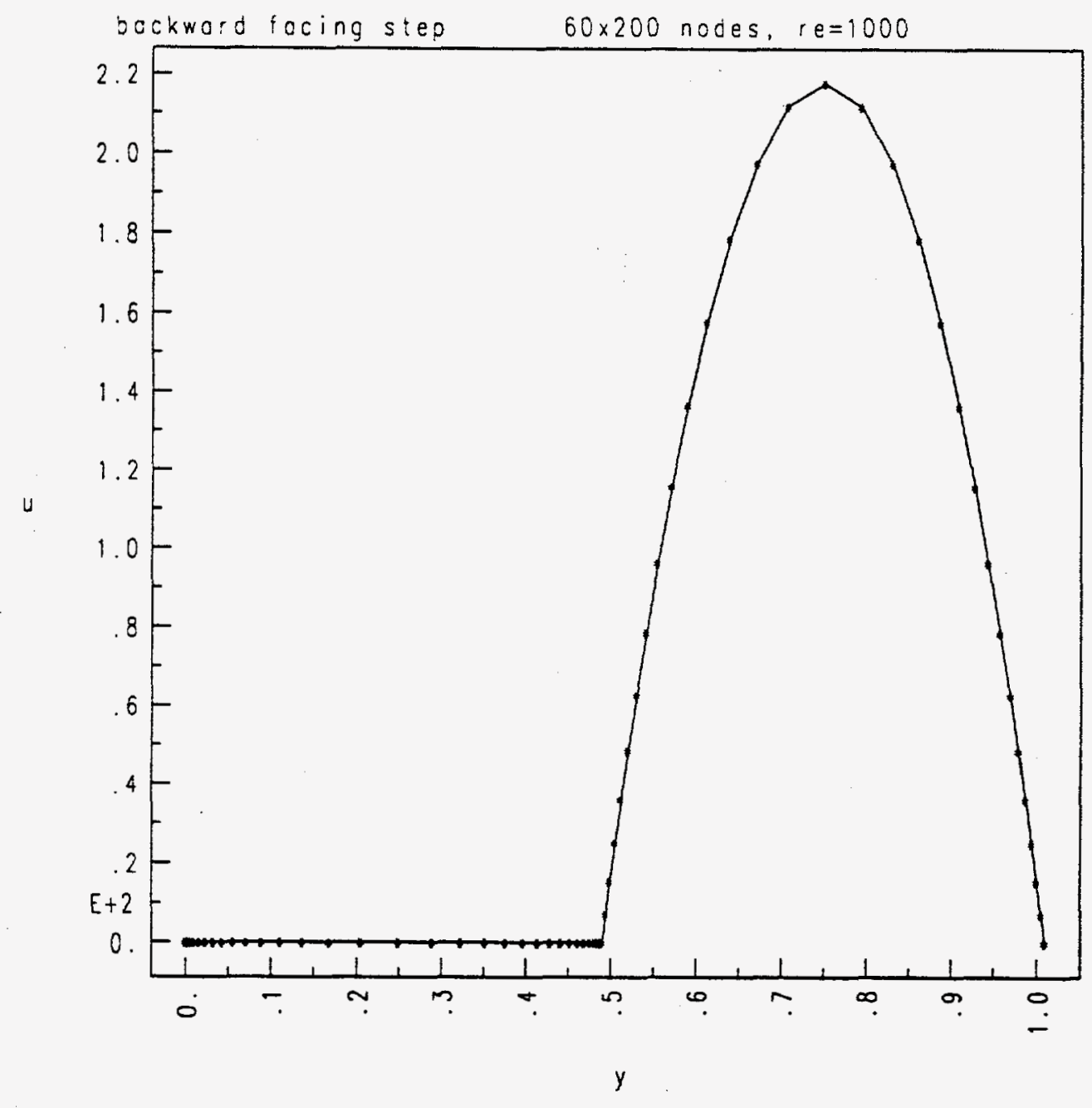

Figure 5.1.3 The specified inlet condition for $R e=1000$ (kinematic viscosity, $v=$ $0.151 \mathrm{~cm}^{2} / \mathrm{s}$ ). 


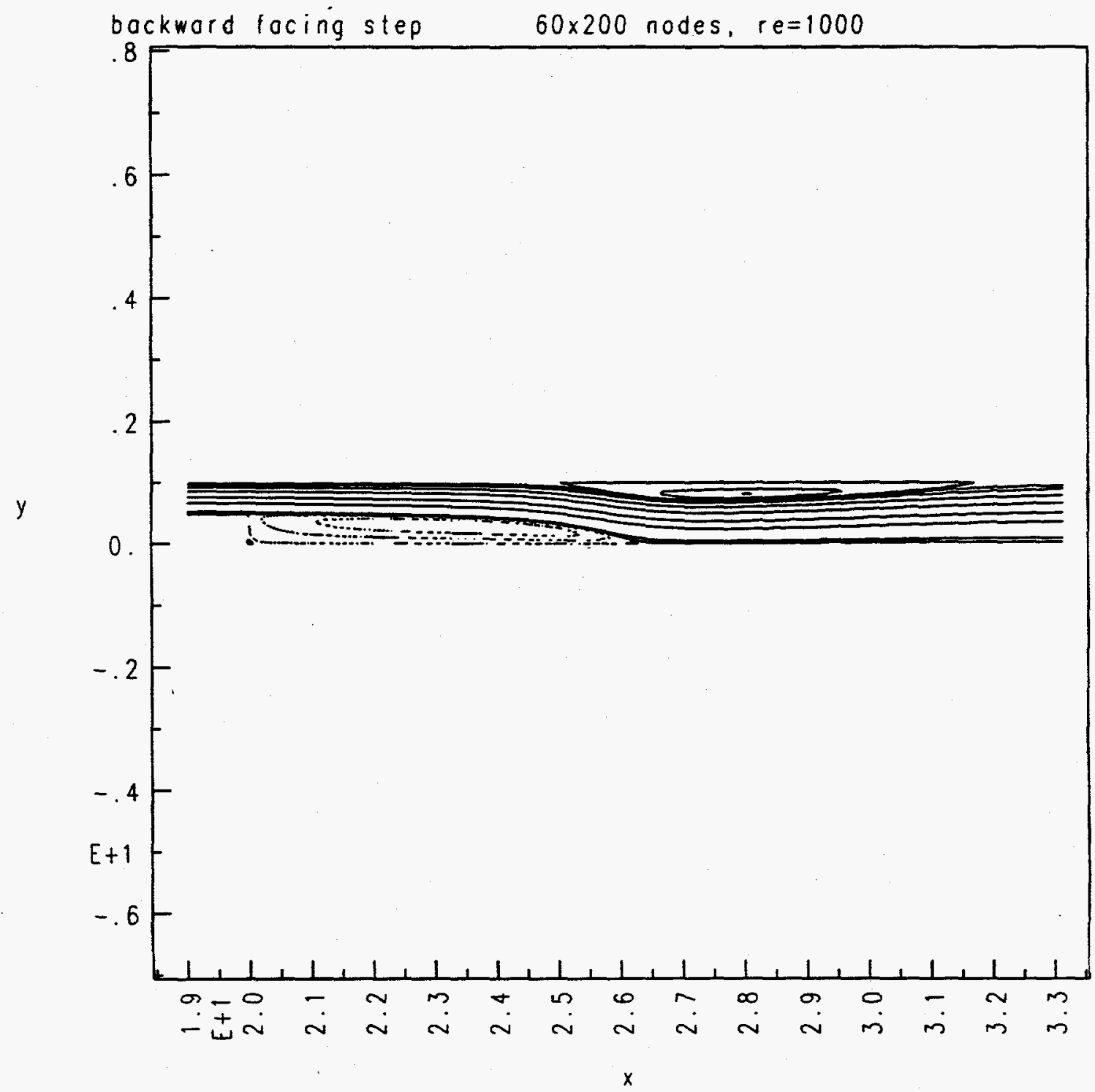

Figure 5.1.4 Streamline plot for $\operatorname{Re}=1000$ using the $60 \times 200$ mesh. 


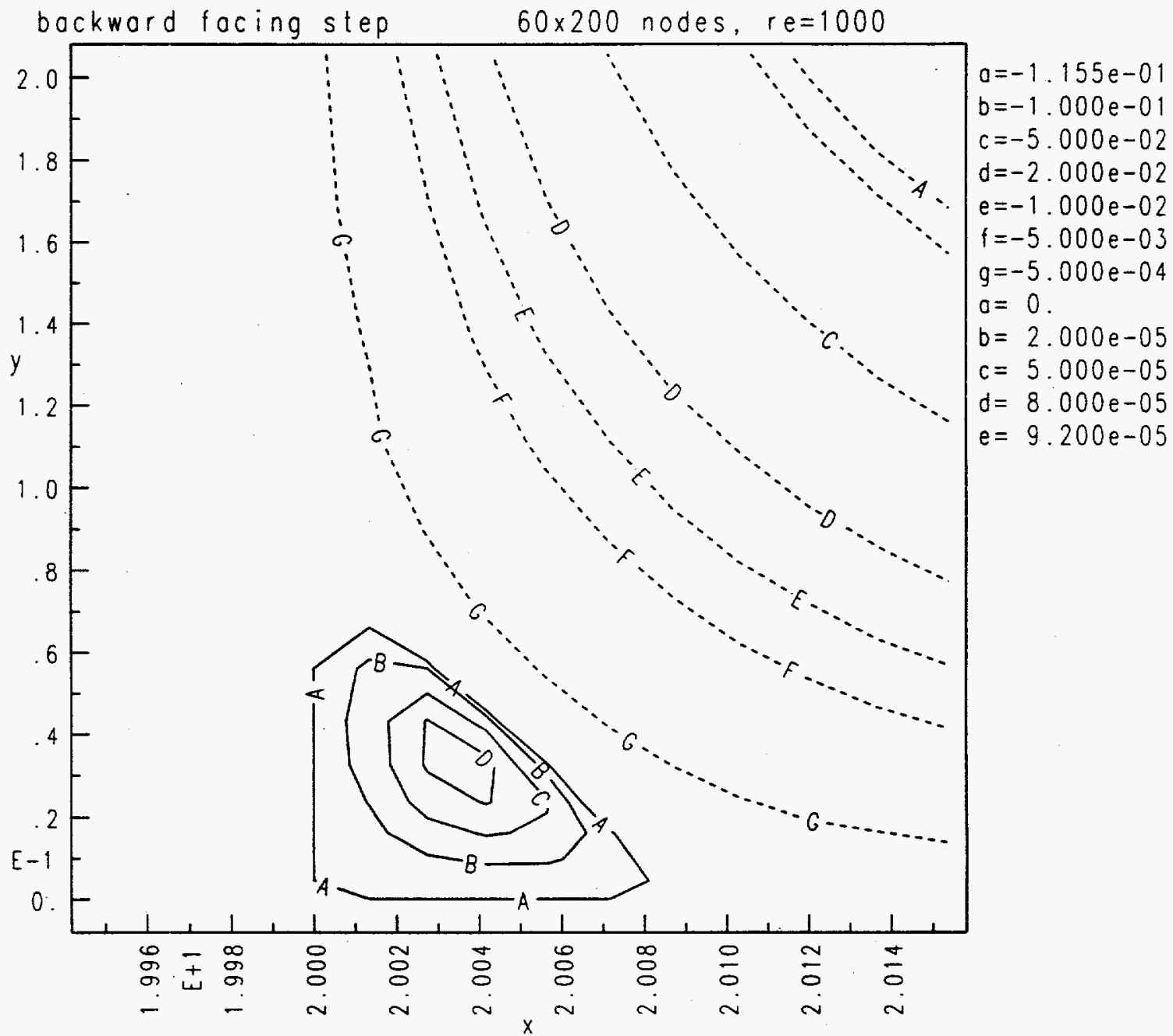

Figure 5.1.5 Streamline plot at base of step for $R e=1000$ using the $60 \times 200$ mesh. 


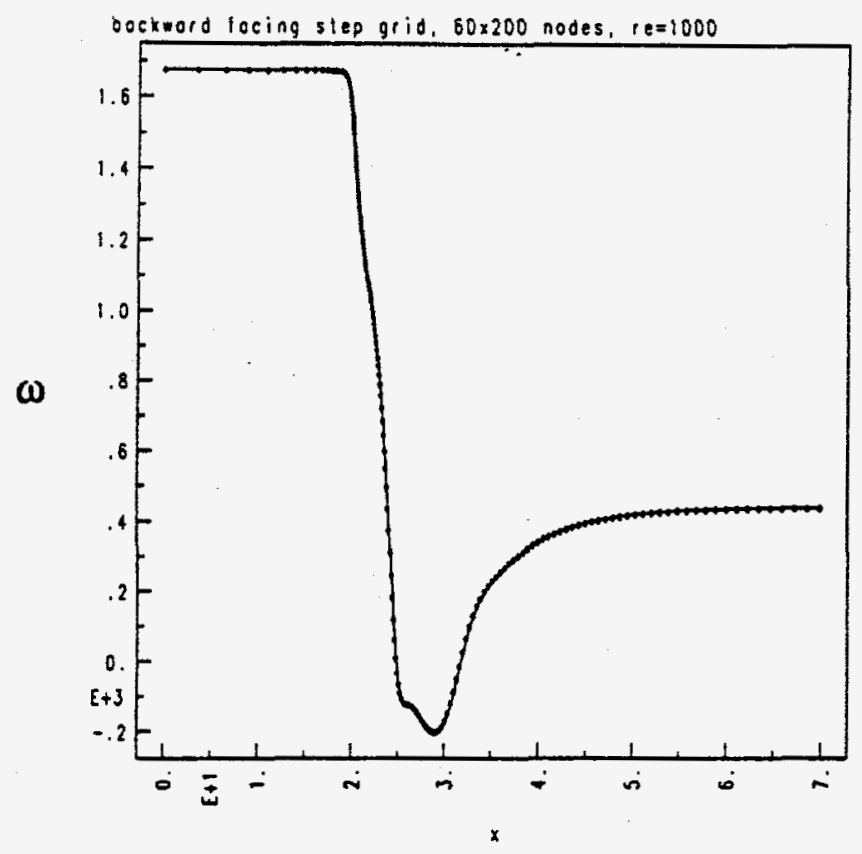

(a)

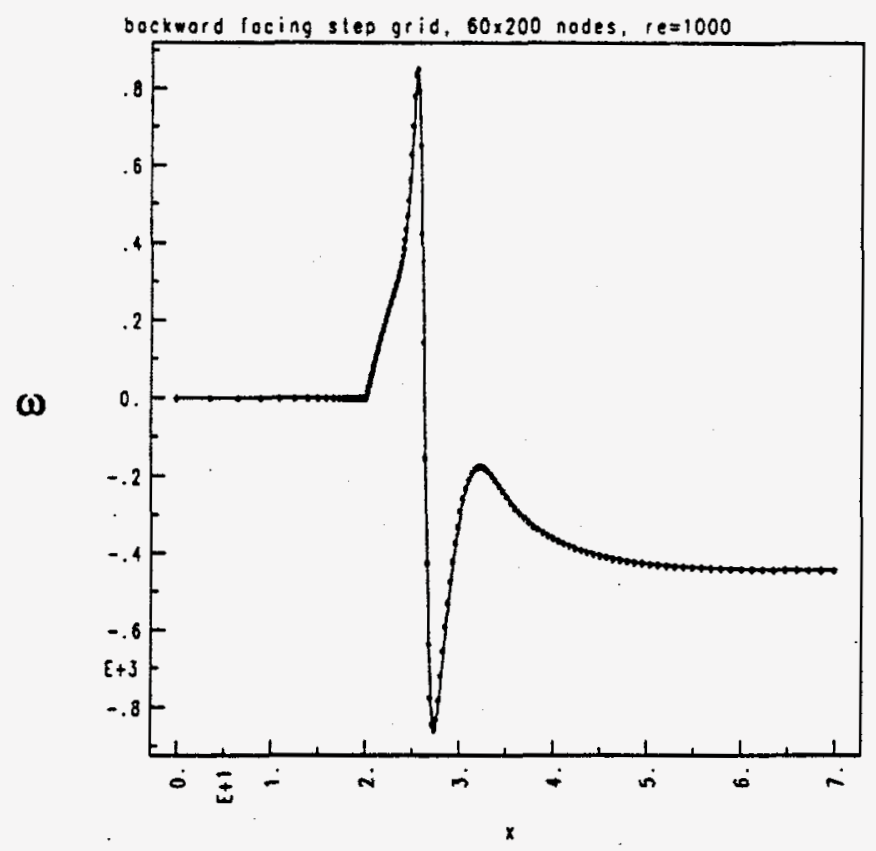

(b)

Figure 5.1.6 Vorticity versus distance along the a) top wall and b) bottom wall for the backward-facing step with $R e=1000$ and the $60 \times 200$ mesh. 


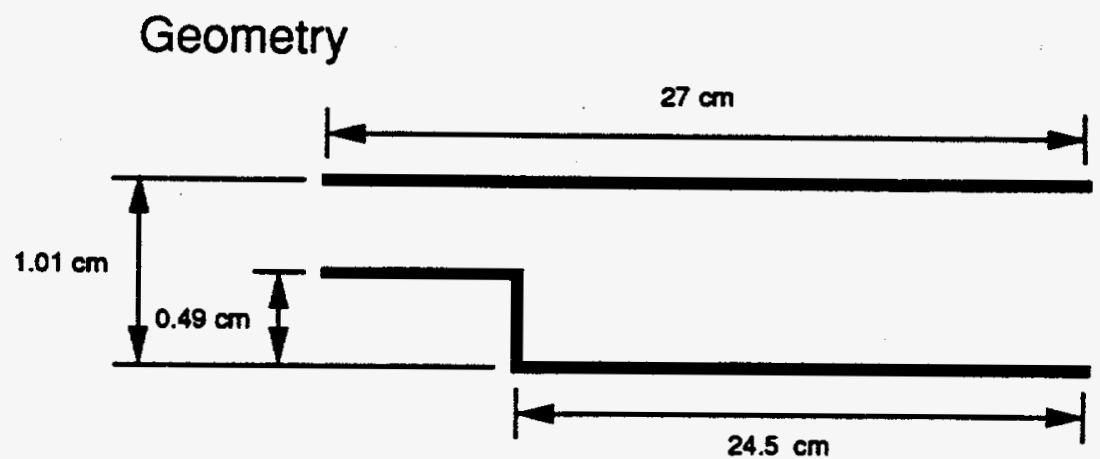

Logical Grid

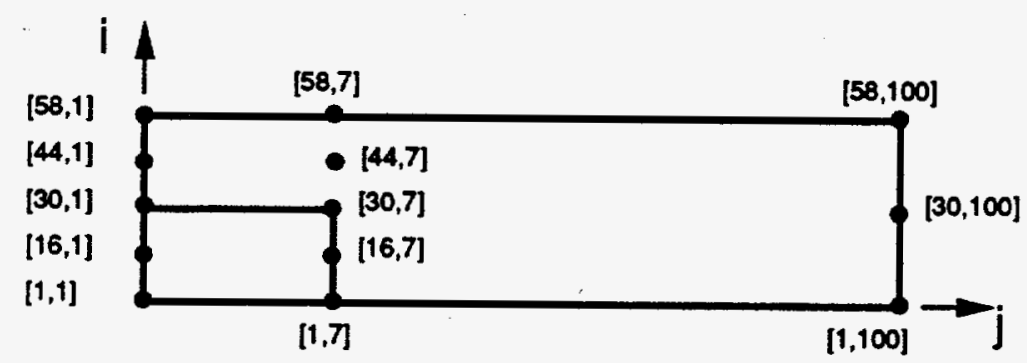

Details of Mesh Near Step

$[30,5]$

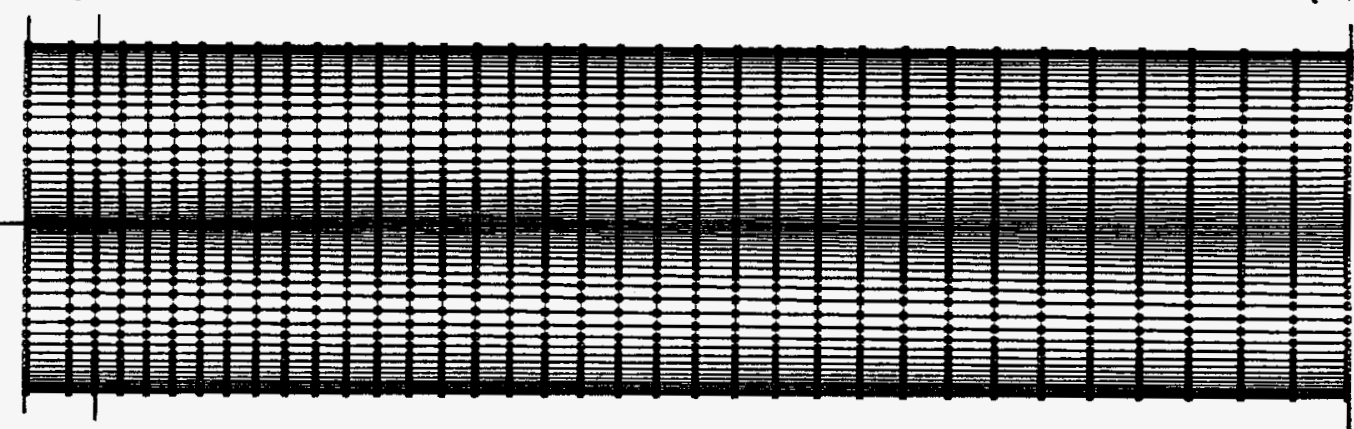

$[1,5] \quad[1,7]$

$[1,40]$

Figure 5.1.7 The $58 \times 100$ mesh and geometry. 


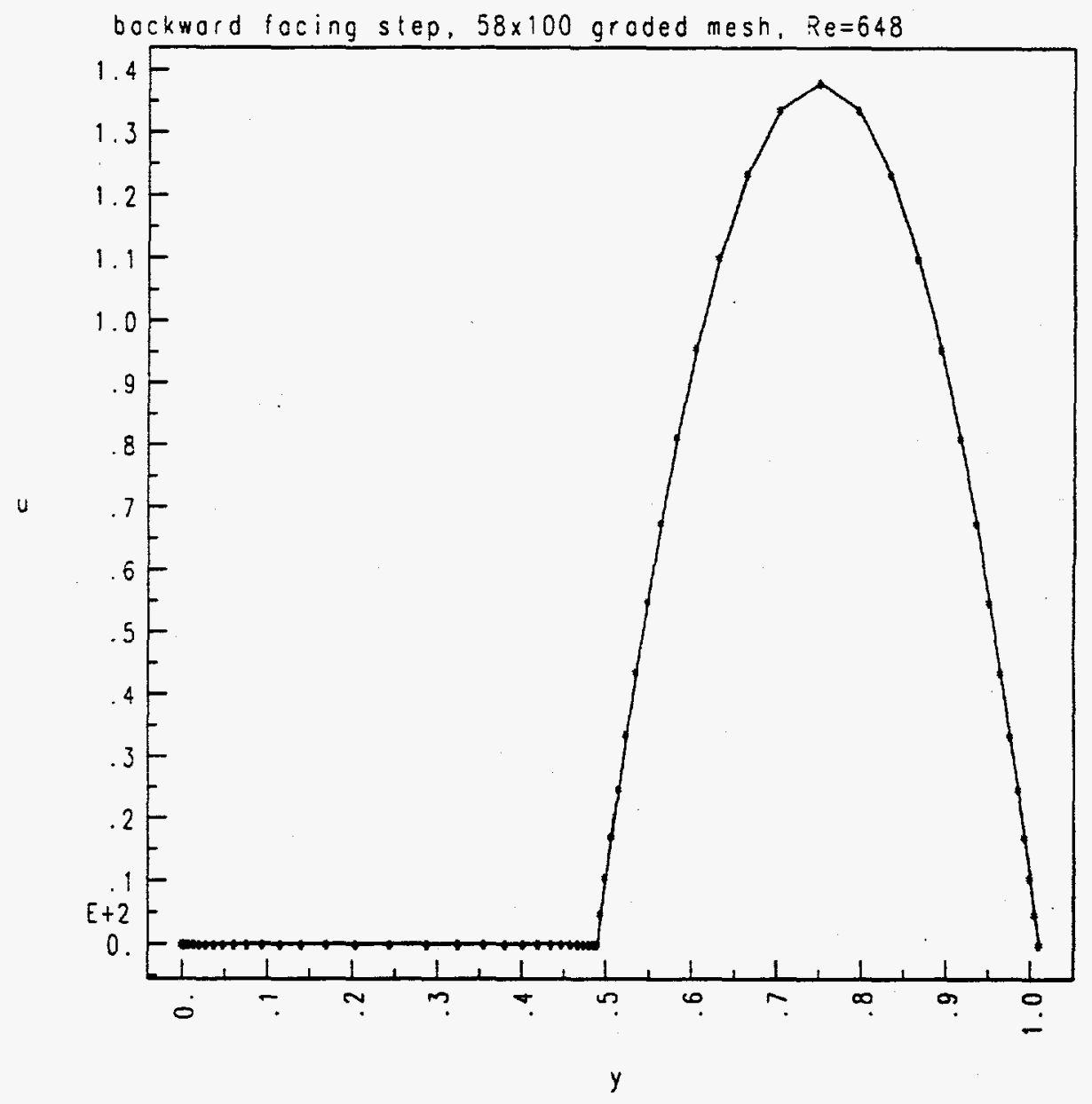

Figure 5.1.8 The specified inlet condition condition for $R e=648$ (kinematic viscosity, $v=0.148 \mathrm{~cm}^{2} / \mathrm{s}$ ). 


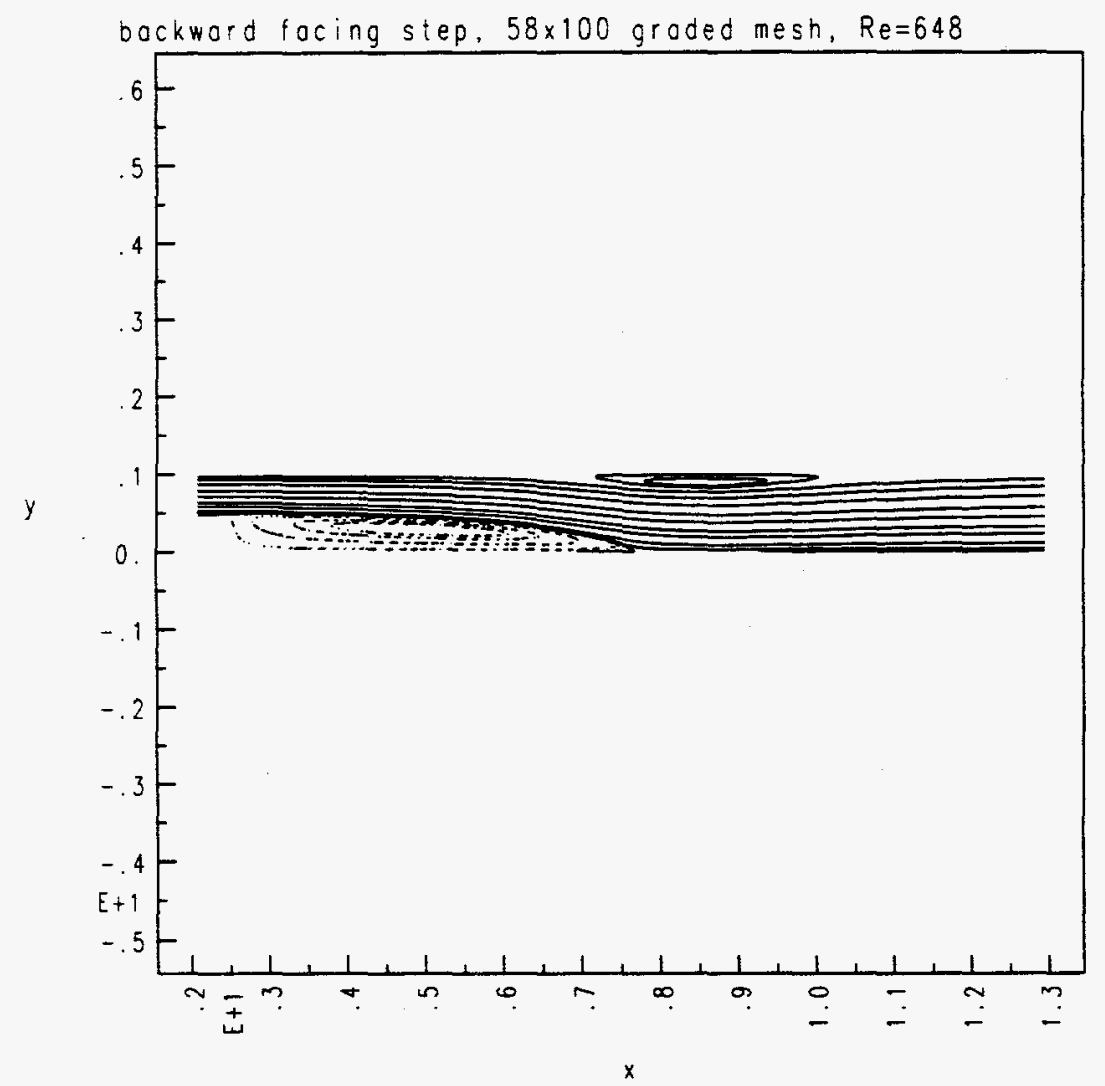

Figure 5.1.9 Streamline plot for $R e=648$ using the $58 \times 100$ mesh. 


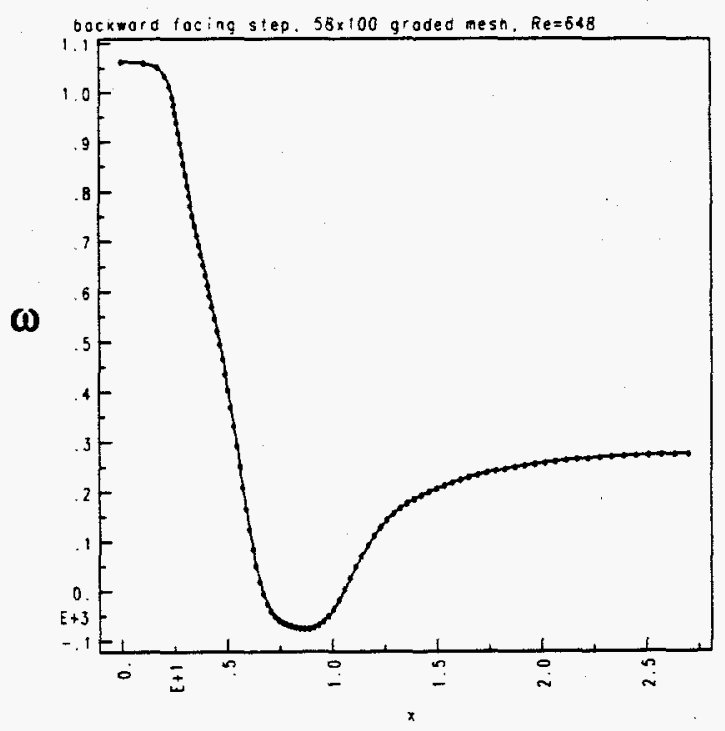

(a)

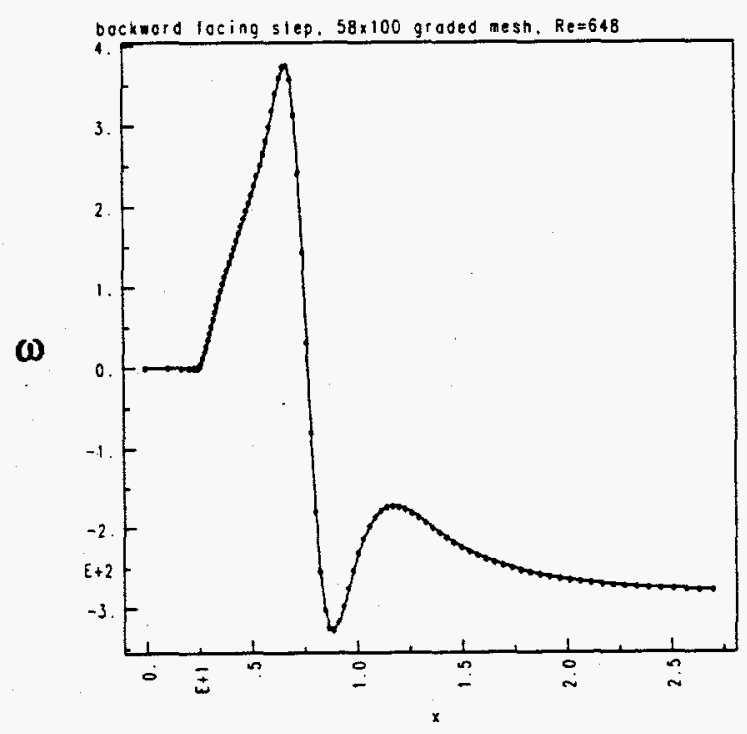

(b)

Figure 5.1.10 Vorticity versus distance along the a) top wall and b) bottom wall for the backward-facing step with $\operatorname{Re}=648$ and the $58 \times 100$ mesh. 


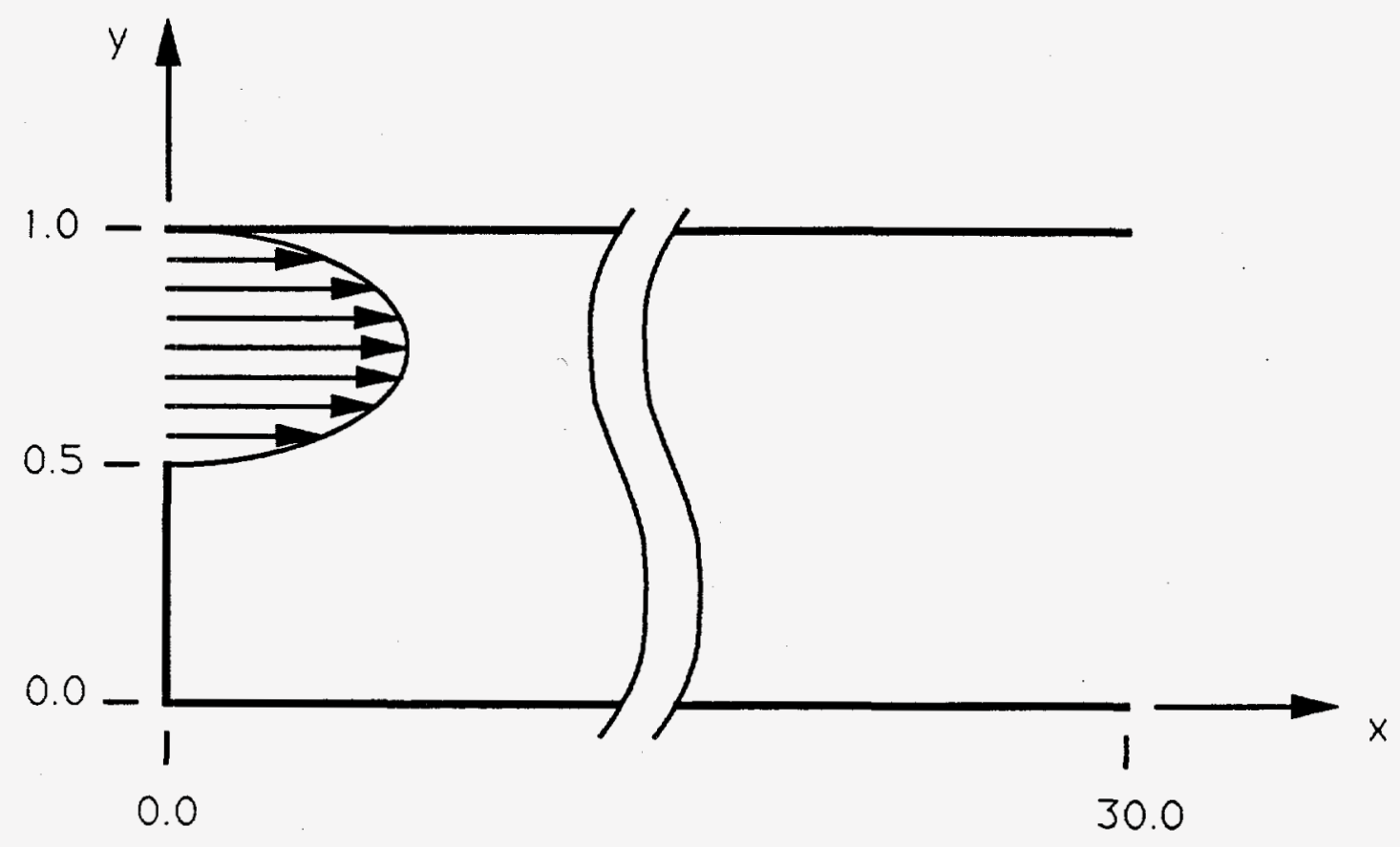

For $0 \leq \mathrm{x} \leq 15 \quad$ uniform grid, $3 / 4$ of total number of elements

For $15 \leq \mathrm{x} \leq 30$ grading factor of 2 (i.e., elements near $x=30$ are twice the length of elements near $x=15$ ), $1 / 4$ of total number of elements

Figure 5.1.11 Details of mesh and geometry used for $\operatorname{Re}=800$ cases (i.e., for comparison to Gartling 1990). 
Table 5.1.1 Comparison of separation and reattachment points downstream of backward-facing step for $\mathrm{Re}=\mathbf{8 0 0}$.

\begin{tabular}{|c|c|c|c|c|c|}
\hline \multirow{2}{*}{$\begin{array}{l}\text { Distance } \\
\text { from } \\
\text { Inlet }\end{array}$} & \multicolumn{2}{|c|}{$\begin{array}{l}\text { Present Prediction } \\
\text { (4 nodes/element) }\end{array}$} & \multicolumn{3}{|c|}{$\begin{array}{l}\text { Gartling (1990) } \\
\text { (9 nodes/element) }\end{array}$} \\
\hline & $\begin{array}{l}8,421 \text { nodes } \\
(8,000 \\
\text { elements }) \\
\end{array}$ & $\begin{array}{l}32,841 \text { nodes } \\
\text { (32,000 } \\
\text { elements) }\end{array}$ & $\begin{array}{l}8,421 \text { nodes } \\
(2,000 \\
\text { elements })\end{array}$ & $\begin{array}{l}32,841 \text { nodes } \\
(8,000 \\
\text { elements }\end{array}$ & \begin{tabular}{|l}
129,681 nodes \\
(32,000 \\
elements $)$ \\
\end{tabular} \\
\hline $\begin{array}{l}x_{1} \\
x_{2} \\
x_{3}\end{array}$ & $\begin{array}{r}5.59 \\
4.35 \\
10.28\end{array}$ & $\begin{array}{r}5.95 \\
4.70 \\
10.43\end{array}$ & $\begin{array}{r}6.07 \\
4.83 \\
10.47\end{array}$ & $\begin{array}{r}6.09 \\
4.85 \\
10.48\end{array}$ & $\begin{array}{r}6.10 \\
4.85 \\
10.48\end{array}$ \\
\hline
\end{tabular}
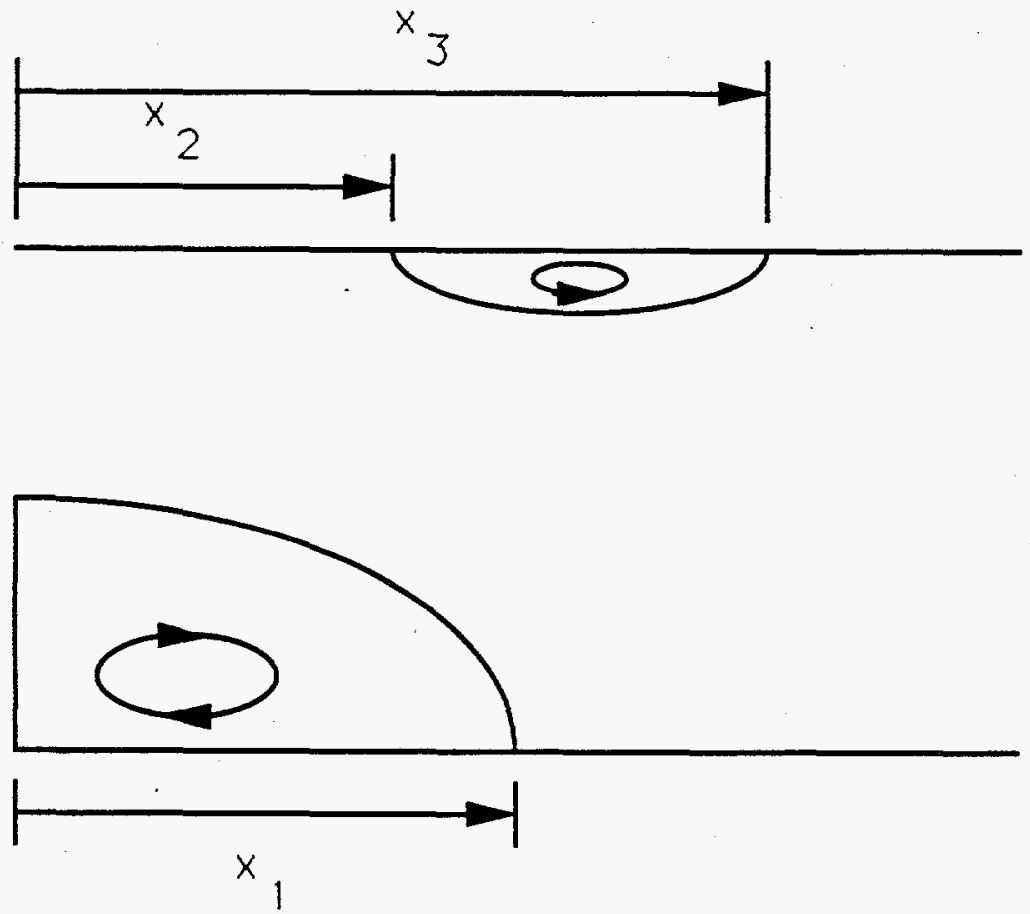


\subsection{An Accurate Solution for the 'Turbulent' Case}

The accurate two-dimensional solution or direct simulation for the 'turbulent' flow case of $\operatorname{Re}=10,000$ was generated using the same code as that used to generate the LES results but without a model for the small-scale motion. Thus, the direct simulation is the (approximate) solution of the Navier-Stokes equations without averaging or filtering. (The equations and code details are given in Appendix D.)

Velocity time histories at chosen points in the flow and a time series of stream function plots (i.e., snapshots of the instantaneous results) for the filtered and unfiltered direct simulation are generated. (See Appendix $\mathrm{G}$ for descripiton of spatial filtering of the direct simulation.) Also included are power spectrum calculations for the velocity time histories. (See Appendix $\mathrm{H}$ for a description of the method used to calculate the power spectra.)

The convergence of the direct simulation is estimated locally by comparing the velocity time histories at selected points in the flow and globally by comparing a series of stream function plots for several grid sizes on a uniformally discretized computational field. Full grid convergence cannot be achieved with the limited computer resources available, but a reasonably accurate solution is achieved for the purposes of this study (i.e., capturing the large-scale motion). The fine mesh was good enough to assert that the direct simulation is a more accurate solution than that achieved with the LES model.

Figure 5.2.1 gives a description of the prescribed inlet, outlet, and boundary conditions. The Reynolds number is based on an average inlet velocity of 1.0 (neglecting wall effects), a channel height of 1.0 , and a kinematic viscosity of $10^{-4}$. The computational field starts at the step. Starting the calculations at the step was found to 
minimize 'wiggles' (i.e., spatial oscillations caused by lack of grid resolution) and reduce the problem size (especially with the structured grid restriction for the code used in this study). A uniform grid is used for all the reported calculations. The inlet velocity condition is essentially flat with a steep ramping from the step corner and upper wall which is smooth enough to avoid 'wiggle' generation and is believed to approximate the turbulant inflow profile. No slip conditions are specified at walls and zero natural boundary conditions are specified at the outlet. The initial condition is a zero velocity field with the imposed boundary conditions; however, the first step in the code is a mass adjustment (as described in Section 3.2.4). Thus, the initial condition is approximately a potential flow solution shown by the stream function plot in Figure 5.2.2a. ${ }^{1}$

To determine the convergence of the solution, several grid sizes were used and compared. The predicted flow field is unsteady with vorticies shedding from the step and propagating downstream (Figure 5.2.2b). A comparison of the $u_{1}$ and $u_{2}$ velocity time histories at two different locations in the flow field is presented in Figures 5.2.3 through 5.2.6. Some of the results were not in phase with each other and the time histories for these cases are shifted in time by matching minimum points of the cycle (as described on the figures).

All of the different mesh size cases were run without subcycling (i.e., updates of the pressure matrix were performed at each time step, as described in Section 3.2.7), except for the finest grid case of a $101 \times 1001$ node grid. Subcycling was required for the $101 \times 1001$ node case because of the large problem size and small step size requirement (i.e., the Courant limit with explicit time integration). However,

${ }^{1}$ The mass adjustment does not enforce that the curl of the velocity field is zero everywhere (i.e., not enforced at the inlet boundary condition), but the vorticity is small. 
subcycling was only used until an approximate periodic solution was achieved and then subcycling was not used to achieve the final periodic solution.

It is clear ftom the velocity time histories (Figures 5.2.3 through 5.2.6) that the flow is approximately periodic (see discussion in Section 2.4.6) and the periodic cycles improve for the finer grids. For the $81 \times 801$ and $101 \times 1001$ node grids the cycle periods agree, at 6.36 time units. For the $u_{1}$ time history at $\left.(x, y)=2.0,0.25\right)$, Figure 5.2.3, with the $81 \times 801$ node grid, the minimum point in the cycle is $35 \%$ higher than that for the $101 \times 1001$ node grid, and the maximum peak for the $81 \times 801$ node grid is $2 \%$ lower than the maximum for the $101 \times 1001$ node grid. The general shape of the time histories for the $81 \times 801$ and $101 \times 1001$ grids compare well, except for some differences in the high frequency motion. Overall, the time histories show that locally the $81 \times 801$ and $101 \times 1001$ grids are in fair agreement in that the large-scale motion and some small-scale motion are being captured.

The results were found to not only be sensitive to the chosen mesh, but they were also sensitive to the chosen time step. In all of the above described results, the Courant limit $(\Delta t<\Delta / v$, for mesh size $\Delta$ and characteristic velocity $v$ ), not the diffusion limit $\left(\Delta t<\Delta^{2} / 2 v\right.$ for viscosity $v$ ), determined the stability time step. ${ }^{1}$ Thus, a larger time step was used for the coarse grids than for the fine grids (see time steps listed on figures). The smallest time step of 0.005 was for the $101 \times 1001$ node grid. In an attempt to determine the effect of time step on accuracy, a $25 \times 241$ and the $41 \times 401$ node grids were run with the 0.005 time step. The $25 \times 241$ grid was unstable (i.e., the velocities grow to infinity). The instability is probably caused by the dependence of the

${ }^{1}$ To generate the results in this study, the time step was first chosen to be slightly greater than the Courant limit and was then progressively halved until a stable solution was achieved (i.e., the velocities no longer grew to infinity). The final time step satisfied the Courant limit. 
balancing tensor diffusivity on $\Delta t$, causing a reduction in the effective diffusion and an unstable character of the differential-algebraic equations. An investigation of this instability is beyond the scope of this research.

The velocity time histories for the $41 \times 401$ grid for time steps 0.02 and 0.005 are compared to the results for the $101 \times 1001$ grid with a 0.005 time step in Figures 5.2.7 through 5.2.10. The predicted period for the $41 \times 401$ grid with $\Delta t=0.02$ is 6.16 and with $\Delta t=0.005$ is 6.47 , which are $3.1 \%$ lower and $1.7 \%$ higher, respectively, than the 6.36 period predicted for the $101 \times 1001$ grid. It appears that while the 0.02 time step for the $41 \times 401$ grid satisfies the stability requirement (i.e., the Courant limit), a smaller time step is needed for accuracy improvement.

We now consider the power spectra of the computed results. Using long duration time histories for the $41 \times 401$ grid at $\Delta t=0.02$ and 0.005 , the corresponding power spectra were calculated. The input time histories and resulting power spectra are shown in Figures 5.2.11 to 5.2.22. (The figure captions indicate the choice of data used to generate each power spectrum.) The power spectrum calculations show that with the smaller time step, more high-frequency (small-scale) motion is being captured. However, the low-frequency (or large-scale) motion is being captured by the coarse mesh at both the 0.02 and 0.005 time steps. It should be noted that the power spectra calculations were performed on a 32 bit machine. Since the input velocities are $O(1)$, the machine precision is $\mathrm{O}\left(10^{-7}\right)$ for the 32 bit machine. Thus, the plotted power spectra below $10^{-7}$ are probably numerical error due to machine precision.

A comparison of the power spectra for the $81 \times 801$ and $101 \times 1001$ grids and the extended time histories used to generate the power spectra are shown in Figures 5.2.23 to 2.2.34. The power spectra further support the above conclusion that locally 
the velocity prediction for the $81 \times 801$ and $101 \times 1001$ grids are in agreement for the low-frequency (or large-scale) motion and some of the high-frequency (or small-scale) motion is being captured with both grids. Therefore, it appears that we have a converged solution for the large-scale motion. Because the finer grid of $101 \times 1001$ nodes with the small time step of 0.005 resolves adequately the large scale motion, we assert that it can be used as an accurate solution to compare to the large-scale motion generated with the LES approach and a coarse grid.

To determine global convergence, we look at a series of stream function plots. Stream function plots for the $81 \times 801$ and $101 \times 1001$ grids (shifted in time in the same manner as described on the time history plots) are shown in Figure 5.2.35. The results are in good agreement. The discrepancies that were present in the local time history plots are less apparent in the global stream function plots. Thus, it seems safe to assume that the $81 \times 801$ grid results can be considered a nearly converged solution in the global sense. Since the $101 \times 1001$ grid case is even better, the results for the $101 \times 1001$ grid will be considered the 'direct' simulation for this study and will be used for comparison to the LES results.

Velocity profiles near the channel outlet for the $101 \times 1001$ grid are shown in Figure 5.2.36 and 5.2.37 for two different snapshots in time. The profile plots are smooth at the outlet and upstream of the outlet. Figure 5.2.37 does show a wiggle at the bottom wall. This appears to be due to poor resolution of the small recirculation zone at the outlet, but only for the first element adjacent to the wall. Based on these results, the zero natural boundary condition at the channel outlet appears to give a smooth well behaved solution.

Before the direct simulation results for the instantaneous flow field, $u_{1}$ and $u_{2}$, 
can be compared to the LES results for the large-scale motion, $\bar{u}_{1}$ and $\bar{u}_{2}$, the direct simulation will need to be filtered. The filter width must coincide with that used in generating the LES results.

The method for filtering the direct simulation results is a cell volume-average approach which is consistent with the cell volume-averaging used to generate the LES results. The details of the filtering method and the implementation in the code are outlined in Appendix G. In summary, the filtering scheme performs a cell volumeaverage over a specified number of elements adjacent to and surrounding each node of the 'direct' simulation. For the code implementation with trilinear or bilinear basis functions in three or two dimensions, respectively, we have

$$
\bar{u}_{\alpha}(x, y, z, t)=\frac{\sum_{n=1}^{N E} A_{n}\left(\sum_{i=1}^{N P E} u_{\alpha_{i}}\right)}{2^{n \operatorname{dim}} \sum_{n=1}^{N E} A_{n}}
$$

where $N E$ is the number of elements within the domain $x-\left(\Delta x_{f} / 2\right)$ to $x+\left(\Delta x_{f} / 2\right), y-\left(\Delta y_{f} / 2\right)$ to $y+\left(\Delta y_{f} / 2\right)$, and $z-\left(\Delta z_{f} / 2\right)$ to $z+\left(\Delta z_{f} / 2\right), N P E$ is the number of points per element, $A_{n}$ is the volume or area in three or two dimensions, respectively, for element $n$, and $n$ dim is the number of dimensions. In this formulation, the assumption is made that the filter width limits fall on element boundaries (see Appendix G). This assumption is not necessary, but it simplifies implementation of the filtering scheme.

It should also be noted that the filtering scheme is only applied to those nodes that are at least one-half the filter width from the boundaries. As shown in Figure 
5.2.38, a 16 element patch (or a filter width of $4 \Delta=4(0.01)=0.04, \Delta=\sqrt{\Delta x \Delta y}$ ) is used to filter the $101 \times 1001$ node 'direct' simulation. The scheme is not applied to the boundary nodes or to the nodes adjacent to the boundaries because one-half the filter width is greater than the distance to the boundaries for these nodes.

The filtered 'direct' simulation velocity time histories are shown in Figures 5.2.39 through 5.2.42 for one period along with the unfiltered results for comparison. The nonfiltered and filtered time histories are almost identical. Only slight differences can be seen at locations of high frequency motion (i.e., at small oscillations or at sharp peaks or valleys). According to these results, a filter width of 0.04 filters out very little of the small-scale motion present in the $101 \times 1001$ grid results. This would suggest that a grid size of 0.04 (or a $25 \times 241$ node mesh) would give a solution in agreement with the 0.01 grid size (the $101 \times 1001$ node mesh). However, as shown in Figures 5.2.3 through 5.2.6, the time histories for the coarse mesh of $41 \times 401$ nodes does not agree with the fine mesh results. Even when the same time step is chosen $(\Delta t=0.005)$, the time histories for the $41 \times 401$ grid do not agree with the time histories for the $101 \times$ 1001 grid, as shown in Figures 5.2.7 through 5.2.10. However, there is an improvement in the power spectra, as shown in Figures 5.2.43 to 5.2.46, when the small time step is used with the $41 \times 401$ grid. Therefore, the agreement between the nonfiltered and filtered velocities for the $101 \times 1001$ grid does not imply that a coarser grid with grid size equal to the filter width will give a converged solution. However, the above results do further indicate that the time step for accuracy is significantly less than the time step required for stability.

The filtering scheme does not necessarily preserve mass consistency. Thus, the stream function plots of the filtered field would not be a valid global solution. 
Time-averaged results were generated for the $101 \times 1001$ grid for two different time durations; 20,000 and 30,000 time steps at $\Delta t=0.005$ (100 and 150 time units). (Start times for the time averages were not from time zero, but after the flow field had established an approximate periodic behavior.) For the period of 6.36 time units we are capturing approximately 15 and 23 cycles for the 20,000 and 30,000 time step averages, respectively. From the stream function plot in Figure 5.2.35, we see that a recirculation zone that forms at the step moves approximately 2 length units in one cycle; i.e., the eddy "speed" is $x / 6.36 \cong 0.3$, as compared with the average velocity of 0.5 . Thus, a step-generated recirculation zone will have traveled approximately 3 and 4.5 times the length of the entire computational field for 20,000 and 30,000 time steps, respectively.

The predicted $\mathrm{x}$ locations for separation and reattachment points are listed in Table 5.2.1. The results show that a 20,000 time step average is adequate at generating the stationary solution. Figure 5.2 .47 is a stream function plot of the time-averaged solution showing the recirculation zones. The reattachment length for the major recirculation zone is 1.697 or approximately 3.4 step heights downstream of the step. Small recirculation zones along the top and bottom walls are too small to be seen on the plot in Figure 5.2.47, but Table 5.2.1 clearly indicates their locations.

Profile plots of the time-averaged $u_{1}$ velocity, $\left\langle u_{1}\right\rangle$, turbulent intensity, $\left\langle u_{1}^{\prime 2}\right\rangle$, turbulent shear $\left\langle u_{1} u_{2}\right\rangle$, turbulent kinetic energy, and dissipation rate (as defined in Appendix B) are shown in Figures 5.2.48 through 5.2.53 for selected locations. The profiles are smooth across the channel indicating good resolution of the mean field quantities.

The time-averaged velocity profiles near the channel outlet are shown in 
Figure 5.2.53. The profile at the outlet $(x=10.00)$ is smooth in the central portion of the channel but the gradient at the wall is very steep and indicates some wiggles. The profiles become smoother at the wall as we move just upstream. This indicates that the 'error' caused by the specified outlet conditions (i.e., zero natural boundary conditions) is confined to the few element rows adjacent to the outlet and is not translated upstream.

In summary, the results for the $101 \times 1001$ grid using $\Delta t=0.005$ will be called the 'direct' simulation in the following sections and will be used for evaluation of the LES results. The above results indicate that it is important to use a consistent time step for all calculations being compared. Therefore, all LES calculations will be performed for $\Delta t=0.005$, which was the time step used in generating the direct simulation. The above results also indicate that a time-average over 20,000 time steps for $\Delta t=0.005$ should be adequate for generating the stationary time-averaged solution. 


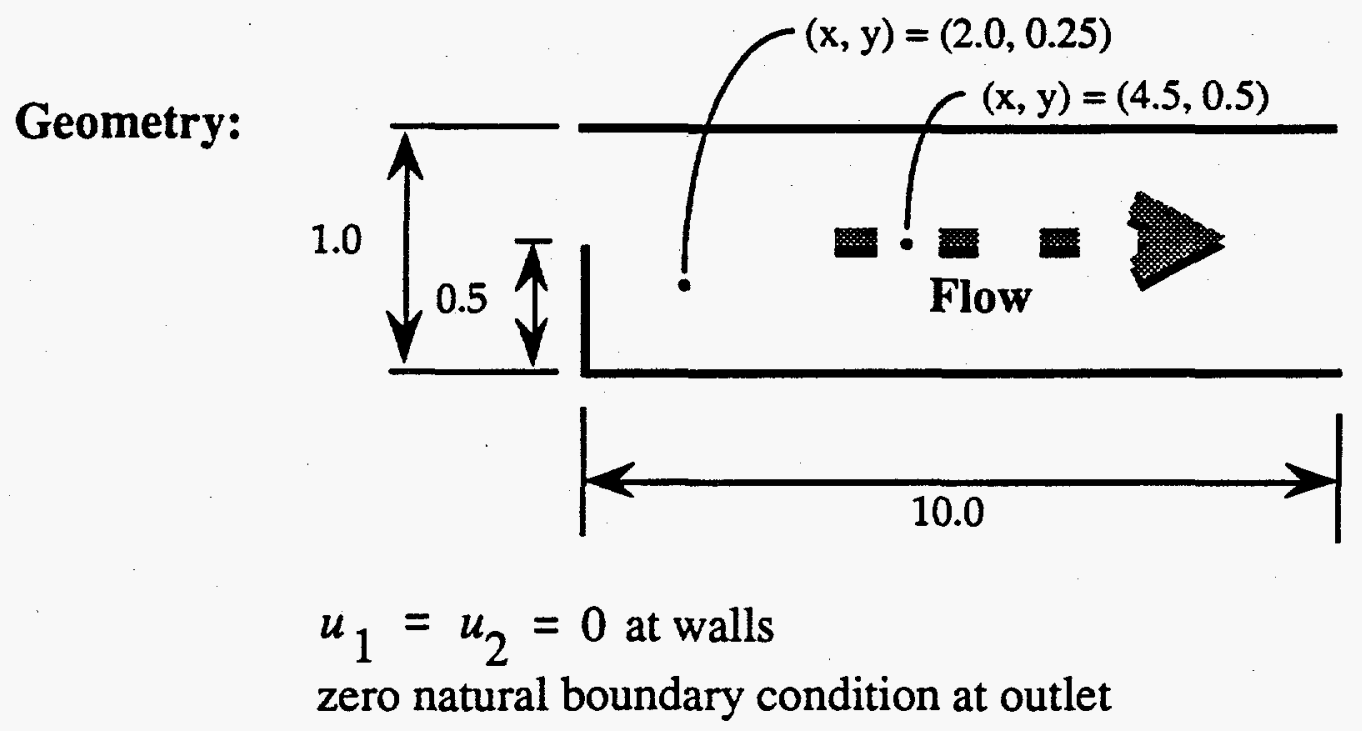

Inlet Condition:

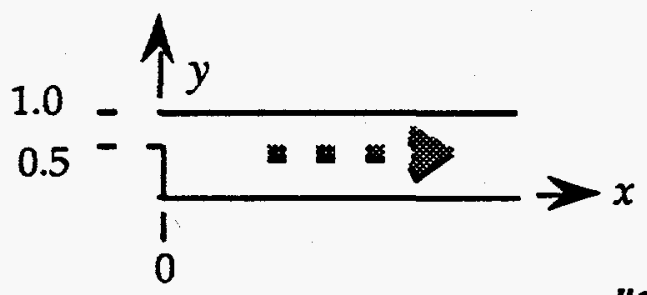

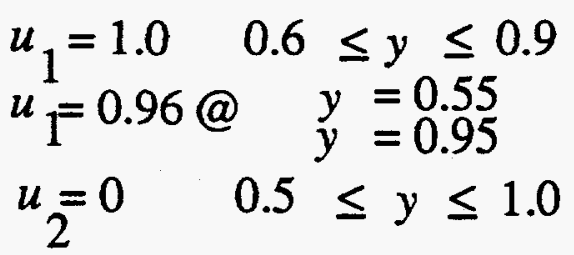

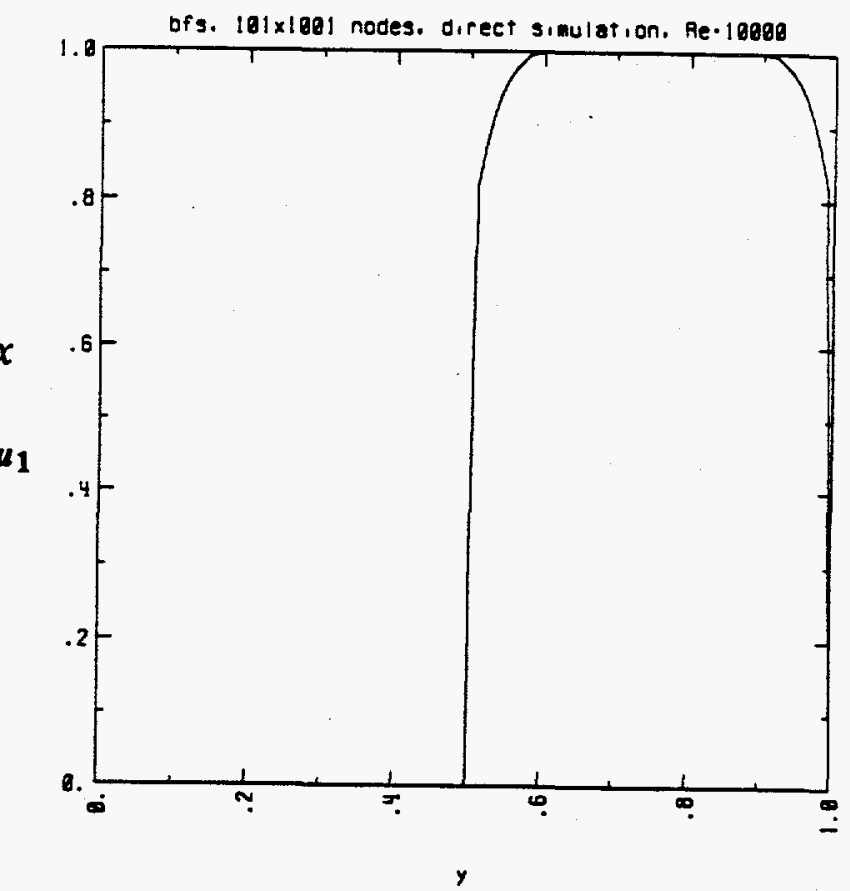

Figure 5.2.1 Description of the geometry and the prescribed inlet, outlet, and boundary conditions for the 'direct' simulation cases. 


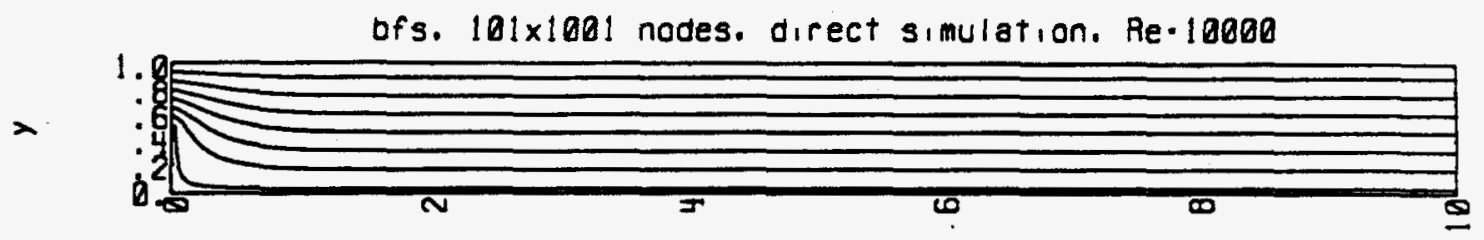

(a)

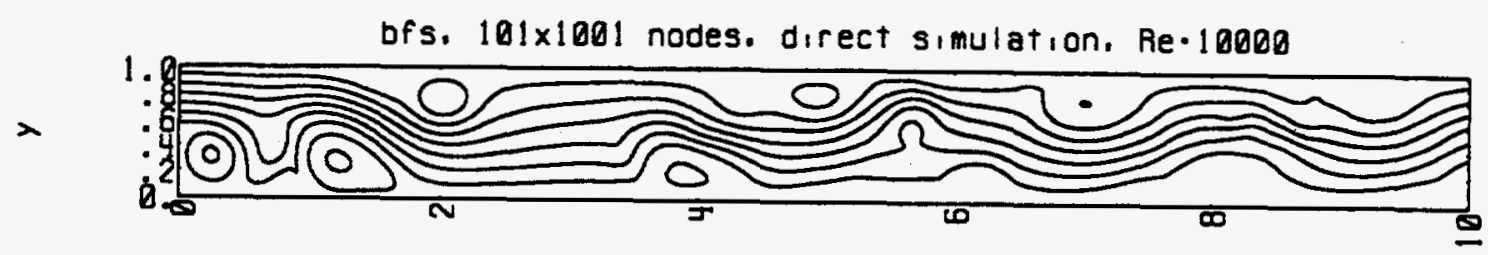

$\mathbf{x}$

(b)

Figure 5.2.2 The (a) mass adjusted solution used as initial condition for 'direct' simulation cases and (b) predicted stream function plot at a snapshot in time. (Shown solution is for the $101 \times 1001$ nodes grid.) 
Grid

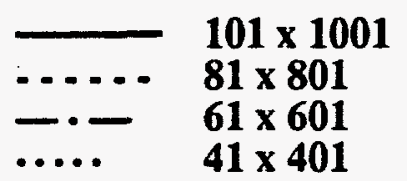

$\Delta t \quad$ Time Span*

0.005

0.010

0.010

0.020
77.0 to 87.0

77.7 to 87.7

77.7 to 87.7

81.55 to 91.55

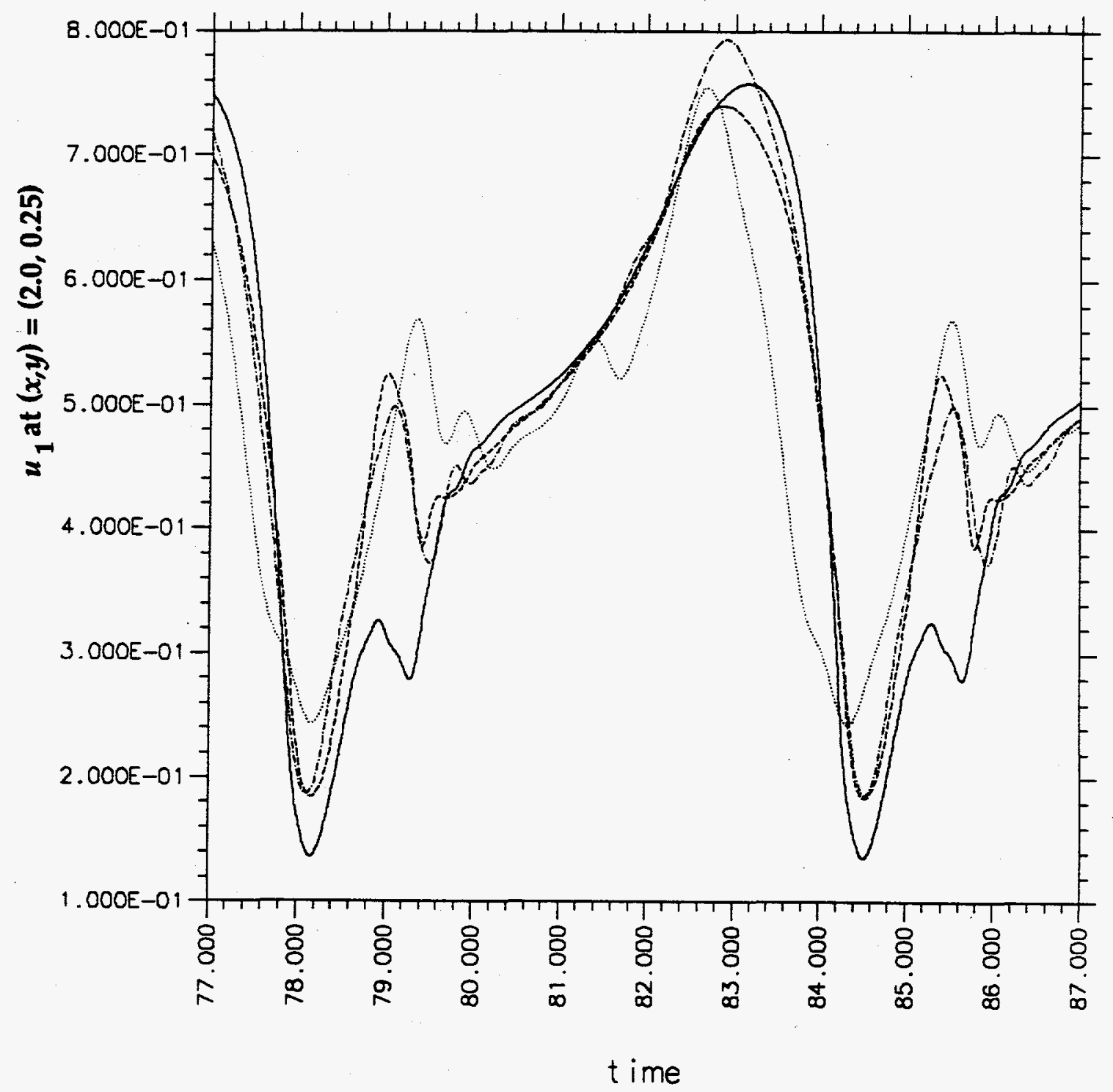

*Time histories are shifted by placing minimum points at time $=\mathbf{7 8 . 1 6}$ (which is the time that the minimum occurred for the 101 x 1001 grid).

Fig. 5.2.3 Comparison of $u_{1}$, velocity time histories for various grid sizes at the point location $(x, y)=(2.0,0.25)$. 
Grid
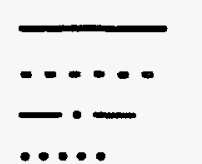

$101 \times 1001$

$81 \times 801$

$61 \times 601$

$41 \times 401$
$\Delta t$

0.005

0.010

0.010

0.020
Time Snan*

77.0 to 87.0

77.7 to 87.7

77.7 to 87.7

81.55 to 91.55

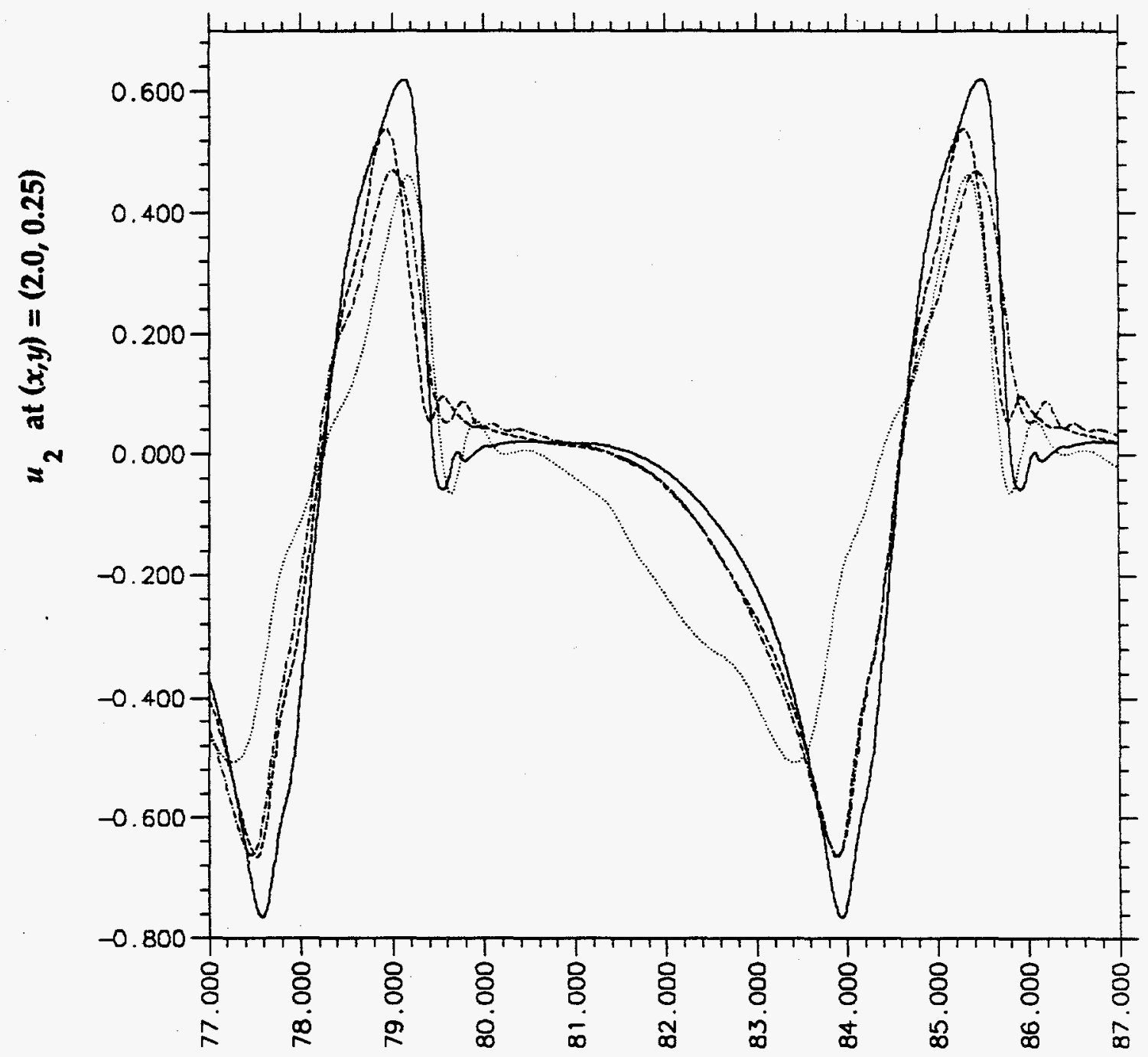

time

*Time histories are shifted by placing minimum points at time $=\mathbf{7 8 . 1 6}$.

Fig. 5.2.4 Comparison of $u_{2}$, velocity time histories for various grid sizes at the point location $(x, y)=(2.0,0.25)$. 
Grid

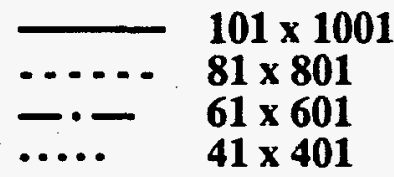

$\Delta t \quad$ TimeSpan*

0.005

0.010

0.010

$\mathbf{0 . 0 2 0}$
77.0 to 87.0

77.7 to 87.7

77.7 to 87.7

81.55 to 91.55

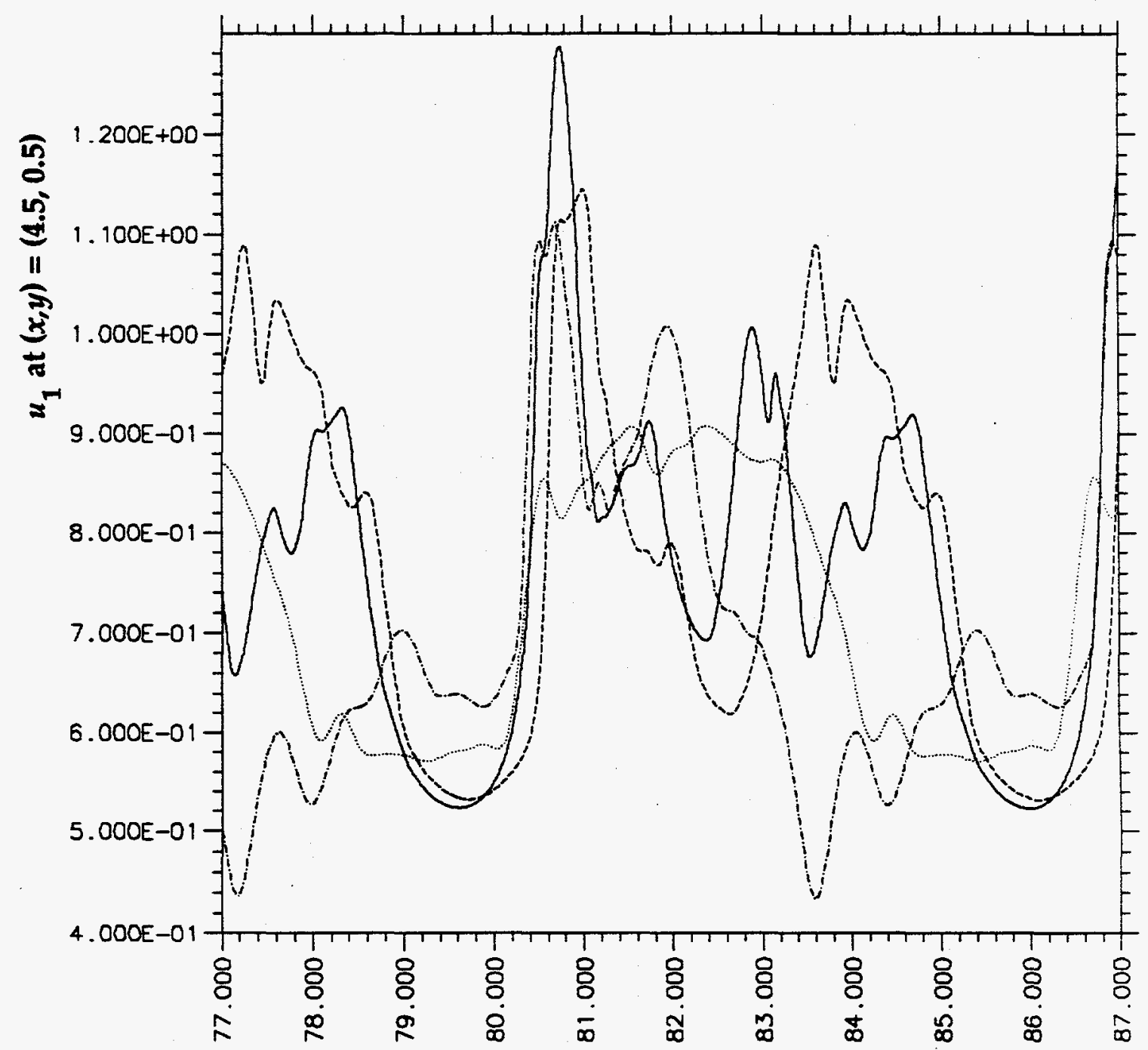

t ime

*Time histories are shifted by placing minimum points at time $=\mathbf{7 8 . 1 6}$.

Fig. 5.2.5 Comparison of $u_{1}$, velocity time histories for various grid sizes at the point location $(x, y)=(4.5,0.5)$. 
Grid

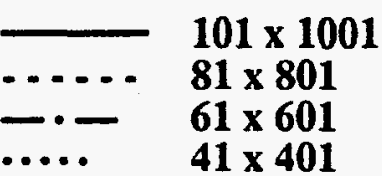

$\Delta t$

0.005

0.010

0.010

0.020
TimeSpan*

77.0 to 87.0

77.7 to 87.7

77.7 to 87.7

81.55 to 91.55

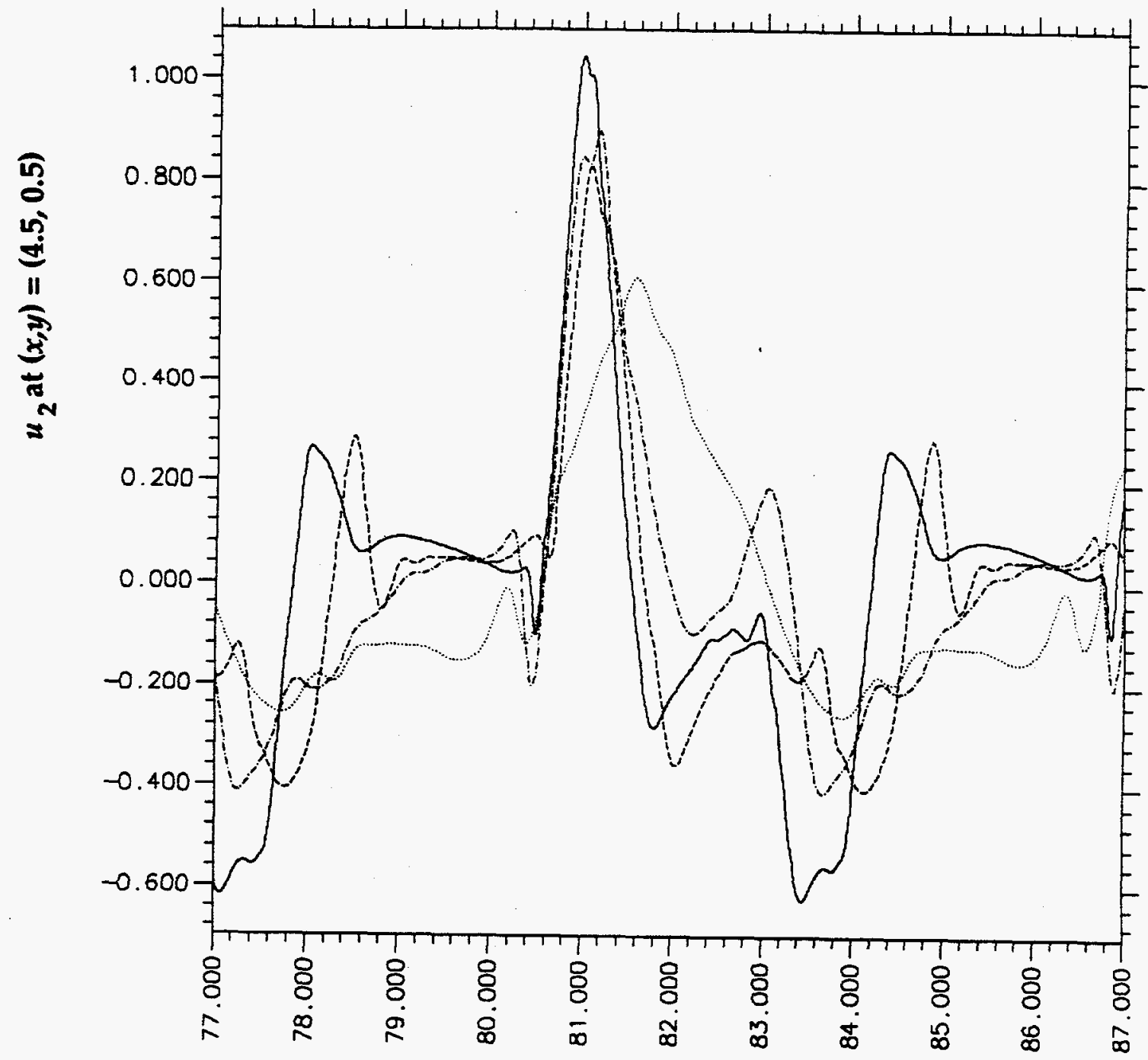

time

*Time histories are shifted by placing minimum points at time $=\mathbf{7 8 . 1 6}$.

Fig. 5.2.6 Comparison of $u_{2}$, velocity time histories for various grid sizes at the point location $(x, y)=(4.5,0.5)$. 
Grid

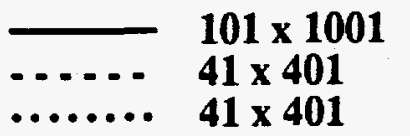

$\Delta$

0.005

0.005

0.020
Time Span*

77.0 to 87.0 165.55 to 175.55 81.55 to 91.55

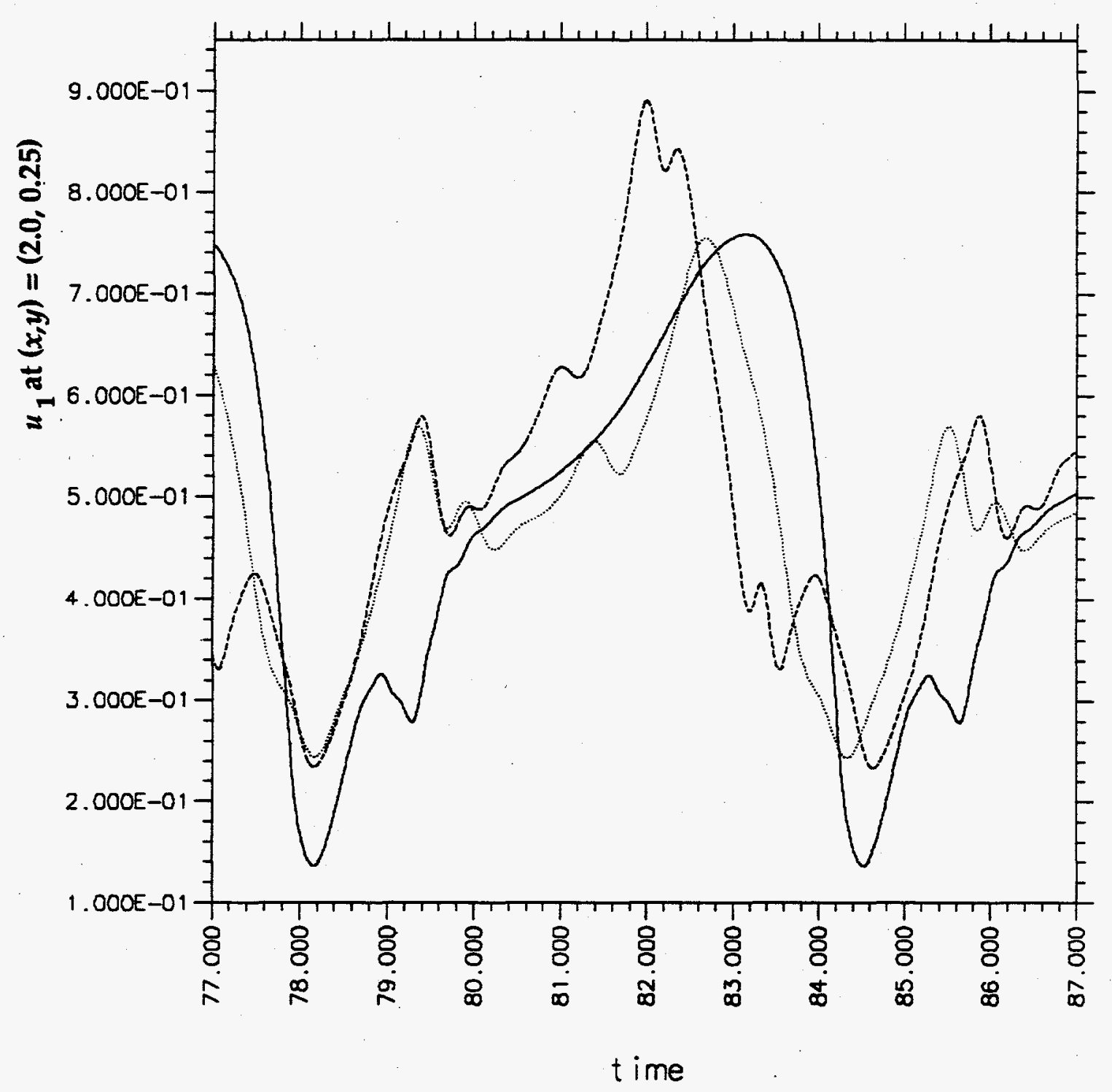

*Time histories are shifted by placing minimum points at time $=78.16$ (which is the time that the minimum occurred for the 101 x 1001 grid).

Fig. 5.2.7 Comparison of $u_{1}$, velocity time histories for the $101 \times 1001$ grid with $\Delta t=0.005$ and for the $41 \times 401$ grid with $\Delta t=0.02$ and 0.005 at the point location $(x, y)=(2.0,0.25)$. 
Grid

$-101 \times 1001$

..... $41 \times 401$

....... $41 \times 401$
$\Delta t$

0.005

0.005

0.020
Time Span*

77.0 to 87.0

165.55 to 175.55

81.55 to 91.55

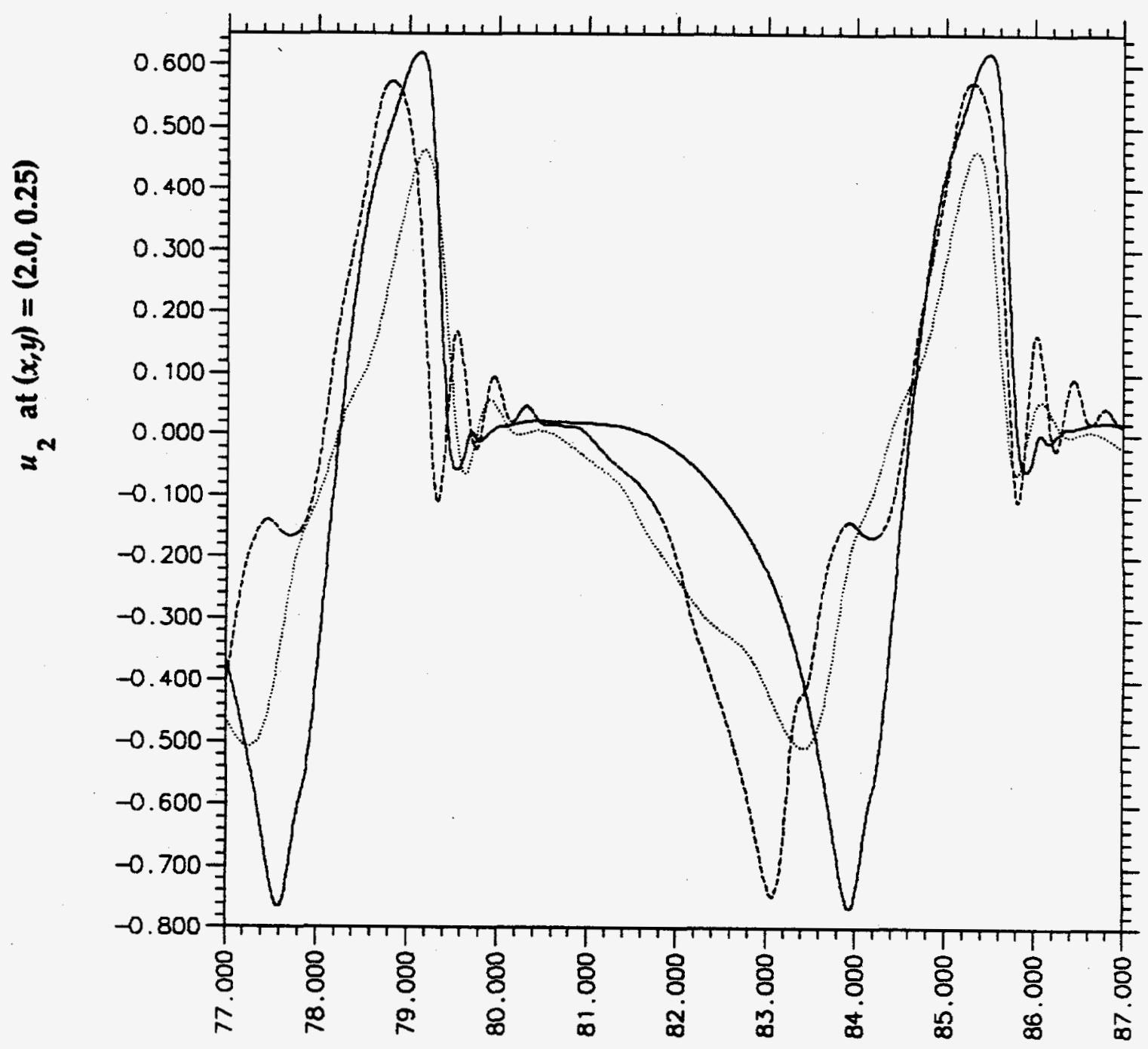

t ime

*Time histories are shifted by placing minimum points at time $=\mathbf{7 8 . 1 6}$.

Fig. 5.2.8 Comparison of $u_{2}$, velocity time histories for the $101 \times 1001$ grid with $\Delta t=0.005$ and for the $41 \times 401$ grid with $\Delta t=0.02$ and 0.005 at the point location $(x, y)=(2.0,0.25)$. 
Grid

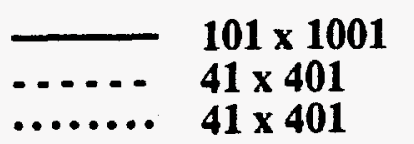

$\Delta t$

0.005

0.005

0.020
Time Span*

77.0 to 87.0 165.55 to 175.55 81.55 to 91.55

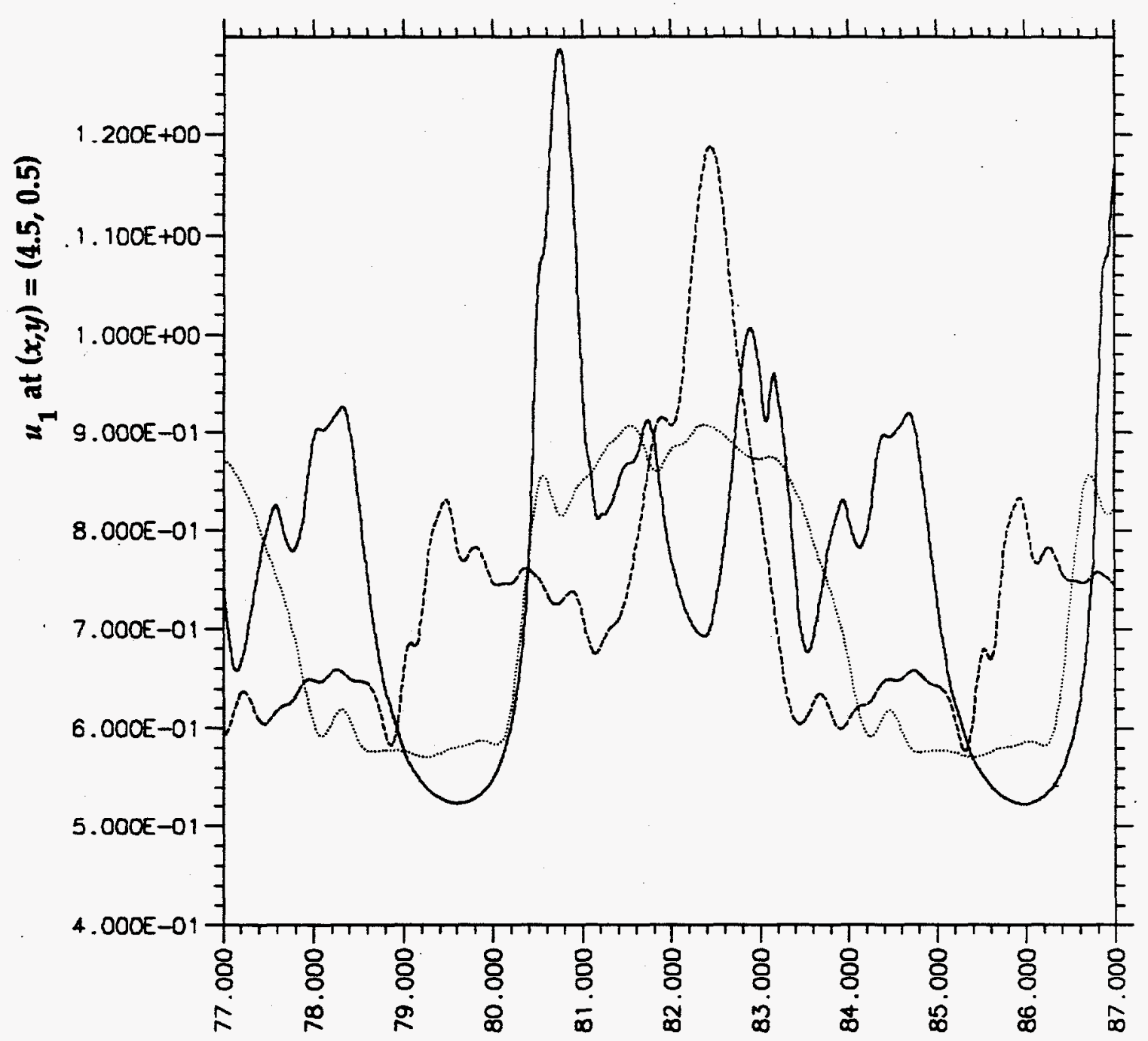

time

*Time histories are shifted by placing minimum points at time $=78.16$.

Fig. 5.2.9 Comparison of $u_{1}$, velocity time histories for the $101 \times 1001$ grid with $\Delta t=0.005$ and for the $41 \times 401$ grid with $\Delta t=0.02$ and 0.005 at the point location $(x, y)=(4.5,0.5)$. 
Grid

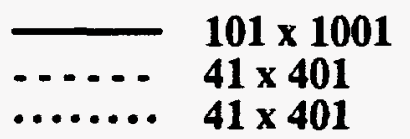

$\Delta$

0.005

0.005

0.020
Time Span*

77.0 to 87.0

165.55 to 175.55

81.55 to 91.55

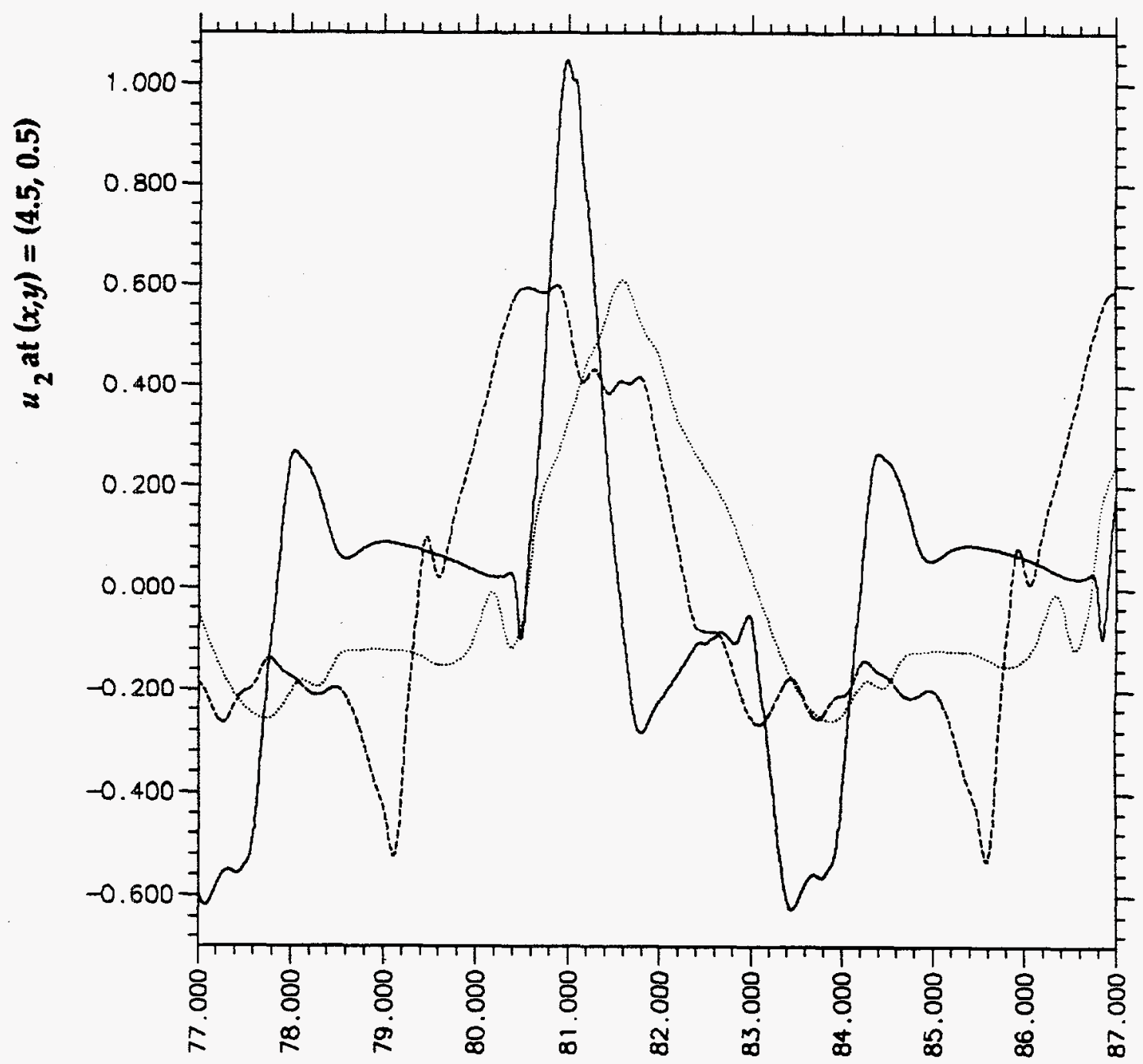

time

*Time histories are shifted by placing minimum points at time $=\mathbf{7 8 . 1 6}$.

Fig. 5.2.10 Comparison of $u_{2}$, velocity time histories for the $101 \times 1001$ grid with $\Delta t=0.005$ and for the $41 \times 401$ grid with $\Delta t=0.02$ and 0.005 at the point location $(x, y)=(4.5,0.5)$. 


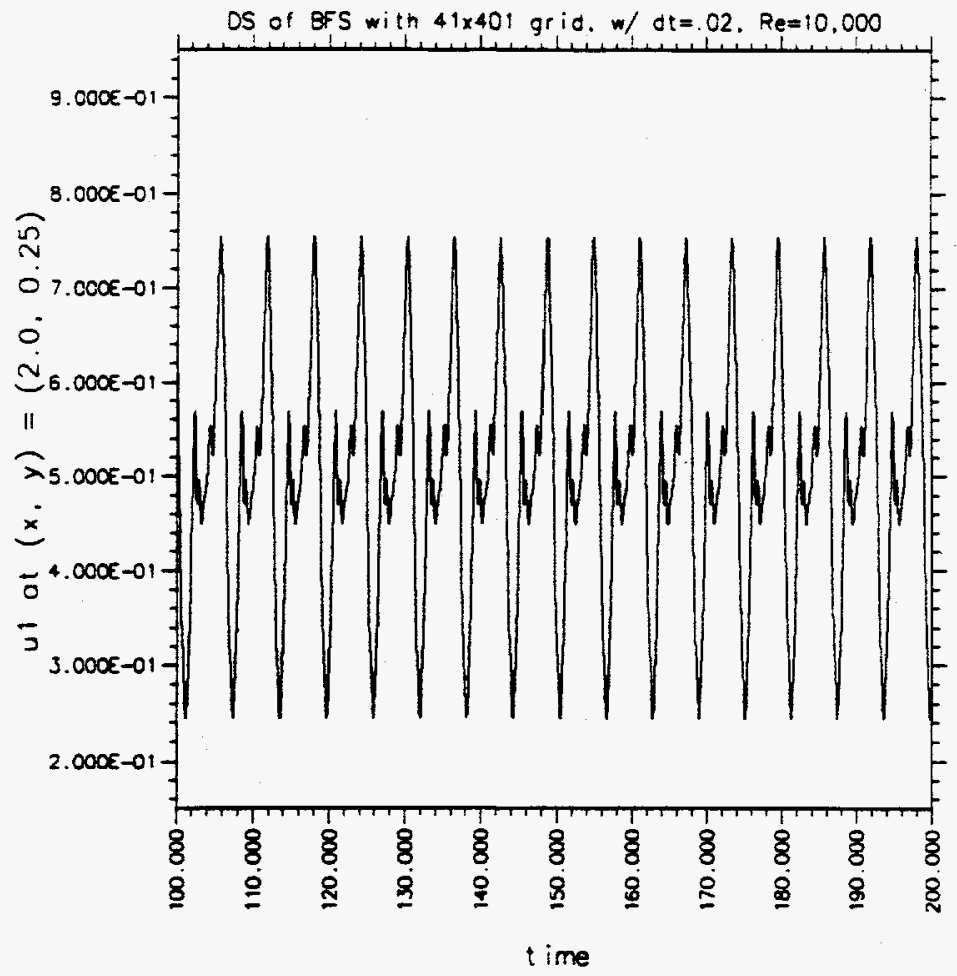

(a)

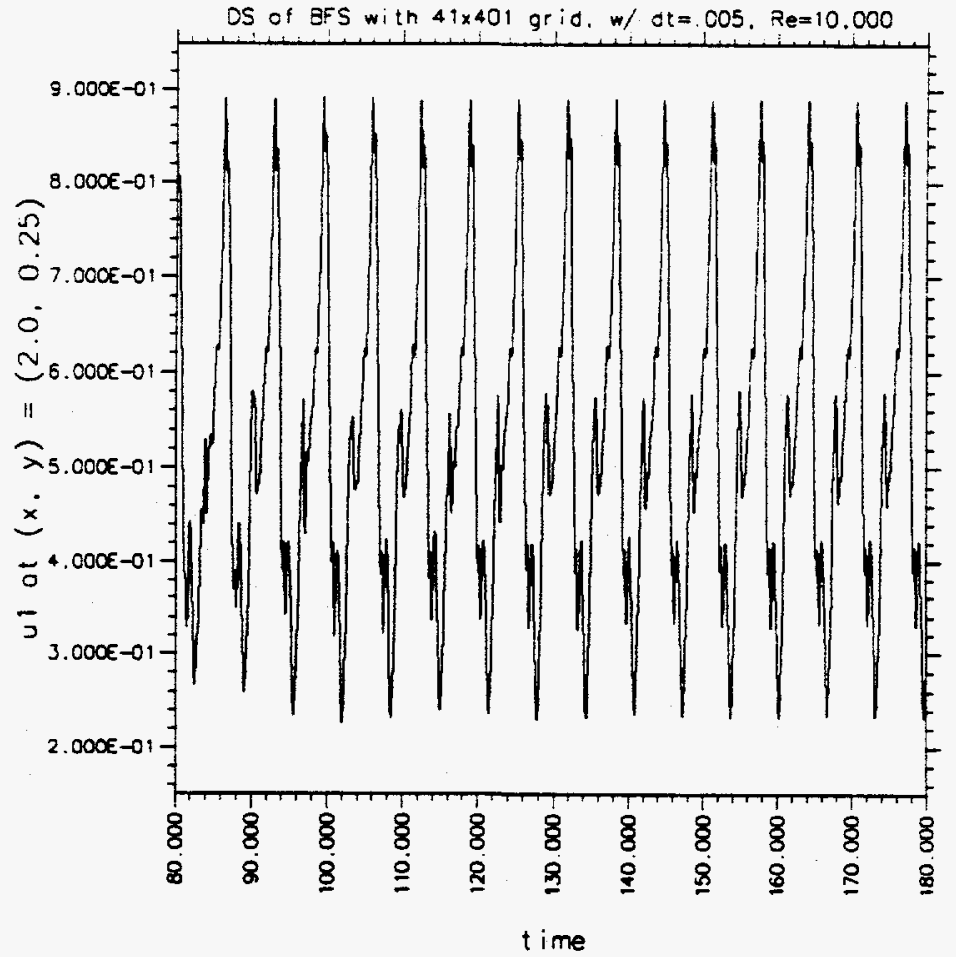

(b)

Figure 5.2.11 Extended time histories of $u_{1}$ at $(x, y)=(2.0,0.25)$ for the $41 x 401$ node grid using a) $\Delta t=0.02$ and b) $\Delta t=0.005$ (shown for the same axis scales). 


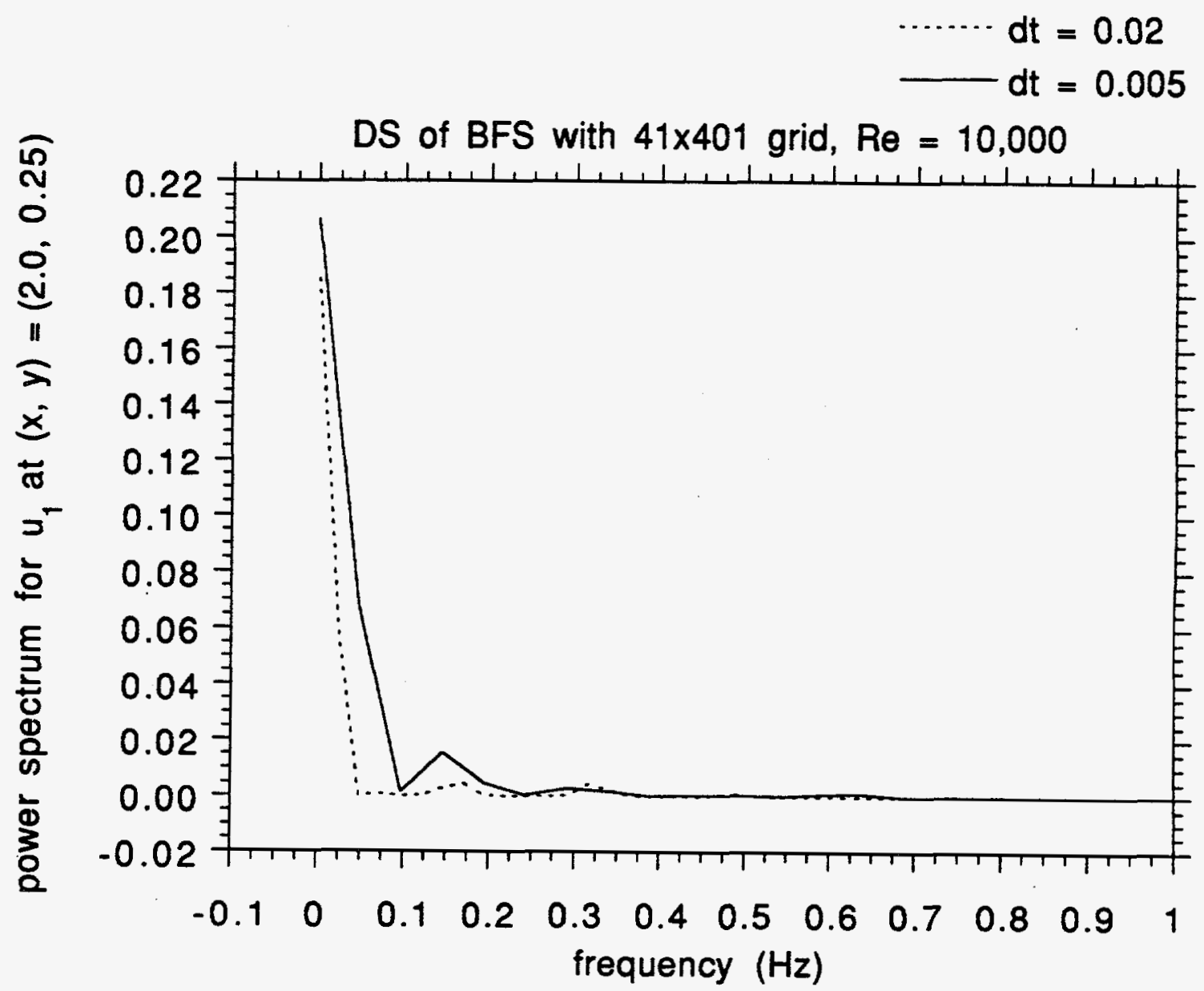

Figure 5.2.12 Power spectrum on linear plot for $u_{1}$ time history at $(x, y)=(2.0$, $0.25)$ for the $41 \times 401$ grid using $\Delta t=0.02(t=100$ to 161.42) and $\Delta t=0.005(t=140$ to 170.715). 

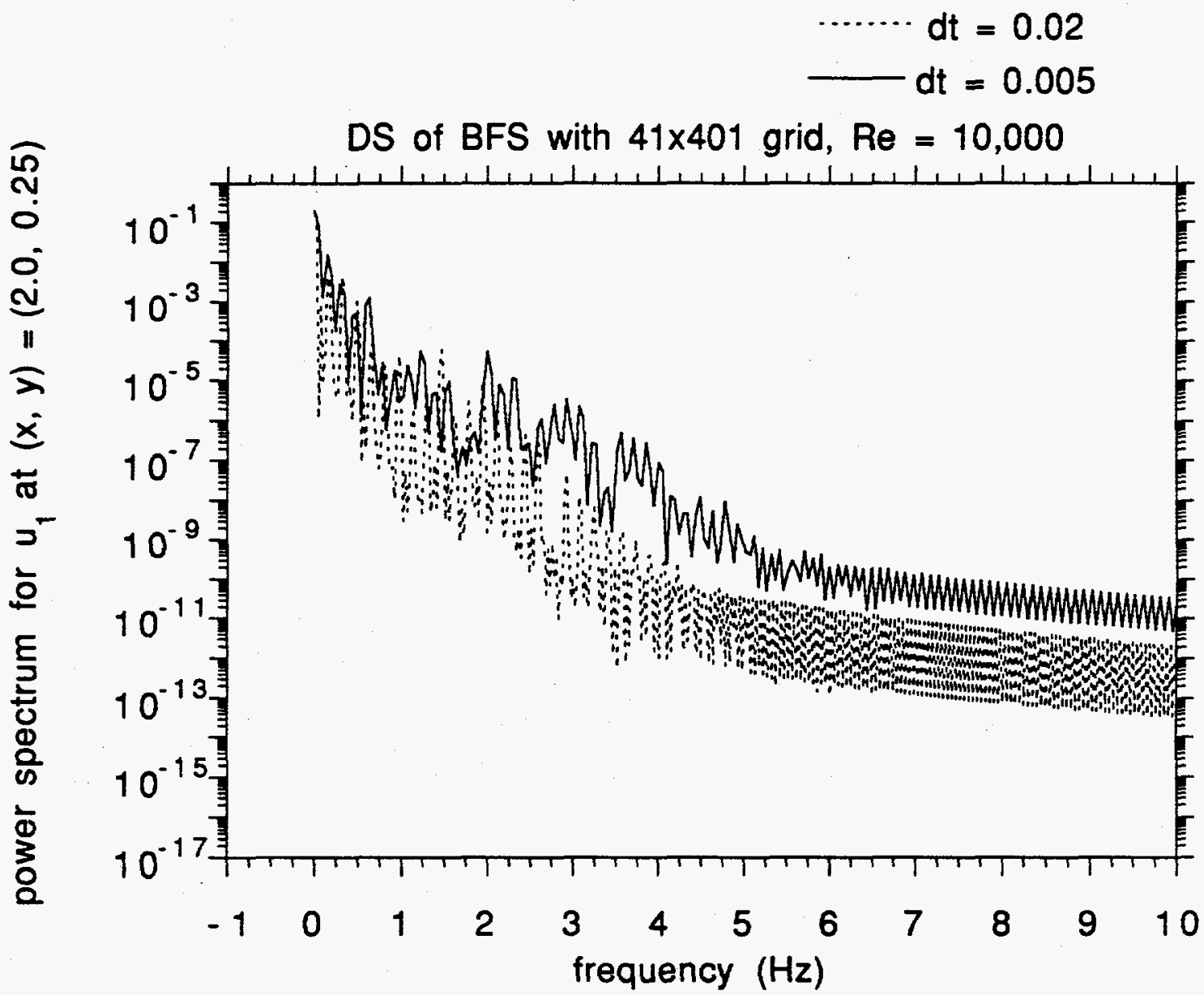

Figure 5.2.13 Power spectrum on log plot for $u_{1}$ time history at $(x, y)=(2.0,0.25)$ for the $41 \times 401$ grid using $\Delta t=0.02(t=100$ to 161.42) and $\Delta t=0.005(t=140$ to 170.715). 


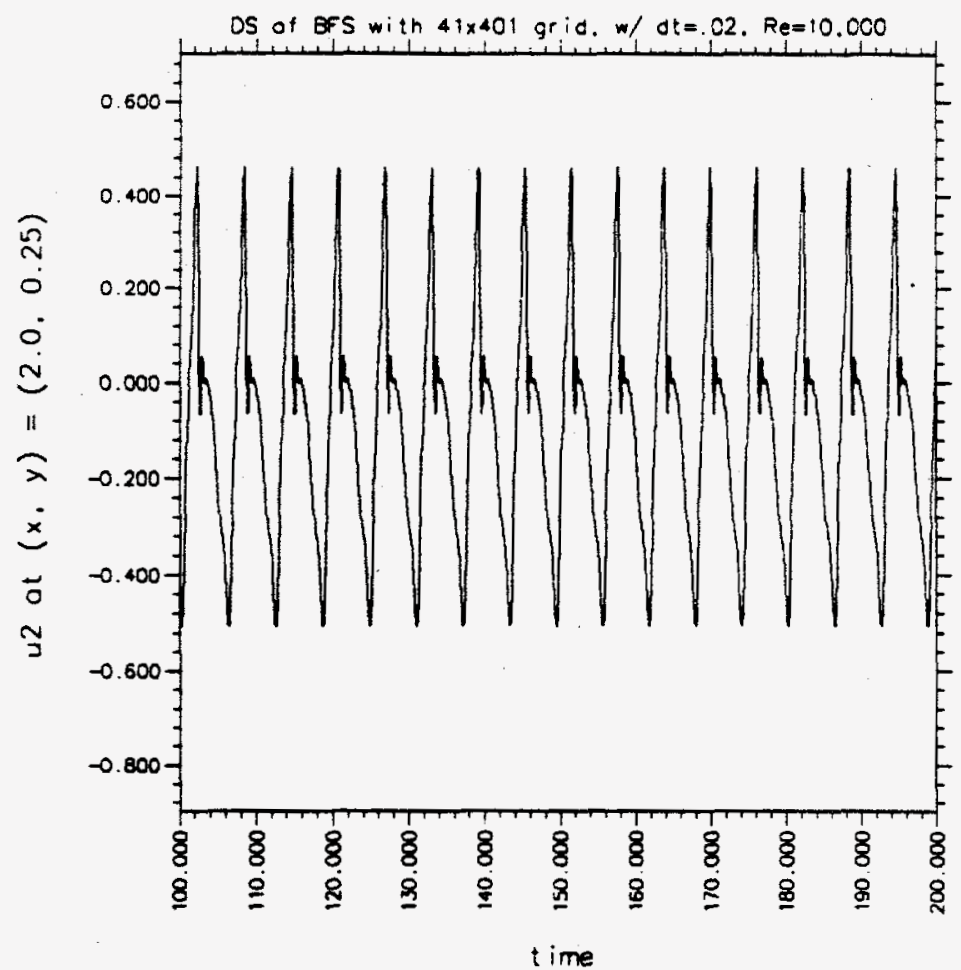

(a)

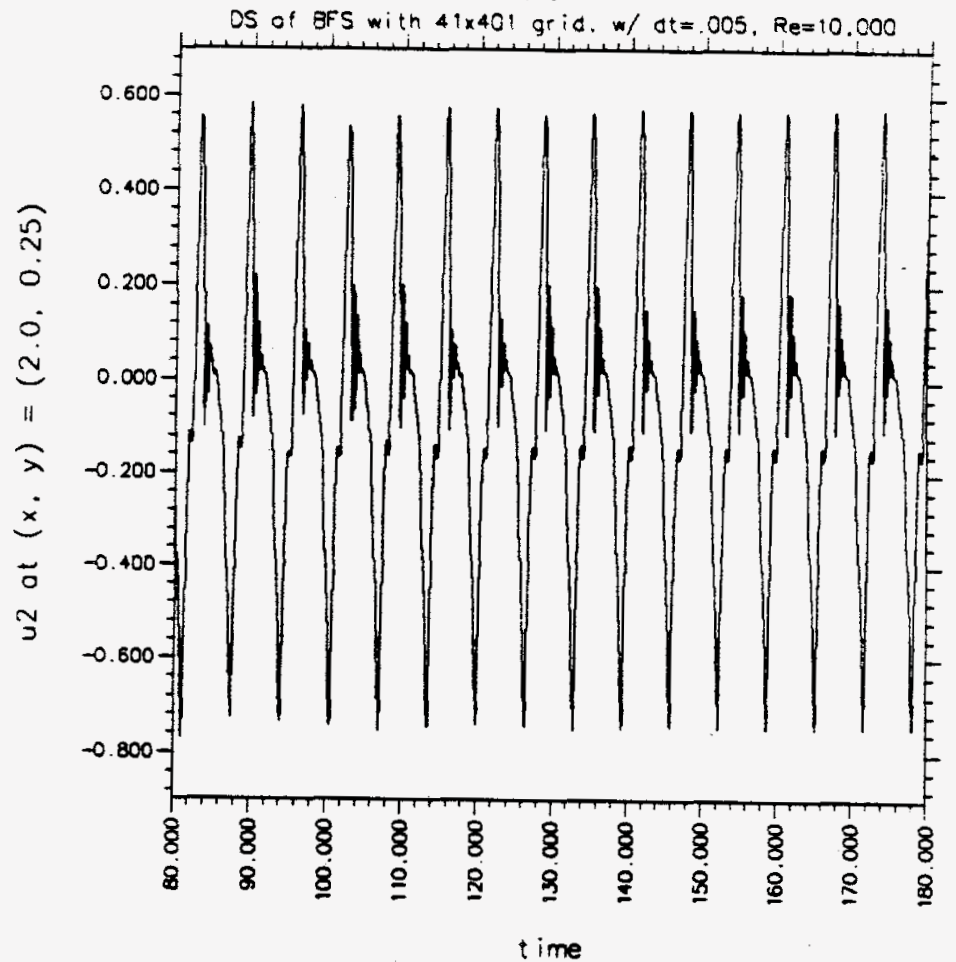

(b)

Figure 5.2.14 Extended time histories of $u_{2}$ at $(x, y)=(2.0,0.25)$ for the $41 \times 401$ node grid using a) $\Delta t=0.02$ and b) $\Delta t=0.005$ (shown for the same axis scales). 


$$
\begin{aligned}
& -\mathrm{dt}=0.005 \\
& \cdots \cdots \mathrm{dt}=0.02
\end{aligned}
$$

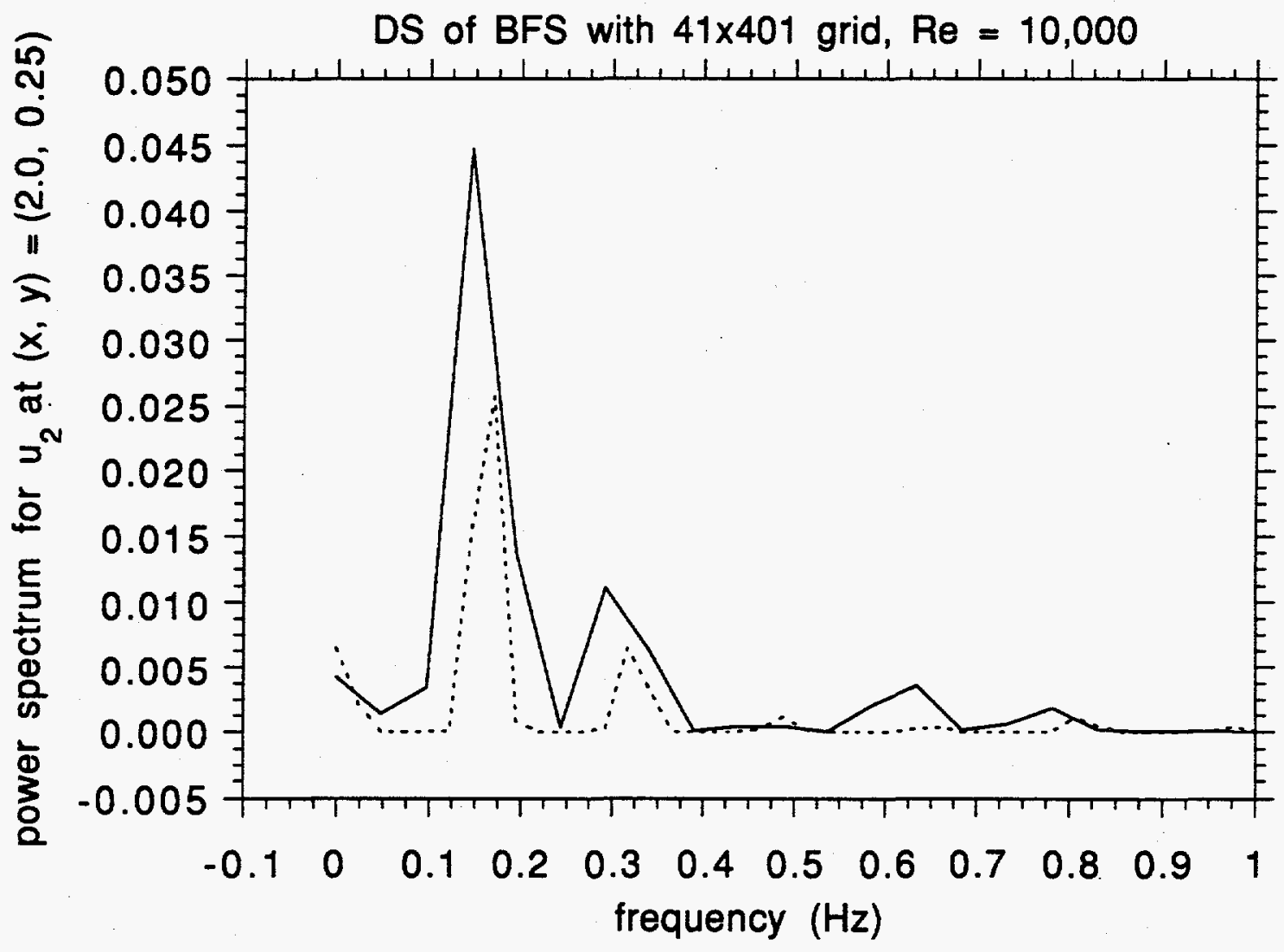

Figure 5.2.15 Power spectrum on linear plot for $u_{2}$ time history at $(x, y)=(2.0$, $0.25)$ for the $41 \times 401$ grid using $\Delta t=0.02(t=100$ to 161.42) and $\Delta t=0.005(t=140$ to 170.715). 

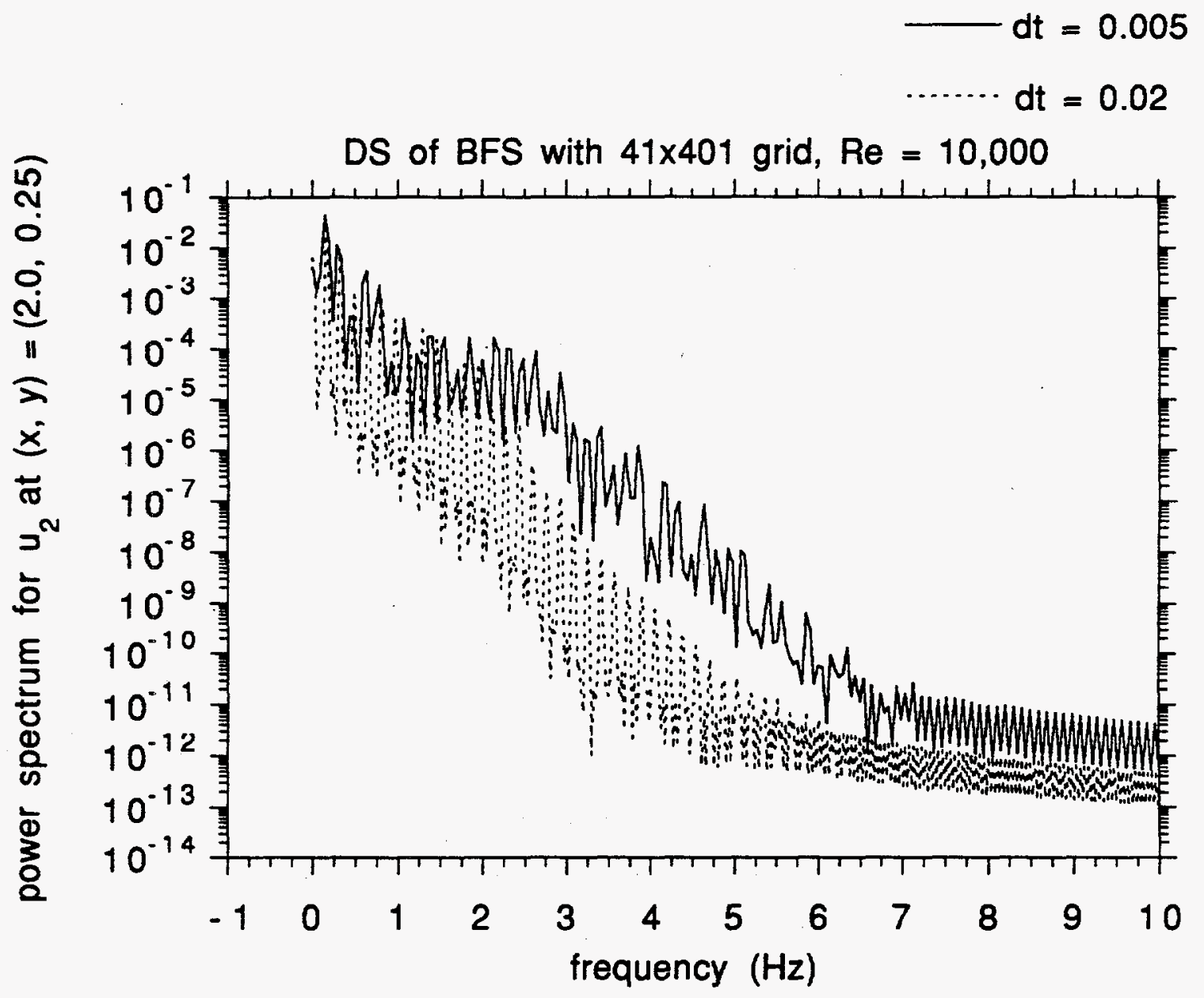

Figure 5.2.16 Power spectrum on log plot for $u_{2}$ time history at $(x, y)=(2.0,0.25)$ for the $41 \times 401$ grid using $\Delta t=0.02(t=100$ to 161.42) and $\Delta t=0.005(t=140$ to 170.715). 


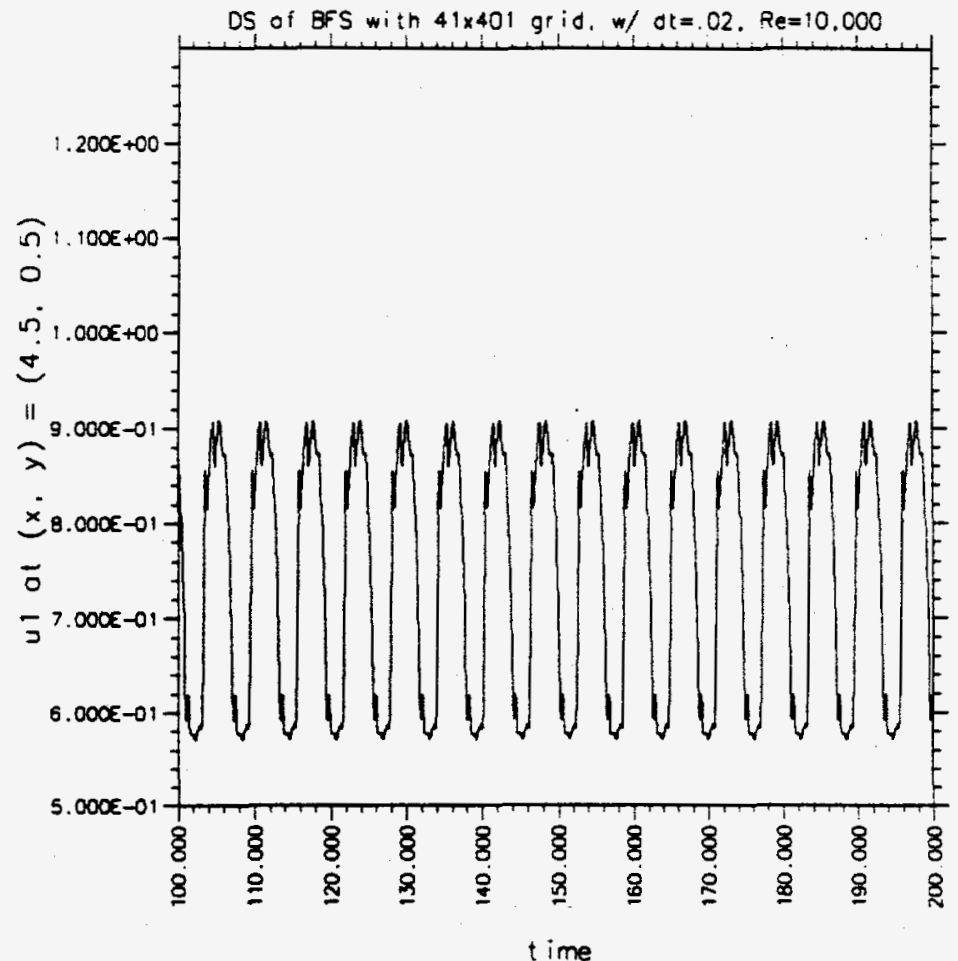

(a)

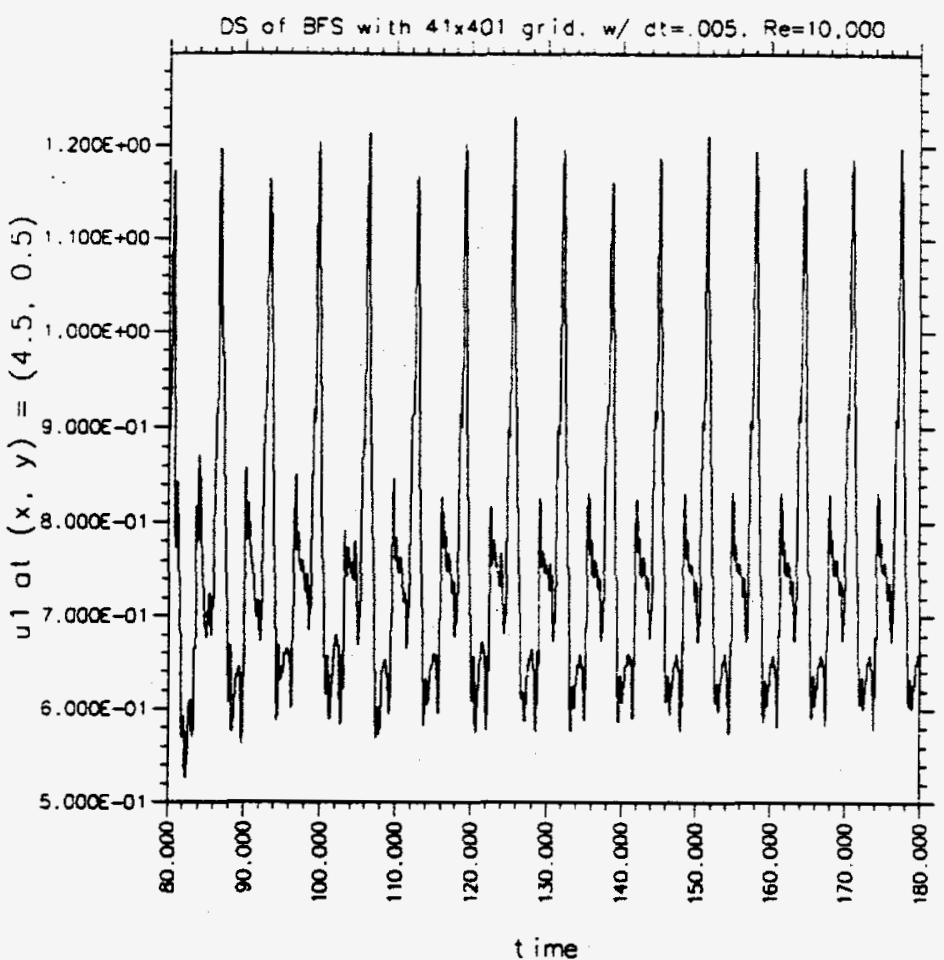

(b)

Figure 5.2.17 Extended time histories of $u_{1}$ at $(x, y)=(4.5,0.5)$ for the $41 \times 401$ node grid using a) $\Delta t=0.02$ and $\mathrm{b}$ ) $\Delta t=0.005$ (shown for the same axis scales). 
$\longrightarrow d t=0.005$

$\cdots \cdots \cdot d t=0.02$

DS of BFS with $41 \times 401$ grid, $R e=10,000$

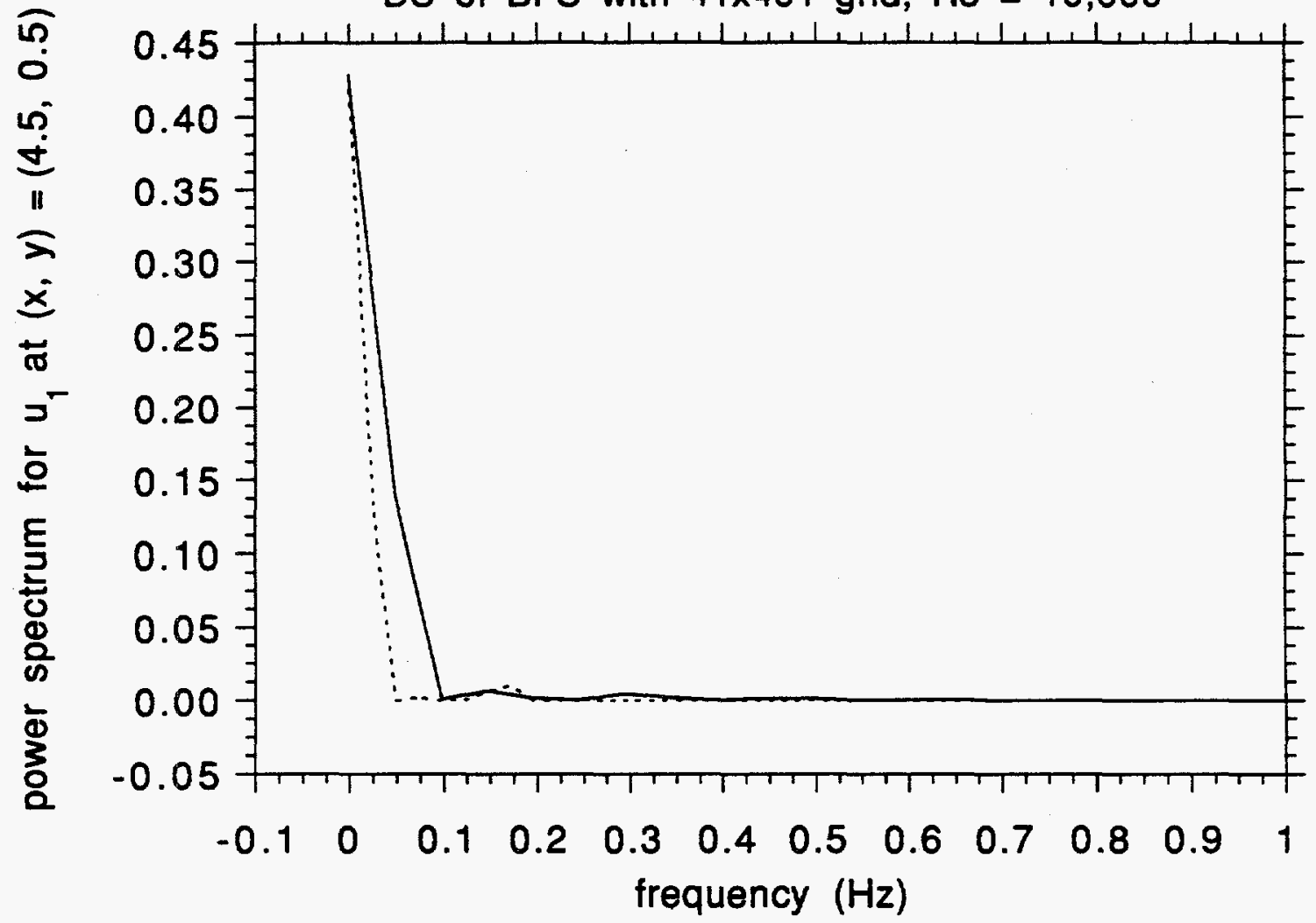

Figure 5.2.18 Power spectrum on linear plot for $u_{1}$ time history at $(x, y)=(4.5,0.5)$ for the $41 \times 401$ grid using $\Delta t=0.02(t=100$ to 161.42) and $\Delta t=0.005(t=140$ to 170.715). 


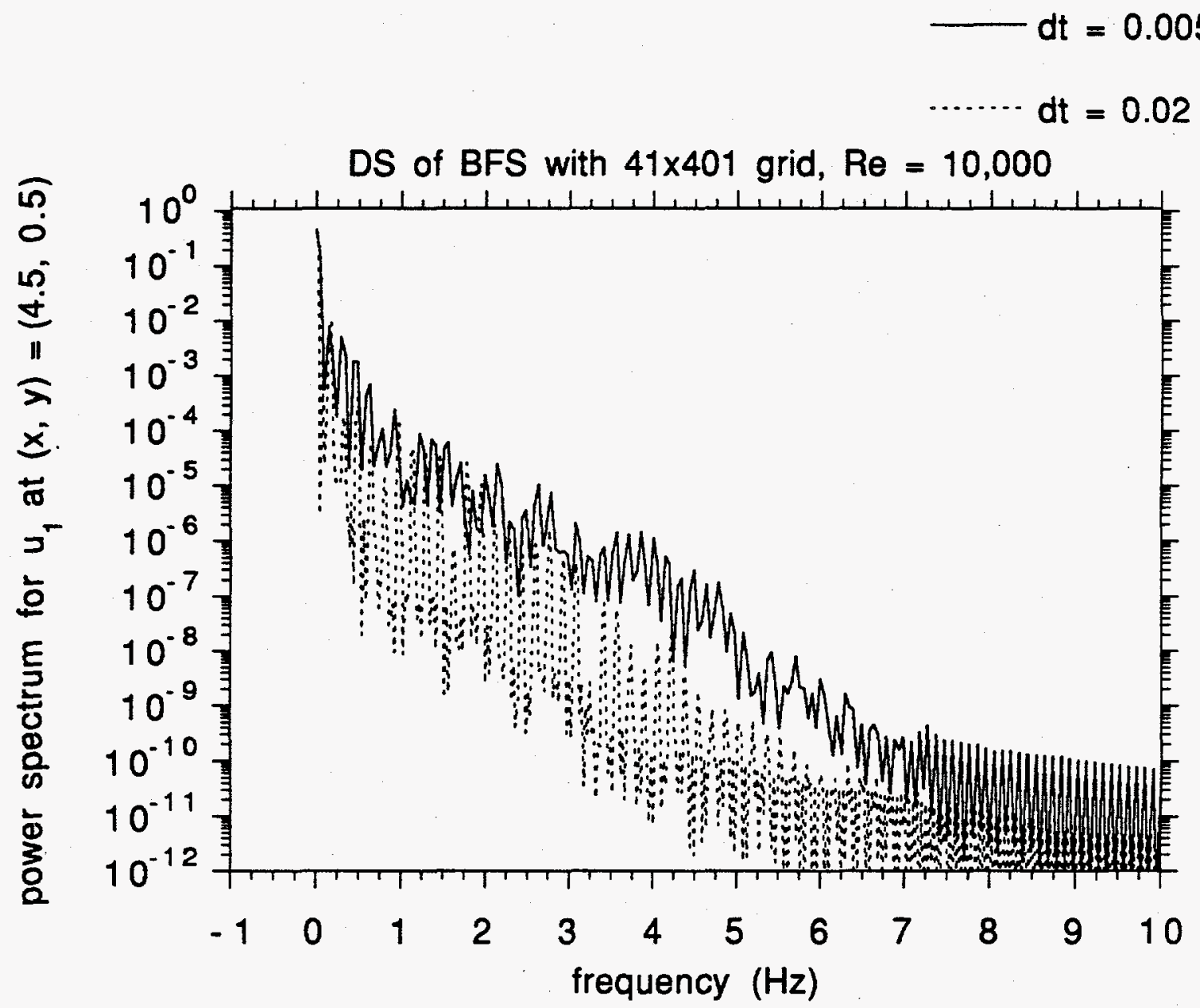

Figure 5.2.19 Power spectrum on log plot for $u_{1}$ time history at $(x, y)=(4.5,0.5)$ for the $41 \times 401$ grid using $\Delta t=0.02(t=100$ to 161.42$)$ and $\Delta t=0.005(t=140$ to 170.715). 


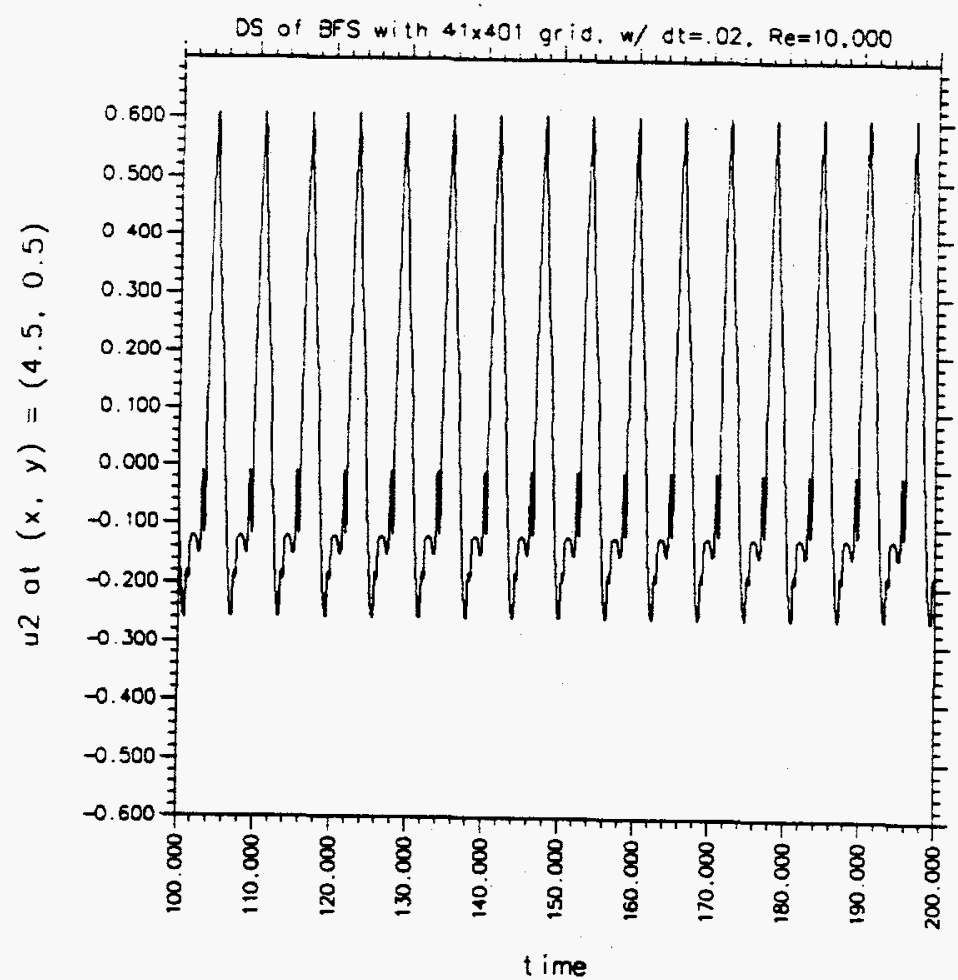

(a)

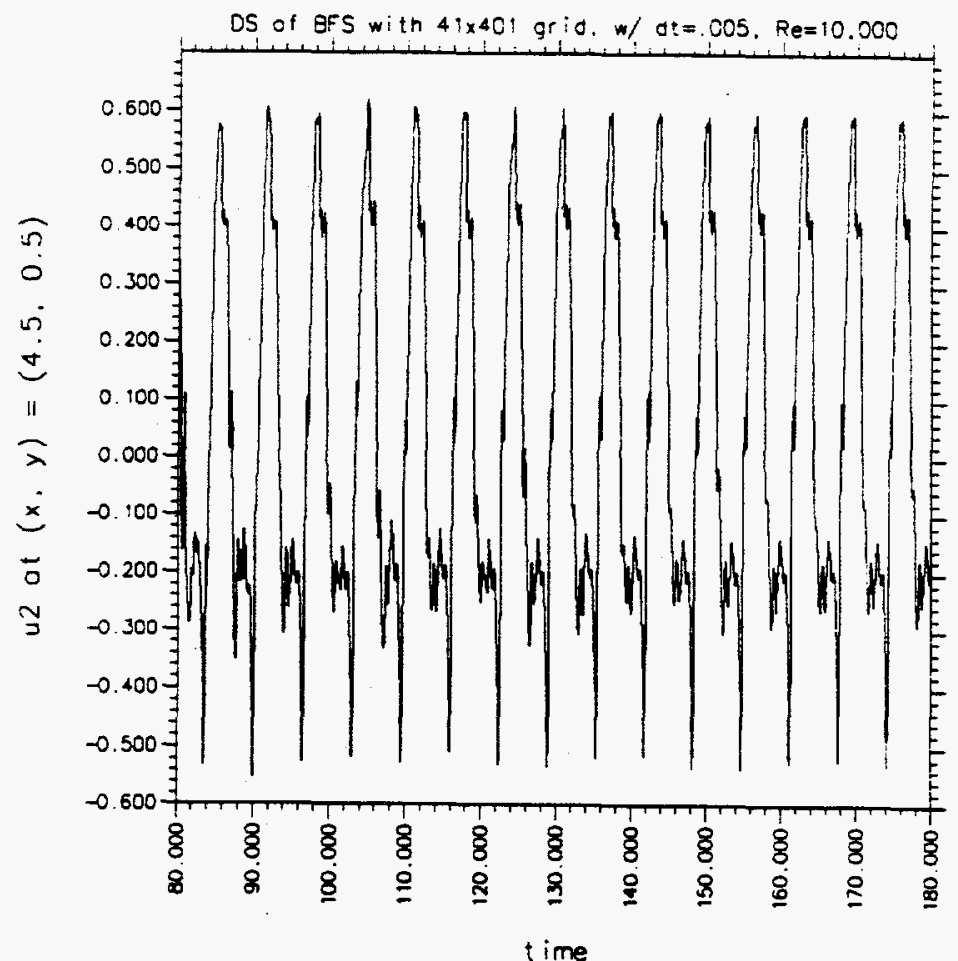

(b)

Figure 5.2.20 Extended time histories of $u_{2}$ at $(x, y)=(4.5,0.5)$ for the $41 \times 401$ node grid using a) $\Delta t=0.02$ and $b$ ) $\Delta t=0.005$ (shown for the same axis scales). 


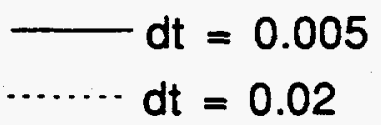

DS of BFS with $41 \times 401$ grid, $R e=10,000$

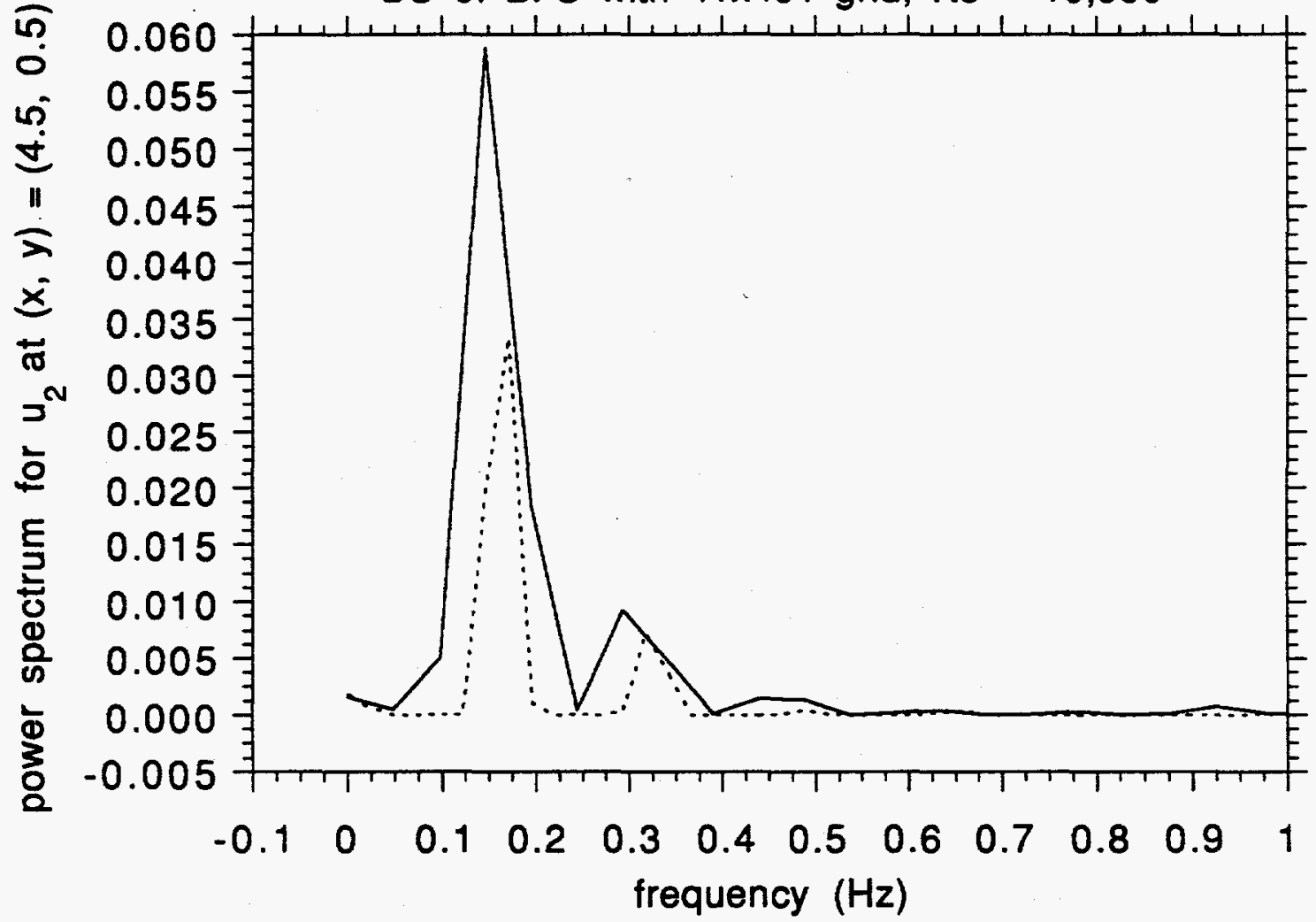

Figure 5.2.21 Power spectrum on linear plot for $u_{2}$ time history at $(x, y)=(4.5,0.5)$ for the $41 \times 401$ grid using $\Delta t=0.02(t=100$ to 161.42) and $\Delta t=0.005(t=140$ to 170.715). 


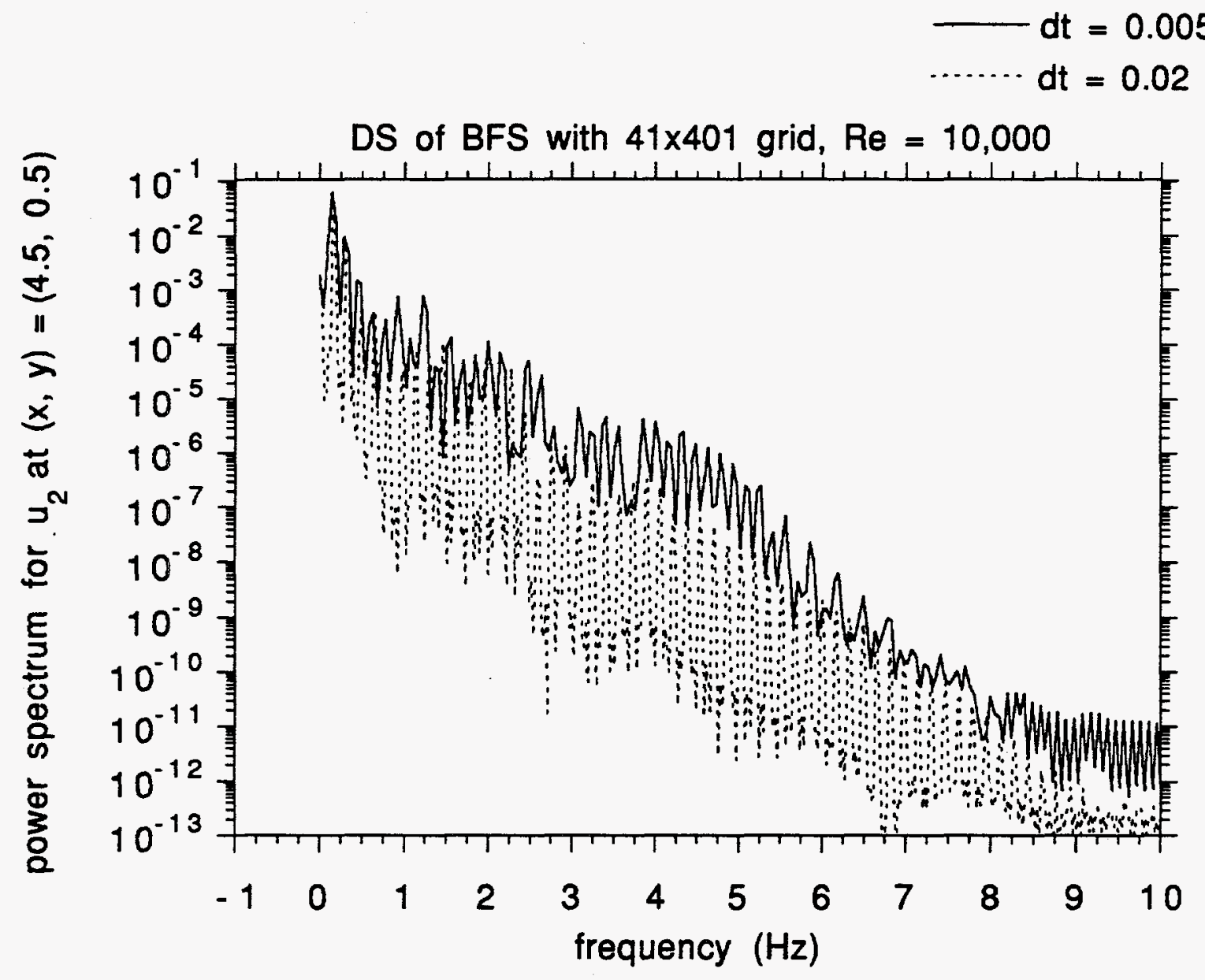

Figure 5.2.22 Power spectrum on $\log$ plot for $u_{2}$ time history at $(x, y)=(4.5,0.5)$ for the $41 \times 401$ grid using $\Delta t=0.02(t=100$ to 161.42$)$ and $\Delta t=0.005(t=140$ to 170.715). 


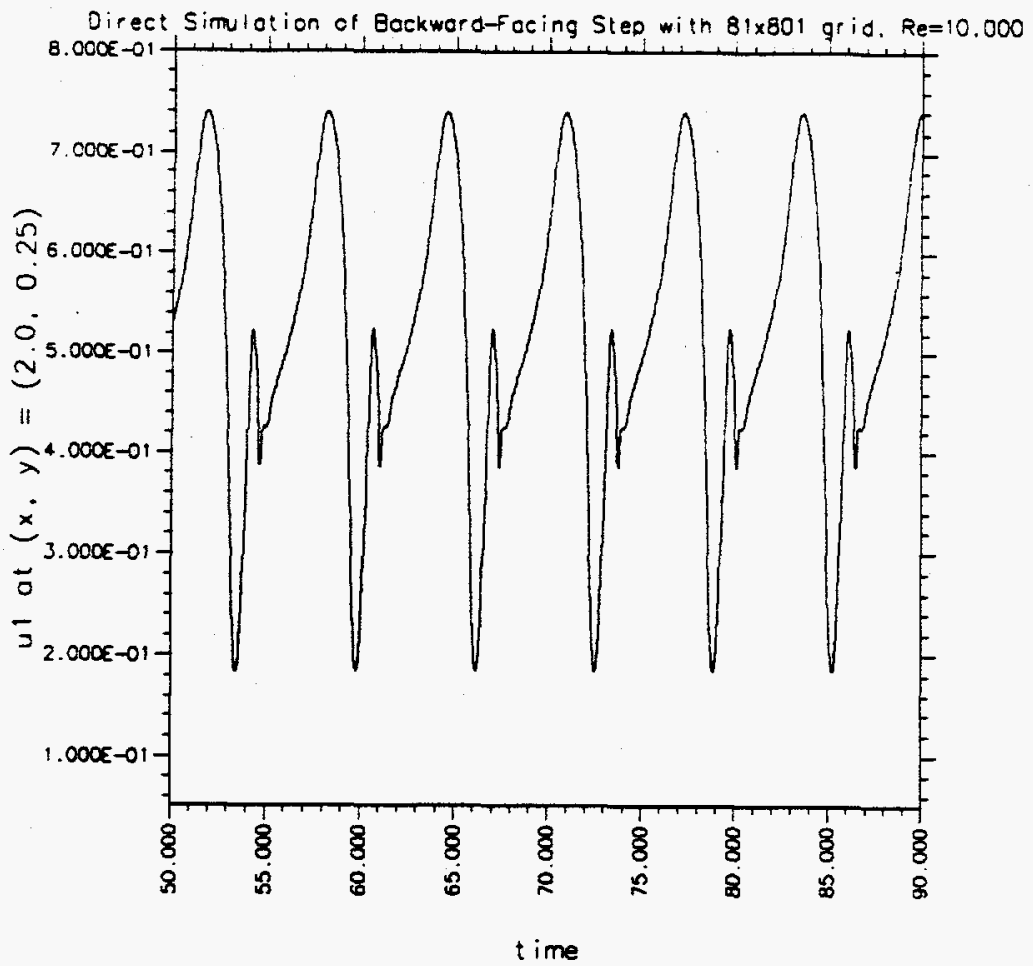

(a)

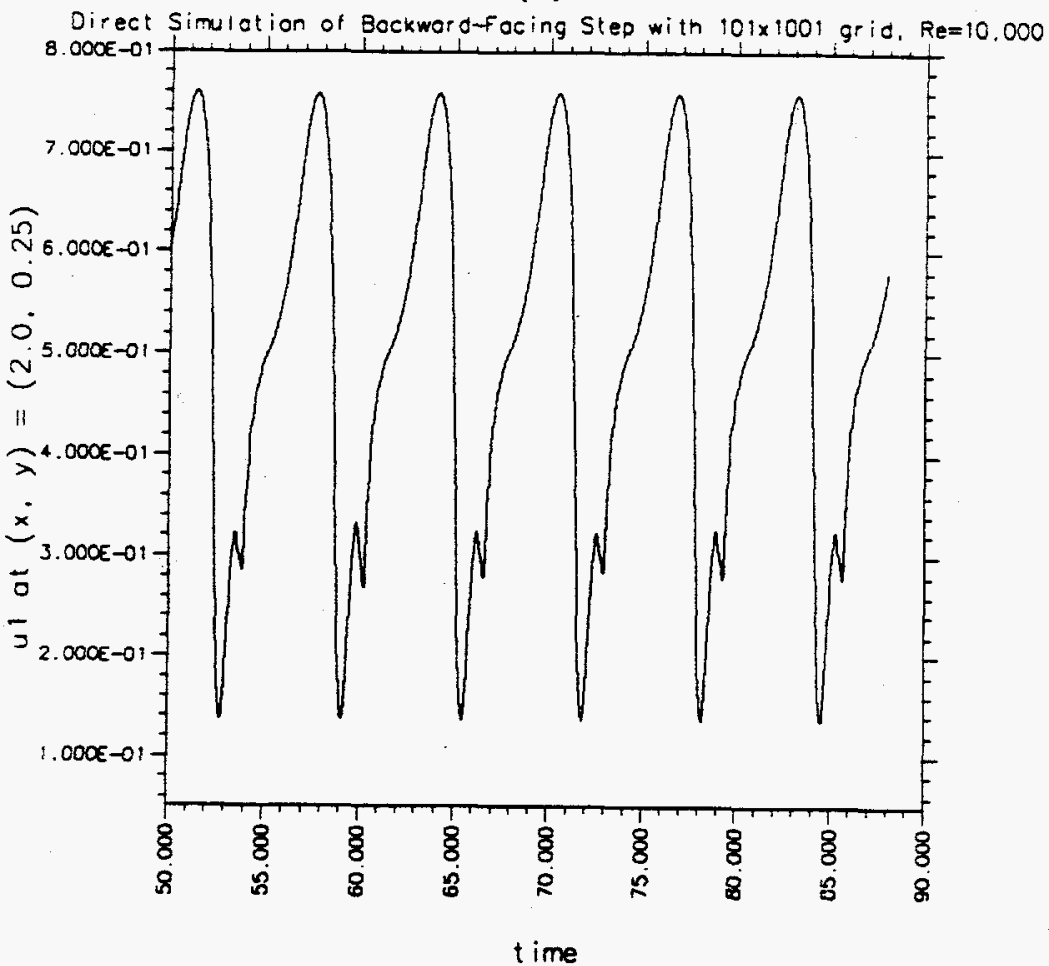

(b)

Figure 5.2.23 Extended time histories of $u_{1}$ at $\left(x_{2} y\right)=(2.0,0.25)$ for a) the $81 \times 801$ grid at $\Delta t=0.01$ and b) the $101 \times 1001$ grid at $\Delta t=0.005$ (shown for the same axis scales). 
$101 \times 1001$ grid with $\mathrm{dt}=0.005$

$81 \times 801$ grid with $\mathrm{dt}=0.01$

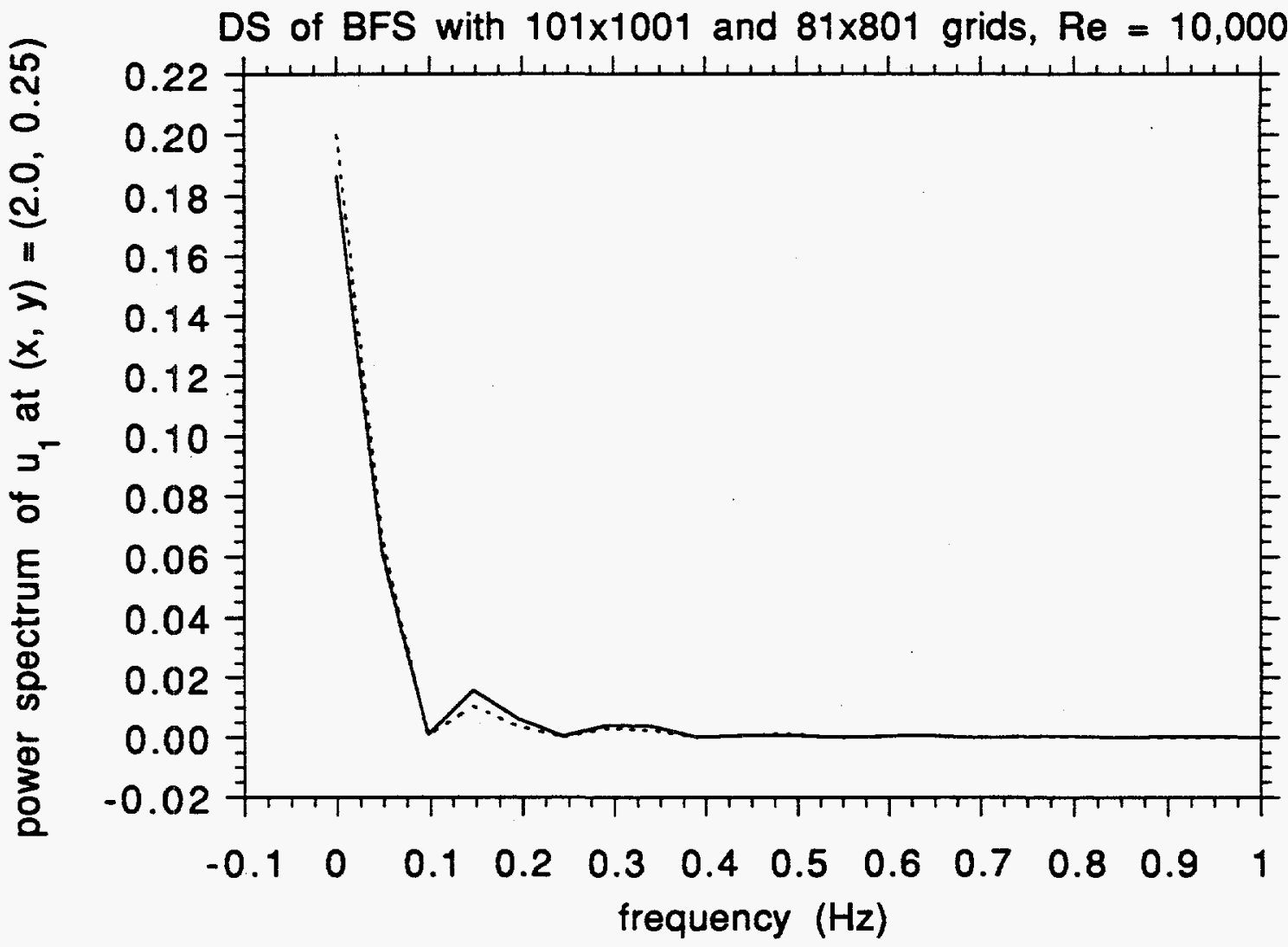

Figure 5.2.24 Power spectrum on linear plot for $u_{1}$ time history at $(x, y)=(2.0$, $0.25)$ for the $81 \times 801$ grid using $\Delta t=0.01(t=50$ to 80.71) and the $101 \times 1001$ grid using $\Delta t=0.005(t=50$ to 80.715$)$. 
$101 \times 1001$ grid with $\mathrm{dt}=0.005$

$81 \times 801$ grid with $d t=0.01$

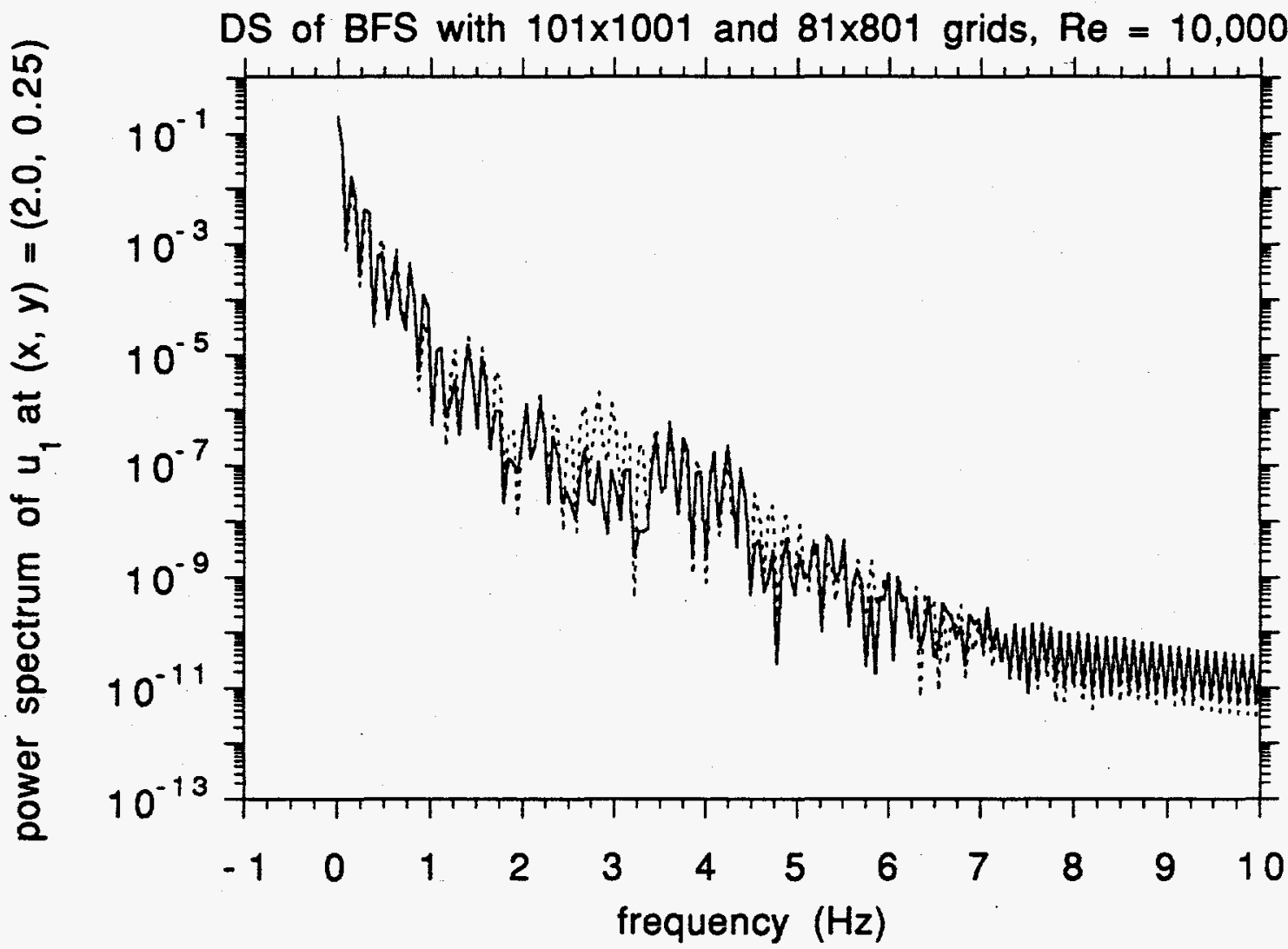

Figure 5.2.25 Power spectrum on log plot for $u_{1}$ time history at $(x, y)=(2.0,0.25)$ for the $81 \times 801$ grid using $\Delta t=0.01(t=50$ to 80.71$)$ and the $101 \times 1001$ grid using $\Delta t=0.005(t=50$ to 80.715$)$. 


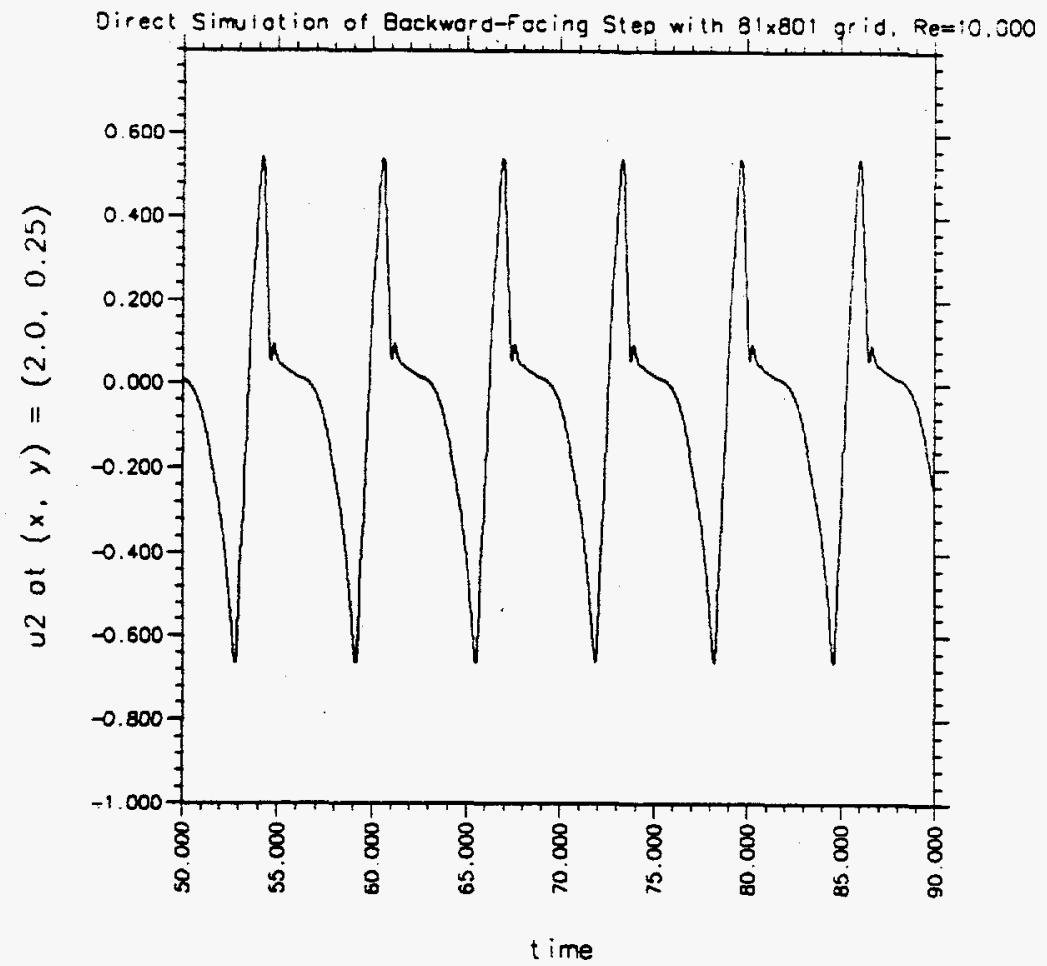

(a)

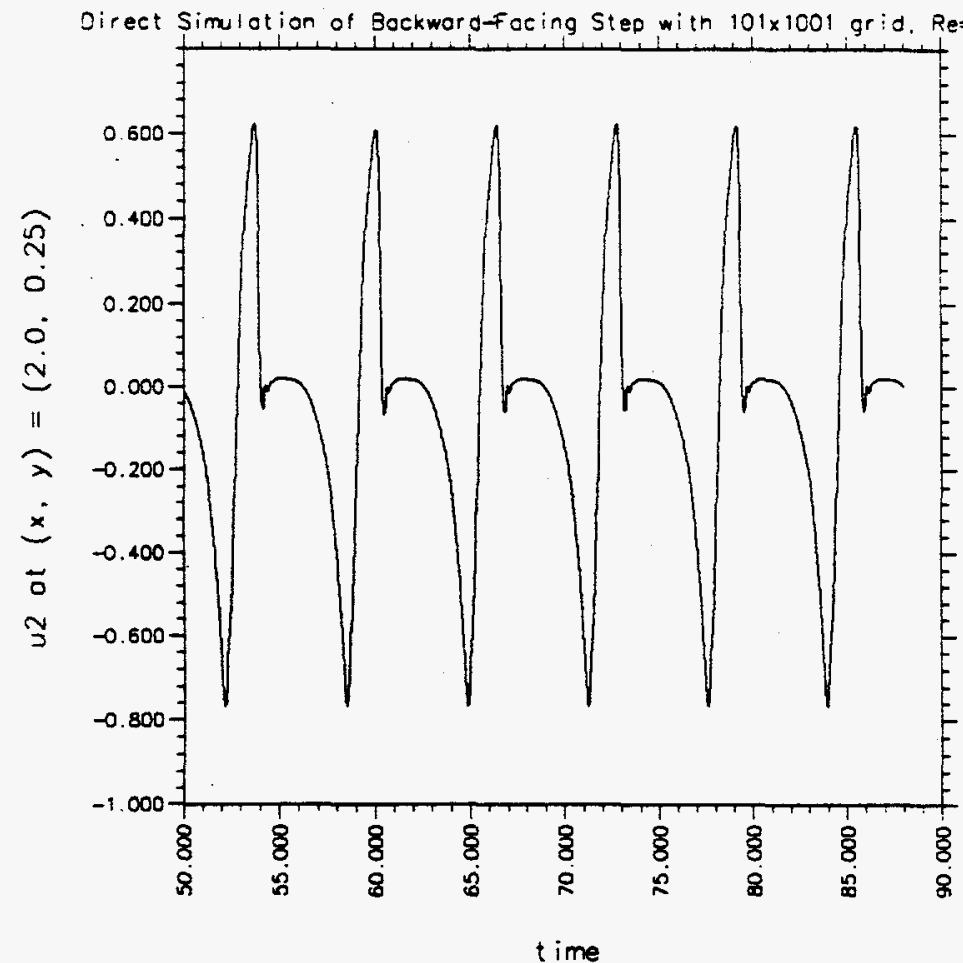

(b)

Figure 5.2.26 Extended time histories of $u_{2}$ at $\left(x_{2}, y\right)=(2.0,0.25)$ for a) the $81 \times 801$ grid at $\Delta t=0.01$ and $b$ ) the $101 \times 1001$ grid at $\Delta t=0.005$ (shown for the same axis scales). 
$-101 \times 1001$ grid with $\mathrm{dt}=0.005$

$\cdots \cdot . \cdot 81 \times 801$ grid with $d t=0.01$

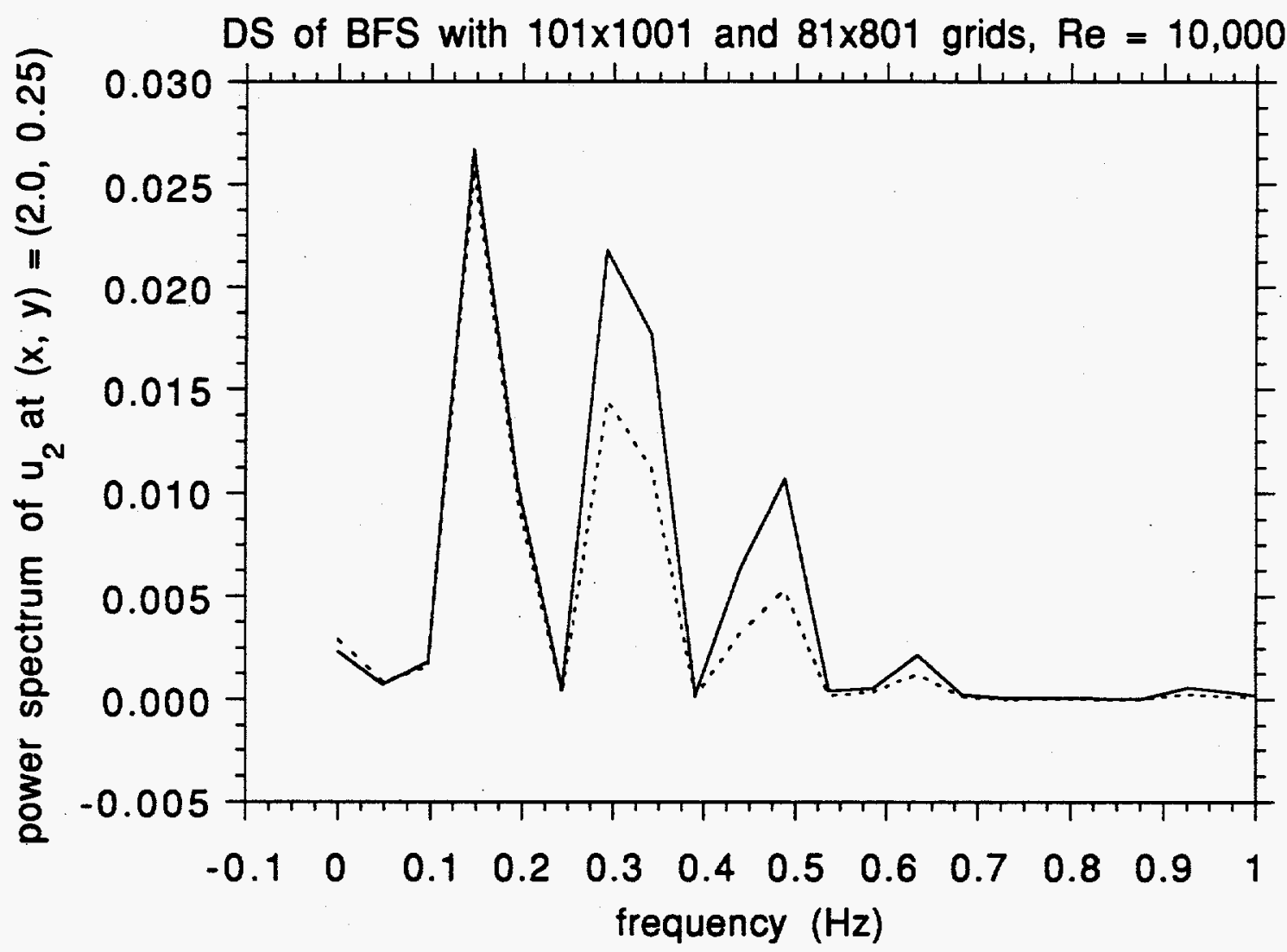

Figure 5.2.27 Power spectrum on linear plot for $u_{2}$ time histories at $(x, y)=(2.0$, $0.25)$ for the $81 \times 801$ grid using $\Delta t=0.01(t=50$ to 80.71) and the $101 \times 1001$ grid using $\Delta t=0.005(t=50$ to 80.715$)$. 
$101 \times 1001$ grid with $d t=0.005$

$81 \times 801$ grid with $d t=0.01$

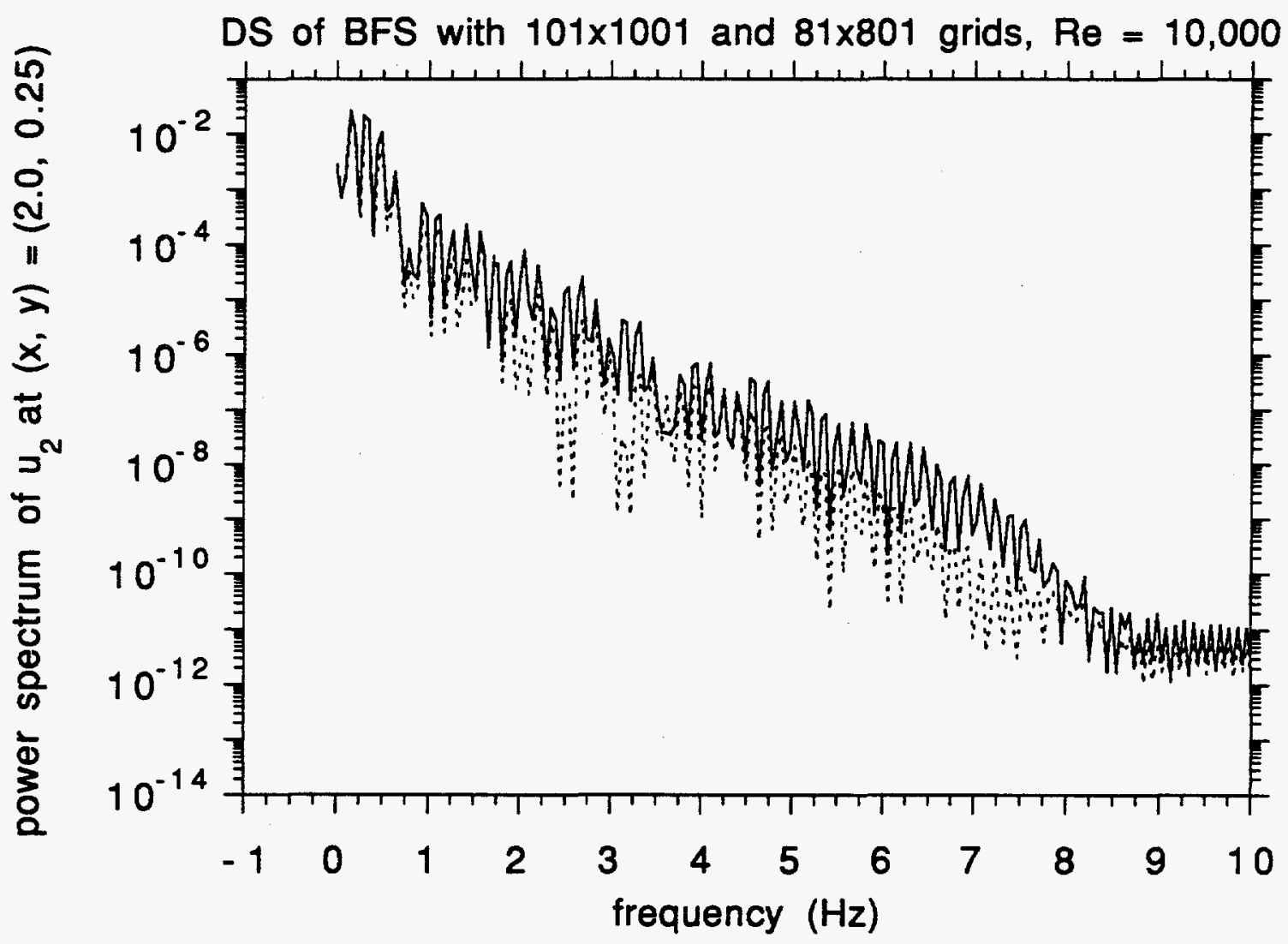

Figure 5.2.28 Power spectrum on $\log$ plot for $u_{2}$ time history at $(x, y)=(2.0,0.25)$ for the $81 \times 801$ grid using $\Delta t=0.01(t=50$ to 80.71$)$ and the $101 \times 1001$ grid using $\Delta t=0.005(t=50$ to 80.715$)$. 


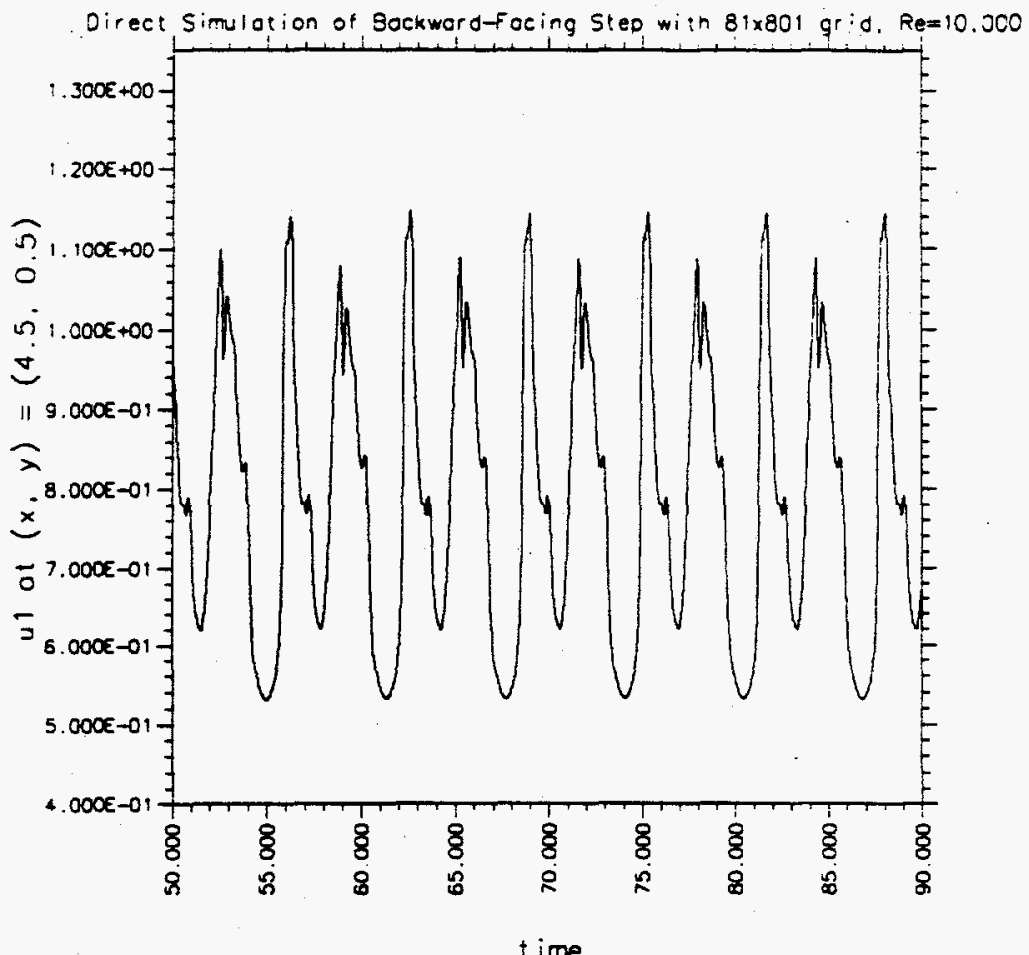

(a)

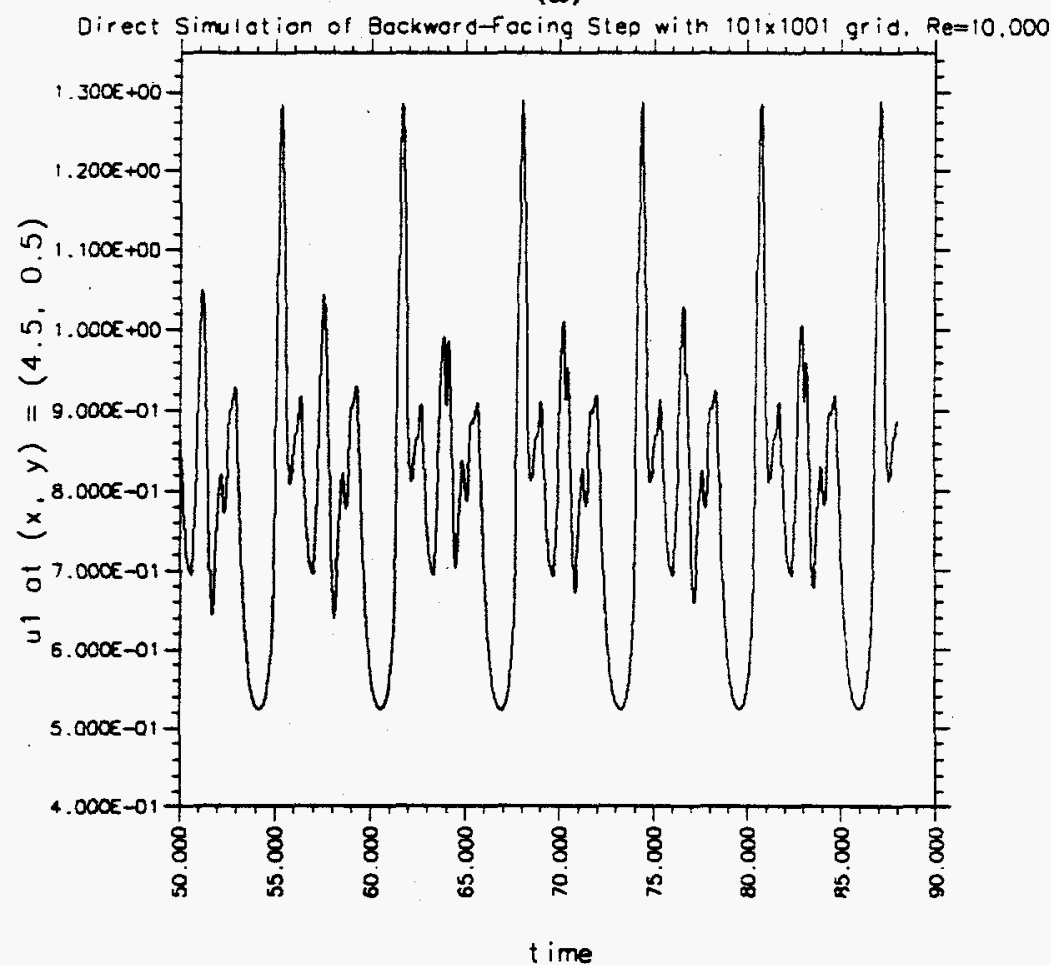

(b)

Figure 5.2.29 Extended time histories of $u_{1}$ at $(x, y)=(4.5,0.5)$ for a) the $81 \times 801$ grid at $\Delta t=0.01$ and $b$ ) the $101 \times 1001$ grid at $\Delta t=0.005$ (shown for the same axis scales). 


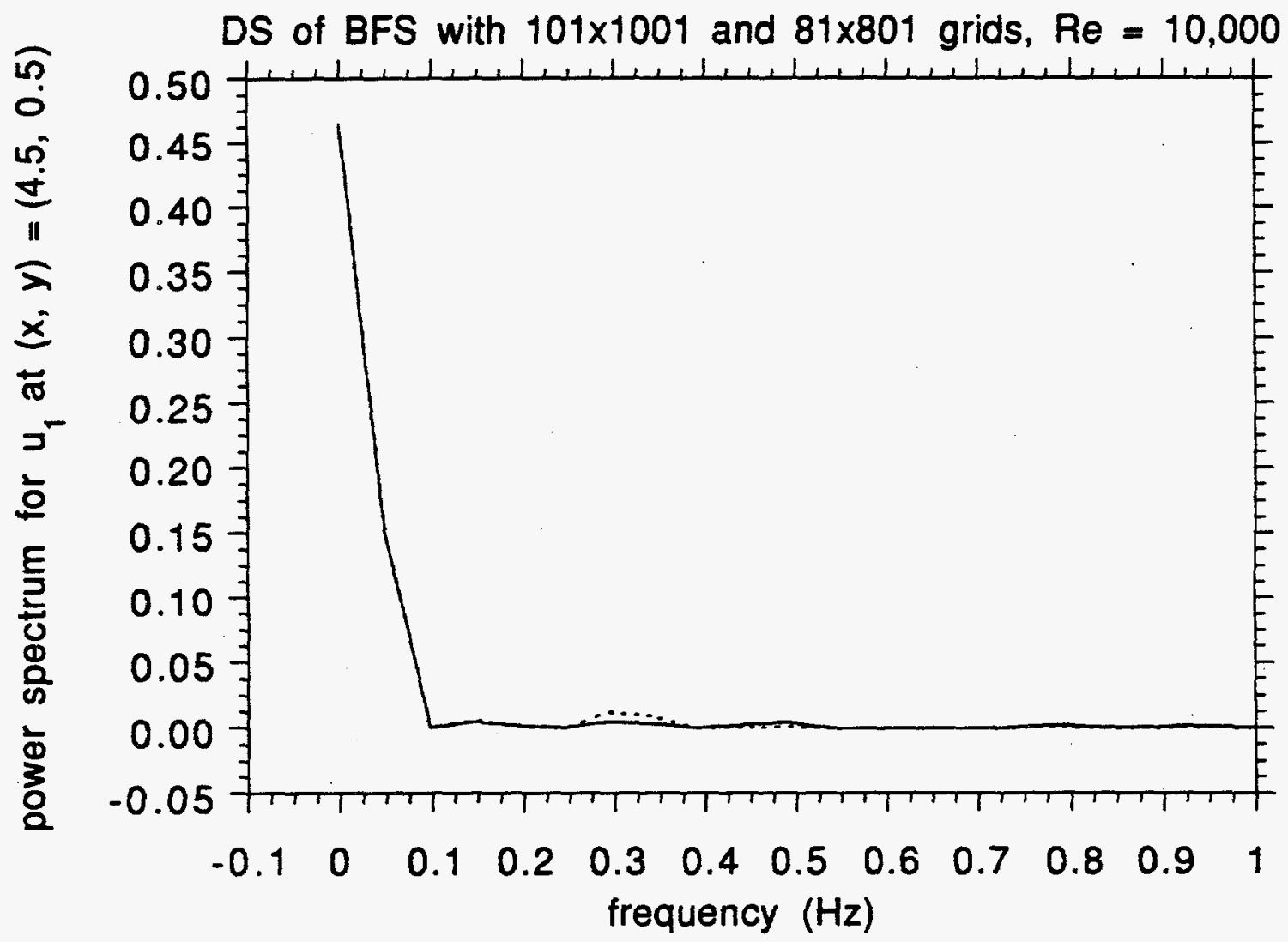

Figure 5.2.30 Power spectrum on linear plot for $u_{1}$ time history at $(x, y)=(4.5,0.5)$ for the $81 \times 801$ grid using $\Delta t=0.01(t=50$ to 80.71$)$ and the $101 \times 1001$ grid using $\Delta t=0.005(t=50$ to 80.715). 
$-101 \times 1001$ grid with $\mathrm{dt}=0.005$

$\cdots \cdots . .21 \times 801$ grid with $\mathrm{dt}=0.01$

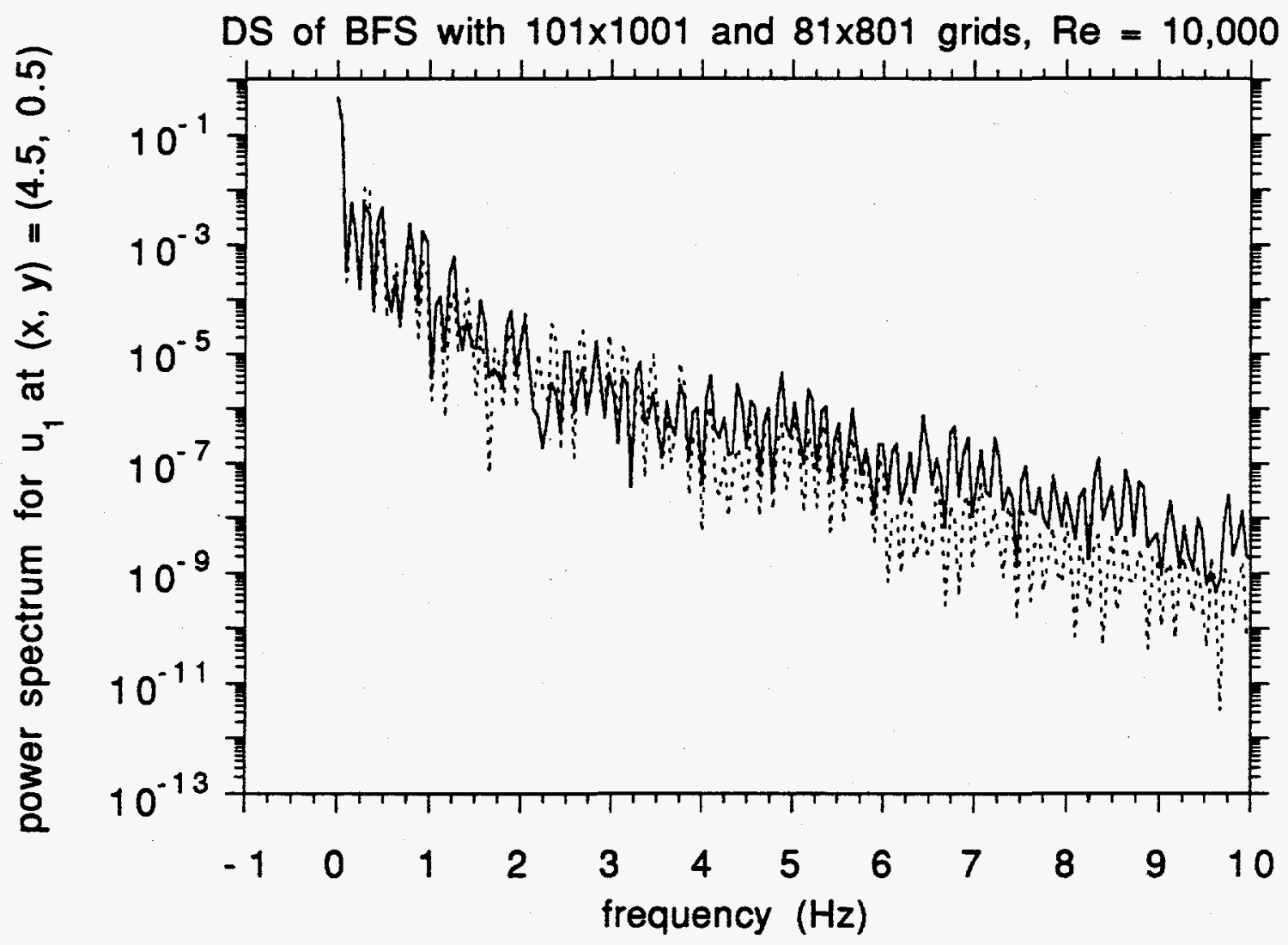

Figure 5.2.31 Power spectrum on log plot for $u_{1}$ time history at $(x, y)=(4.5,0.5)$ for the $81 \times 801$ grid using $\Delta t=0.01(t=50$ to 80.71$)$ and the $101 \times 1001$ grid using $\Delta t=$ $0.005(t=50$ to 80.715$)$. 


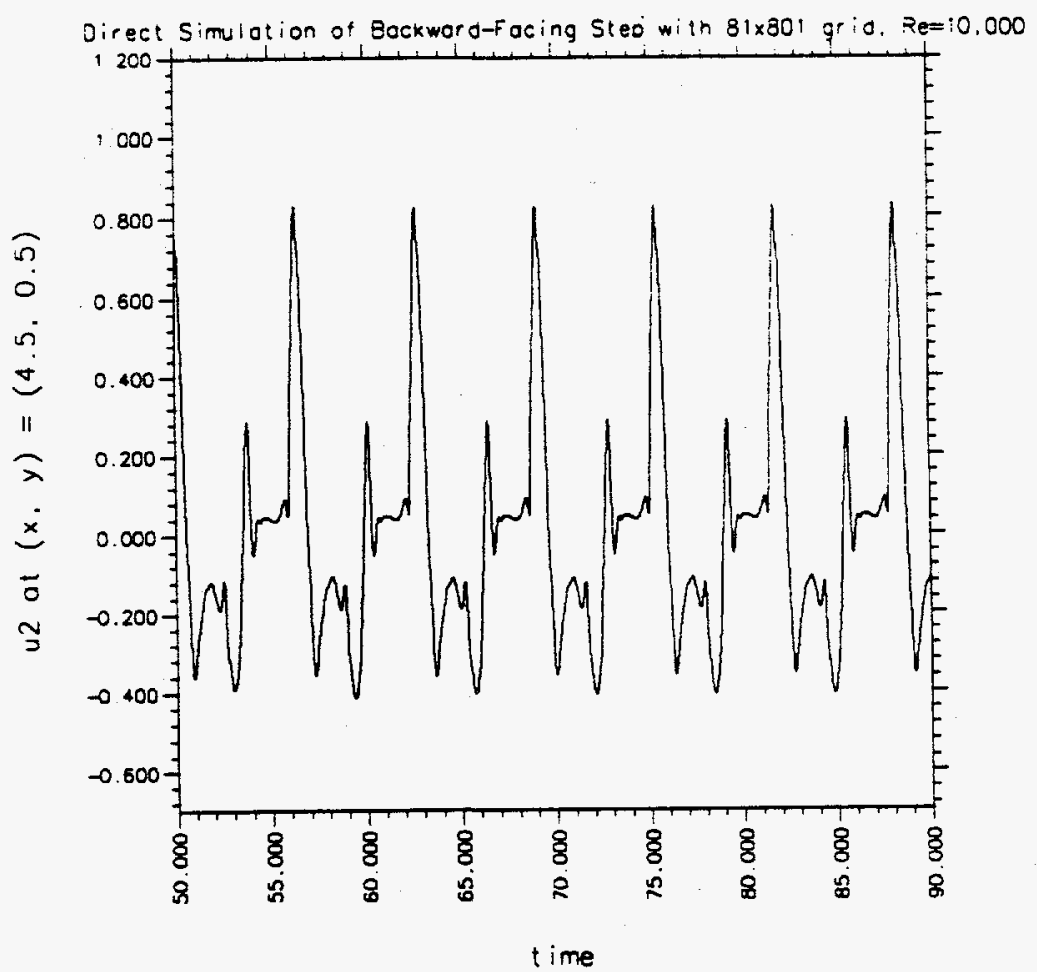

(a)

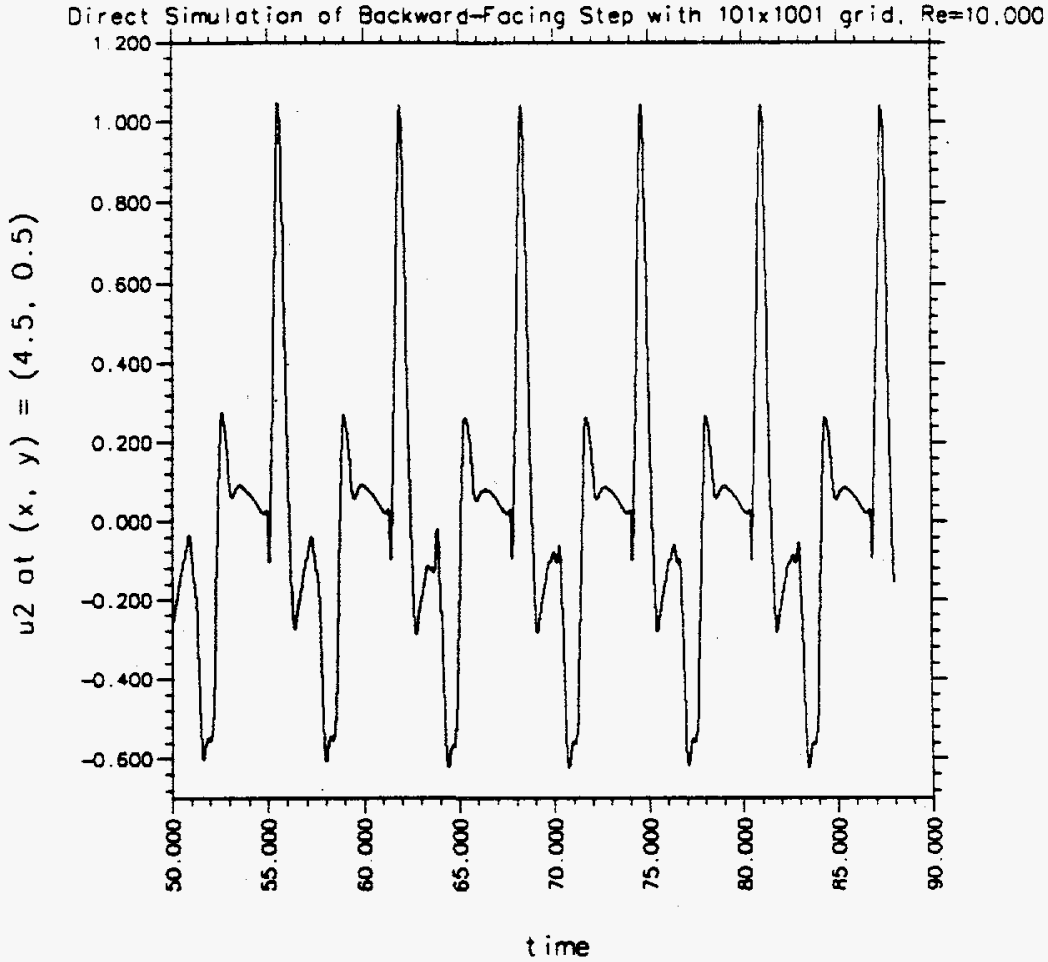

(b)

Figure 5.2.32 Extended time histories of $u_{2}$ at $(x, y)=(4.5,0.5)$ for a) the $81 \times 801$ grid at $\Delta t=0.01$ and $b$ ) the $101 \times 1001$ grid at $\Delta t=0.005$ (shown for the same axis scales). 
$-101 \times 1001$ grid with $d t=0.005$ $81 \times 801$ grid with $d t=0.01$

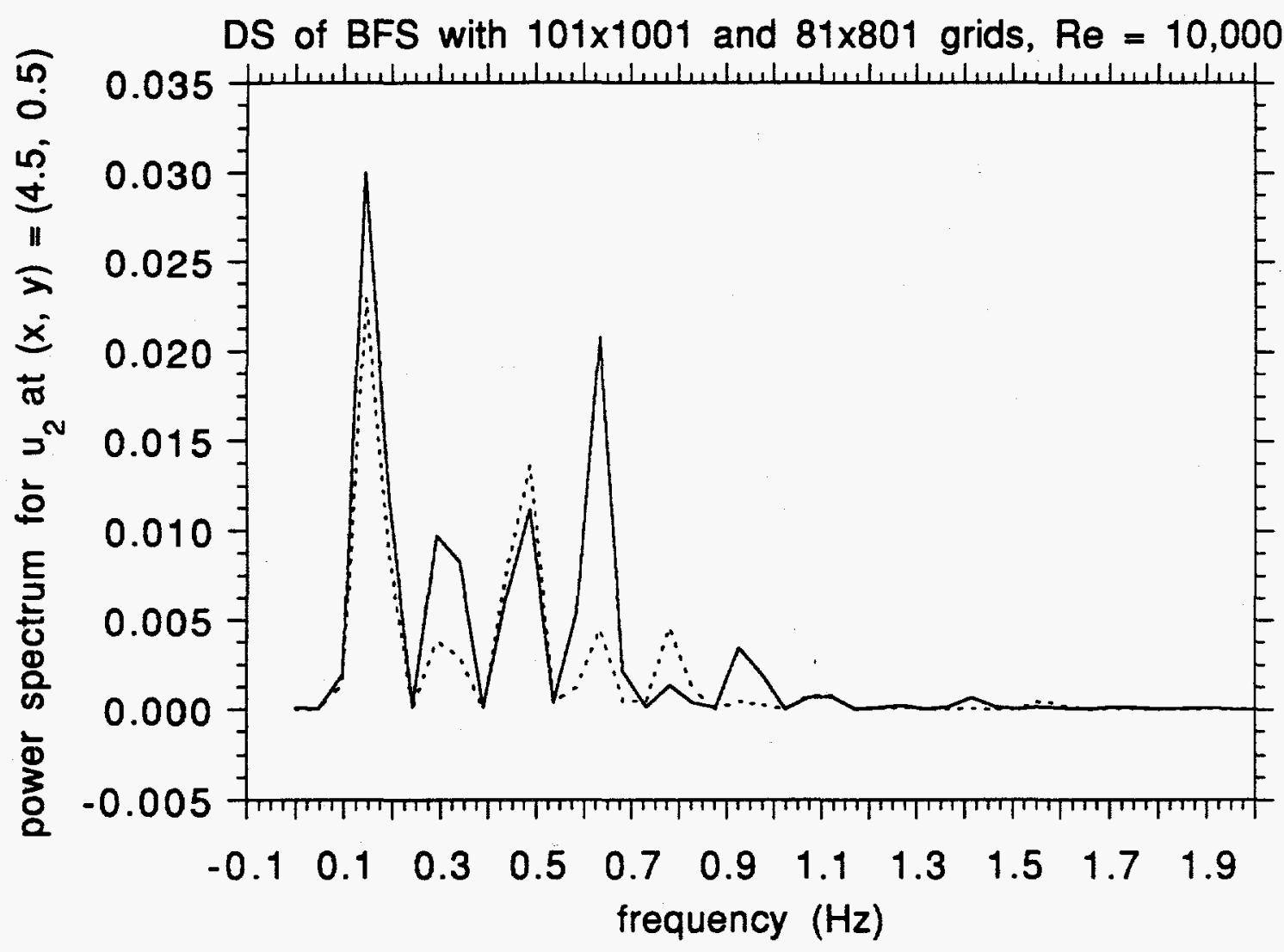

Figure 5.2.33 Power spectrum on linear plot for $u_{2}$ time history at $(x, y)=(4.5,0.5)$ for the $81 \times 801$ grid using $\Delta t=0.01(t=50$ to 80.71$)$ and the $101 \times 1001$ grid using $\Delta t=0.005(t=50$ to 80.715$)$. 
$101 \times 1001$ grid with $d t=0.005$

$81 \times 801$ grid with $d t=0.01$

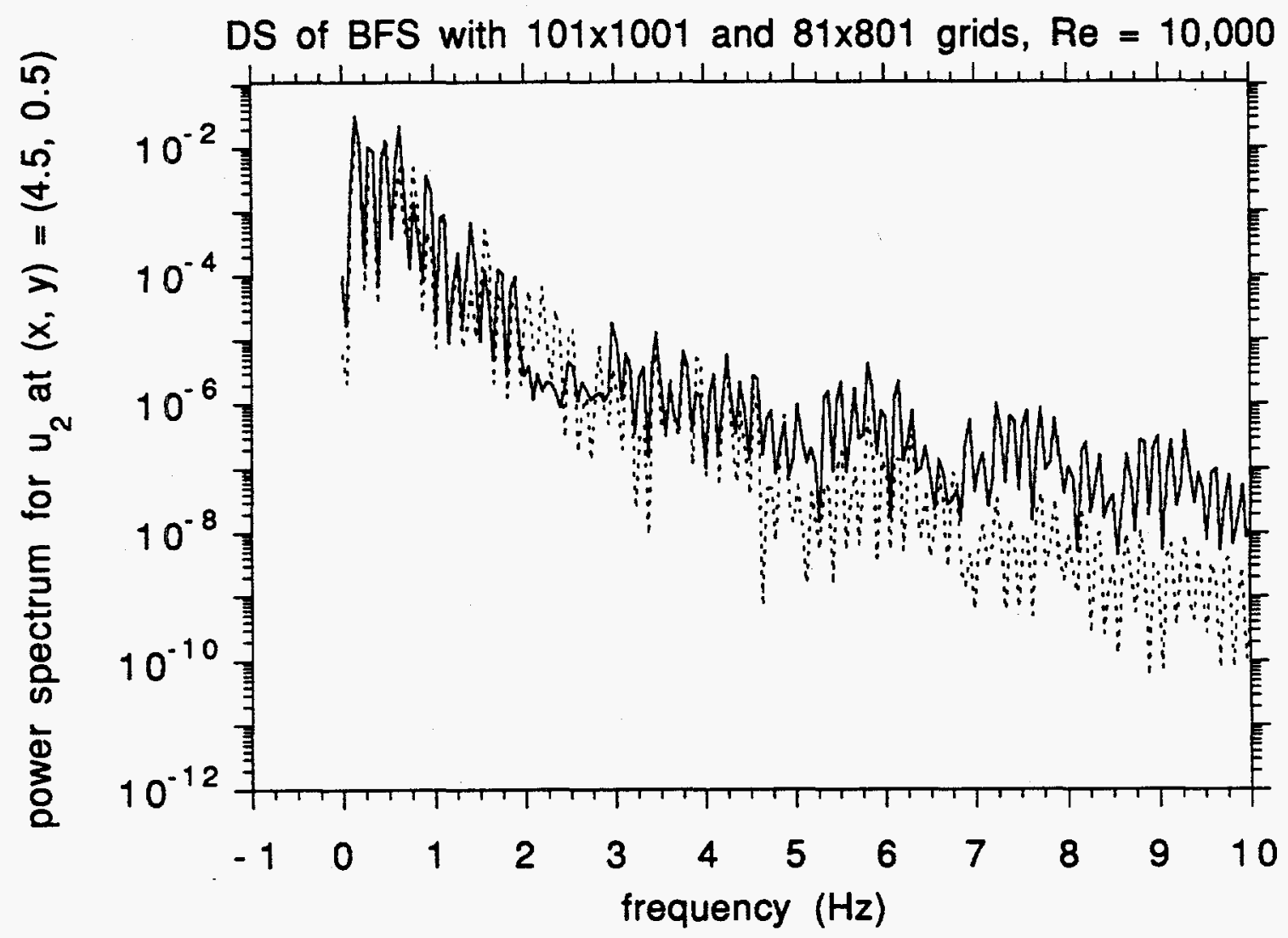

Figure 5.2.34 Power spectrum on $\log$ plot for $u_{2}$ time history at $(x, y)=(4.5,0.5)$ for the $81 \times 801$ grid using $\Delta t=0.01(t=50$ to 80.71) and the $101 \times 1001$ grid using $\Delta t=$ $0.005(t=50$ to 80.715). 
Time
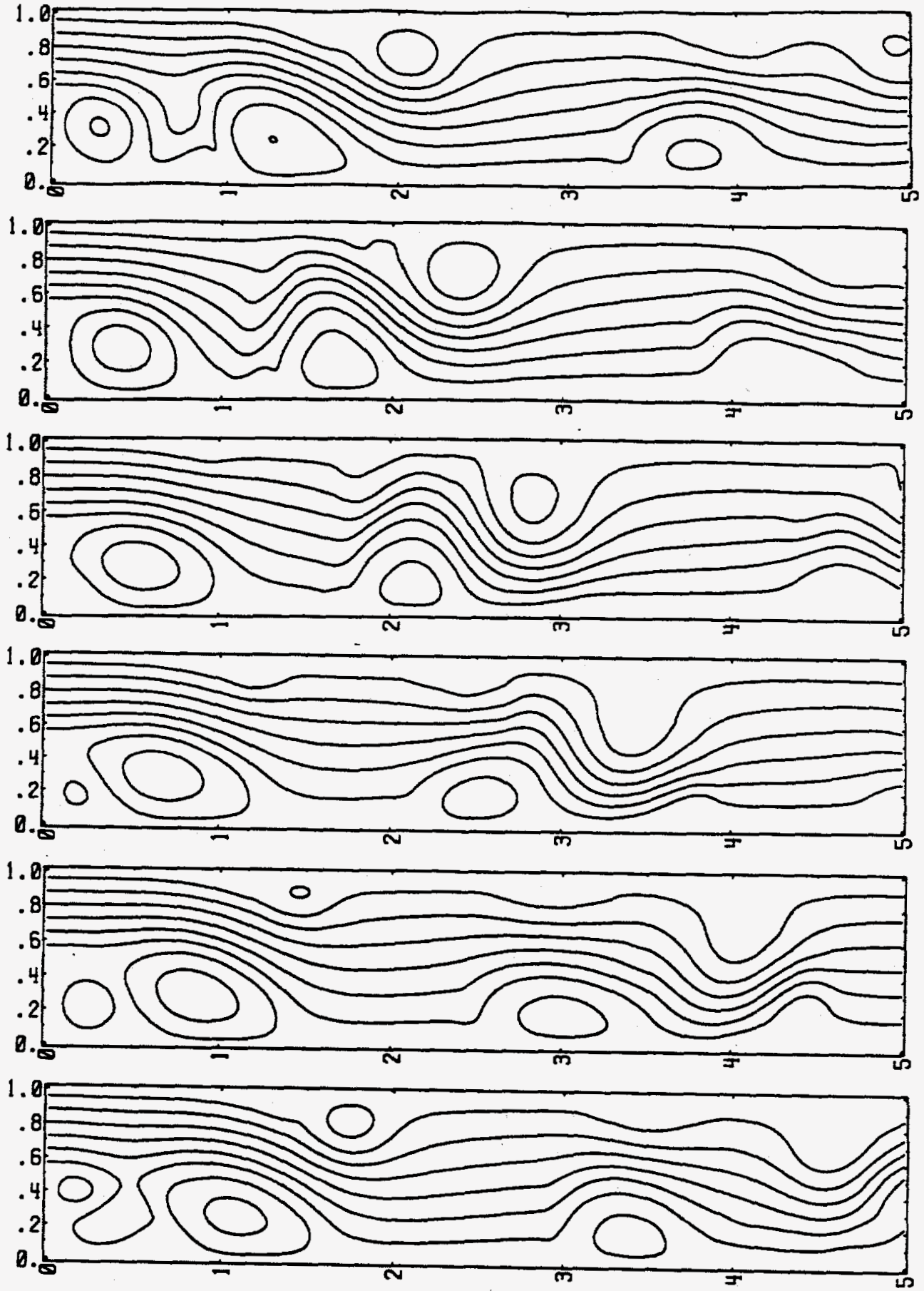

Figure 5.2.35a Time series of stream function plots showing only half of field length for the $81 \times 801$ grid at $0,1 / 6,2 / 6,3 / 6,4 / 6$, and $5 / 6$ of cycle. 
Time
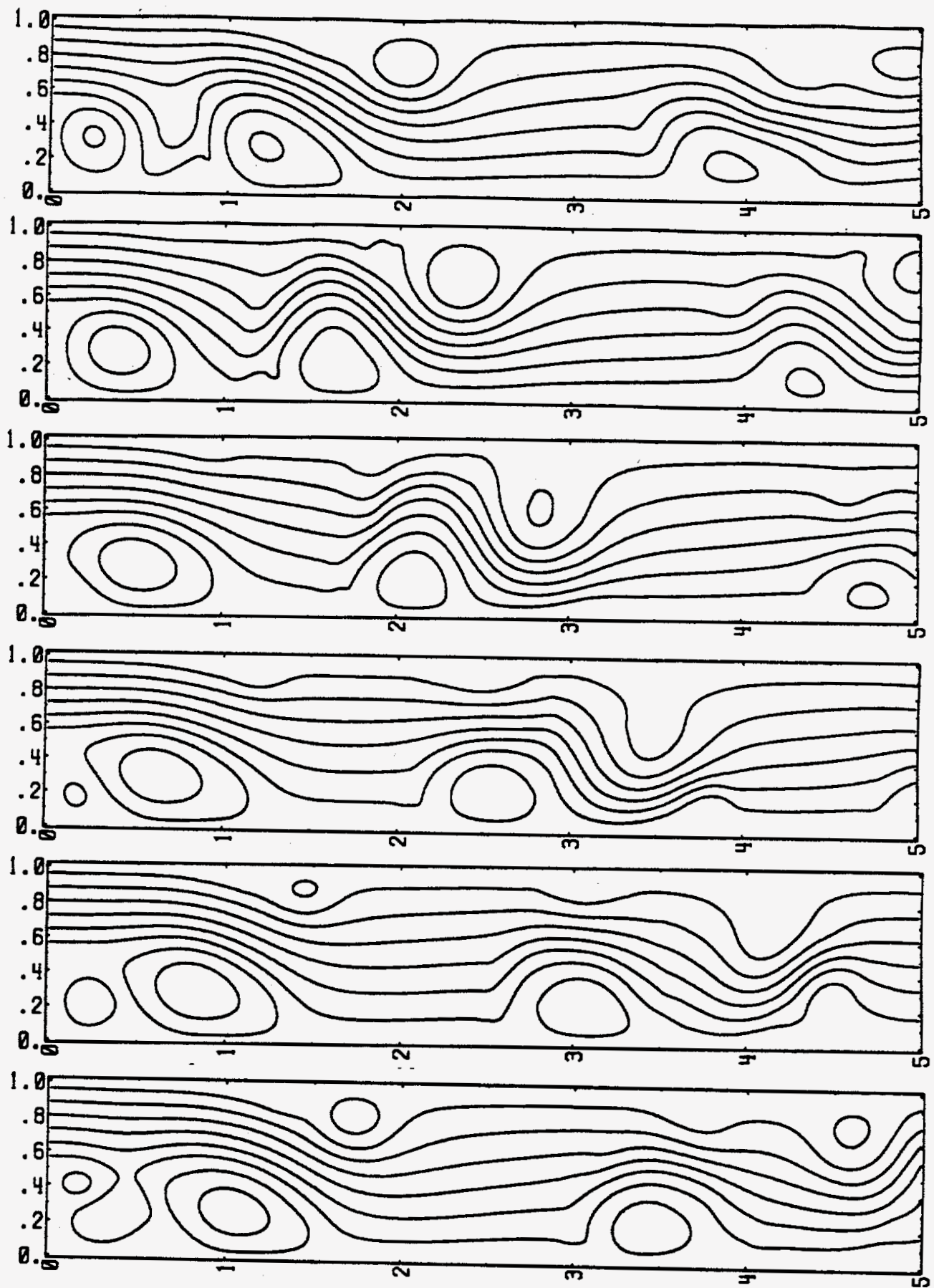

Figure 5.2.35b Time series of stream function plots showing only half of field length for the $101 \times 1001$ grid at $0,1 / 6,2 / 6,3 / 6,4 / 6$, and $5 / 6$ of cycle. 
Time
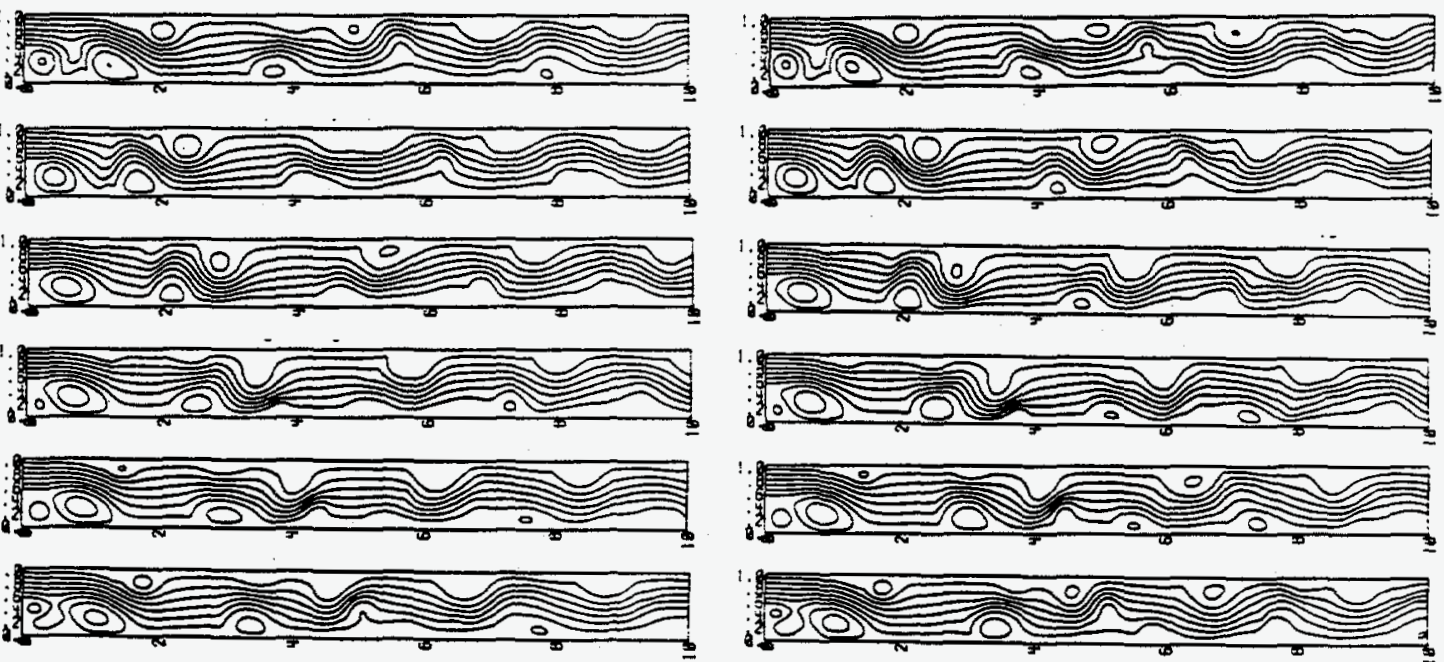

$81 \times 801$ grid

$101 \times 1001$ grid

Figure 5.2.35c Comparison of time series of stream function plots for the $81 \times 801$ and $101 \times 1001$ grids. (One cycle is shown for the same specified stream function levels for each case at $0,1 / 6,2 / 6,3 / 6,4 / 6$, and $5 / 6$ of cycle.) 


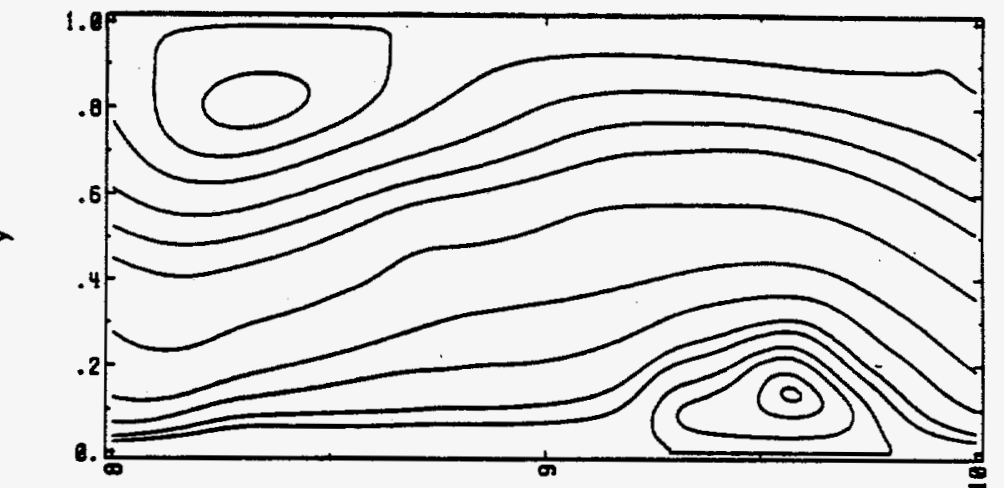

$x=9.97$

$x=9.98$
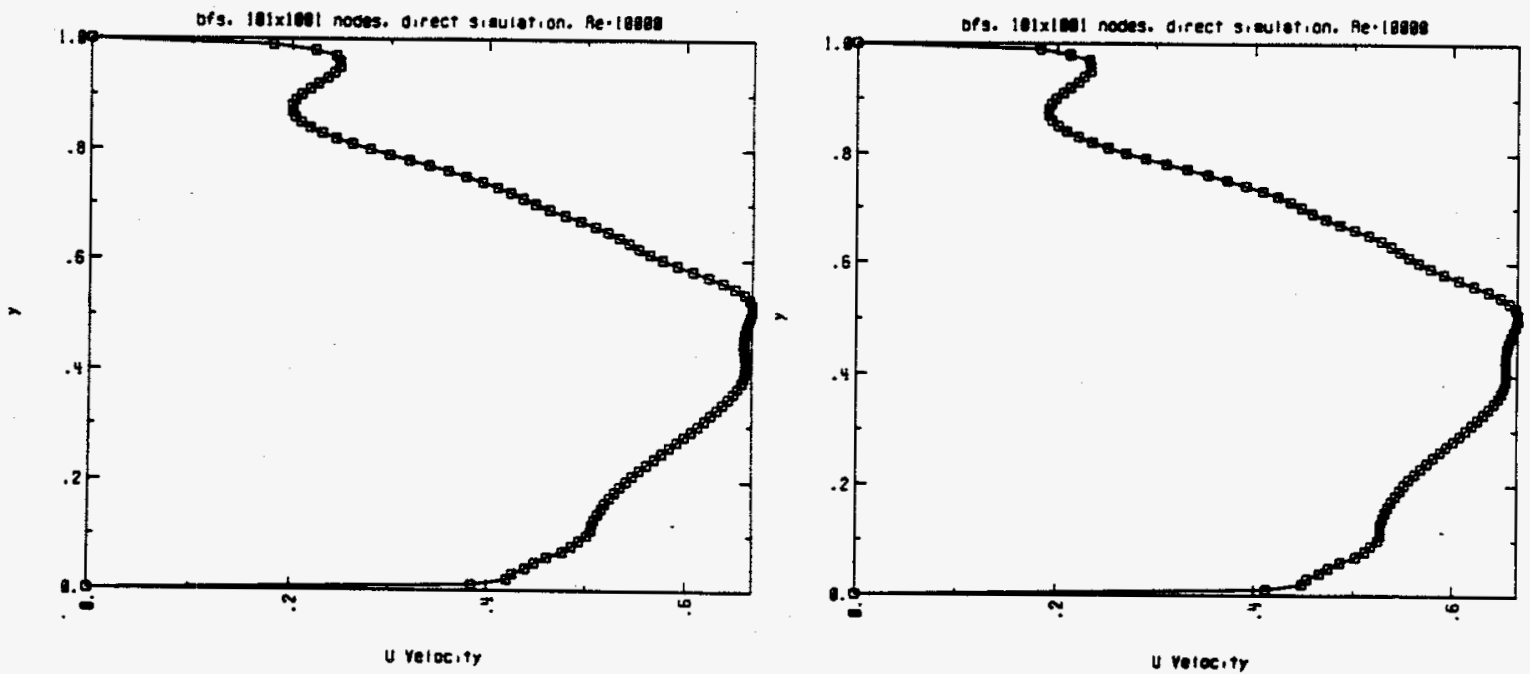

$$
x=9.99
$$

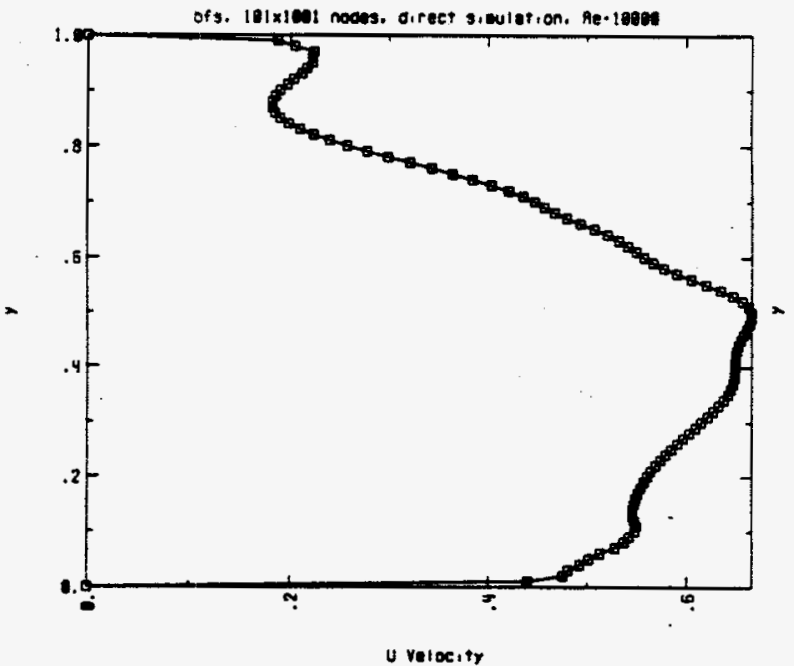

$$
\mathbf{x}=\mathbf{1 0 . 0 0}
$$

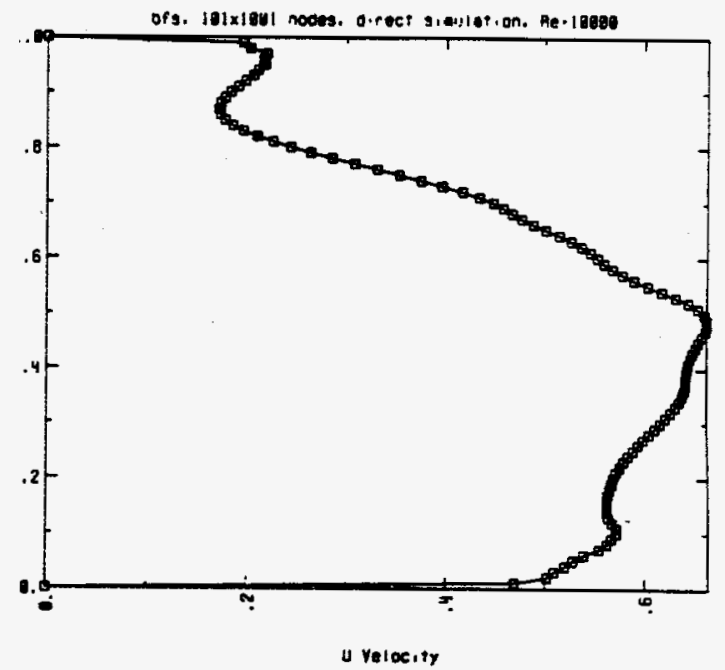

Figure 5.2.36 The predicted $u_{1}$ velocity profiles at the channel outlet for the $101 \times 1001$ grid with $\Delta t=0.005$ at the snapshot in time shown by the stream function plot. 


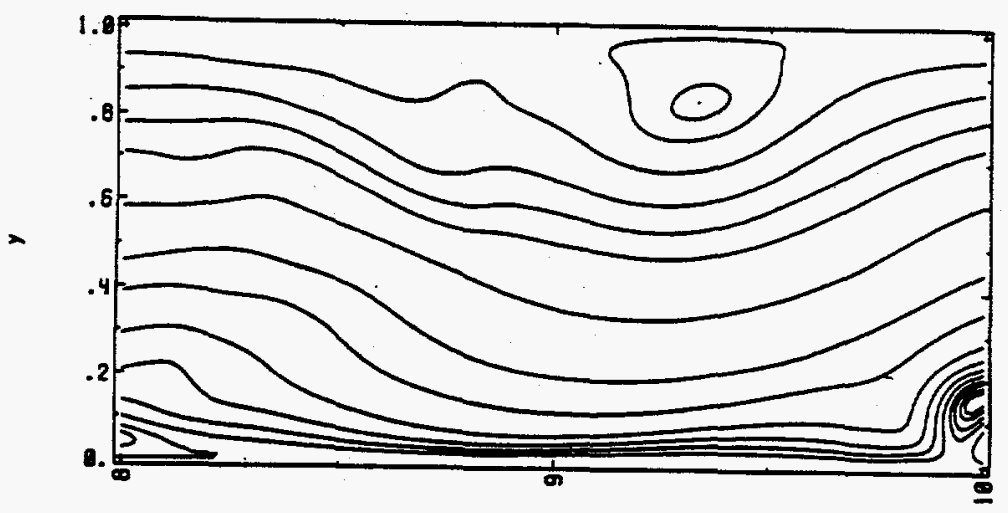

$x=9.97$

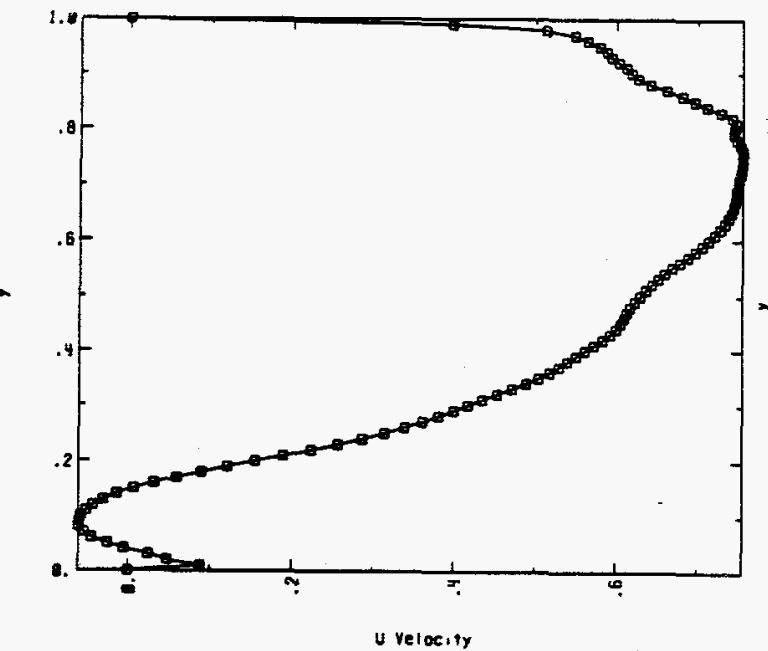

$x=9.99$

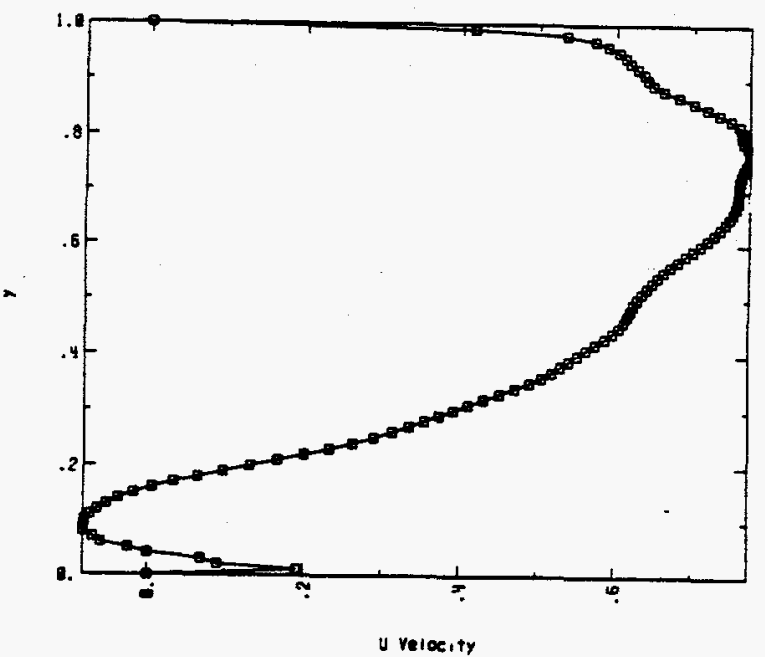

$x=9.98$

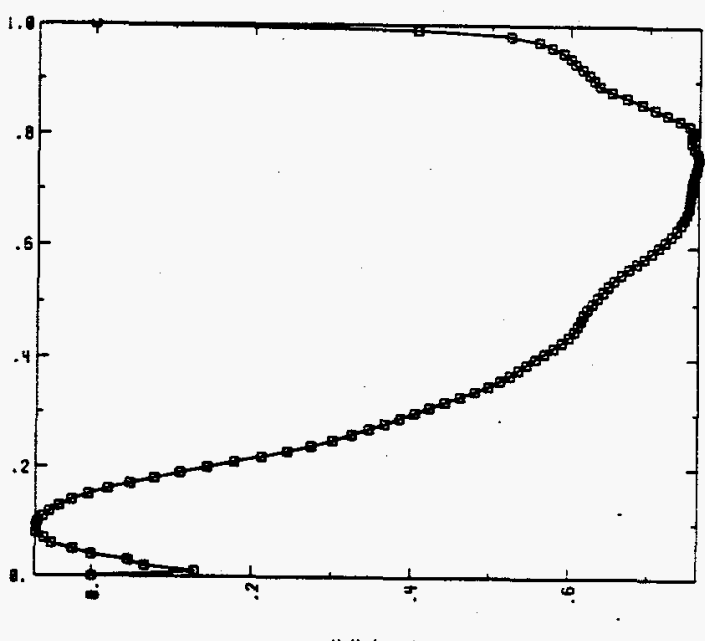

$x=10.00$

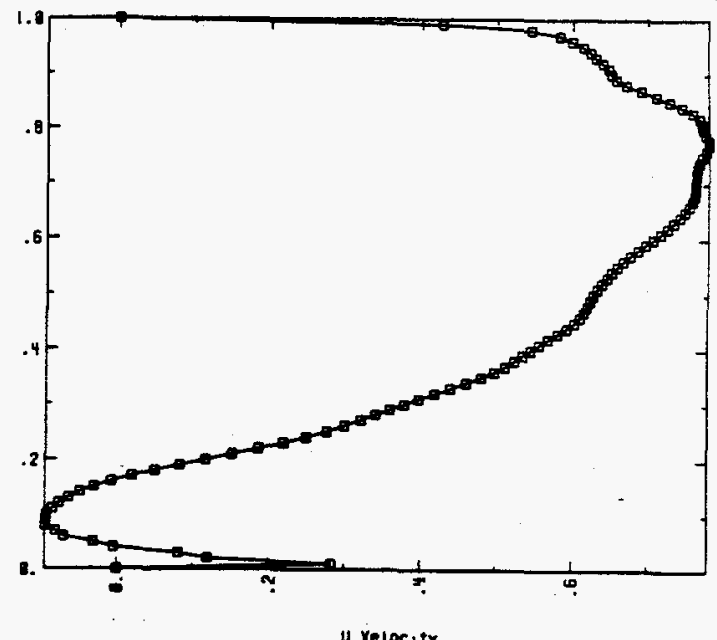

Figure 5.2.37 The predicted $u_{1}$ velocity profiles at the channel outlet for the $101 \times 1001$ grid with $\Delta t=0.005$ at the snapshot in time shown by the stream function plot. 


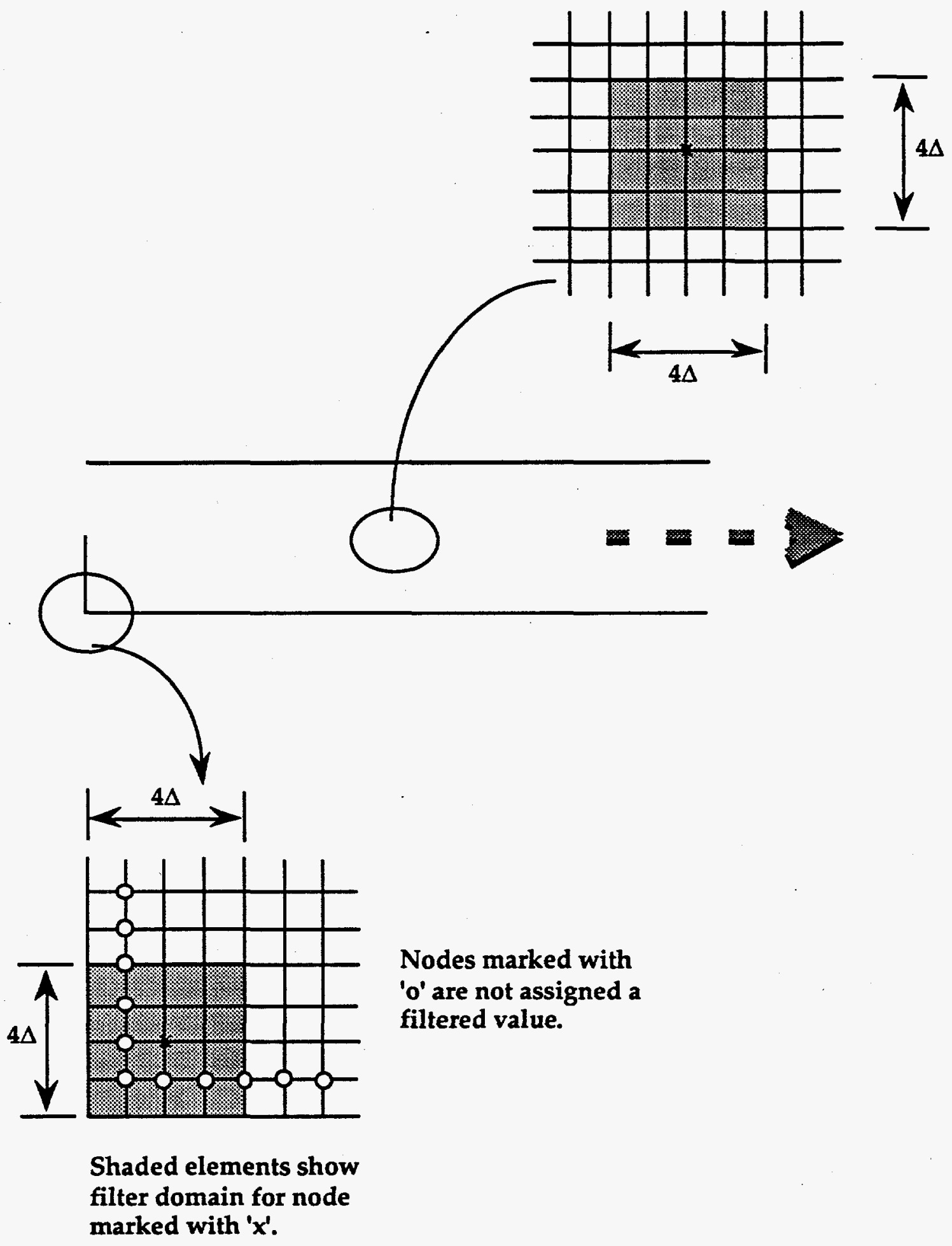

Figure 5.2.38 Description of cell volume-averaging scheme for a 16 element patch $\left(\Delta_{f}=4 \Delta\right)$ applied to the direct simulation. 


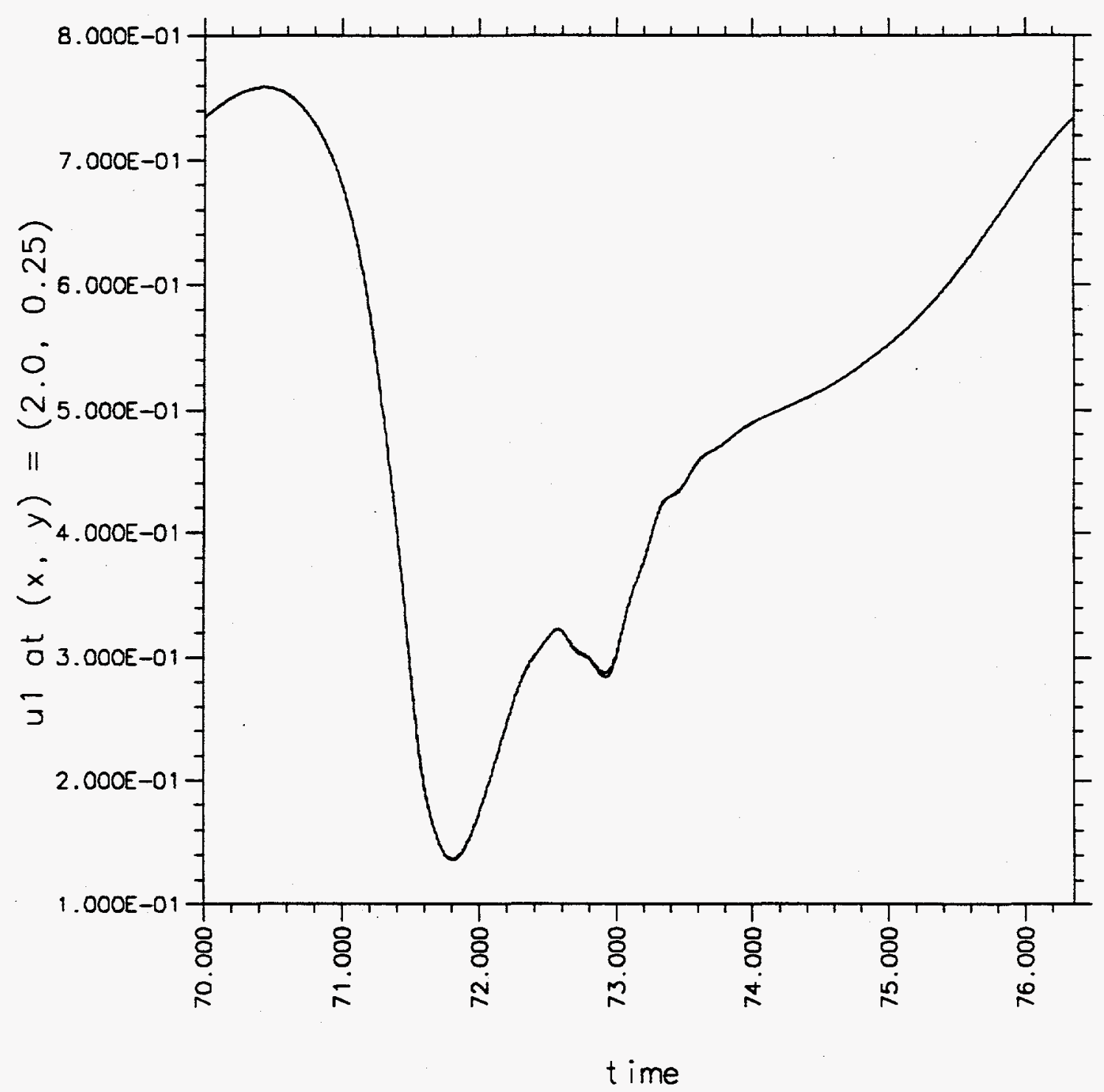

Fig. 5.2.39 Comparison of the unfiltered $(\Delta=0.01)$ and filtered $\left(\Delta_{f}=4 \Delta=0.04\right) u_{1}$ velocity time histories for the $101 \times 1001$ grid at the point location $(x, y)=(2.0$, 0.25). 
$101 \times 1001$ Direct Simulation Filtered $\left(\Delta_{f}=0.04\right.$ or 16 element filter)

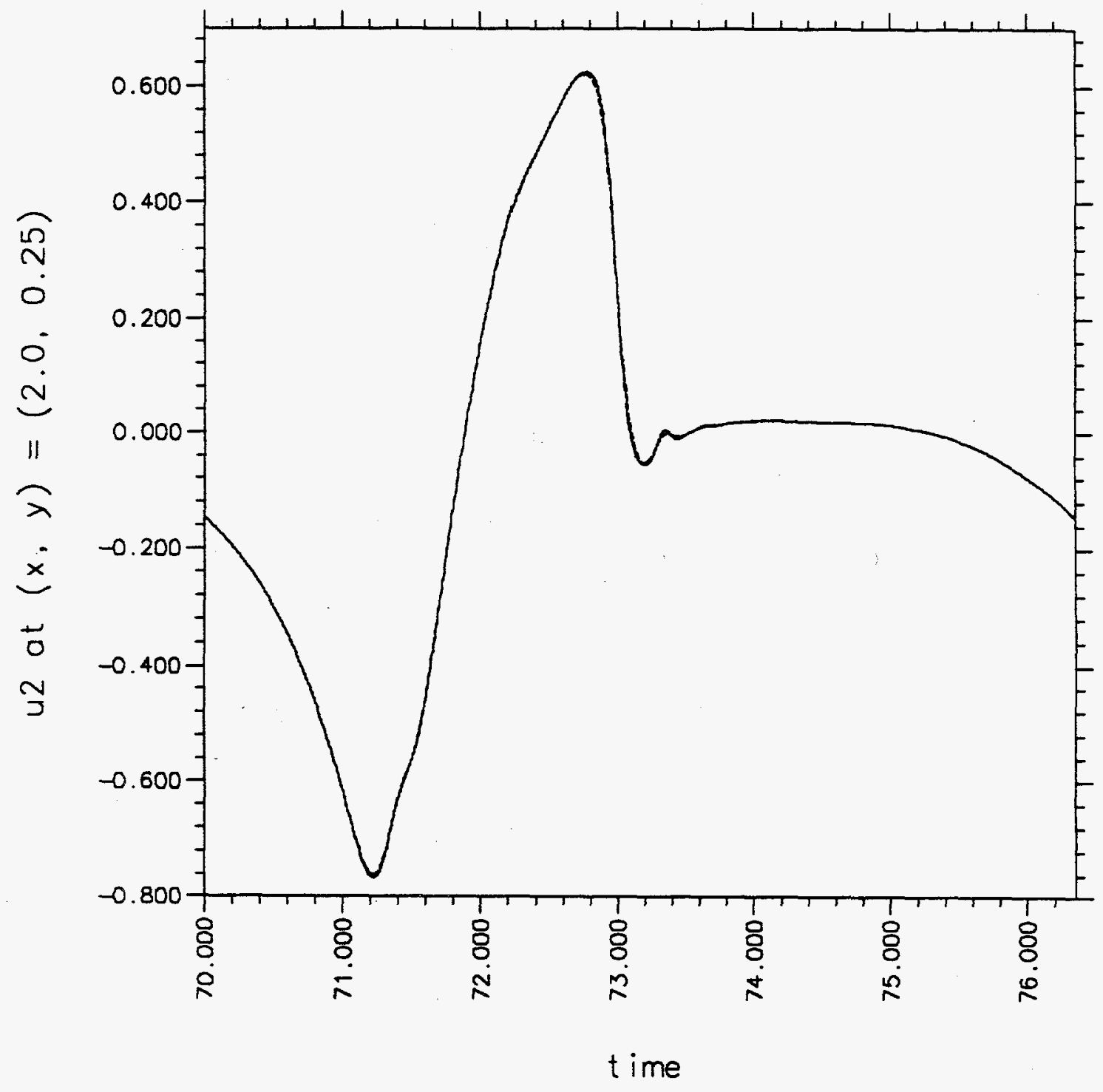

Fig. 5.2.40 Comparison of the unfiltered $(\Delta=0.01)$ and filtered $\left(\Delta_{f}=4 \Delta=0.04\right) u_{2}$ velocity time histories for the $101 \times 1001$ grid at the point location $(x, y)=(2.0$, 0.25). 


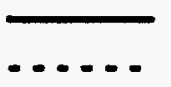

$101 \times 1001$ Direct Simulation Filtered $\left(\Delta_{f}=0.04\right.$ or 16 element filter)

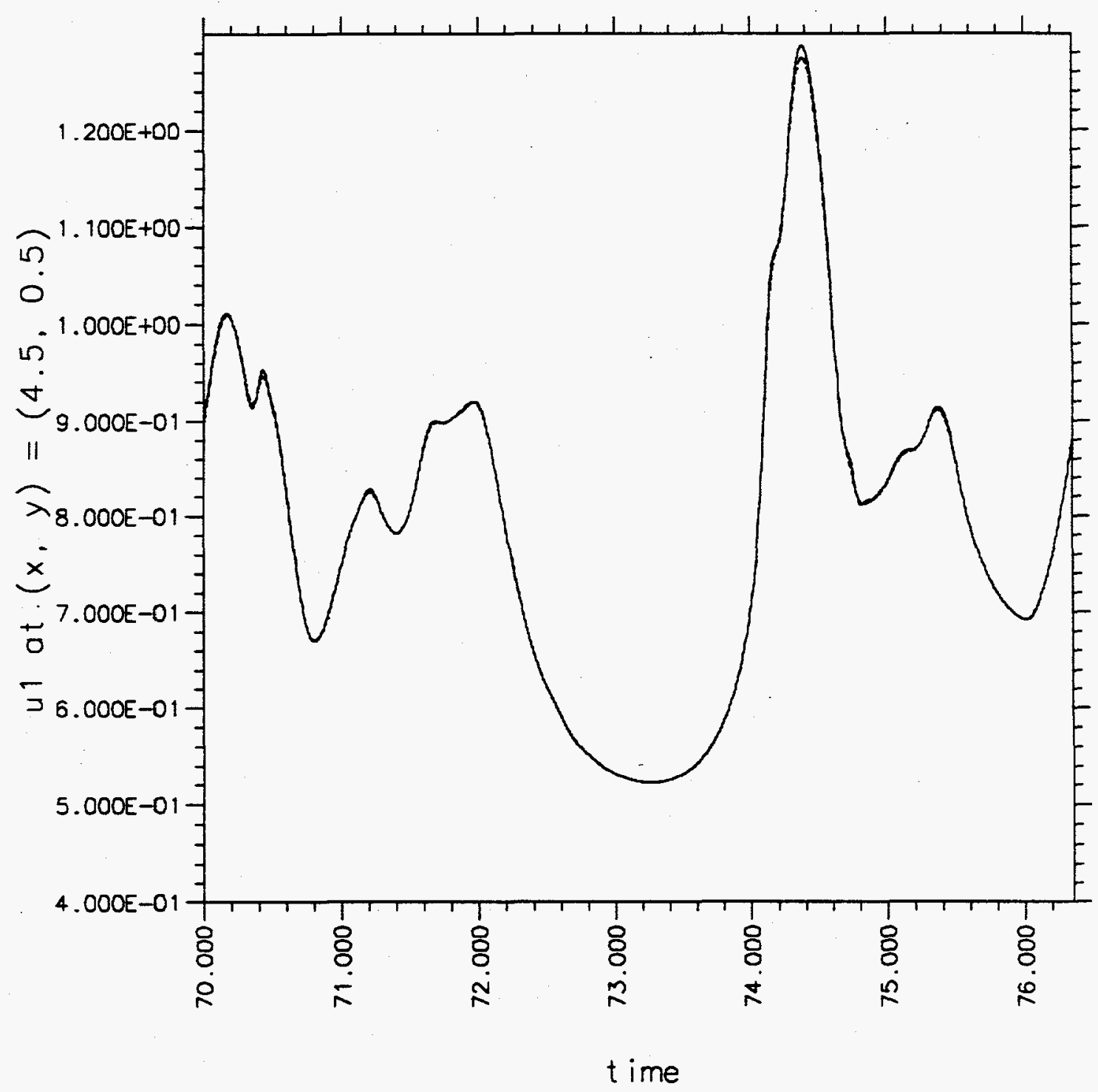

Fig. 5.2.41 Comparison of the unfiltered $(\Delta=0.01)$ and filtered $\left(\Delta_{f}=4 \Delta=0.04\right) u_{1}$ velocity time histories for the $101 \times 1001$ grid at the point location $(x, y)=(4.5,0.5)$. 
$101 \times 1001$ Direct Simulation

Filtered $\left(\Delta_{f}=0.04\right.$ or

16 element filter)

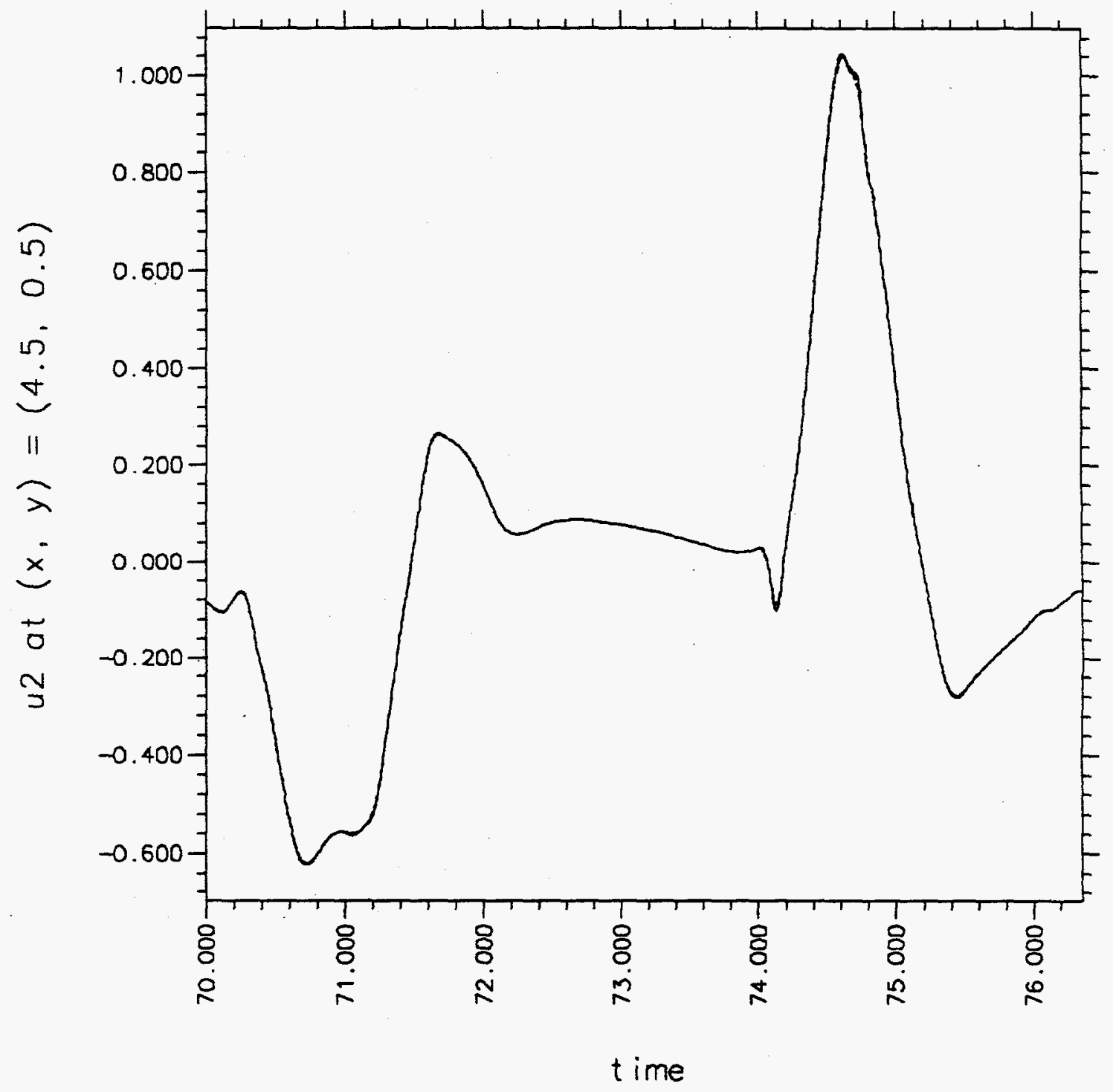

Fig. 5.2.42 Comparison of the unfiltered $(\Delta=0.01)$ and filtered $\left(\Delta_{f}=4 \Delta=0.04\right) u_{2}$ velocity time histories for the $101 \times 1001$ grid at the point location $(x, y)=(4.5,0.5)$. 

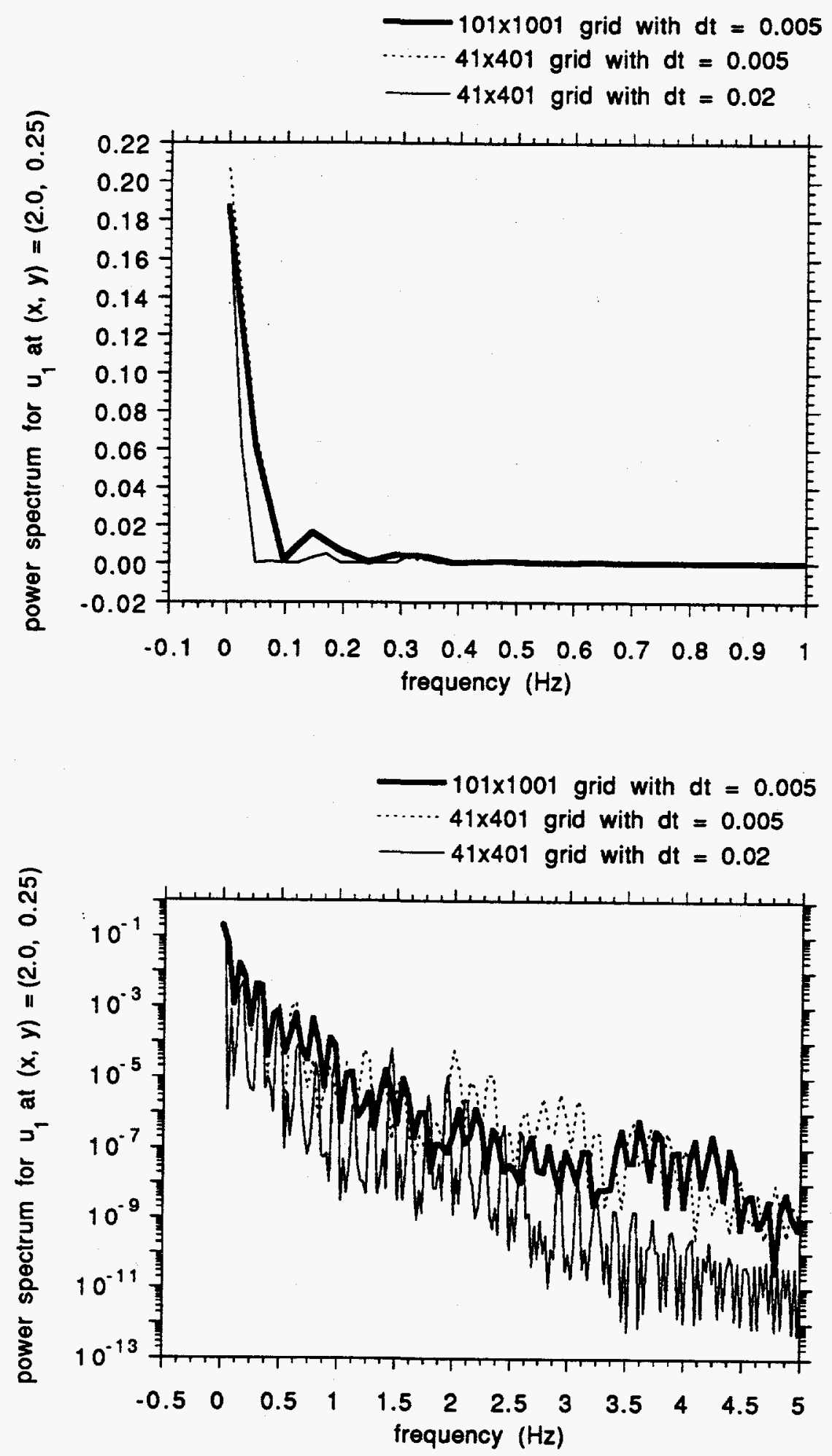

Figure 5.2.43 Power spectrum on a linear and a log plot for $u_{1}$ time history at $(x, y)=(2.0,0.25)$ for the $41 \times 401$ grid using $\Delta t=0.02(t=100$ to 161.42) and $\Delta t=$ $0.005(t=140$ to 170.715$)$ and for the $101 \times 1001$ grid using $\Delta t=0.005(t=50$ to 80.715). 

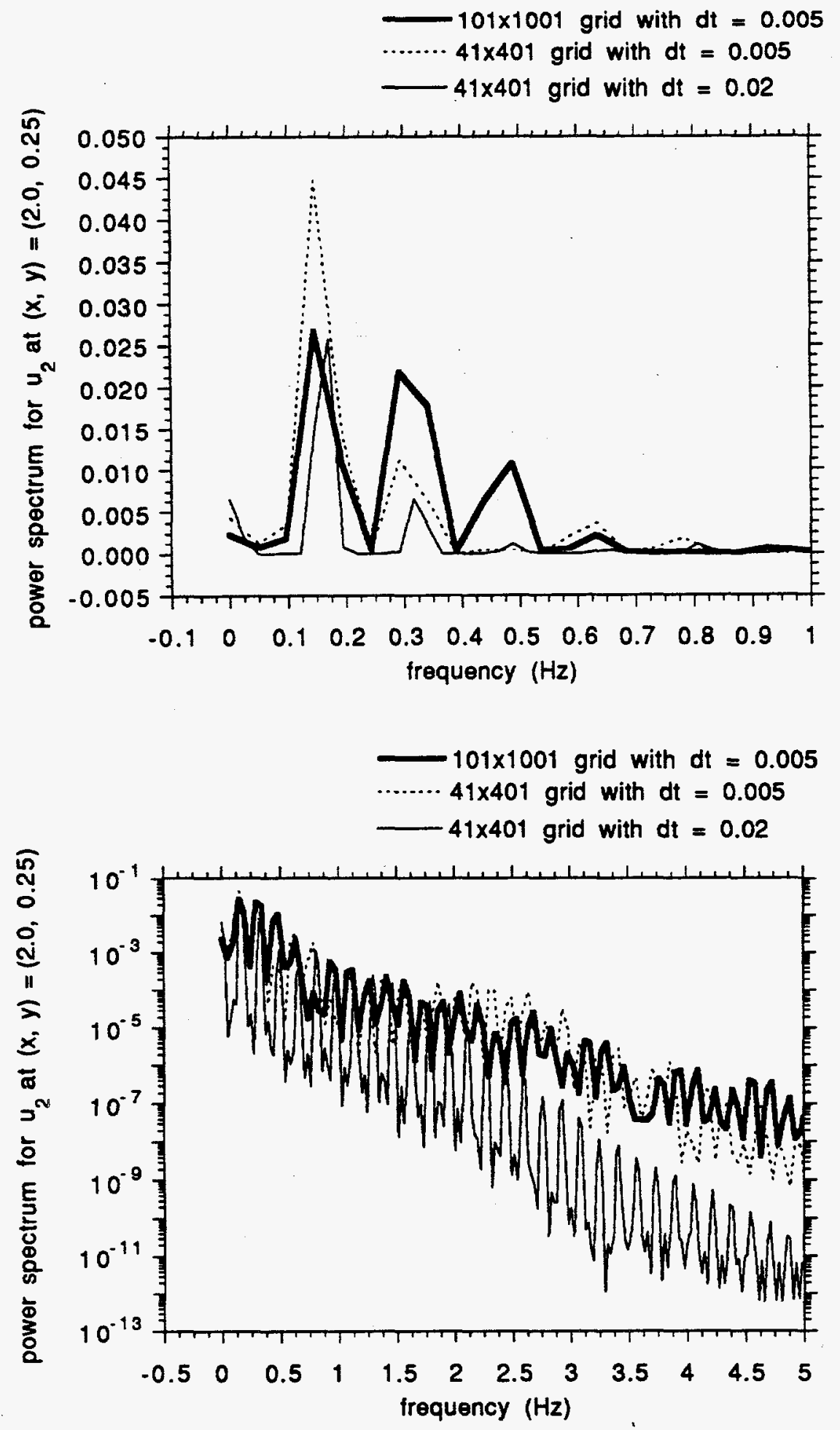

Figure 5.2.44 Power spectrum on a linear and a $\log$ plot for $u_{2}$ time history at $(\mathrm{x}, \mathrm{y})=(2.0,0.25)$ for the $41 \times 401$ grid using $\Delta t=0.02(t=100$ to 161.42) and $\Delta t=$ $0.005(t=140$ to 170.715$)$ and for the $101 \times 1001$ grid using $\Delta t=0.005(t=50$ to 80.715). 
- $101 \times 1001$ grid with $\mathrm{dt}=0.005$

$41 \times 401$ grid with $d t=0.005$
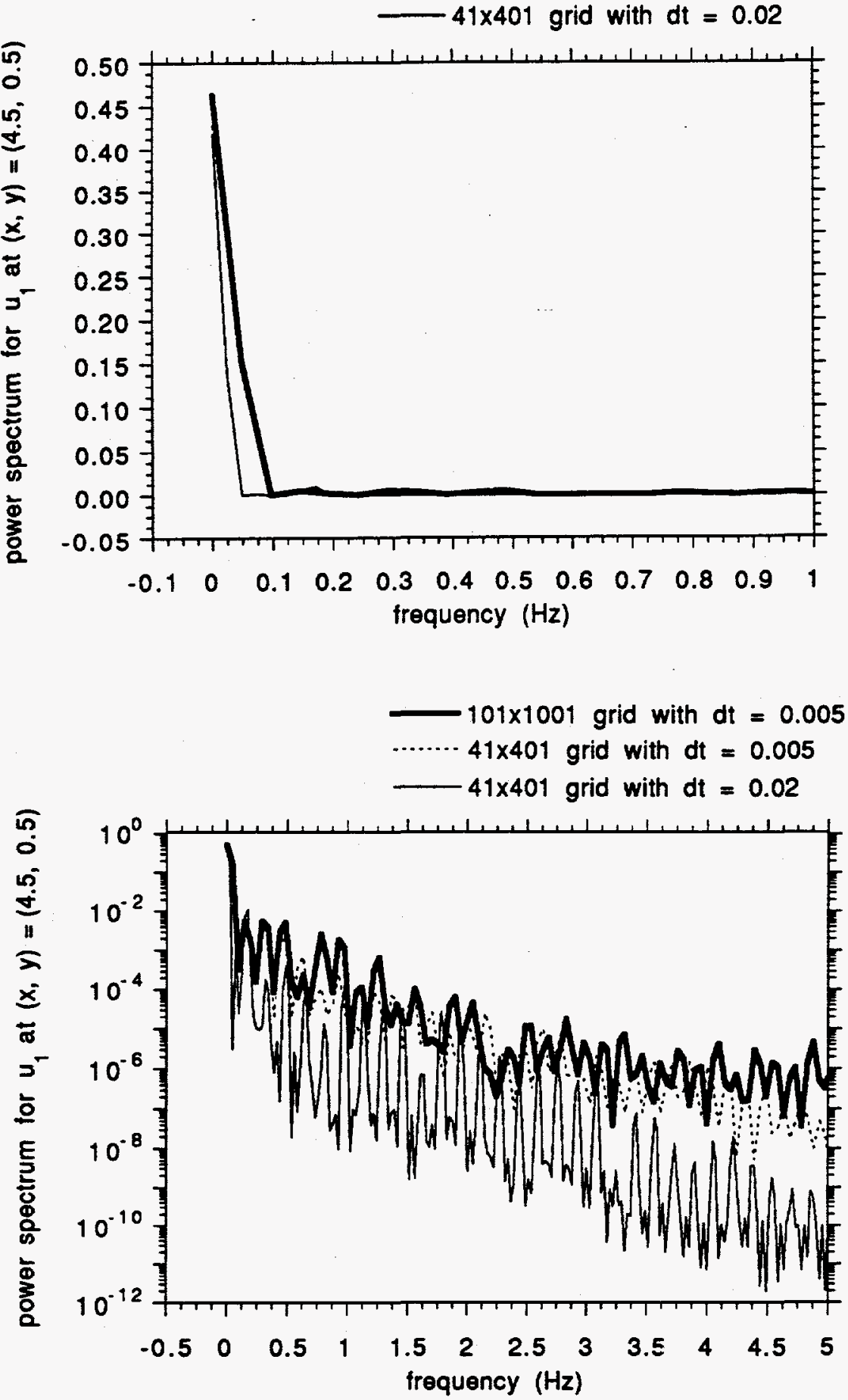

Figure 5.2.45 Power spectrum on a linear and a $\log$ plot for $u_{1}$ time history at $(\mathrm{x}, \mathrm{y})=(4.5,0.5)$ for the $41 \times 401$ grid using $\Delta t=0.02(t=100$ to 161.42) and $\Delta t=$ $0.005(t=140$ to 170.715$)$ and for the $101 \times 1001$ grid using $\Delta t=0.005(t=50$ to 80.715). 

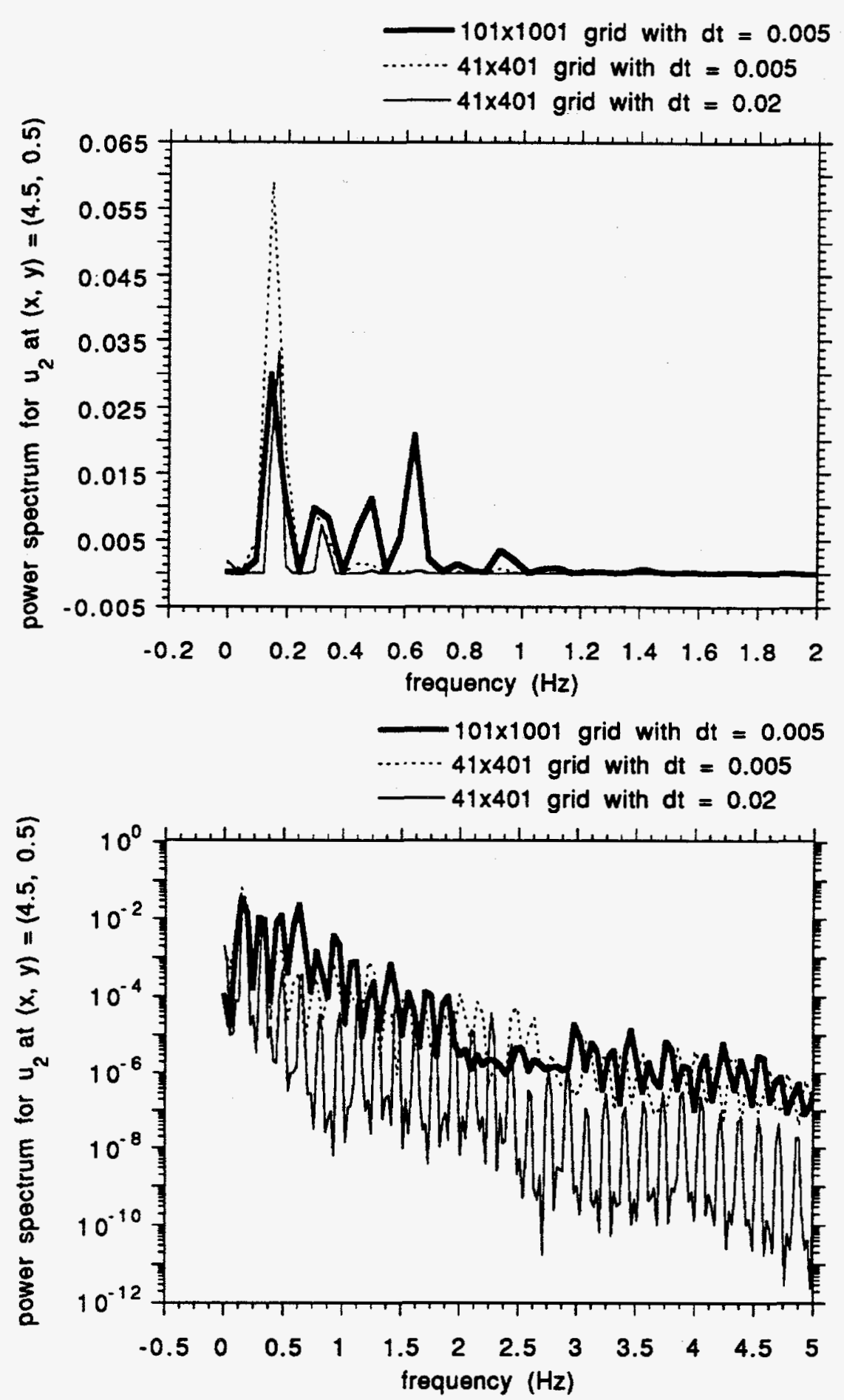

Figure 5.2.46 Power spectrum on a linear and a log plot for $u_{2}$ time history at $(x, y)=(4.5,0.5)$ for the $41 \times 401$ grid using $\Delta t=0.02(t=100$ to 161.42$)$ and $\Delta t=$ $0.005(t=140$ to 170.715$)$ and for the $101 \times 1001$ grid using $\Delta t=0.005(t=50$ to 80.715). 
Table 5.2.1 Time averaged results for the $101 \times 1001$ grid and $\Delta t=0.005$.

\begin{tabular}{|c|c|c|c|}
\hline & \multicolumn{2}{|c|}{$\begin{array}{l}\mathrm{x} \text {-location for separation } \\
\text { and reattachment points }\end{array}$} & \multirow[b]{2}{*}{$\begin{array}{c}\text { relative change } \\
\text { between cases } \\
(\%)\end{array}$} \\
\hline ? & $\begin{array}{l}\text { Time } \\
\text { Average for } \\
20,000 \text { time } \\
\text { steps }\end{array}$ & $\begin{array}{l}\text { Time } \\
\text { Average for } \\
30,000 \text { time } \\
\text { steps }\end{array}$ & \\
\hline \multirow[t]{5}{*}{ Bottom Wall } & 0.091 & 0.091 & 0.0 \\
\hline & 0.366 & 0.367 & 0.3 \\
\hline & 1.697 & 1.697 & 0.0 \\
\hline & 7.356 & 7.359 & $<0.1$ \\
\hline & 7.493 & 7.484 & 0.1 \\
\hline \multirow[t]{8}{*}{ Top Wall } & 1.024 & 1.024 & 0.0 \\
\hline & 1.576 & 1.566 & 0.6 \\
\hline & 2.130 & 2.117 & 0.6 \\
\hline & 2.299 & 2.306 & 0.3 \\
\hline & 4.877 & 4.869 & 0.2 \\
\hline & 5.345 & 5.344 & $<0.1$ \\
\hline & 6.747 & 6.740 & 0.1 \\
\hline & 6.904 & 6.922 & 0.3 \\
\hline
\end{tabular}




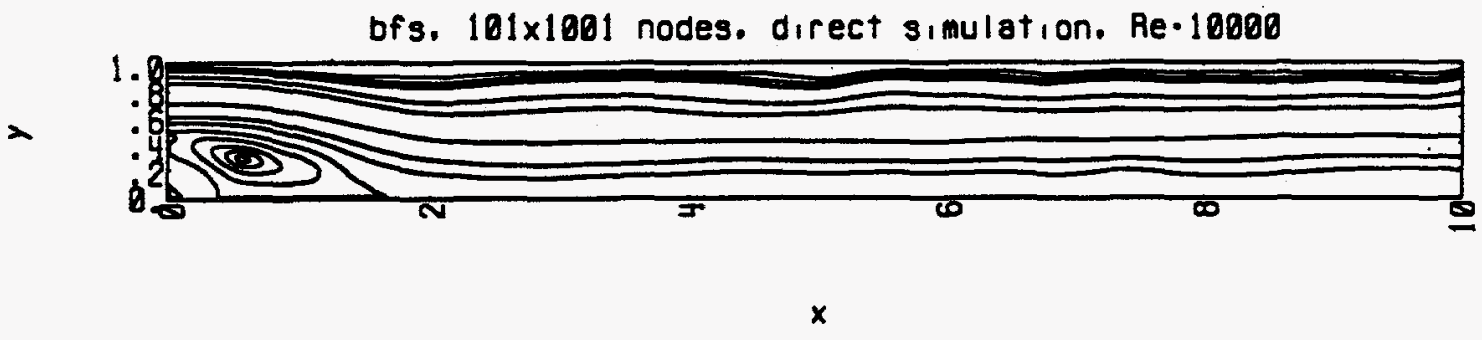

Entire Computational Field

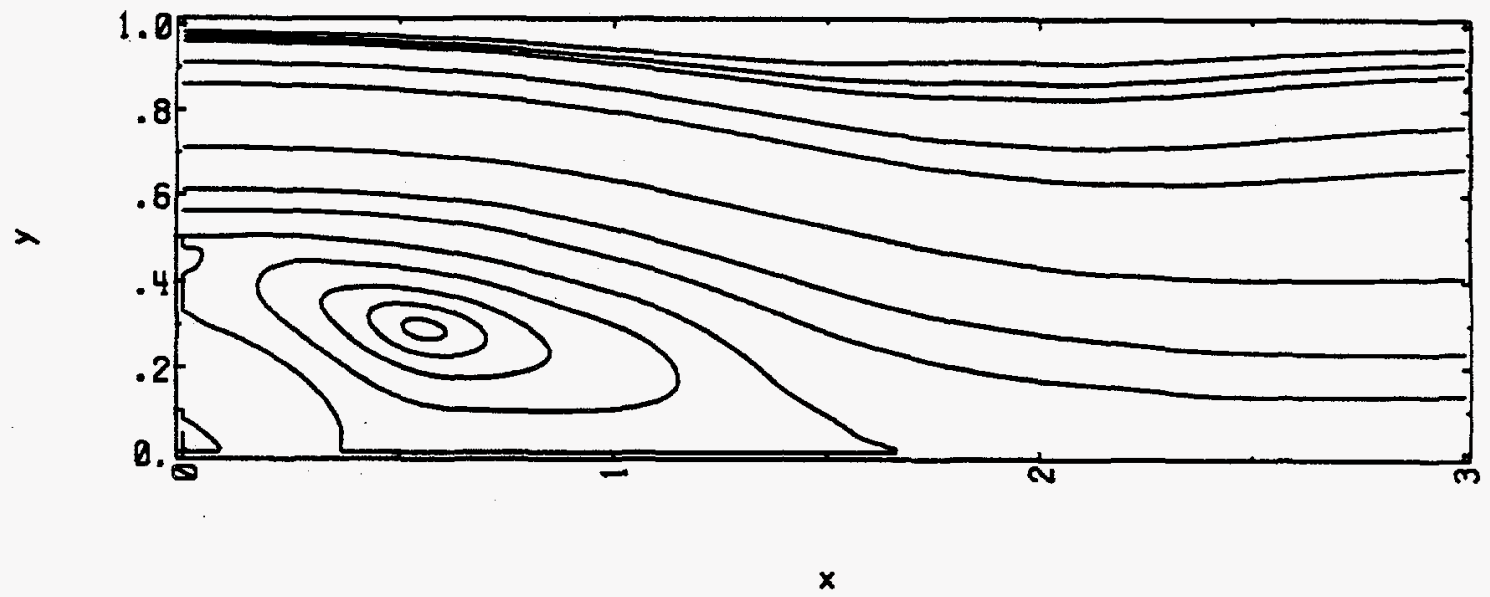

Enlargement of Major Recirculation Zone

Figure 5.2.47 Time-averaged stream function for the $101 \times 1001$ grid and $\Delta t=$ 0.005 for a time-averaging duration of 30,000 time steps. (Small recirculation zones along the top and bottom walls are too small to be seen on these plots.) 
bfs. $101 \times 1001$ nodes. direct simulation. Re 10000
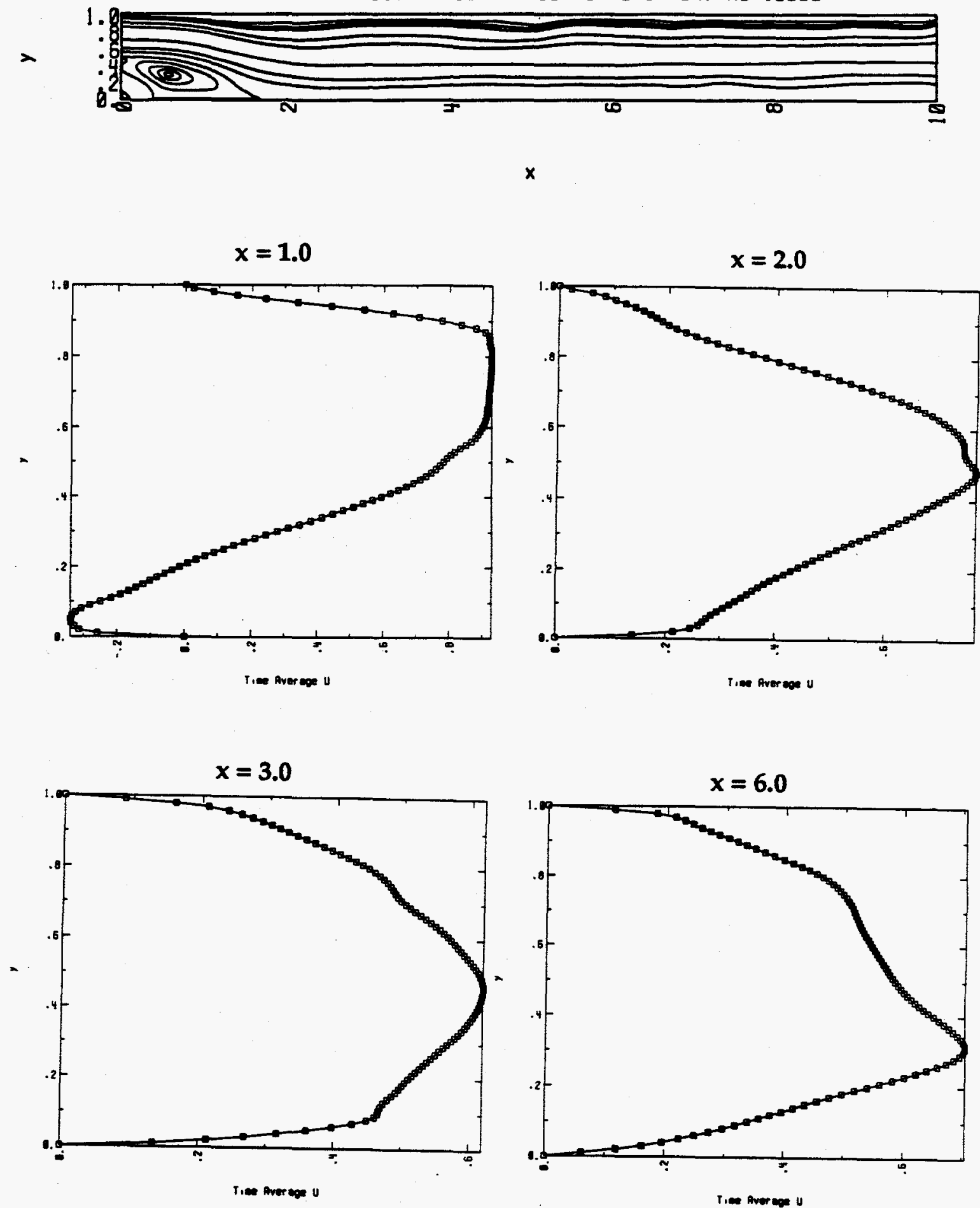

Figure 5.2.48 The time-averaged $u_{1}$ velocity, $\left\langle u_{1}\right\rangle$, at selected locations for the $101 \times 1001$ grid and $\Delta t=0.005$. 

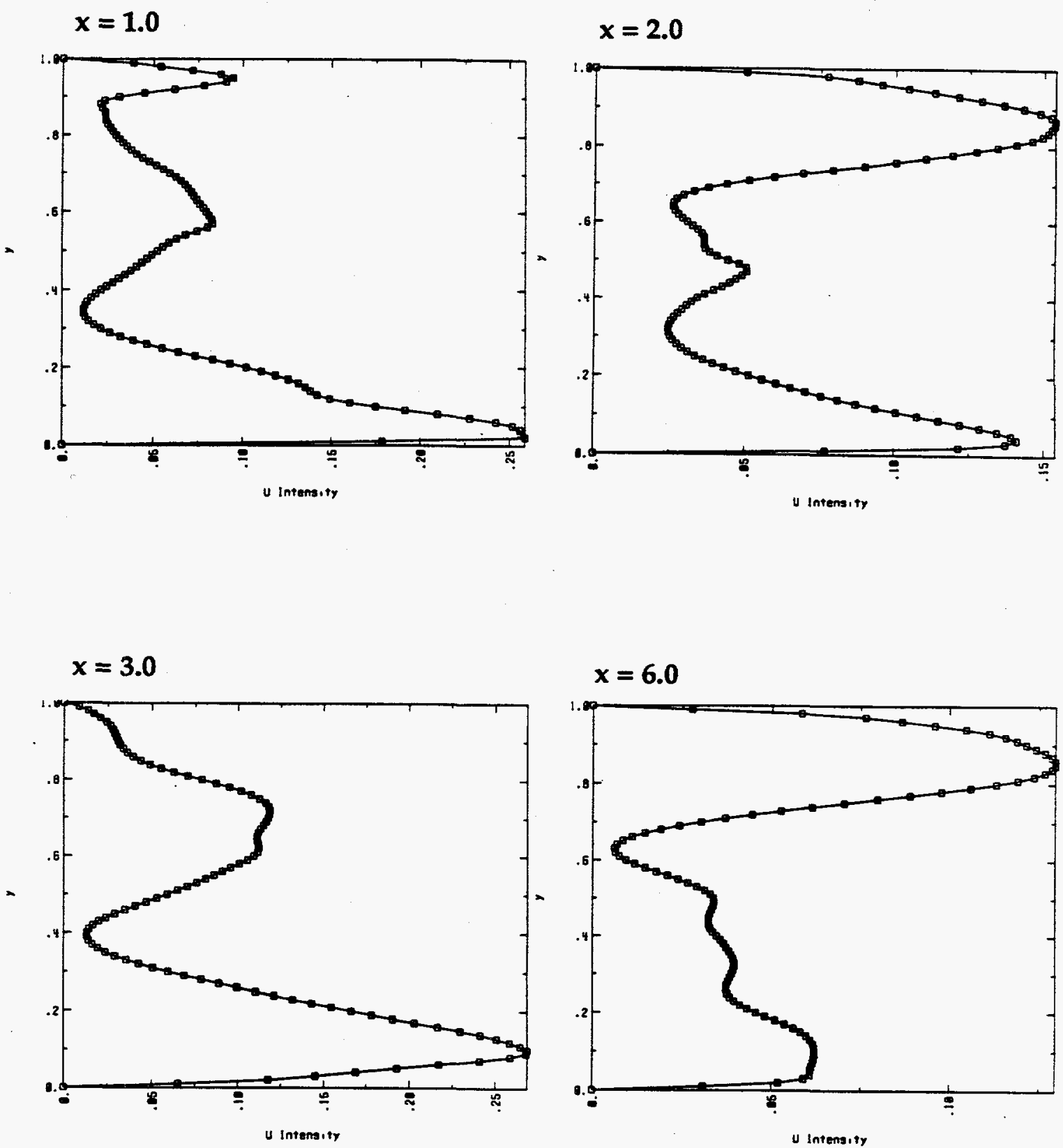

Figure 5.2.49 The turbulent intensity, $\left\langle u_{1}^{\prime 2}\right\rangle$, at selected locations for the $101 \times 1001$ grid and $\Delta t=0.005$. 
$x=1.0$

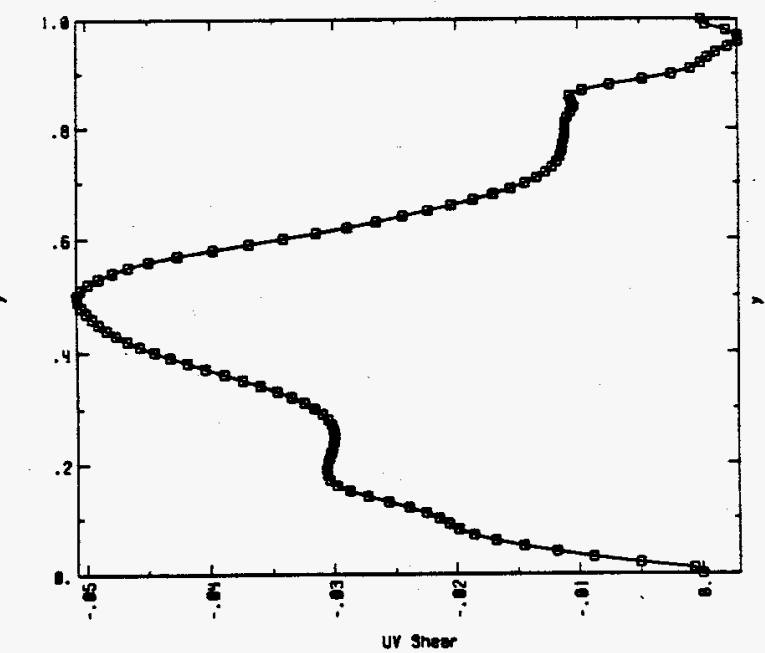

$x=3.0$

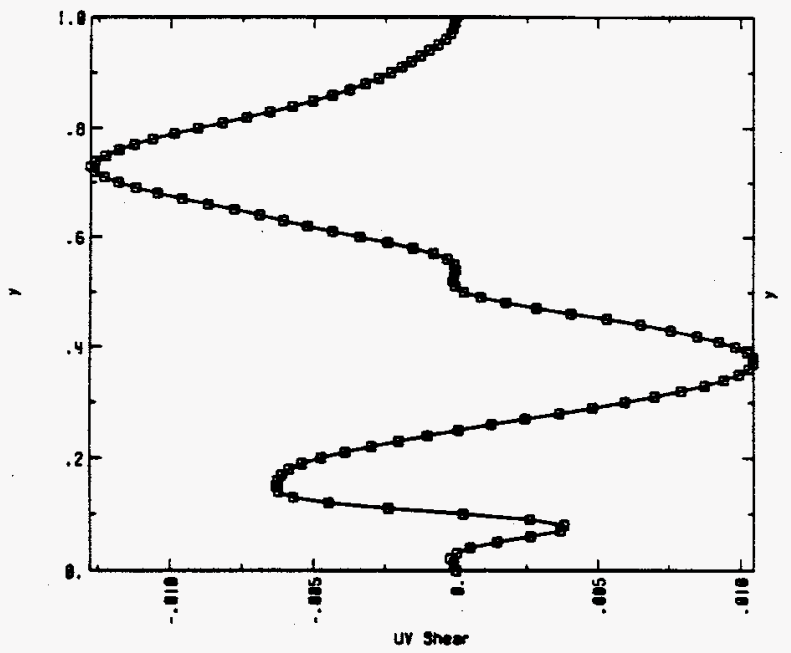

$x=\mathbf{2 . 0}$

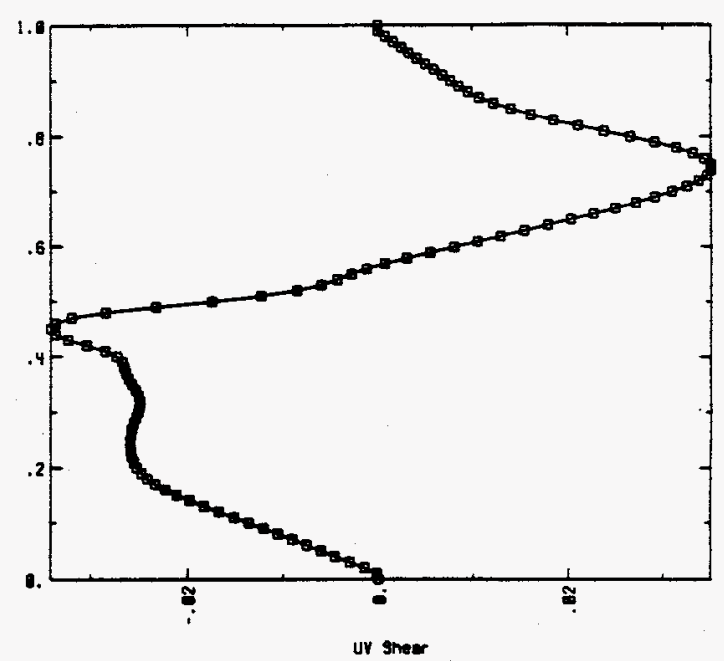

$x=6.0$

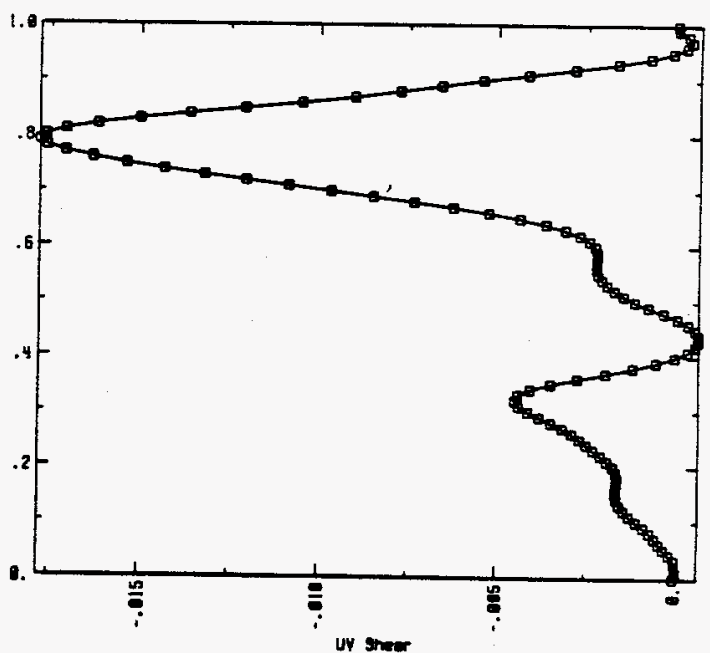

Figure 5.2.50 The turbulent shear, $\left\langle u_{1}^{\prime \prime} u_{2}^{\prime \prime}\right\rangle$, at selected locations for the $101 \mathrm{x}$ 1001 grid and $\Delta t=0.005$. 

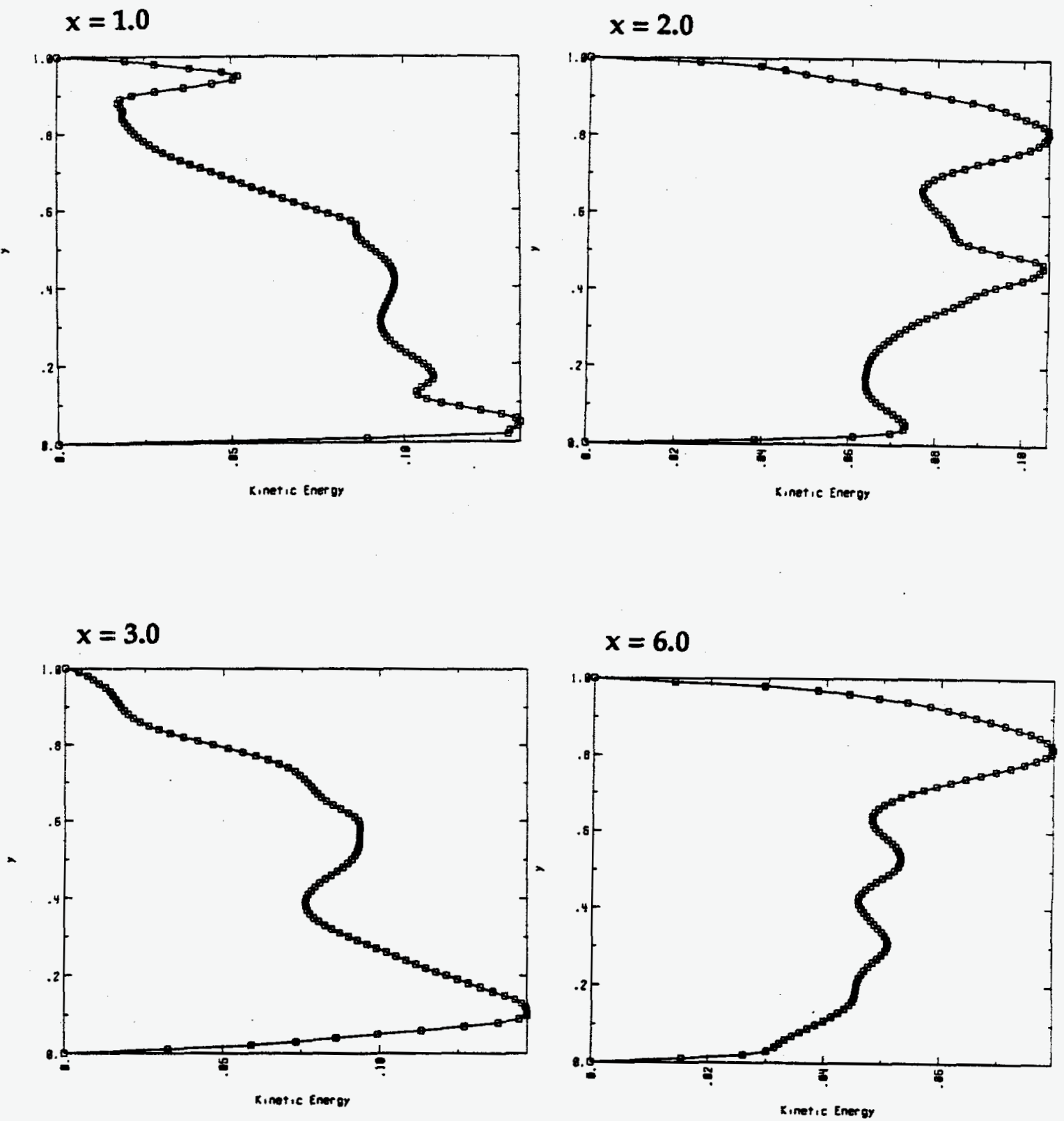

Figure 5.2.51 The turbulent kinetic energy at selected locations for the $101 \times 1001$ grid and $\Delta t=0.005$. 
$x=1.0$

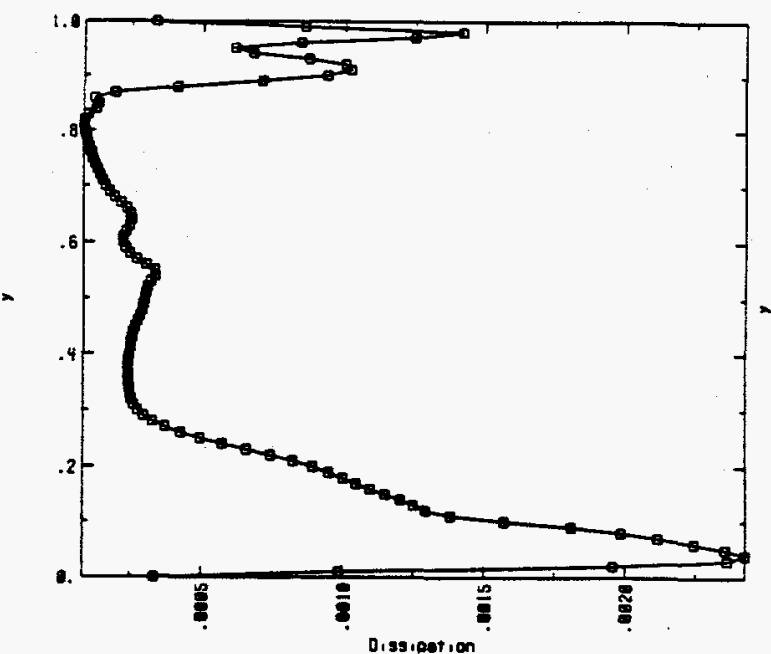

Dissicetion

$$
\mathbf{x}=\mathbf{3 . 0}
$$

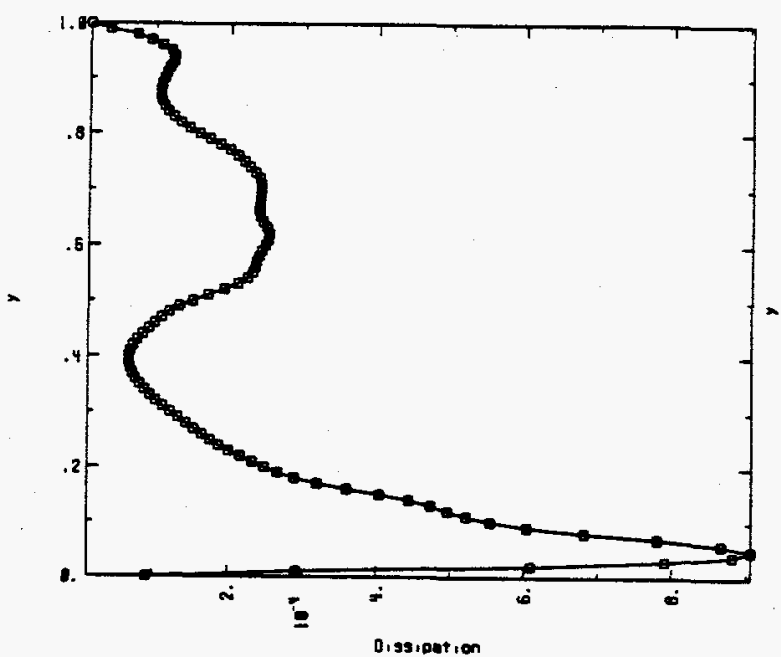

$x=\mathbf{2 . 0}$

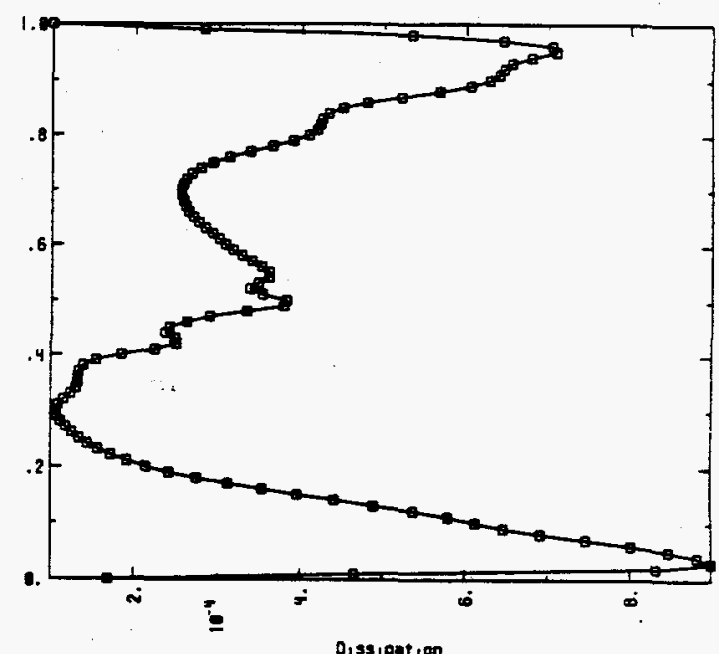

$x=6.0$

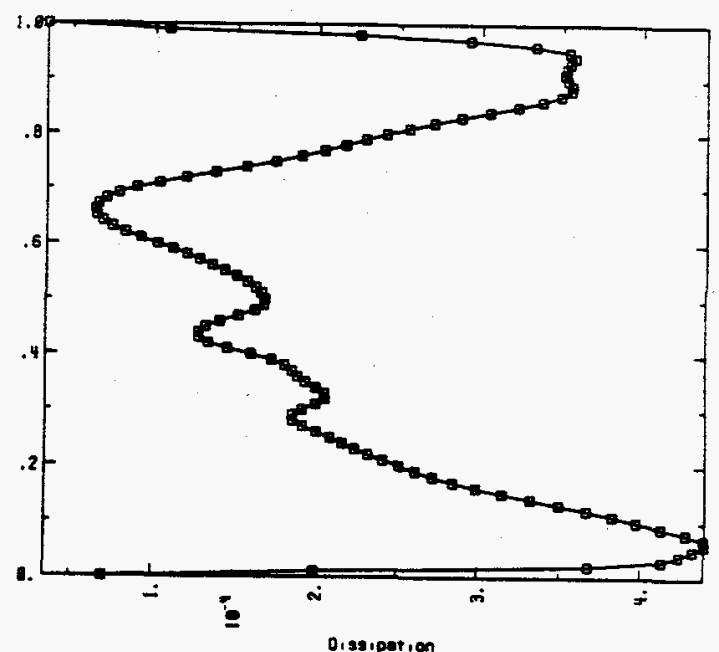

Figure 5.2.52 The turbulent rate of dissipation at selected locations for the $101 \times 1001$ grid and $\Delta t=0.005$. 


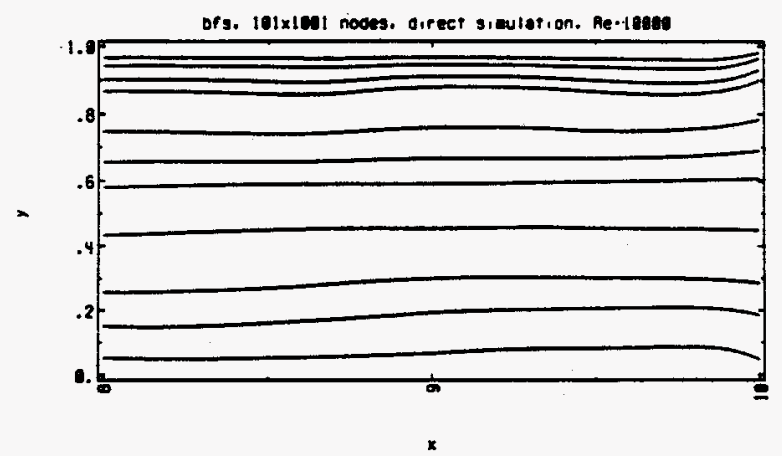

$$
x=9.97
$$

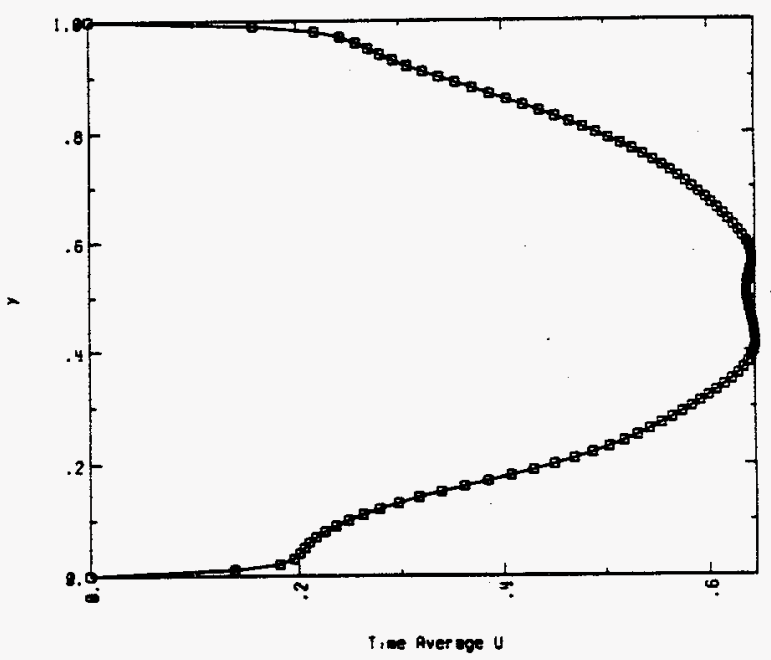

$$
x=9.99
$$

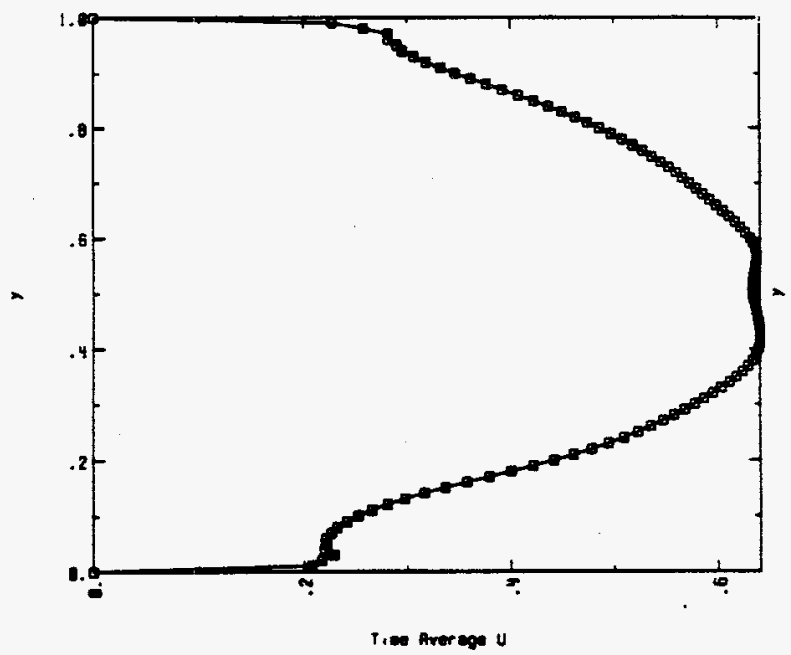

$x=9.98$

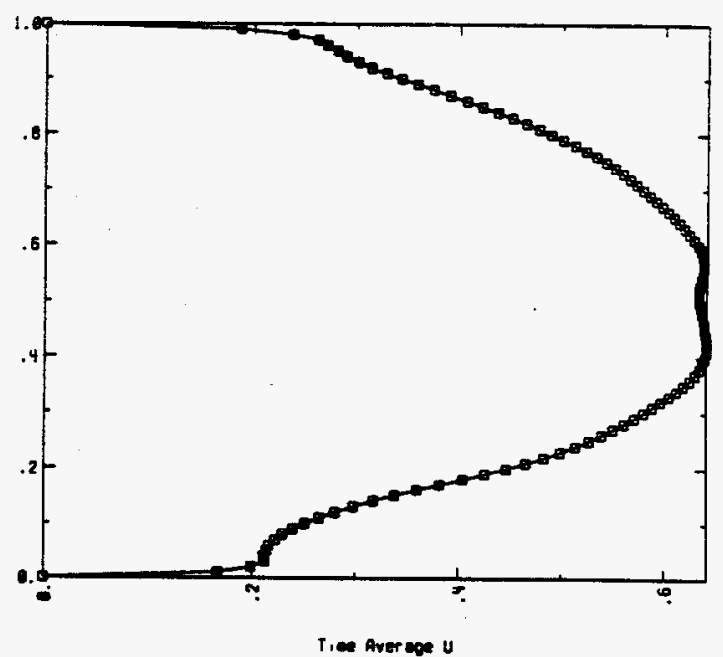

$$
x=\mathbf{1 0 . 0 0}
$$

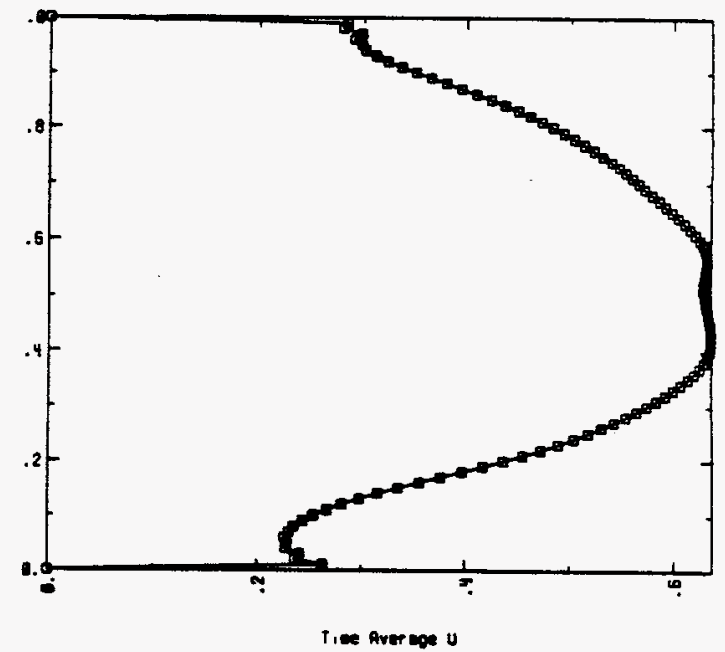

Figure 5.2.53 The time-averaged $u_{1}$ velocity, $\left\langle u_{1}\right\rangle$, at the channel outlet for the $101 \times 1001$ grid and $\Delta t=0.005$. 


\subsection{LES Results}

The following sections include LES results with and without explicit filtering of the advection term for the 'turbulent' flow case of $\operatorname{Re}=10,000$. The small-scale motion is approximated using the vorticity model with the model constant $C_{\omega}=0.2154$. As explained in Appendix $\mathrm{E}$, the value of the vorticity model constant $C_{\omega}$ is set equivalent to a value of the Smagorinsky constant $C$ to the $2 / 3$ power (i.e., $C_{\omega}=C^{2 / 3}$ ). As explained in Section 2.4.4, there is no standard value for $C$ and researchers have chosen different values with various degrees of success. The Smagorinsky constant used by Deardorf (1970a) for a channel flow was $C=0.1$ and is chosen for use in this study. Thus, we have $C_{\omega}=(0.1)^{2 / 3}=0.2154$.

Velocity time histories at chosen points, their corresponding power spectra, and a time series of stream function plots and $v_{T}$ contour plots are generated using the LES approach and compared to the direct simulation result described in Section 5.2. The LES results are evaluated locally by comparing the velocity time histories and their corresponding power spectra and globally by comparing a series of stream function plots to the direct simulation. Time-averaged statistics are also presented.

The prescribed inlet, outlet, and boundary conditions are the same as those used for the direct simulation and are described in Figure 5.2.1 and in Section 5.2. The computational field starts at the step. An everywhere-uniform grid and a non-uniform grid with grading normal to the wall are used for the reported calculations. The initial condition is approximately a potential flow solution as shown in Figure 5.2.2. All cases were run without subcycling. A constant time step of 0.005 was used for all calculations, which is consistent with that used for the direct simulation. 


\subsubsection{LES Without Explicit Filtering of the Advection Term}

The LES results for an everywhere-uniform $25 \times 241$ node grid and $\Delta t=0.005$ using the SGS vorticity model with $C_{\omega}=0.2154$ and $\Delta_{f}=\Delta$ are given in Figures 5.3.1 through 5.3.24 and Tables 5.3.1 and 5.3.2.

In Figure 5.3.1, the LES $\bar{u}_{1}$ and $\bar{u}_{2}$ velocity time histories are compared to the direct simulation (DS) for two locations in the flow field for one DS time cycle (6.36 time units). The LES results are not in phase with the DS so the LES time histories are shifted in time by matching a minimum point in the LES cycle with that in the DS cycle for the $\bar{u}_{1}$ time history at $(x, y)=(2.0,0.25)$. Extended time histories are shown in Figures 5.3.2a, 4a, 6a, and 8a. The character of the LES and DS time histories are similar (i.e., approximate periodicity with some similar peaks and valleys) and the average magnitudes appear to be about the same, but there is not strong agreement between the LES and DS time histories.

The extended time histories for the LES and DS results show clearly that the LES results are only approximately periodic and there is variation from cycle to cycle. A closer look shows that every other cycle are in closer agreement than every consecutive cycle. Checking the LES predicted times at which a minimum for $\bar{u}_{1}$ at $(x, y)=(2.0,0.25)$ is reached (Figure 5.3.2a), we see that the period of every other cycle is in closer agreement than that for every consecutive cycle (see Table 5.3.1). The LES cycle period with the $25 \times 241$ grid varies from approximately 5.0 to 5.6 which is $21 \%$ to $12 \%$ lower than the 6.36 cycle for the DS.

Using the extended time histories for the LES and DS results, the corresponding power spectra were calculated and compared (Figures 5.3.3, 5, 7, and 9). As expected, 
the power spectra indicate good agreement between the LES and DS results at low frequencies (or large scales), but the LES approach does not capture the high-frequency motion (or small-scale motion). The LES results in the low frequency end of the spectra indicate a shift to high frequencies, which is due to the shorter cycle (or higher vortex shedding frequency) predicted by the LES approach.

The time series of stream function plots in Figure 5.3.10 clearly show that the LES approach does capture the character of the flow very well. The LES predicted formation of vortices at the step and top wall and the propagation of these vortices downstream is in agreement with the DS results. However, the LES predicted vortex shedding frequency appears to be slightly higher than that observed for the DS. This observation coincides with the shorter LES cycle period.

A time series of contour plots for the SGS turbulent viscosity, $v_{T}$, calculated with the vorticity model are shown in Figure 5.3.11 along with the corresponding stream function plots. The magnitude and variation of $v_{T}$ for a snapshot in time is shown with profile plots for selected locations in Figure 5.3.12. There are higher gradients in $v_{T}$ at walls and in the recirculation zones closest to the step compared to the gradients in $v_{T}$ in the core of the flow. The larger $v_{T}$ values at the wall and in the recirculation regions are due to the higher vorticity gradients at these locations (i.e., $v_{T}$ $\left.\propto \partial \omega / \partial x_{\beta}\right)$. Figure 5.3.13 shows the variation in vorticity at selected locations for the same snapshot in time as that shown in Figure 5.3.12 (points on the curves indicate grid point locations). In summary, the turbulent eddy viscosity $v_{T}$ is highest at the walls, but $v_{T}$ is on the order of one-tenth of the molecular viscosity throughout most of the flow.

Profile plots of the $\bar{u}_{1}$ velocity are given in Figure 5.3.14 for the same snapshot in time as that for figures 5.3 .13 and 5.3.12. The velocity wiggles at the walls are 
relatively small. These wiggles indicate that further grid resolution is needed at the wall. Since the velocity profiles are smoothed away from the first wall grid point, it appears that more resolution is needed only in the elements adjacent to the wall.

To further investigate if finer discretization at the wall will remove some of the wiggles at the wall, we can take a closer look at the velocity profiles normal and tangent to the wall. The $\bar{u}_{1}$ velocity profiles along the top and bottom walls (i.e., actually one grid point from wall) are shown in Figure 5.3.15 again for the same snapshot in time. A portion of these profiles from $x=0.0$ to 1.0 are shown in Figure 5.3.16 along with a profile plot in the cross-stream direction at $x=0.5$. Comparing the velocity gradient normal and tangential to the wall at $x=0.5$, we see that the velocity gradient in the normal direction $\left(\partial \bar{u}_{1} / \partial x_{2}\right)$ is approximately 4 to 12 times greater than that in the tangential direction $\left(\partial \bar{u}_{1} / \partial x_{1}\right)$. This implies that a finer grid in the normal direction at the wall could eliminate the wiggles observed at the wall. This was done with the addition of more elements and mesh grading at the wall. The results are given at the end of this section.

Velocity profiles near the channel outlet are shown in Figure 5.3.17. The profile plot at the channel outlet $(x=10.00)$ shows wiggles at the walls and is not entirely smooth in the central portion of the channel. However, as we move just upstream, the velocity profiles are smooth. As for the DS, this indicates an 'error' in the specified outlet conditions (i.e., zero natural boundary conditions) which is confined to the few element rows adjacent to the outlet and is not translated upstream.

Time-averaged LES results were generated for two different time durations; 10,000 and 20,000 time steps at $\Delta t=0.005$ (50 and 100 time units, respectively). Since the cycle duration varies from approximately 5.0 to 5.6 time units, we are capturing at 
least 9 and 18 cycles for the 10,000 and 20,000 time steps, respectively. From the stream function plots for the instantaneous results (Figure 5.3.10), we estimate that a step-generated recirculation zone travels approximately 2 length units per cycle; i.e., the eddy speed is $2 / 5.0=0.40$ to $2 / 5.6 \equiv 0.36$. Thus, a step-generated recirculation zone will have traveled approximately 1.5 to 3 times the length of the computational field (10.0 length units) in 10,000 to 20,000 time steps.

The LES predicted $x$ locations for the separation and reattachment points are listed in Table 5.3.2. Figure 5.3.18 is a stream function plot of the time-averaged LES result showing the predicted recirculation zones. The reattachment length for the major recirculation zone is 2.037 or approximately 4.1 step heights downstream of the step. This length is approximately $20 \%$ longer that the DS length (1.697 length units or 3.4 step heights).

Profile plots of the time-averaged $\bar{u}_{1}$ velocity, the turbulent intensity, shear, kinetic energy, and dissipation rate (as defined in Appendix B) are shown in Figures 5.3.19 to 5.3.24 for selected locations. The profiles are fairly smooth across the channel, but do show some wiggles and larger gradients in the wall regions.

The time-averaged velocity profiles near the channel outlet are shown in Figure 5.3.24. Similar to the instantaneous profiles shown in Figure 5.3.17, the time-averaged profiles at the channel outlet have wiggles at the walls and are not entirely smooth across the channel. The profiles do become smoother as we move just upstream of the outlet.

To eliminate the wiggles in the wall regions and hopefully improve the LES prediction, more elements were added with grading in the wall region. The 
computational field of $33 \times 245$ nodes is described in Figure 5.3.25. The two rows of uniform elements adjacent to the walls in the $25 \times 241$ node grid were replaced with 6 graded elements. This mesh refinement brings the total number of elements up from 5,760 to 7,808 , a $36 \%$ increase in number of elements. The LES results for the $33 x$ 245 node grid and $\Delta t=0.005$ using the SGS vorticity model with $C_{\omega}=0.2154$ and $\Delta_{f}=$ $\Delta$ are given in Figures 5.3.26 through 5.3 47 and Table 5.3.3.

In Figure 5.3.26, the LES $\bar{u}_{1}$ and $\bar{u}_{2}$ velocity time histories are compared to the DS for two locations in the flow field for one DS time cycle (6.36 time units). The LES results are shifted in time by matching a minimum point in the LES and DS cycle for the $\bar{u}_{1}$ time history at $(x, y)=(2.0,0.25)$. Extended time histories are shown in Figures 5.3.27a, 29a, 31a, and 33a. There is a definite improvement with the $33 \times 245$ grid compared to the $25 \times 241$ grid. The LES and DS time histories are in closer agreement. The LES results are periodic with a 6.29 cycle period. This is only $1 \%$ shorter than the DS 6.36 cycle. The LES magnitudes for the peaks and valleys on the time history plots do not agree with the DS, but the character of the histories are in closer agreement for the refined grid.

The extended time histories were used to calculate the power spectra for the $33 \mathrm{x}$ 245 grid (Figures 5.3.28, 30, 32, and 34). The LES and DS results agree for the dominant lowest frequency and show an improvement over the $25 \times 241$ grid. This is due to the close agreement in the DS and LES periodic cycle. However, there appears to be some discrepancies in the next dominant higher frequencies. The DS and LES predicted dominant frequency values agree (i.e., the peaks occur at the same frequency values), but there are some discrepancies in the power spectrum magnitudes for the dominant frequencies just above the lowest dominant frequency. Overall, the $33 \times 245$ grid shows an improvement over the $25 \times 241$ grid because of the agreement in the 
dominant frequencies. It could also be possible that further refinement (e.g., mesh grading at the wall) with the DS would show a change in the DS power spectrum magnitudes. (However, such a refinement would be very computationally expensive.)

The time series of stream function plots in Figure 5.3.35 show very good agreement between the DS and LES. The LES calculations accurately capture the vortex shedding from the step and upper wall and the propagation of these vortices downstream.

A time series of contour plots for the SGS turbulent viscosity, $v_{T}$, are shown in Figure 5.3.36 along with the corresponding stream function plots. The magnitude and variation of $v_{T}$ for a snapshot in time is shown with profile plots for selected locations in Figure 5.3.37. Since $v_{T}$ is proportional to the grid size, its magnitude at the wall is smaller with the refined grid. Figure 5.3.38 shows the variation in vorticity at selected locations for the same snapshot in time as that shown in Figure 5.3.37. The refined grid captures the steep vorticity gradients in the wall region without any apparent wiggles in the solution.

Profile plots of the $\bar{u}_{1}$ velocity are given in Figure 5.3.39 for a snapshot in time. The profiles are smooth at the walls as well as in the central portion of the channel. The grid refinement has eliminated the wiggles at the walls that were present with the $25 \mathrm{x}$ 241 grid.

Velocity profiles near the channel outlet are shown in Figure 5.3.40. Again, the grid refinement has eliminated the wiggles at the wall and also in the central portion of the channel that were present with the $25 \times 241$ grid. This result implies that the zero natural boundary condition is a useful specified outlet condition for the LES and that 
wiggles in the velocity profiles can be reduced with mesh refinement.

Time-averaged LES results with the $33 \times 245$ grid were generated for two different time durations; 10,000 and 20,000 time steps at $\Delta t=0.005$. The LES predicted $\mathbf{x}$ locations for the separation and reattachment points are listed in Table 5.3.3. Figure 5.3.41 is a stream function plot of the time-average LES showing the predicted recirculation zones. The reattachment length for the major recirculation zone is 1.543 or approximately 3.1 step heights downstream of the step. This length is only $9 \%$ shorter than the DS length.

Profile plots of the time-averaged $\bar{u}_{1}$ velocity, the turbulent intensity, shear, kinetic energy, and dissipation rate are shown in Figures 5.3.42 through 5.3.47 for selected locations. The grid refinement has eliminated the wiggles at the walls that were present with the $25 \times 241$ grid. Even the parts of the profiles away from the walls, where the grid resolution was unchanged, are smoother.

To clearly show the improvement in the solution by the addition of the SGS model, we ran the $33 \times 245$ grid without the SGS model (i.e., a direct simulation). These results are presented in Figures 5.3.48 through 5.3.53 and Table 5.3.4. The extended time histories in Figures 5.3.48 to 5.3.51 show that without the SGS model, the results are not periodic and do not compare well to the DS. The predicted velocity profiles at selected locations for a snapshot in time shown in Figure 5.3.52 indicate some wiggles in the wall region at $x=1.0$ and 3.0. Wiggles were not present when the SGS model was used (see Figure 5.3.39). Time-averaged results for 10,000 and 20,000 time steps at $\Delta t$ $=0.005$ are presented in Table 5.3.4 and Figure 5.3.53. The separation and reattachment lengths vary significantly between the 10,000 and 20,000 time step averages. Longer averaging times would be needed before the stationary solution could 
definitely be established. When the SGS model was used, almost no variation in the separation and reattachment locations was observed between the two averaging durations (Table 5.3.3). This indicates that the SGS model improves the solution stability while accurately capturing the large-scale motion.

In summary, grid resolution in wall regions with grading normal to walls resulted in a significant improvement in the LES. It can be concluded that an accurate solution can be generated using the LES approach that compares very well with a DS, with a $92 \%$ reduction in elements from the DS to the LES grid (i.e., 100,000 elements for the DS and 7,808 elements for the LES). 

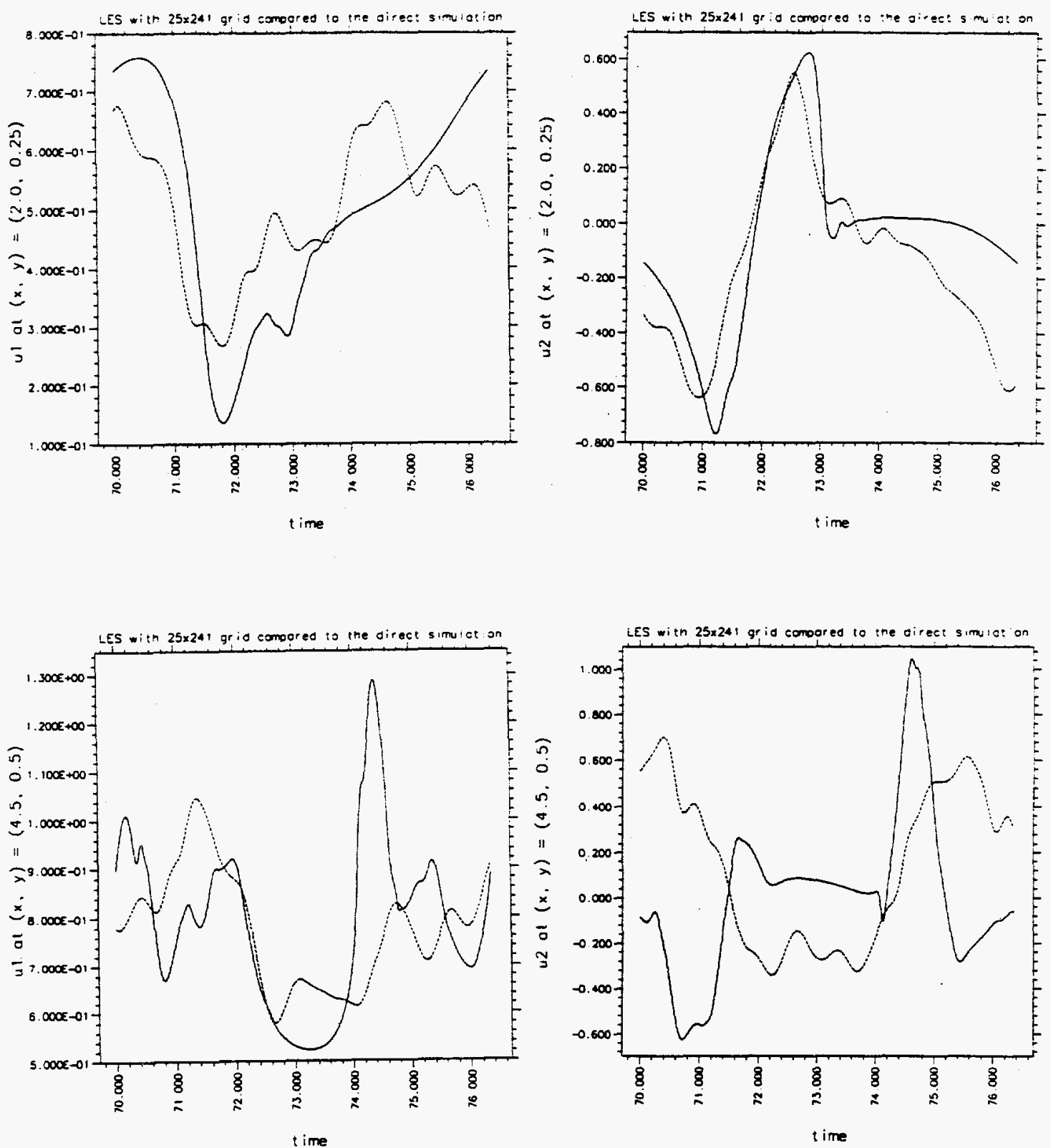

Fig. 5.3.1 Comparison of LES time histories for the $25 \times 241$ grid, $\Delta t=0.005$, $C_{\omega}=.2154$, and $\Delta_{f}=\Delta$ to the direct simulation for one cycle. (One cycle is 6.36 time units for the direct simulation.) The LES curve has been shifted in time so that the minimum $\bar{u}_{1}$ values at $(x, y)=(2.0,0.25)$ are shown to occur at the same time. 


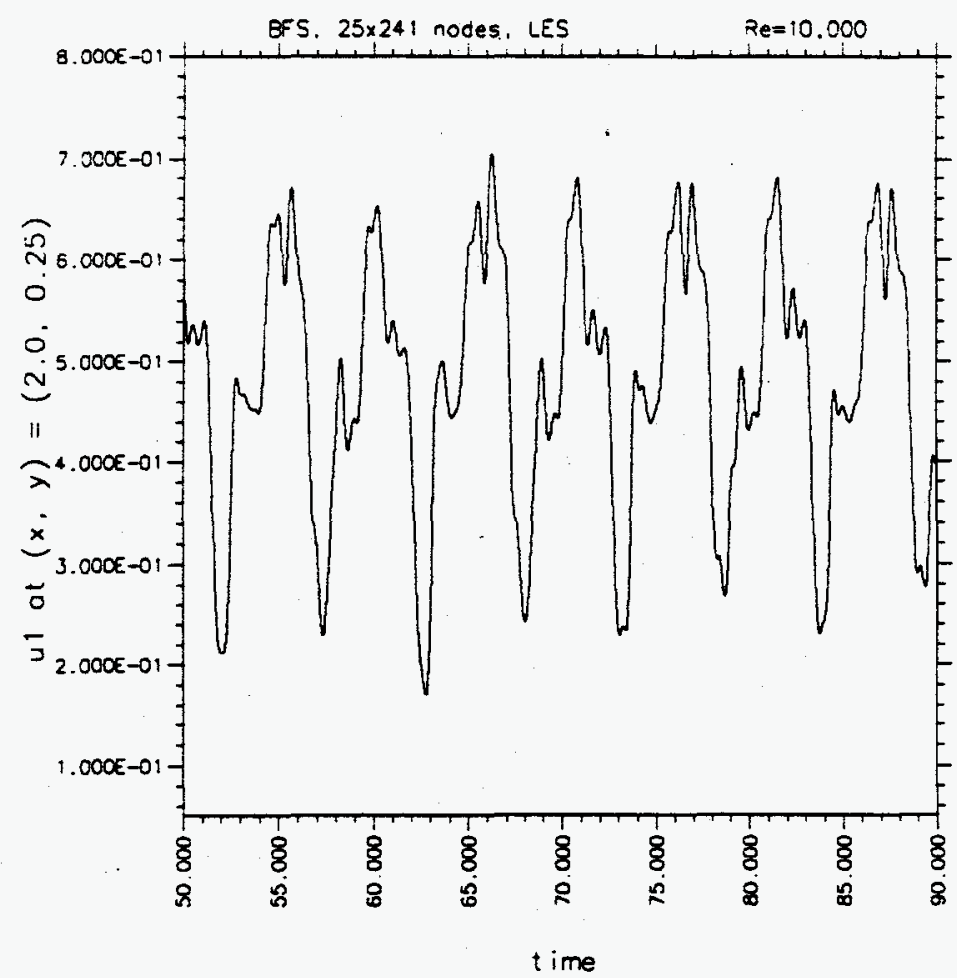

(a)

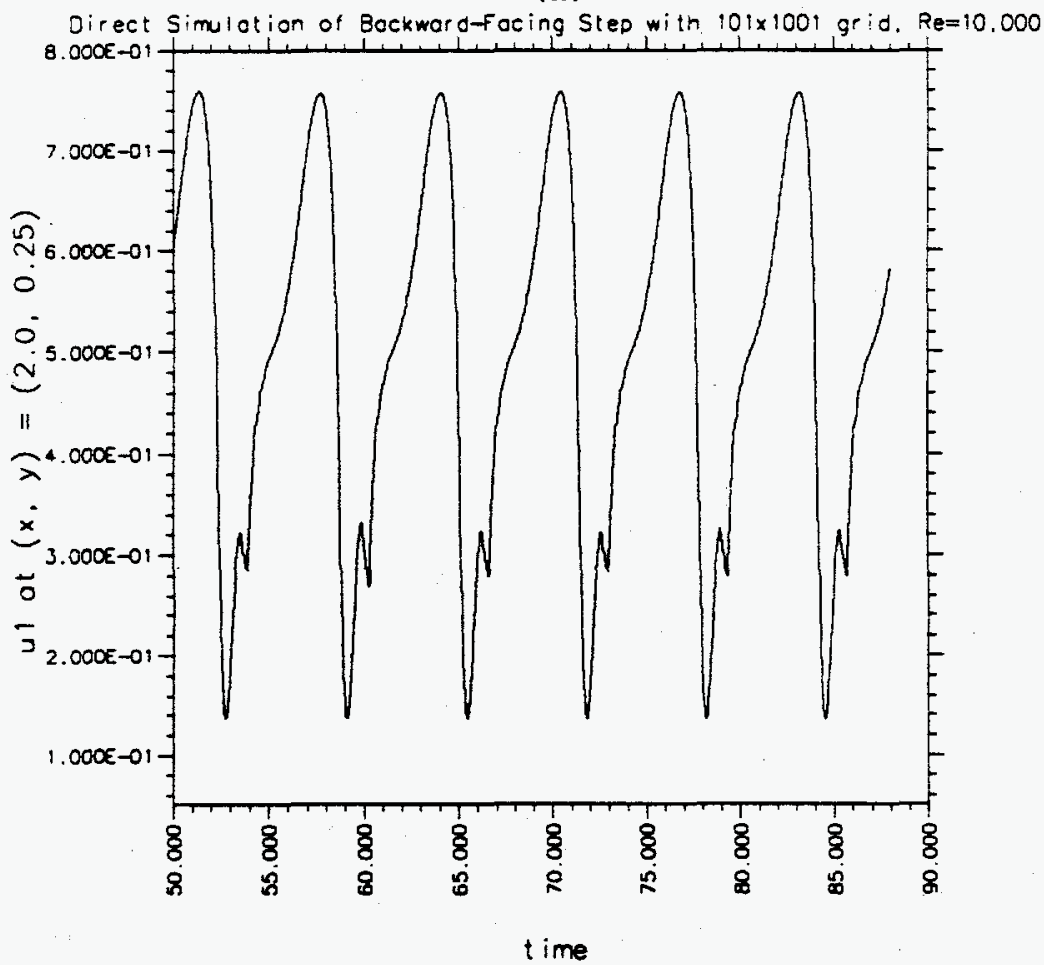

(b)

Fig. 5.3.2 Extended time histories of $\bar{u}_{1}$ at $(x, y)=(2.0,0.25)$ for a) the $25 \times 241$ grid, $\Delta t=0.005$, and the SGS vorticity model with $C_{\omega}=.2154$ and $\Delta_{f}=\Delta$ and b) the direct simulation (shown for the same axis scales). 
Table 5.3.1 LES Predicted Cycle Periods Using $\bar{u}_{1}$ Time History at $(x, y)=(2.0,0.25)$.

\begin{tabular}{|c|c|}
\hline $\begin{array}{l}\text { Times at Which } \bar{u}_{1} \\
\text { Minimum Reached }\end{array}$ & $\begin{array}{c}\text { Period of Time Between } \bar{u}_{1} \\
\text { Minimums }\end{array}$ \\
\hline 68.025 & 5.040 \\
73.065 & 5.625 \\
78.690 & 5.045 \\
83.735 & 5.610 \\
89.345 & \\
\hline
\end{tabular}



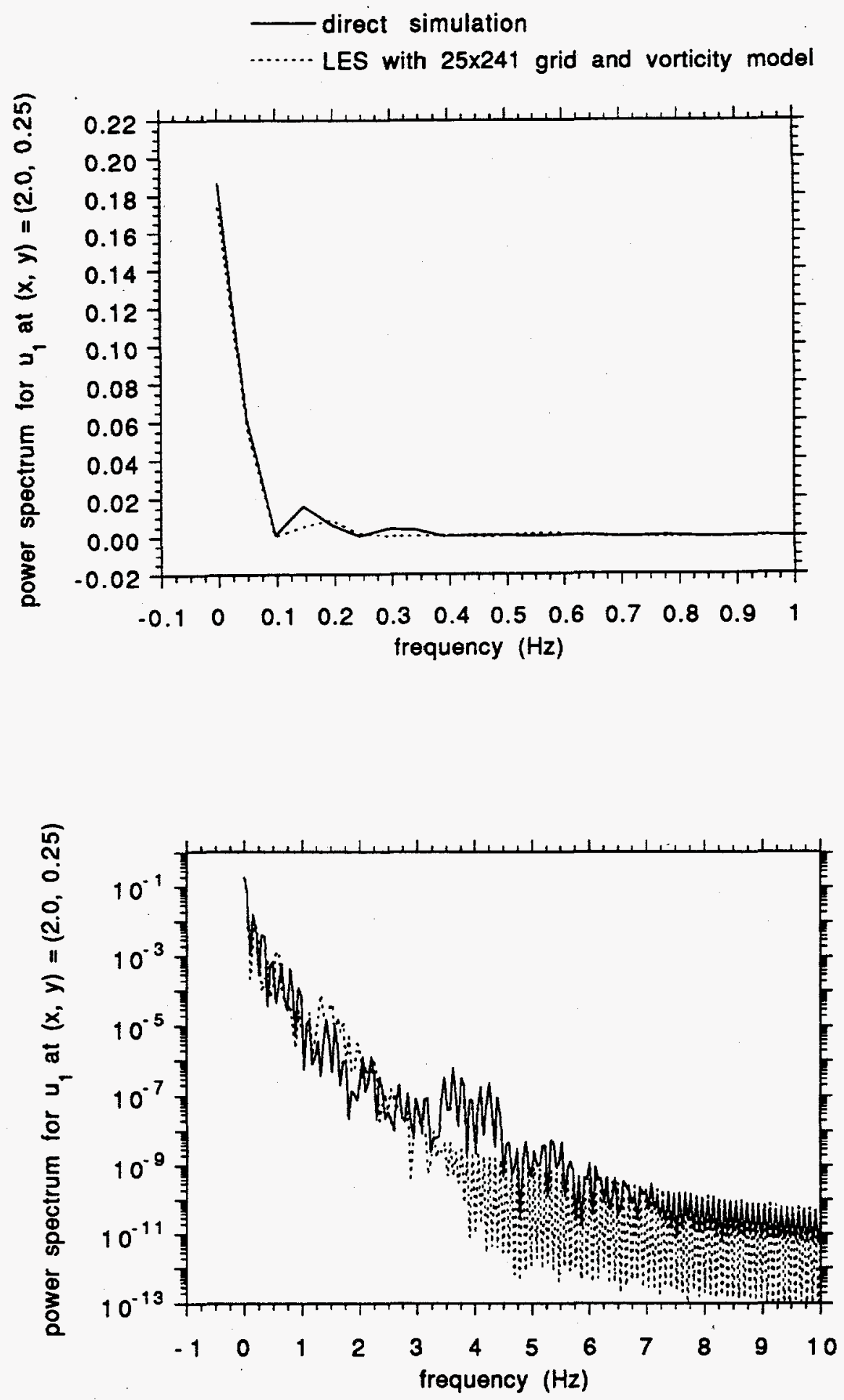

Fig. 5.3.3 Power spectrum on a linear and a log plot for $\bar{u}_{1}$ time history at $(x, y)=$ $(2.0,0.25)$ for the $25 \times 241$ grid, $\Delta t=0.005$, and the SGS vorticity model with $C_{\omega}=.2154$ and $\Delta_{f}=\Delta(t=50$ to 80.715) compared to the direct simulation. 


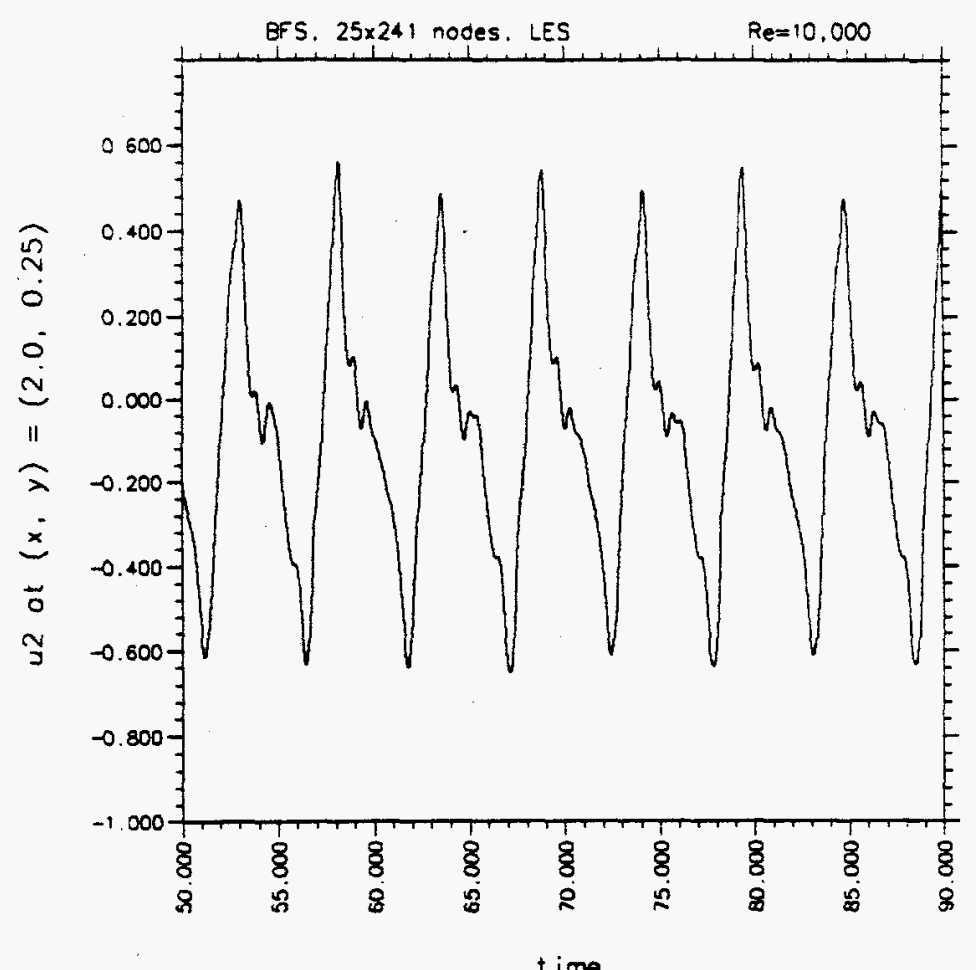

(a)

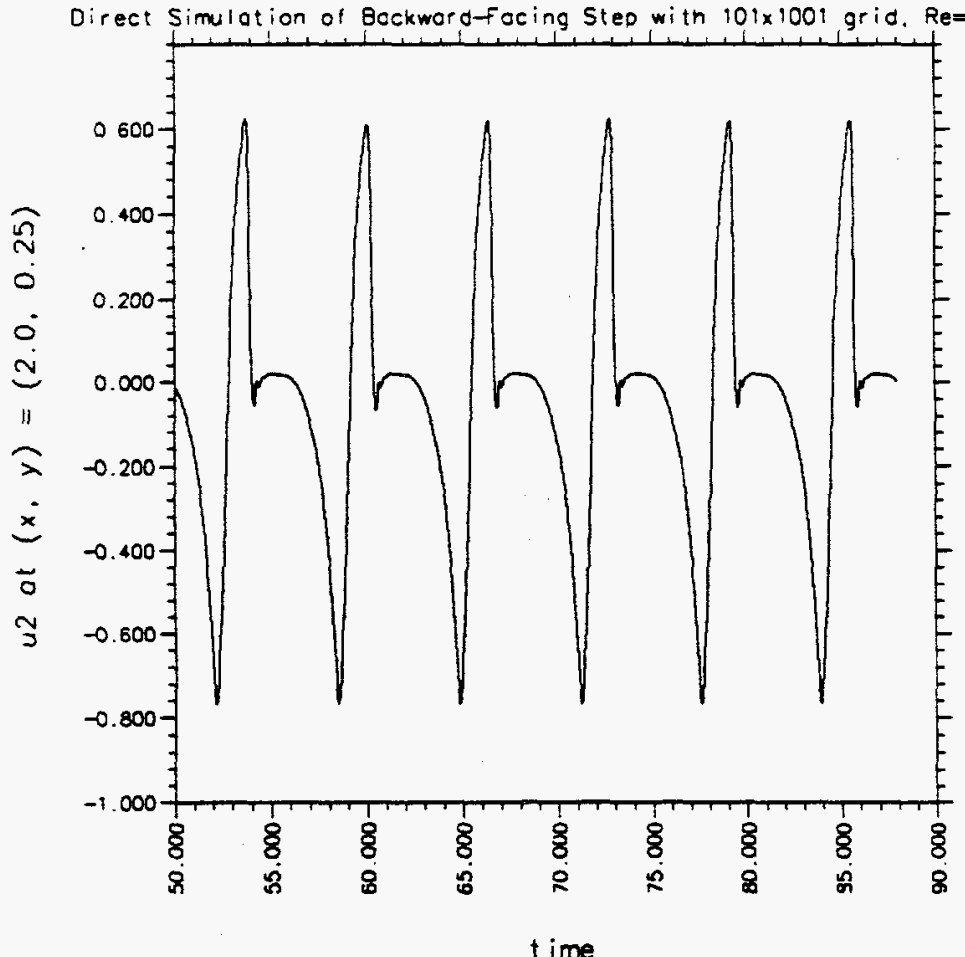

(b)

Fig. 5.3.4 Extended time histories of $\bar{u}_{2}$ at $(x, y)=(2.0,0.25)$ for a) the $25 \times 241$ grid, $\Delta t=0.005$, and the SGS vorticity model with $C_{\omega}=.2154$ and $\Delta_{f}=\Delta$ and b) the direct simulation (shown for the same axis scales). 

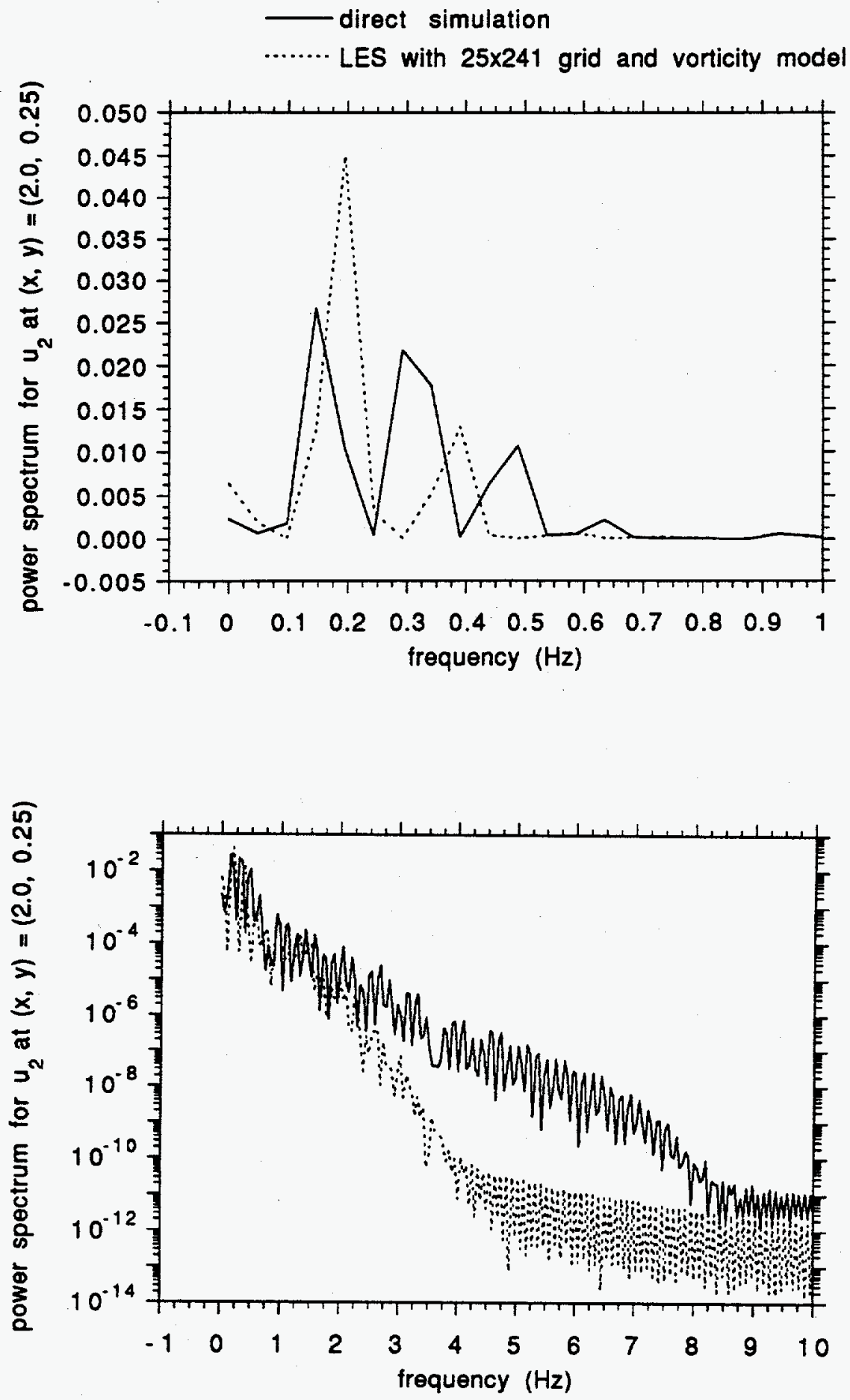

Fig. 5.3.5 Power spectrum on a linear and a $\log$ plot for $\bar{u}_{2}$ time history at $(x, y)=$ $(2.0,0.25)$ for the $25 \times 241$ grid, $\Delta t=0.005$, and the SGS vorticity model with $C_{\omega}=.2154$ and $\Delta_{f}=\Delta(t=50$ to 80.715) compared to the direct simulation. 


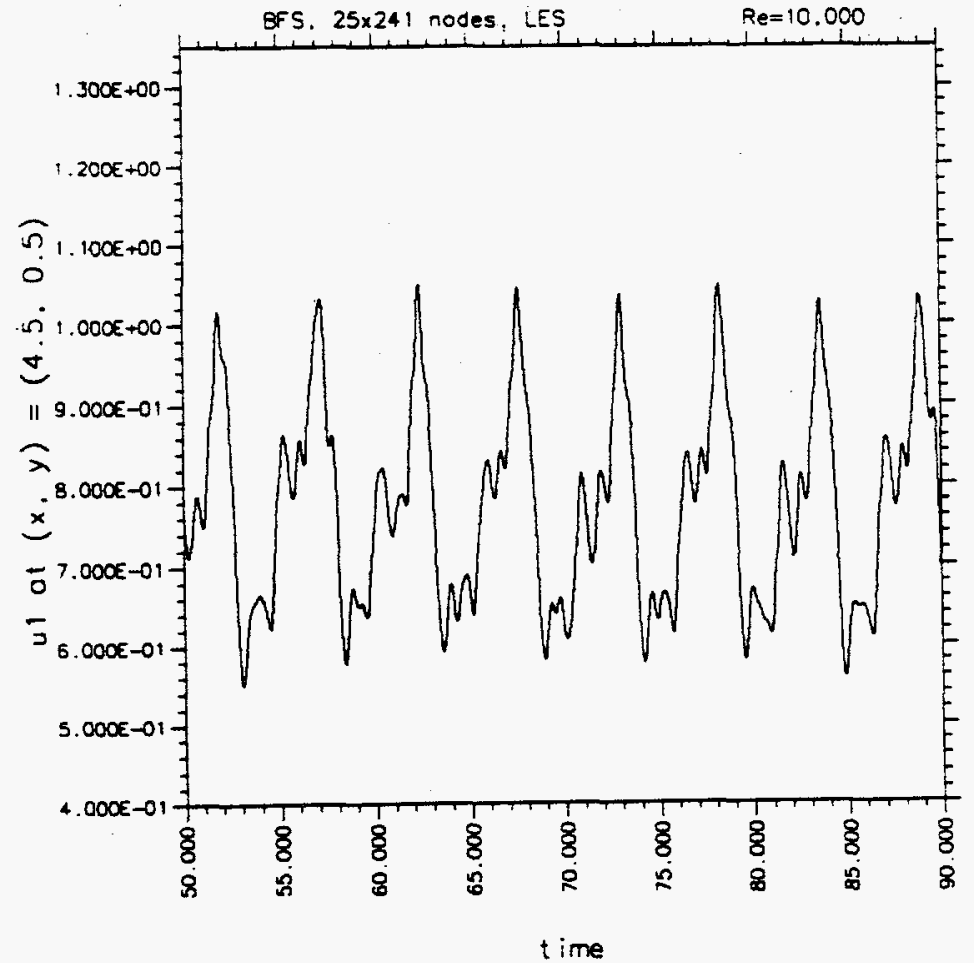

(a)

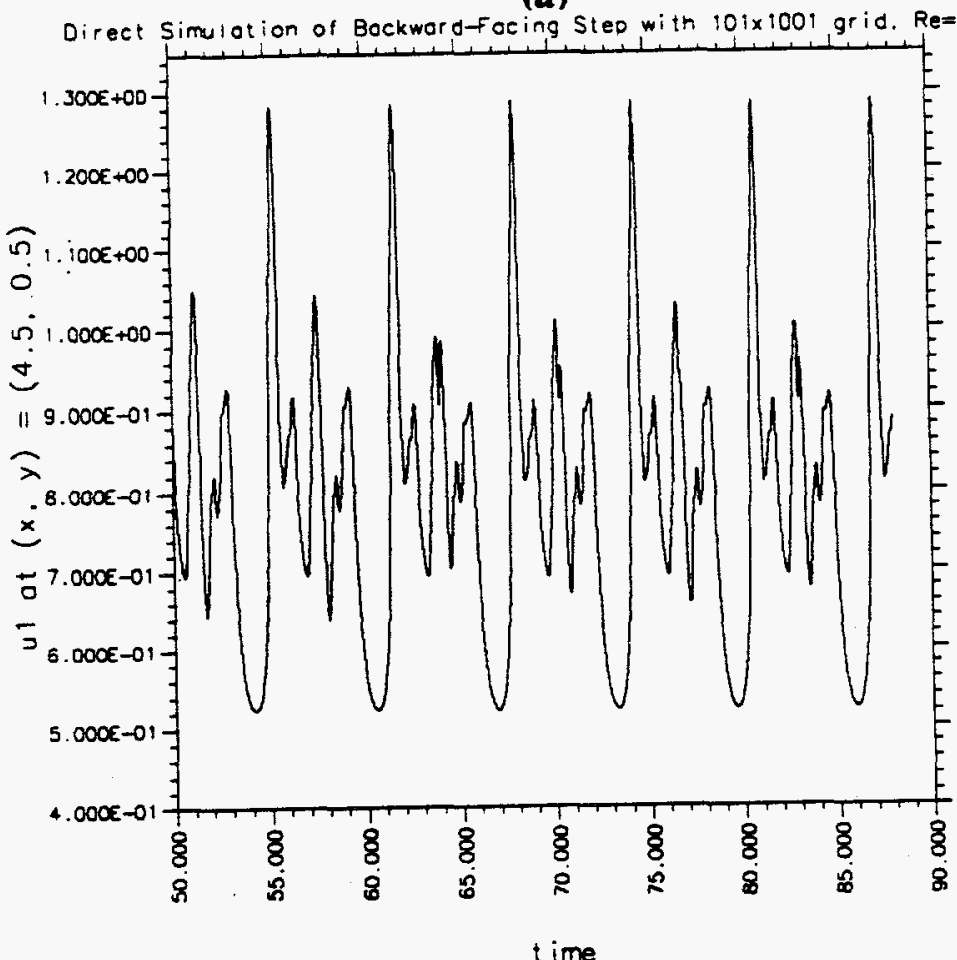

(b)

Fig. 5.3.6 Extended time histories of $\bar{u}_{1}$ at $(x, y)=(4.5,0.5)$ for a) the $25 \times 241$ grid, $\Delta t=0.005$, and the SGS vorticity model with $C_{\omega}=.2154$ and $\Delta_{f}=\Delta$ and $b$ ) the direct simulation. 

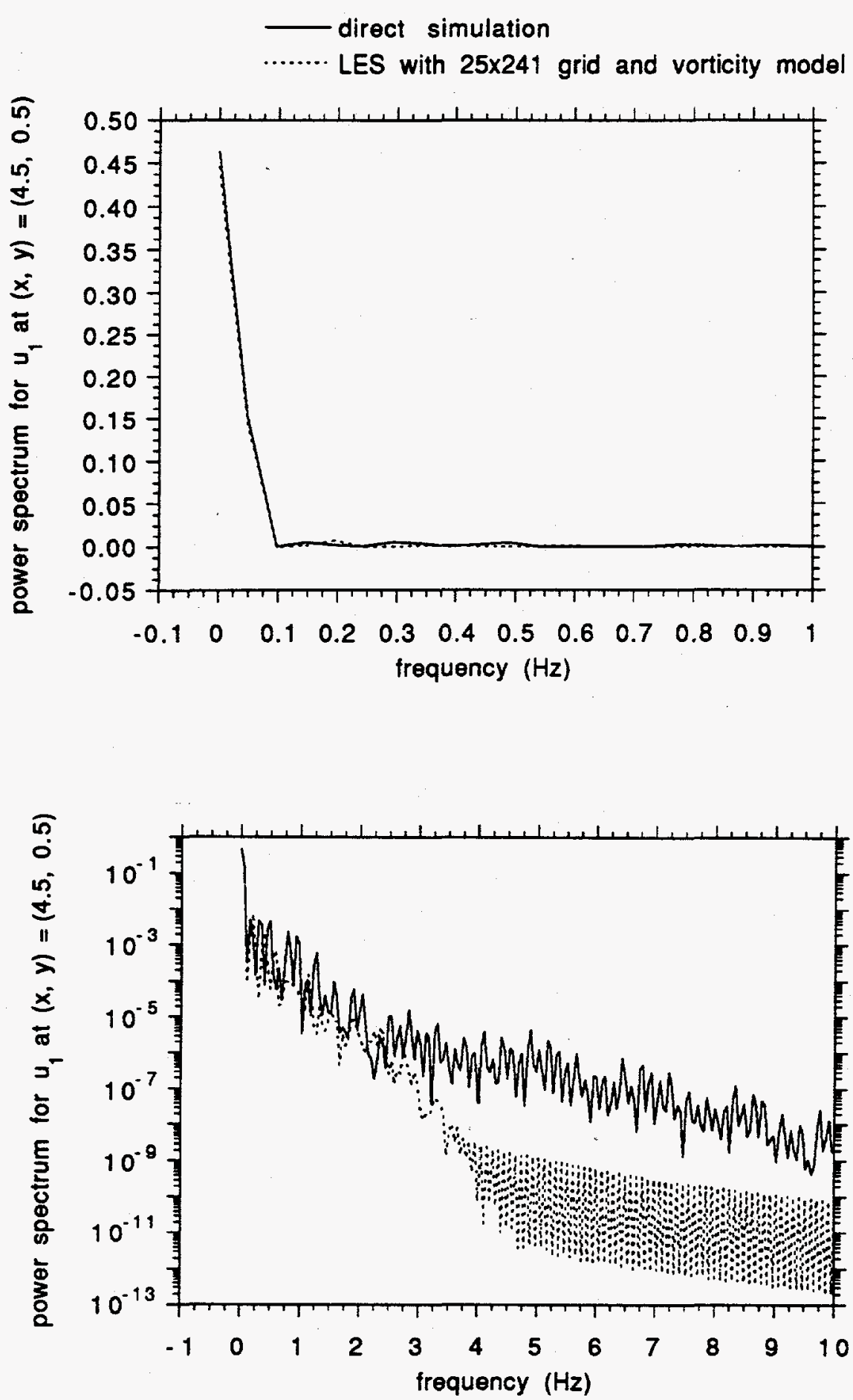

Fig. 5.3.7 Power spectrum on a linear and a log plot for $\bar{u}_{1}$ time history at $(x, y)=$ $(4.5,0.5)$ for the $25 \times 241$ grid, $\Delta t=0.005$, and the SGS vorticity model with $C_{\omega}=0.2154$ and $\Delta f=\Delta(t=50$ to 80.715$)$ compared to the direct simulation. 


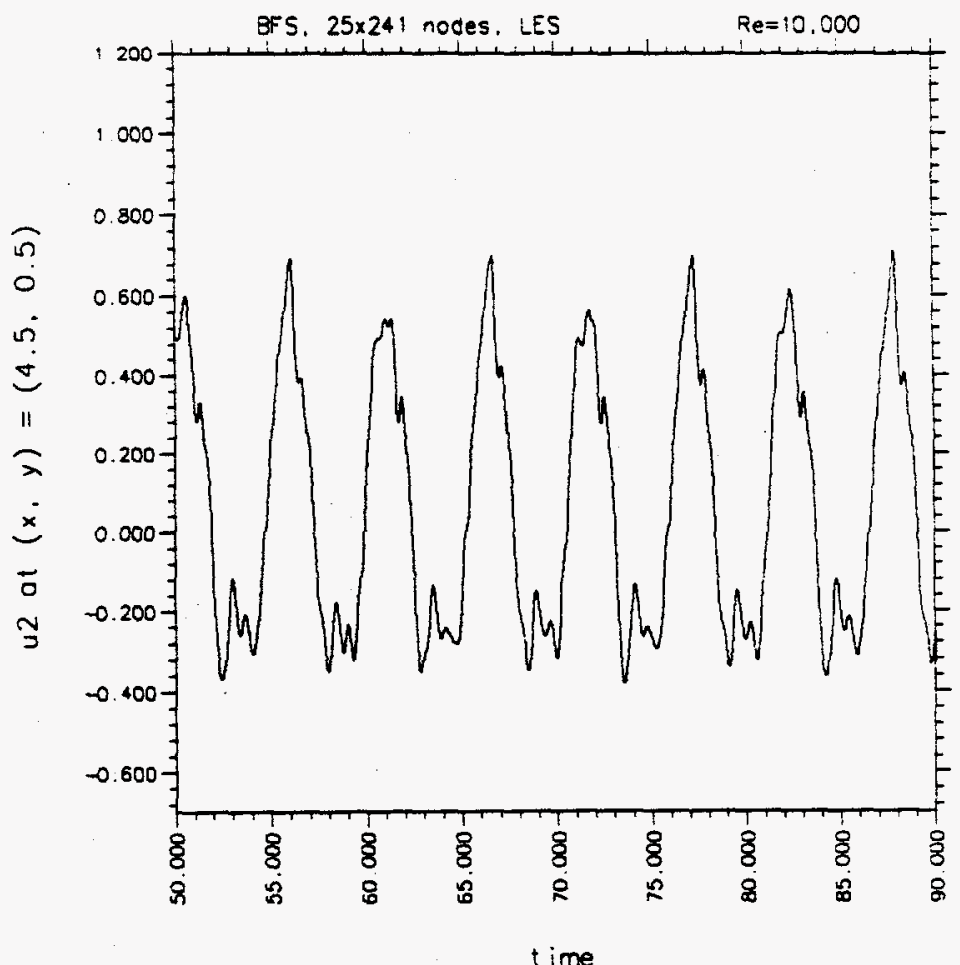

(a)

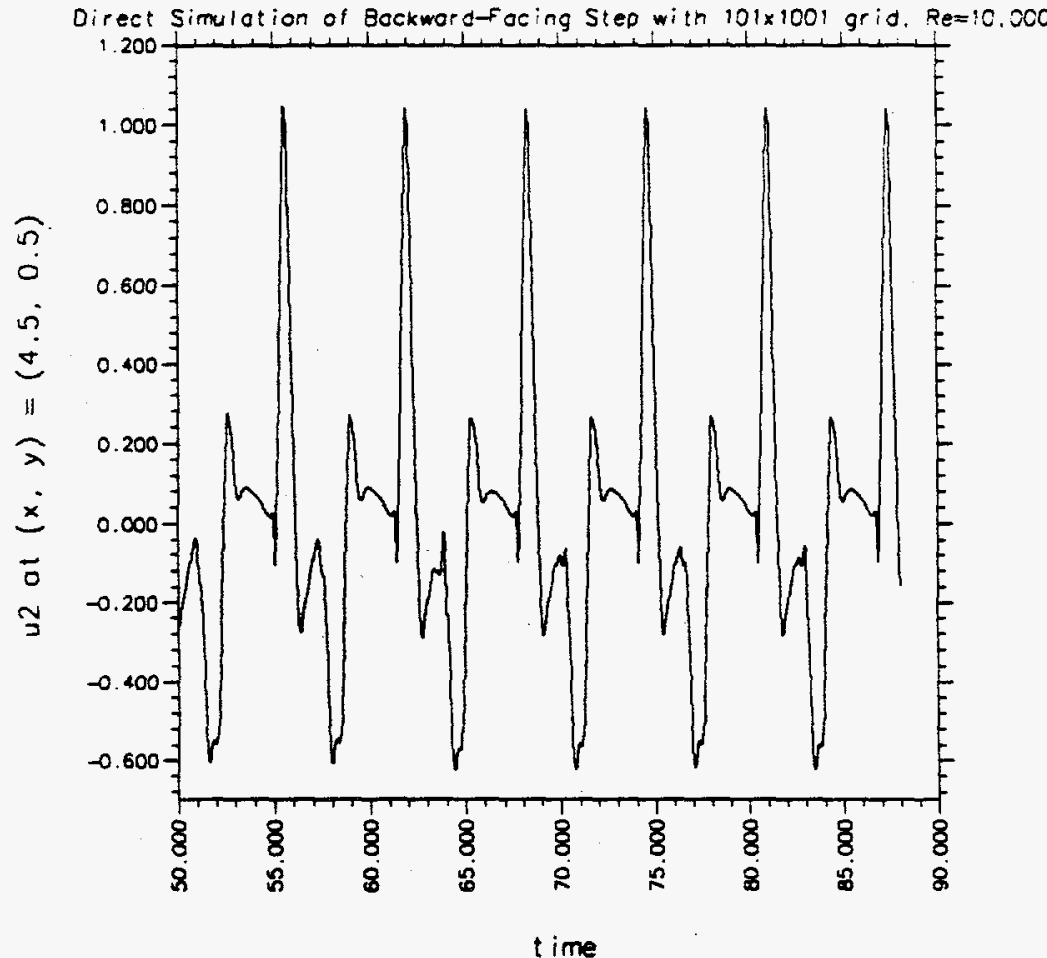

(b)

Fig. 5.3.8 Extended time histories of $\bar{u}_{2}$ at $(x, y)=(4.5,0.5)$ for a) the $25 \times 241$ grid, $\Delta t=0.005$, and the SGS vorticity model with $C_{\omega}=.2154$ and $\Delta_{f}=\Delta$ and b) the direct simulation. 

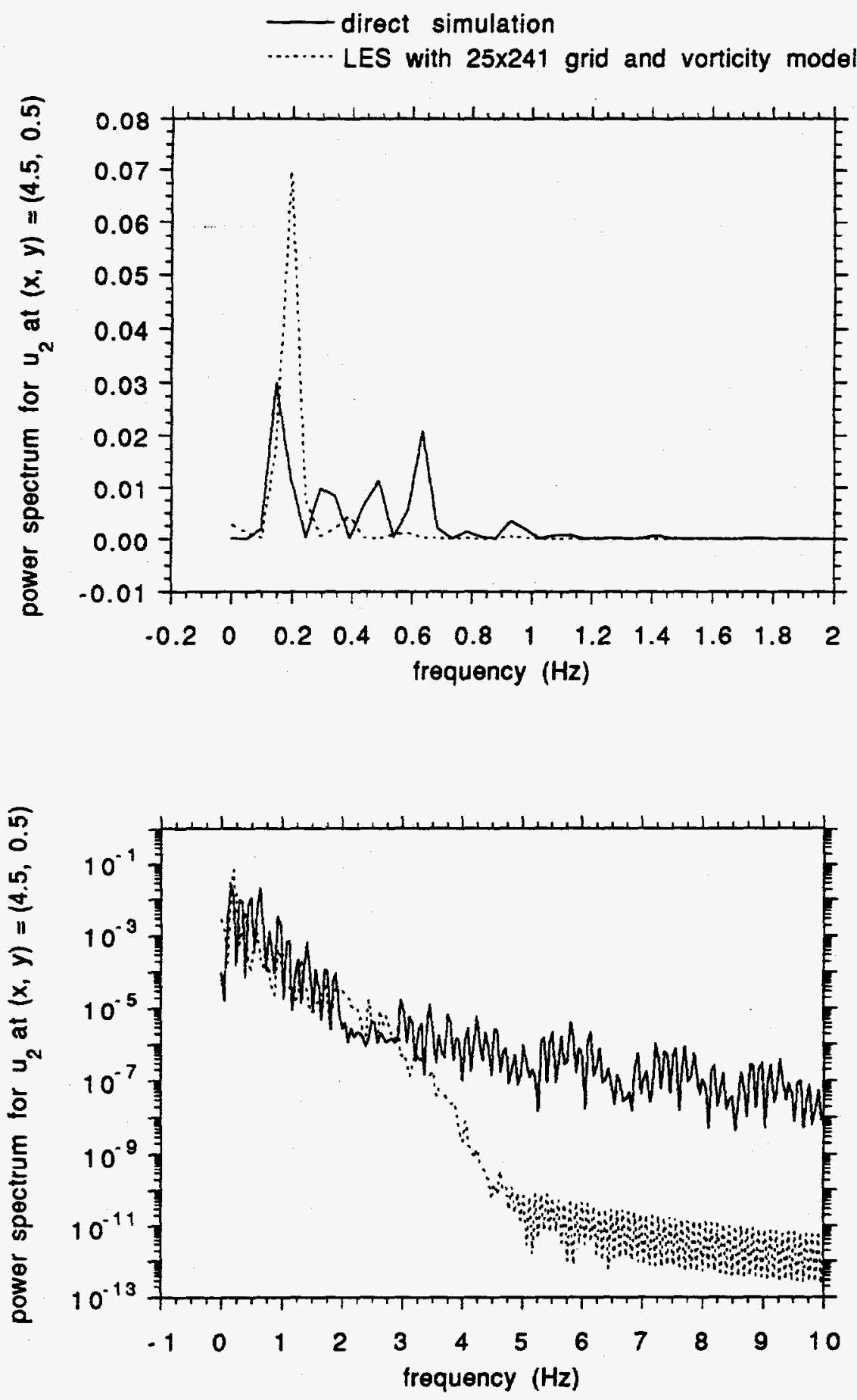

Fig. 5.3.9 Power spectrum on linear and a log plot for $\bar{u}_{2}$ time history at $(x, y)=$ $(4.5,0.5)$ for the $25 \times 241$ grid, $\Delta t=0.005$, and the SGS vorticity model with $C_{\omega}=.2154$ and $\Delta_{f}=\Delta(t=50$ to 80.715$)$ compared to the direct simulation. 
$\frac{\text { Time }}{1}$
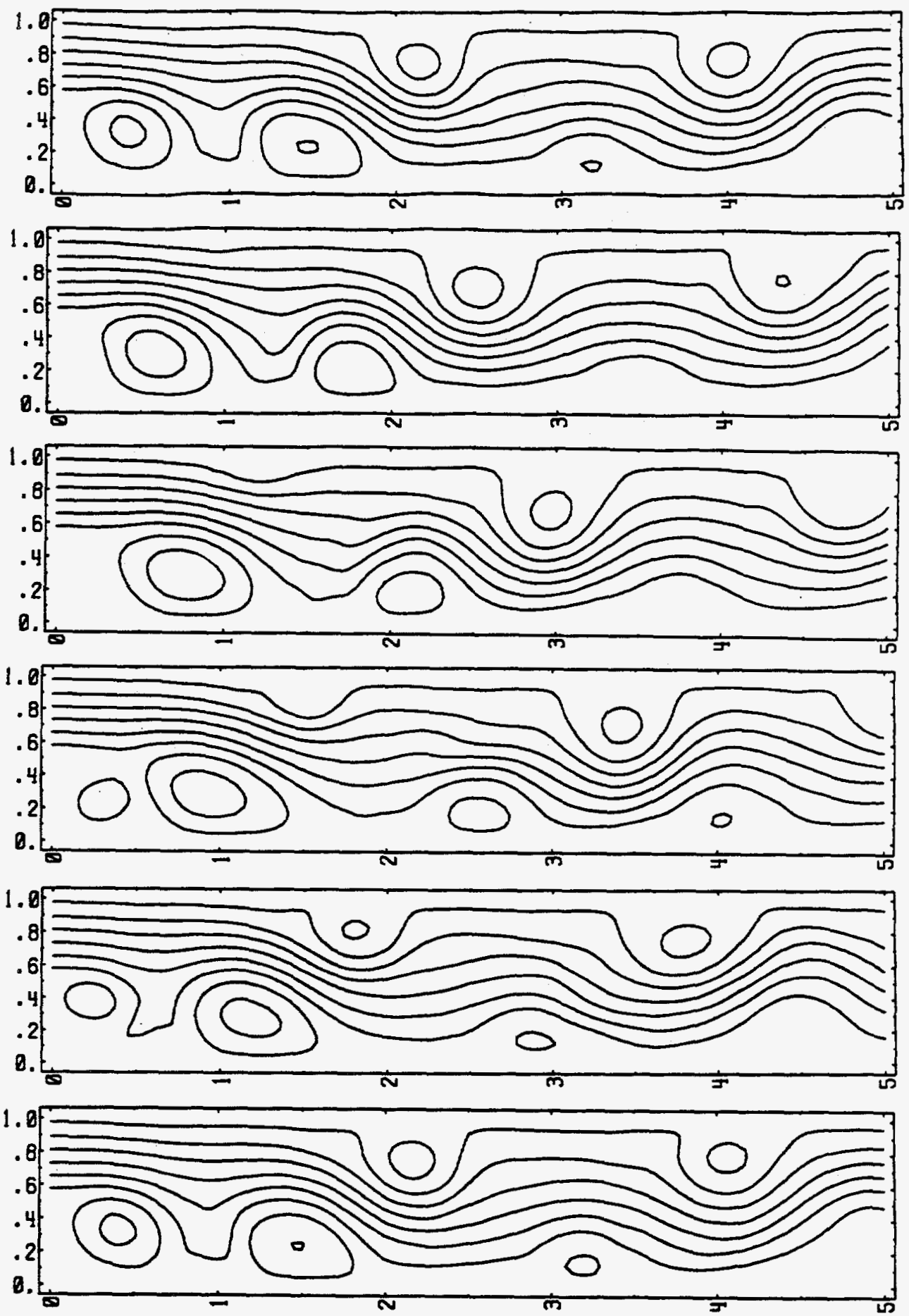

Fig. 5.3.10a Time series of stream function plots showing only half of field length for the $25 \times 241$ grid, $\Delta t=0.005$, and the SGS vorticity model with $C_{\omega}=.2154$ and $\Delta_{f}=\Delta$ at $0,1 / 6,2 / 6,3 / 6,4 / 6$, and $5 / 6$ of DS cycle. 

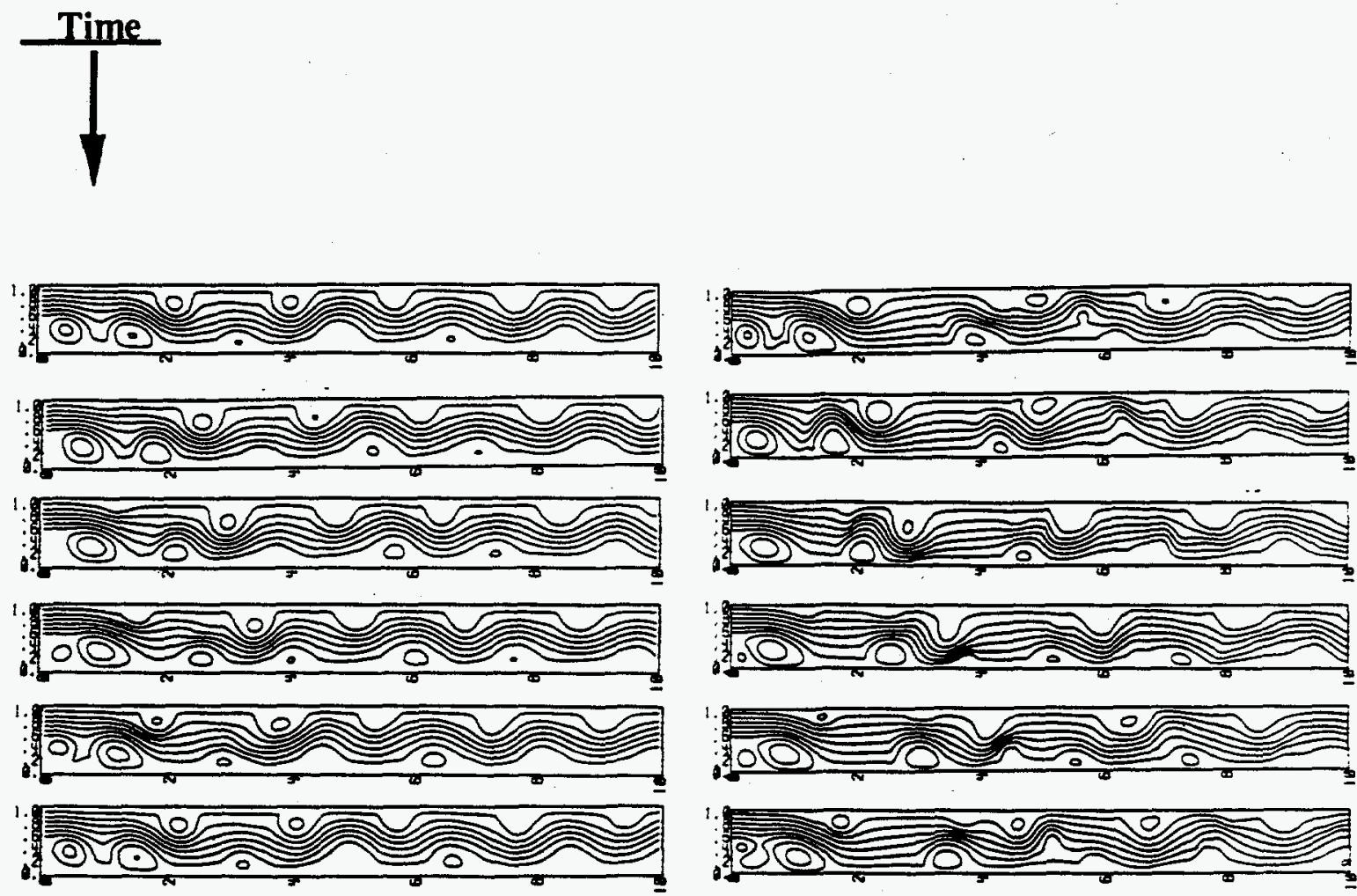

LES with $25 \times 241$ grid

Direct Simulation

Fig. 5.3.10b Time series of stream function plots for the $25 \times 241$ grid, $\Delta t=0.005$, and the SGS vorticity model with $C_{\omega}=.2154$ and $\Delta_{f}=\Delta$ compared to the direct simulation. (One cycle is shown for the same specified stream function levels for each case at $0,1 / 6,2 / 6,3 / 6,4 / 6$, and $5 / 6$ of a DS cycle.) 
Time
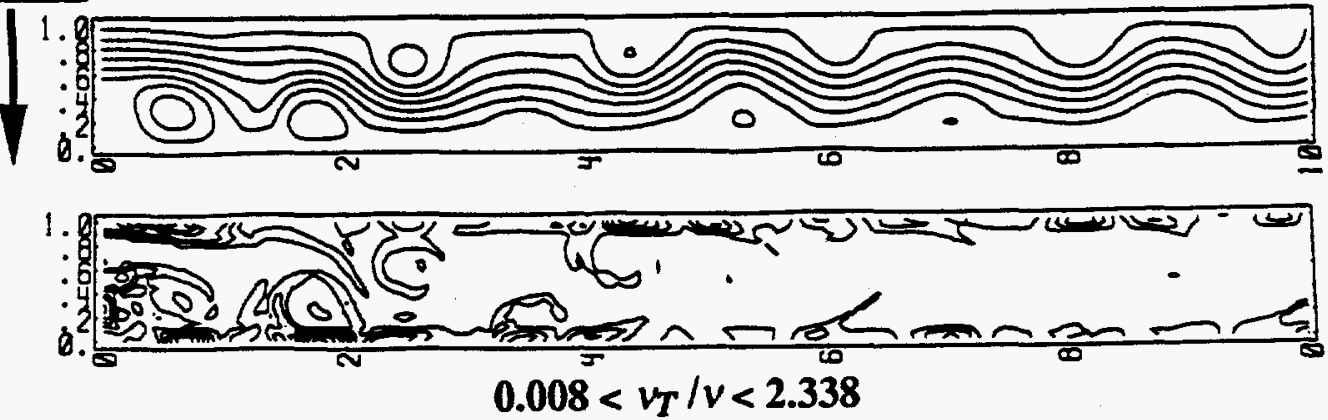

\section{$0.008<v_{T} / v<2.338$}
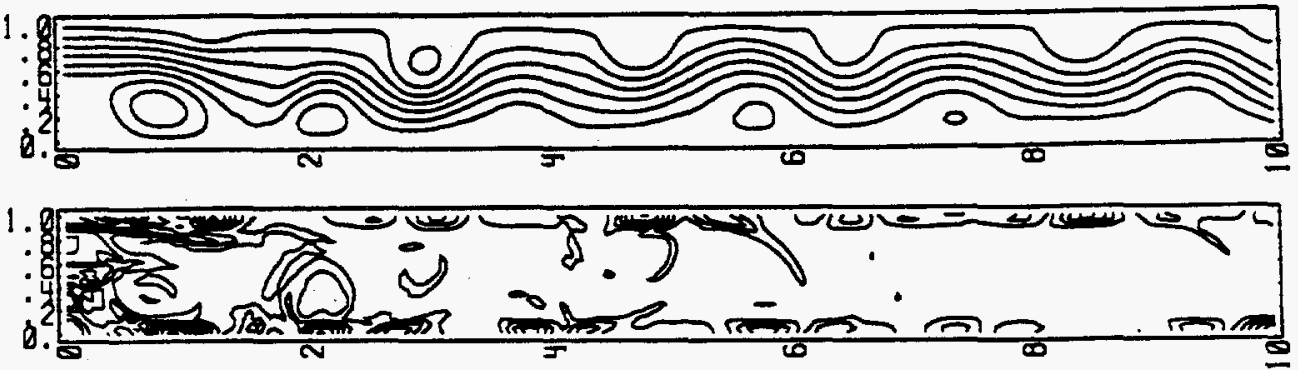

$0.002<v_{T} / v<2.585$
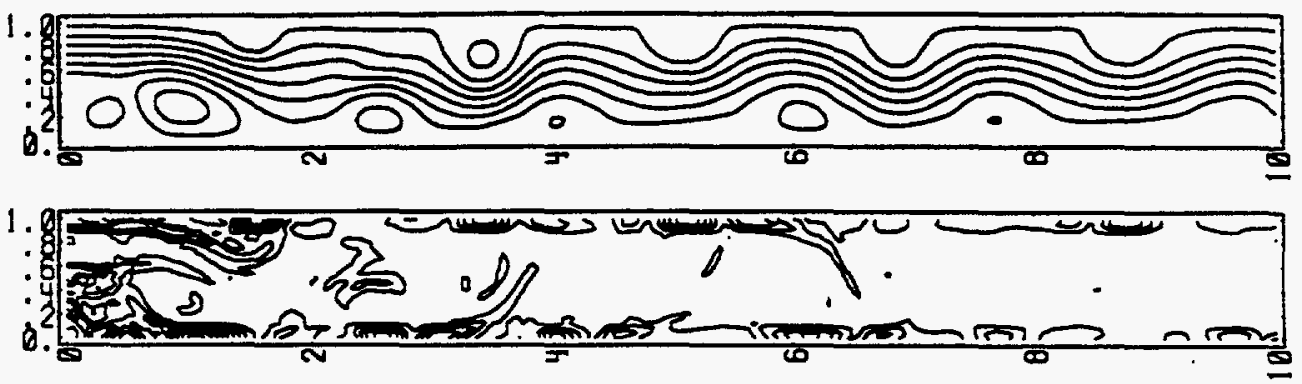

$0.004<v_{T} / v<2.622$
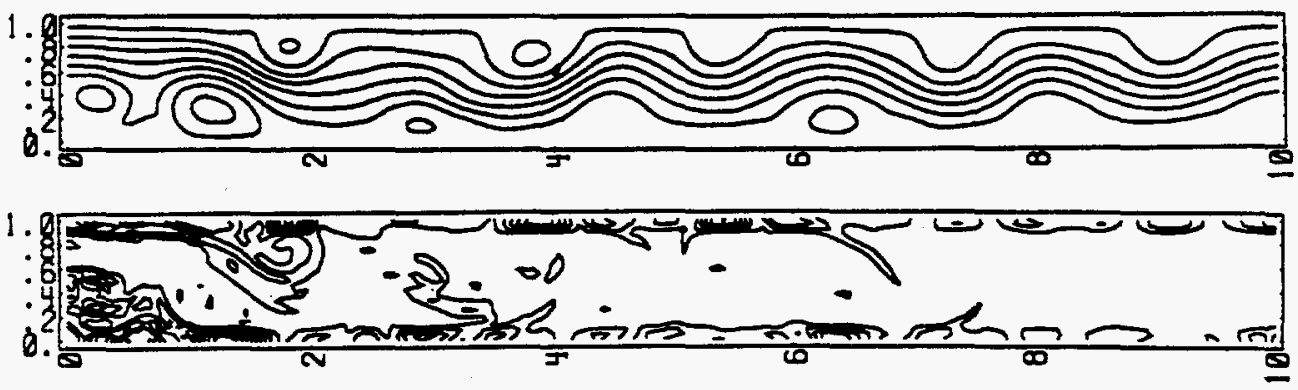

$0.004<v_{T} / v<2.740$

Fig. 5.3.11 Time series of $v_{T}$ contours compared to stream function plots for the $25 \times 241$ grid, $\Delta t=0.005$, and the SGS vorticity model with $C_{\omega}=0.2154$ and $\Delta_{f}=\Delta$ (second through fifth stream function plots in figures 5.3.10). 

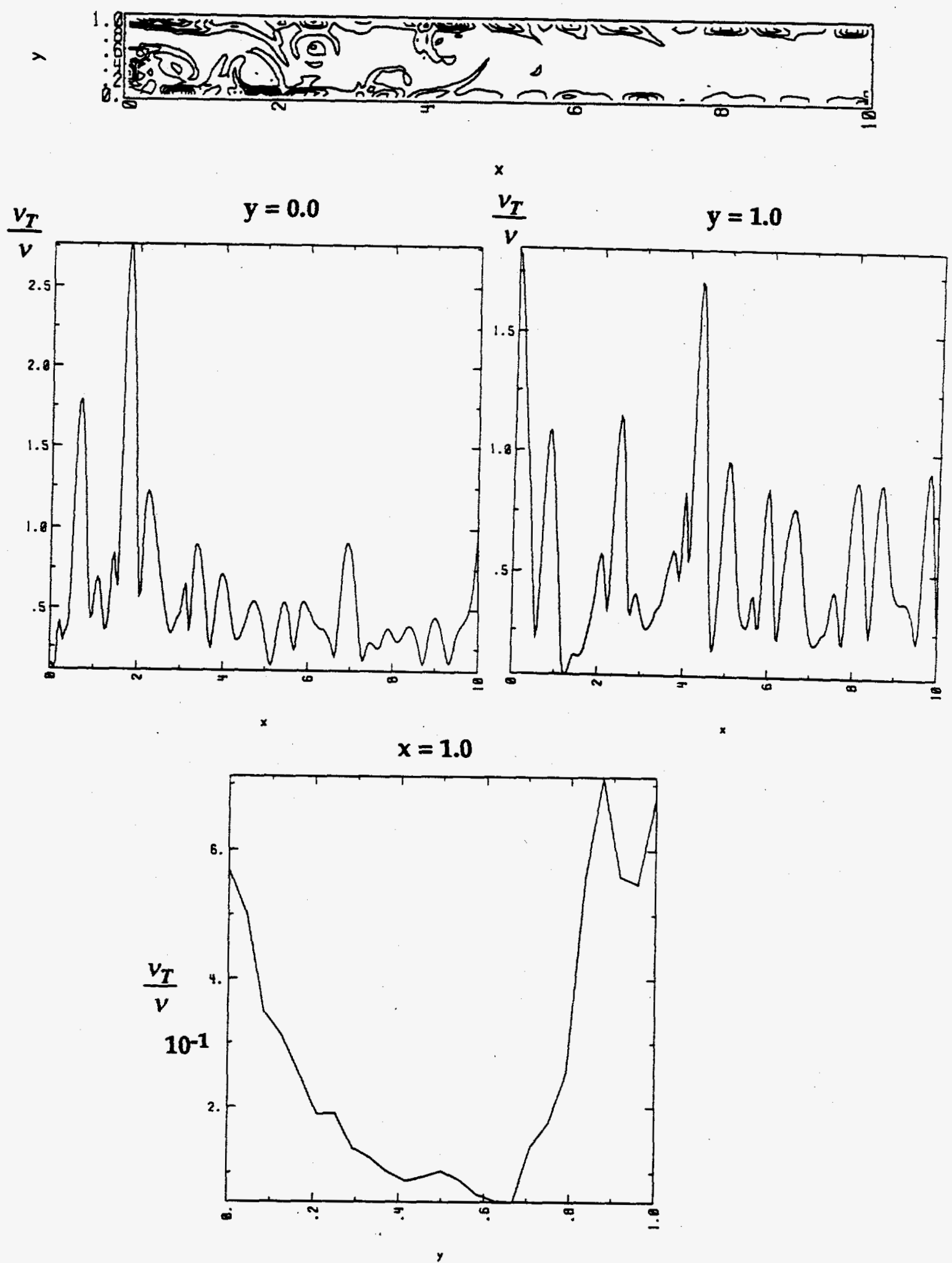

Fig. 5.3.12 A vT contour plot and profile plots at selected locations for the $25 \times 241$ grid, $\Delta t=0.005$, and the SGS vorticity model with $C_{\omega}=0.2154$ and $\Delta_{f}=\Delta$. 
$\mathbf{x}=\mathbf{1 . 0}$

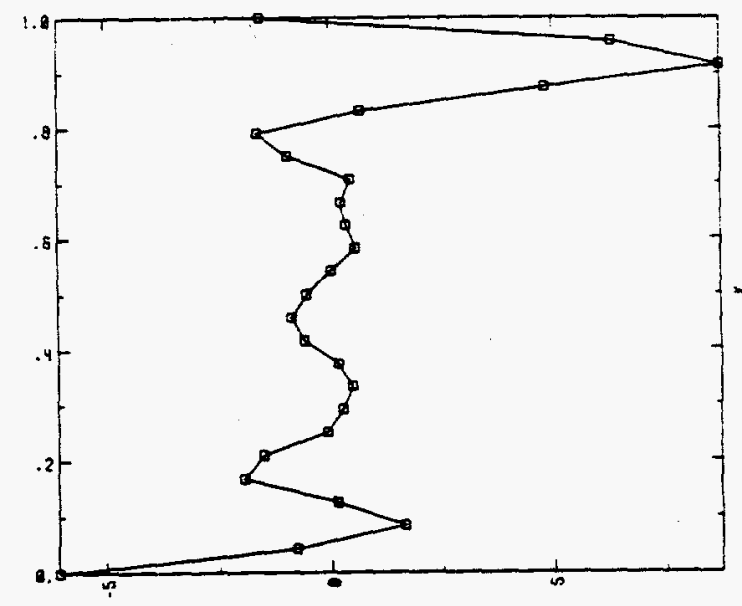

Vorticity

$$
x=3.0
$$

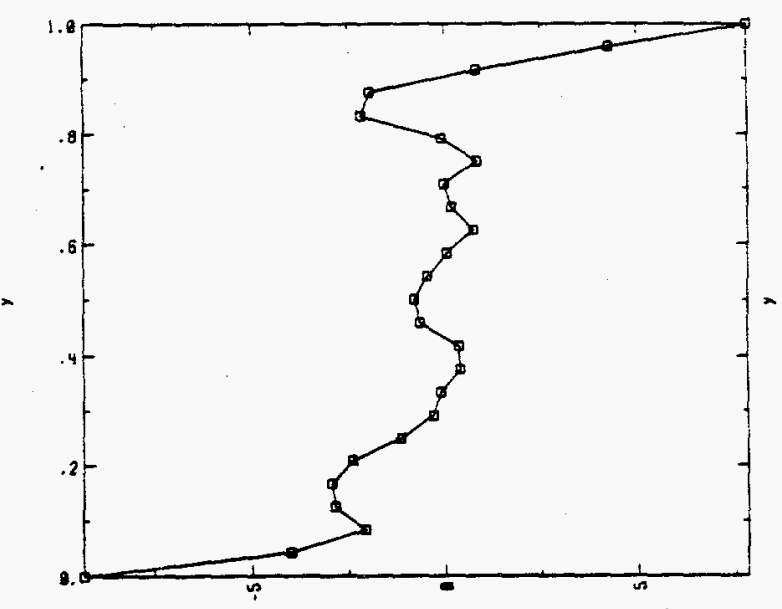

Yorticity $x=2.0$

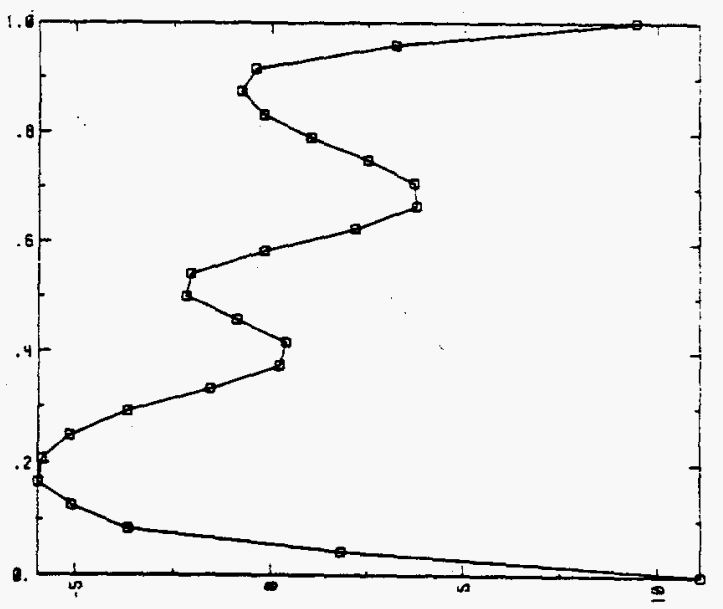

vorticity

$x=6.0$

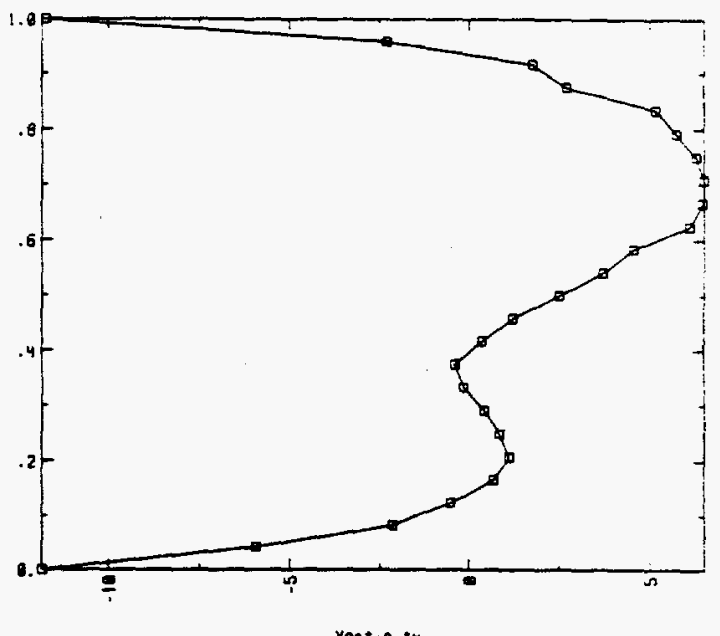

Yorticity

Fig. 5.3.13 Profile plots of the LES predicted vorticity at selected locations for the $25 \times 241$ grid, $\Delta t=0.005$, and the SGS vorticity model with $C_{\omega}=0.2154$ and $\Delta_{f}=\Delta$ at a snapshot in time (same time as that for Figures 5.3.12 through 5.3.17). 


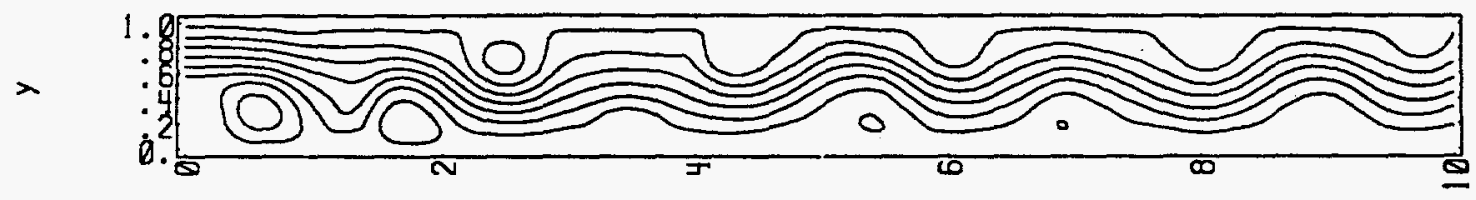

x

$$
\mathbf{x}=\mathbf{1 . 0}
$$

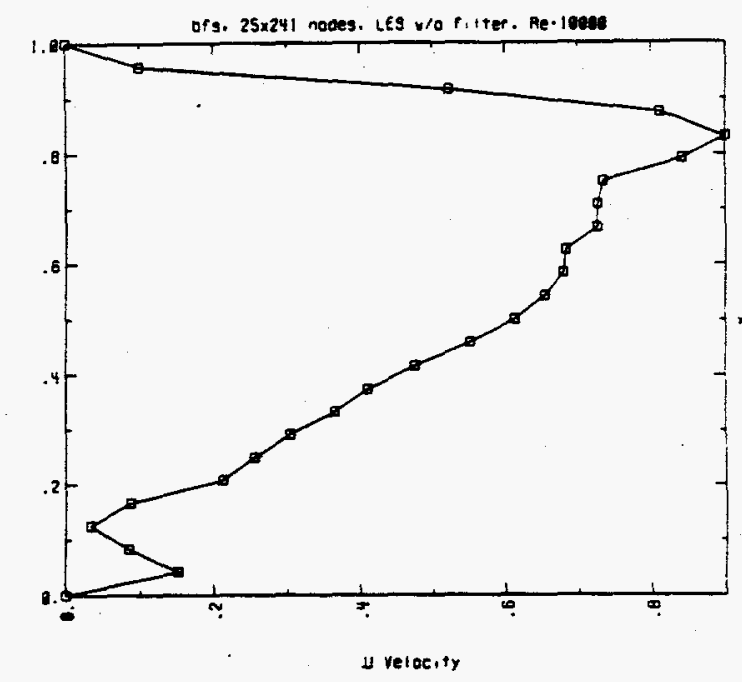

$\mathbf{x}=\mathbf{3 . 0}$

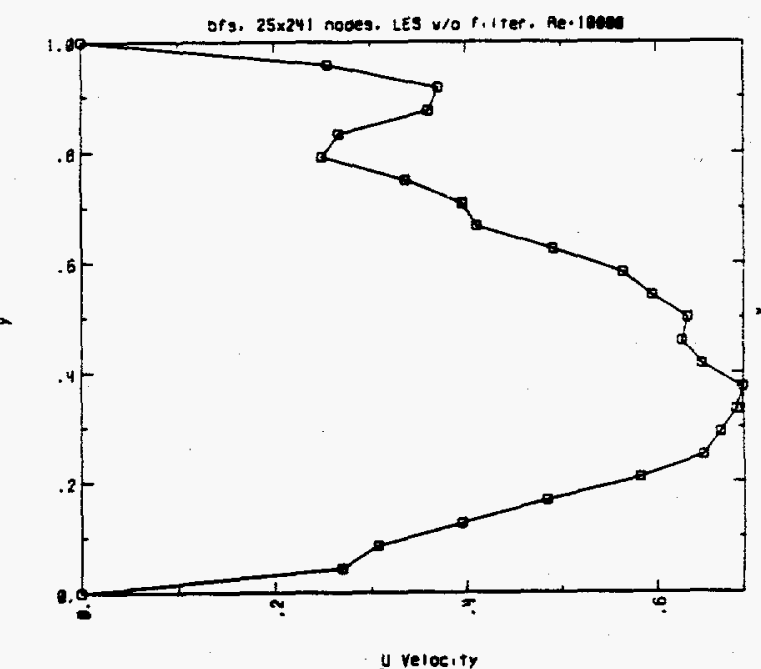

$\mathbf{x}=\mathbf{2 . 0}$
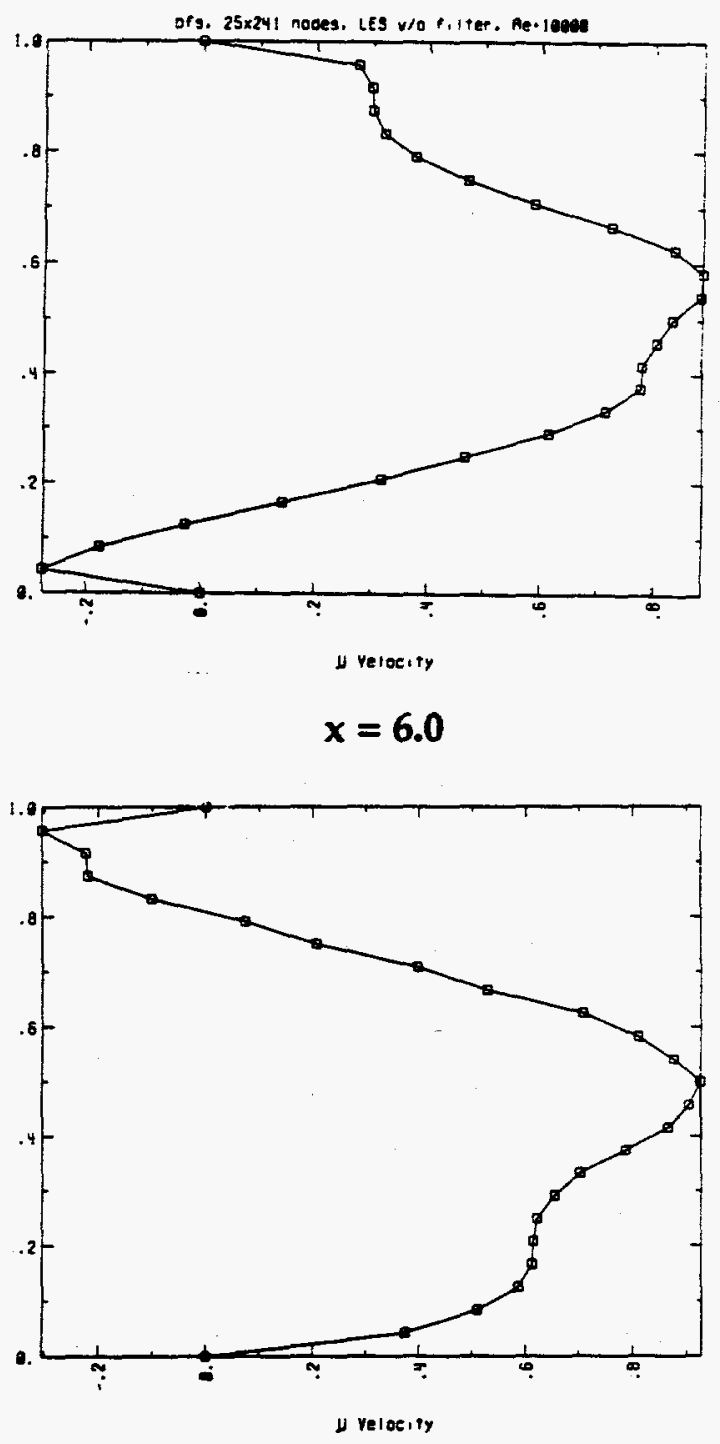

Fig. 5.3.14 The LES predicted $\bar{u}_{1}$ velocity profiles at selected locations for the $25 \mathrm{x}$ 241 grid, $\Delta t=0.005$, and the SGS vorticity model with $C_{\omega}=0.2154$ and $\Delta_{f}=\Delta$ at a snapshot in time (same time as that for Figures 5.3.12 through 5.3.17). 
Top

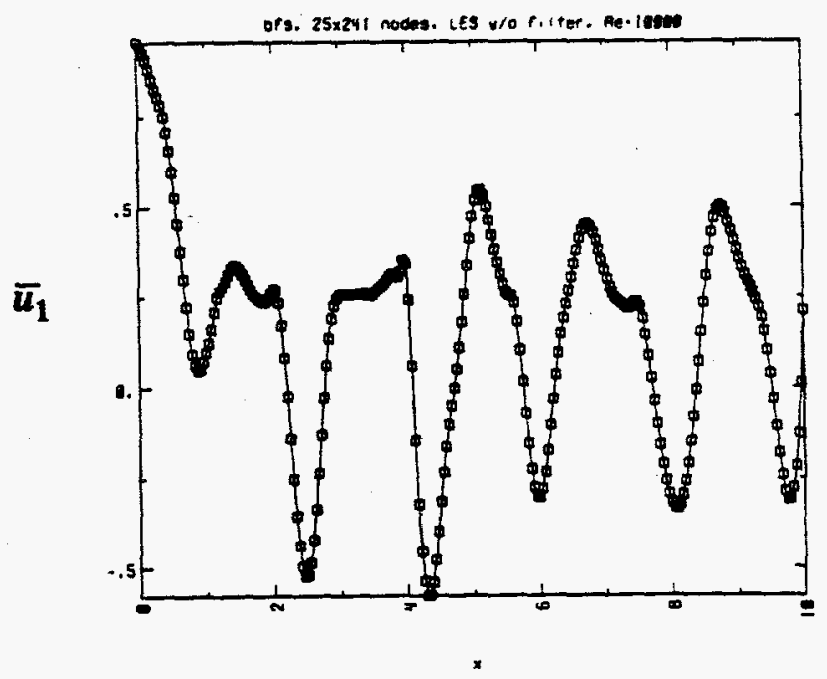

Bottom

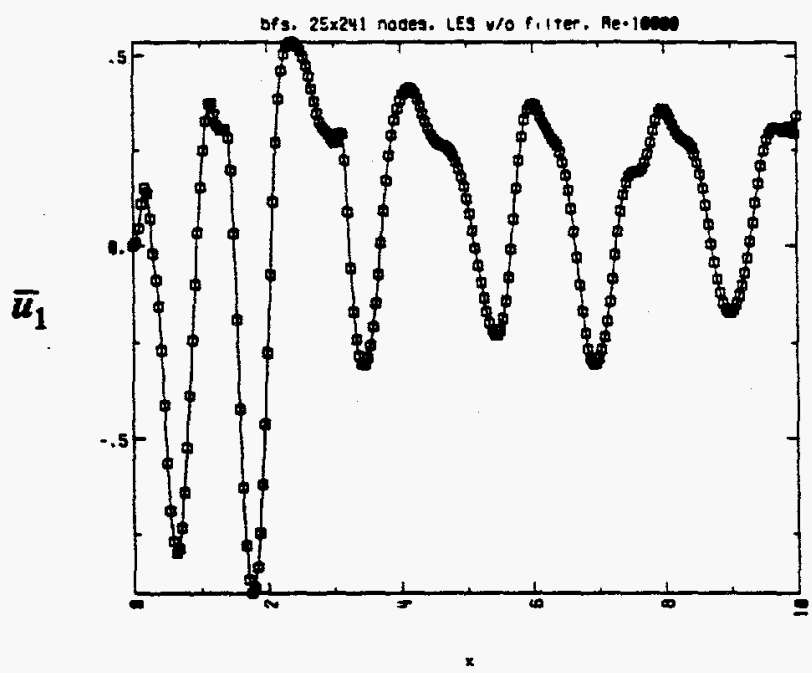

Fig. 5.3.15 The $\bar{u}_{1}$ velocity profiles in the streamwise $x$-direction along the top and bottom walls (i.e., one cell length from wall) at a snapshot in time (same time as that for Figures 5.3.12 through 5.3.17). 
Top Wall

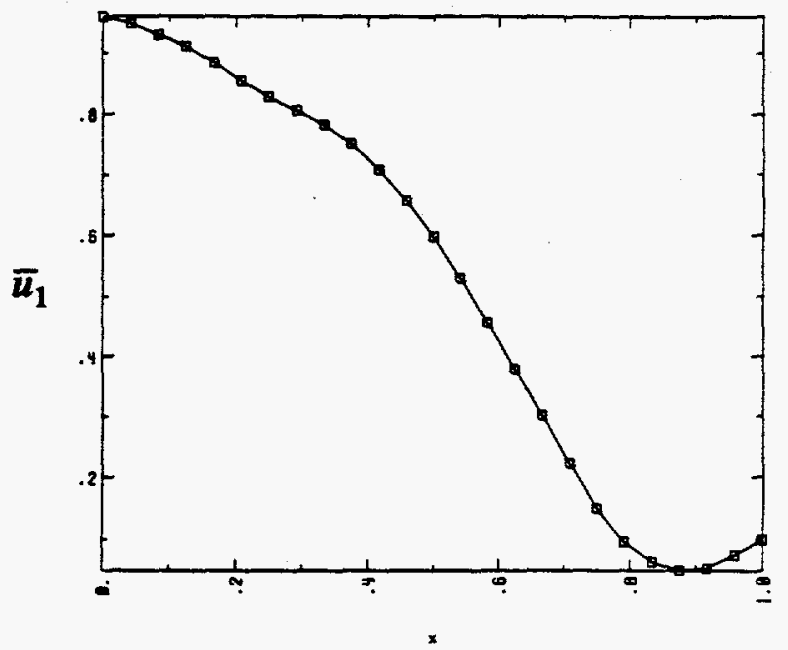

Bottom Wall

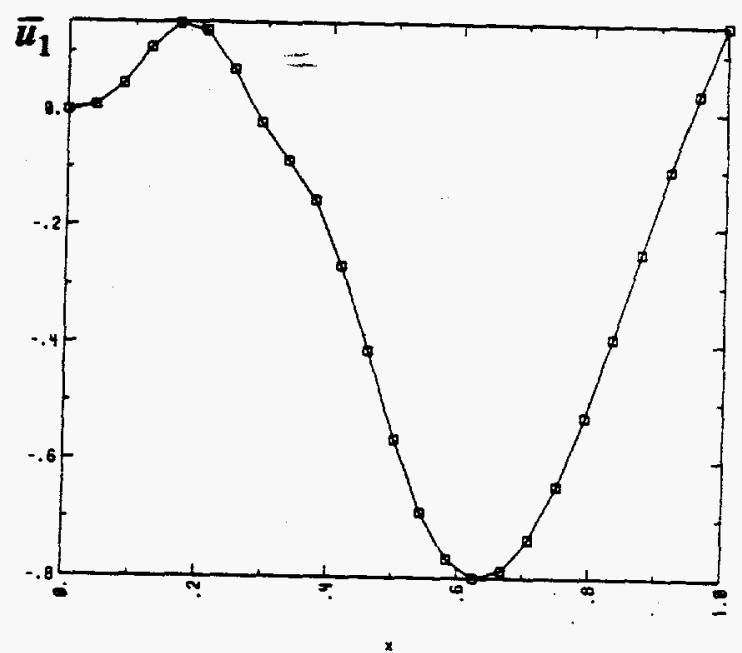

$x=0.5$

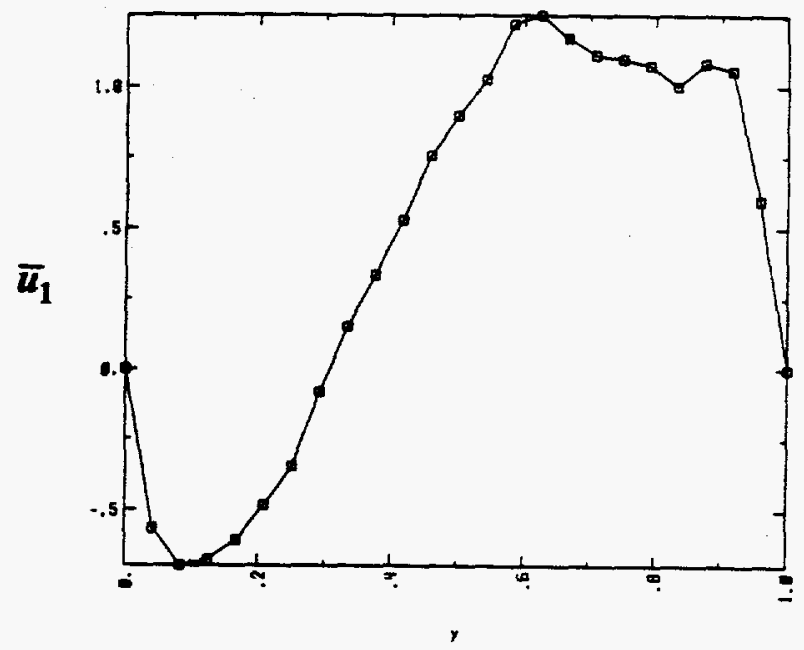

Fig. 5.3.16 Comparison of $\bar{u}_{1}$ velocity profiles in the streamwise x-direction along the top and bottom walls for $y=0.0$ to 1.0 to the $\bar{u}_{1}$ velocity profile in the crossstream $y$-direction at $x=0.5$ at a snapshot in time (same time as that for Figures 5.3.12 through 5.3.17). 

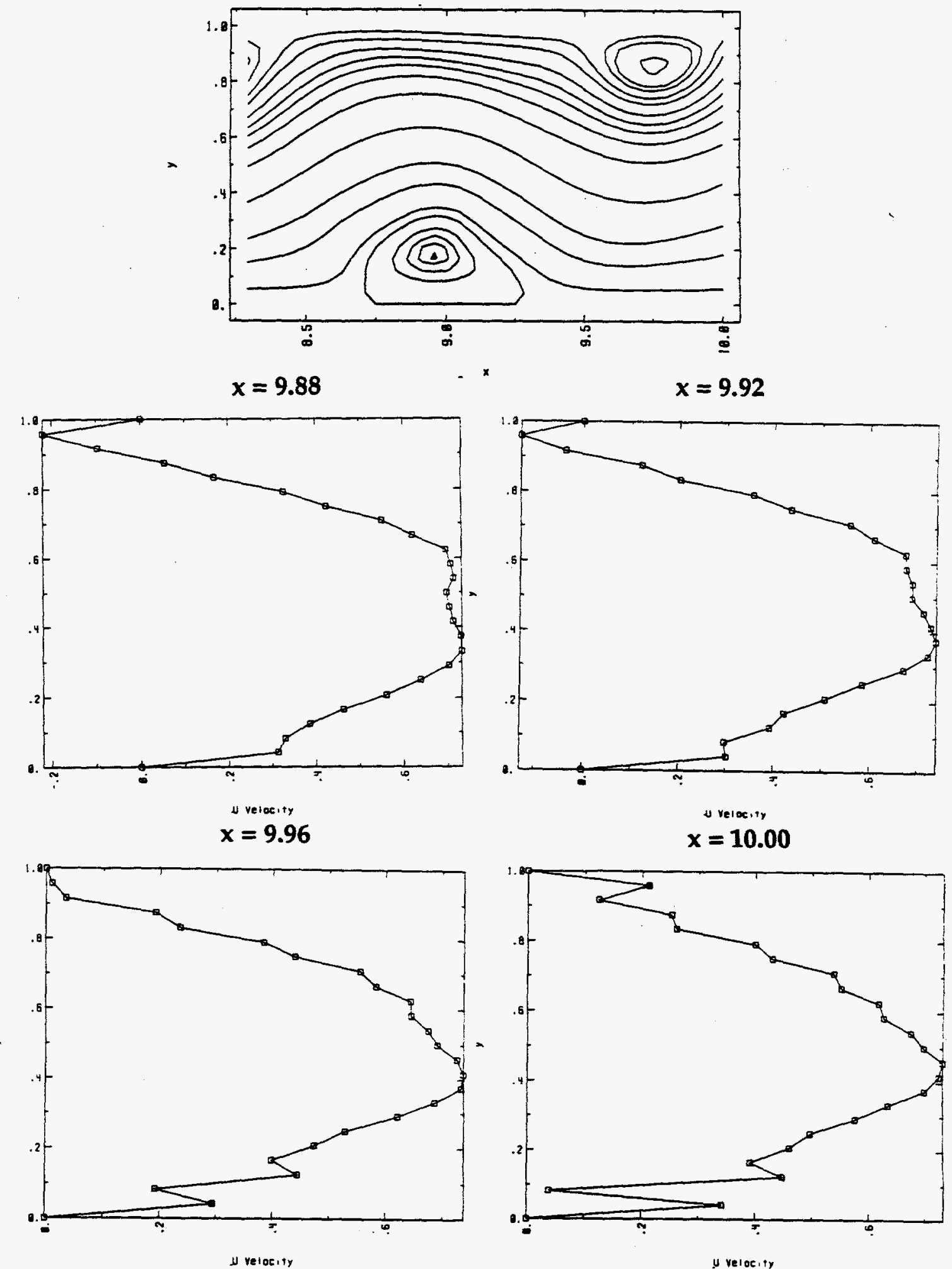

Fig. 5.3.17 The LES predicted $\overline{\boldsymbol{u}}_{1}$ velocity profiles at channel outlet for the $25 \mathrm{x}$ 241 grid, $\Delta t=0.005$ and the SGS vorticity model with $C_{\omega}=0.2154$ and $\Delta f=\Delta$ at a snapshot in time (same time as that for Figures 5.3.12 through 5.3.17). 
Table 5.3.2 Time-averaged LES results for the $25 \times 241$ grid, $\Delta t=0.005$, and the SGS vorticity model with $C_{\omega}=0.2154$ and $\Delta_{f}=\Delta$.

\begin{tabular}{||c|c|c|c|}
\hline & \multicolumn{2}{|c|}{$\begin{array}{c}\text { x-Iocation for separation } \\
\text { and reattachment points }\end{array}$} & $\begin{array}{c}\text { celative change } \\
\text { retween cases } \\
(\%)\end{array}$ \\
\cline { 2 - 4 } & $\begin{array}{c}\text { time average } \\
\text { for 10,000 } \\
\text { time steps }\end{array}$ & $\begin{array}{c}\text { time average } \\
\text { for 20,000 } \\
\text { time steps }\end{array}$ & \\
\hline \multirow{3}{*}{ Bottom Wall } & 0.063 & 0.062 & 1.6 \\
& 0.472 & 0.476 & 0.8 \\
& 2.048 & 2.037 & 0.5 \\
& 6.266 & 6.171 & 1.5 \\
& 6.839 & 6.347 & 7.8 \\
& 1.442 & 1.409 & 2.3 \\
& 2.172 & 2.076 & 4.6 \\
& 3.916 & 3.921 & 0.1 \\
& 4.458 & 4.447 & 0.3 \\
\hline
\end{tabular}




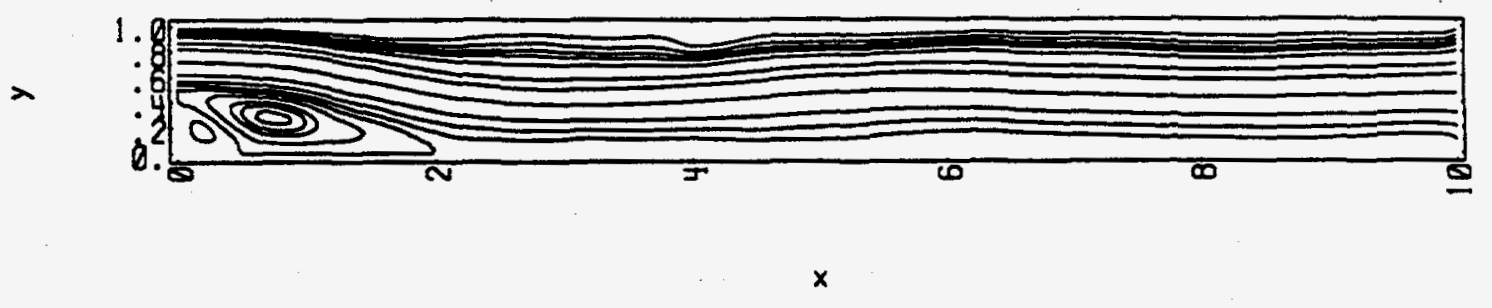

Entire Computational Field

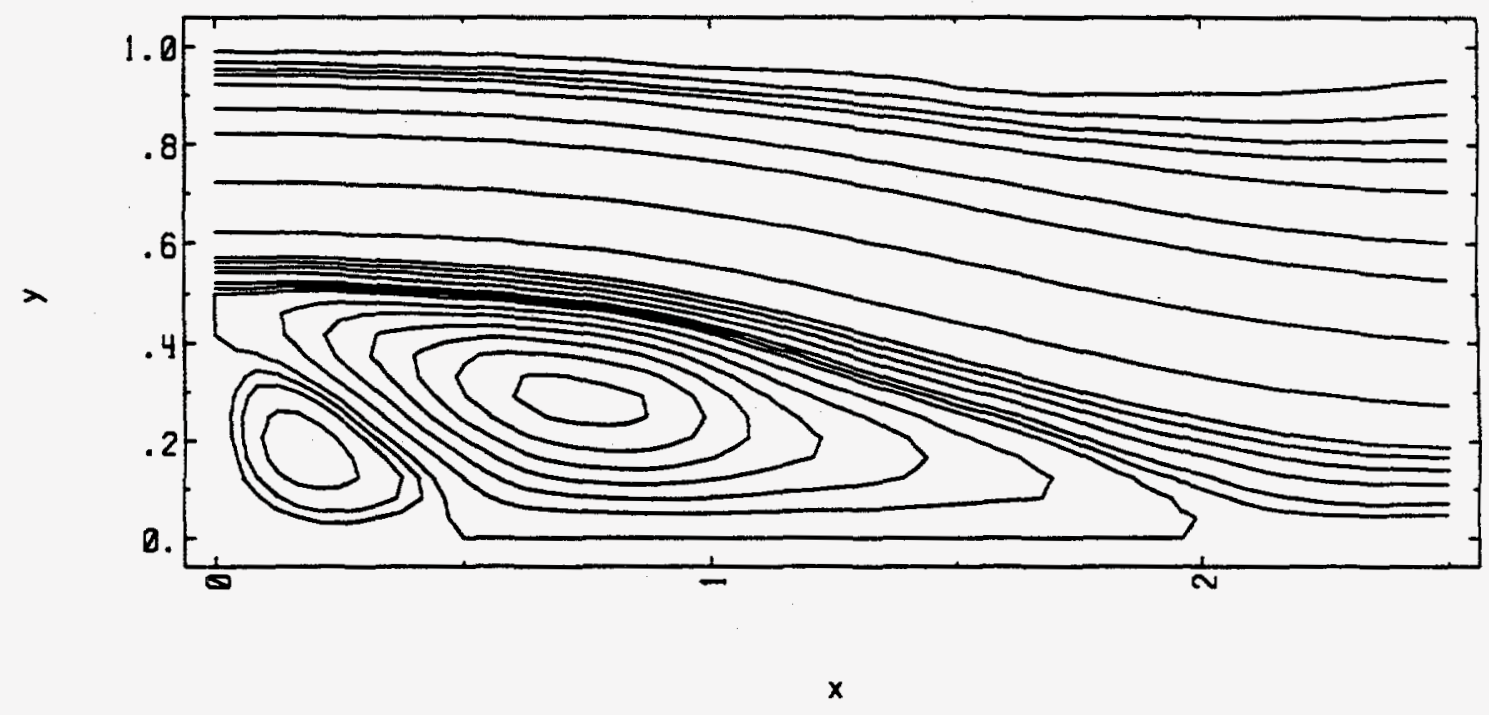

Enlargement of Major Recirculation Zone

Figure 5.3.18 Time-average LES stream function for the $25 \times 241$ grid, $\Delta t=0.005$, and the SGS vorticity model with $C_{\omega}=0.2154$ and $\Delta_{f}=\Delta$ for a time-averaging duration of 20,000 time steps. 


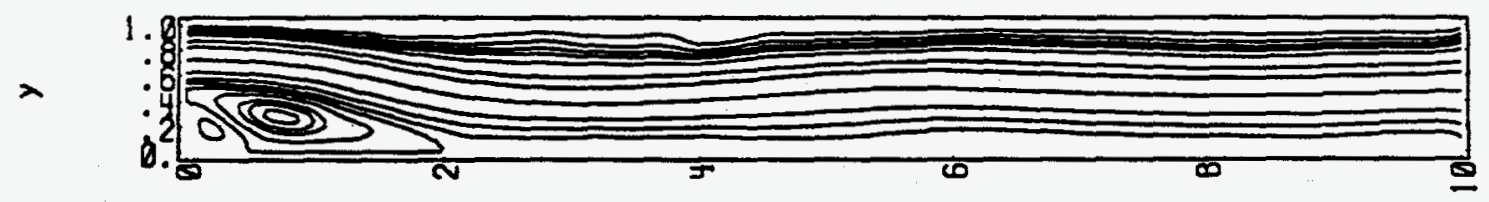

$\mathbf{x}=\mathbf{1 . 0}$
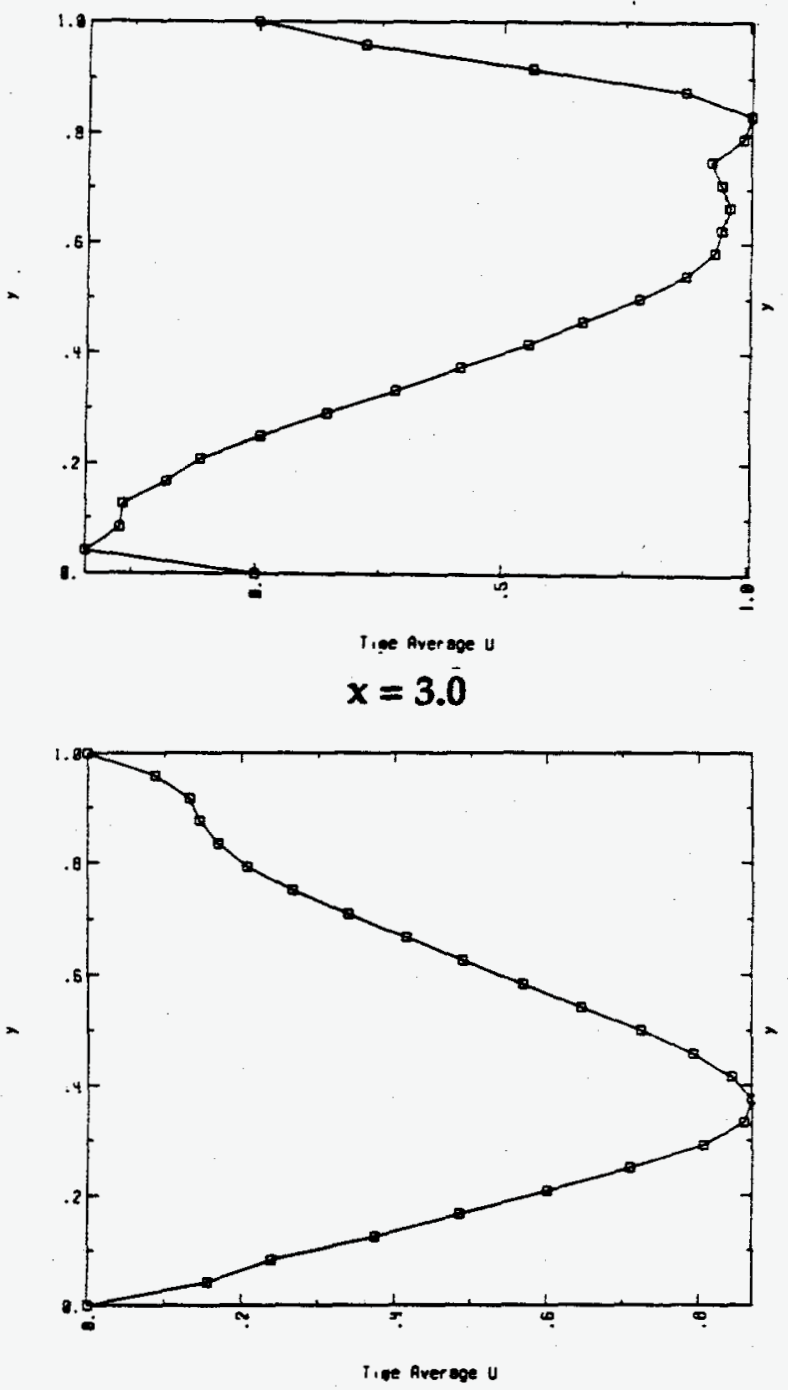

$x=2.0$

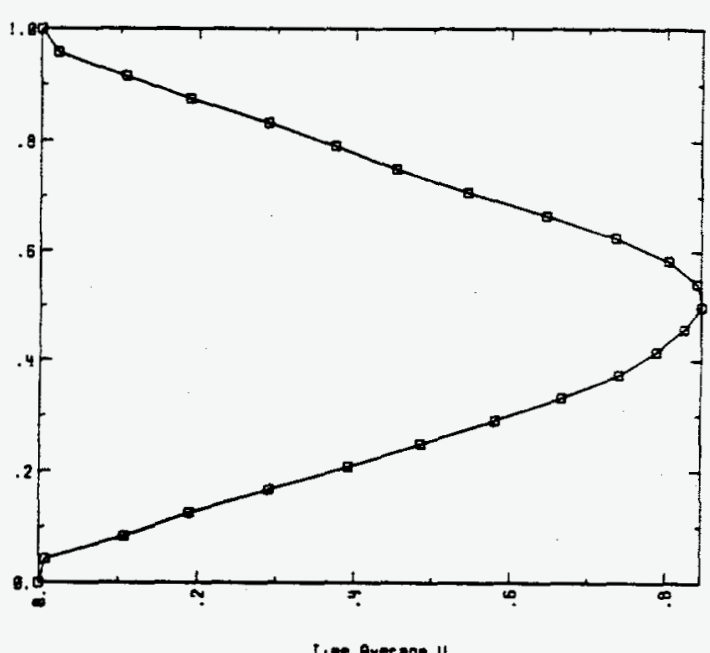

$x=6.0$

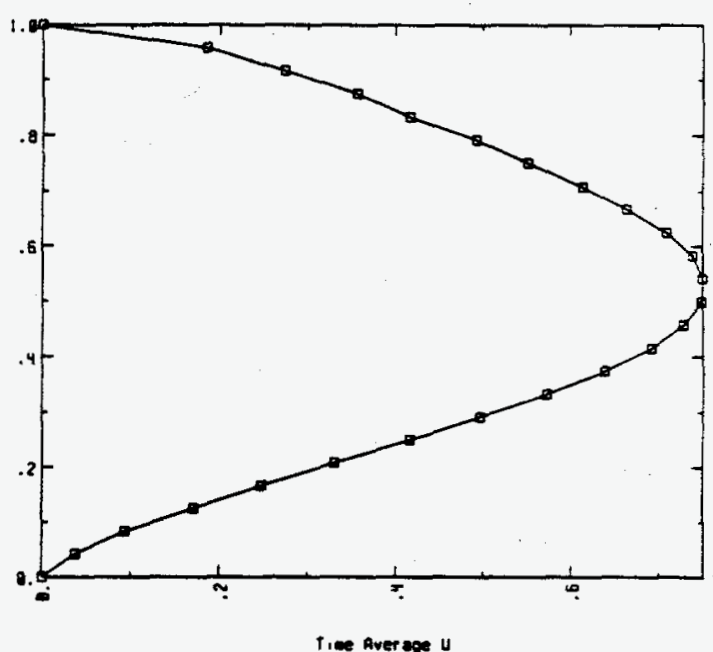

Figure 5.3.19 The LES predicted time-averaged $\bar{u}_{1}$ velocity, $<\bar{u}_{1}>$ for the $25 \times 241$ grid, $\Delta t=0.005$, and the SGS vorticity model with $C_{\omega}=0.2154$ and $\Delta_{f}=\Delta$. 
$\mathbf{x}=\mathbf{1 . 0}$

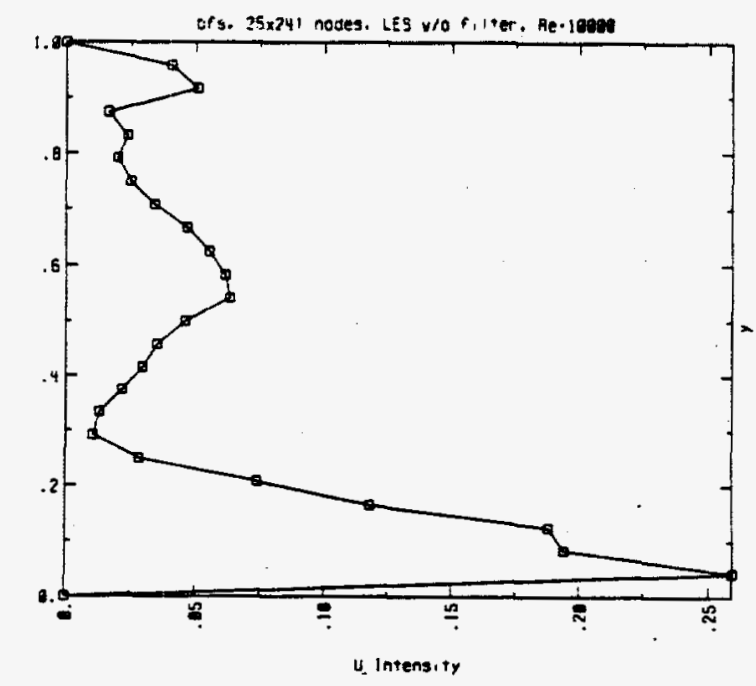

$x=\mathbf{3 . 0}$

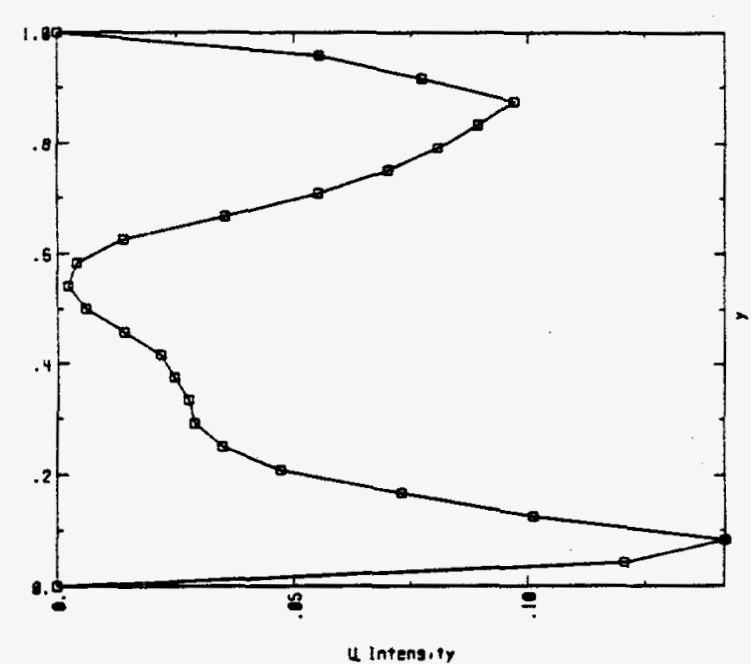

$x=\mathbf{2 . 0}$

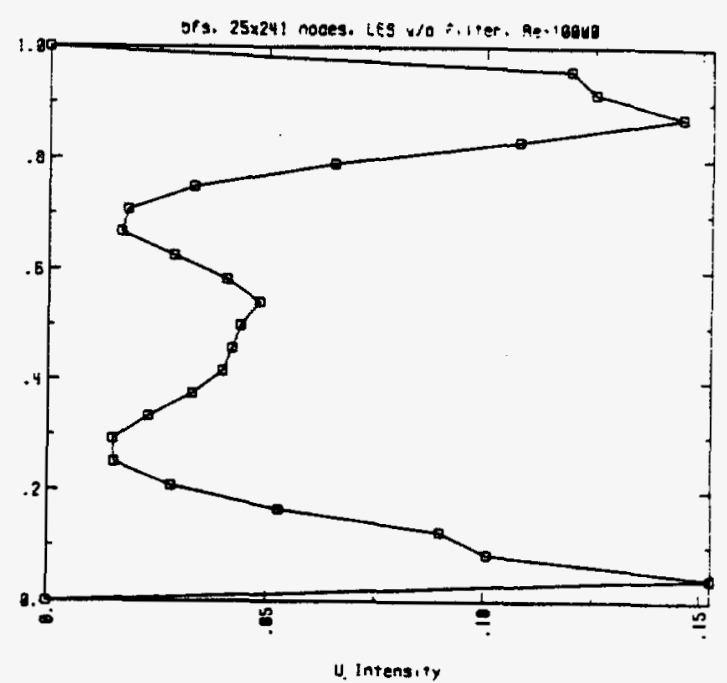

$x=6.0$

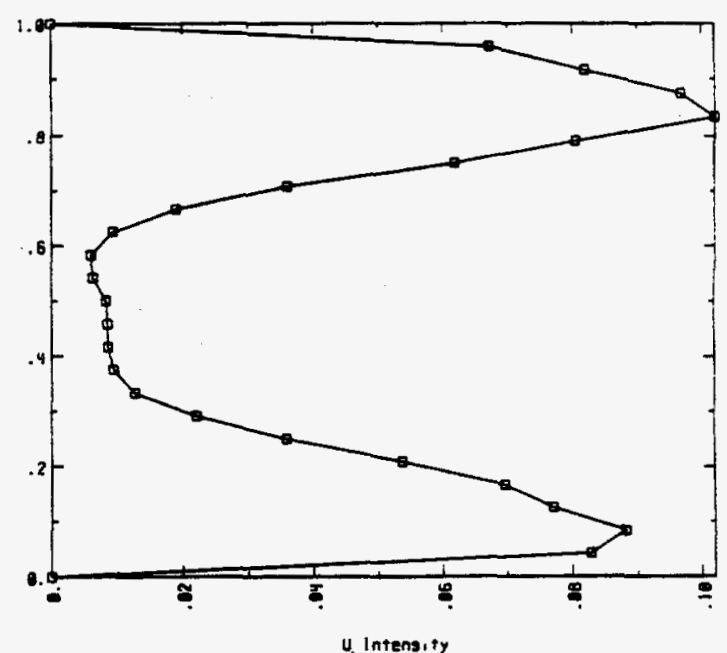

Figure 5.3.20 The LES predicted turbulent intensity, $\left\langle\bar{u}_{1}{ }^{2}\right\rangle$, at selected locations for the $25 \times 241$ grid, $\Delta t=0.005$, and the SGS vorticity model with $C_{\omega}=0.2154$ and $\Delta f=\Delta$. 
$x=1.0$

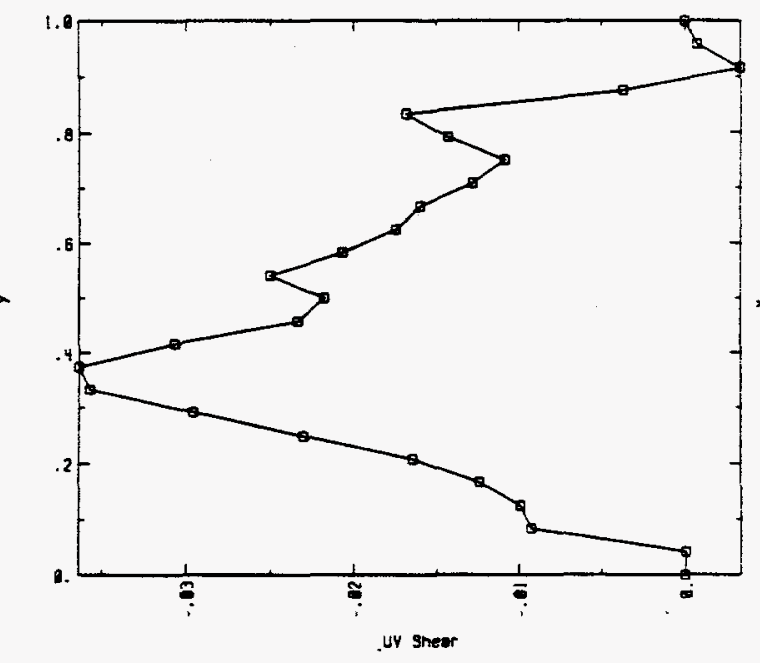

$\mathbf{x}=\mathbf{3 . 0}$

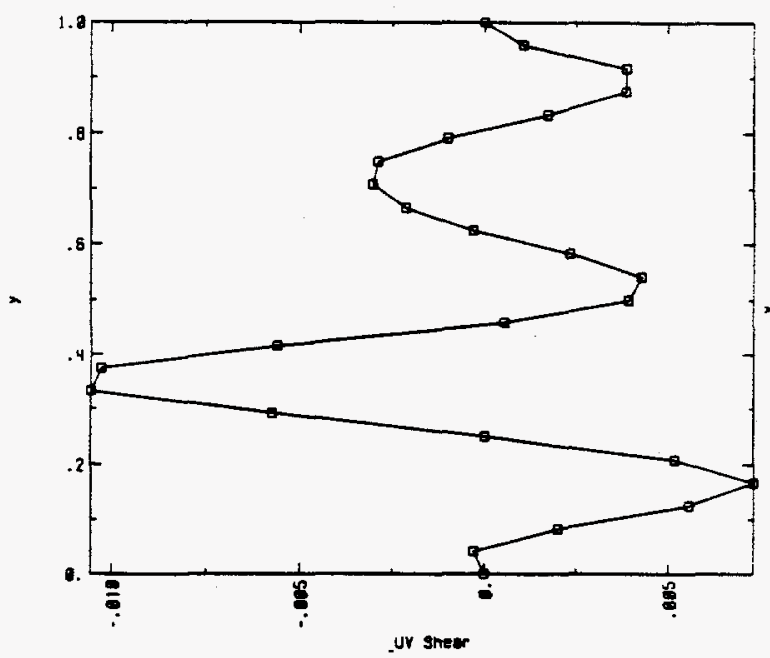

$\mathbf{x}=\mathbf{2 . 0}$

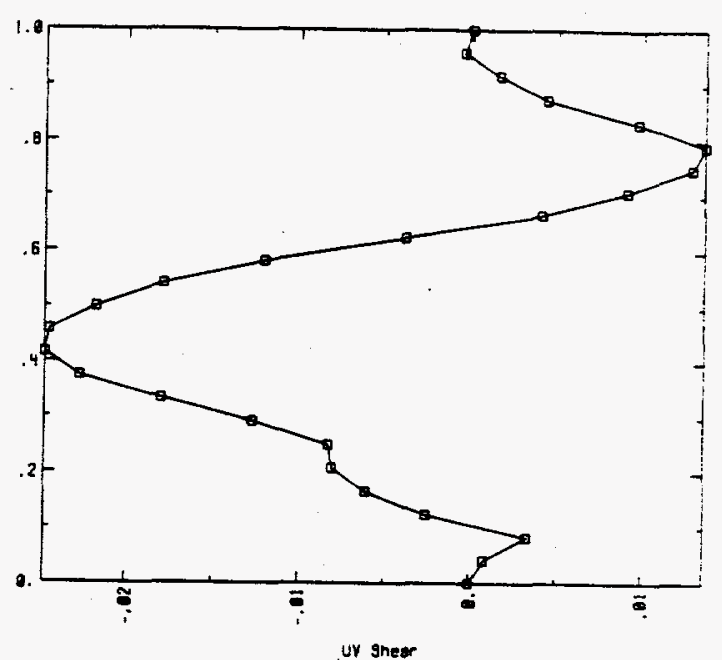

$x=6.0$

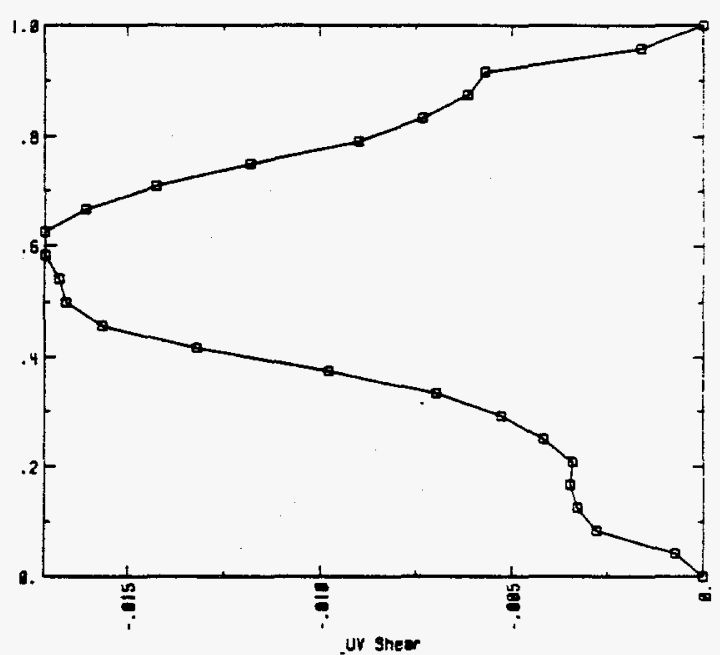

Figure 5.3.21 The LES predicted turbulent shear, $\left\langle\bar{u}_{1}^{\prime \prime} \bar{u}_{2}^{\prime \prime}\right\rangle$, at selected locations for the $25 \times 241$ grid, $\Delta t=0.005$, and the SGS vorticity model with $C_{\omega}=0.2154$ and $\Delta_{f}=\Delta$. 

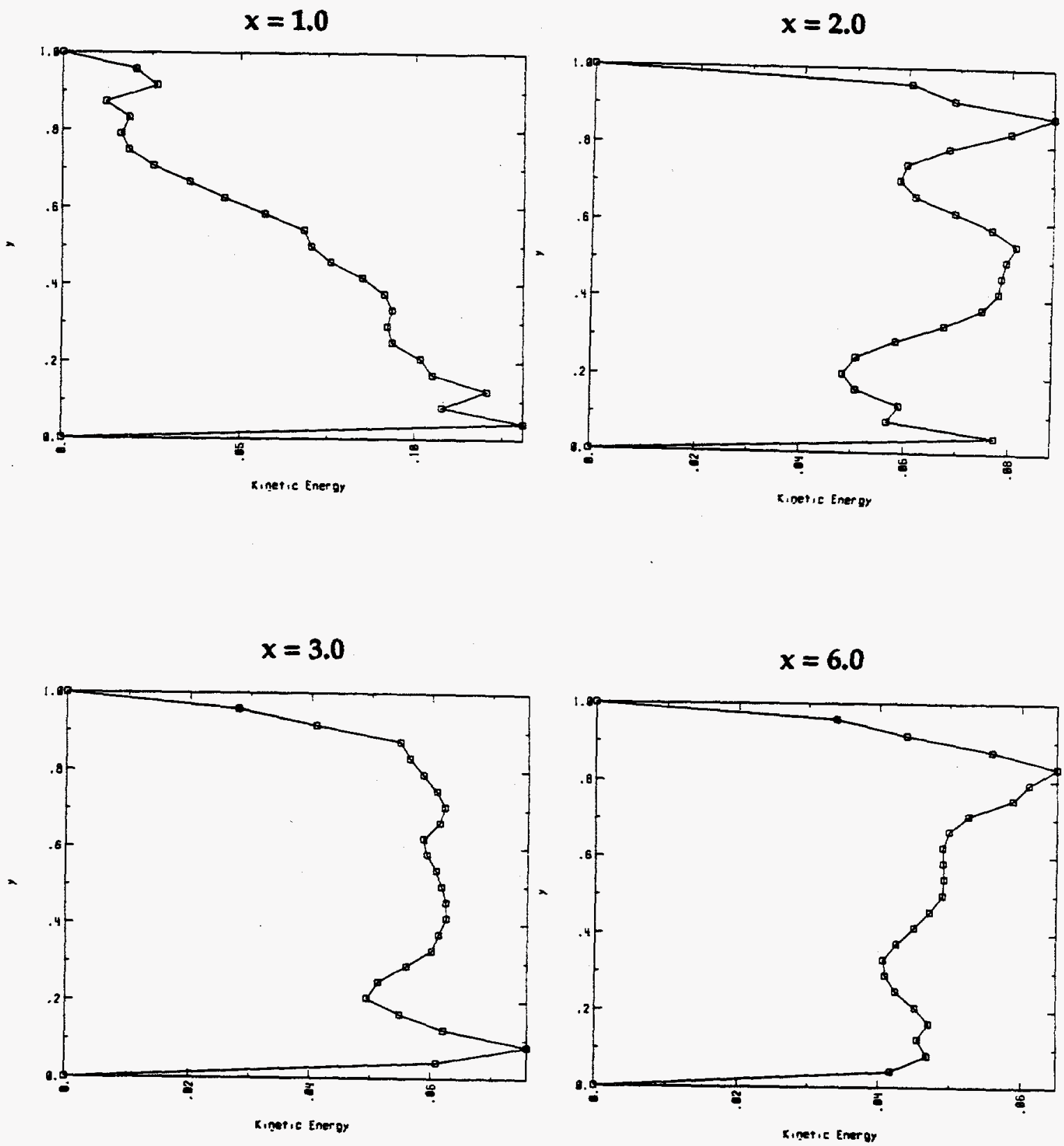

Figure 5.3.22 The LES predicted turbulent kinetic energy at selected locations for the $25 \times 241$ grid, $\Delta t=0.005$, and the SGS vorticity model with $C_{\omega}=0.2154$ and $\Delta_{f}$ $=\Delta$. 
$x=1.0$

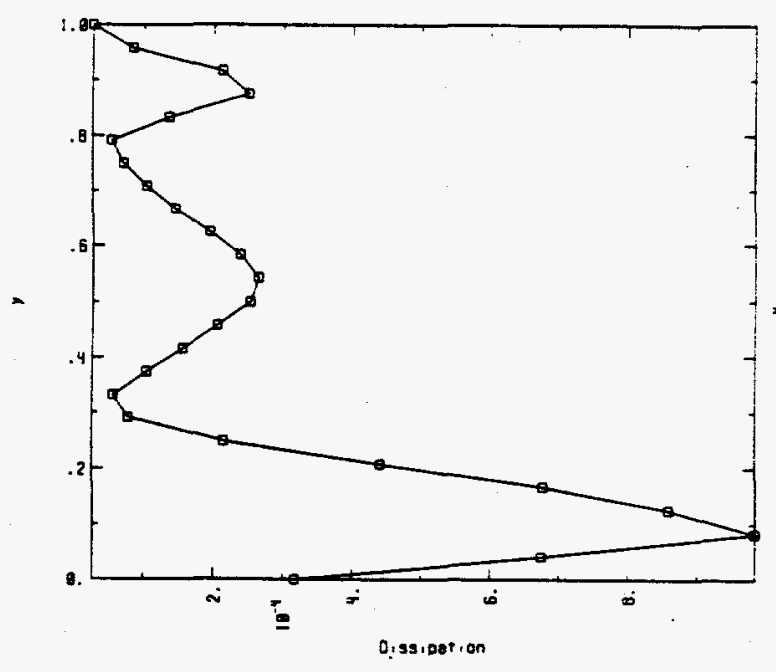

$x=\mathbf{3 . 0}$

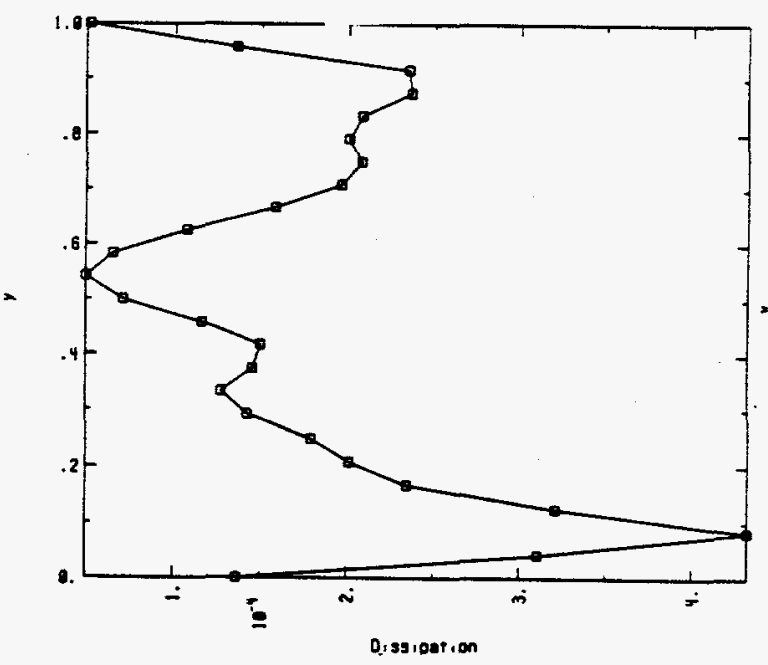

$x=\mathbf{2 . 0}$

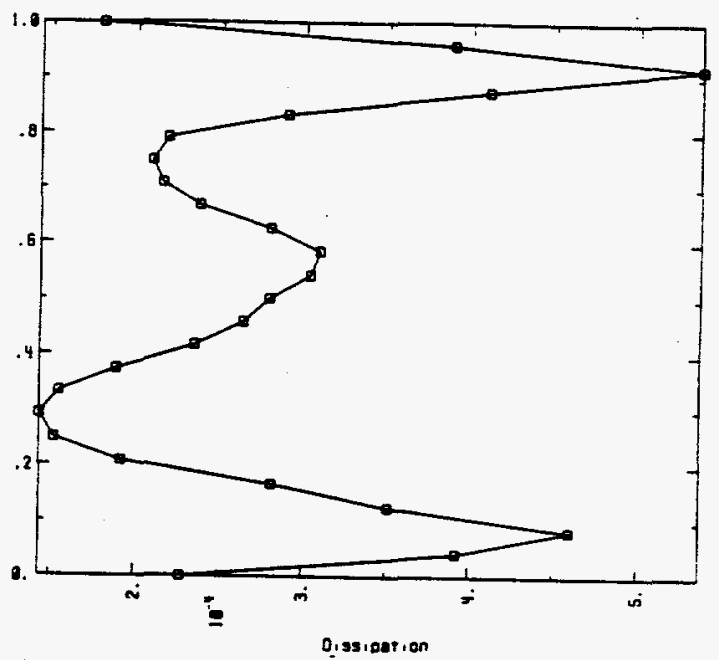

$$
x=6.0
$$

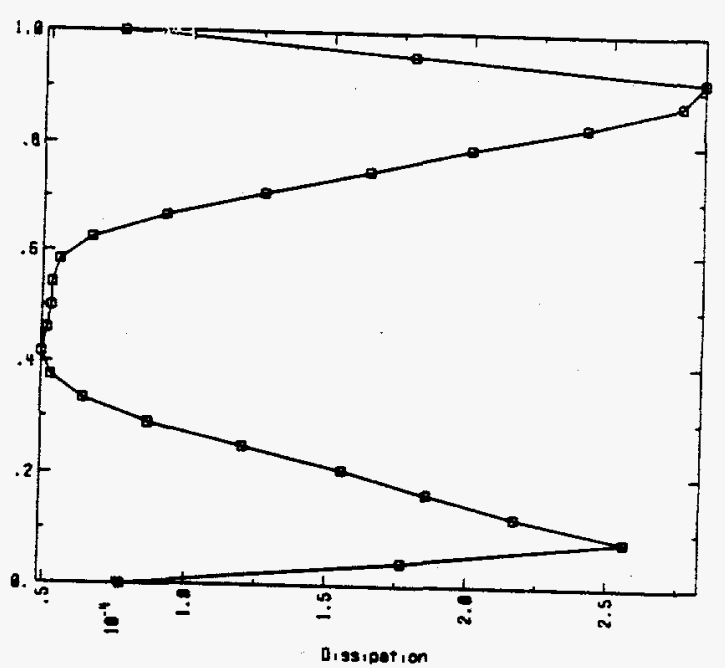

Figure 5.3.23 The LES predicted, turbulent rate of dissipation at selected locations for the $25 \times 241$ grid, $\Delta t=0.005$, and the SGS vorticity model with $C_{\omega}=0.2154$ and $\Delta_{f}=\Delta$. 


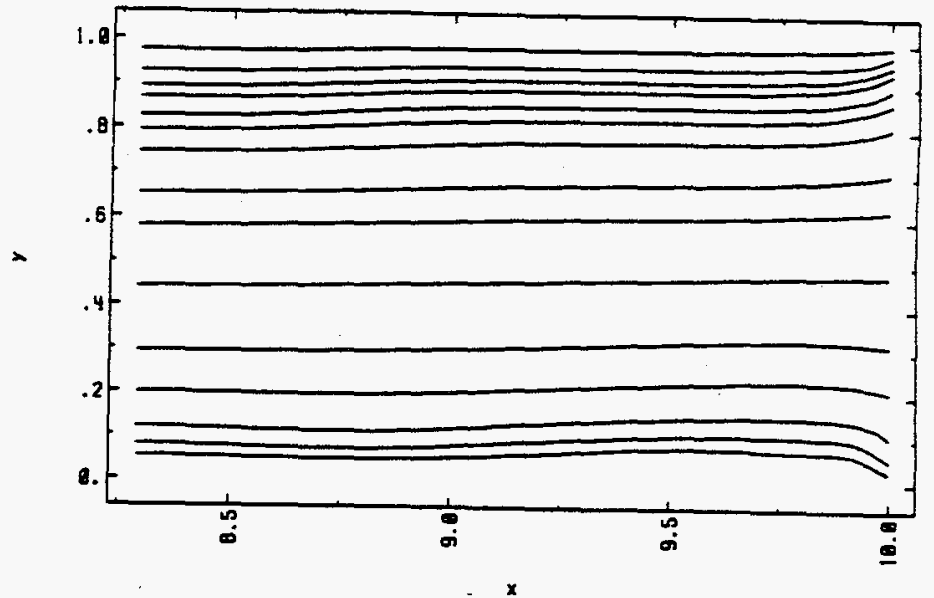

$x=9.98$

$x=9.92$
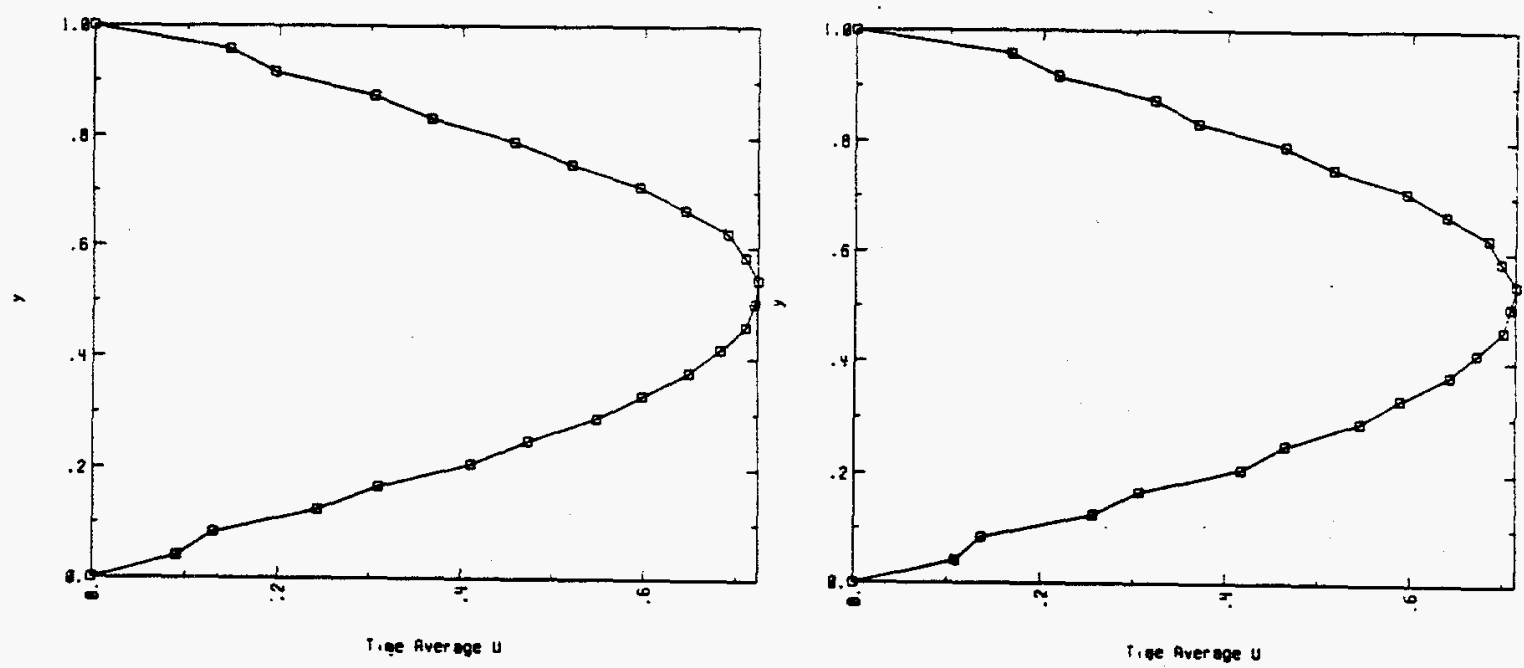

$x=9.96$
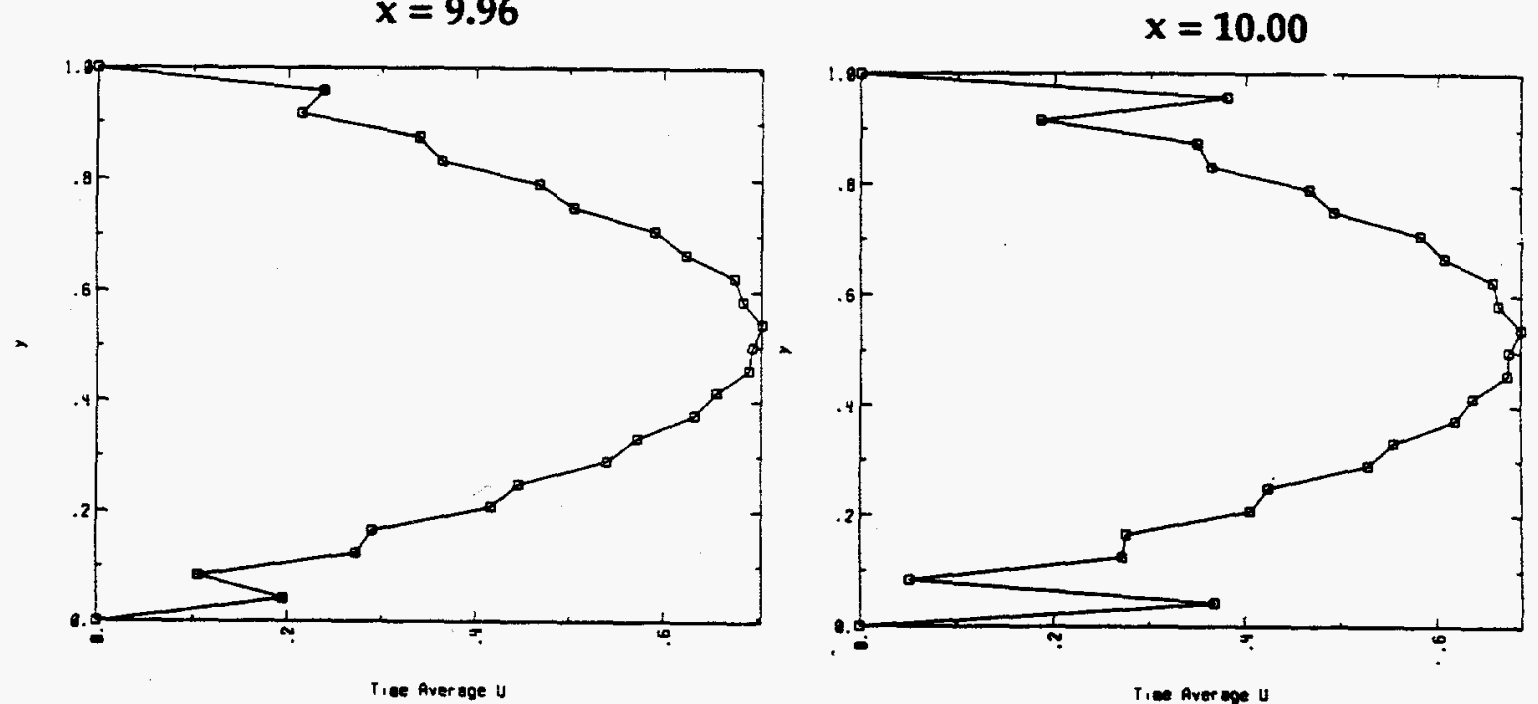

Figure 5.3.24 The LES predicted time-averaged $\bar{u}_{1}$ velocity, $\left\langle\bar{u}_{1}\right\rangle$, at the channel outlet for the $25 \times 241$ grid, $\Delta t=0.005$, and the SGS vorticity model with $C_{\omega}=0.2154$ and $\Delta_{f}=\Delta$ 
Entire Computational Field

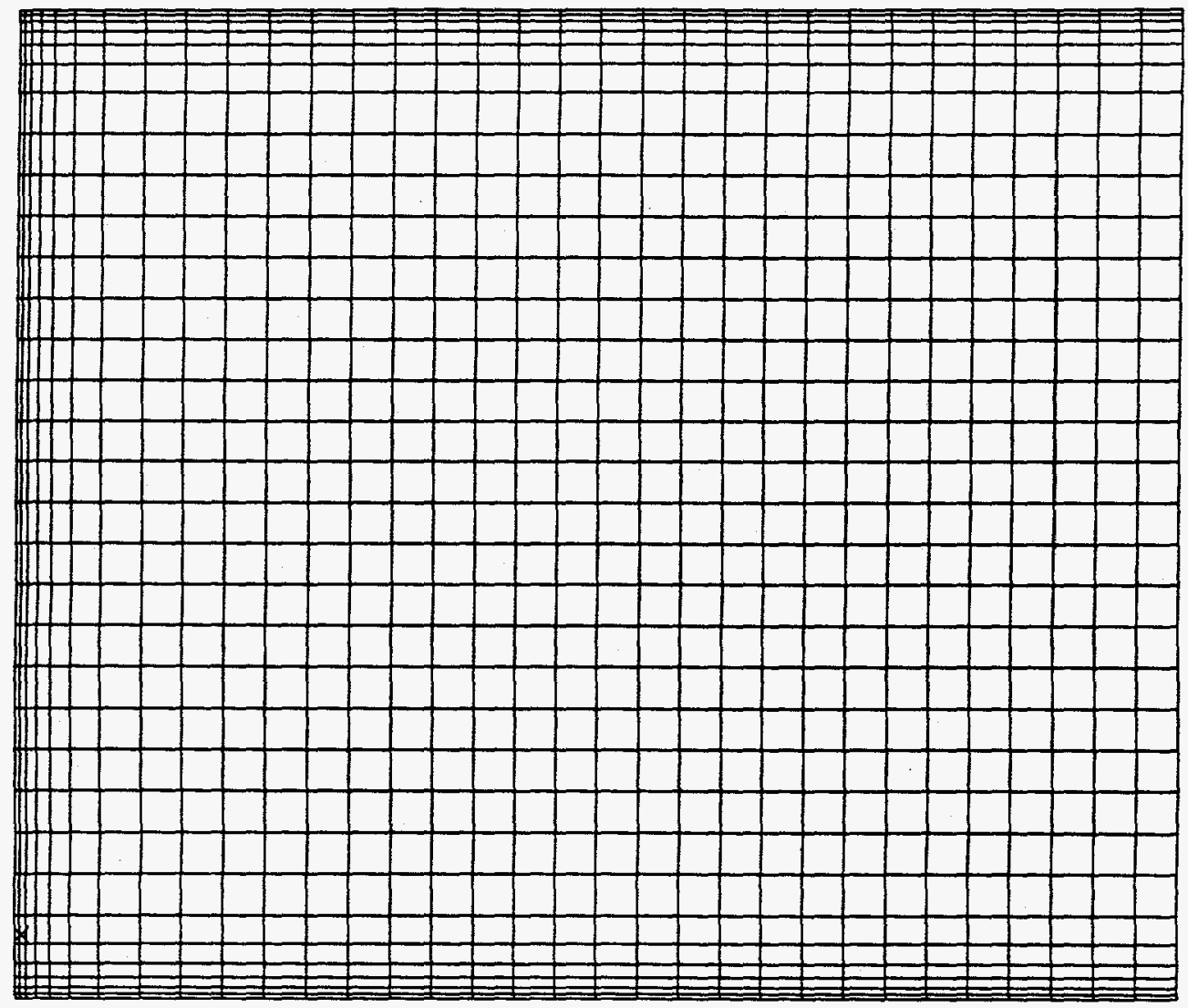

Entrance Region Showing 6 to 1 Mesh Grading Normal to Walls for the 6 elements adjacent to the wall

Figure 5.3.25 Geometry and mesh description for the $33 \times 245$ node grid. The sixth element from wall is six times bigger in direction normal to wall compared to the element adjacent to wall $\left(\Delta x_{\min }=\Delta y_{\min }=0.0047\right)$. 

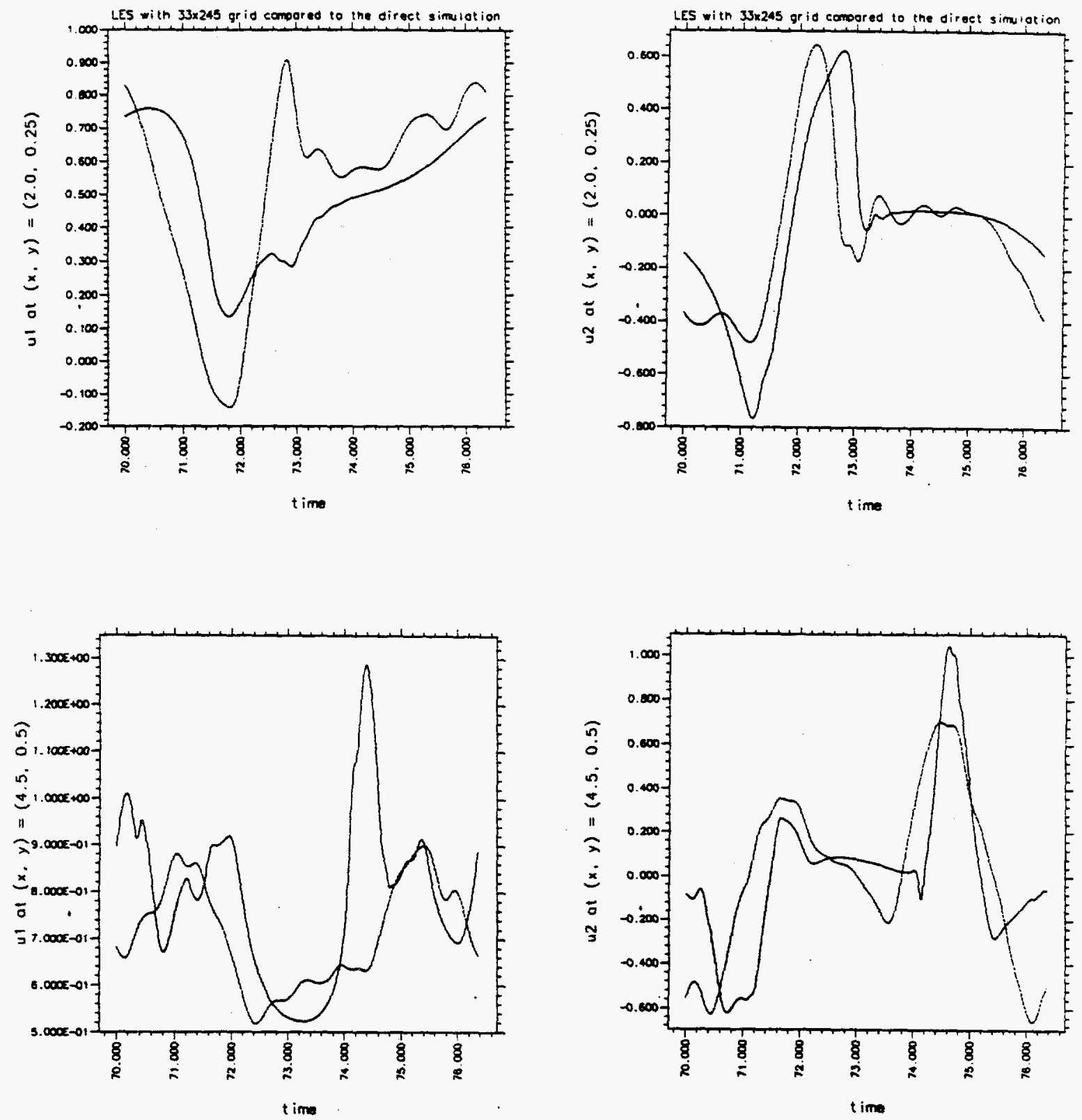

Fig. 5.3.26 Comparison of LES time histories for the $33 \times 245$ grid, $\Delta t=0.005$, $C_{\omega}=.2154$, and $\Delta f=\Delta$ to the direct simulation for one cycle. (One cycle is 6.36 time units for the direct simulation.) The LES curve has been shifted in time so that the minimum $\bar{u}_{1}$ values at $(x, y)=(2.0,0.25)$ are shown to occur at the same time. 


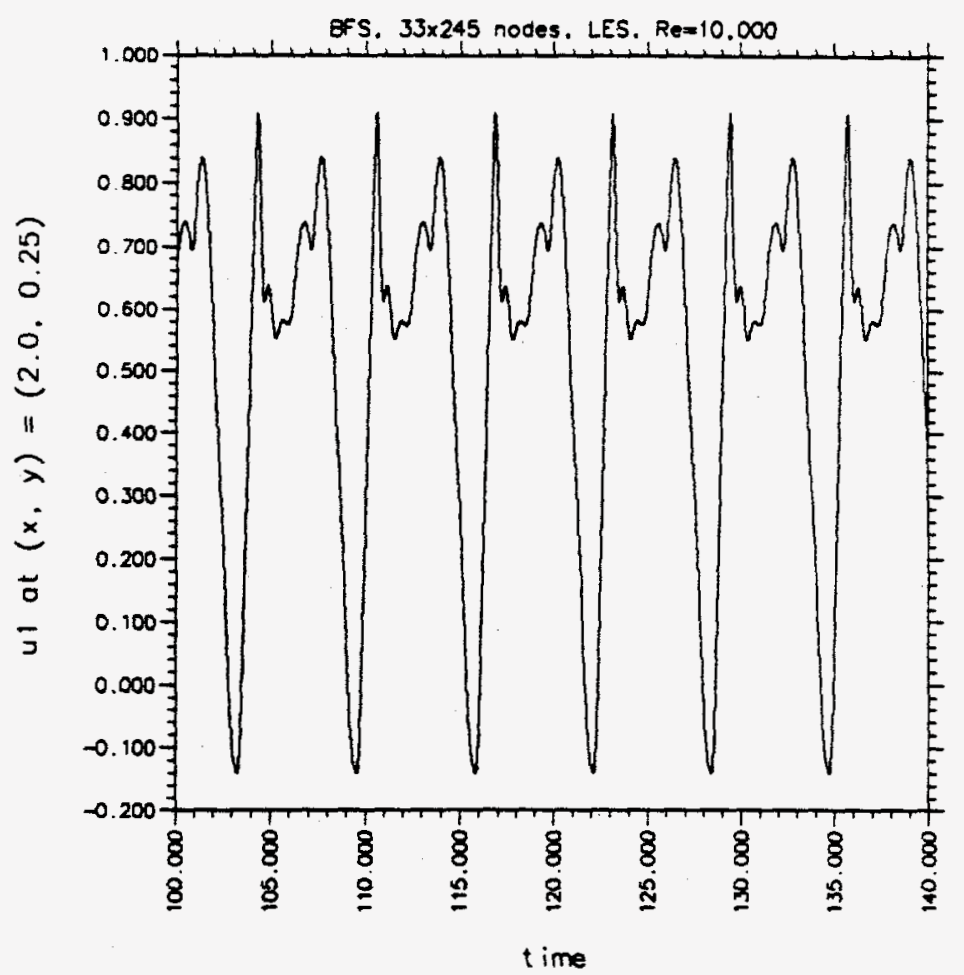

(a)

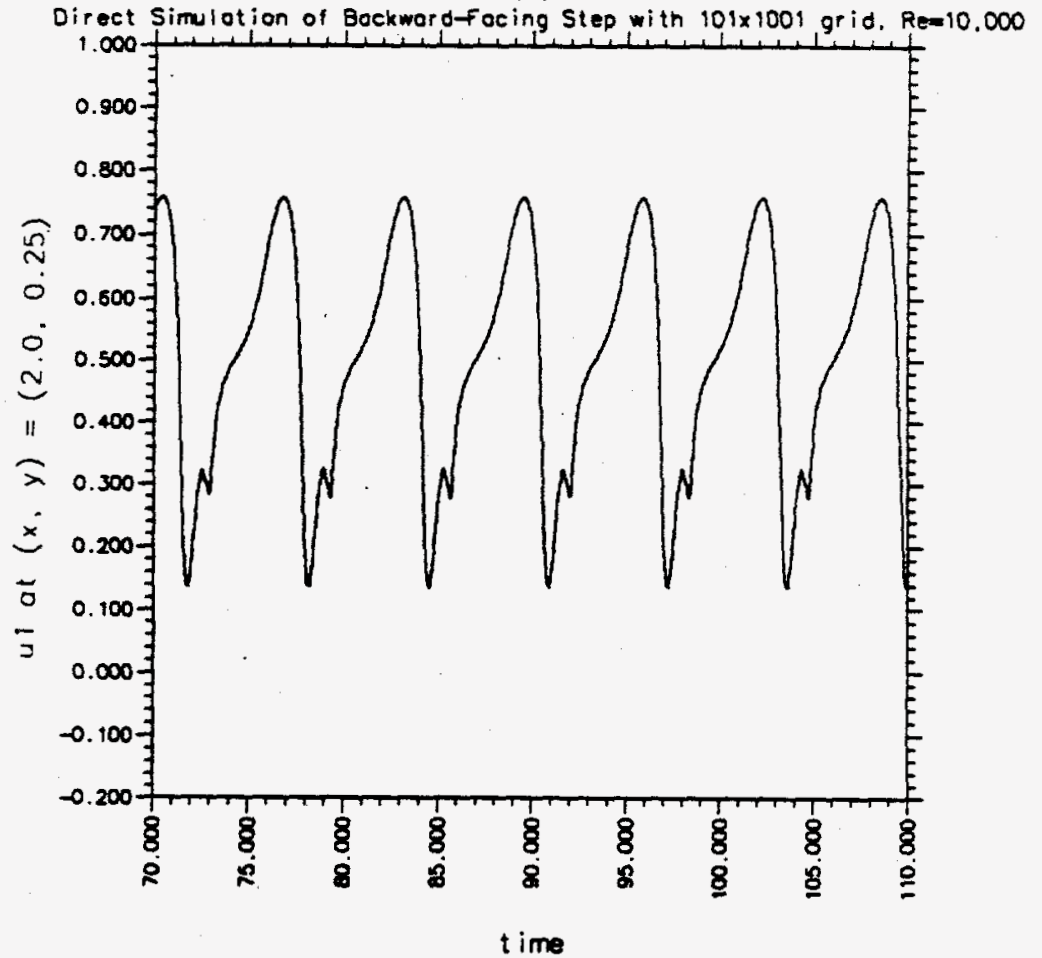

(b)

Fig. 5.3.27 Extended time histories of $\bar{u}_{1}$ at $(x, y)=(2.0,0.25)$ for a) the $33 \times 245$ grid, $\Delta t=0.005$, and the SGS vorticity model with $C_{\omega}=.2154$ and $\Delta_{f}=\Delta$ and b) the direct simulation (shown for the same axis scales). 

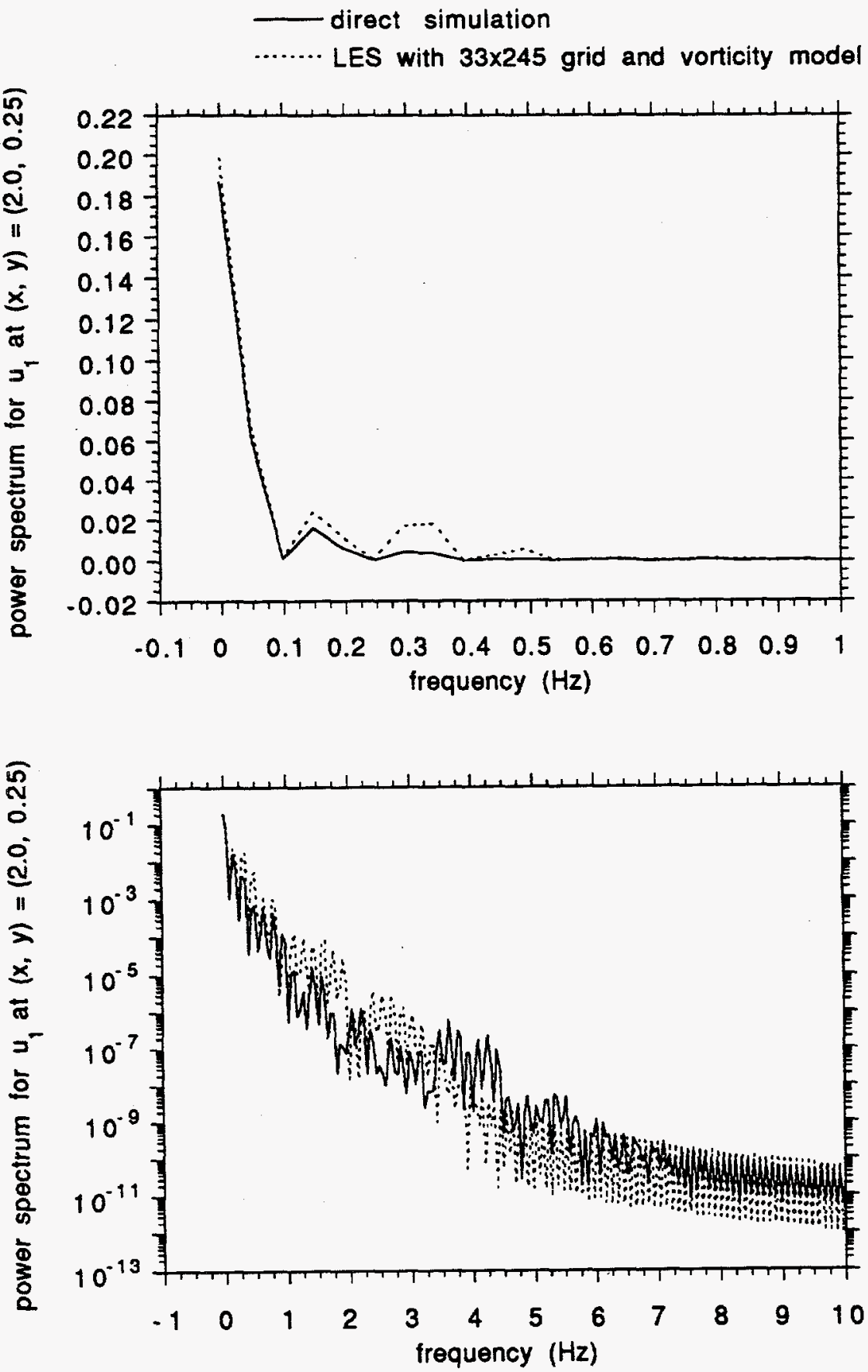

Fig. 5.3.28 Power spectrum on a linear and a log plot for $\bar{u}_{1}$ time history at $(x, y)=$ $(2.0,0.25)$ for the $33 \times 245$ grid, $\Delta t=0.005$, and the SGS vorticity model with $C_{\omega}=.2154$ and $\Delta_{f}=\Delta(t=100$ to 130.715$)$ compared to the direct simulation. 


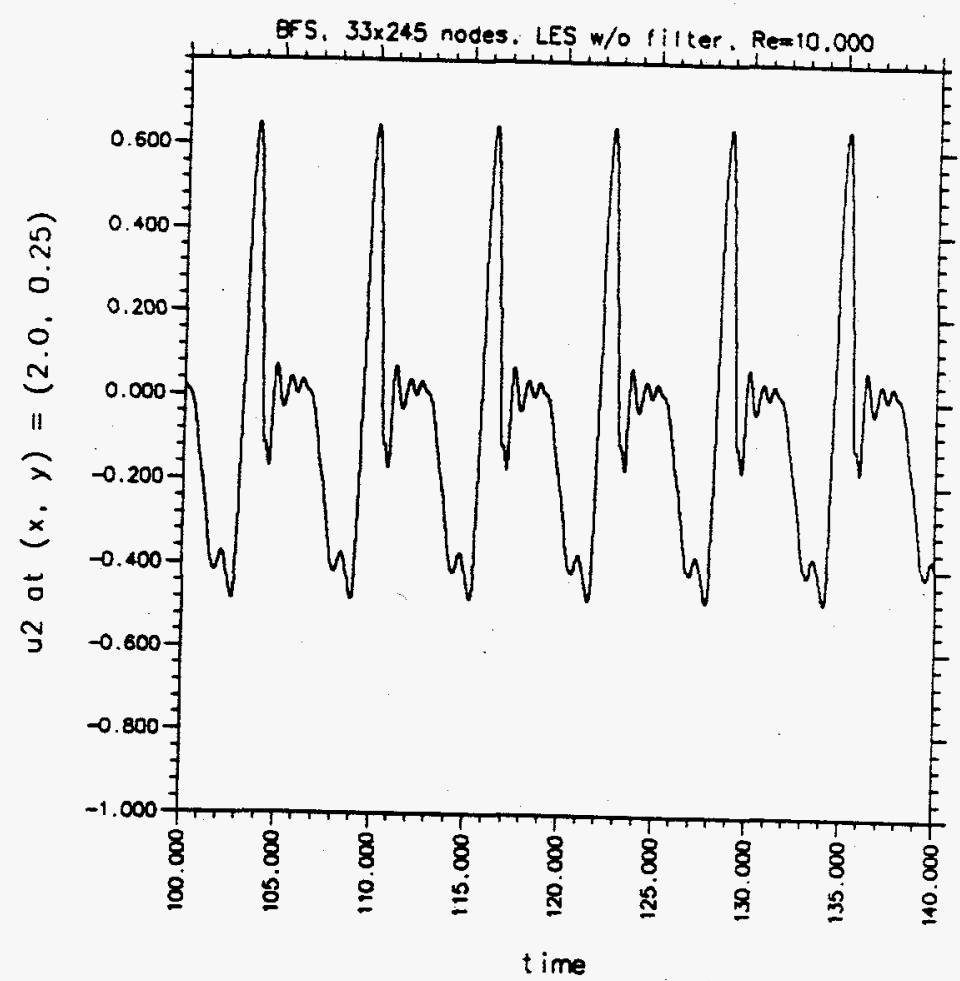

(a)

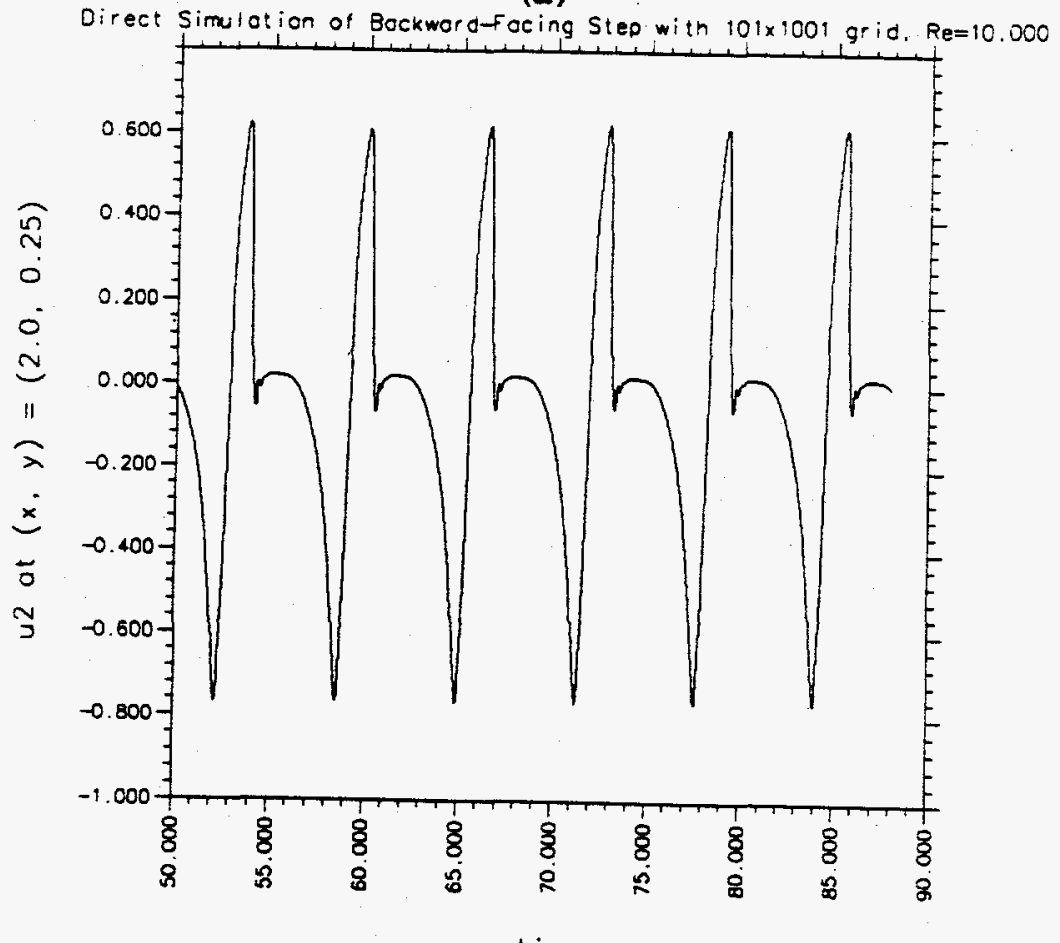

(b)

Fig. 5.3.29 Extended time histories of $\bar{u}_{2}$ time history at $(x, y)=(2.0,0.25)$ for a) the $33 \times 245$ grid, $\Delta t=0.005$, and the SGS vorticity model with $C_{\omega}=.2154$ and $\Delta_{f}=$ $\Delta$ and b) the direct simulation (shown for same axis scales). 

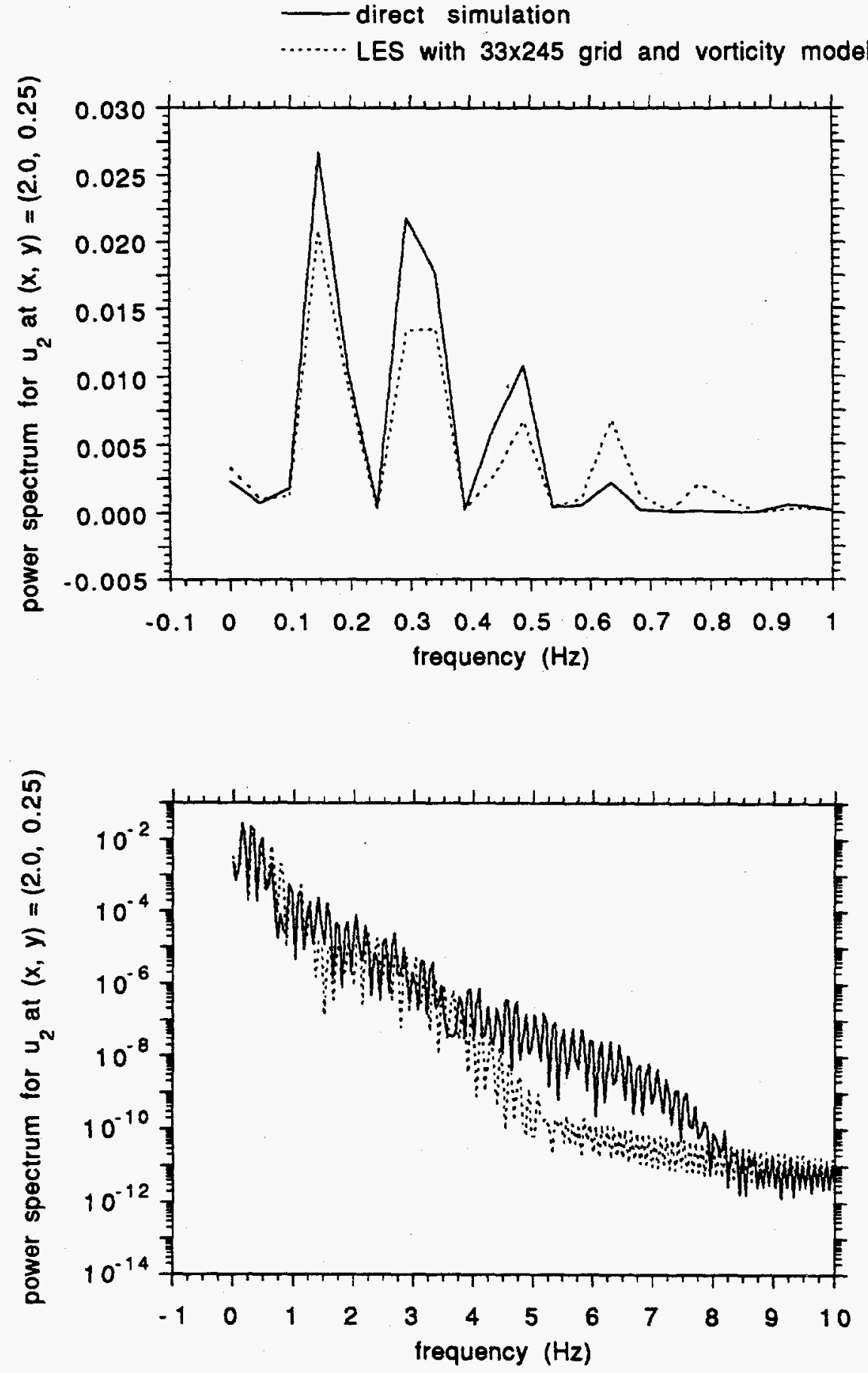

Fig. 5.3.30 Power spectrum on a linear and a log plot for $\bar{u}_{2}$ time history at $(x, y)=$ $(2.0,0.25)$ for the $33 \times 245$ grid, $\Delta t=0.005$, and the SGS vorticity model with $C_{\omega}=.2154$ and $\Delta_{f}=\Delta(t=100$ to 130.715$)$ compared to the direct simulation. 


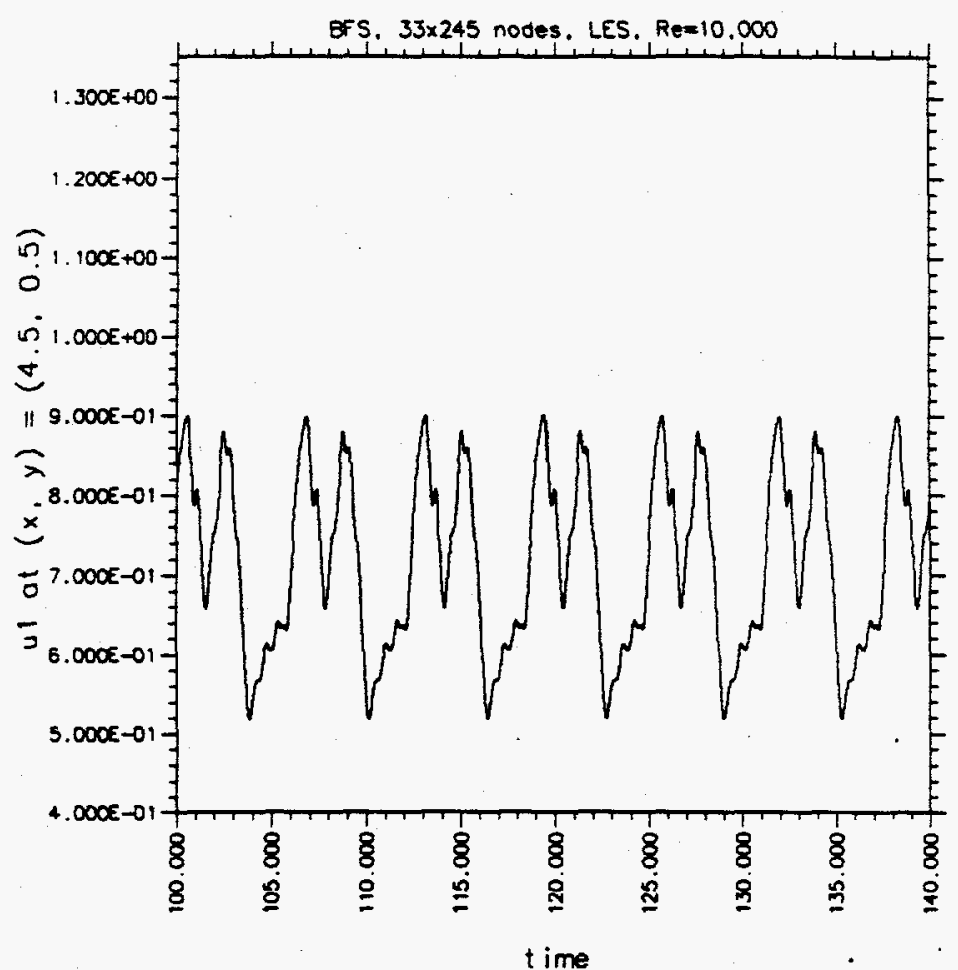

(a)

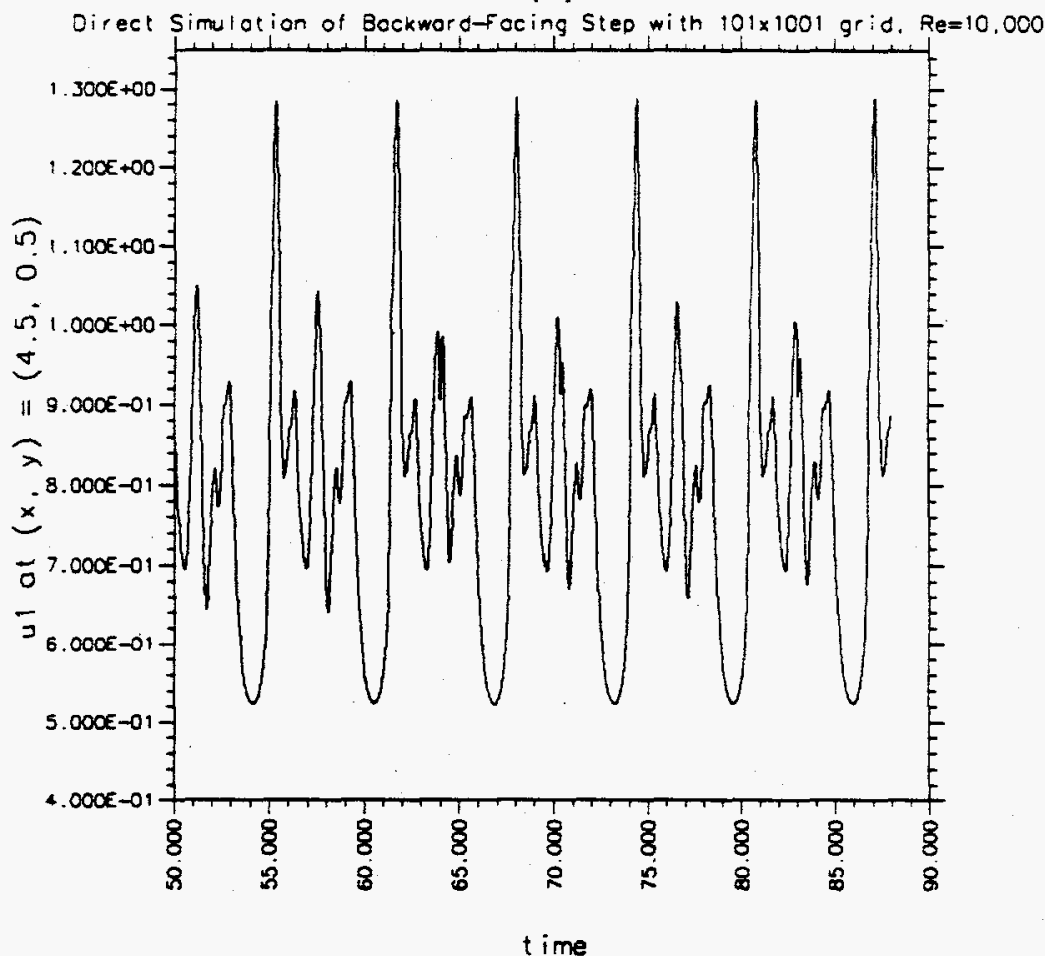

(b)

Fig. 5.3.31 Extended time histories of $\bar{u}_{1}$ time history at $(x, y)=(4.5,0.5)$ for a) the $33 \times 245$ grid, $\Delta t=0.005$, and the SGS vorticity model with $C_{\omega}=.2154$ and $\Delta_{f}=\Delta$ and b) the direct simulation. 

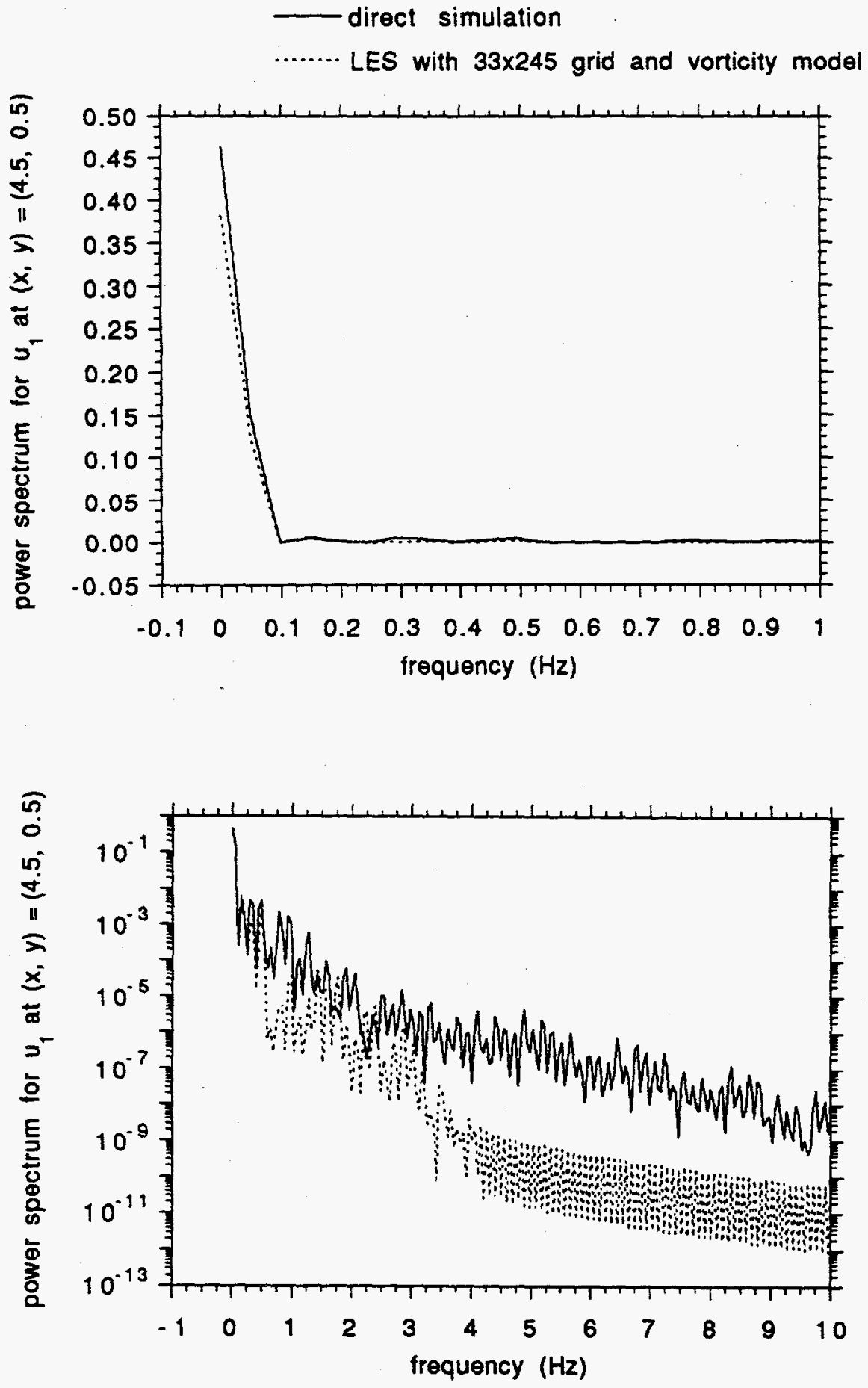

Fig. 5.3.32 Power spectrum on a linear and a $\log$ plot for $\bar{u}_{1}$ time history at $(x, y)=$ $(4.5,0.5)$ for the $33 \times 245$ grid, $\Delta t=0.005$, and the SGS vorticity model with $C_{\omega}=0.2154$ and $\Delta_{f}=\Delta(t=100$ to 130.715$)$ compared to the direct simulation. 


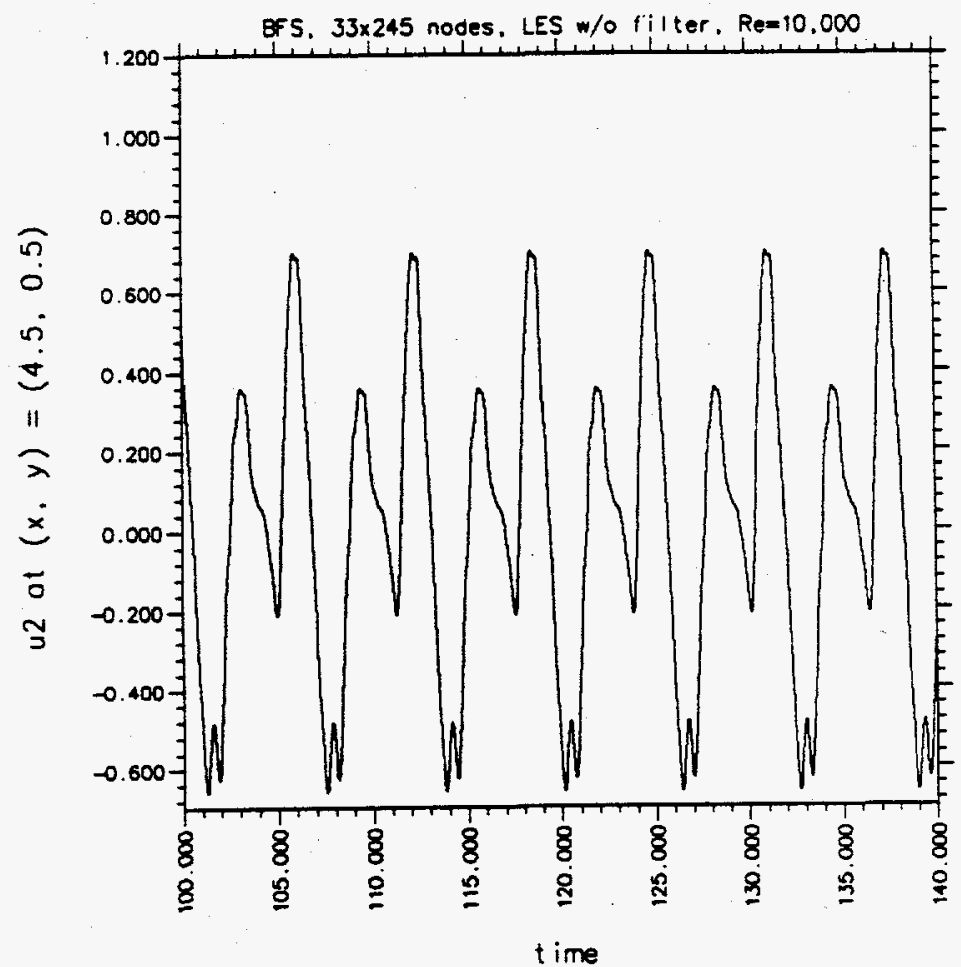

(a)

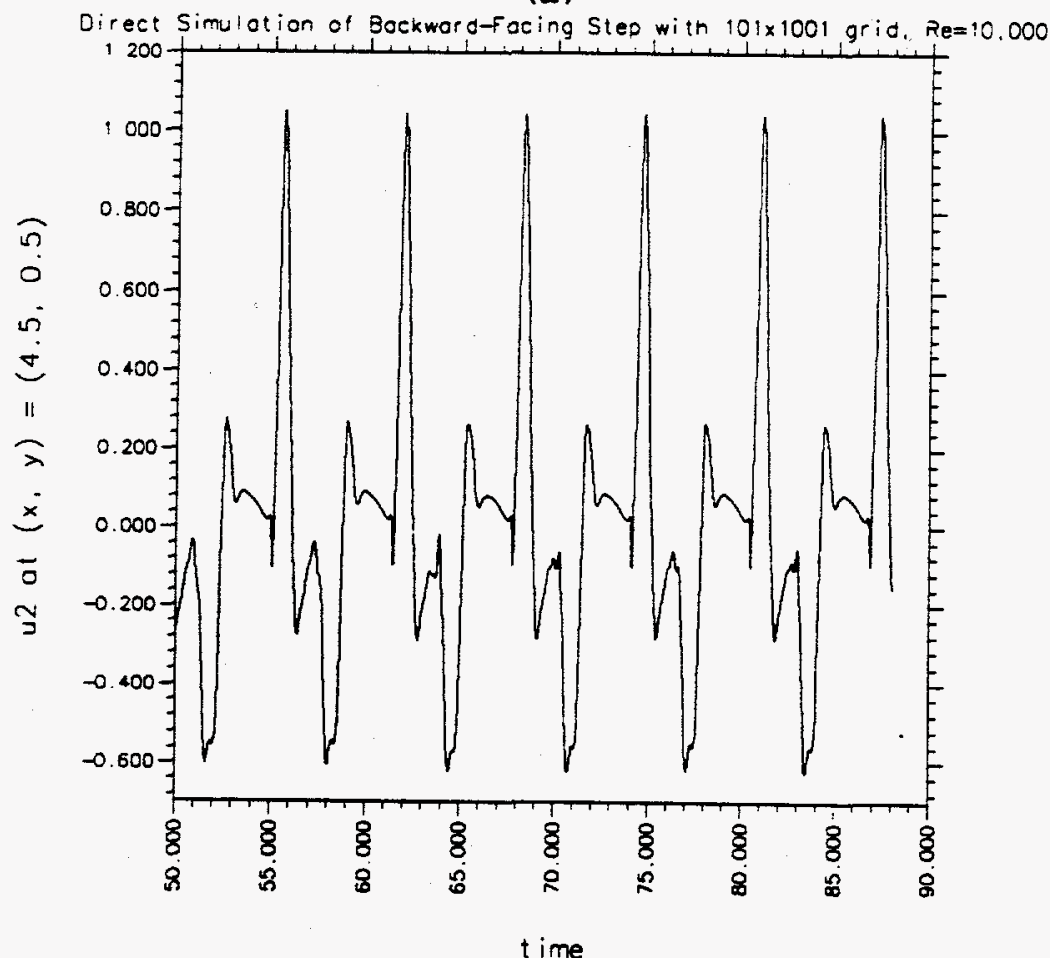

(b)

Fig. 5.3.33 Extended time histories of $\bar{u}_{2}$ at $(x, y)=(4.5,0.5)$ for the $33 \times 245$ grid, $\Delta t=0.005$, and the SGS vorticity model with $C_{\omega}=.2154$ and $\Delta_{f}=\Delta$ and b) the direct simulation 

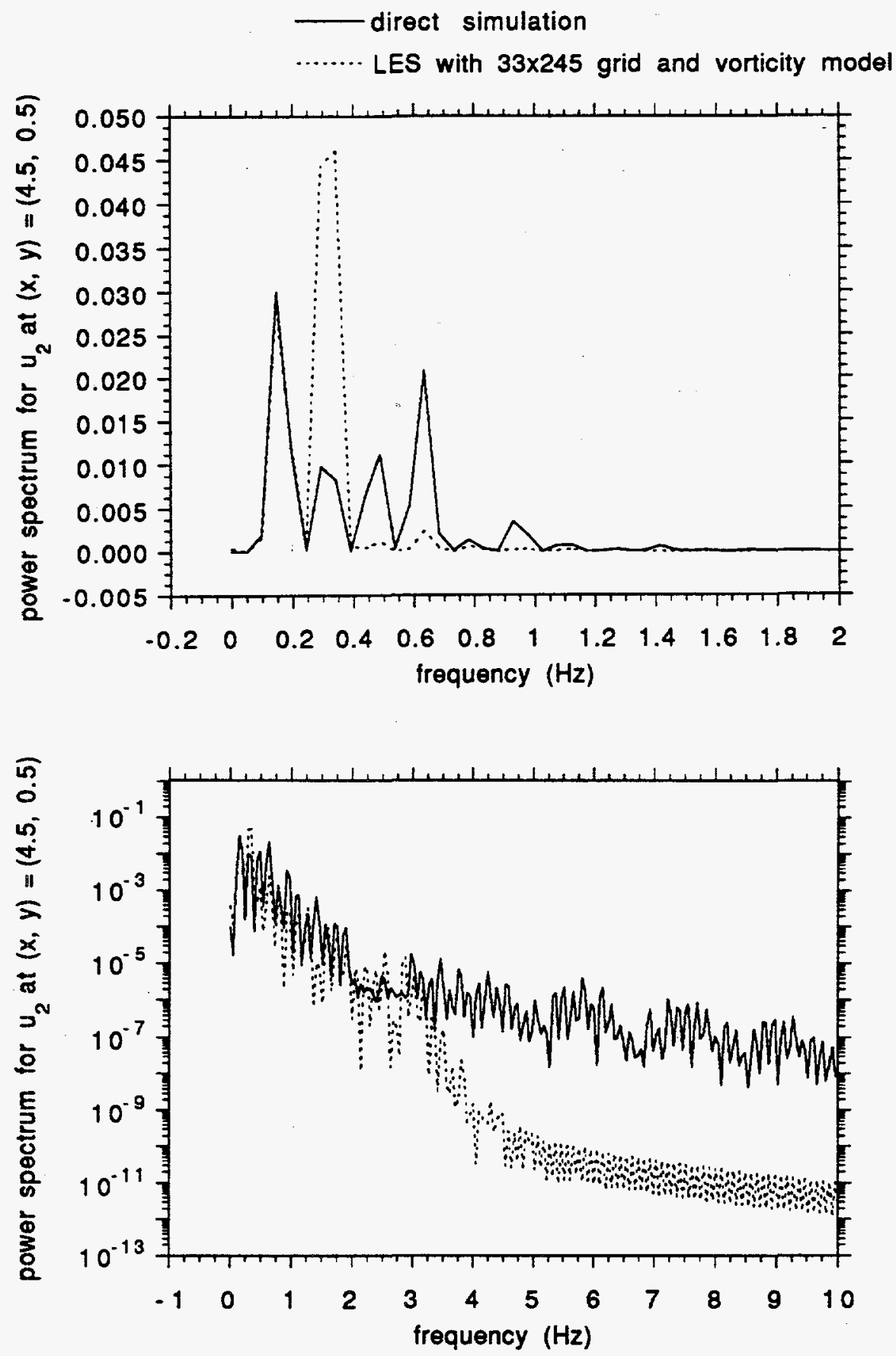

Fig. 5.3.34 Power spectrum on a linear and a log plot for $\bar{u}_{2}$ time history at $(x, y)=$ $(4.5,0.5)$ for the $33 \times 245$ grid, $\Delta t=0.005$, and the SGS vorticity model with $C_{\omega}=.2154$ and $\Delta_{f}=\Delta(t=100$ to 130.715$)$ compared to the direct simulation. 

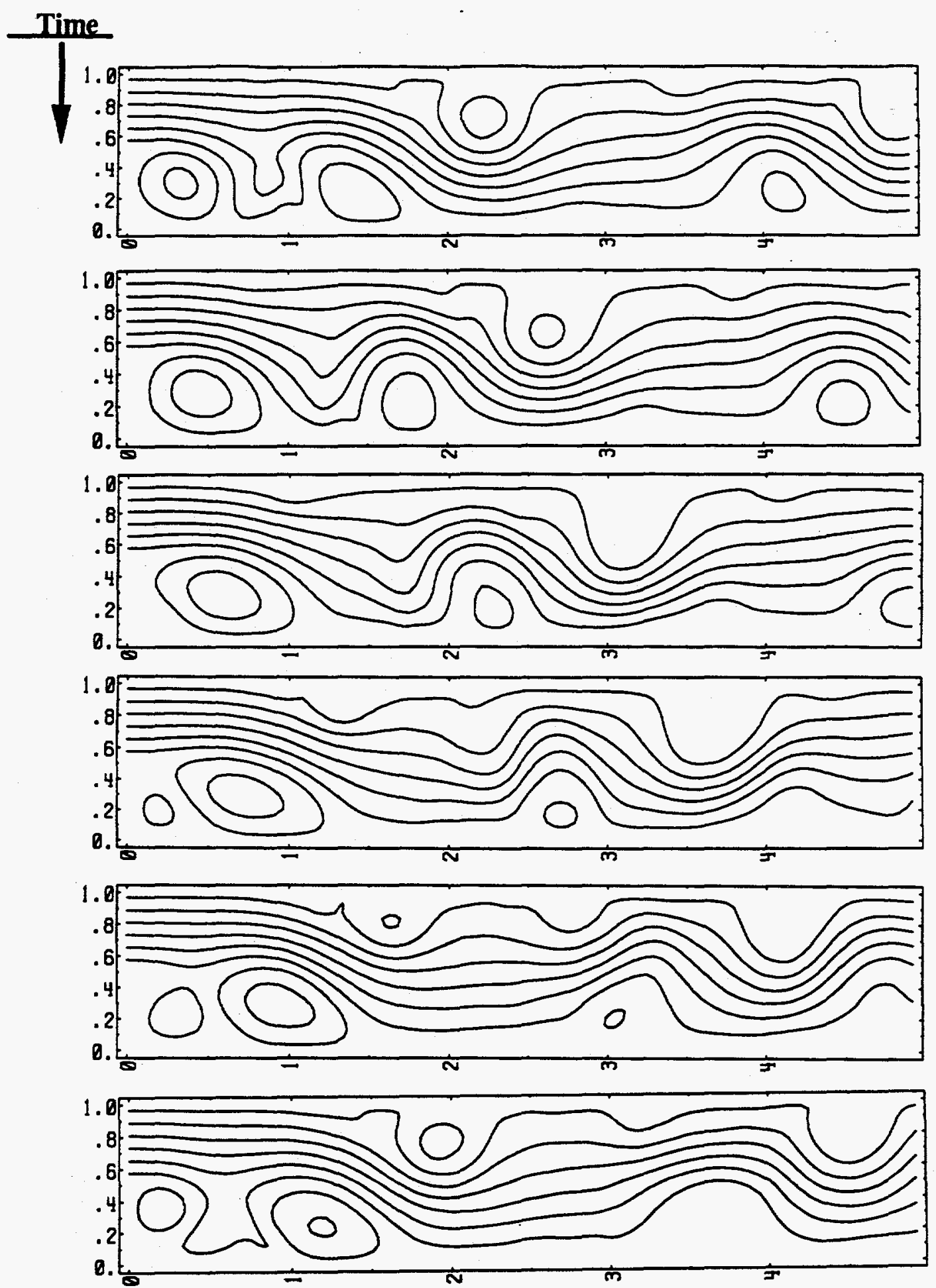

Fig. 5.3.35a Time series of stream function plots showing only half of field length for the $33 \times 245$ grid, $\Delta t=0.005$, and the SGS vorticity model with $C_{\omega}=.2154$ and $\Delta_{f}=\Delta$ at $0,1 / 6,2 / 6,3 / 6,4 / 6$, and $5 / 6$ of a DS cycle. 

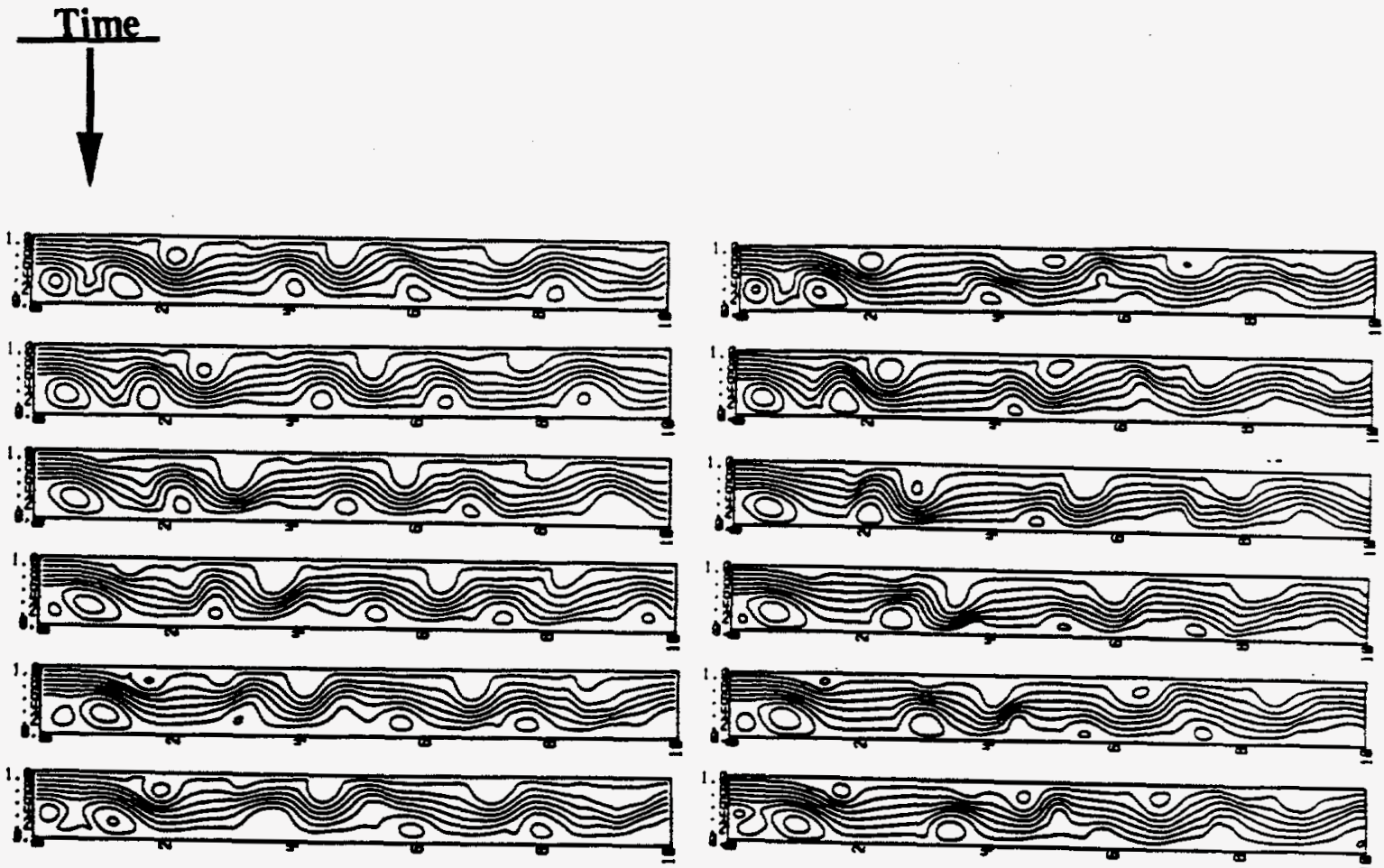

LES with $33 \times 245$ grid

Direct Simulation

Fig. 5.3.35b Time series of stream function plots for the $33 \times 245$ grid, $\Delta t=0.005$, and the SGS vorticity model with $C_{\omega}=.2154$ and $\Delta_{f}=\Delta$ compared to the direct simulation. (One cycle is shown for the same specified stream function levels for each case at $0,1 / 6,2 / 6,3 / 6,4 / 6$, and $5 / 6$ of a DS cycle.) 
Time
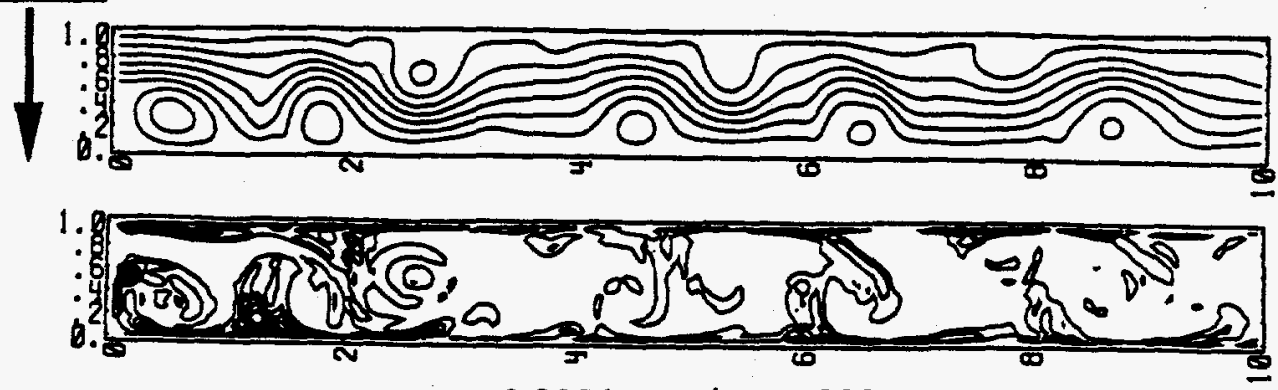

$0.0001<v_{T} / v<2.000$
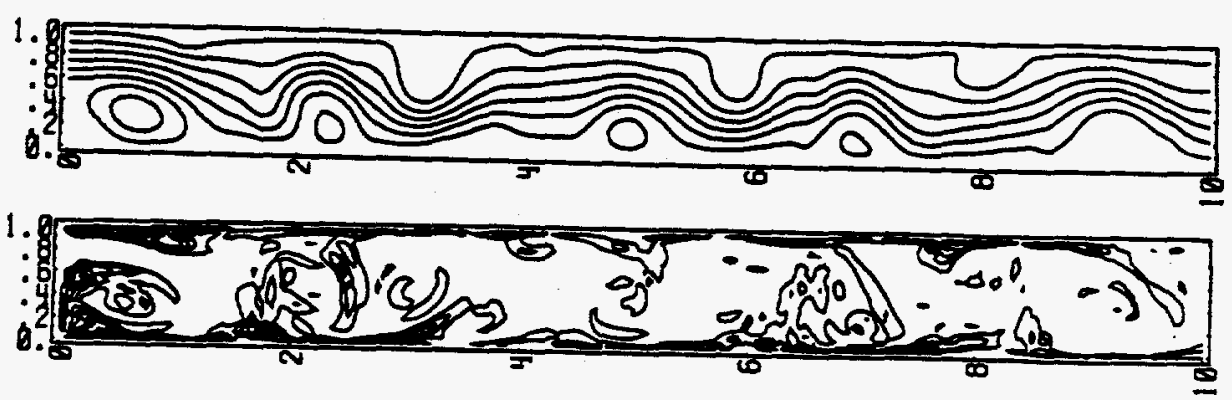

$0.0002<v_{T} / v<2.101$
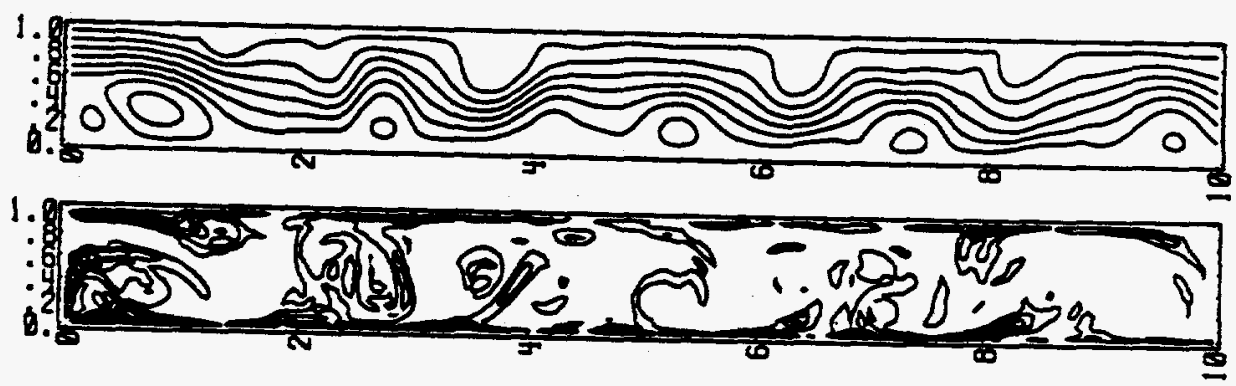

$0.0001<v_{T} / v<1.975$
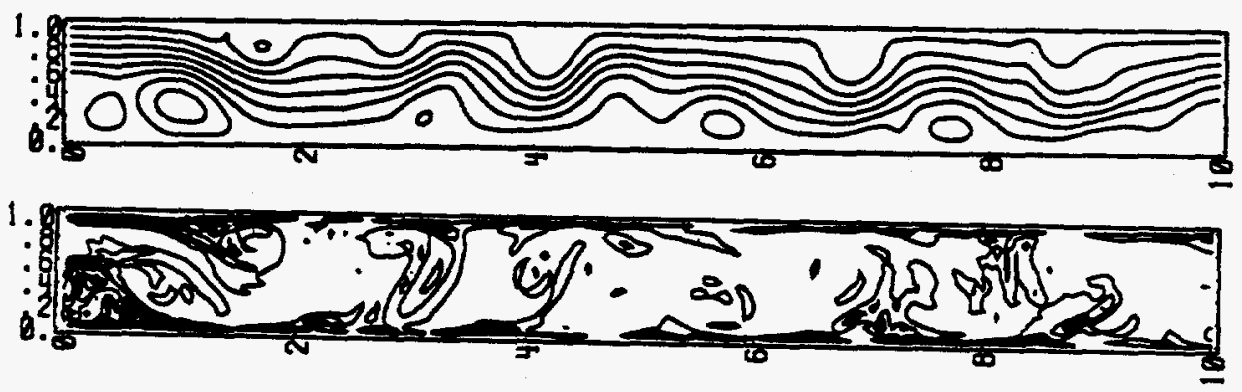

$0.0003<v_{T} / v<2.255$

Fig. 5.3.36 Time series of $v_{T}$ compared to stream function plots for the $33 \times 245$ grid, $\Delta t=0.005$, and the SGS vorticity model with $C_{\omega}=0.2154$ and $\Delta_{f}=\Delta$ (second through fifth stream function plots in Figure 5.3.35a). 

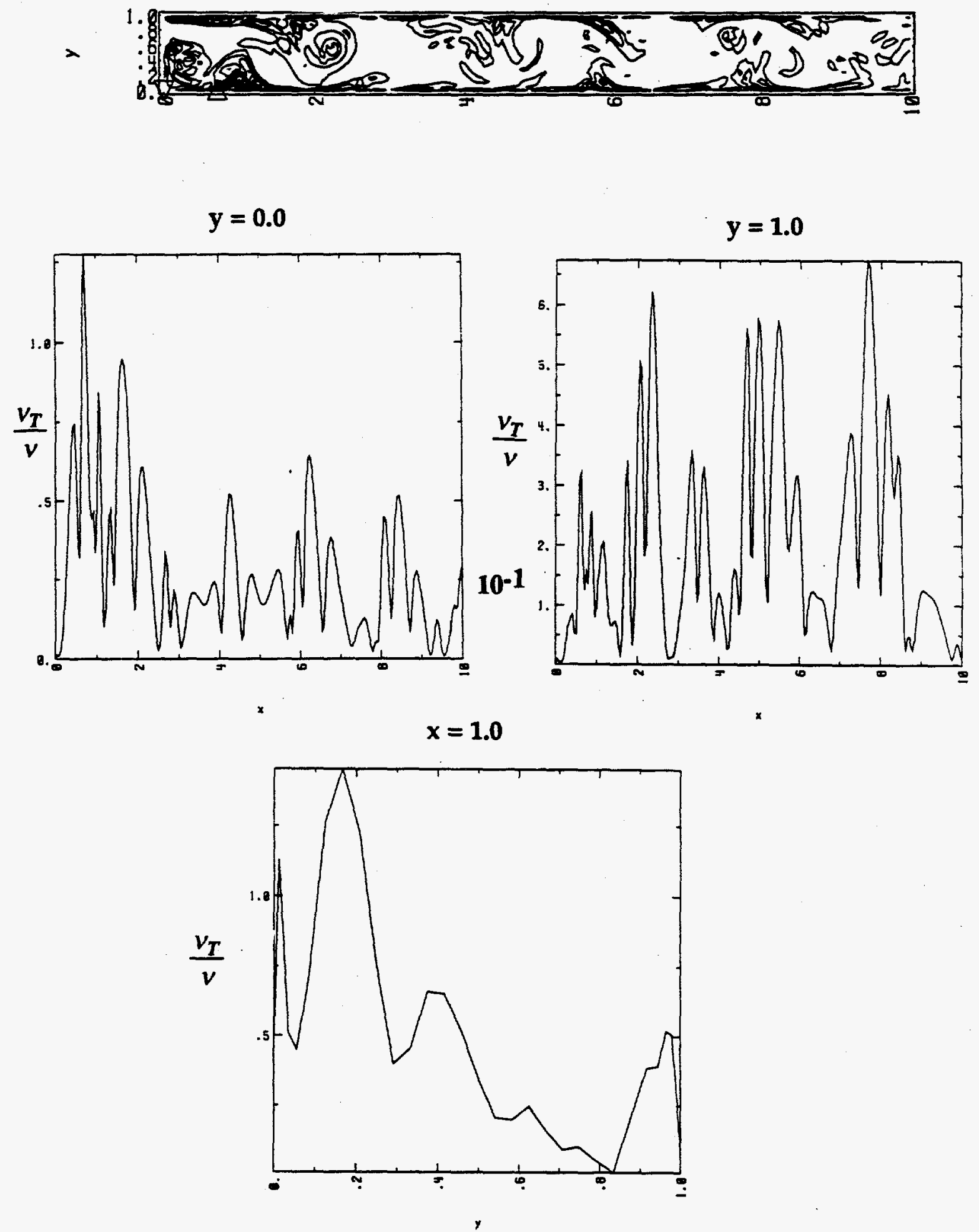

Fig. 5.3.37 A $v_{T}$ contour plot and profile plots at selected locations for the $33 \mathrm{x}$ 245 grid, $\Delta t=0.005$, and the SGS vorticity model with $C_{\omega}=0.2154$ and $\Delta_{f}=\Delta$ at the contour plot snapshot in time. 
$\mathbf{x}=\mathbf{1 . 0}$

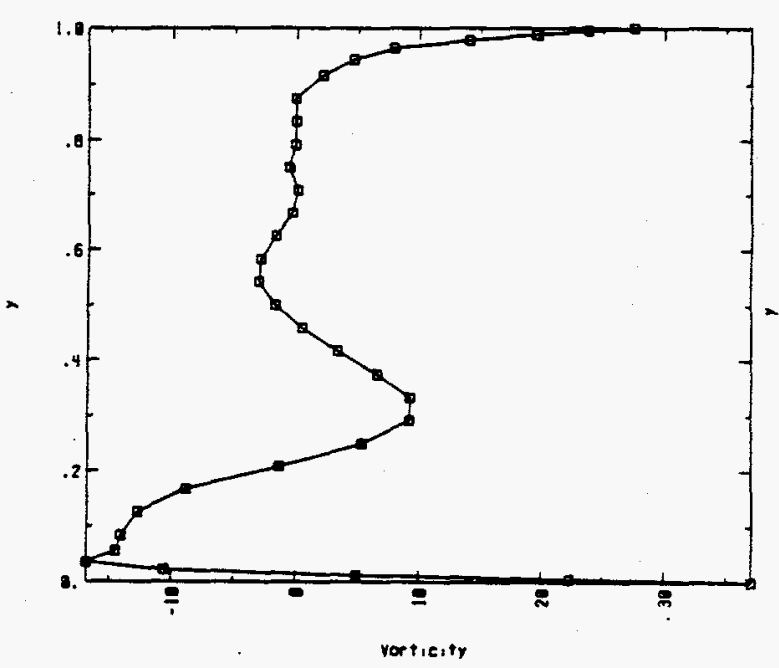

$\mathbf{x}=\mathbf{3 . 0}$

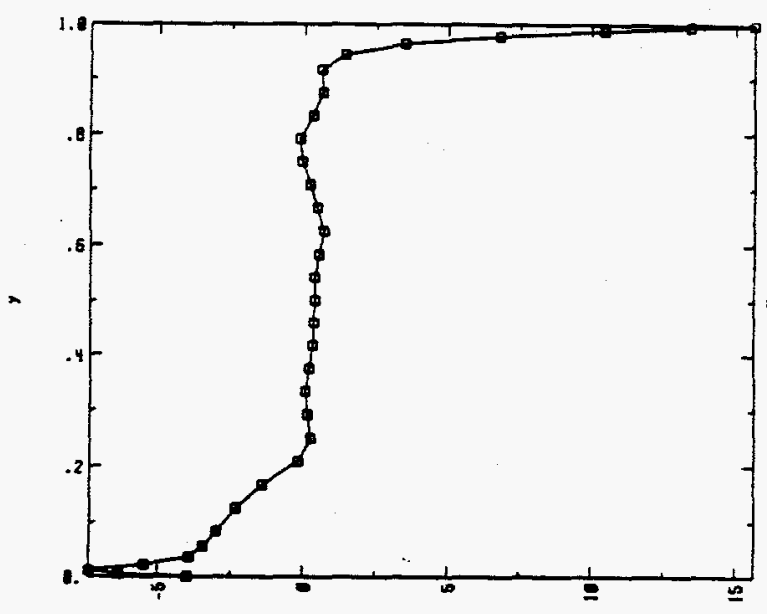

verticity $\mathbf{x}=\mathbf{2 . 0}$

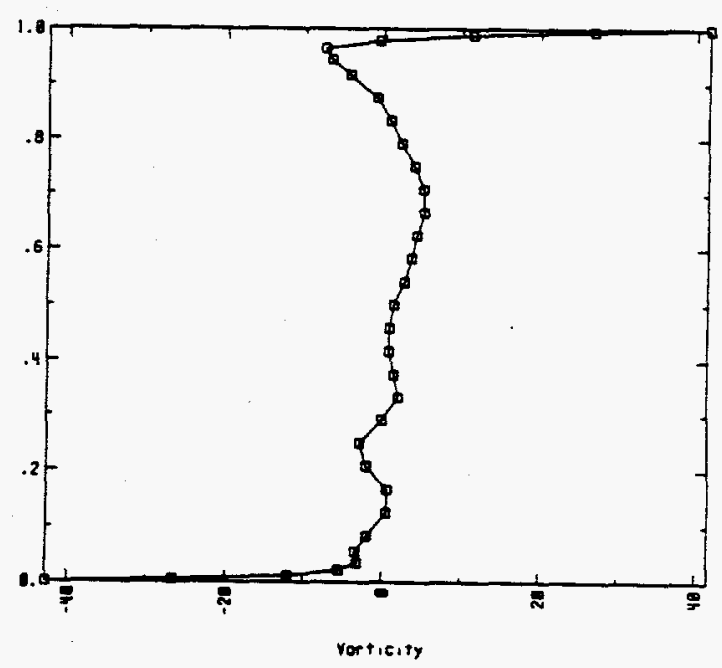

$x=6.0$

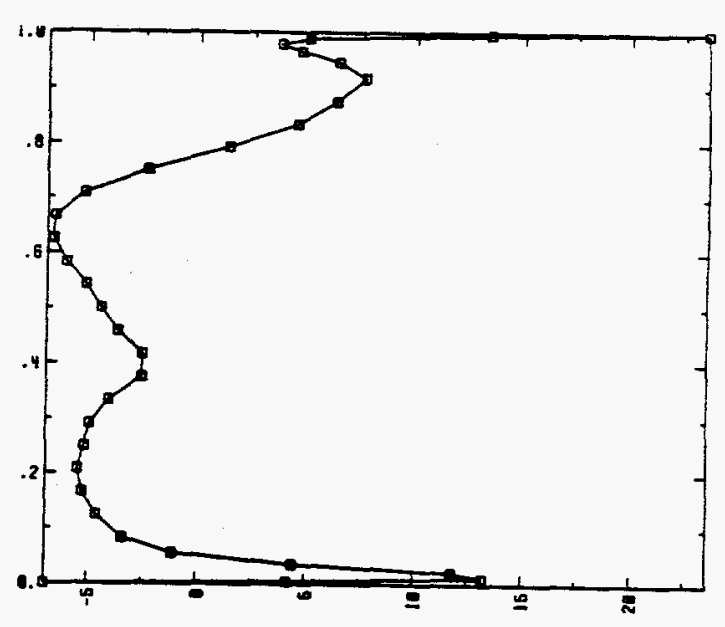

Vortieipy

Fig. 5.3.38 Profile plots of the LES predicted vorticity at selected locations for the $33 \times 245$ grid, $\Delta t=0.005$, and the SGS vorticity model with $C_{\omega}=.2154$ and $\Delta_{f}=\Delta$ at a snapshot in time (same time as that for Figures 5.3.37 through 5.3.39). 


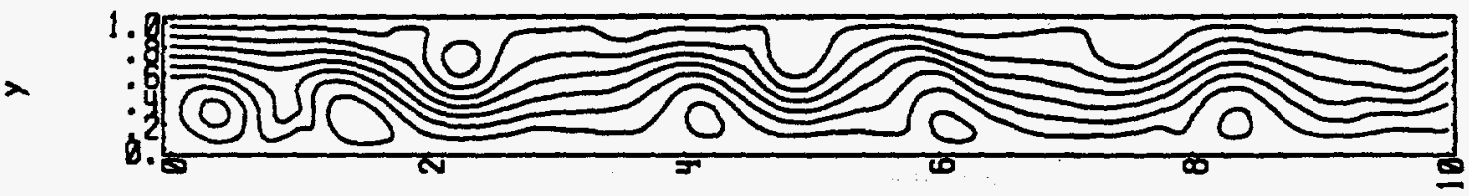

$$
\mathbf{x}=\mathbf{1 . 0}
$$

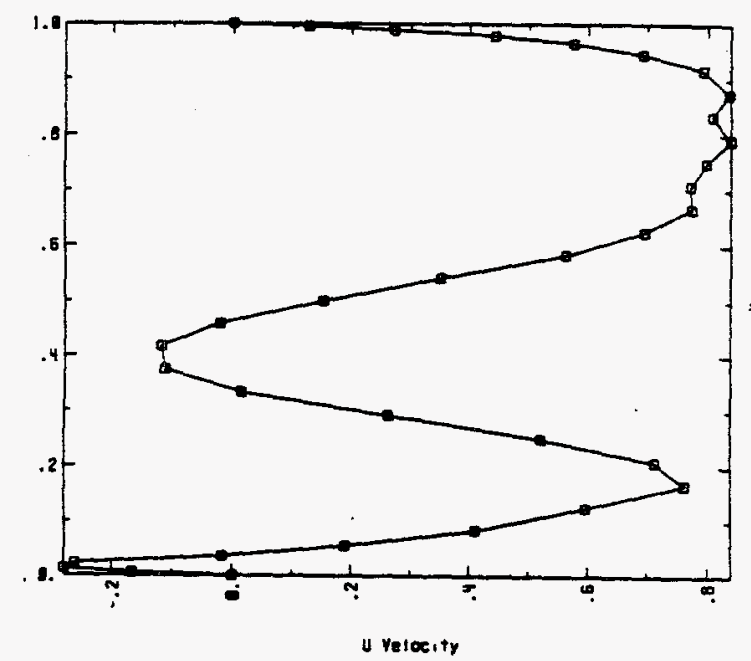

$\mathbf{x}=\mathbf{3 . 0}$

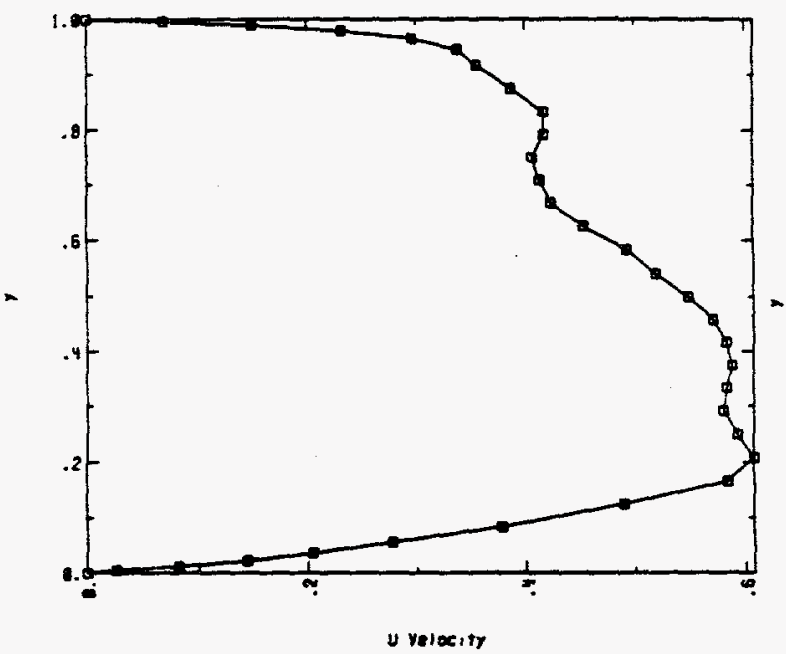

$x=\mathbf{2 . 0}$
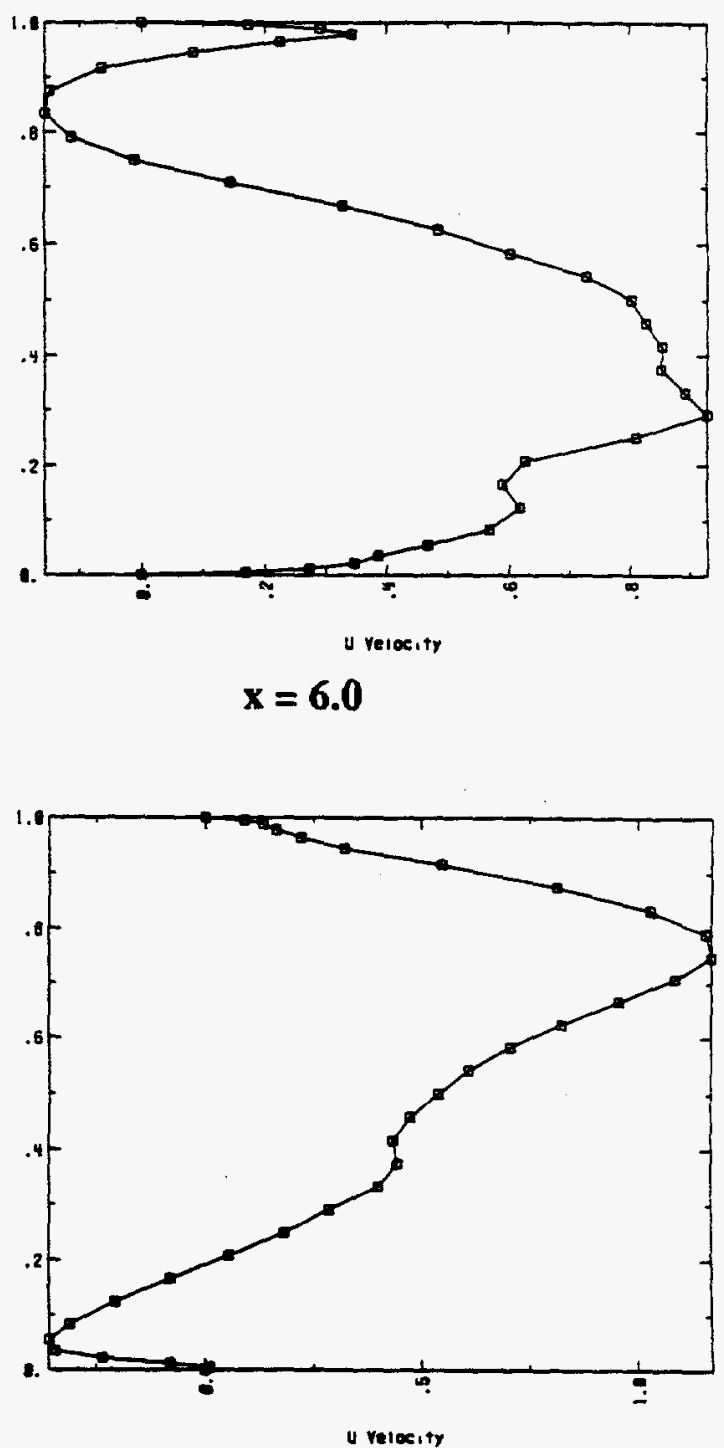

Fig. 5.3.39 The LES predicted $\bar{u}_{1}$ velocity profiles at selected locations for the $33 \mathrm{x}$ 245 grid, $\Delta t=0.005$, and the SGS vorticity model with $C_{\omega}=0.2154$ and $\Delta_{f}=\Delta$ at the shown stream function snapshot in time. 


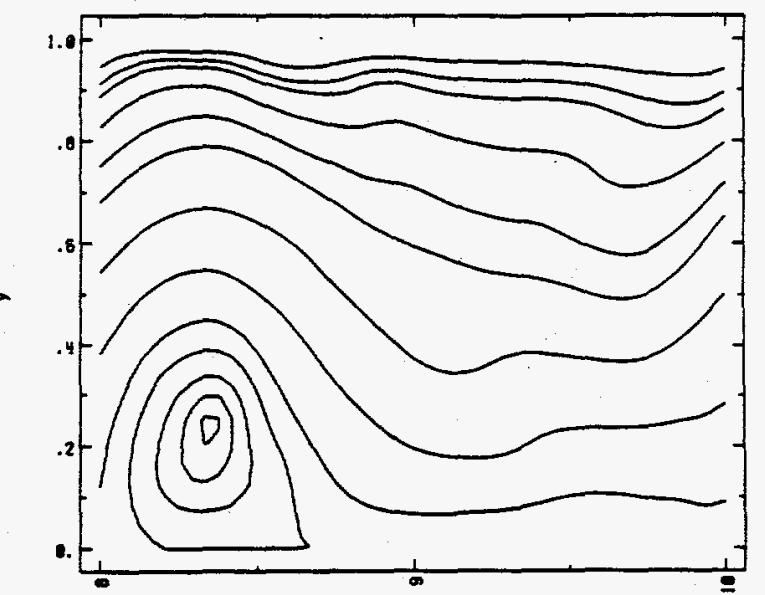

$x=9.88$
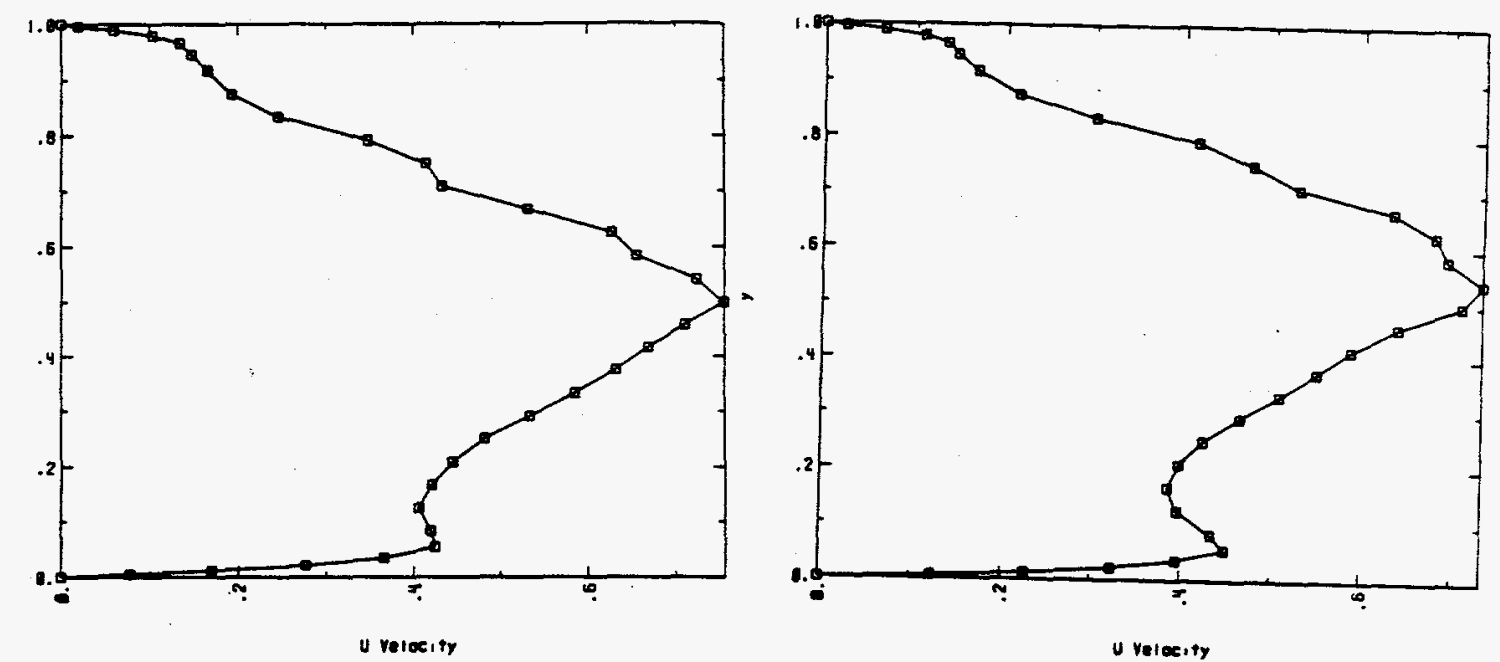

$x=9.96$
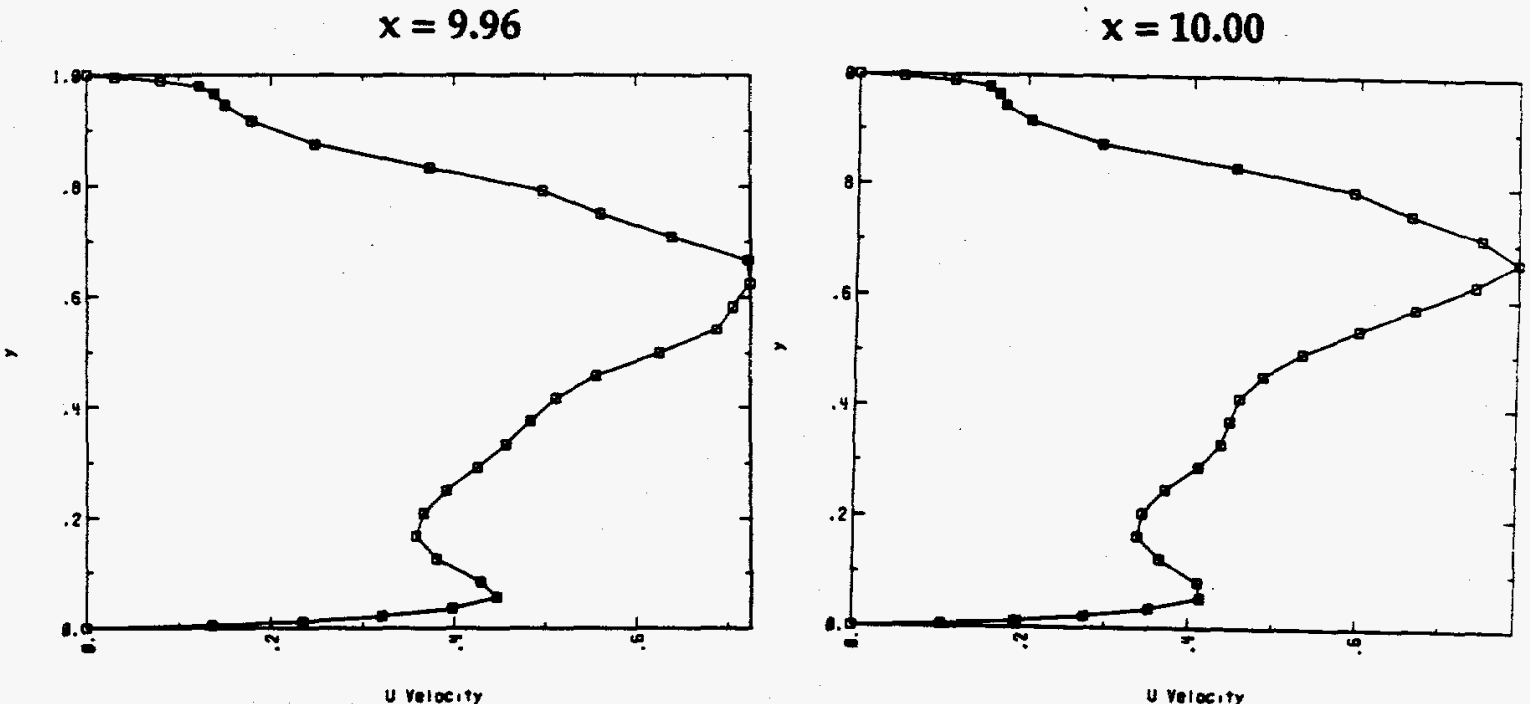

Fig. 5.3.40 The LES predicted $\bar{u}_{1}$ velocity profiles at channel outlet for the $33 \mathrm{x}$ 245 grid, $\Delta t=0.005$, and the SGS vorticity model with $C_{\omega}=0.2154$ and $\Delta_{f}=\Delta$ at the shown stream function plot snapshot in time. 
Table 5.3.3 Time-averaged LES for the $33 \times 245$ grid, $\Delta t=0.005$, and the SGS vorticity model with $C_{\omega}=0.2154$ and $\Delta_{f}=\Delta$.

\begin{tabular}{|c|c|c|c|}
\hline & \multicolumn{2}{|c|}{$\begin{array}{c}\text { X-location for separation } \\
\text { and reattachment points }\end{array}$} & $\begin{array}{c}\text { relative change } \\
\text { between cases } \\
(\%)\end{array}$ \\
\cline { 2 - 4 } & $\begin{array}{l}\text { time average } \\
\text { for 10,000 } \\
\text { time steps }\end{array}$ & $\begin{array}{l}\text { time average } \\
\text { for 20,000 } \\
\text { time steps }\end{array}$ & \\
\hline \multirow{3}{*}{ Bottom Wall } & 0.010 & 0.010 & 0.0 \\
& 0.084 & 0.084 & 0.0 \\
& 0.420 & 0.420 & 0.0 \\
& 1.543 & 1.543 & 0.0 \\
& 0.990 & 0.990 & 0.0 \\
& 1.061 & 1.062 & 0.1 \\
& 7.797 & 7.798 & $<0.1$ \\
& 8.130 & 8.129 & $<0.1$ \\
\hline
\end{tabular}




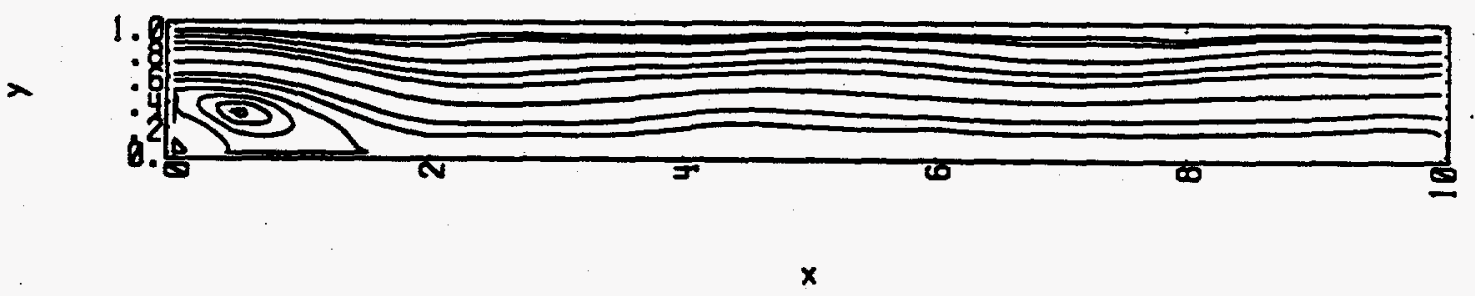

Entire Computational Field

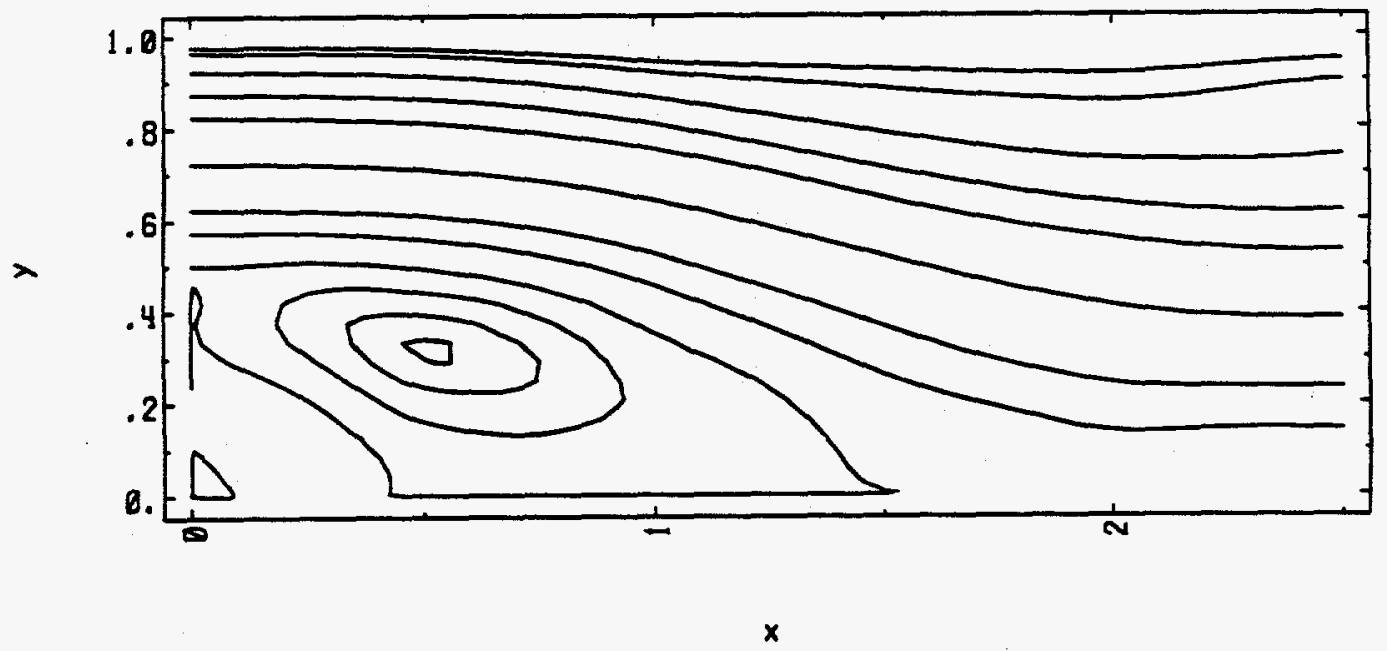

Enlargement of Major Recirculation Zone

Figure 5.3.41 Time-average LES stream function for the $33 \times 245$ grid, $\Delta t=0.005$, and the SGS vorticity model with $C_{\omega}=0.2154$ and $\Delta_{f}=\Delta$ for a time-averaging duration of 20,000 time steps. 

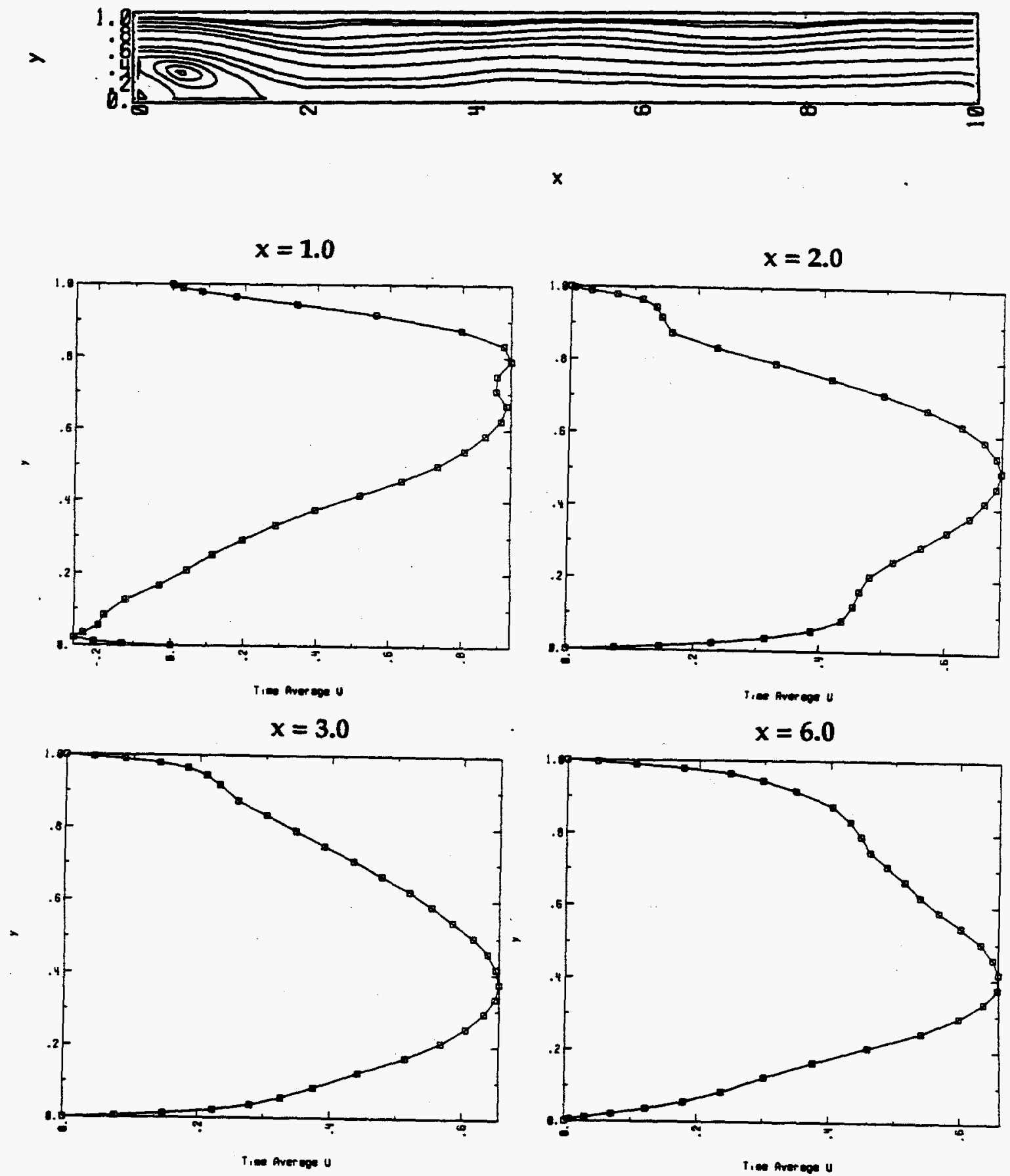

Fig. 5.3.42 The LES predicted time-averaged $\bar{u}_{1}$ velocity, $\left\langle\bar{u}_{1}\right\rangle$, at selected locations for the $33 \times 245$ grid, $\Delta t=0.005$, and the SGS vorticity model with $C_{\omega}=0.2154$ and $\Delta_{f}=\Delta$ 
$\mathbf{x}=\mathbf{1 . 0}$

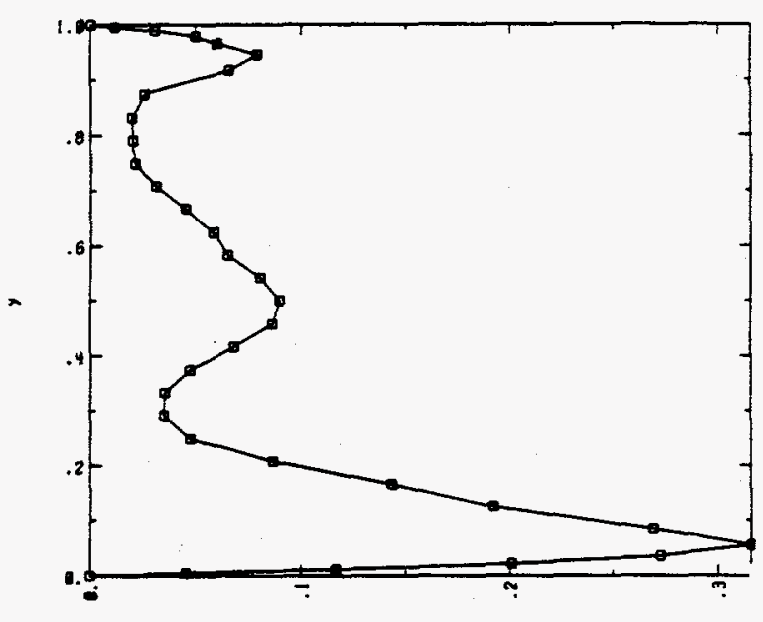

U Intengity

$\mathbf{x}=\mathbf{3 . 0}$

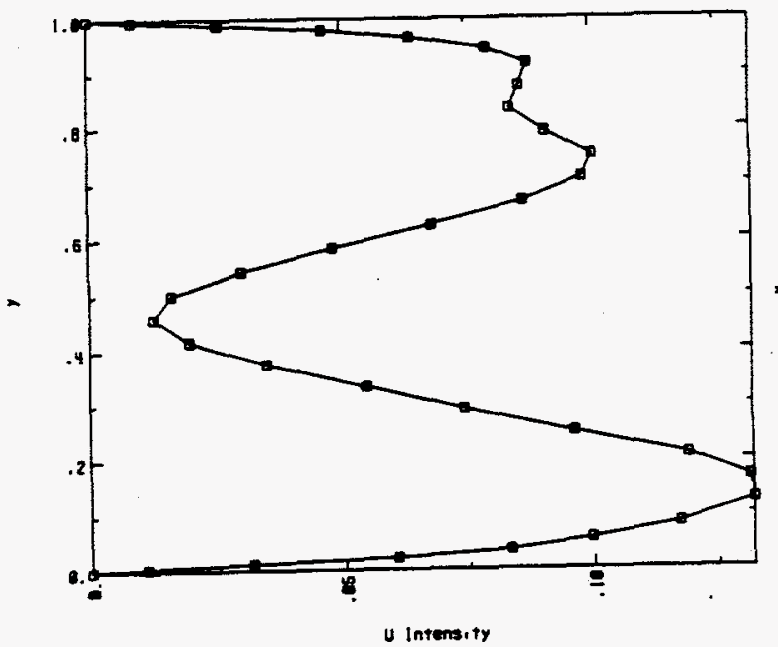

$x=2.0$

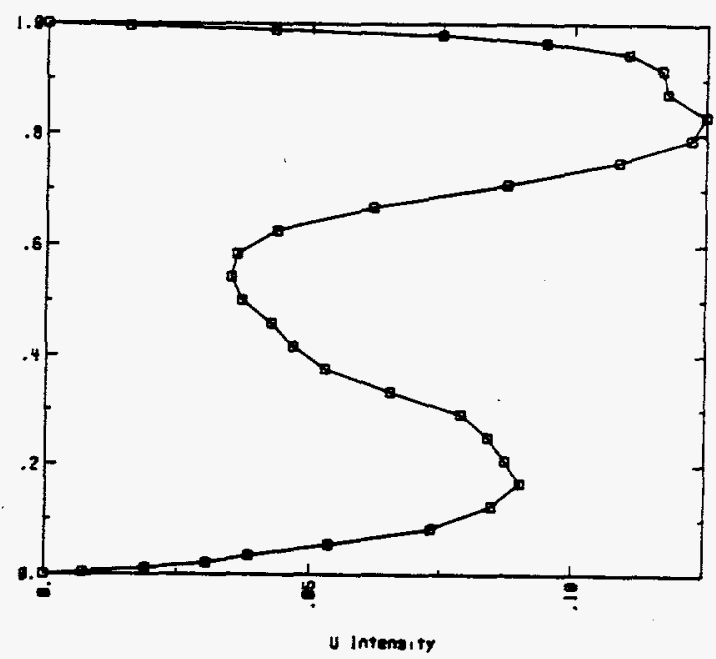

$x=6.0$

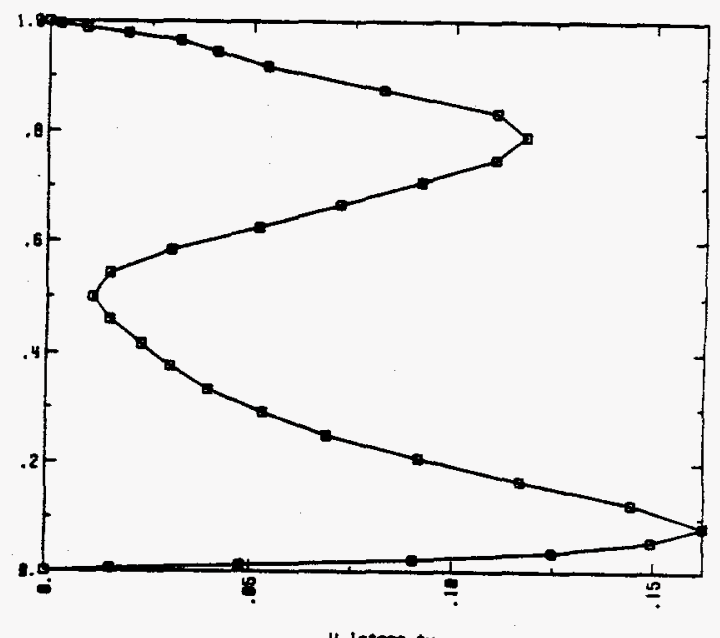

Figure 5.3.43 The LES predicted turbulent intensity, $\left\langle\bar{u}_{1}=2\right.$, , at selected locations for the $33 \times 245$ grid, $\Delta t=0.005$, and the SGS vorticity model with $C_{\omega}=0.2154$ and $\Delta_{f}=\Delta$. 
$x=1.0$

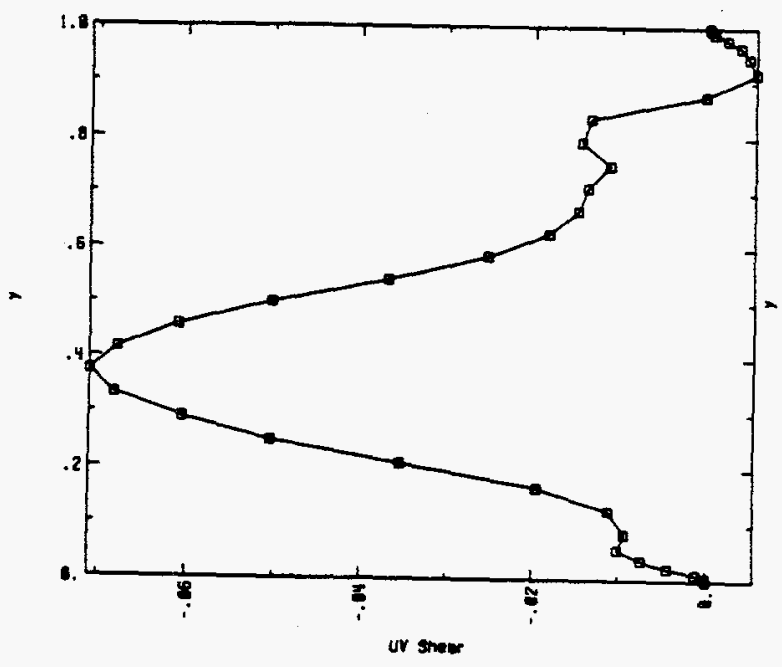

$x=\mathbf{3 . 0}$

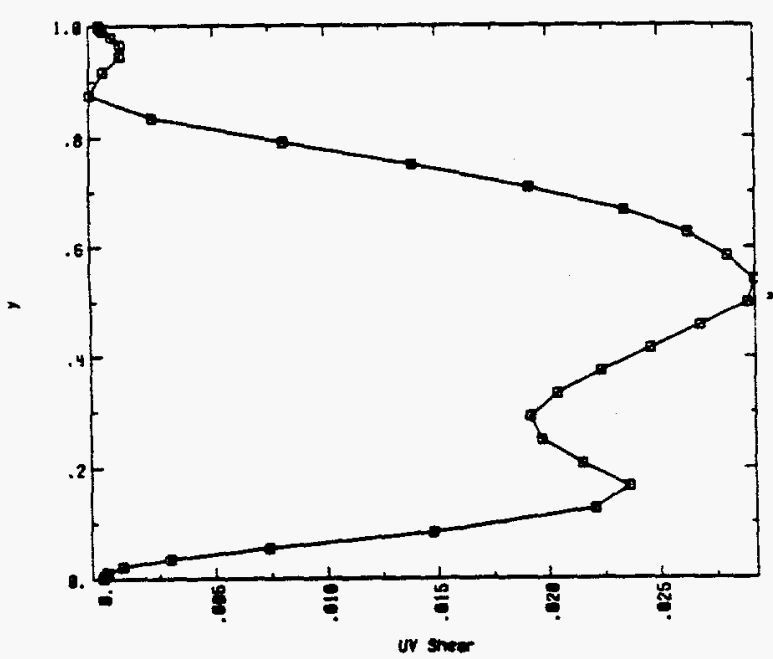

$x=2.0$

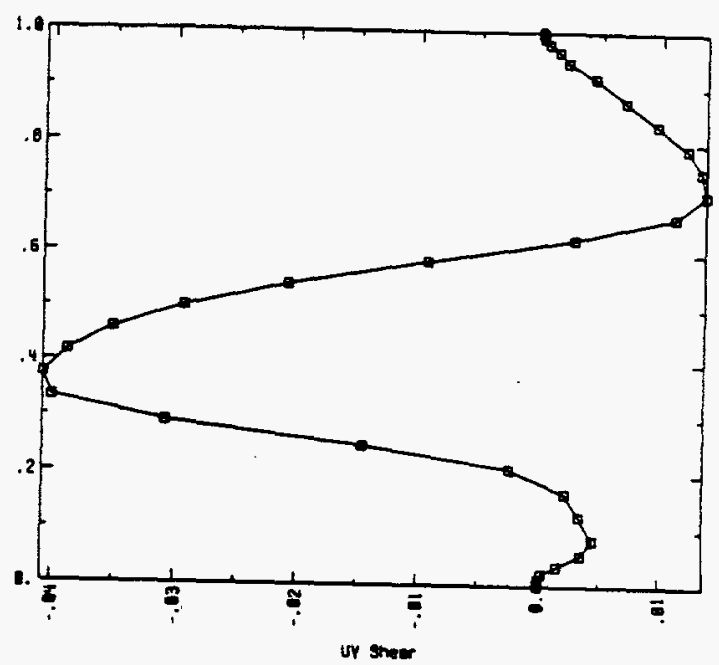

$x=6.0$

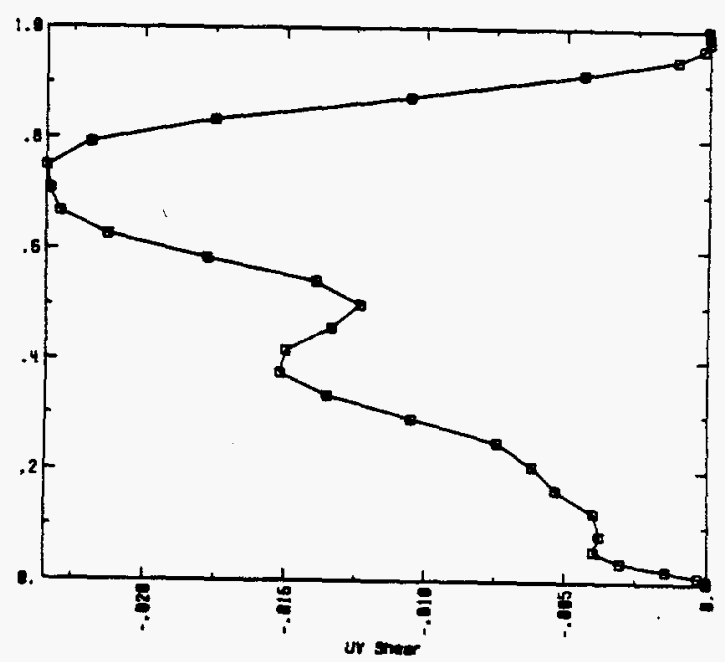

Figure 5.3.44 The LES predicted turbulent shear, $\left\langle\bar{u}_{1} " \bar{u}_{2}^{\prime \prime}\right\rangle$, at selected locations for the $33 \times 245$ grid, $\Delta t=0.005$, and the SGS vorticity model with $C_{\omega}=0.2154$ and $\Delta f=\Delta$. 

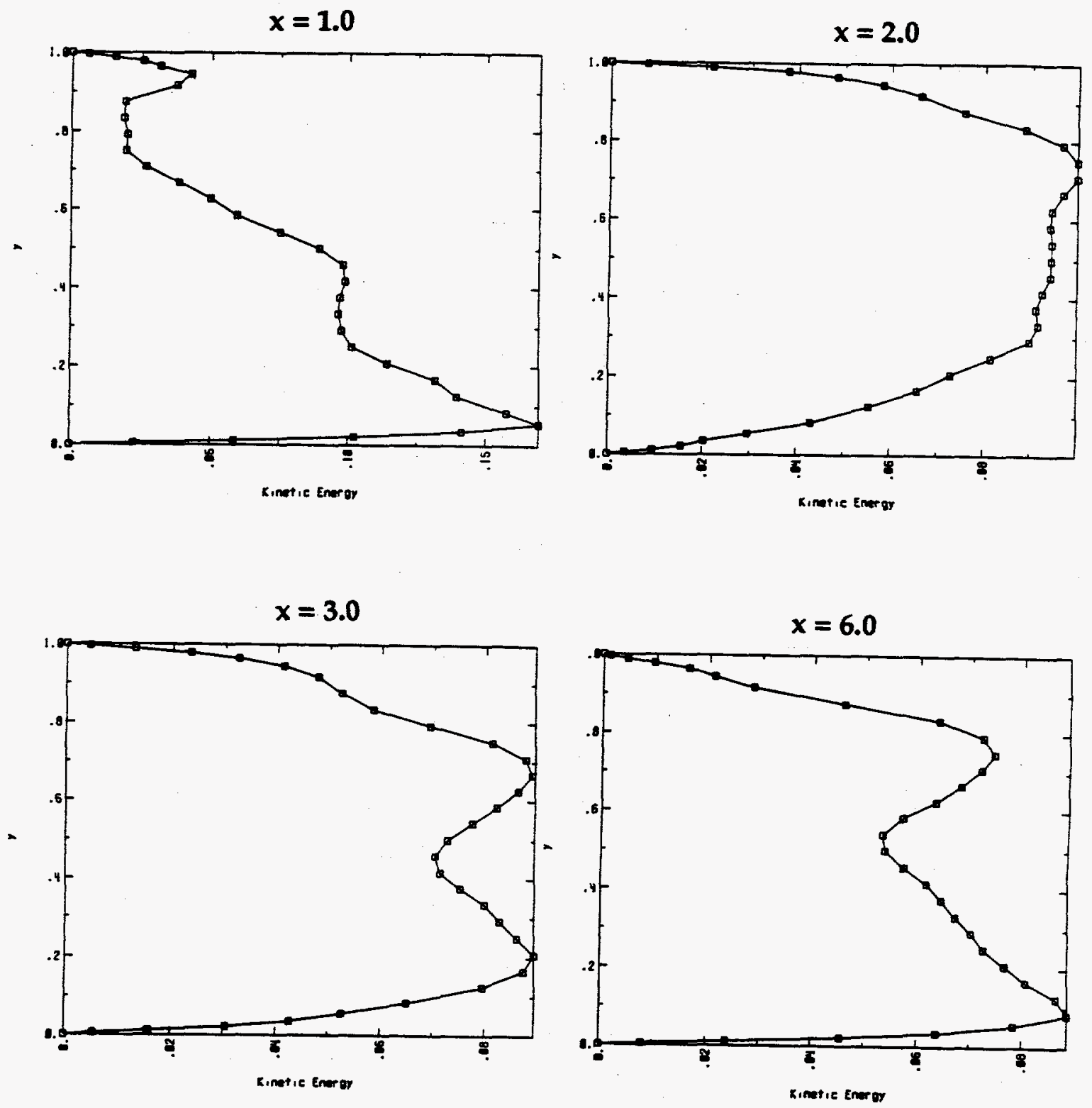

Figure 5.3.45 The LES predicted turbulent kinetic energy at selected locations for the $33 \times 245$ grid, $\Delta t=0.005$, and the SGS vorticity model with $C_{\omega}=0.2154$ and $\Delta_{f}=\Delta$. 
$\mathbf{x}=\mathbf{1 . 0}$

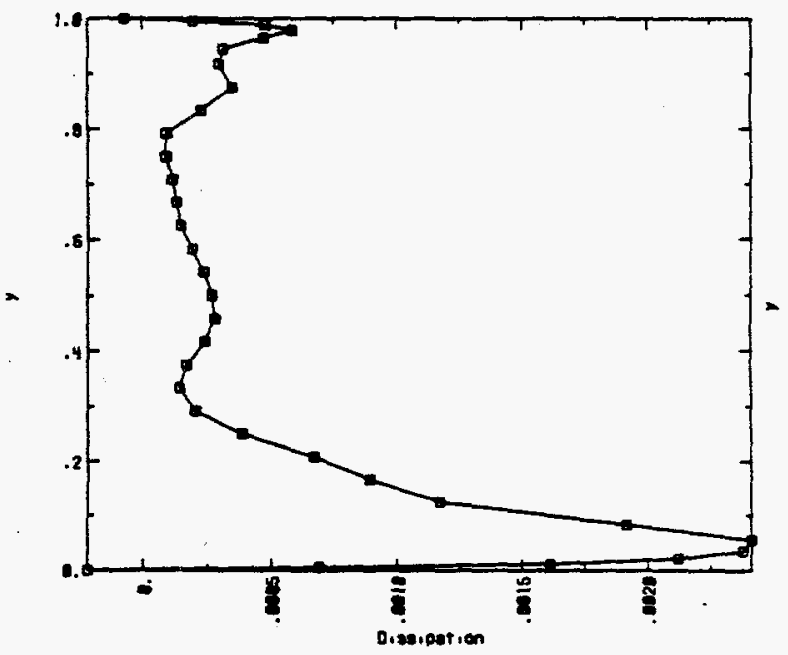

$\mathbf{x}=\mathbf{3 . 0}$

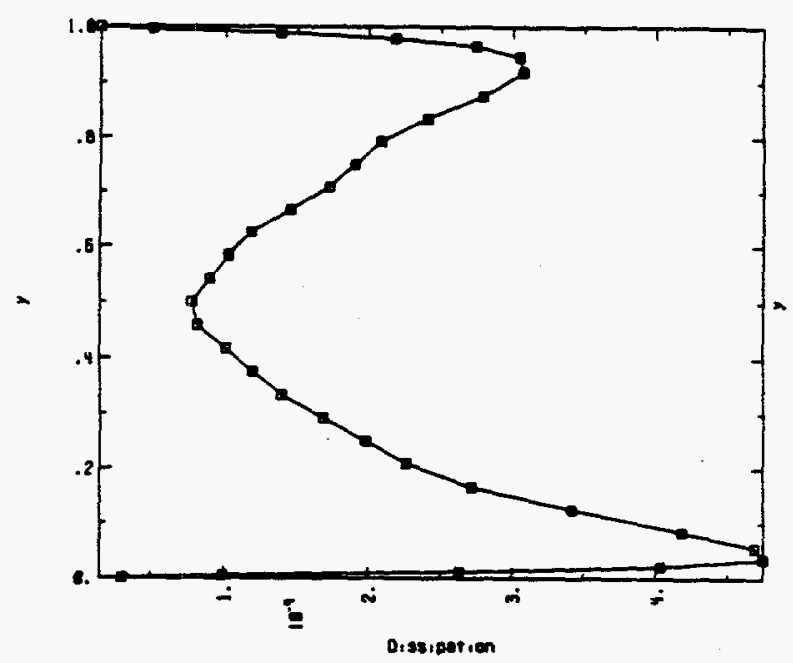

$\mathbf{x}=\mathbf{2 . 0}$

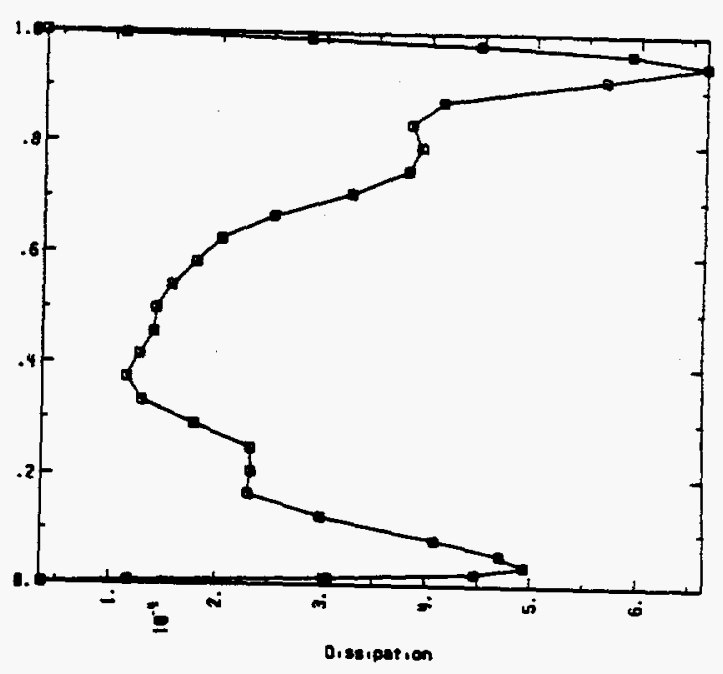

$x=6.0$

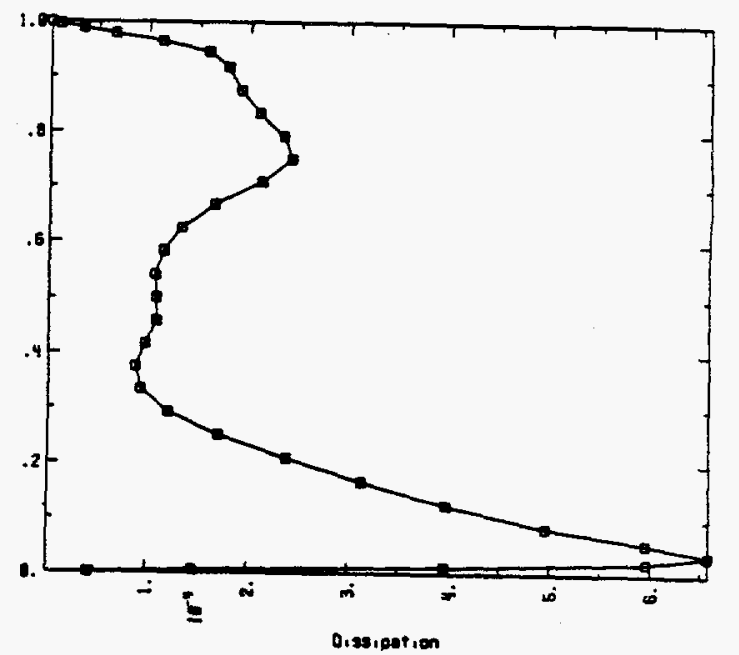

Figure 5.3.46 The LES predicted turbulent rate of dissipation at selected locations for the $33 \times 245$ grid, $\Delta t=0.005$, and the SGS vorticity model with $C_{\omega}=0.2154$ and $\Delta f=\Delta$. 

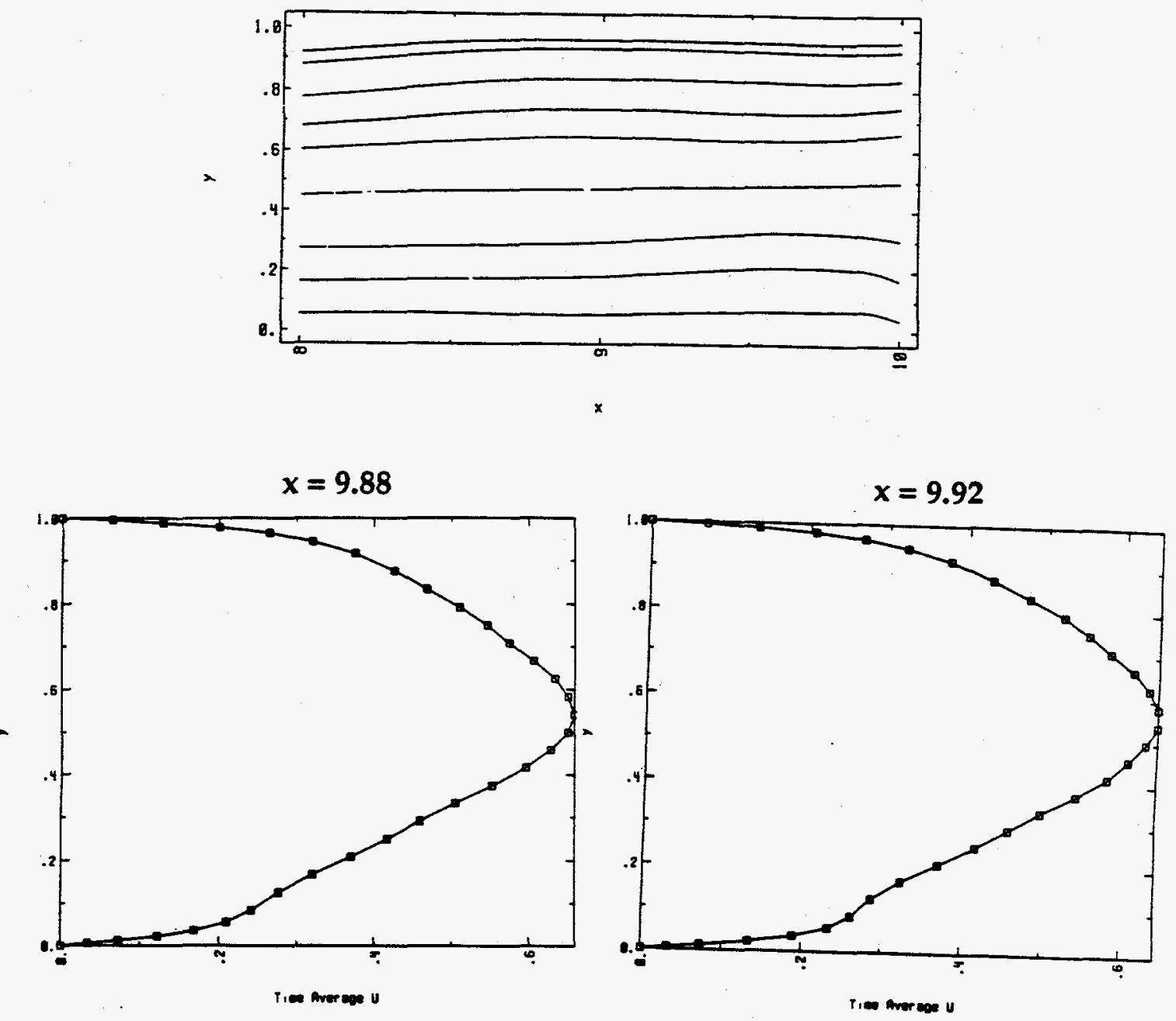

$x=9.96$
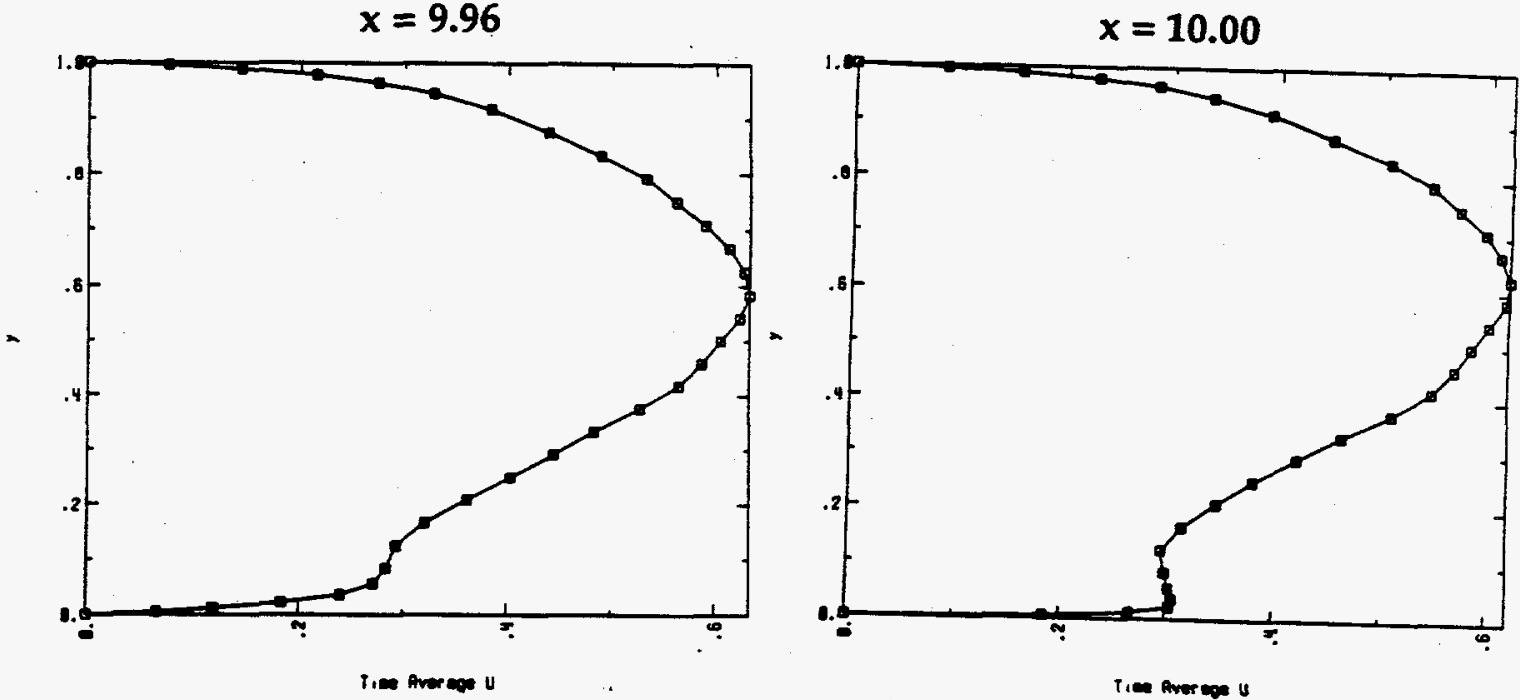

Figure 5.3.47 The LES predicted time-averaged velocity, $\left\langle\bar{u}_{1}\right\rangle$, at the channel outlet for the $33 \times 245$ grid, $\Delta t=0.005$, and the SGS vorticity model with $C_{\omega}=0.2154$ and $\Delta_{f}=\Delta$ 


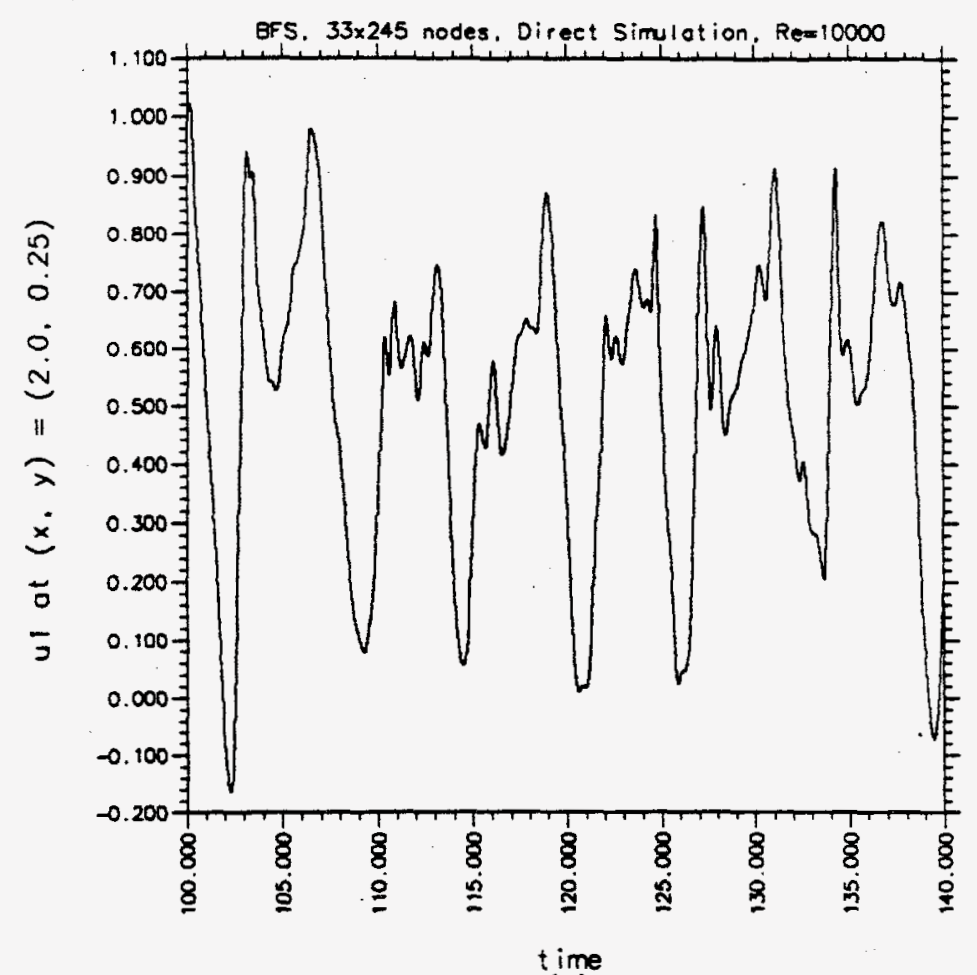

(a)

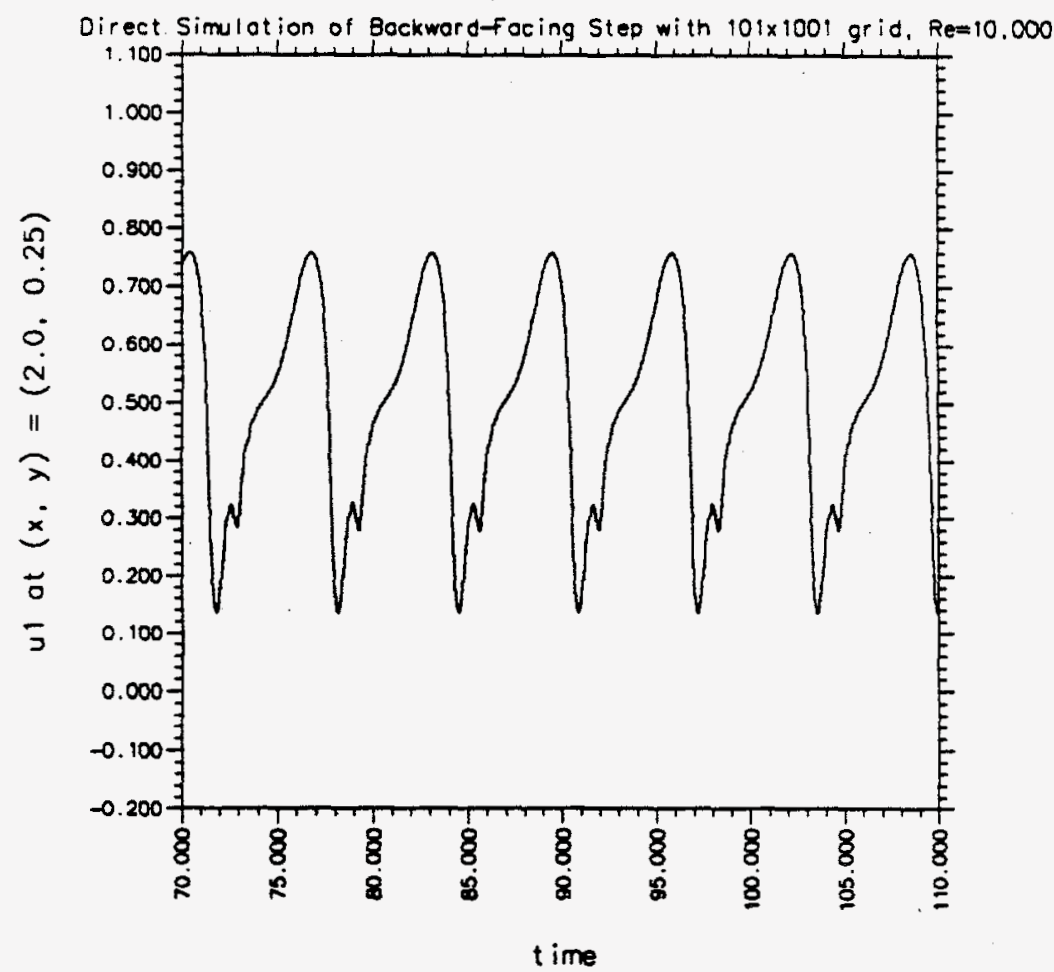

(b)

Fig. 5.3.48 Extended time histories of $u_{1}$ at $(x, y)=(2.0,0.25)$ for a) a DS with the $33 \times 245$ grid and $\Delta t=0.005$ and $b$ ) the DS with the $101 \times 1001$ grid. 


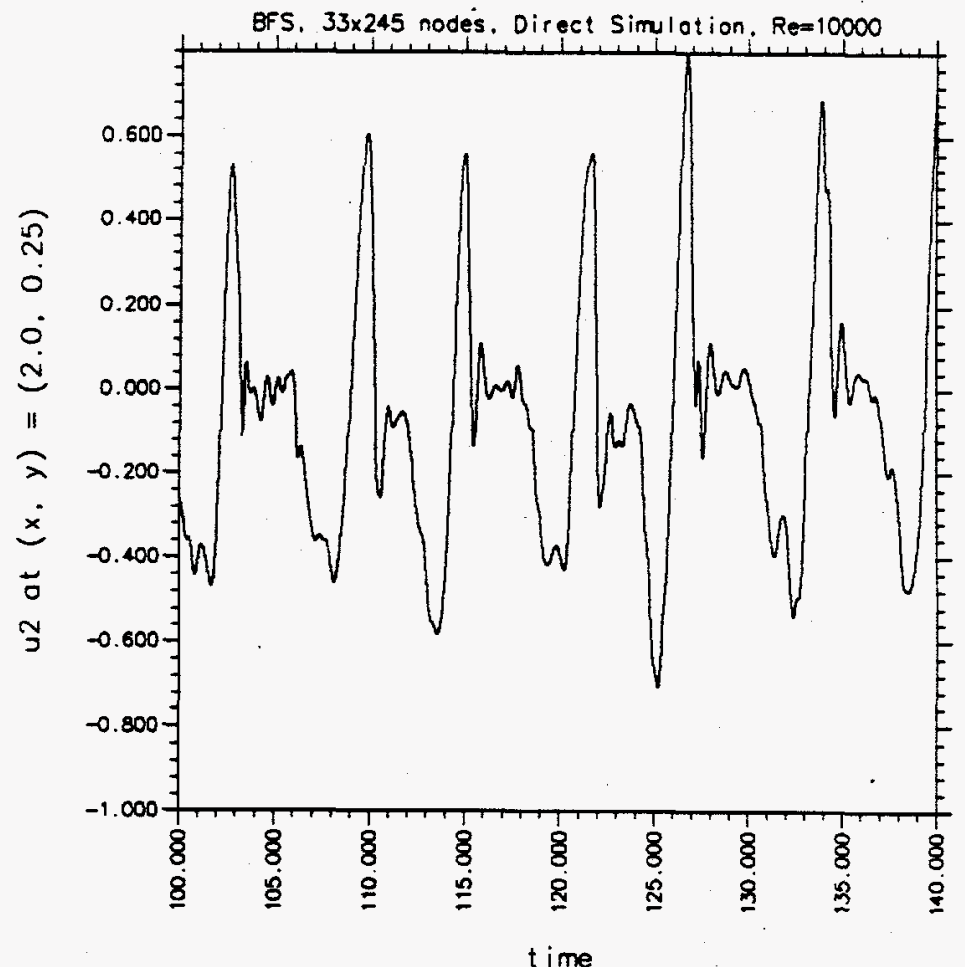

(a)

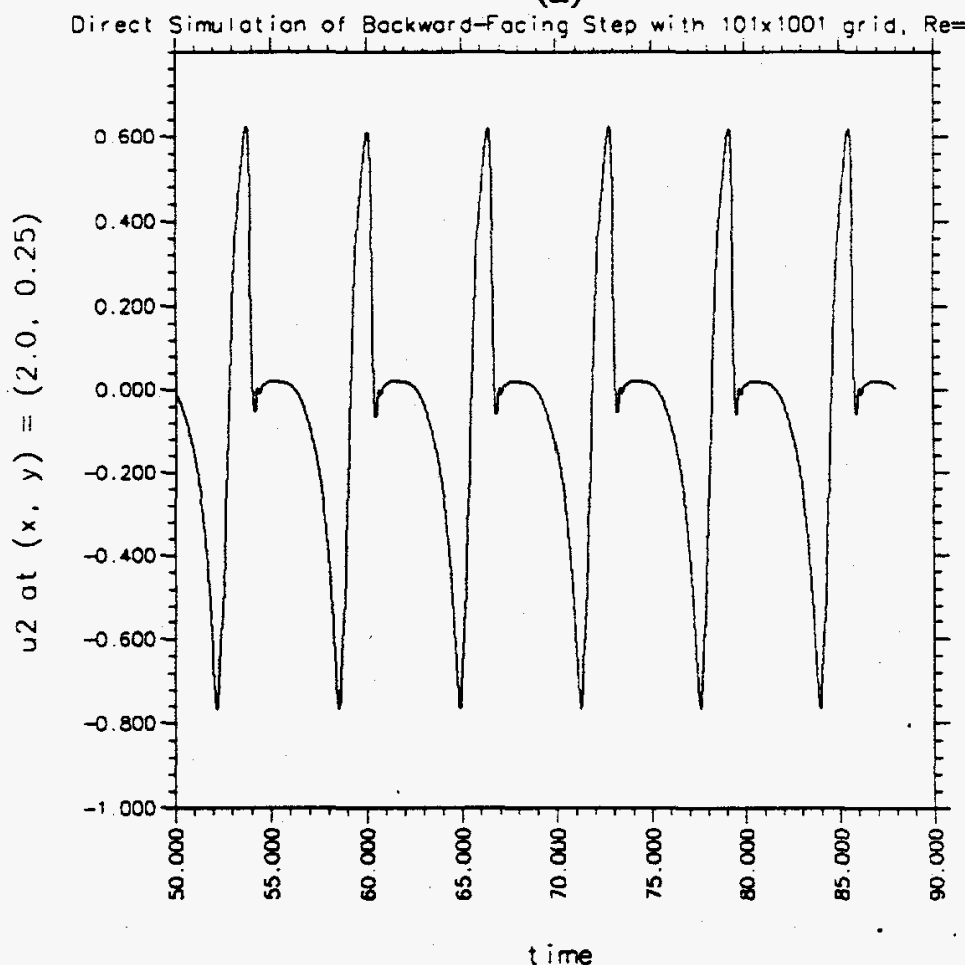

(b)

Fig. 5.3.49 Extended time histories of $u_{2}$ at $(x, y)=(2.0,0.25)$ for a) a DS with the $33 \times 245$ grid and $\Delta t=0.005$ and $b$ ) the DS with the $101 \times 1001$ grid. 


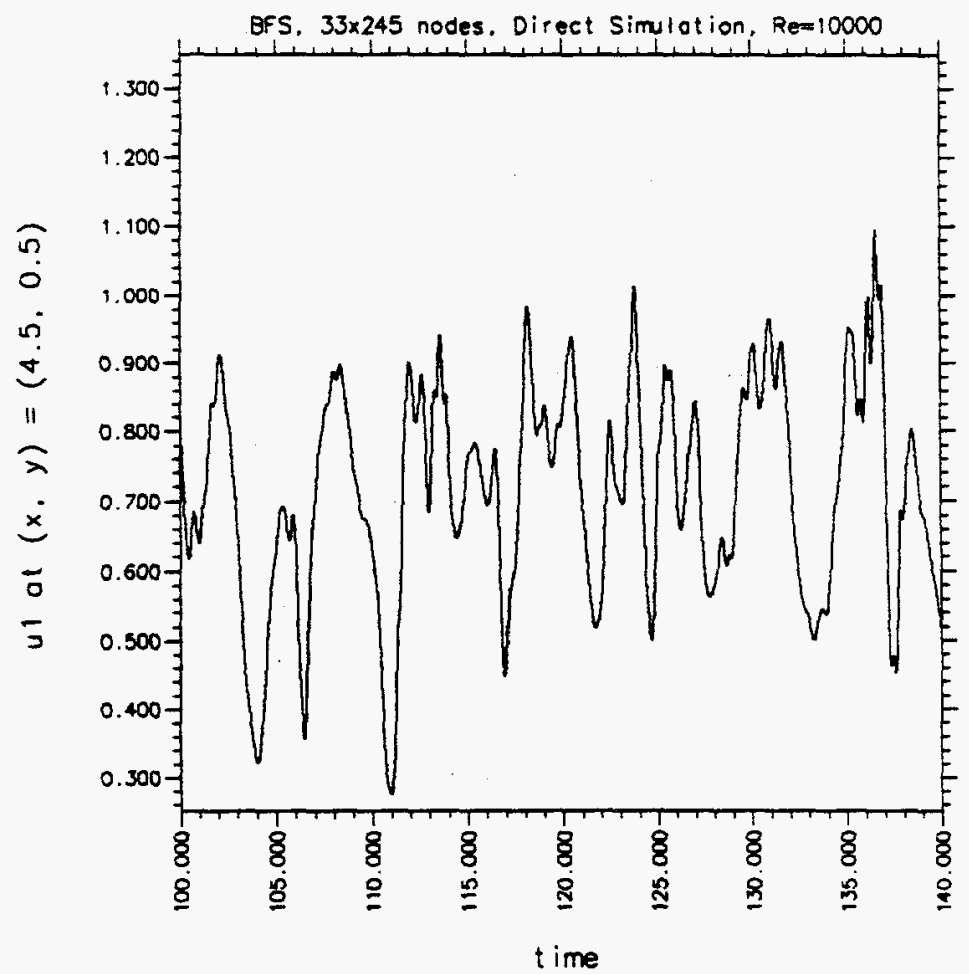

(a)

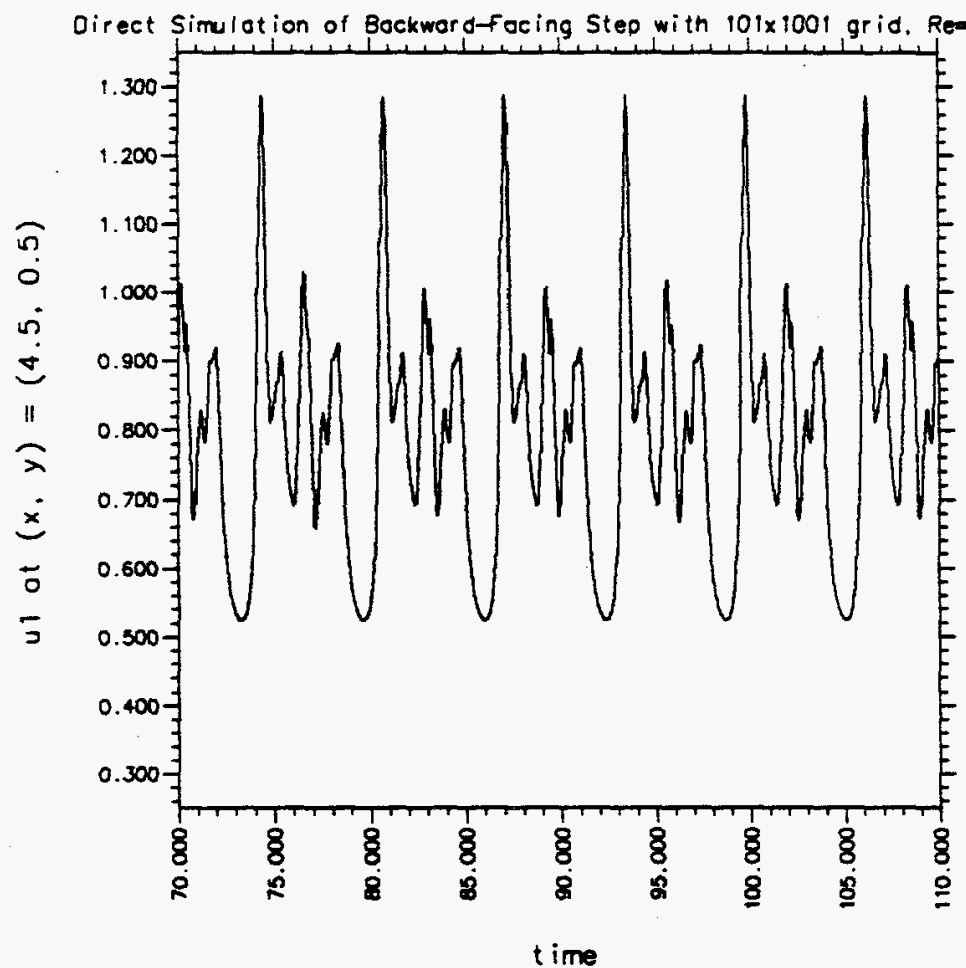

(b)

Fig. 5.3.50 Extended time histories of $u_{1}$ at $(x, y)=(4.5,0.5)$ for a) a DS with the $33 \times 245$ grid and $\Delta t=0.005$ and $b$ ) the DS with the $101 \times 1001$ grid. 


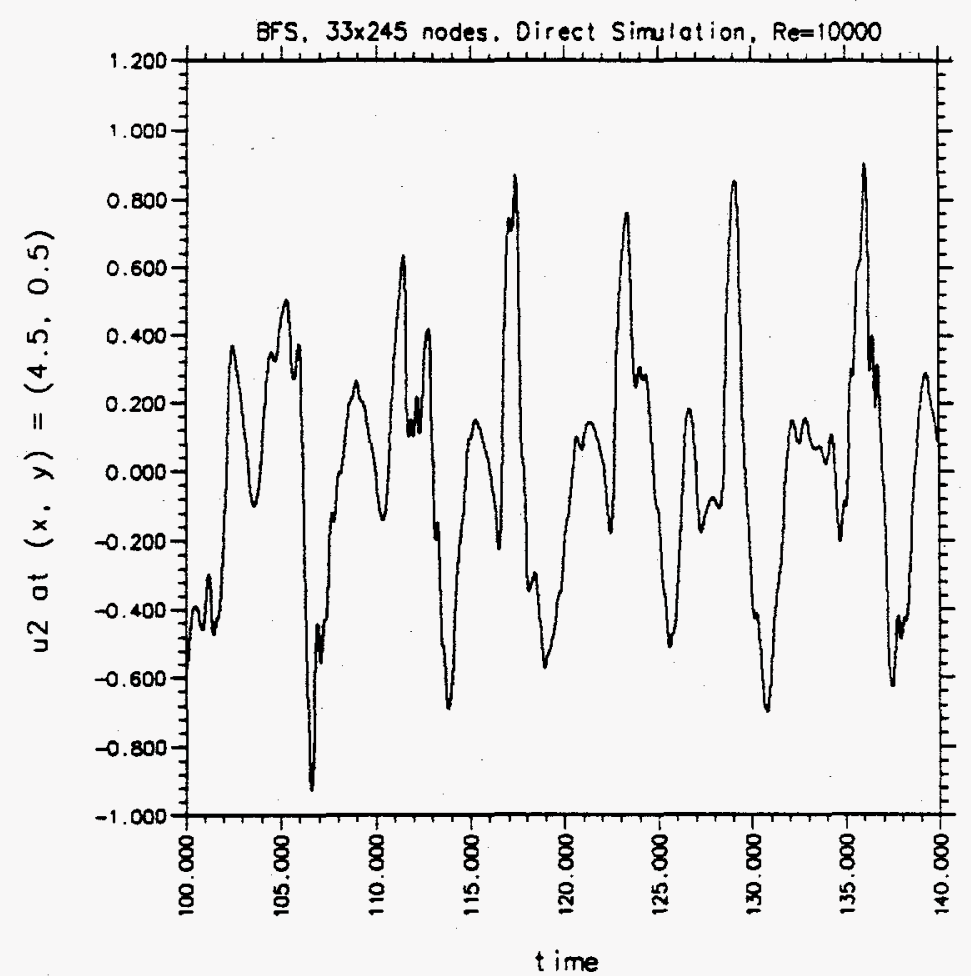

(a)

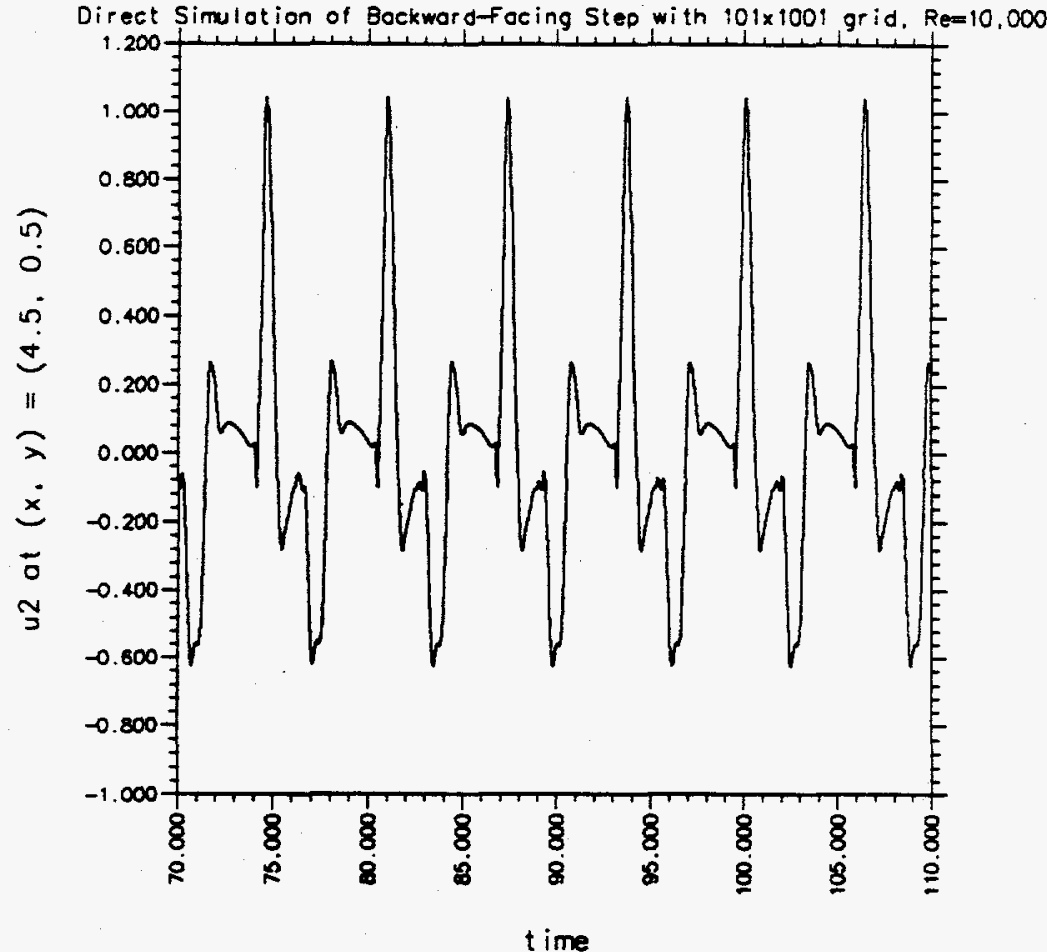

(b)

Fig. 5.3.51 Extended time histories of $u_{2}$ at $(x, y)=(4.5,0.5)$ for a) a DS with the $33 \times 245$ grid and $\Delta t=0.005$ and b) the DS with the $101 \times 1001$ grid. 
BF3. $33 \times 245$ nodes, Direct Simulation, Re 10000

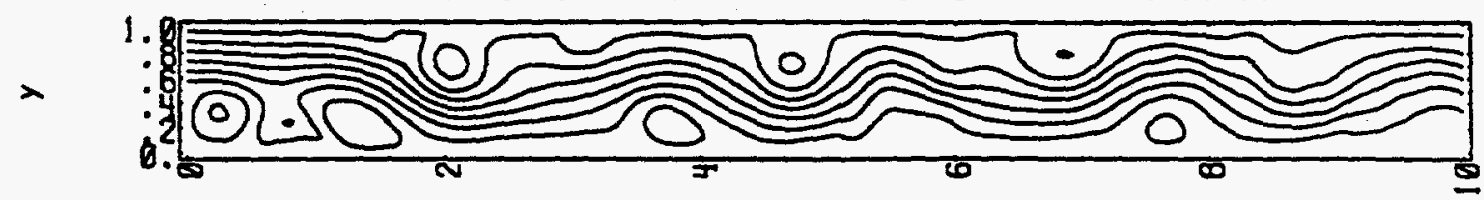

$x$

$\mathbf{x}=\mathbf{1 . 0}$

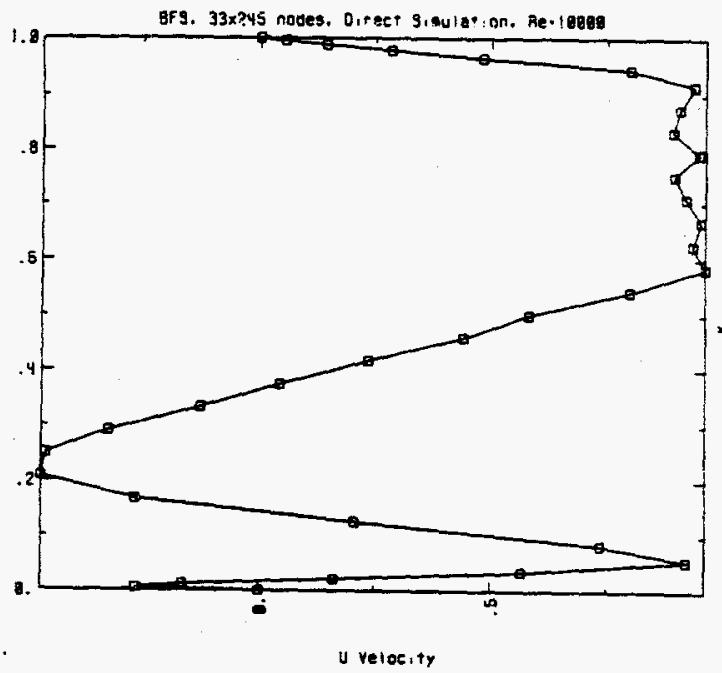

$\mathbf{x}=\mathbf{3 . 0}$

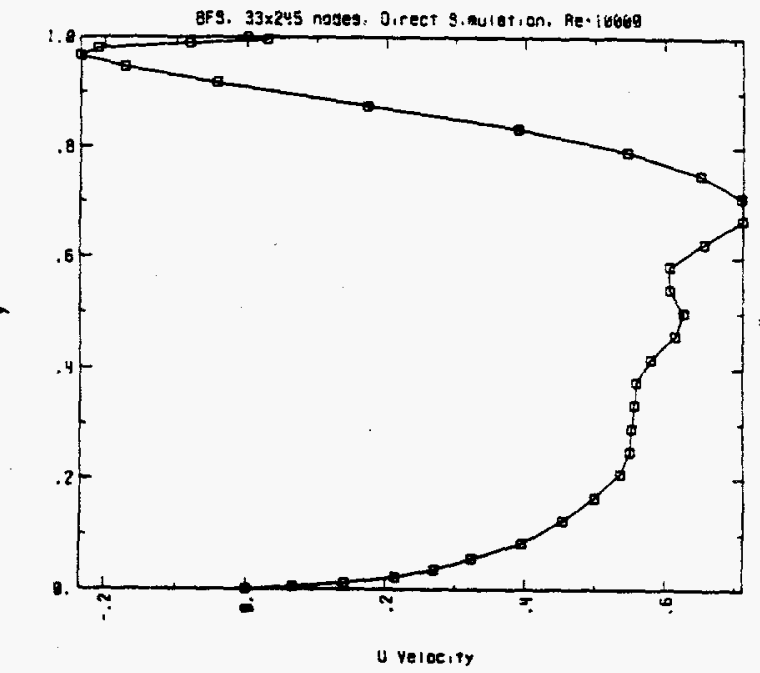

$\mathbf{x}=\mathbf{2 . 0}$

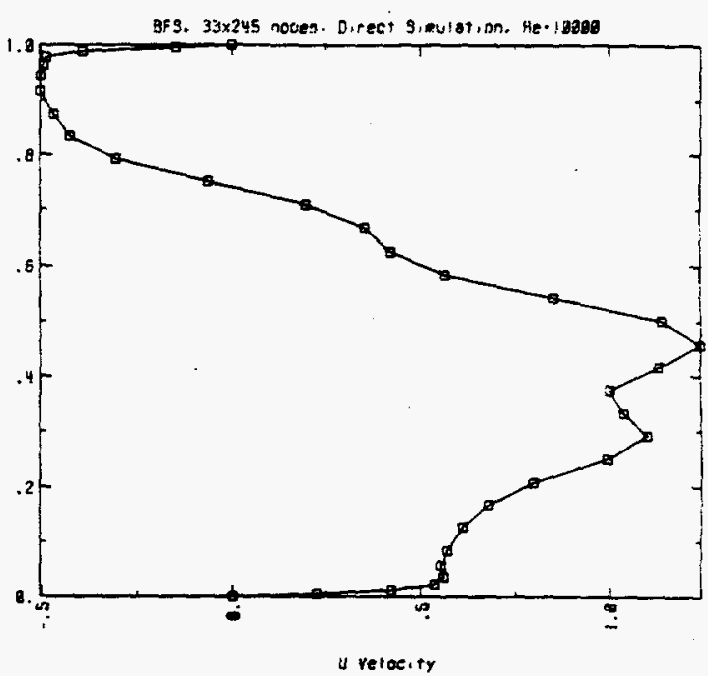

$x=6.0$

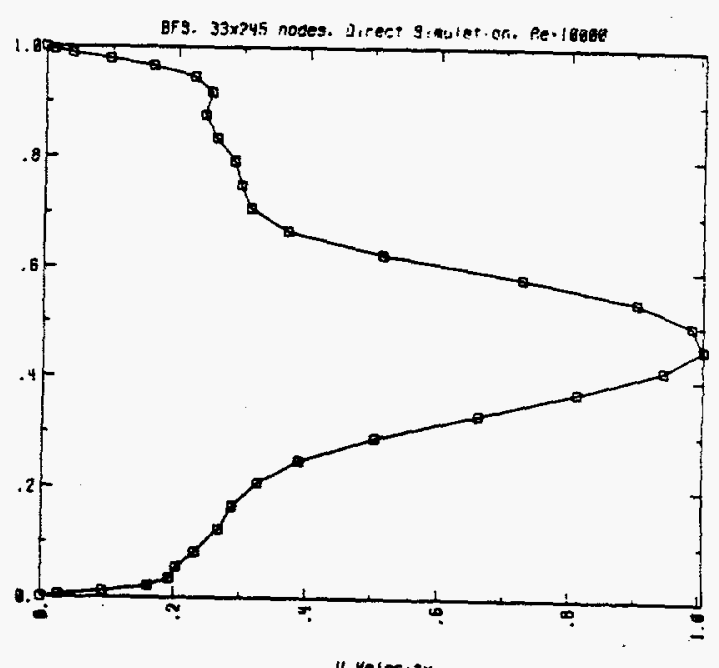

4 Vefocity

Fig. 5.3.52 The DS $u_{1}$ velocity profiles at selected locations for the $33 \times 245$ grid and $\Delta t=0.005$ at the shown streamfunction snapshot in time. 
Table 5.3.4 Time-averaged DS for the $33 \times 245$ grid and $\Delta t=0.005$.

\begin{tabular}{|l|c|c|c|}
\hline \multirow{2}{*}{} & \multicolumn{2}{|c|}{$\begin{array}{c}\text { x-location for separation } \\
\text { and reattachment points }\end{array}$} & $\begin{array}{c}\text { relative change } \\
\text { between cases } \\
(\%)\end{array}$ \\
\cline { 2 - 4 } & $\begin{array}{c}\text { time average } \\
\text { for 10,000 } \\
\text { time steps }\end{array}$ & $\begin{array}{l}\text { time average } \\
\text { for 20,000 } \\
\text { time steps }\end{array}$ & \\
\hline \hline \multirow{2}{*}{ Bottom Wall } & 0.005 & 0.007 & 28.6 \\
& 0.077 & 0.064 & 20.3 \\
& 0.695 & 0.460 & 51.1 \\
& 2.459 & 1.745 & 40.9 \\
& & 0.902 & \\
& & 1.124 & 27.7 \\
& 2.072 & 1.622 & 22.8 \\
\hline
\end{tabular}




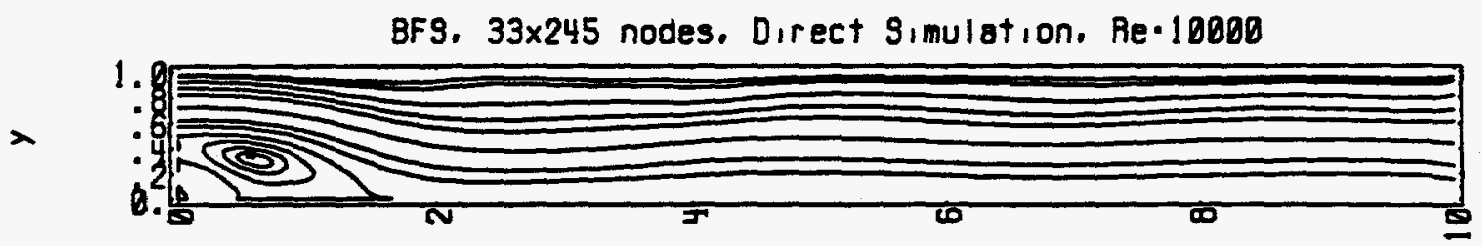

$\mathbf{x}$

Entire Computational Field

BFS. $33 \times 245$ nodes. Direct Simulation. Re-10080

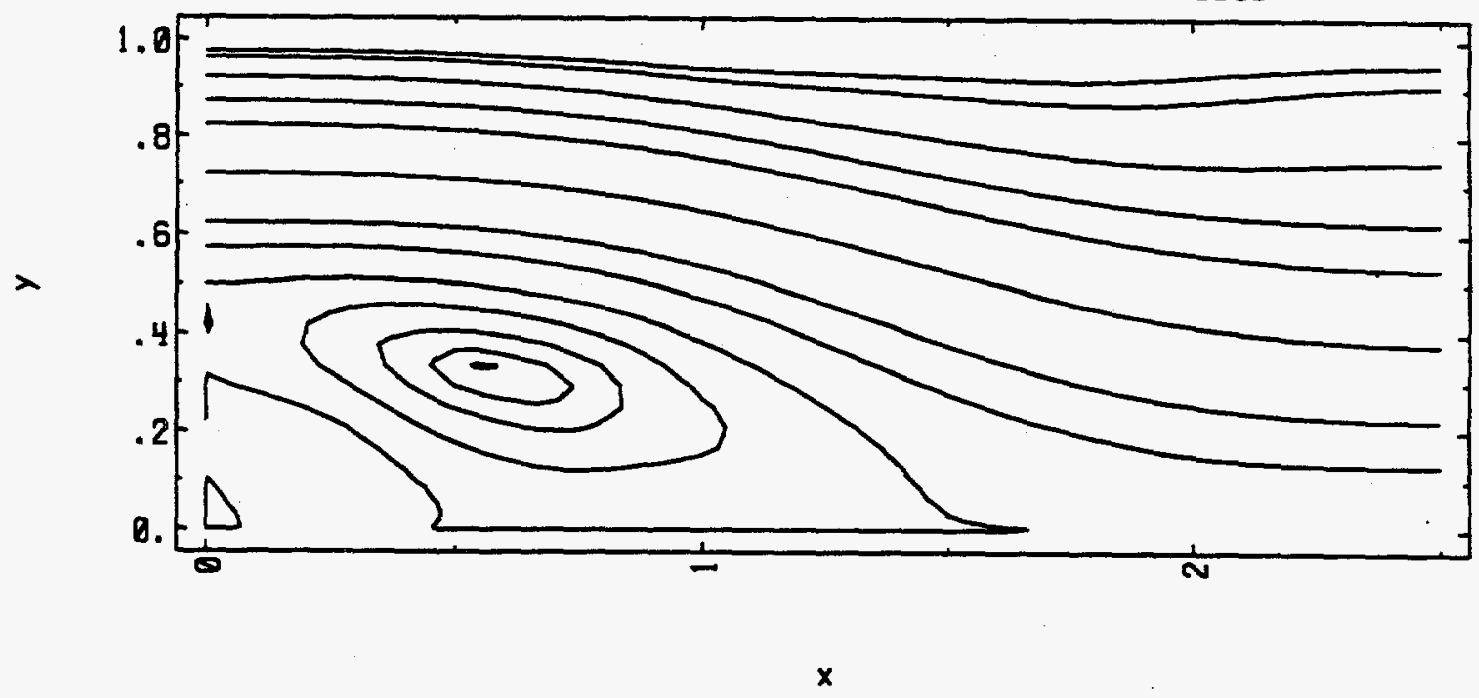

Enlargement of Major Recirculation Zone

Figure 5.3.53 Time-average DS stream function for the $33 \times 245$ grid and $\Delta t=$ 0.005 for a time-averaging duration of 20,000 time steps. 


\subsubsection{LES With Explicit Filtering of the Advection Term}

In Section 5.3.1, the LES calculations were performed for a SGS filter width equivalent to the grid size $(\Delta f=\Delta)$. It was shown in Section 3.3.2 that by using onepoint Gaussian quadrature and the centroid advection velocity simplification, the 'exact' advection term with explicit filtering reduces to the approximate advection term without explicit filtering. Therefore, with the finite element approach used here, the calculations in Section 5.3.1 are the results for explicit filtering at a filter width equivalent to the grid size. In this section, we attempt to choose a filter width that is greater than the grid size for the explicit filtering of the advection term. The discrete form of the explicitly filtered advection term using the centroid advection velocity simplification and one-point Gaussian quadrature is developed in Section 3.3.2 and the final form is equation 3.11. It should also be noted that the length scale used in the SGS vorticity model is $l=\left(C_{\omega} \Delta f\right)^{3}$.

For $\Delta_{f}=\Delta$ the best results were found with the $33 \times 245$ grid with mesh grading only in the wall region and a core uniform grid size of $\Delta=0.0417$. In this section, we solve the backward-facing step problem with $\Delta f=3 \Delta$ for two different grid sizes. (The choice of $\Delta f=3 \Delta$ or an odd integer factor of $\Delta$ is done for ease in calculation and programming, as explained in Section 3.3.2.)

For the first case we use the $33 \times 245$ node grid so that the filter width (away for the walls) is $\Delta f=3 \Delta=3(0.0417)=0.125$. In this case, we are seeking the potential benefits of explicit filtering with $\Delta f=3 \Delta$ as compared to the case in Section 5.3.1 with $\Delta f=\Delta$ for approximately the same computational effort. For the second case we chose $\Delta f=0.0417=3 \Delta$ so that the core uniform grid size is $\Delta=0.0417 / 3=0.0139$. The wall area is more finely discretized with mesh grading normal to the walls. The computational field is a $75 \times 722$ node grid with 3 graded elements at the wall with a 2 to 
1 grading ratio (i.e., the third element farthest from the wall is twice as big as the element adjacent to the wall). The $75 \times 722$ grid is shown in Figure 5.3.54. This grid represents approximately a factor of 6 increase in the total number of elements over the total for the $33 \times 245$ grid. Since the filter width for the $75 \times 722$ grid with $\Delta f=3 \Delta$ is the same as that for the $33 \times 245$ grid with $\Delta f=\Delta$, the demands on the SGS model should be the same in both cases. Any improvements in the solution with the $75 \times 722$ grid should be due to the improvement in numerical discretization.

We first present the results for the $33 \times 245$ grid with $\Delta f=3 \Delta=0.125$. Extended time histories are compared to the DS in Figures 5.3.55, 57, 59, and 61. The LES results with $\Delta f=3 \Delta$ are approximately periodic, but the velocity magnitudes and the time history characteristics are much different than the DS and the LES with $\Delta f=\Delta$ (see Figures 5.3.27, 29, 31, and 33). The LES results with $\Delta f=3 \Delta$ are significantly damped near the step at $(x, y)=(2.0,0.25)$ with less damping downstream at $(x, y)=(4.5,0.5)$. The power spectrum indicates that the LES is not accurately capturing the large-scale motion (Figures 5.3.56, 58, 60, and 62).

At approximately 216 time units (approximately 43,200 steps for $\Delta t=0.005$ ) the LES with the $33 \times 245$ grid and $\Delta f=3 \Delta$ goes unstable (i.e., the velocity tends to infinity). Stream function plots and corresponding contour plots of $v_{T}$ for 6.36 time units (i.e., the DS cycle) just before the run goes unstable are shown in Figure 5.3.63. The stream function plots show an elongated recirculation zone unlike that for the DS or the LES with $\Delta f=\Delta$ (see Figure 5.3.35) ${ }^{1}$. The stream function plots show coalescing of the recirculation zones at about 6 to 7 step heights downstream, which was not seen with the DS. The contours for $v_{T}$ with $\Delta_{f}=3 \Delta$ have much different

\footnotetext{
${ }^{1}$ Because the LES for $\Delta f=3 \Delta$ is significantly different than the DS, no attempt is made to shift the LES in time for direct comparison to the DS.
} 
characteristics than those for $\Delta f=\Delta$ with the $33 \times 245$ grid (see Figure 5.3.36). The magnitude and variation of $v_{T}$ for a snapshot in time is shown with profile plots for selected locations in Figure 5.3.64. Since $v_{T}$ is proportional to $\Delta_{f}{ }^{3}$, we see an increase in magnitude for $\Delta f=3 \Delta$ in the core of the flow over the case with $\Delta f=\Delta$ (see Figure 5.3.37 for comparison). It should be noted that filtering is performed on elements whose centroid is at least one-half the filter width form the boundaries. Therefore, $v_{T}$ values in the wall region are unaffected unless the wall vorticity gradients are altered.

The instability with the $33 \times 245$ grid for $\Delta f=3 \Delta$ is caused by the large effective viscostiy (i.e., $v_{e f f}=v+v_{T}$ ) and a subsequent reduction in the stability time step. In the cases for $\Delta f=\Delta$, the stability time step was Courant limit controlled (i.e., $\Delta t<\Delta / V$ for grid size $\Delta$ and characteristic velocity $V$. In this case, with $\Delta f=3 \Delta$, the stability time step is diffusion limit controlled (i.e., $\Delta t<\Delta 2 / 2 v$ eff). The smallest nodal spacing for the $33 \times 245$ grid is $\Delta=0.0047$ at the walls and the maximum $\nu_{T}$ value is $8.903 \times 10^{-3}\left(v=10^{-4}\right)$ for the contour plots in Figure 5.3.63. Thus, the estimated diffusion limit is

$$
\Delta t_{\text {diff }}<\frac{(0.0047)^{2}}{2\left(8.908 \times 10^{-3}+1 \times 10^{-4}\right)} \equiv 0.001
$$

The estimated Courant limit for characteristic velocity $V=1.0$ is

$$
\Delta t_{\text {courear }}<\frac{0.0047}{1} \cong 0.005 \text {. }
$$

The time step used in the above reported claculations for the $33 \times 245$ grid with $\Delta f=3 \Delta$ was $\Delta t=0.005$. Thus, the run goes unstable because the time step exceeded the stability time step. 
In summary, the $33 \times 245$ grid with $\Delta f=\Delta$ and $\Delta t=0.005$ gave results that compared very well with the DS, however, the same grid with $\Delta f=3 \Delta$ not only gave highly damped results that compared poorly to the DS but a smaller stability time step is required. Therefore, setting $\Delta f=3 \Delta$ gave a much poorer result compared to $\Delta f=\Delta$ for the same field discretization.

We next present the results for the $75 \times 722$ grid with $\Delta f=3 \Delta=0.0417$. Extended time histories are compared to the DS in Figures 5.3.65, 67, 69, and 71. The LES results are approximately periodic, but the velocity magnitudes and time history characteristics are different than the DS. The variation in the period length is shown in Table 5.3.5. The power spectrum for the LES time histories is compared to the DS power spectrum in Figures $5.3 .66,68,70,72$. The power spectrum for $\bar{u}_{1}$ at $(x, y)=$ $(2.0,0.25)$ and $(4.5,0.5)$ are in fair agreement with the DS at large scales (i.e., low frequencies). However, the LES result shows higher values at small scales (i.e., high frequencies). This may be due to the unstable characteristic (i.e., high frequency oscillations) of the LES time histories. The power spectra for $\bar{w}_{2}$ show large differences between the LES and DS results. One interesting feature of the $\bar{\varkappa}_{2}$ time history at $(x, y)$ $=(4.5,0.5)$ (Figure 5.3.71) is the jump to lower velocity magnitudes that occurs at approximately 65 time units. This unusual characteristic was not seen in any of the previously presented DS or LES results.

At approximately 107 time units, the LES with the $75 \times 722$ grid and $\Delta f=3 \Delta$ goes unstable. Stream function plots and corresponding contour plots of $v_{T}$ for 6.36 time units, just before the run goes unstable, are shown in Figure 5.3.73. The time period for these LES stream function plots was chosen so that they could be compared to the DS results and LES results in Figure 5.3 .35 (i.e., the LES results for the $75 \times 722$ grid are shifted in time such that a $\bar{u}_{1}$ minimum at $(x, y)=(2.0,0.25)$ matches with the 
time that the $\bar{u}_{1}$ minimum occurs in the DS). The stream function plots are in good agreement with the DS.

The $v_{T}$ contours for the $75 \times 722$ grid with $\Delta_{f}=3 \Delta$ in Figure 5.3.73 are much different than those for the $33 \times 245$ grid with $\Delta f=\Delta$ in Figure 5.3.36 and with $\Delta f=3 \Delta$ in Figure 5.3.63. The magnitude and variation of $v_{T}$ for a snapshot in time is shown with profile plots for selected locations in Figure 5.3.74.

The instability with the $75 \times 722$ grid for $\Delta f=3 \Delta$ is caused by the large effective viscosity and a subsequent reduction in the stability time step. The smallest nodal spacing for the $75 \times 722$ grid is $\Delta=0.0063$ at the walls and the maximum $v_{T}$ value is $4.645 \times 10^{-3}$ for the contour plots shown in Figure 5.3.73. Thus, the estimated diffusion limit is

$$
\Delta t_{\text {diff }}<\frac{(0.0063)^{2}}{2\left(4.645 \times 10^{-3}+1 \times 10^{-4}\right)}=0.004 \text {. }
$$

The estimated Courant limit for $V=1.0$ is

$$
\Delta t_{\text {Courent }}<\frac{0.0063}{1}=0.006 \text {. }
$$

The time step used for the caluclations with the $75 \times 722$ grid and $\Delta f=3 \Delta$ was $\Delta t=$ 0.005 . Thus, the run goes unstable because the time step exceeded the stability time step.

The solution for the finer $75 \times 722$ grid with $\Delta_{f}=3 \Delta$ is an improvement over that for the $33 \times 245$ grid with $\Delta_{f}=3 \Delta$, but neither solution gives an improved solution over that for the $33 \times 245$ grid with $\Delta f=\Delta$. The reduction in the stability time step with $\Delta f=3 \Delta$ is also an undesirable result. 
258

In summary, the method used here to choose a filter width that is greater than the grid size for the explicit filtering of the advection term, as described in Section 3.3.2, gave poorer results than those for $\Delta f=\Delta$ and requires more computational effort. Evaluation of the results for two grid sizes of a $33 \times 245$ grid and a $75 \times 722$ grid with $\Delta_{f}$ $=3 \Delta$ for both cases indicates that finer discretization is required for the $\Delta f=3 \Delta$ to obtain similar accuracy as achieved with $\Delta f=\Delta$. Therefore, there appears to be no benefit in choosing the filter width greater than the grid size with the numerical approach used in this study (ie., the explicitly filtered advection term using the centroid advection velocity simplification and 1-point Gaussian quadrature). 


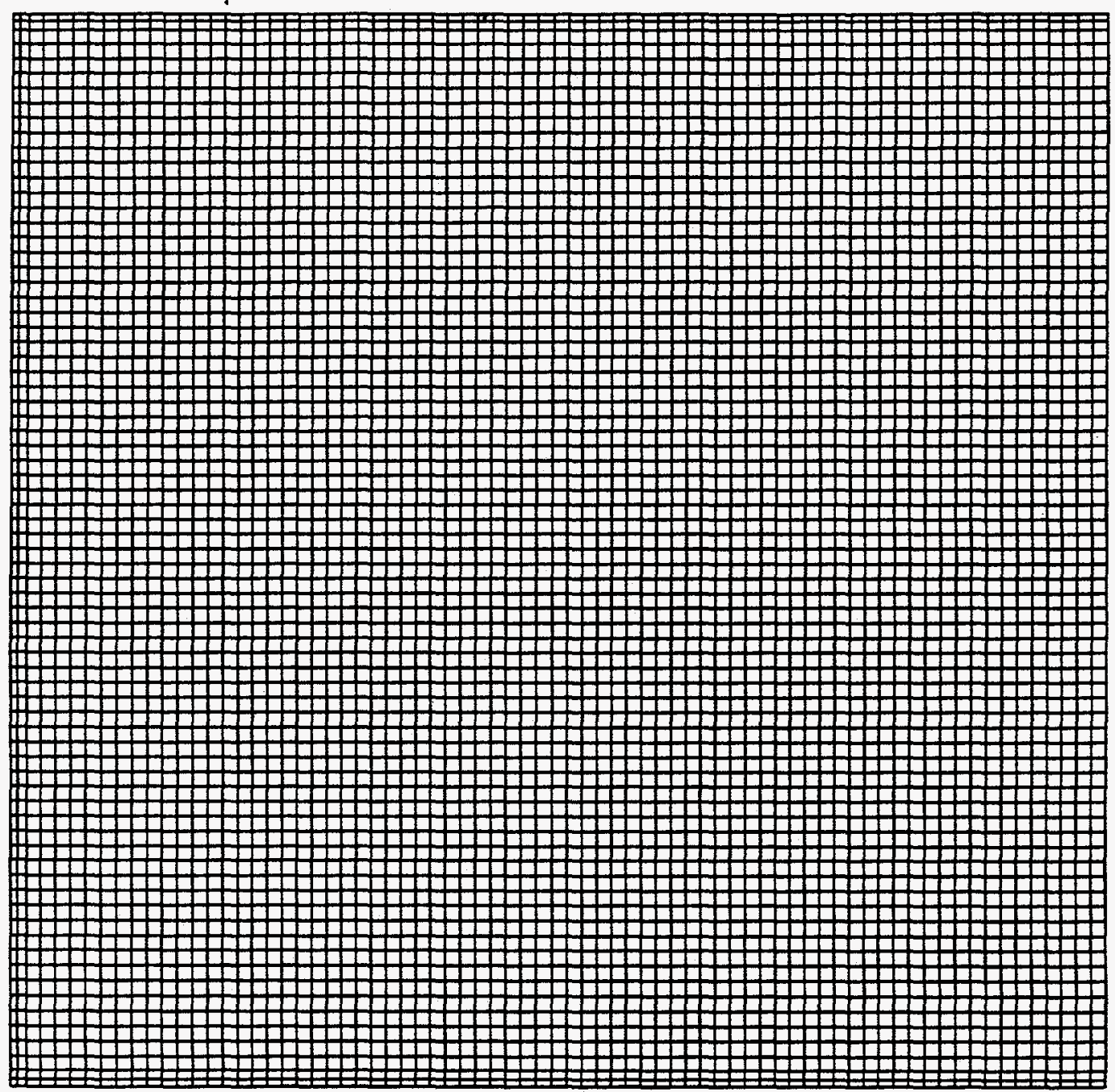

Entrance Region Showing 2 to 1 Mesh Grading Normal to Walls

$\left(\Delta x_{\min }=\Delta y_{\min }=0.0063\right)$

Figure 5.3.54 Geometry and mesh description for the 75x 722 node grid with 3 graded elements at wall. 


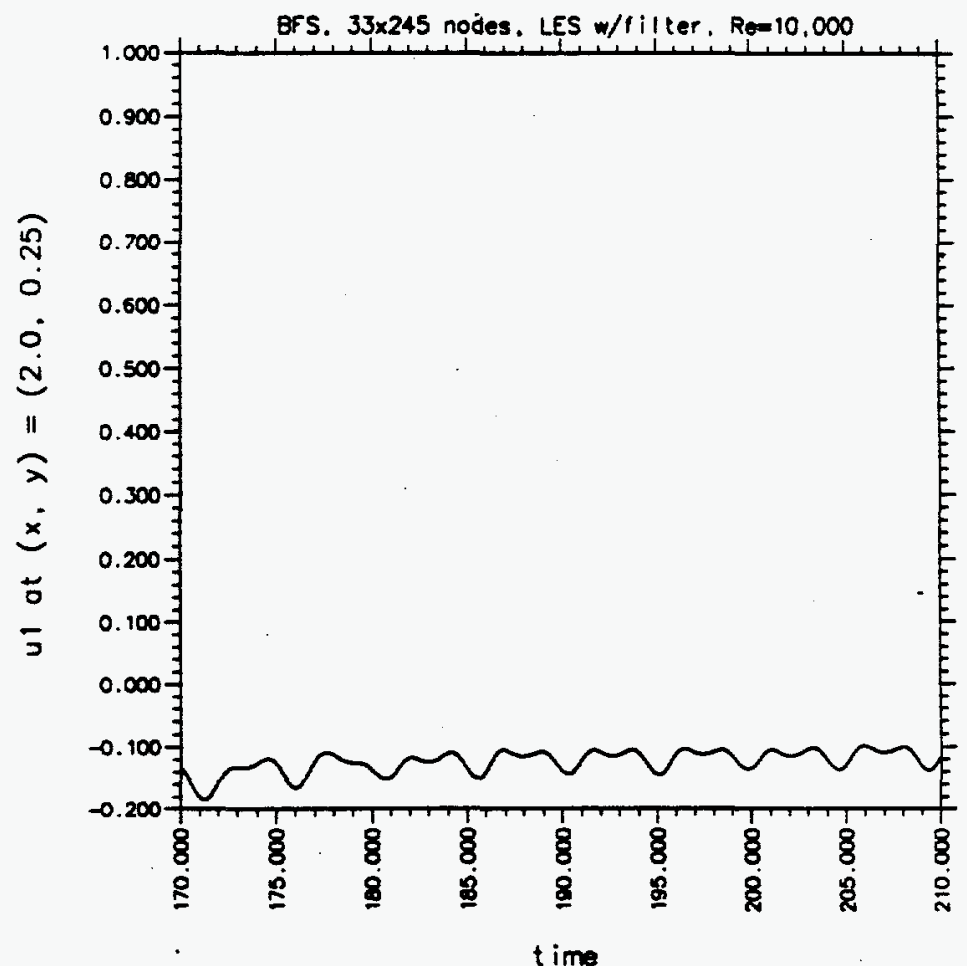

(a)

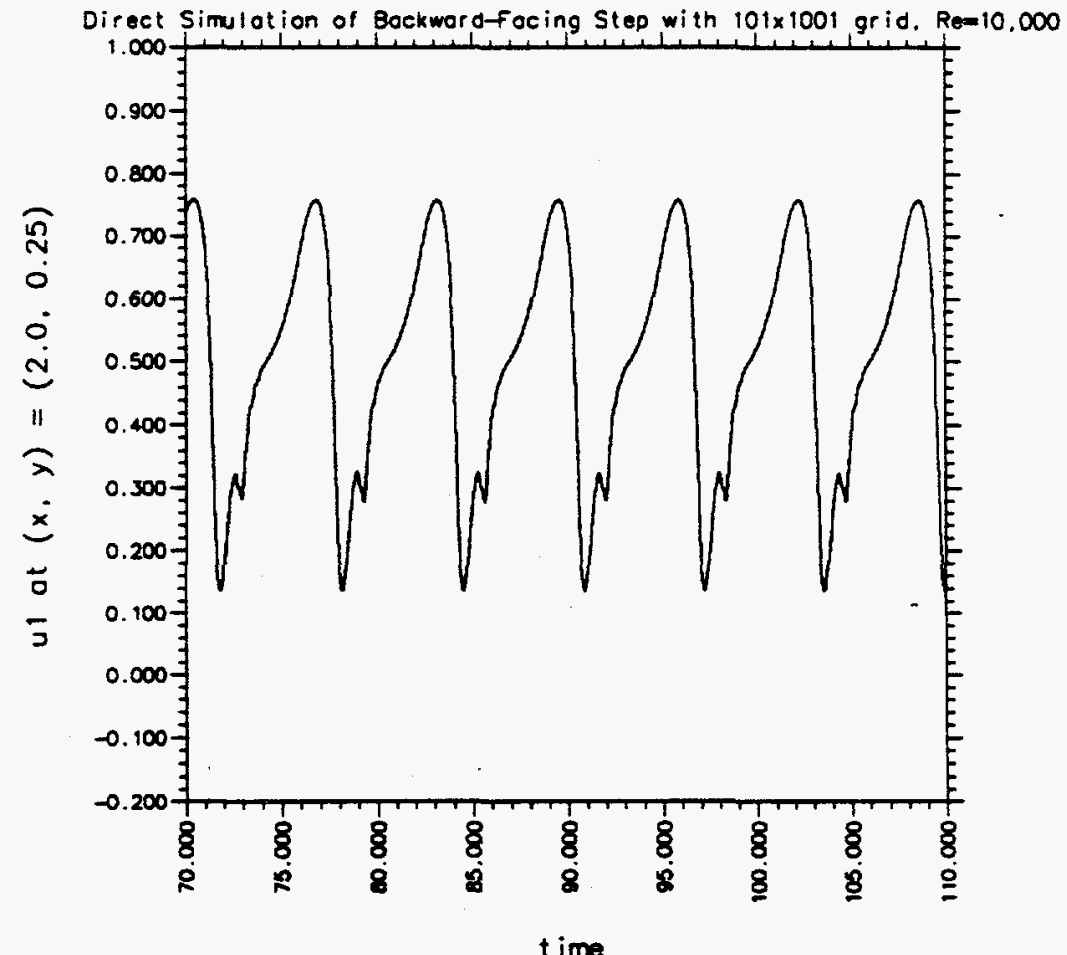

(b)

Fig. 5.3.55 Extended time histories of $\bar{u}_{1}$ at $(x, y)=(2.0,0.25)$ for a) the $33 \times 245$ grid with $\Delta_{f}=3 \Delta$ and $b$ ) the direct simulation. 

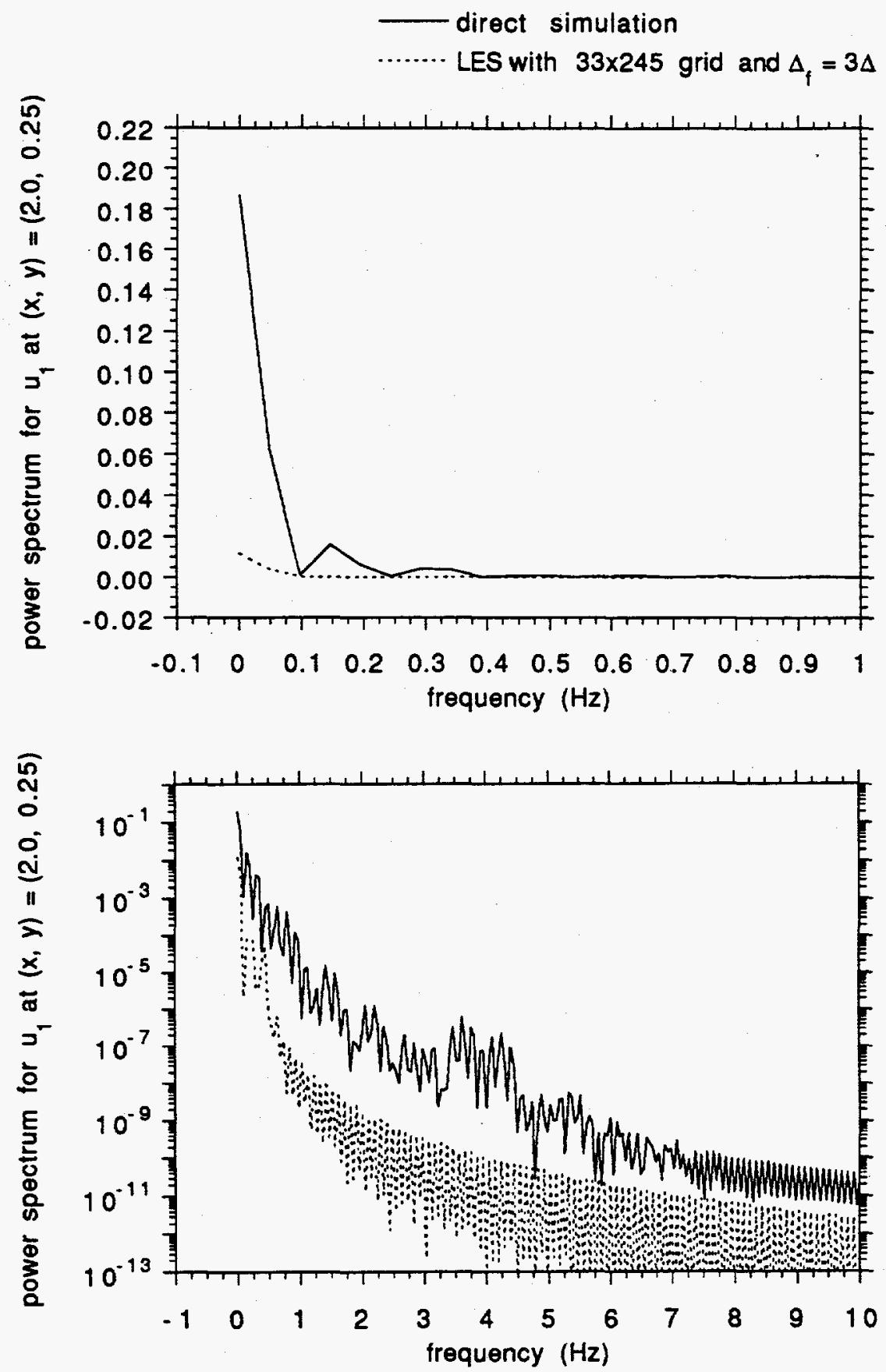

Fig. 5.3.56 Power spectrum on a linear and a log plot for $\bar{u}_{1}$ time history at $(x, y)=$ $(2.0,0.25)$ for the $33 \times 245$ grid with $\Delta t=0.005$ and $\Delta_{f}=3 \Delta(t=170$ to 200.715) compared to the direct simulation. 


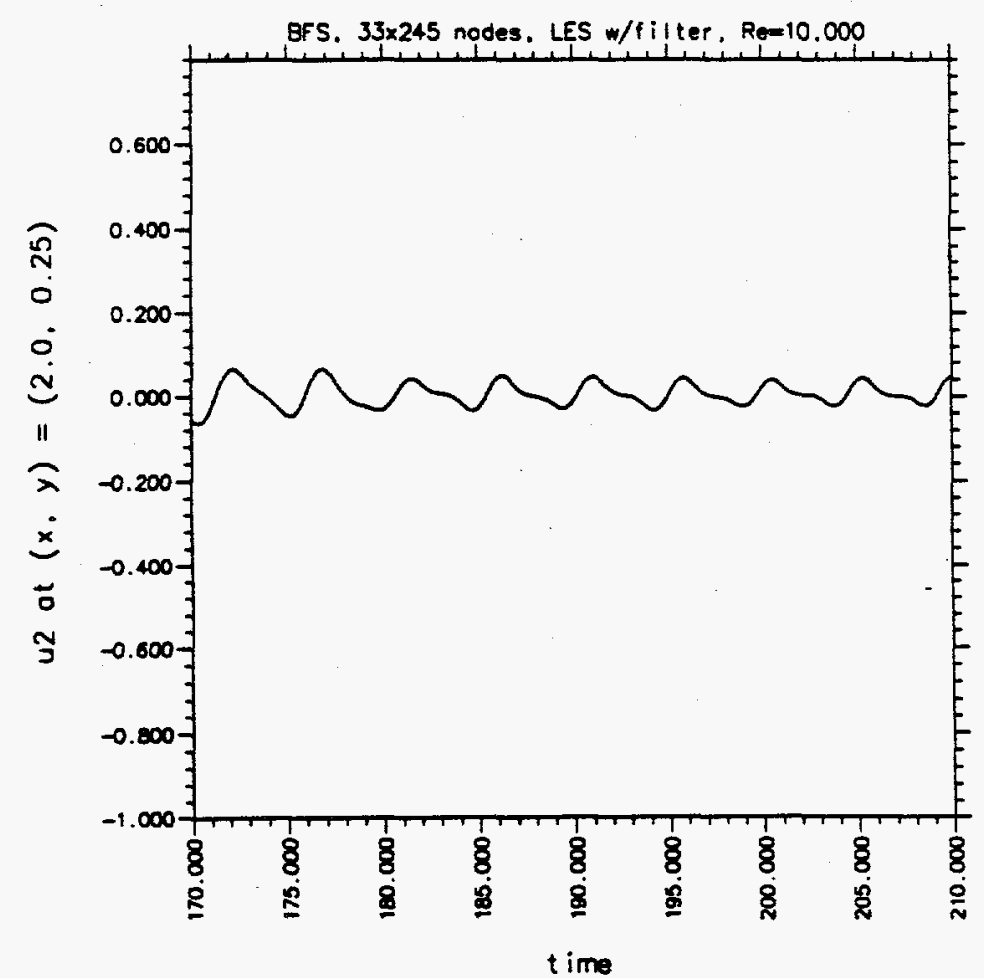

(a)

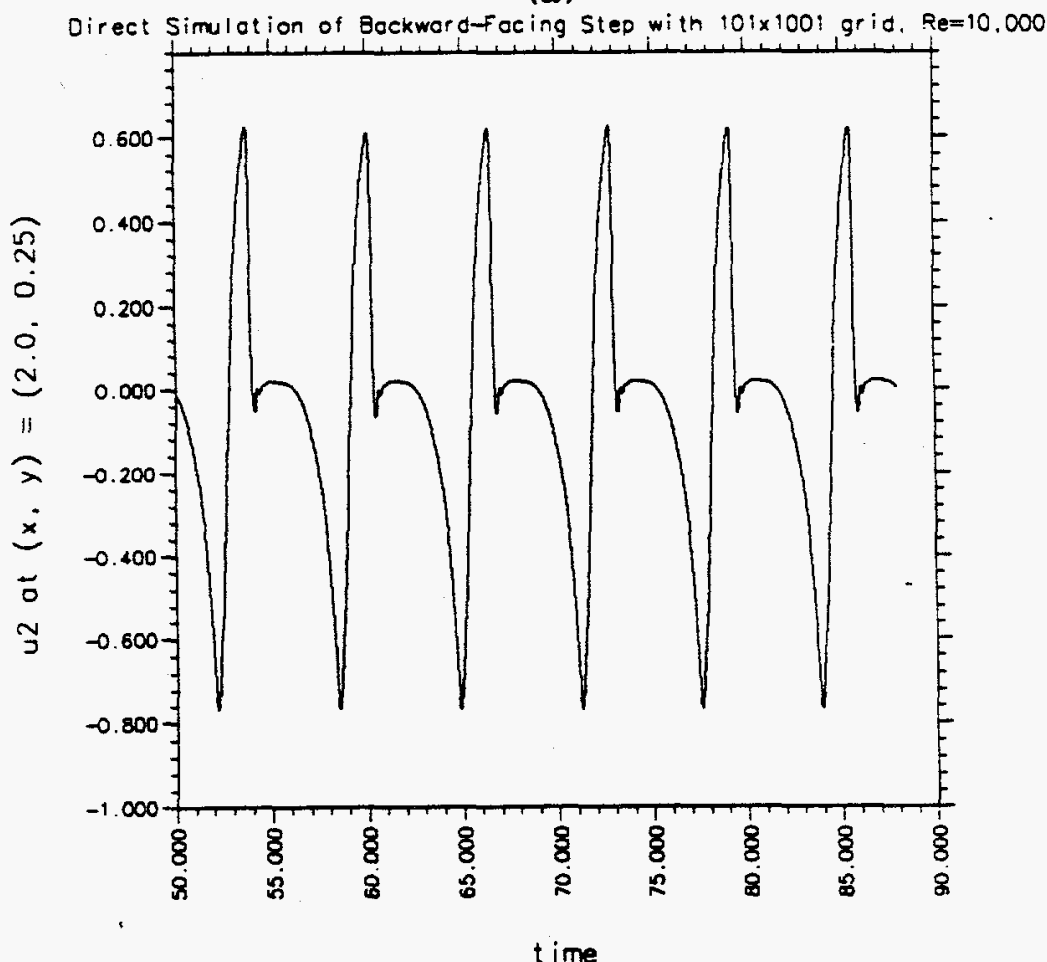

(b)

Fig. 5.3.57 Extended time histories of $\bar{u}_{2}$ time history at $(x, y)=(2.0,0.25)$ for a) the $33 \times 245$ grid with $\Delta_{f}=3 \Delta$ and b) the direct simulation. 

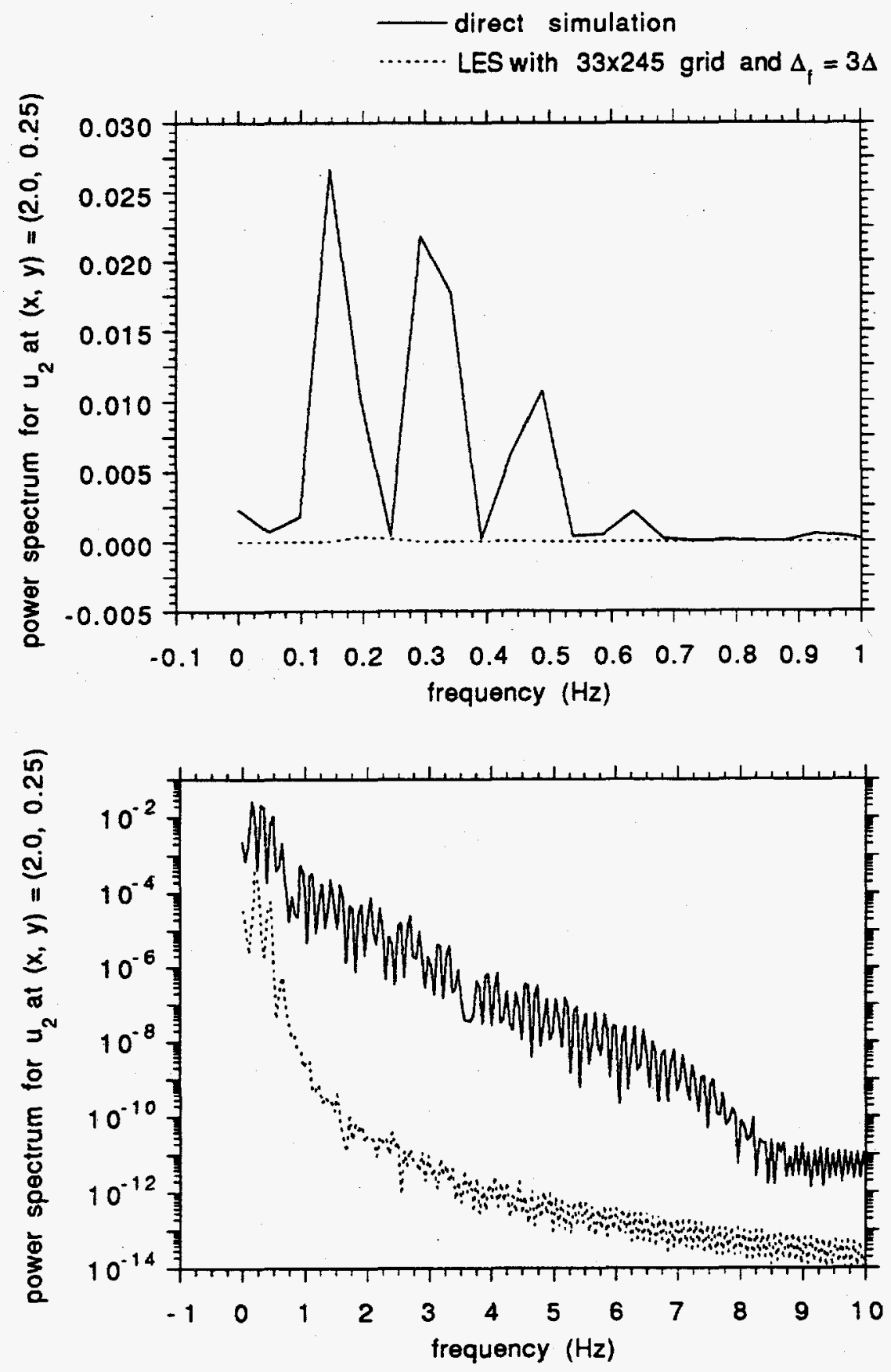

Fig. 5.3.58 Power spectrum on a linear and a $\log$ plot for $\bar{u}_{2}$ time history at $(x, y)=$ $(2.0,0.25)$ for the $33 \times 245$ grid with $\Delta t=0.005$ and $\Delta_{f}=3 \Delta(t=170$ to 200.715) compared to the direct simulation. 


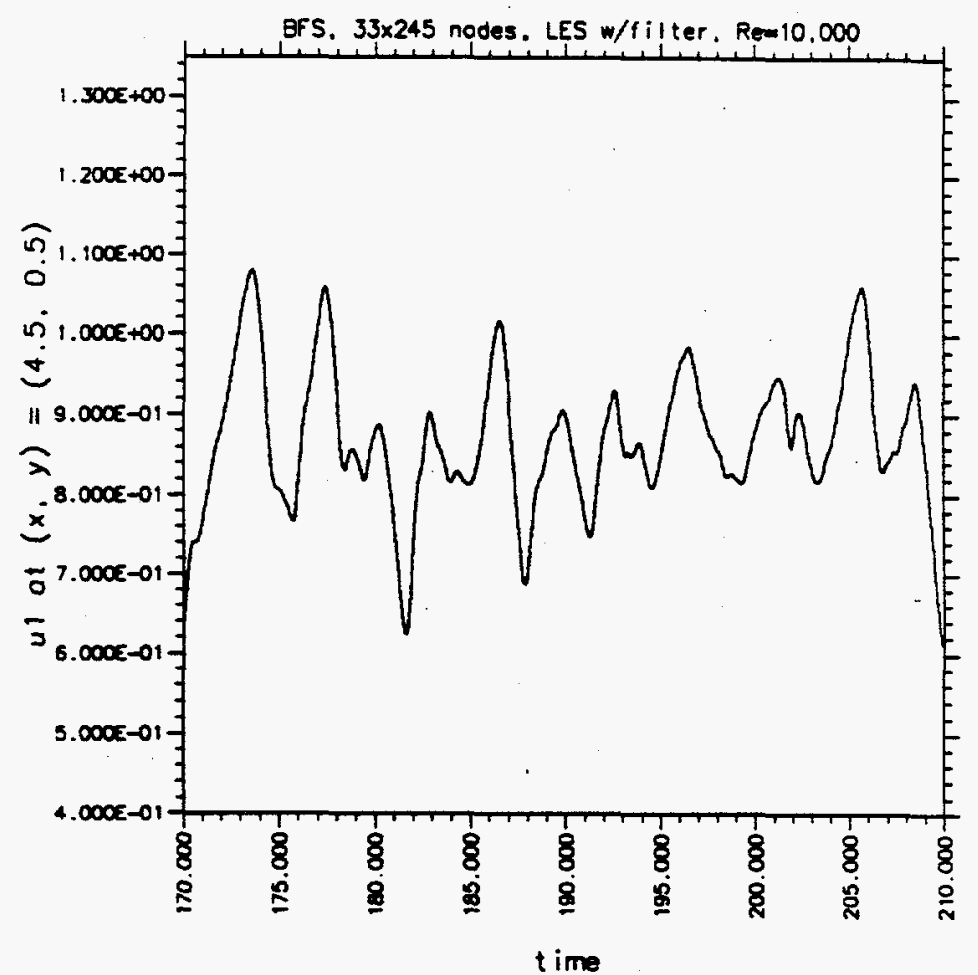

(a)

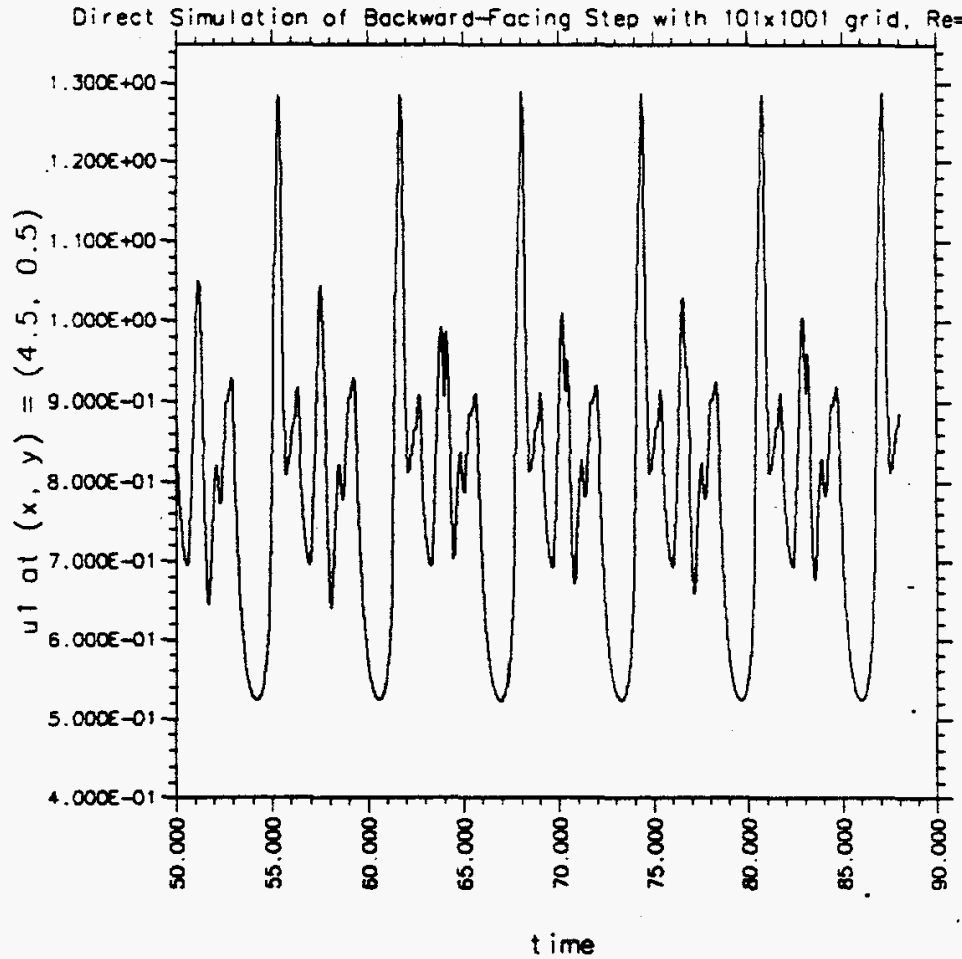

(b)

Fig. 5.3.59 Extended time histories of $\bar{u}_{1}$ time history at $\left(x_{2}, y\right)=(4.5,0.5)$ for a) the $33 \times 245$ grid with $\Delta_{f}=3 \Delta$ and b) the direct simulation. 

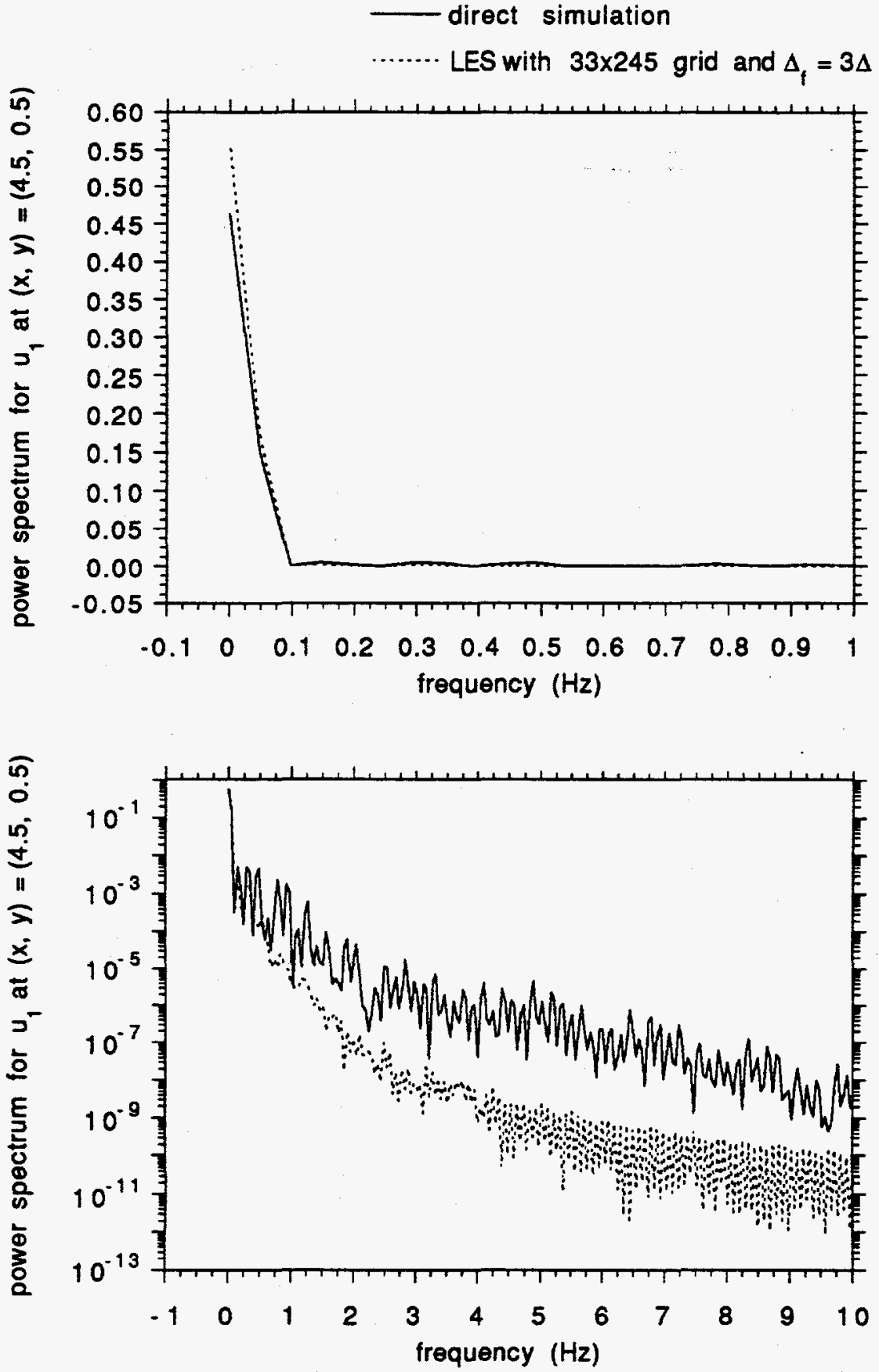

Fig. 5.3.60 Power spectrum on a linear and a log plot for $\bar{u}_{1}$ time history at $\left(x_{2} y\right)=$ $(4.5,0.5)$ for the $33 \times 245$ grid with $\Delta t=0.005$ and $\Delta_{f}=3 \Delta(t=170$ to 200.715) compared to the direct simulation. 


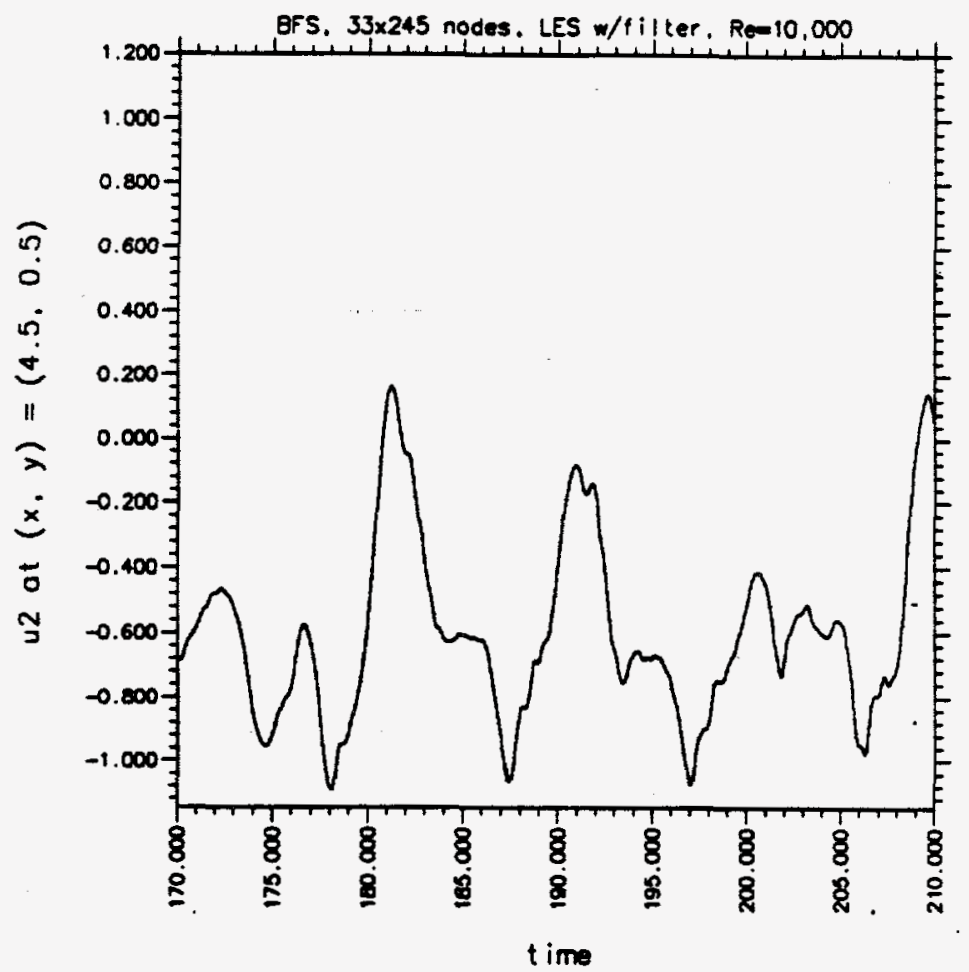

(a)

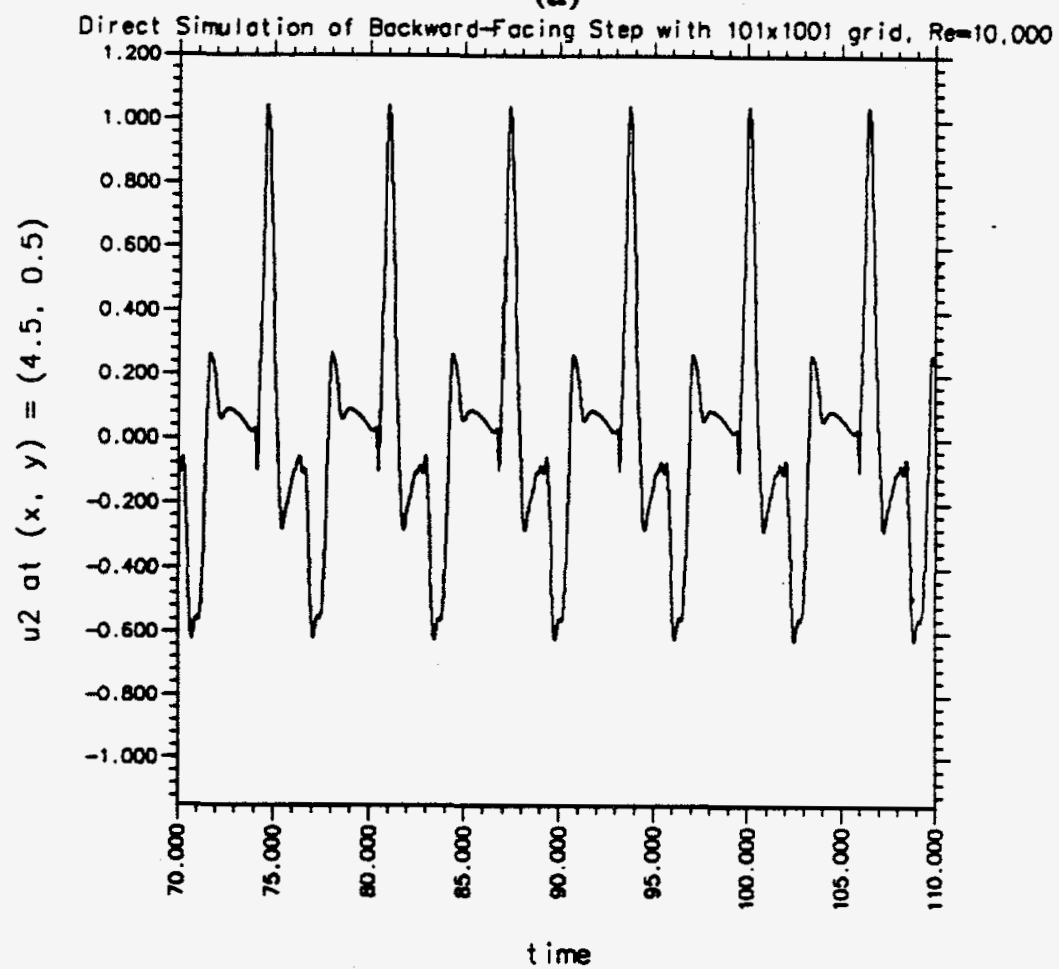

(b)

Fig. 5.3.61 Extended time histories of $\overline{u_{2}}$ at $(x, y)=(4.5,0.5)$ for a) the $33 \times 245$ grid with $\Delta_{f}=3 \Delta$ and b) the direct simulation. 

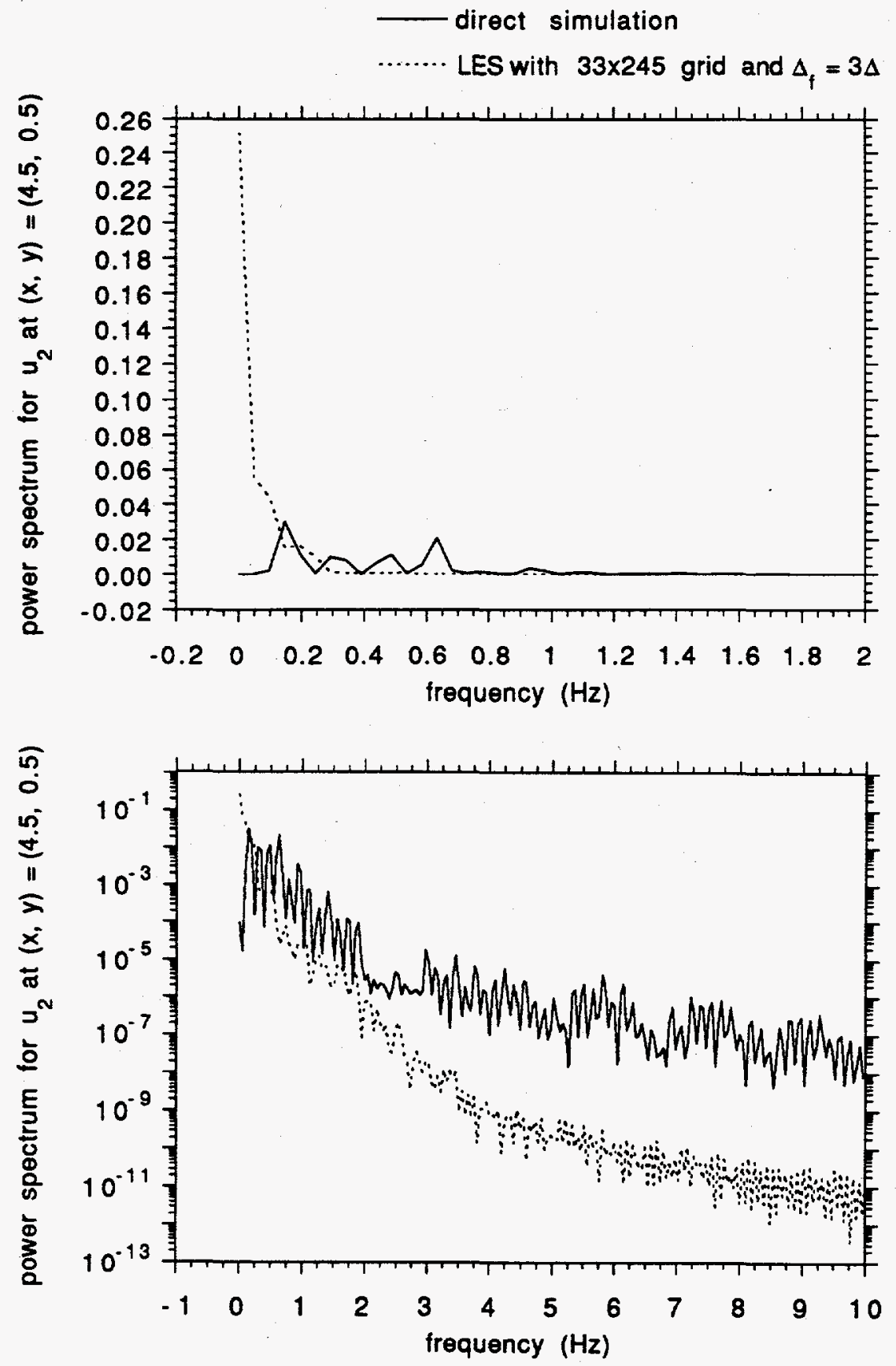

Fig. 5.3.62 Power spectrum on a linear and a log plot for $\bar{u}_{2}$ time history at $(x, y)=$ $(4.5,0.5)$ for the $33 \times 245$ grid with $\Delta t=0.005$ and $\Delta_{f}=3 \Delta(t=170$ to 200.715) compared to the direct simulation. 

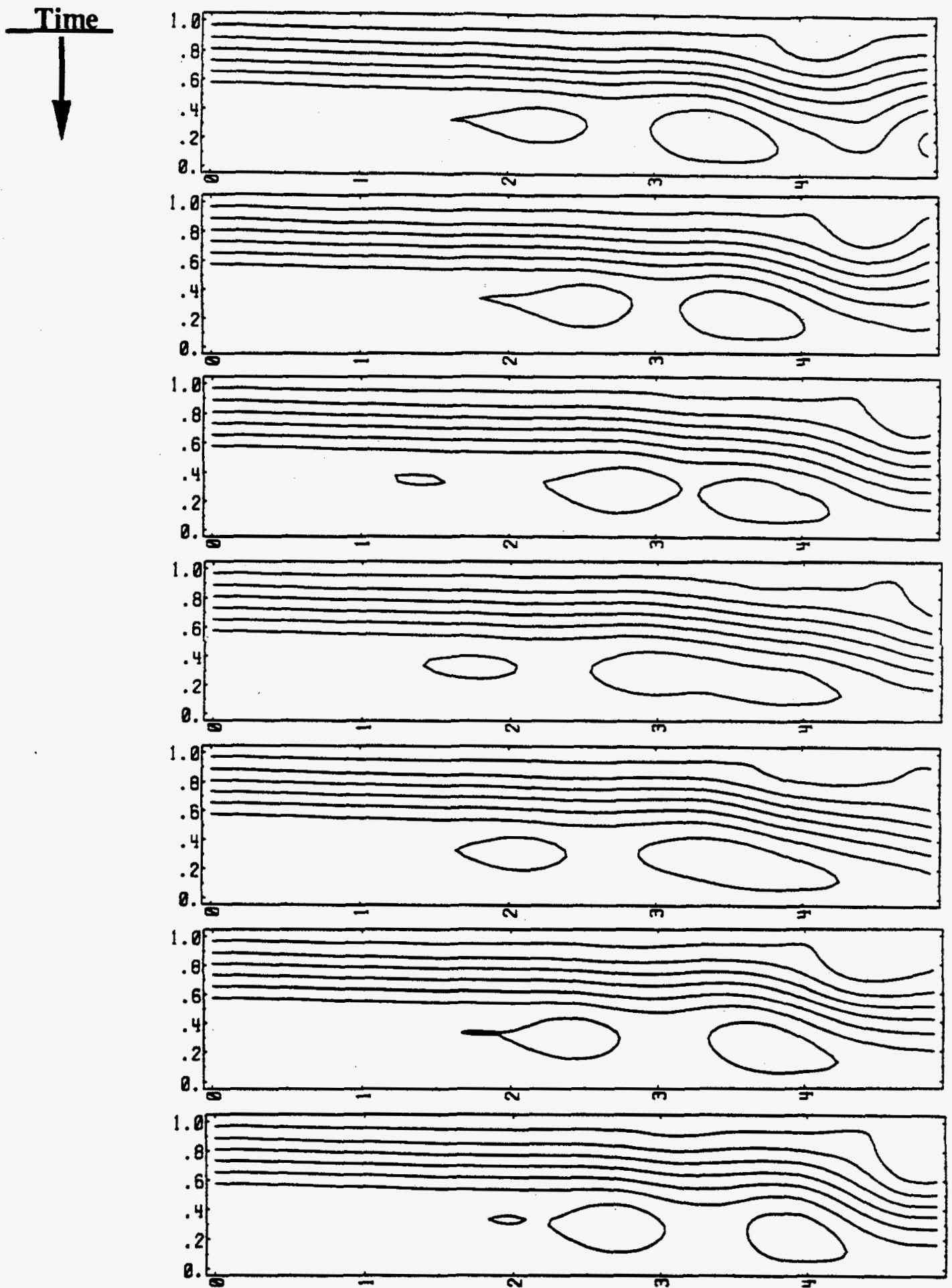

Fig. 5.3.63a Time series of stream function plots showing only half of the field length for the $33 \times 245$ grid with $\Delta_{f}=3 \Delta$ at $0,1 / 6,2 / 6,3 / 6,4 / 6,5 / 6$, and $6 / 6$ fo a DS cycle. 
Time
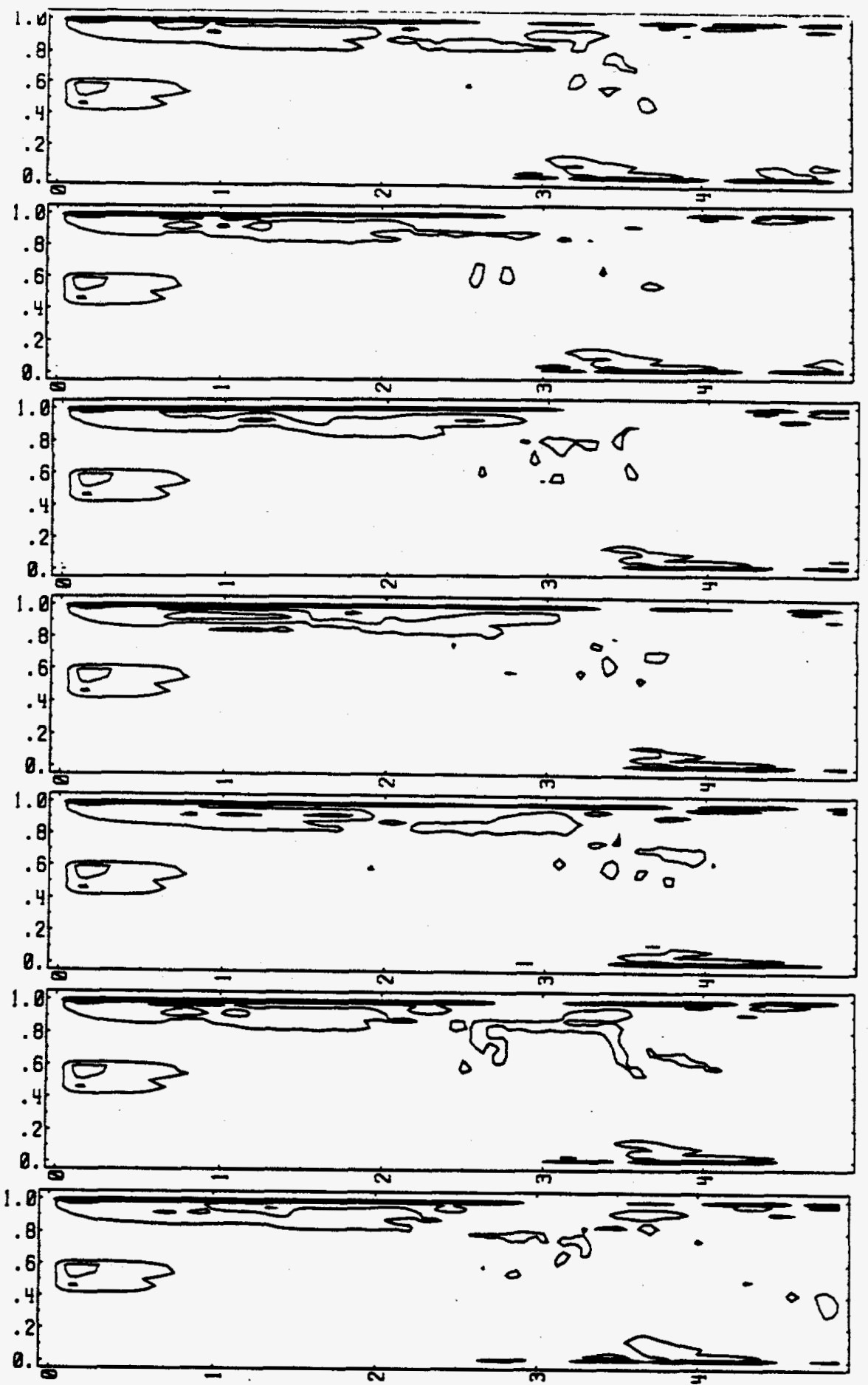

$$
0.00003<v T^{\prime} v<89.03
$$

Fig. 5.3.63b Time series of contour plots for $v_{t}$ showing only half of the field length for the $33 \times 245$ grid with $\Delta_{f}=3 \Delta$ at $0,1 / 6,2 / 6,3 / 6,4 / 6,5 / 6$, and $6 / 6$ of a DS cycle. 
Time
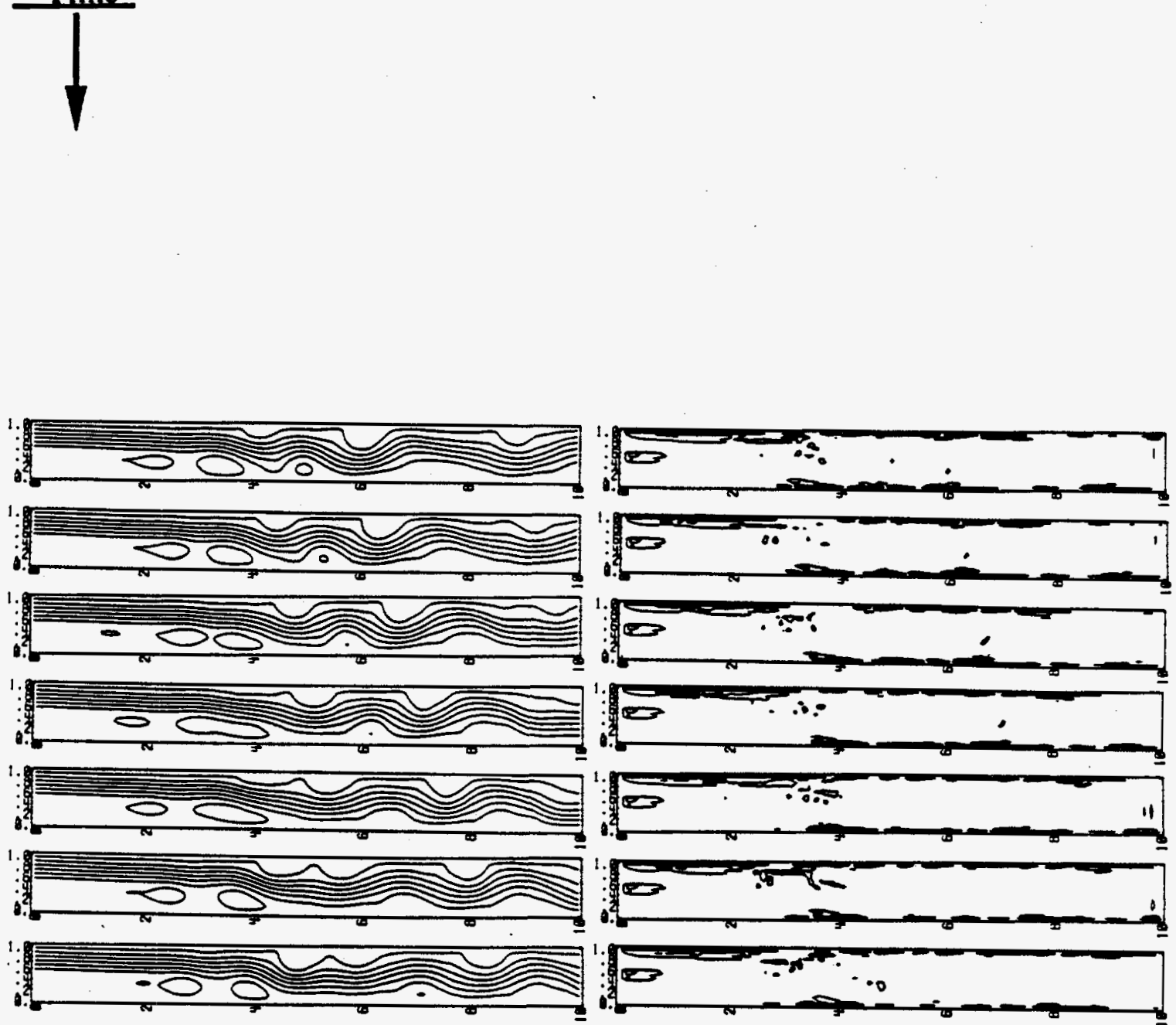

Stream Function

$v T$ contours

$0.00003<v_{T} / v<89.03$

Maximum $v_{T}$ at $(x, y)=(.2083, .9885)$

Fig. 5.3.63c Comparison of a time series of stream function plots and contour plots for $v_{T}$ for the $33 \times 245$ grid with $\Delta_{f}=3 \Delta$ (one cycle is shown at $0,1 / 6,2 / 6,3 / 6,4 / 6$, $5 / 6$, and $6 / 6$ of a DS cycle). 
BF9. $33 \times 245$ nodes. LES w/f, Iter. Re 10000

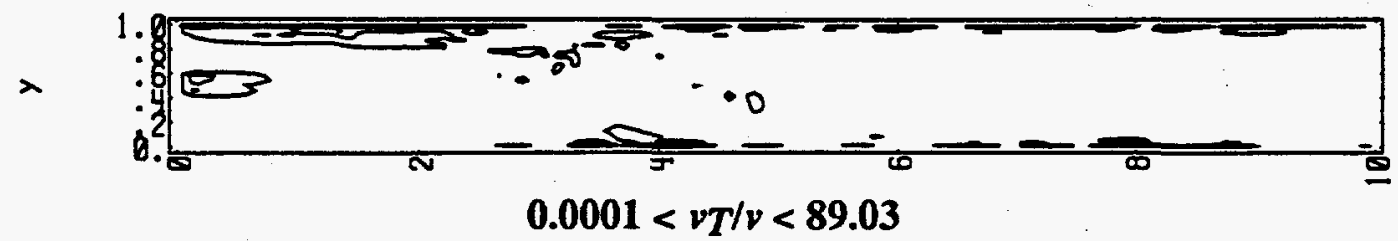

$\mathbf{y}=\mathbf{0 . 0}$

$\mathbf{y}=\mathbf{1 . 0}$
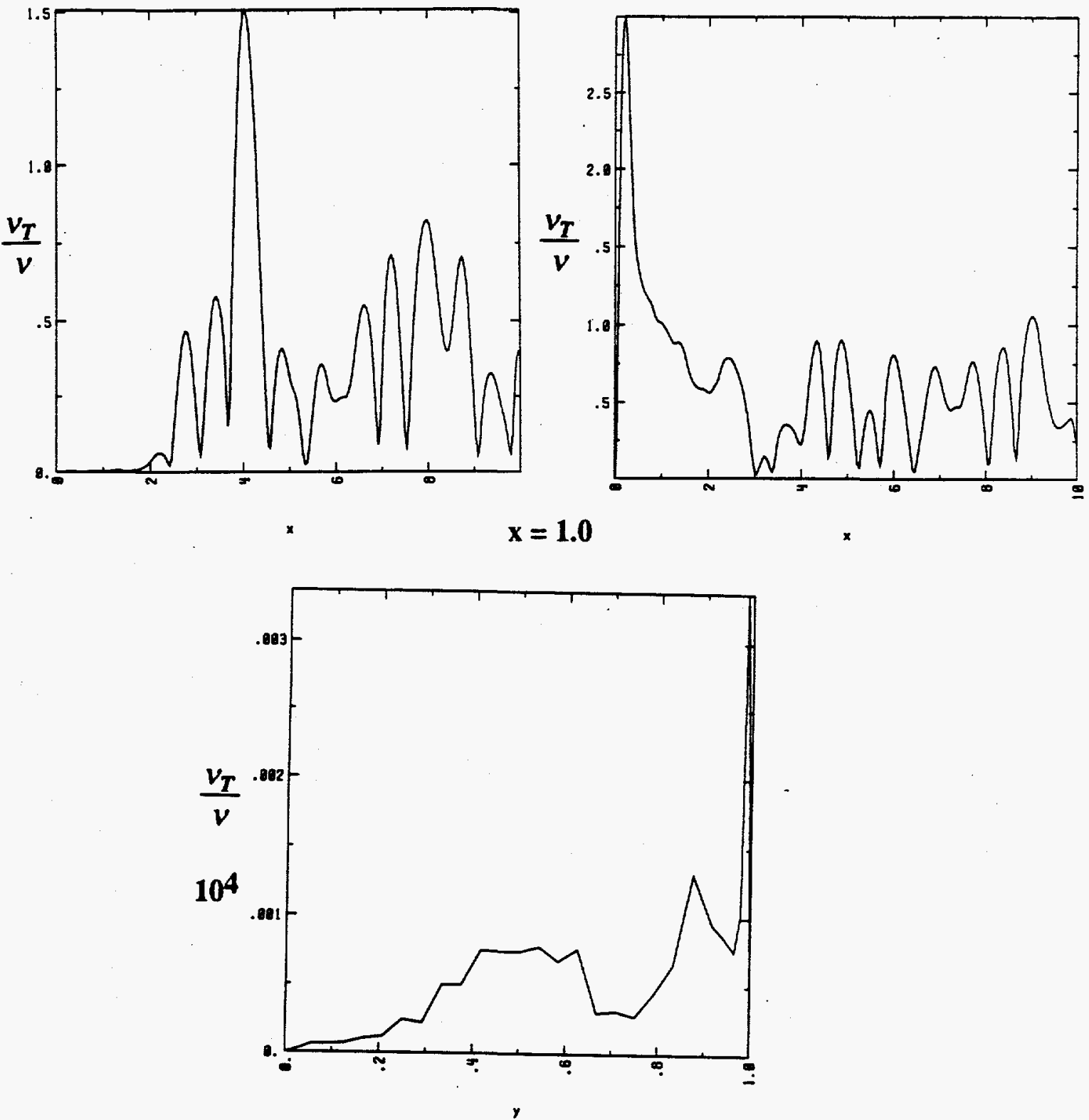

Fig. 5.3.64 A $v_{T}$ contour plot and profile plots at selected locations for the $33 \mathrm{x}$ 245 grid with $\Delta_{f}=3 \Delta$ at a snap shot in time. 


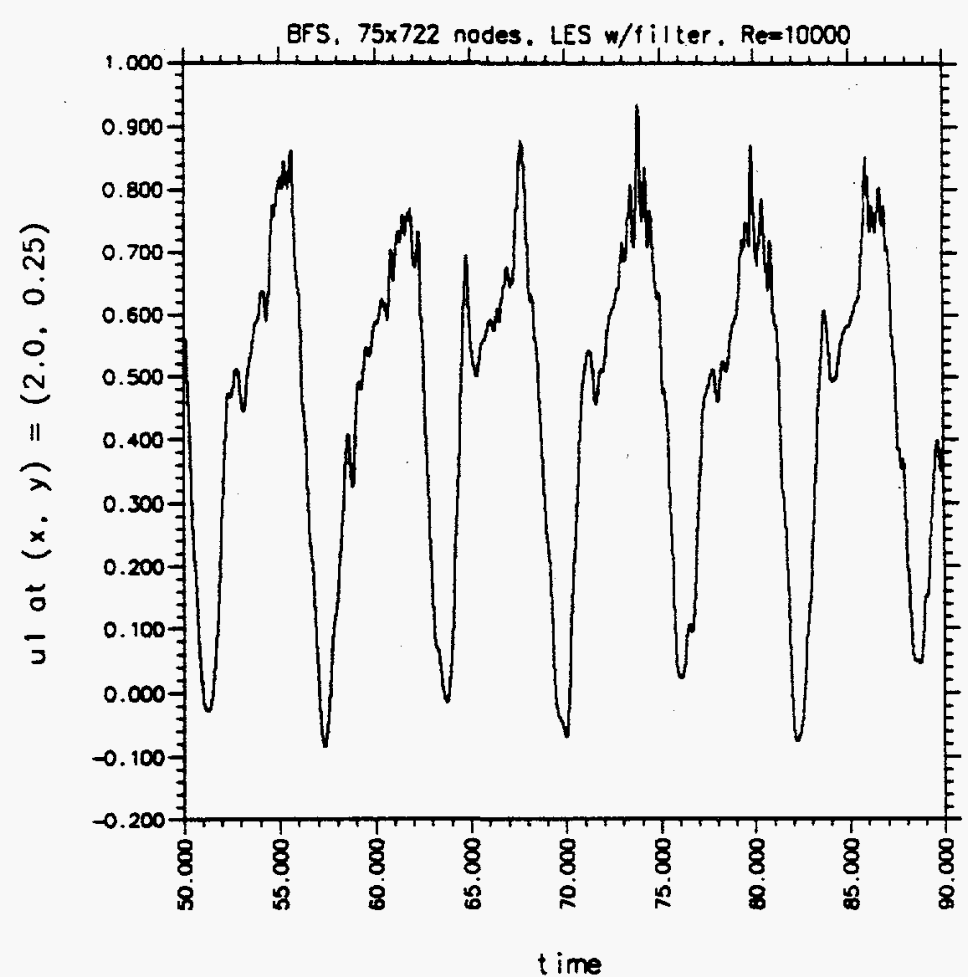

(a)

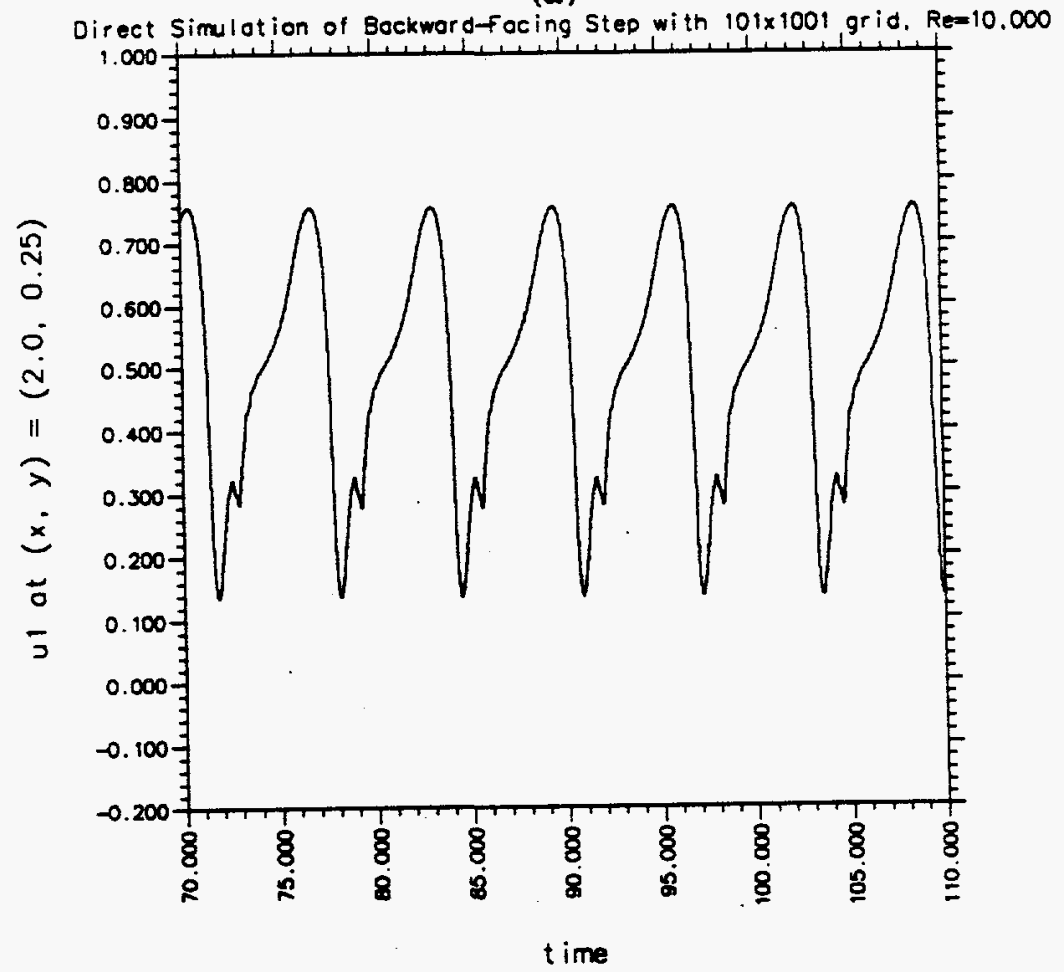

(b)

Fig. 5.3.65 Extended time histories of $\bar{u}_{1}$ at $(x, y)=(2.0,0.25)$ for a) the $75 \times 722$ grid with $\Delta_{f}=3 \Delta$ and b) the direct simulation. 

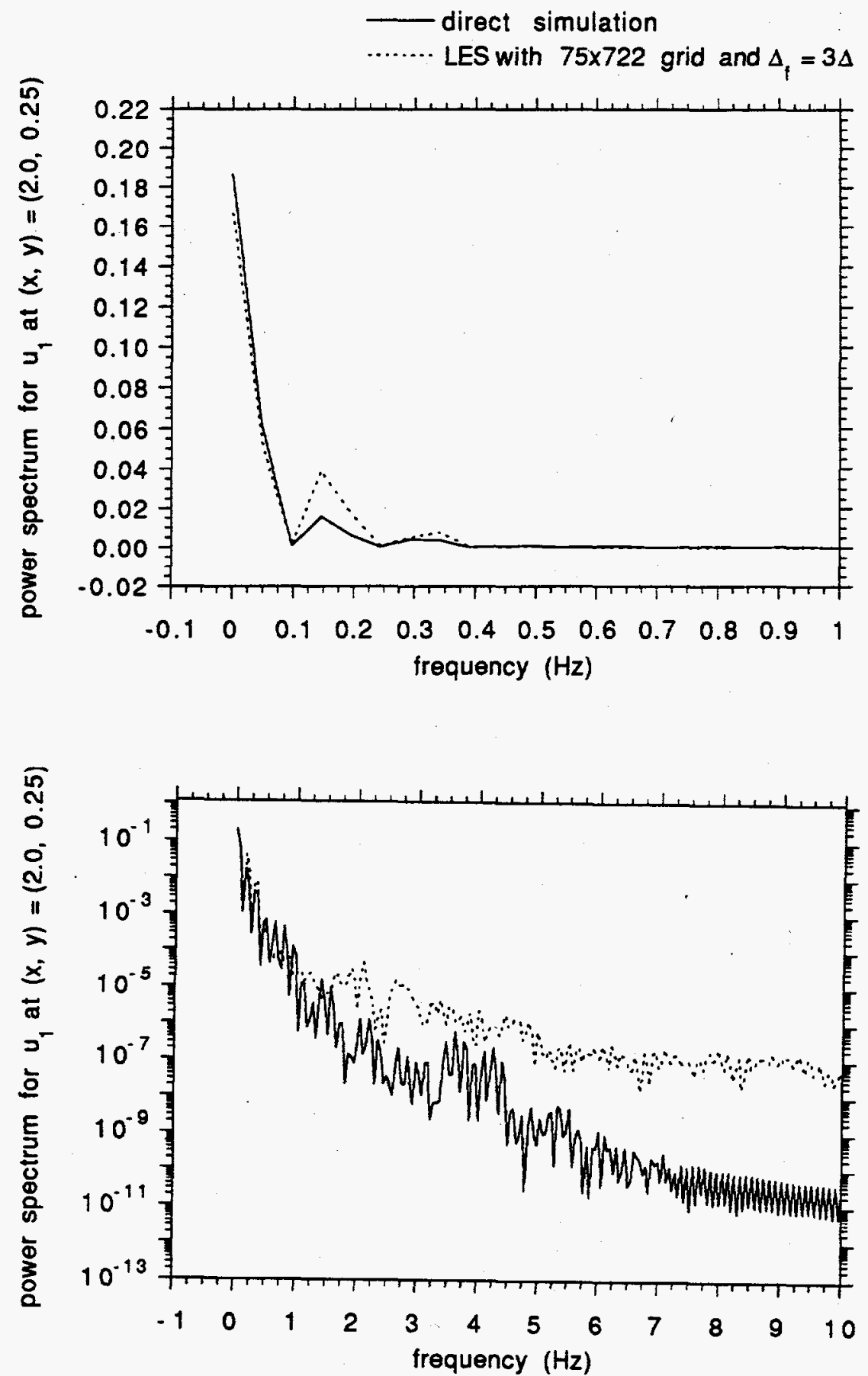

Fig. 5.3.66 Power spectrum on a linear and a $\log$ plot for $\bar{u}_{1}$ time history at $(x, y)=$ $(2.0,0.25)$ for the $75 \times 722$ grid with $\Delta t=0.005$ and $\Delta_{f}=3 \Delta(t=70$ to 100.715) compared to the direct simulation. 


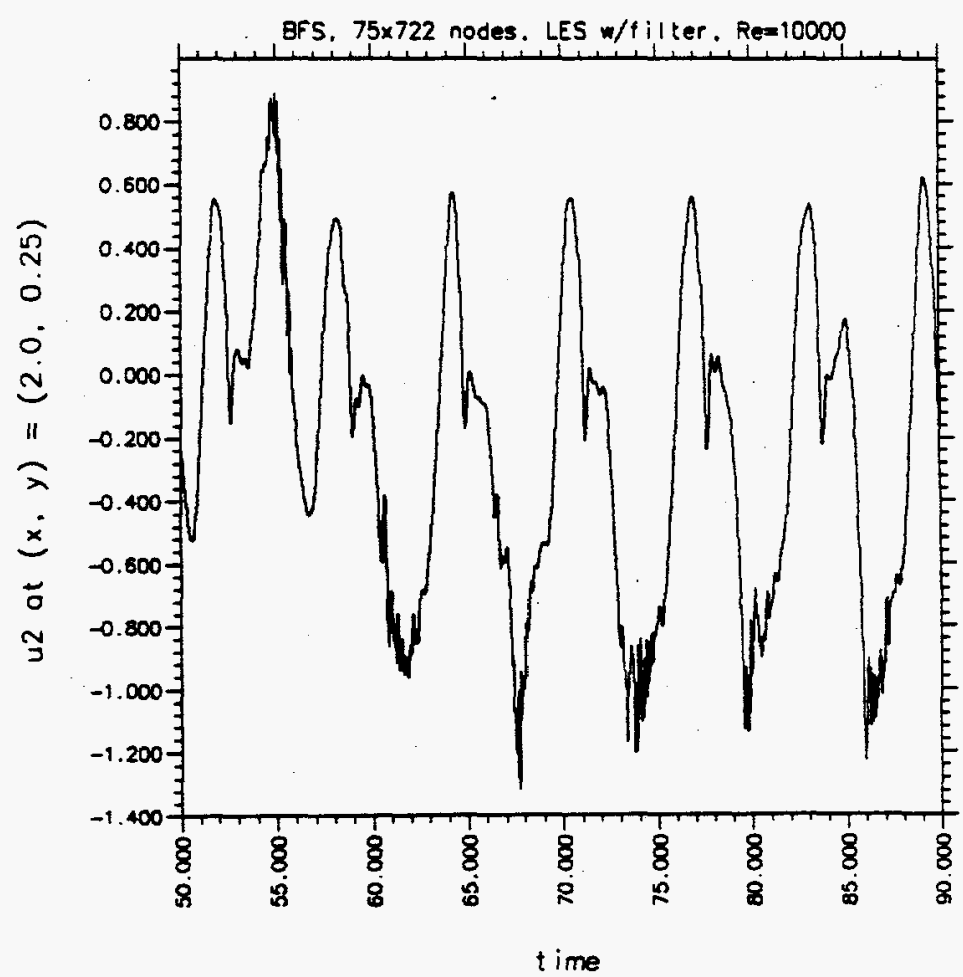

(a)

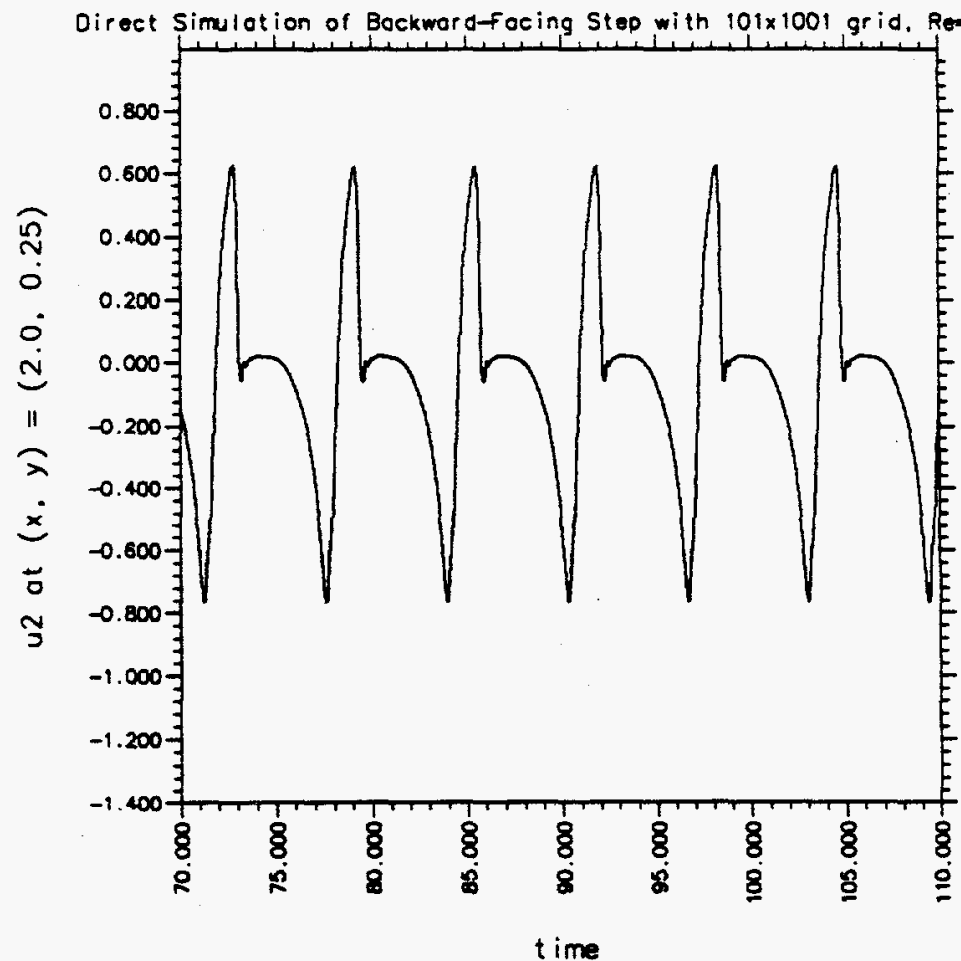

(b)

Fig. 5.3.67 Extended time histories of $\bar{u}_{2}$ time history at $(x, y)=(2.0,0.25)$ for a) the $75 \times 722$ grid with $\Delta_{f}=3 \Delta$ and $b$ ) the direct simulation. 

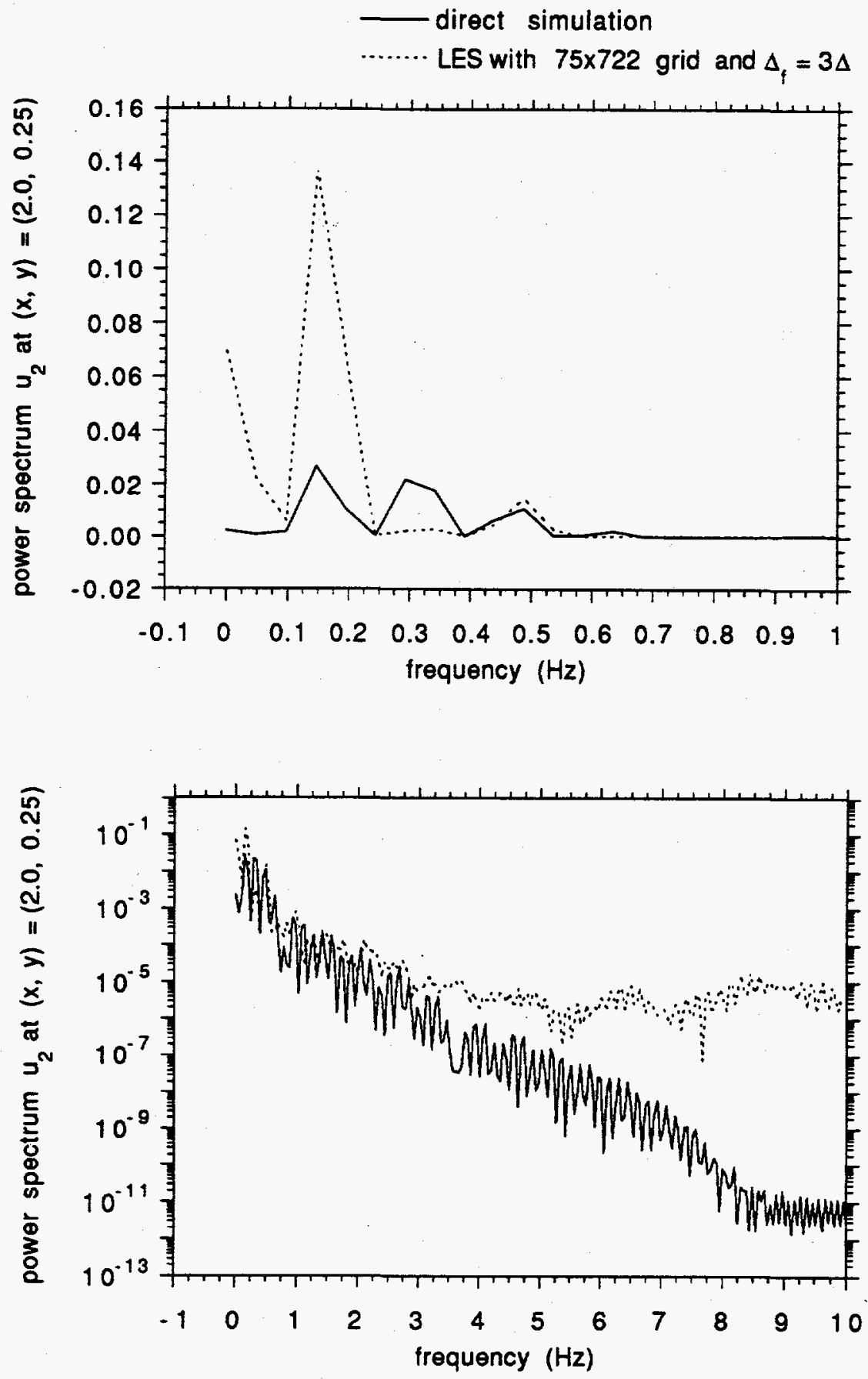

Fig. 5.3.68 Power spectrum on a linear and a log plot for $\bar{u}_{2}$ time history at $(x, y)=$ $(2.0,0.25)$ for the $75 \times 722$ grid with $\Delta t=0.005$ and $\Delta_{f}=3 \Delta(t=70$ to 100.715) compared to the direct simulation. 


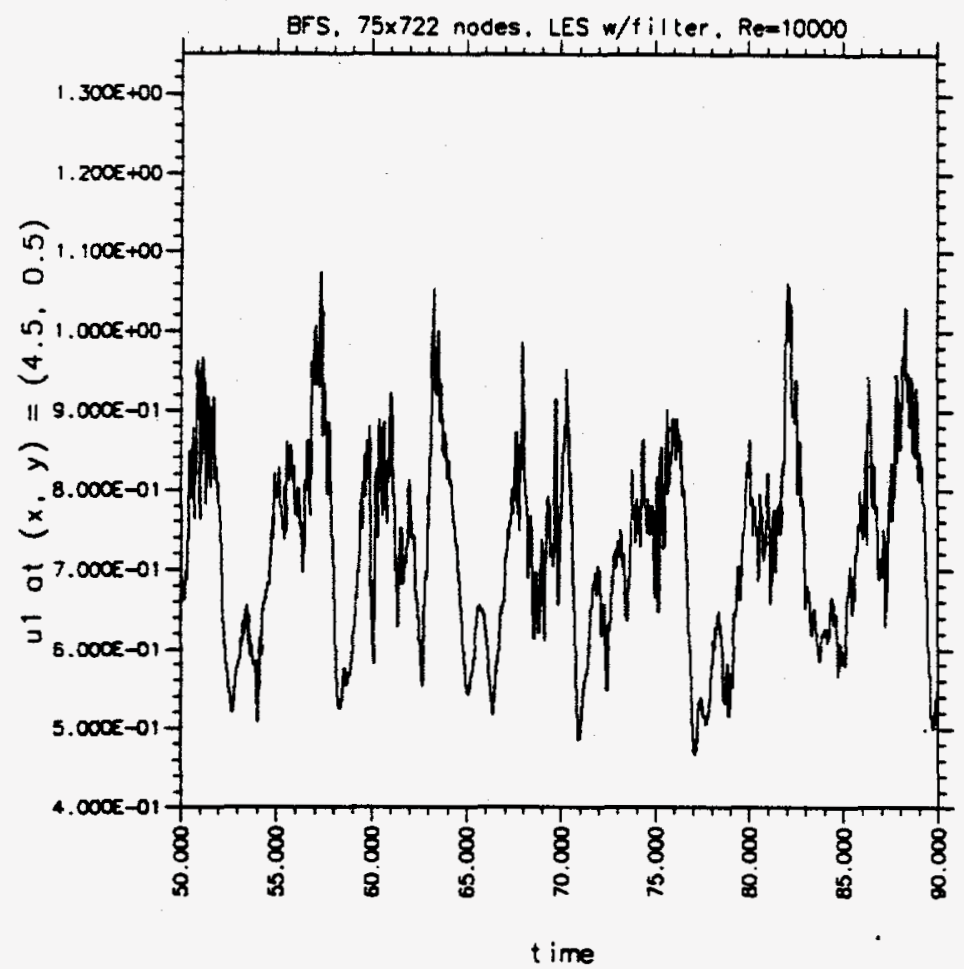

(a)

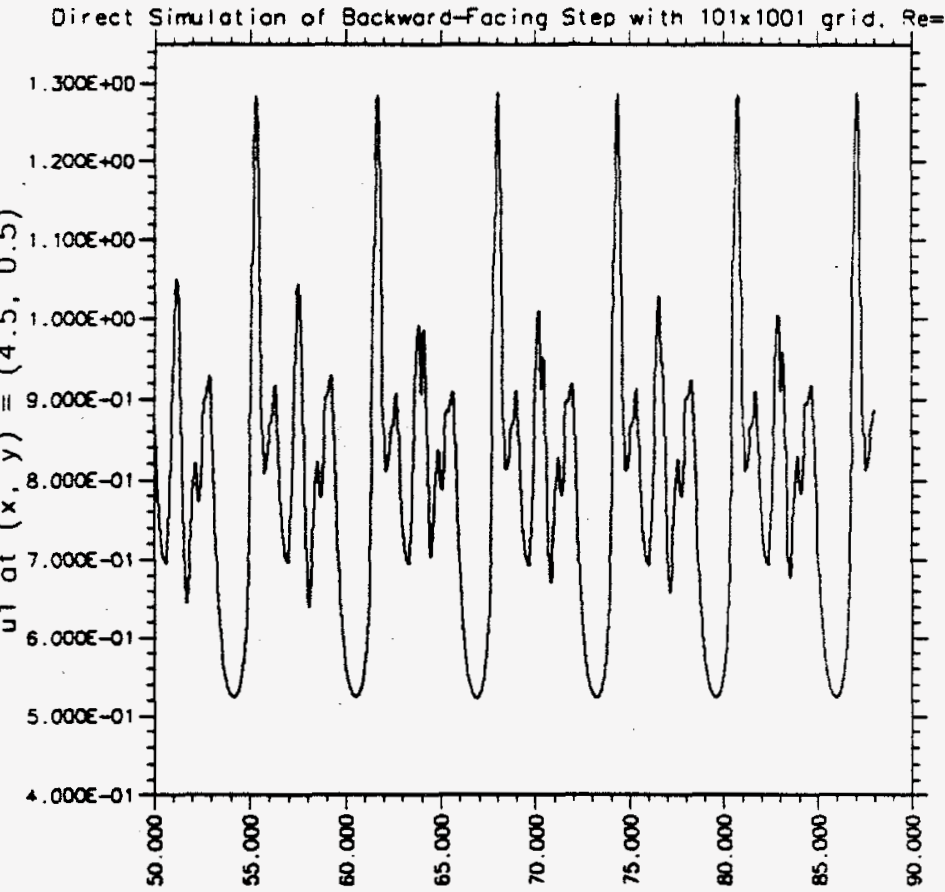

t ime

(b)

Fig. 5.3.69 Extended time histories of $\bar{u}_{1}$ time history at $(x, y)=(4.5,0.5)$ for a) the $75 \times 722$ grid with $\Delta_{f}=3 \Delta$ and b) the direct simulation. 

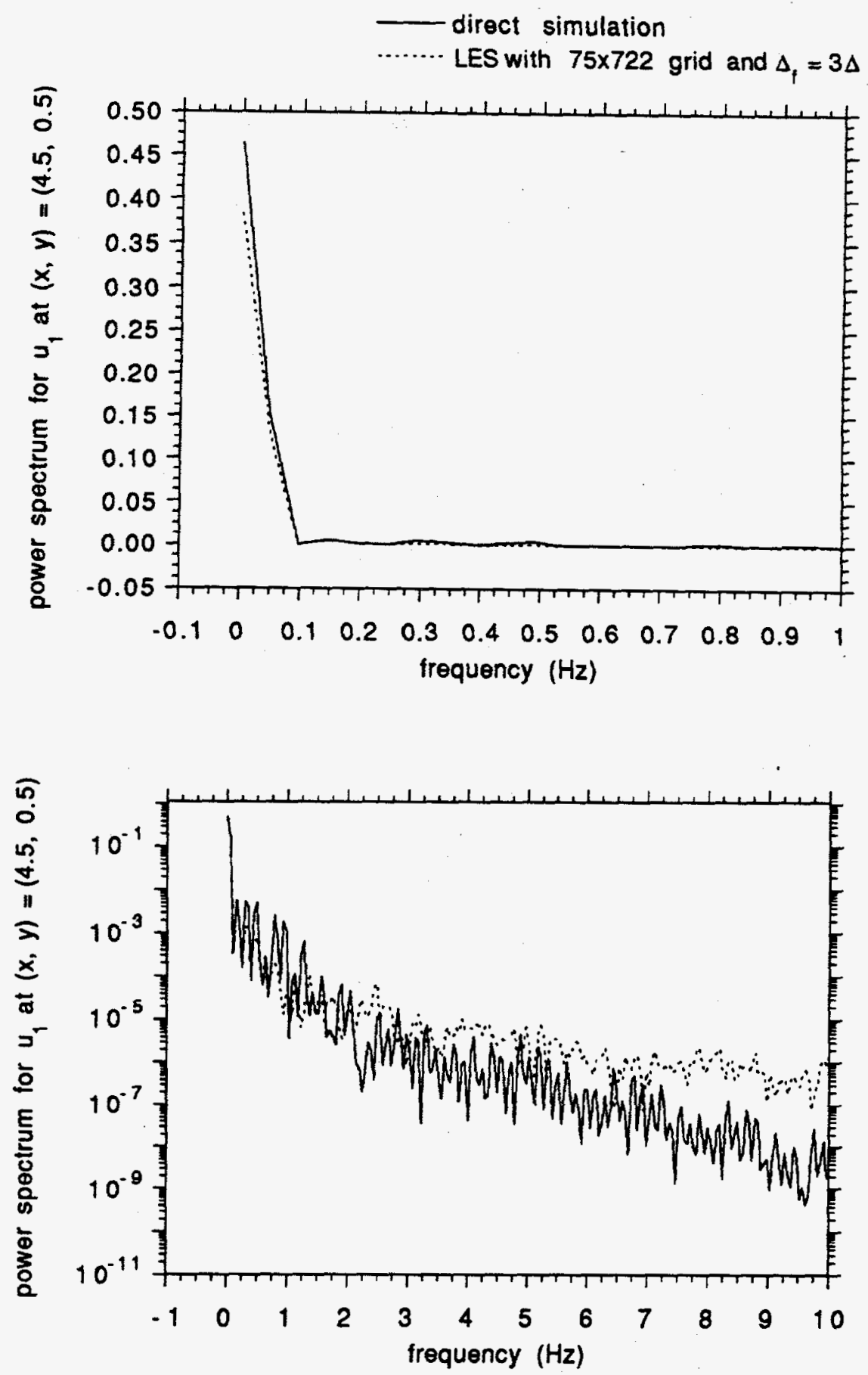

Fig. 5.3.70 Power spectrum on a linear and a log plot for $\bar{u}_{1}$ time history at $\left(x_{2} y\right)=$ $(4.5,0.5)$ for the $75 \times 722$ grid with $\Delta t=0.005$ and $\Delta_{f}=3 \Delta(t=70$ to 100.715) compared to the direct simulation. 


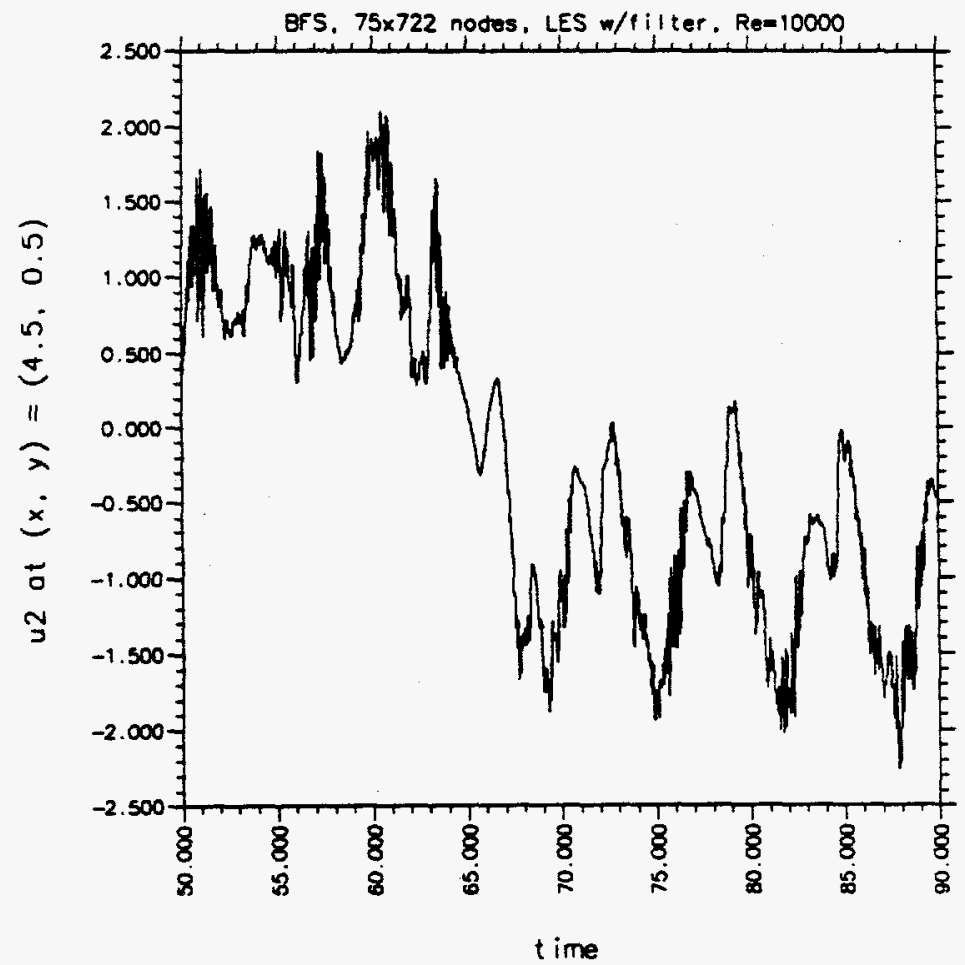

(a)

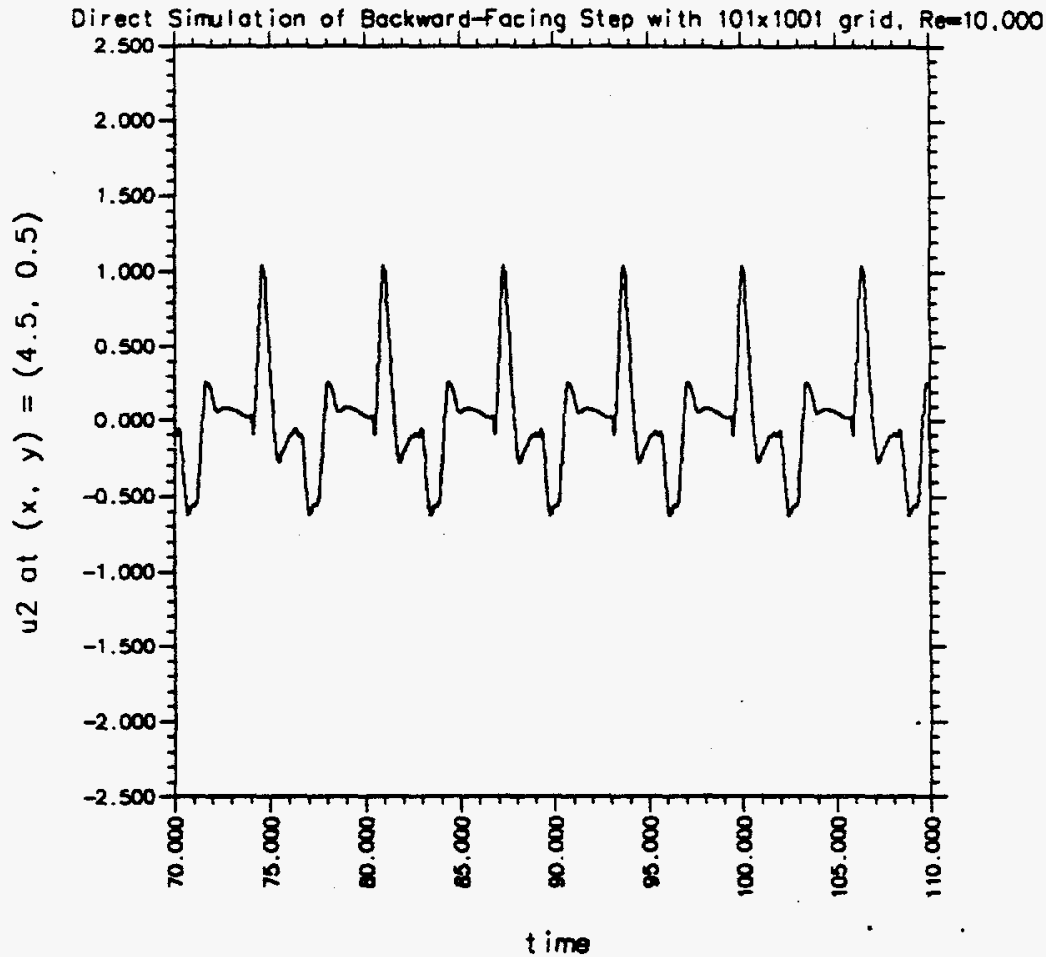

(b)

Fig. 5.3.71 Extended time histories of $\bar{u}_{2}$ at $(x, y)=(4.5,0.5)$ for a) the $75 \times 722$ grid with $\Delta_{f}=3 \Delta$ and b) the direct simulation. 

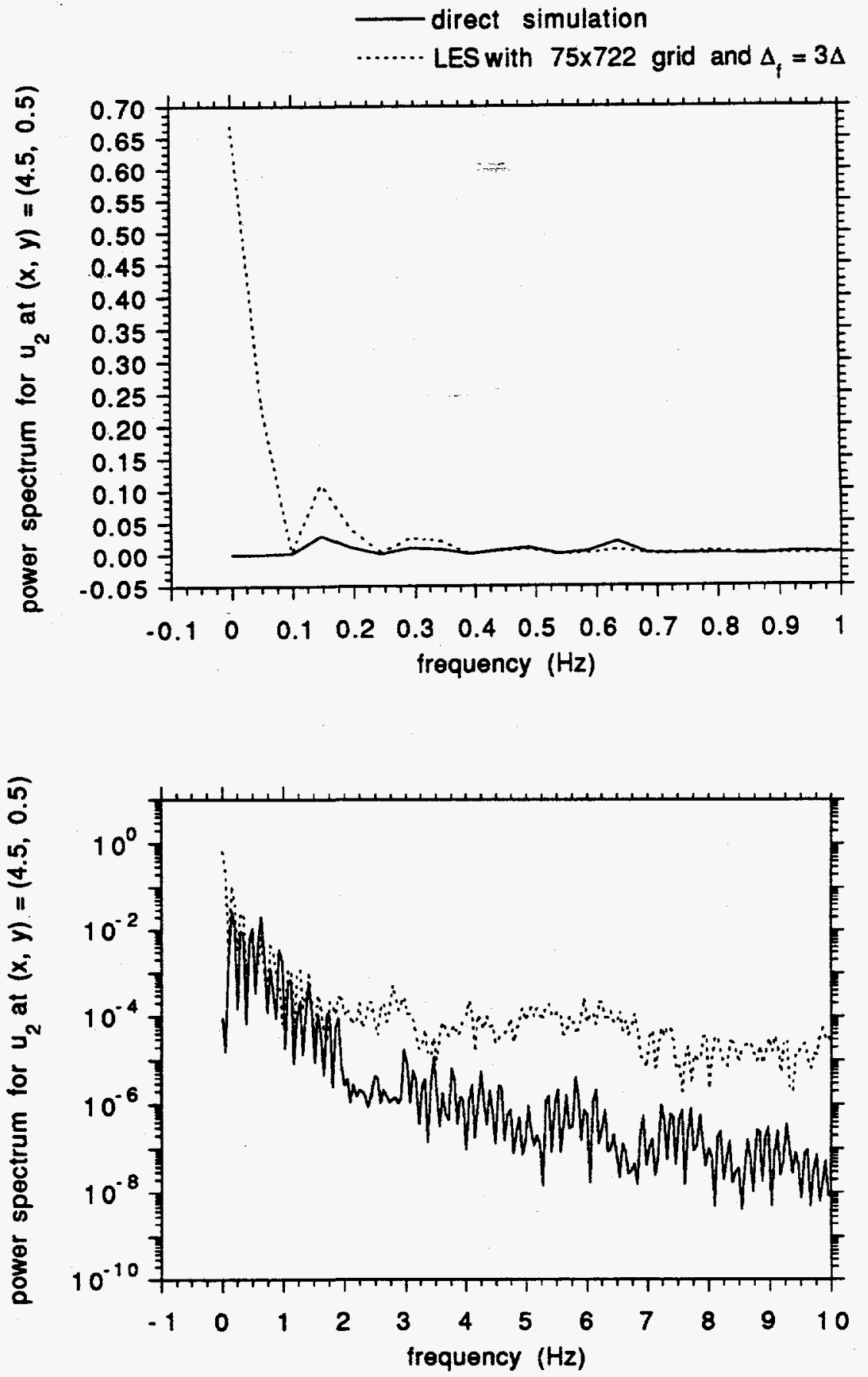

Fig. 5.3.72 Power spectrum on a linear and a log plot for $\bar{u}_{2}$ time history at $(x, y)=$ $(4.5,0.5)$ for the $75 \times 722$ grid with $\Delta t=0.005$ and $\Delta_{f}=3 \Delta(t=70$ to 100.715$)$ compared to the direct simulation. 
Table 5.3.5 LES Predicted Cycle Periods Using $\bar{u}_{1}$ Time History at $(x, y)=(2.0,0.25)$ for the $75 \times 722$ grid with $\Delta_{f}=3 \Delta$.

\begin{tabular}{|c|c|}
\hline $\begin{array}{l}\text { Times at Which } \bar{u}_{1} \\
\text { Minimum Reached }\end{array}$ & $\begin{array}{l}\text { Period of Time Between } \bar{u}_{1} \\
\text { Minimums }\end{array}$ \\
\hline \hline 76.105 & 6.075 \\
82.180 & 6.530 \\
88.710 & 5.970 \\
94.680 & 6.085 \\
100.765 & \\
\hline
\end{tabular}



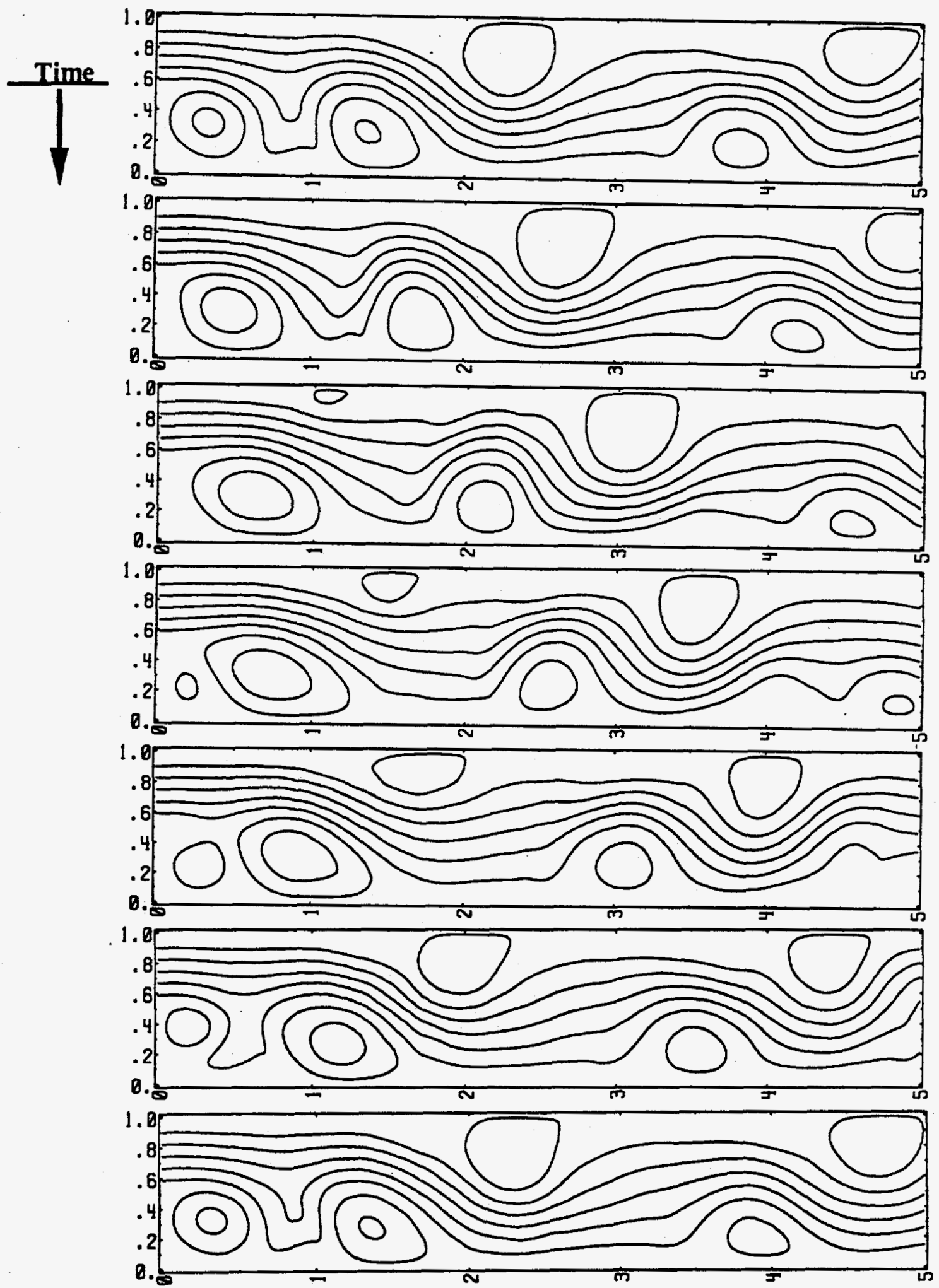

Fig. 5.3.73a Time series of stream function plots showing only half of the field length for the $75 \times 722$ grid with $\Delta_{f}=3 \Delta$ at $0,1 / 6,2 / 6,3 / 6,4 / 6,5 / 6$, and $6 / 6$ of a DS cycle. 

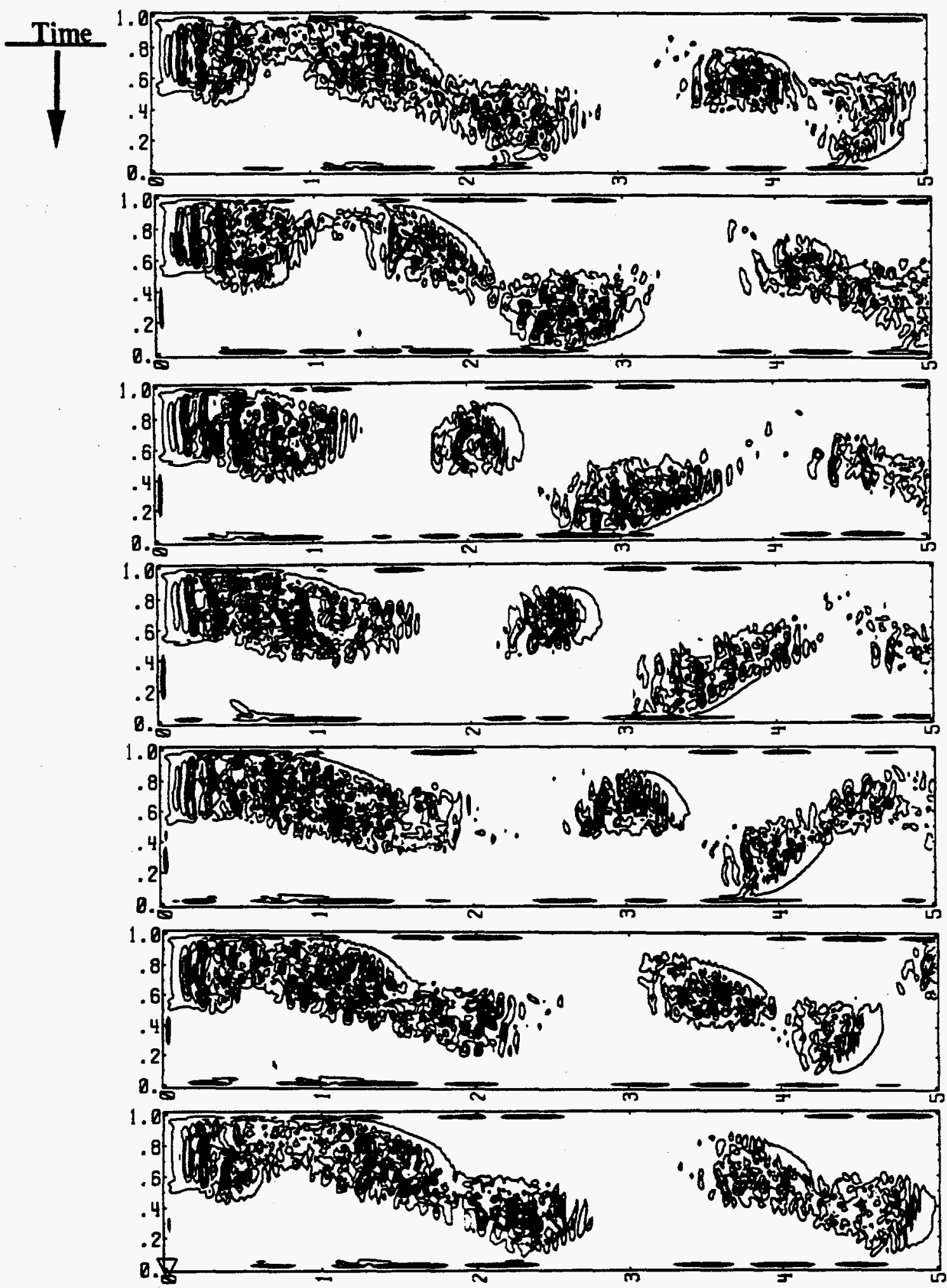

Fig. 5.3.73b Time series of $\boldsymbol{v} T$ contour plots showing only half of the field length for the $75 \times 722$ grid with $\Delta_{f}=3 \Delta$ at $0,1 / 6,2 / 6,3 / 6,4 / 6,5 / 6$, and $6 / 6$ of a DS cycle. 
Time
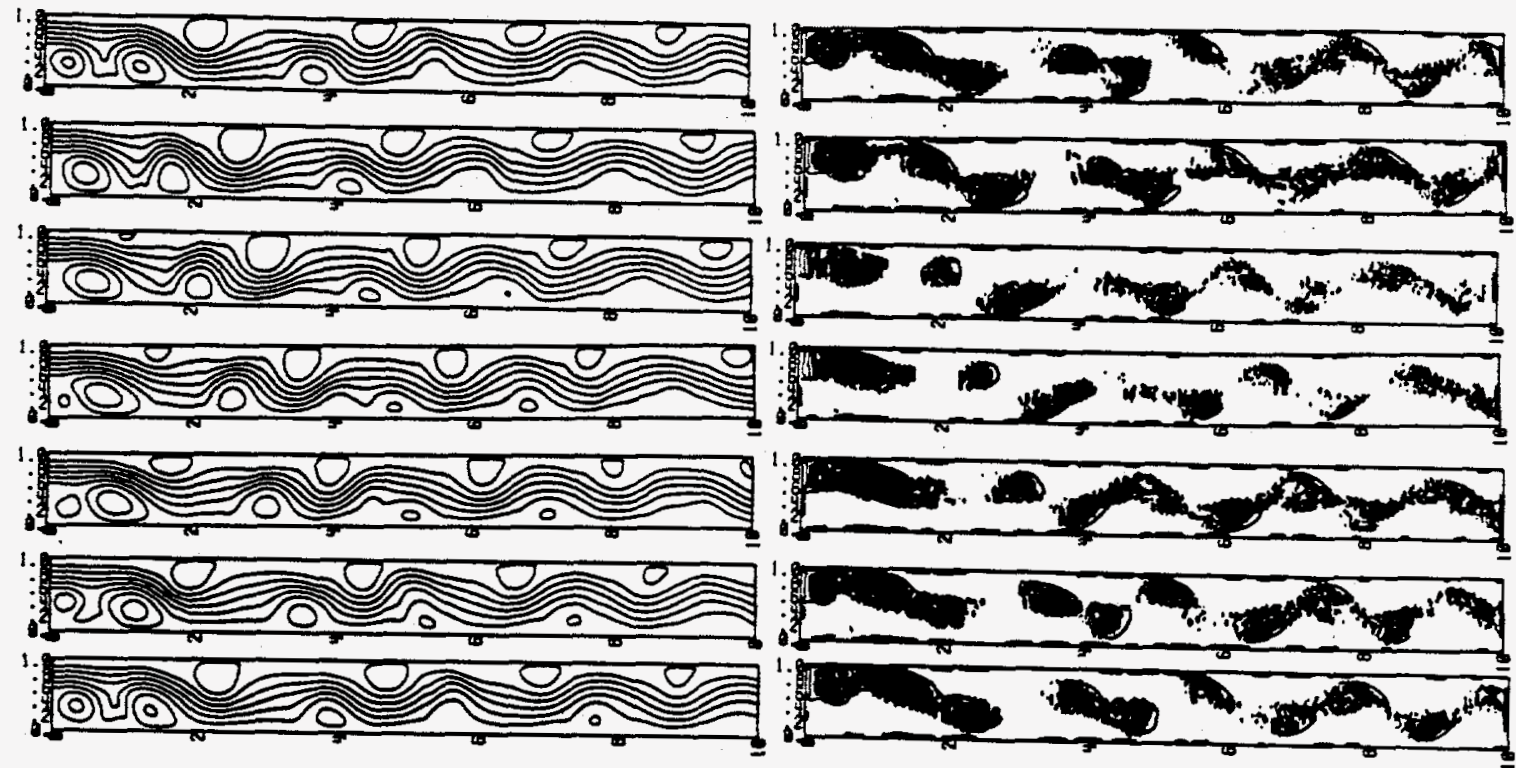

Stream Function

$v_{T}$ contours

$0.0001<v_{T} / v<46.45$

Maximum $v_{T}$ at $(\mathrm{x}, \mathrm{y})=(.73613, .68055)$

Fig. 5.3.73c Comparison of a time series of stream function plots and contour plots for $v_{T}$ for the $75 \times 722$ grid with $\Delta_{f}=3 \Delta$ (one cycle is shown at $0,1 / 6,2 / 6,3 / 6,4 / 6$, $5 / 6$, and $6 / 6$ of a DS cycle). 


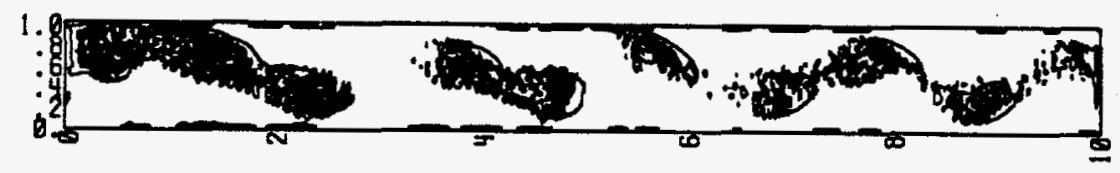

$.0002<v T / v<29.48$

$x=1.0$

$x=\mathbf{2 . 0}$
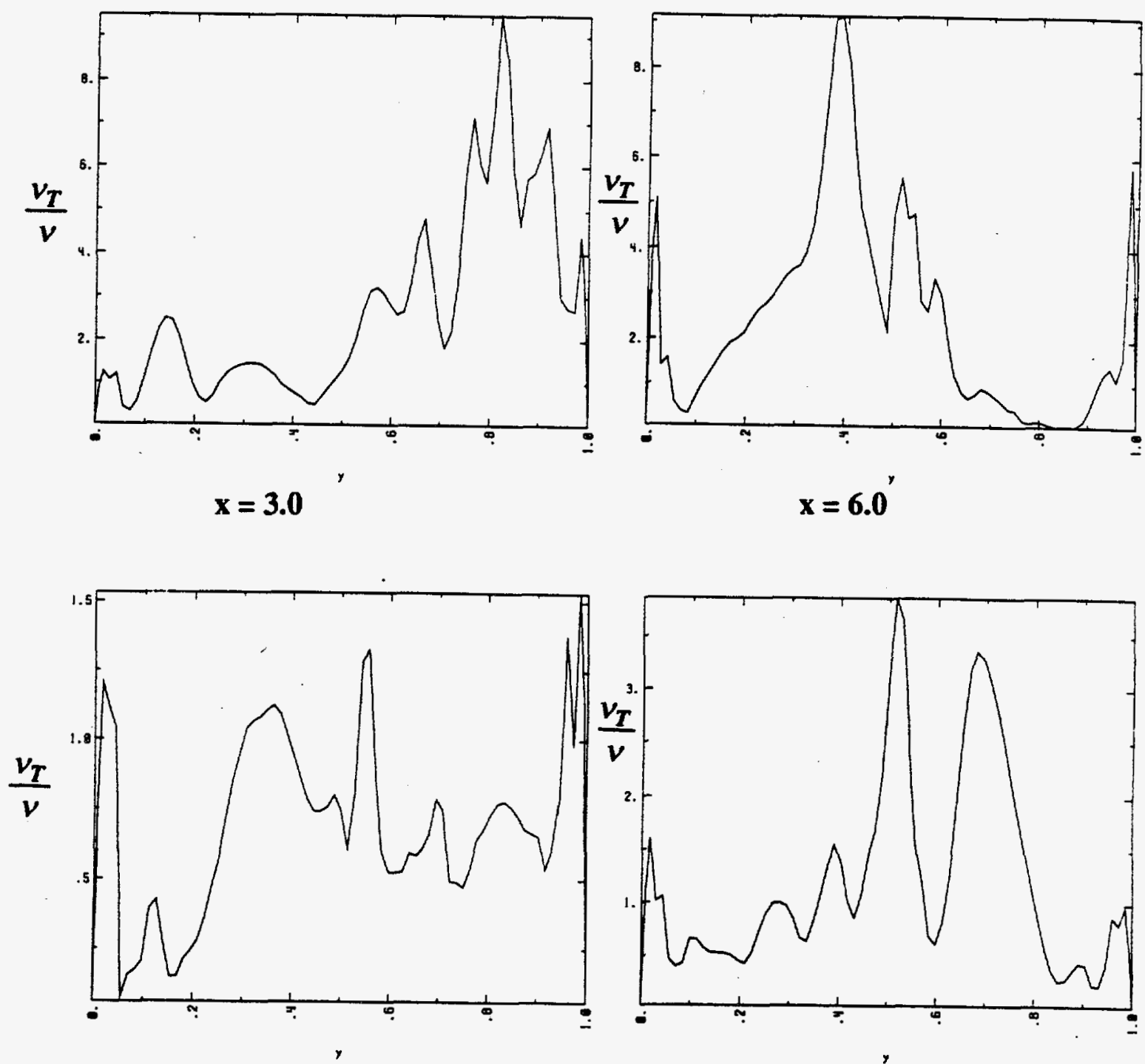

Fig. 5.3.74 A $v_{T}$ contour plot and profile plots at selected locations for the $75 \mathrm{x}$ 722 grid with $\Delta_{f}=3 \Delta$ at a snapshot in time. 


\section{Summary and Conclusions}

In this study, an LES model was successfully developed, implemented into a computer code, validated, and tested for the case of a two-dimensional backward-facing step. The LES method was used in conjunction with a FEM numerical scheme to show that FEM is a viable approach for the solution of the LES equations.

A modified Galerkin finite element method was used with quadrilateral elements with piecewise continuous multilinear basis functions for velocity and piecewise-constant basis functions for pressure. To reduce run time cost, a lumped mass matrix was used and the coefficient matrices were computed using 1-point quadrature. The hour-glass correction term and BTD terms used for the laminar flow case were used for the LES formulation. The pressure Poisson equation was solved using a direct skyline solver and a forward (explicit) Euler time integration scheme was used for the velocity solution.

Two-dimensional turbulence theory was used in the formulation of the continuous and discrete equations and in the formulation of the SGS model so that all of the code development was consistent with the two-dimensional assumption. Current SGS models were investigated and a two-dimensional model called the vorticity model was developed for application into the finite element code.

The discrete LES equations were developed using FEM and implemented into an existing laminar finite element code. It was shown that, with FEM, the equations can be filtered over a width $\Delta f>\Delta$ and the numerical scheme does not necessarily imply the filter width. In theory, the filtering method and filter width can be chosen just as is done with the spectral method approach. The key to this flexibility is in the fact that 
even though the method is grid based, the flow variables are defined continuously at all points in the flow field and not just at discrete points. The finite element method in general allows for the exact solution of the advection term by calculation of the derivative of the filtered product of the basis functions.

Cell volume-averaging was used in this study because there are currently no available filtering schemes for wall bounded flows (i.e., current published literature does not include the application of a filter normal to the walls of a bounded domain). A scheme for explicitly solving the LES advection term with the finite element method was developed and implemented in the code. The analytical approach for choosing a filter width in the solution of the advection term was simplified because of the centroid advection velocity simplification and the use of one-point quadrature in the existing code. In summary, for $\Delta f=\Delta$ the exact advection term reduces to that for the approximated advection term with this finite element approach. Filter widths at the grid size and greater than the grid size were chosen.

Wall models were investigated for the case of separating flow. A new approach of using the SGS model to the wall was used for solving the two-dimensional backward-facing step problem.

For the laminar verification tests presented in this study, a zero natural boundary condition at the outlet gives accurate results. In the discretized LES formulation, the natural boundary condition included the SGS motion (equation 3.1b). Zero natural boundary conditions for the LES outflow condition were used and were found to give accurate results.

The coding was verified for the laminar case by comparison to the numerical 
and experimental results of other researchers. Methods for validating an LES were discussed and an approach was developed. The method consists of evaluating both the transient and the time-averaged results. Snapshots of stream function plots for the LES and a direct simulation (DS) were compared for one periodic cycle. The LES and DS predicted velocity time histories and their power spectra were also compared. Before the DS for the instantaneous flow field was compared to the LES results for the largescale motion, the DS was filtered. A cell volume-averaging scheme for filtering the DS was developed and used.

For the DS, the results were found to not only be sensitive to grid size, but they were also sensitive to the chosen time step. The Courant limit determines the stability time step, but a significantly smaller time step was needed for accuracy. For Re.= 10,000 we obtain a converged solution for the large-scale motion for a grid of $101 \times 1001$ nodes with a time step of 0.005 . The DS nonfiltered and filtered time histories were almost identical. However, it was shown that this agreement between the nonfiltered and filtered velocities does not imply that a coarser grid will give a converged solution. Time averaging the DS over 20,000 time steps for $\Delta t=0.005$ generated a stationary time-averaged solution.

For the LES with $\Delta f=\Delta$, grid resolution in wall regions with grading normal to walls results in a significant improvement over an everywhere-uniform grid. It was concluded that an accurate solution that compares with the DS can be generated using the LES approach with a $92 \%$ reduction in elements from the DS to the LES grid (i.e., 100,000 elements for the DS and 7,808 elements for the LES).

When the chosen filter width was greater than the grid size $(\Delta f=3 \Delta)$, the results were unstable. Thus, the method developed here for explicitly filtering the advection 
term with the centroid advection velocity simplification and one-point quadrature was unstable. Evaluation of the results before the runs go unstable for two grid sizes (a $33 \times 245$ node grid and a $75 \times 722$ node grid) indicates that finer discretization was required for $\Delta f=3 \Delta$ to obtain the same accuracy achieved with $\Delta f=\Delta$. Therefore, there appears to be no benefit in choosing the filter width greater that the grid size with the numerical approach used in this study.

In this study, it was shown that the LES approach in conjunction with a finite element numerical scheme can accurately predict the transient, complex flow over a backward-facing step. Comparing the computational effort required for the LES solution with the DS, the LES solution is generated at a significant reduction in computational effort. These results support the hypothesis that the LES/FEM approach is a viable method for solving turbulent flows around complex geometries with efficient and effective use of resources. LES provides a high degree of accuracy with a minimum of empiricism, and the finite element method is ideal for the modeling of complex geometries of practical interest.

Future efforts should be the expansion of the code capability to threedimensional simulations. This should first involve implementation and exercising of the Smagorninsky model, followed by implementation and exercising of Germano's model. It is important that we clearly evaluate simulation improvement with Germano's model over the Smagorinsky model because Germano's model is more difficult to implement and use and requires more computational effort over that for the Smagorinsky model (as explained in Section 2.4.4). Wall model development should also be part of future efforts. The approach used here (i.e., using the LES model to the wall) could be further investigated for higher Reynolds number cases. Engineering problems of practical interest can only be considered with adequate wall 
approximations, since resolution of the wall region usually requires excessive computer resources. Future efforts may also include an investigation and development of filtering schemes for wall-bounded flows. 


\section{References}

Adams, E. W. and Johnson, J. P. 1988a, "Effects of the Separating Shear Layer on the Reattachment Flow Structure, Part 2: Reattachment Length and Wall Shear Stress", Experiments in Fluids, 6, pp. 493-499.

Adams, E. W. and Johnston, J.P. 1988b, "Flow structure in the Near-Wall Zone of a Turbulent Separated Flow", AIAA Journal, 26, No. 8, August, pp. 932-939.

Anderson, D. A., Tannehill, J. C., and Pletcher, R. H. 1984, Computational Fluid Mechanics and Heat Transfer, Hemisphere Publ. Corp., pp. 252-254.

Armaly, B.F., Durst, F., Pereira, J.C.F., Schönung, B. 1983, "Experimental and Theoretical Investigation of Backward Facing Step Flow", J. Fluid Mech., 127, pp. 473-496.

Baetke, F., Werner, H., and Wengle, H. 1987, "Computation of Turbulent Flow Around a Cube on a Vector Computer", Proc. 6th Symp. Turbulent Shear Flows, Toulouse, France, Sept. 7-9.

Bardina, J., Ferziger, J. H., and Reynolds, W. C. 1980, "Improved Subgrid Scale Models for Large Eddy Simulation", AIAA, paper 80-1357.

Bardina, J., Ferziger, J.H. and Reynolds, W.C. 1983, "Improved Turbulence Models Based on Large Eddy Simulation of Homogeneous, Incompressible, Turbulent Flows," Report TF-19, Thermosciences Div., Dept. of Mech. Engrg., Stanford Univ., Stanford, CA.

Becker, E. B. Carey, G. F., and Oden, J. T. 1981, Finite Elements: An Introduction, 1, Prentice-Hall.

Berbee, J.G. and Ellzey, J.L. 1989, "The Effect of Aspect Ratio on the Flow Over a Reward-Facing Step", Experiments in Fluids, 7, No. 7, July, pp. 447-452.

Cardot, B. and Pironneau, O. 1988, "Simulation of Turbulence with Transient Mean with the k- $\varepsilon$ Model", proceddings from the Winter Annual Meeting of ASME, Chicago, Illinois, Nove. 27-Dec. 2, 1988, pp. 1-10.

Carey, G. F. and Oden, J. T. 1986, Finite Elements: Fluid Mechanics, 6, Prentice-Hall.

Clark, R.A., Ferziger, J.H., and Reynolds, W.C. 1979, "Evaluation of Subgrid-Scale Models Using an Accurately Simulated Turbulent Flow", J. Fluid Mech., 91, Part 1, pp. 1-16.

Cook, R. D., Malkus, D. S., and Plesha, M. E. 1989, Concepts and Applications of Finite Element Analysis, John Wiley \& Sons.

Deardorff, J.W. 1970a, "A Numerical Study of Three-Dimensional Turbulent Channel Flow at Large Reynolds Numbers", J. of Fluid Mech., 41, Part 2, pp. 453-480.

Deardorff, J.W. 1970b, "A Three-Dimensional Numerical Investigation of the Idealized Planetary Boundary Layer", Geophys. Fluid Dyn., 1, p. 377. 
Durst, F. and Pereira, J.C.F. 1988, "Time-Dependent Laminar Backward-Facing Step Flow in a Two-Dimensional Duct", Journal of Fluids Engineering, 110, pp. 289296.

Eaton, J. K. and Johnston, J. P. 1980, "Turbulent Flow Reattachment: An Experimental Study of the Flow and Structure Behind a Backward-Facing Step", Report MD39, Stanford Univ., Stanford, CA.

Ferziger, J. H. 1977, "Large Eddy Numerical Simulations of Turbulent Flows", AIAA Journal, 15, No. 9, September 1977, pp. 1261-1267.

Ferziger, J. H. 1981, "Higher-Level Simulations of Turbulent Flows", Report TF-16, Thermosciences Div., Dept. of Mech. Engrg., Stanford University, Stanford, CA.

Ferziger, J. H. 1990, "Approaches to Turbulent Flow Computation: Applications to Flow Over Obstacles", Journal of Wind Engineering and Industrial Aerodynamics, 35, pp. 1-19.

Ferziger, J.H. 1987, "Simulation of Incompressible Turbulent Flows", Journal of Computational Physics, 69, p. 1-48.

Findikakis, A. N. and Street, R. L. 1980, "Numerical Simulation of Turbulent Stratified Flows in Surface Water Impoundments", Proc. of the Spec. Conf. on Comput. and Phys. Model in Hydraul. Eng., Chicago, Illinois, August 6-8, 1980, Published by ASCE, New York, NY, pp. 249-260.

Findikakis, A. N., Franzini, J. B., and Street, R. L. 1978, "Simulation of Stratified Turbulent Flows in Closed Water Bodies Using the Finite Element Method", Proceedings from Finite Elements in Water Resources Conference, July 1978, London, England, pp. 3/23-44.

Fox, D.G. and Deardorff, J.W. 1972, "Computer Methods for Simulation of Multidimensional Turbulence", J. Comput. Phys., 11, pp. 612-619.

Franke, R. and Rodi, W. 1991, "Calculation of Vortex Shedding Past a Square Cylinder with Various Turbulence Models", Proc. 8th Symp. Turbulent Shear Flows, Munich, Germany, Sept. 1991.

Gartling, D. K. 1990, "A Test Problem for Outflow Boundary Conditions-Flow over a Backward-Facing Step", International Journal for Numerical Methods in Fluids, 11, p. 953-967.

Germano, M. 1986a, "Differential Filters for the Large Eddy Numerical Simulation of Turbulent Flows", Physics of Fluids, 29, No. 6, June, pp. 1755-1757.

Germano, M. 1986b, "Differential Filters of Elliptic Type", Physics of Fluids, 29, No. 6, June, pp. 1757-1758.

Germano, M. 1990, "Averaging Invariance of the Turbulent Equations and Similar Subgrid-Scale Modeling", NASA Ames/Stanford University Center for Turbulence Research, Manuscript 116. 
Germano, M. Piomelli, U., Moin, P., Cabot, W. H. 1990, "A Dynamic Subgrid-Scale Eddy Viscosity Model", NASA Ames/Stanford Univ. Center for Turbulence Research Proceedings of the Summer Program 1990, pp. 5-17.

Ghia, H. N., Oswald, G. A., and Ghia, U., 1987 "A Direct Method for the Solution of Unsteady Two-Dimensional Incompressible Navier-Stokes Equations", 2nd Symp. on Num. Phys. Aspects of Aerodynamic Flows, California State University, Long Beach.

Gottlieb, D.M. and Orszag, S.A. 1977, SIAM J. Sci. Stat. Comput., 19.

Goudreau, G. and Hallquist, J. 1982, "Recent Developments in Large-Scale Finite Element Lagrangian Hydrocode Technology", Comp. Meth. Appl. Mech. Eng., 33, p. 725.

Gresho, P. M. 1985, "Contribution to von Karman Institute Lecture Series on "Computational Fluid Dynamics": Advection-Diffusion and Navier-Stokes Equations", LLNL Report UCRL-92275.

Gresho, P. M. 1986, "Time Integration and Conjugate Gradient Methods for the Incompressible Navier-Stokes Equations", Proceedings from the 6th FEWR Conference in Lisbon, June 1986, Springer-Verlag, pp. 3-29.

Gresho, P. M. 1991, "Incompressible Fluid Dynamics: Some Fundamental Formulation Issues", Annual Reviews of Fluid Mechanics, 23, pp. 413-453.

Gresho, P. M. and Sani, R. L. 1987, "On Pressure Boundary Conditions for the Incompressible Navier-Stokes Equations", International Journal for Numerical Methods in Fluids, 7, p. 1111.

Gresho, P.M., Chan, S.T., Lee, R.L., and Upson, C.D. 1984, "A Modified Finite Element Method For Solving the Time-Dependent Incompressible NavierStokes Equations. Part 1: Theory", Int. J. Num. Meth. in Fluids, 4, pp. 557-598.

Hinze, J. 1975, Turbulence, McGraw-Hill.

Hood, P. and Taylor, C. 1974, "Navier-Stokes Equations Using Mixed Interpolation", International Symposium on the Finite Element Method in Flow Problems, Proceedings, January 1974, Swansea, UK.

Hughes, T. J. R. and Brooks, A. 1982, "Streamline Upwind/Petrov-Galerkin Formulations for Convection Dominated Flows with Particular Emphasis on the Incompressible Navier-Stokes Equations", Computer Methods in Applied Mechanics and Engineering, 32, pp. 199-259.

Hughes, T. J. R., Franca, L. P., Hulbert, G. M., Johan, Z., and Shakib, F. 1988, "'"The Galerkin/Least Squares Method for Advection-Diffusive Equations", in Recent Developments in Computational Fluid Dynamics, T. E. Tezduyar and T. J. R. Hughes, ed., AMD, 95, pp. 75-91.

Hutton, A.G., Smith, R.M., and Hickmott, S. 1987, "The Computation of Turbulent Flows of Industrial Complexity by the Finite Element Method-Progress and 
Prospects", Finite Elements in Fluids, 7, edited by Gallagher, R.H., Glowinski, R., Gresho, P.M., Oden, J.T., and Zienkiewics, O.C., John Wiley \& Sons Ltd., 1987, pp. 289-309.

Jones, W.P. and Launder, B.E. 1972, "The Prediction of Laminarization with a TwoEquation Model of Turbulence", Int. J. Heat and Mass Transfer, 15, p. 301.

Kim, J. and Moin, P. 1985, "Application of a Fractional-Step Method to Incompressible Navier-Stokes Equations", Journal of Computational Physics, 59, No. 2, June 1985, pp. 308-323.

Kim, J. and Moin, P.J. 1985, "Application of a Fractional-Step Method to Incompressible Navier-Stokes Equations", J. Comput. Phys., 54, pp. 308-323.

Kim, J., Kline, S.J., and Johnston, J.P. 1980, "Investigation of a Reattaching Turbulent Shear Layer: Flow over a Backward-Facing Step", Transactions of the ASME, 102, September, pp. 302-308.

Kobayashi, T., Morinishi, Y., and Oh, K-J 1992, "Large-Eddy Simulation of BackwardFacing Step Flow", Communications in Applied Numerical Methods, 8, pp. 431441.

Kobayashi, T., Morinishi, Y., and Taniguchi, N. 1989, "Applications of LES to Complicated Flow Fields", International Seminar on Supercomputing in Fluid Flow, Boston.

Kondo, N., Tosaka, N., and Nishimura, T. 1986, "Finite Element Analysis for Turbulent Flows-Elastic Shell Interaction", Proceedings from Sixth International Symposium on Finite Element Methods in Flow Problems, 16-20 June 1986, Antibes, France, pp. 177-181.

Kondoh, T. and Nagano, Y. 1989, "Numerical Investigation of a Backward-Facing Step", Proceedings from International Symp. on Comput. Fluid Dynamics, 3rd Nagoya, Japan, Aug. 28-31, pp. 299-304.

Kwak, D., Reynolds, W.C., and Ferziger, J.H. 1975, "Three Dimensional Time Dependent Computation of Turbulent Flow", Report TF-5, Thermosciences Div., Dept. of Mech. Engrg, Stanford Univ., Stanford, CA.

Lasher, W. C. and Taulbee, D. B. 1989, "The Effect of Near-Wall Treatment on the Behavior of Reynolds-Stress Turbulence Models for Reattaching Flows", Proc. 7th Symp. Turbulent Shear Flows, Stanford Univ., Stanford, CA, Aug. 21-23.

Le, H., Moin, P., and Kim, J. 1990, "Direct Numerical Simulation of Turbulence Over a Backward-Facing Step", Abstract submitted to 43rd Annual Meeting of the Division of Fluid Dynamics, Ithaca, New York, Nov. 18-20.

Leith, C.E. 1965, Methods in Computational Physics, 4, New York Academic.

Leith, C.E. 1990, "Stochastic Backscatter in a Subgrid-Scale Model: Plane Shear Mixing Layer", Physics of Fluids A, 2, No. 3, March 1990.

Leonard, A. 1974, "Energy Cascade in Large-Eddy Simulations of Turbulent Fluid 
Flows", Adv. Geophys., 18A, pp. 237-248.

Lilly, D. K. 1967, "The Representation of Small-Scale Turbulence in Numerical Experiments", Proc. IBM Scientific Computing on Environmental Sciences, IBM, White Plains, NY.

Machenhauer, B. 1979, "The Spectral Model, Numerical Methods used in Atmospheric Models", GARP Report, 17, II, pp. 121-275.

Mansour, N. N. and Moin, P. 1983, "Computation of Turbulent Flows Over a Backward-Facing Step", Fourth Symp. on Turbulent Shear Flows, Karlsruhe.

Mason, P.J. and Callen, N.S. 1986 "On the Magnitude of the Subgrid-Scale Eddy Coefficient in Large-Eddy Simulation of the Turbulent Channel Flow", J. Fluid Mech., 162, pp. 439-462.

Moin, P., Kim, J. J. 1981, "Numerical Investigation of Turbulent Channel Flow", J. Fluid Mech., 118, pp. 341-377.

Moin, P., Reynolds, W.C., and Ferziger, J. H. 1978, "Large Eddy Simulation of Turbulent Channel Flow", Rept TF-12, Dept. of Mech. Engr., Stanford Univ.

Monin, A.S. and Yaglom, A.M. 1971, Statistical Fluid Mechanics, 1, Cambridge Mass: MIT Press, 769 pp.

Murakami, S. and Mochida, A. 1988, "3-D Numerical Simulation of Airflow Around a Cubic Model by Means of the k-e Model", J. of Wind Engineering and Industrial Aerodynamics, 31, pp. 283-303.

Murakami, S., Mochida, A., and Hibi, K. 1987, "Three-Dimensional Numerical Simulation of Air Flow around a Cubic Model by Means of Large Eddy Simulation", Journal of Wind Engineering and Industrial Aerodynamics, 25, pp. 291-305.

Neto, A. S., Grand, D., and Lesieur, M. 1991a, "Simulation numérique bidimensionnelle d'un ecoulement turbulent stratifiè derrière une marche", Int. J. Heat Mass Transfer, 34, No. 8, pp. 1999-2011.

Neto, A. S., Grand, D., Métais, O., Lesieur, M. 1991b, "Large-Eddy Simulation of the Turbulent Flow in the Downstream Region of a Backward-Facing Step", Lecture Series 1991-03, New Approaches in the Description and Modeling of Turbulence, May 6-8, 1991, von Karman Institute for Fluid Dynamics, Chaussee de Waterloo, 72, B-1640 Rhode Saint Genese, Belgium.

Orszag, S. A. 1972, "Comparison of Pseudospectral and Spectral Approximation", Studies in Applied Mechanics.

Orszag, S.A. 1971, "Numerical Simulation of Incompressible Flows Within Simple Boundaries : Accuracy", J. Fluid Mech., 49, P1, pp. 75-112.

Piomelli, U., Cabot, W. H., Moin, P., and Lee, S. 1990, "Subgrid-Scale Backscatter in Transitional and Turbulent Flows", NASA Ames/Stanford University Center for 
Turbulence Research Proceedings of the Summer Program 1990, pp. 19-30.

Piomelli, U., Ferziger, J.H., and Moin, P. 1987, "Models for Large Eddy Simulations of Turbulent Channel Flows including Transpiration", Rept TF-32, Dept. of Mech. Engr., Stanford Univ.

Piomelli, U., Moin, P., and Ferziger, J. H. 1988, "Model Consistency in Large Eddy Simulation of Turbulent Channel Flows", Phys. Fluids, 31, No. 7, July, pp. 1884-1891.

Reddy, J. N. 1984, An Introduction to the Finite Element Method, McGraw-Hill.

Reynolds, O. 1895, Phil. Trans. A, 186, p.123.

Reynolds, W.C. 1989 "The Potential and Limitations of Direct and Large Eddy Simulations", Position paper, Whither Turbulence Workshop, Cornell University, March 22-24, pp. 1-30.

Robinson, S. K., Kline, S. J., and Spalart, P. R. 1989, "A Review of Quasi-Coherent Structures in a Numerically Simulated Turbulent Boundary Layer", NASA Technical Memorandum 102191.

Robinson, S.K. 1989, "A Review of Vortex Structures and Associated Coherent Motions in Turbulent Boundary Layers", Proceedings of the Second IUTAM Symposium on Structure of Turbulence and Drag Reduction, Federal Institute of Technology, Zurich, Switzerland, July 25-28, 1989.

Rodi, Wolfgang 1980, Turbulence Models and Their Application in Hydraulics-A State of the Art Review, Presented by the IAHR-Sectioon on Fundamentals of Division II: Experimental and Mathematical Fluid Dynamics.

Rogallo, R.S. and Moin, P. 1984, "Numerical Simulation of Turbulent Flows", Ann. Rev. Fluid Mech., 16, pp. 99-137.

Ruderich, R. and Fernholz, H.H. 1986, "An Experimental Investigation of a Turbulent Shear Flow with Separation, Reverse Flow, and Reattachment", J. of Fluid Mech., 163, pp. 283-322.

Samagio, A. and Vlachos, N.S. 1988, "Experimental and Numerical Investigation of Three-Dimensional Turbulent Flow in a Backward-Facing Step", Proceeding from Comput. Engr. 1988, July 31-Aug. 4 at San Francisco, CA, Publ. by ASME, New York, NY, pp. 465-473.

Sandham, N.D. and Reynolds, W.C. 1987, "Some Inlet Plan Effects on the Numerically Simulated Spatially-Developing Mixing Layer", Proc. Sixth Symp. Turb. Shear Flows, Toulouse, France, Sept. 7-9.

Sani, R. L., Gresho, P. M., Lee, R. L., and Griffiths, D. F. 1981, "The Cause and Cure (?) of the Spurious Pressures Generated by Certain FEM Solutions of the Incompressible Navier-Stokes Equations: Part 1", International Journal for Numerical Methods in Fluids, 1, pp. 17-43.

Schmitt, L. and Friedrich, R. 1987, "Large-Eddy Simulation of Turbulent Backward 
Facing Step Flow", Proceedings from Seventh GAMM-Conference on Numerical Methods in Fluid Mechanics, Louvain-la-Neuve, Belgium, Sept. 911,1987 , Brunswick, Federal Republic of Germany, Friedr. Vieweg und Sohn, 1988, pp. 355-362.

Schumann, U. 1975, "Subgrid Scale Model for Finite Difference Simulations of Turbulent Flows in Plane Channels and Annuli", J. of Computational Physics, 18 , p. $376-404$.

Smagorinsky, J. 1963, "General Circulation Experiments with the Primitive Equations,

I. The Basic Experiment", Monthly Weather Review, 91, No. 3, pp. 99-164.

Smagorinsky, J., Manabe, S., and Holloway, J. L. 1965, "Numerical Results from a Nine-Level General Circulation Model of the Atmosphere", Monthly Weather Review, 93, No. 12, pp. 727-768.

Spalart, P.R. 1988, "Direct Numerical Simulation of a Turbulent Boundary Layer up to $\mathrm{R}_{\mathrm{q}}=1410^{\prime \prime}, \mathrm{J}$. Fluid Mech., 187, p. 61.

Speziale, C.G. 1985, "Galilean Invariance of Subgrid-Scale Stress Models in the LargeEddy Simulation of Turbulence", J. Fluid Mech., 156, pp.55-62.

Speziale, C.G. and Ngo, Tuan 1988, "Numerical Solution of Turbulent Flow Past a Backward Facing Step Using a Nonlinear k-e Model", Int. J. Engng. Sci., 26, No. 10, pp. 1099-1112.

Taylor, R., Wilson, E., and Sackett, S. 1981, "Direct Solution of Equations by Frontal and Variable Band, Active Column Methods", in Nonlinear Finite Element Analysis in Structural Mechanics, Springer-Verlag, 521 p.

Tennekes, H. and Lumley, J. L. 1972, A First Course in Turbulence, MIT Press.

Tuerpe, D. R., Rodriguez, D. J., Gresho, P. M., and Sani, R. L. 1978, "Mass-Consistent Wind Field Modeling: Finite Difference vis-a-vis Finite Element", Proc. 9th International Technical Meeting on Air Pollution Modeling and Its Application, Toronto, Canada, August 28-31, 1978.

Werner, H. and Wengle H. 1989, "Large-Eddy Simulation of Turbulent Flow over a Square Rib in a Channel", Proceedings from 7th Symposium on Turbulent Shear Flows, Stanford University, Aug. 21-23, Section 10.2, pp. 1-6.

Wyngaard, J.C., ed. 1984, Large-Eddy Simulation: Guidelines for Its Application to Planetary Boundary Layer Research, Michaels Communications, August 1984, $122 \mathrm{p}$. 


\section{Appendix A}

\section{Details of Equation Formulation}

The most general form (applicable to compressible and incompressible flow) of the Navier-Stokes equations is

Mass Balance

$$
\frac{\partial \rho}{\partial t}+\frac{\partial}{\partial x_{\alpha}}\left(\rho u_{\alpha}\right)=0
$$

Momentum Balance

$$
\rho \frac{\partial u_{\alpha}}{\partial t}+\rho u_{\beta} \frac{\partial u_{\alpha}}{\partial x_{\beta}}=-\frac{\partial p}{\partial x_{\alpha}}+\frac{\partial}{\partial x_{\beta}} \tau_{\alpha \beta}
$$

where $p$ includes the gravitational body force, $\rho g_{\alpha}$,

$$
\tau_{\alpha \beta}=\mu\left(\frac{\partial u_{\alpha}}{\partial x_{\beta}}+\frac{\partial u_{\beta}}{\partial x_{\alpha}}-\frac{2}{3} \delta_{\alpha \beta} \frac{\partial u_{\gamma}}{\partial x_{\gamma}}\right)
$$

and repeated indices imply summation.

If we assume that density changes in time and space are negligible, the flow is called incompressible. The incompressible flow equations are 


$$
\begin{gathered}
\frac{\partial u_{\alpha}}{\partial x_{\alpha}}=0 \\
\frac{\partial u_{\alpha}}{\partial t}+\frac{\partial}{\partial x_{\beta}} u_{\alpha} u_{\beta}=-\frac{1}{\rho} \frac{\partial p}{\partial x_{\alpha}}+\frac{\partial}{\partial x_{\beta}} \tau_{\alpha \beta}
\end{gathered}
$$

where

$$
\tau_{\alpha \beta}=v\left(\frac{\partial u_{\alpha}}{\partial x_{\beta}}+\frac{\partial u_{\beta}}{\partial x_{\alpha}}\right)
$$

If we make the additional assumption that $v$ is a constant, the equations reduce to

$$
\begin{gathered}
\frac{\partial u_{\alpha}}{\partial x_{\alpha}}=0 \\
\frac{\partial u_{\alpha}}{\partial t}+\frac{\partial}{\partial x_{\beta}} u_{\alpha} u_{\beta}=-\frac{1}{\rho} \frac{\partial p}{\partial x_{\alpha}}+v \frac{\partial^{2}}{\partial x_{\beta}^{2}} u_{\alpha}
\end{gathered}
$$

which is applicable to both laminar and turbulent flows.

The first step in an LES formulation is to define the resolved (large-scale) velocity field and a filter (which is done in Section 2.4.2). The result is that the instantaneous velocity, $u_{\alpha}$, is decomposed into a resolved field, $\bar{u}_{\alpha}$, and an SGS field, $u_{\alpha}^{\prime}$;

$$
u_{\alpha}=\bar{u}_{\alpha}+u_{\alpha}^{\prime}
$$

We will filter the equations using the filter properties 


$$
\frac{\overline{\partial f}}{\partial t}=\frac{\partial \bar{f}}{\partial t} \text { and } \frac{\overline{\partial f}}{\partial x_{\beta}}=\frac{\partial \bar{f}}{\partial x_{\beta}}
$$

where the function $f=f(x, y, z, t)$, in general. The resulting equations are:

$$
\begin{gathered}
\frac{\partial \bar{u}_{\alpha}}{\partial x_{\alpha}}=0 \\
\frac{\partial \bar{u}_{\alpha}}{\partial t}+\frac{\partial \overline{u_{\alpha} u_{\beta}}}{\partial x_{\beta}}=-\frac{1}{\rho} \frac{\partial \bar{p}}{\partial x_{\alpha}}+v \frac{\partial^{2}}{\partial x_{\beta}^{2}} \bar{u}_{\alpha}
\end{gathered}
$$

For the advection term,

$$
\begin{aligned}
\overline{u_{\alpha} u_{\beta}} & =\overline{\left(\bar{u}_{\alpha}+\dot{u_{\alpha}}\right)\left(\bar{u}_{\beta}+\dot{u_{\beta}}\right)} \\
& =\overline{\bar{u}_{\alpha} \bar{u}_{\beta}}+\overline{u_{\alpha} \overline{u_{\beta}}}+\overline{\overline{u_{\alpha}} \dot{u_{\beta}}}+\overline{u_{\alpha} \dot{u_{\beta}}} .
\end{aligned}
$$

Substituting and rearranging

$$
\frac{\partial \bar{u}_{\alpha}}{\partial t}+\frac{\partial}{\partial x_{\beta}} \overline{\bar{u}_{\alpha} \bar{u}_{\beta}}=-\frac{1}{\rho} \frac{\partial \bar{p}}{\partial x_{\alpha}}+v \frac{\partial^{2}}{\partial x_{\beta}^{2}} \bar{u}_{\alpha}-\frac{\partial}{\partial x_{\beta}}\left(\overline{u_{\alpha} \bar{u}_{\beta}}+\overline{\bar{u}_{\alpha} u_{\beta}}+\overline{u_{\alpha} \bar{u}_{\beta}}\right)
$$

This is equation 2.3 and is the form of the momentum equation discretized in Section 3.0.

Another form that will be used in this study for calculations with the Smagorinsky model is equation 2.2. For reasons discussed in the text, it is derived by adding and subtracting $\delta_{\alpha \beta} \overline{u_{\gamma}^{\prime} u_{\gamma}^{\prime}} / 3$ from the right side, and adding $\partial \bar{u}_{\alpha} \bar{u}_{\beta} / \partial x_{\beta}$ to 
300

both sides of the equation, resulting in

$$
\begin{aligned}
\frac{\partial \bar{u}_{\alpha}}{\partial t}+ & \frac{\partial}{\partial x_{\beta}} \bar{u}_{\alpha} \bar{u}_{\beta}=-\frac{1}{\rho} \frac{\partial}{\partial x_{\alpha}}\left(\bar{p}+\frac{1}{3} \rho \delta_{\alpha \beta} \overline{\bar{u}_{\gamma}^{\prime} u_{\gamma}^{\prime}}\right) \\
& +v \frac{\partial 2}{\partial x_{\beta}^{2}} \bar{u}_{\alpha}-\frac{\partial}{\partial x_{\beta}}\left(\overline{\bar{u}_{\alpha} \bar{u}_{\beta}}-\bar{u}_{\alpha} \bar{u}_{\beta}\right)-\frac{\partial}{\partial x_{\beta}}\left(\overline{\overline{u_{\alpha} \bar{u}_{\beta}}}+\overline{\bar{u}_{\alpha} u_{\beta}^{\prime}}\right) \\
& -\frac{\partial}{\partial x_{\beta}}\left(\overline{\bar{u}_{\alpha}^{\prime} u_{\beta}^{\prime}}-\frac{1}{3} \delta_{\alpha \beta} \overline{u_{\gamma}^{\prime} \bar{u}_{\gamma}^{\prime}}\right) .
\end{aligned}
$$




\section{Appendix B}

\section{Time Averaging of Instantaneous Quantities}

The following describes how time averages of the instantaneous quantities were generated during program execution (i.e., while generating the instantaneous quantities, instead of as a postprocessing operation). The time averages are calculated during program execution to avoid storing values at each time step and thus to reduce memory and storage requirements. The method used in calculating the time-average velocities, turbulent intensities, $\left\langle u^{\prime \prime}\right\rangle,\left\langle v^{\prime \prime} 2\right\rangle$ and $\left\langle w^{\prime \prime} 2\right\rangle$, turbulent shear, $\left\langle u^{\prime \prime} v^{\prime \prime}\right\rangle$, and the dissipation rate are outlined. (The brackets $<$ represent a time average.) The described averaging methods were implemented in the code used for this study for twodimensional problem solutions. Also presented is the method used to take the time averages for a number of consecutive intervals and calculate the time average for the sum of the intervals.

\section{Time-Average Definition}

As explained in Section $2.3^{1}$, the velocity field can be decomposed into a mean velocity <u> (time, space, or ensemble averaged) and a fluctuating velocity, $u$ ", such that the instantaneous velocity $u$ is defined as

\footnotetext{
${ }^{1}$ In Section 2.3, we use the notation $u_{i}$ to represent the $x_{i}$ component of the velocity where $i=1,2$, and 3 . We use the notation $u, v$, and $w$ for the three velocity components in this appendix to avoid confusion with the summation index used here.
} 


$$
u=<u>+u
$$

The averaging operation $\left\langle>\right.$ is defined such that $\left\langle u^{\prime \prime}\right\rangle=0$. If the turbulence flow field is stationary (i.e., statistically steady), averaging with respect to time can be used (Hinze 1975 or Lumley 1972). By definition,

$$
<u(\underline{x})>=\lim _{T \rightarrow \infty} \frac{1}{2 T} \int_{-T}^{+T} u(\underline{x}, t) d t
$$

For a finite value of the averaging interval $T$, we define the averaging operator by

$$
<u(\underline{x})>=\frac{1}{T} \int_{t-\frac{T}{2}}^{t+\frac{T}{2}} u\left(\underline{x}, t^{\prime}\right) d t^{\prime}
$$

with the condition that $T_{1} \ll T \ll T_{2}$ (Hinze 1975) where the lower value $T_{1}$ is the time scale for the turbulence and the upper value $T_{2}$ is the period for slow variations in the flow field that we do not regard as part of the turbulence. If the averaging interval $T$ meets the above condition, the average value should be independent of when you start the average. Thus, $\partial<u>/ \partial t$ is zero (or negligibly small) in the interval $T$ and even zero over several $T$ intervals (since $T \ll T_{2}$ ).

In the numerical simulation, the appropriate choice of the averaging interval $T$ (i.e., such that $T_{1} \ll T \ll T_{2}$ ) is not known until after the instantaneous velocities have been calculated over a relatively long time interval (i.e., for several $T_{2}$ intervals). However, to avoid storing the velocity field at every time step for postprocessing of the average quantities, we calculate the averages during program execution (i.e., while we calculate the instantaneous velocity). The following describes the method used to 
calculate time averages during program execution for a chosen (a priori) interval $T$. If this chosen interval does not produce a time invariant average value (or one that varies only slightly over several $T$ intervals), the values for interval $T$ can be postprocessed. As described in the following sections, the averages for $k$ intervals of time duration $T$ can be used to generate values for an interval $k T$, where $k$ is a positive integer.

If the chosen $T$ is too small and the time average varies more than desired between adjacent intervals, the calculated time average is dependent on where the interval is started. The time-average velocity is then

$$
\langle u(\underline{x}, t)\rangle=\frac{1}{T} \int_{t-\frac{T}{2}}^{t+\frac{T}{2}} u\left(\underline{x}, t^{\prime}\right) d t^{\prime}
$$

where the value is defined at time $t$ which is the midpoint for the interval $T$. Although the calculated time-average velocities, turbulent intensities, shear and dissipation are not the desired result, they can be postprocessed to obtain the time average for a longer time interval.

\section{Time-Averaged Velocities}

The time-averaged velocity $\langle u\rangle$ for an interval $T$ is defined as

$$
<u(\underline{x}, t)>=\frac{1}{T} \int_{t-\frac{T}{2}}^{t+\frac{T}{2}} u\left(\underline{x}, t^{\prime}\right) d t^{t}
$$

and is the value at the interval midpoint $t$. The above integration can be approximated with the discrete summation 


$$
\begin{aligned}
& \int_{t-\frac{T}{2}}^{t+\frac{T}{2}} u\left(\underline{x}, t^{\prime}\right) d t^{\prime}=\int_{t-\frac{T}{2}}^{t-\frac{T}{2}+\Delta t} 1 u\left(\underline{x}, t^{\prime}\right) d t^{\prime}+\int_{t-\frac{T}{2}+\Delta t_{1}}^{t-\frac{T}{2}+\Delta t}+\Delta t z u\left(\underline{x}, t^{\prime}\right) d t^{\prime}+\ldots+\int_{t+\frac{T}{2}-\Delta t}^{t+\frac{T}{2}} u\left(\underline{x}, t^{\prime}\right) d t^{\prime} \\
& \cong u\left(\underline{x}, t-\frac{T}{2}+\frac{\Delta t_{1}}{2}\right) \Delta t_{1}+u\left(\underline{x}, t-\frac{T}{2}+\Delta t_{1}+\frac{\Delta t_{2}}{2}\right) \Delta t_{2}+\ldots+u\left(\underline{x}, t+\frac{T}{2}-\frac{\Delta t_{n}}{2}\right) \Delta t_{n},
\end{aligned}
$$

The above discrete summation (i.e., midpoint rule) is used for the time integration in the numerical calculation, and the averaging is performed using a constant time step to give

$$
\langle u(\underline{x}, t)\rangle \cong \frac{\sum_{i=1}^{n} u\left(\underline{x}, t_{i}\right) \cdot \Delta t}{\sum_{i=1}^{n} \Delta t}=\frac{\sum_{i=1}^{n} u_{i}}{n}
$$

where $u_{i}$ is the value of $u$ at time step $i$ and at time $t_{i}, n$ is the number of time steps, and $\Delta t$ is the integration step size which is constant in the existing code (i.e., $\Sigma \Delta t=n \Delta t=$ $T$ ). This is the time-averaged velocity at the midpoint of the interval and is calculated for every node. Because the summed values are at the midpoint of the interval (according to the midpoint rule), the start of the averaging interval is $t-(T / 2)=t_{1}-\left(\Delta t_{1} / 2\right)$ and the end of the averaging interval is $t+(T / 2)=t_{n}+\left(\Delta t_{n} / 2\right)$.

If a longer time interval for averaging is desired, say $k T$ where $k$ is an integer, the values for interval $k T$ can be postprocessed using the following calculation. For time interval $k T$, the time-average velocity is

$$
\begin{aligned}
& \langle u(\underline{x}, t)\rangle=\frac{1}{k T} \int_{t-\frac{k T}{2}}^{t+\frac{k T}{2}} u\left(\underline{x}, t^{\prime}\right) d t^{\prime} \cong \frac{\sum_{i=1}^{k n} u_{i}}{k n}=\frac{\sum_{i=1}^{n} u_{i}+\sum_{i=n+1}^{2 n} u_{i}+\ldots+\sum_{i=(k-1) n+1}^{k n} u_{i}}{k n} \\
& =\frac{1}{k}\left(\langle u\rangle_{1}+\langle u\rangle_{2}+\ldots+\langle u\rangle_{k}\right)=\frac{\sum_{i=1}^{k n}\langle u\rangle_{i}}{k}
\end{aligned}
$$


where $\langle u\rangle_{i}$ is the time averaged velocity for the time interval $t+(i-1) T-\frac{T}{2}$ to $t+(i-1) T+\frac{T}{2}$.

\section{Turbulent Intensities}

The $x$-component of the velocity fluctuation $u^{n}$ is defined as

$$
u^{*}=u-\langle u>
$$

and the $\mathrm{x}$-component of the turbulent intensity is

$$
<u^{\prime 2}>=\frac{1}{T} \int_{t-\frac{T}{2}}^{t+\frac{T}{2}} u^{\prime 2} d t^{\prime}=\frac{1}{T} \int_{t-\frac{T}{2}}^{t+\frac{T}{2}}(u-<u>)^{2} d t^{\prime}
$$

If we chose $T$ such that $T_{1} \ll T \ll T_{2}$, we have

$$
<\langle u>><u>
$$

and for the turbulence intensity

$$
\begin{aligned}
& \left.\left.<u^{n 2}\right\rangle=\left\langle(u-\langle u\rangle)^{2}\right\rangle=\left\langle u^{2}-2<u\right\rangle u+\langle u\rangle^{2}\right\rangle \\
& \left.=\left\langle u^{2}\right\rangle-2<u\right\rangle\langle u\rangle+\langle u\rangle^{2}=\left\langle u^{2}\right\rangle-\langle u\rangle^{2} .
\end{aligned}
$$

In the numerical calculation, we use the discrete summation for the time integration of $u^{2}$ and thus the expression for calculating $\left\langle u^{\prime \prime}\right\rangle$ is 


$$
<u^{-2}>\cong \frac{\sum_{i=1}^{n} u_{i}^{2}}{n}-<u>^{2} .
$$

If a longer time interval for averaging is desired, say $k T$ where $k$ is an integer, the intermediate values for interval $k T$ can be postprocessed using the following calculation. For time interval $k T$, the turbulent intensity is

$$
<u^{22}>=\frac{1}{k T} \int_{t-\frac{k T}{2}}^{t+\frac{k T}{2}} u^{22} d t^{\prime}
$$

Thus, we have

$$
<u^{\prime 2}>\cong \frac{\sum_{i=1}^{k n} u_{i}^{2}}{k n}-<u>^{2}
$$

where

$$
\frac{\sum_{i=1}^{k n} u_{i}^{2}}{k n}=\frac{1}{k}\left(\frac{\sum_{i=1}^{n} u_{i}^{2}}{n}+\frac{\sum_{i=n+1}^{2 n} u_{i}^{2}}{n}+\ldots+\frac{\sum_{i=(k-1)_{n+1}}^{k n} u_{i}^{2}}{n}\right)
$$

and

$$
<u>^{2} \cong \frac{1}{k^{2}}\left(<u>_{1}+<u>_{2}+\ldots+<u>_{k}\right)^{2}
$$

There is no need to save (for postprocessing) the sum of the squared velocity because we can calculate it from the output average velocity and turbulent intensity as follows: 


$$
\begin{aligned}
& \frac{\sum_{i=1}^{n} u_{i}^{2}}{n}=\left\langle u^{n 2}\right\rangle_{1}+\langle u\rangle_{1}^{2}, \\
& \frac{\sum_{i=n+1}^{2 n} u_{i}^{2}}{n}=\left\langle u^{\prime 2}\right\rangle_{2}+\langle u\rangle_{2}^{2} \text {, etc. }
\end{aligned}
$$

Thus, the turbulent intensity for the interval $t-\frac{k T}{2}$ to $t+\frac{k T}{2}$ can be calculated (i.e.

postprocessed) from the $k$ intermediate intervals using the following expression

$$
\begin{aligned}
\left\langle u^{-2}\right\rangle & \cong \frac{1}{k}\left(\left\langle u^{-2}\right\rangle_{1}+\langle u\rangle_{1}^{2}+\left\langle u^{-2}\right\rangle_{2}+\langle u\rangle_{2}^{2}\right. \\
& \left.+\ldots+\left\langle u^{-2}\right\rangle_{k}+\langle u\rangle_{k}^{2}\right)-\frac{1}{k^{2}}\left(\langle u\rangle_{1}+\langle u\rangle_{2}+\ldots+\langle u\rangle_{k}\right)^{2}
\end{aligned}
$$

or in summation notation,

$$
\left\langle u^{-2}\right\rangle \cong \frac{1}{k}\left(\sum_{i=1}^{k}\left\langle u^{-2}\right\rangle_{i}+\sum_{i=1}^{k}\langle u\rangle_{i}^{2}-\frac{1}{k}\left(\sum_{i=1}^{k}\langle u\rangle_{i}\right)^{2}\right)
$$

\section{Turbulent Shear}

The turbulent shear is defined as

$$
\left\langle u^{\prime} v^{\prime \prime}\right\rangle=\frac{1}{T} \int_{1-\frac{T}{2}}^{t+\frac{T}{2}} u^{\prime} v^{\prime} d t^{\prime}=\frac{1}{T} \int_{t-\frac{T}{2}}^{t+\frac{T}{2}}(u-\langle u\rangle)(v-\langle v\rangle) d t^{\prime}
$$

and in the numerical calculation, we have 


$$
\left\langle u^{\prime \prime} v^{\prime \prime}\right\rangle \cong \frac{\sum_{i=1}^{n} u_{i} v_{i}}{n}-\langle u\rangle\langle v\rangle \text {. }
$$

If a longer time interval for averaging is desired, say $k T$ where $k$ is an integer, the values for interval $k T$ can be postprocessed using the following calculation. For the time interval $k T$

$$
\left\langle u^{\prime \prime} v^{\prime \prime}\right\rangle=\frac{1}{k T} \int_{t-\frac{k T}{2}}^{t+\frac{k T}{2}} u^{\prime \prime} v^{\prime \prime} d t^{\prime} \equiv \frac{\sum_{i=1}^{k n} u_{i} v_{i}}{k n}-\langle u\rangle\langle v\rangle
$$

where

$$
\sum_{i=1}^{k n} u_{i} v_{i}=\frac{1}{k}\left(\frac{\sum_{i=1}^{n} u_{i} v_{i}}{n} \frac{\sum_{i=n+1}^{2 n} u_{i} v_{i}}{n}+\ldots+\frac{\sum_{i=(k-1) n+1}^{k n} u_{i} v_{i}}{n}\right)
$$

and

$$
\langle u\rangle\langle v\rangle \cong \frac{1}{k^{2}}\left(\langle u\rangle_{1}+\langle u\rangle_{2}+\ldots+\langle u\rangle_{k}\right)\left(\langle v\rangle_{1}+\langle v\rangle_{2}+\ldots+\langle v\rangle_{k}\right) .
$$

There is no need to save the sums of the velocity products (i.e., $\Sigma u_{i} v_{i}$ ), because we can calculate them from the output turbulent shear as follows 


$$
\begin{aligned}
& \left.\frac{\sum_{i=1}^{n} u_{i} v_{i}}{n}=\left\langle u^{\prime \prime} v^{\prime \prime}\right\rangle_{1}+\langle u\rangle_{1}<v\right\rangle_{1}, \\
& \frac{\sum_{i=n+1}^{2 n} u_{i} v_{i}}{n}=\left\langle u^{\prime \prime} v^{\prime \prime}\right\rangle_{2}+\langle u\rangle_{2}\langle v\rangle_{2}, \text { etc. }
\end{aligned}
$$

Thus, the turbulent shear for the interval $t-\frac{k T}{2}$ to $t+\frac{k T}{2}$ can be calculated (i.e., postprocessed) from the $k$ intermediate intervals using the following expression

$$
\begin{aligned}
\langle u \dot{v}\rangle & \cong \frac{1}{k}\left(\langle u \dot{v}\rangle_{1}+\langle u\rangle_{1}<v\right\rangle_{1}+\langle u v\rangle_{2}+\langle u\rangle_{2}\langle v\rangle_{2} \\
& \left.\left.+\ldots+\langle u v\rangle_{k}+\langle u\rangle_{k}<v\right\rangle_{k}\right) \\
& -\frac{1}{k^{2}}\left(\langle u\rangle_{1}+\langle u\rangle_{2}+\ldots+\langle u\rangle_{k}\right)\left(\langle v\rangle_{1}+\langle v\rangle_{2}+\ldots+\langle v\rangle_{k}\right)
\end{aligned}
$$

or in summation notation,

$$
\begin{aligned}
\left\langle u v^{\prime \prime}\right. & =\frac{1}{k}\left(\sum_{i=1}^{k}\left\langle u^{\prime \prime} v^{\prime \prime}\right\rangle_{i}+\sum_{i=1}^{k}\langle u\rangle_{i}<v\right\rangle_{i} \\
& \left.-\frac{1}{k}\left(\sum_{i=1}^{k}\langle u\rangle_{i}\right)\left(\sum_{i=1}^{k}\langle v\rangle_{i}\right)\right) .
\end{aligned}
$$

\section{Turbulent Kinetic Energy}

The turbulent kinetic energy is defined as

$$
K=\frac{1}{2}\left(<u^{-2}>+<v^{-2}>+<w^{n 2}>\right)
$$


This quantity did not have to be calculated during calculation of the instantaneous velocity, but was done for convenience.

If a larger time interval is desired, say $k T$, the value is calculated using the above expression with the intensity values at the intermediate intervals.

\section{Energy Dissipation}

The rate of turbulent energy dissipation is defined as

$$
\begin{aligned}
\varepsilon & =v<\left(\frac{\partial u^{\prime \prime}}{\partial x}\right)^{2}+\left(\frac{\partial v^{\prime \prime}}{\partial y}\right)^{2}+\left(\frac{\partial w^{\prime \prime}}{\partial z}\right)^{2}> \\
& =v \frac{1}{T} \int_{1-\frac{T}{2}}^{t+\frac{T}{2}}\left[\left(\frac{\partial u^{\prime \prime}}{\partial x}\right)^{2}+\left(\frac{\partial v^{\prime \prime}}{\partial y}\right)^{2}+\left(\frac{\partial w^{\prime \prime}}{\partial z}\right)^{2}\right] d t^{\prime}
\end{aligned}
$$

where $v$ is the kinematic viscosity and

$$
\begin{aligned}
<\left(\frac{\partial u^{\prime \prime}}{\partial x}\right)^{2} & >=<\left(\frac{\partial}{\partial x}(u-\langle u>))^{2}>\right. \\
& =<\left(\frac{\partial u}{\partial x}\right)^{2}-2 \frac{\partial\langle u\rangle \frac{\partial u}{\partial x}+\left(\frac{\partial\langle u\rangle}{\partial x}\right)^{2}>}{\partial x} \\
& =<\left(\frac{\partial u}{\partial x}\right)^{2}>-\left(\frac{\partial}{\partial x}\langle u\rangle\right)^{2} .
\end{aligned}
$$

In the numerical calculation, we use a summation for the time integration so that

$$
<\left(\frac{\partial u^{-}}{\partial x}\right)^{2}>\equiv \frac{\sum_{i=1}^{n}\left(\frac{\partial u_{i}}{\partial x}\right)^{2}}{n}-\left(\frac{\partial<u>}{\partial x}\right)^{2} .
$$


Substituting the above result into the expression for $\varepsilon$ and similar expressions for the other terms, we obtain the expression for $\varepsilon$,

$$
\begin{aligned}
\varepsilon & =v\left\{\frac{\sum_{i=1}^{n}\left(\frac{\partial u_{i}}{\partial x}\right)^{2}}{n}-\left(\frac{\partial<u>}{\partial x}\right)^{2}+\frac{\sum_{i=1}^{n}\left(\frac{\partial v_{i}}{\partial y}\right)^{2}}{n}-\left(\frac{\partial<v>}{\partial y}\right)^{2}\right. \\
& \left.+\frac{\sum_{i=1}^{n}\left(\frac{\partial w_{i}}{\partial z}\right)^{2}}{n}-\left(\frac{\partial<w>}{\partial z}\right)^{2}\right\} .
\end{aligned}
$$

If a longer time interval for averaging is desired, say $k T$ where $k$ is an integer, the values for $\varepsilon$ can be postprocessed using the results for consecutive $T$ intervals. For the interval $k T$ we have

$$
\varepsilon=v \frac{1}{k T} \int_{t-\frac{k T}{2}}^{t+\frac{k T}{2}}\left[\left(\frac{\partial u^{\prime \prime}}{\partial x}\right)^{2}+\left(\frac{\partial v^{\prime \prime}}{\partial y}\right)^{2}+\left(\frac{\partial w^{\prime \prime}}{\partial z}\right)^{2}\right] d t^{\prime}
$$

Using a summation for the time integration in the numerical calculation, we have 


$$
\begin{aligned}
& \varepsilon \equiv v\left\{\frac{\sum_{i=1}^{n}\left[\left(\frac{\partial u_{i}}{\partial x}\right)^{2}+\left(\frac{\partial v_{i}}{\partial y}\right)^{2}+\left(\frac{\partial w_{i}}{\partial z}\right)^{2}\right]}{k n}\right. \\
& +\ldots+\frac{\sum_{i=(k-1) n+1}^{k n}\left[\left(\frac{\partial u_{i}}{\partial x}\right)^{2}+\left(\frac{\partial v_{i}}{\partial y}\right)^{2}+\left(\frac{\partial w_{i}}{\partial z}\right)^{2}\right]}{k n} \\
& \left.-\left(\frac{\partial<u>}{\partial x}\right)^{2}-\left(\frac{\partial<v>^{2}}{\partial y}\right)-\left(\frac{\partial<w>}{\partial z}\right)^{2}\right\}
\end{aligned}
$$

For the first term we can use

$$
\begin{aligned}
\frac{\sum_{i=1}^{n}\left[\left(\frac{\partial u_{i}}{\partial x}\right)^{2}+\left(\frac{\partial v_{i}}{\partial y}\right)^{2}+\left(\frac{\partial w_{i}}{\partial z}\right)^{2}\right]}{n} & =\frac{\varepsilon_{1}}{v}+\left(\frac{\partial\langle u\rangle_{1}}{\partial x}\right)^{2}+\left(\frac{\partial\langle v\rangle_{1}}{\partial y}\right)^{2}+\left(\frac{\partial<w>_{1}}{\partial z}\right)^{2}
\end{aligned}
$$

where $\varepsilon_{1}$ is the dissipation calculated for the interval $t-\frac{T}{2}$ to $t+\frac{T}{2}$. Thus, for the interval $t-\frac{k T}{2}$ to $t+\frac{k T}{2}$, we have

$$
\begin{gathered}
\varepsilon \cong v\left\{\frac{1}{k} \sum_{i=1}^{k}\left[\frac{\varepsilon_{i}}{v}+\left(\frac{\partial\langle u\rangle_{i}}{\partial x}\right)^{2}+\left(\frac{\partial\langle v\rangle_{i}}{\partial y}\right)^{2}+\left(\frac{\partial\langle w\rangle_{i}}{\partial z}\right)^{2}\right]\right. \\
-\left(\frac{\partial}{\partial x}\langle u>)^{2}-\left(\frac{\partial}{\partial y}\langle v\rangle\right)^{2}-\left(\frac{\partial}{\partial z}\langle w\rangle\right)^{2}\right\} .
\end{gathered}
$$


Table B1 shows a summary of the storage requirements and calculated timeaveraged quantities.

Table B1. Summary of Storage and Output Requirements

For each node and each time average interval:

\begin{tabular}{|c|c|}
\hline $\begin{array}{c}\text { Running Sum from } \\
t-\frac{T}{2} \text { to } t+\frac{T}{2}\end{array}$ & Output \\
\hline$\sum_{i} u_{i}, \sum_{i} v_{i}, \sum_{i} w_{i}$ & $\langle u\rangle,\langle v\rangle,\langle w\rangle$ \\
$\sum_{i} u_{i}^{2}, \sum_{i} v_{i}^{2}, \sum_{i} w_{i}^{2}$ & $\left\langle u^{-2}\right\rangle,\left\langle v^{-2}\right\rangle,\left\langle w^{-2}\right\rangle, k$ \\
$\sum_{i} u_{i} v_{i}$, & $\left\langle u^{-1} v^{-1}\right\rangle$ \\
$\sum_{i}\left[\left(\frac{\partial u_{i}}{\partial x}\right)^{2}+\left(\frac{\partial v_{i}}{\partial y}\right)^{2}+\left(\frac{\partial w_{i}}{\partial z}\right)^{2}\right]$ & \\
\hline
\end{tabular}




\section{Appendix C}

Methodology for Developing the Element

Coefficient Matrices

\section{Transformation to Local Coordinates}

A coordinate transformation is required to rewrite the integrals in terms of the local coordinates $\xi, \eta$, and $\zeta$. For example, using

$$
d x d y d z=|J| d \xi d \eta d \zeta
$$

where $J$ is the Jacobian matrix defined as

$$
[J]=\left[\begin{array}{lll}
\frac{\partial x}{\partial \xi} & \frac{\partial y}{\partial \xi} & \frac{\partial z}{\partial \xi} \\
\frac{\partial x}{\partial \eta} & \frac{\partial y}{\partial \eta} & \frac{\partial z}{\partial \eta} \\
\frac{\partial x}{\partial \zeta} & \frac{\partial y}{\partial \zeta} & \frac{\partial z}{\partial \zeta}
\end{array}\right]
$$

and using the chain rule,

$$
\frac{\partial \phi_{i}}{\partial x}=\frac{\partial \xi}{\partial x} \frac{\partial \phi_{i}}{\partial \xi}+\frac{\partial \eta}{\partial x} \frac{\partial \phi_{i}}{\partial \eta}+\frac{\partial \zeta}{\partial x} \frac{\partial \phi_{i}}{\partial \zeta}
$$

we have 


$$
\begin{aligned}
C_{i_{1}}^{e} & =-\int_{\Omega^{\cdot}} \frac{\partial \phi_{i}}{\partial x} \psi_{j} d x d y d z=-\int_{\Omega^{\cdot}}\left(\frac{\partial \xi}{\partial x} \frac{\partial \phi_{i}}{\partial \xi}+\frac{\partial \eta}{\partial x} \frac{\partial \phi_{i}}{\partial \eta}+\frac{\partial \zeta}{\partial x} \frac{\partial \phi_{i}}{\partial \zeta}\right) \psi_{j}(\xi, \eta, \zeta)|J| d \xi d \eta d \zeta \\
& \equiv-\int_{\Omega^{*}} F(\xi, \eta, \zeta) d \xi d \eta d \zeta .
\end{aligned}
$$

where $|J|$ is the determinant of the Jacobian.

In two-dimensions (and similarily in three-dimensions),

$$
|J|=\left|\begin{array}{l}
\frac{\partial z}{\partial \xi} \frac{\partial \eta}{\partial \xi} \\
\frac{\partial z}{\partial \eta} \frac{\partial y}{\partial \eta}
\end{array}\right|=\frac{\partial x}{\partial \xi} \frac{\partial y}{\partial \eta}-\frac{\partial x}{\partial \eta} \frac{\partial y}{\partial \xi}
$$

and

$$
\begin{aligned}
& \frac{\partial \xi}{\partial x}=\frac{1}{|J|} \frac{\partial y}{\partial \eta}, \frac{\partial \eta}{\partial x}=\frac{-1}{|J|} \frac{\partial y}{\partial \xi}, \\
& \frac{\partial \xi}{\partial y}=\frac{-1}{|J|} \frac{\partial x}{\partial \eta}, \frac{\partial \eta}{\partial y}=\frac{1}{|J|} \frac{\partial x}{\partial \xi} .
\end{aligned}
$$

\section{Gaussian Quadrature}

The quadrature formulas for numerical integration over a rectangular element (where, for convenience, the element $(\xi, \eta, \zeta)$ coordinate limits are from -1 to +1 ) are

$$
\begin{aligned}
\int_{\Omega_{a}} F(\xi, \eta, \zeta) d \xi d \eta d \zeta & =\int_{-1}^{1} \int_{-1}^{1} \int_{-1}^{1} F(\xi, \eta, \zeta) d \xi d \eta d \zeta \\
& \cong \sum_{I=1}^{L} \sum_{J=1}^{M} \sum_{K=1}^{N} F\left(\xi_{l}, \eta_{J}, \zeta_{K}\right) W_{l} W_{J} W_{K}
\end{aligned}
$$

where $F(\xi, \eta, \zeta)$ is the transformed integrand (see example above), $L, M$, and $N$ are the number of quadrature points in the $\xi, \eta$, and $\zeta$ directions, $\left(\xi_{l}, \eta_{J}, \zeta_{K}\right)$ is a Gauss point 
location, and $W_{I}, W_{J}$, and $W_{K}$ are the Gauss weights (for 1-point quadrature $W_{i}=2$, and for 2-point quadrature $W_{i}=1, i=I, J$, and $K$ ).

For 1-point quadrature $L=M=N=1,\left(\xi_{1}, \eta_{1}, \zeta_{1}\right)=(0,0,0)$ (the element centroid), and $W_{I} W_{J} W_{K}=2^{3}=8$. In this case, then,

$$
\int_{\Omega_{e}} F(\xi, \eta, \zeta) d \xi d \eta d \zeta=8 F(0,0,0)
$$

\section{Averaging Operator Definition at Element Level}

The averaging operator is defined as

$$
\bar{f}^{e}(x, y, z, t)=\frac{1}{\Delta x_{f} \Delta y_{f} \Delta z_{f}} \int_{z-\frac{\Delta z_{f}}{2}}^{z+\frac{\Delta z_{f}}{2}} \int_{y-\frac{\Delta y_{f}}{2}}^{y+\frac{\Delta y_{f}}{2}} \int_{x-\frac{\Delta x_{f}}{2}}^{x+\frac{\Delta x_{f}}{2}} f^{e}(\xi, \eta, \zeta, t) d \xi d \eta d \zeta
$$

(Note that here the local coordinates $(\xi, \eta, \zeta)$ span the filter space $\Delta f$.)

Transforming to local coordinates we have

$d x d y d z=|J| d \xi d \eta d \zeta$

If we assume that $|J|$ is constant over the finite volume $\Delta \xi_{f} \Delta \eta_{f} \Delta \zeta_{f}$, then

$$
\int_{z-\frac{\Delta z_{f}}{2}}^{z+\frac{\Delta z_{f}}{2}} \int_{y-\frac{\Delta y_{f}}{2}}^{y+\frac{\Delta y_{f}}{2}} \int_{x-\frac{\Delta x_{f}}{2}}^{x+\frac{\Delta x_{f}}{2}} d x d y d z \equiv|J| \int_{\zeta-\frac{\Delta \zeta_{f}}{2}}^{\zeta+\frac{\Delta \zeta_{f}}{2}} \int_{\eta-\frac{\Delta \eta_{f}}{2}}^{\eta+\frac{\Delta \eta_{f}}{2}} \int_{\xi-\frac{\Delta \xi_{f}}{2}}^{\xi+\frac{\Delta \xi_{f}}{2}} d \xi d \eta d \zeta
$$

and

$$
\Delta x_{f} \Delta y_{f} \Delta z_{f} \cong|J| \Delta \xi_{f} \Delta \eta_{f} \Delta \zeta_{f}
$$


Substituting and transforming to local coordinates

$$
\begin{aligned}
\bar{f}^{e}(x, y, z, t) & \cong \frac{1}{|J| \Delta \xi_{f} \Delta \eta_{f} \Delta \zeta_{f}} \int_{\zeta-\frac{\Delta \zeta_{f}}{2}}^{\zeta+\frac{\Delta \zeta_{f}}{2}} \int_{\eta-\frac{\Delta \eta_{f}}{2}}^{\eta+\frac{\Delta \eta_{f}}{2}} \int_{\xi-\frac{\Delta \xi_{f}}{2}}^{\xi+\frac{\Delta \xi_{f}}{2}} \hat{f}(\alpha, \gamma, \rho, t)|J| d \alpha d \gamma d \rho \\
& \cong \overline{\hat{f}}(\xi, \eta, \zeta, t)
\end{aligned}
$$

where $\hat{f}$ is $f^{*}$ transformed to local coordinates. Thus, the result of the averaging operator applied to the function in global coordinates is approximately equal to the result of the averaging operator applied to the function in local coordinates. The two are identical only for a uniform mesh (i.e., for $|J|=$ constant).

In applying the averaging operator to the incompressible Navier-Stokes equations, it is assumed that the operator commutes with differentiation (i.e., $\overline{\partial u_{\alpha} / \partial x_{\beta}}=\partial \bar{u}_{\alpha} / \partial x_{\beta}$ and $\left.\overline{\partial u_{\alpha} / \partial t}=\partial \bar{u}_{\alpha} / \partial t\right)$. Leibnitz' rule can be used to show that the above defined averaging operator commutes with differentiation for a constant filter width (i.e., $\Delta x_{f}=$ constant, $\Delta y_{f}=$ constant, and $\Delta z_{f}=$ constant). For instance, in one dimension, if the filter width is not constant, $\Delta x_{f}=\Delta x_{f}(x)$ (i.e., a function of $x$ ), then 


$$
\begin{aligned}
& \frac{\partial \bar{f}}{\partial x}=\frac{\partial}{\partial x}\left\{\frac{1}{\Delta x_{f}} \int_{x-\frac{\Delta x_{f}}{2}}^{x+\frac{\Delta x_{f}}{2}} f(\alpha) d \alpha\right\} \\
& =\frac{1}{\Delta x_{f}}\left\{\int_{x-\frac{\Delta x_{f}}{2}}^{x+\frac{\Delta x_{f}}{\partial x}} \frac{\partial}{\partial x}(\alpha) d \alpha+f\left(x+\frac{\Delta x_{f}}{2}\right) \frac{\partial}{\partial x}\left(x+\frac{\Delta x_{f}}{2}\right)-f\left(x-\frac{\Delta x_{f}}{2}\right) \frac{\partial}{\partial x}\left(x-\frac{\Delta x_{f}}{2}\right)\right\} \\
& -\frac{1}{\Delta x_{f}^{2}} \frac{\partial \Delta x_{f}}{\partial x} \int_{x-\frac{\Delta x_{f}}{2}}^{x+\frac{\Delta x_{f}}{2}} f(\alpha) d \alpha \\
& =\frac{1}{\Delta x_{f}}\left\{f\left(x+\frac{\Delta x_{f}}{2}\right)-f\left(x-\frac{\Delta x_{f}}{2}\right)\right\}+\frac{1}{2 \Delta x_{f}}\left\{f\left(x+\frac{\Delta x_{f}}{2}\right)+f\left(x-\frac{\Delta x_{f}}{2}\right)\right\} \frac{\partial \Delta x_{f}}{\partial x} \\
& -\frac{1}{\Delta x_{f}} \bar{f}(x) \frac{\partial \Delta x_{f}}{\partial x} \\
& =\frac{1}{\Delta x_{f}} \int_{x-\frac{\Delta x_{f}}{2}}^{x+\frac{\Delta x_{f}}{2}} \frac{\partial}{\partial \alpha} f(\alpha) d \alpha+\frac{1}{2 \Delta x_{f}}\left\{f\left(x+\frac{\Delta x_{f}}{2}\right)+f\left(x-\frac{\Delta x_{f}}{2}\right)-2 \bar{f}(x)\right\} \frac{\partial \Delta x_{f}}{\partial x} \\
& =\frac{\overline{\partial f}}{\partial x}+\frac{1}{2 \Delta x_{f}}\left\{f\left(x+\frac{\Delta x_{f}}{2}\right)+f\left(x-\frac{\Delta x_{f}}{2}\right)-2 \bar{f}(x)\right\} \frac{\partial \Delta x_{f}}{\partial x}
\end{aligned}
$$

The second term on the right represents the error in assuming that the averaging or filtering operator commutes with differentiation for a variable width filter. However, if the filter width changes smoothly such that $\partial \Delta x_{f} / \partial x$ is small, the error will be small. (Since the error term looks like a second-order difference operator, the error is approximately $O\left(\Delta x_{f}^{2}\right) \partial \Delta x_{f} / \partial x$.) Variable filter widths may result, in practice, when the filter width is determined from the computational mesh (i.e., $\Delta x_{f} \propto \Delta x$ ) and a graded mesh is used.

A sufficient condition for validity of Leibnitz' rule is that $\partial \bar{u}_{\alpha} / \partial x_{\beta}$ and $\partial \bar{u}_{\alpha} / \partial t$ be continuous over the domain of integration. Considering this condition and that with 
the Galerkin finite element method (GFEM), we substitute the expansions

$$
\bar{u}_{\alpha}^{h}(\underline{x}, t)=\sum_{j=1}^{N} \bar{u}_{\alpha}^{j}(t) \phi_{j}(\underline{x})
$$

where the basis function $\phi_{j}(x)$ are piecewise-continuous polynomials, we see that, $\partial \bar{u}_{\alpha}^{h} / \partial x_{\beta}$ is not continuous over the domain of integration for the averaging operator (i.e., $\partial \bar{u}_{\alpha}^{k} / \partial x_{\beta}$ is discontinuous at element boundaries). To show that the averaging operator commutes with differentiation for the GFEM expansion of $\bar{u}_{\alpha}$ into basis functions which are piecewise-continuous polynomials, we investigate the basis functions in one dimension.

Applying the averaging operator to the piecewise linear basis functions $\phi_{j}(x)$ for the one-dimensional example case in Figure $\mathrm{Cl}(\mathrm{a})$ and averaging over 2 grid lengths ( $l_{f}$ $=2 h$ ), results in a bell-shaped curve over 4 grid lengths (Figure C1(b)) (e.g., $\bar{\phi}_{3}(x)$ spans from node 1 to node 5). The bell curve is made up of piecewise-continuous quadratic functions of $x$.

Applying the averaging operator to the product $\phi_{j} \phi_{k}$ for the one-dimensional example and averaging over 2 grid lengths, results in curves that span 3 or 4 grid lengths (Figure C2). The curves are made up of piecewise-continuous cubic functions of $x$ or piecewise-constant values.

In summary, the averaging operator applied to one-dimensional piecewise-linear basis functions results in higher order piecewise-continuous functions that span a greater number of grid lengths than the number averaging over. 
For combinations of $j, k$ it can be shown for the one-dimensional example case with piecewise-linear basis functions that

$$
\frac{\overline{\partial \phi_{j} \phi_{k}}}{\partial x}=\frac{\partial}{\partial x} \overline{\phi_{j} \phi_{k}}
$$

For example, for the one-dimensional case in Figure $\mathrm{Cl}$ (a) (for a constant filter width)

$$
\phi_{2} \phi_{3}=\left\{\begin{array}{cl}
\left(\frac{x_{3}-x}{h}\right)\left(1+\frac{x-x_{3}}{h}\right) & \text { for } x_{2}<x<x_{3} \\
0 & \text { for } x<x_{2} \text { and } x>x_{3}
\end{array}\right.
$$

If the filter width is $2 h$, we have for $x_{1} \leq x \leq x_{2}$

$$
\overline{\phi_{2} \phi_{3}}=\frac{1}{2 h} \int_{x_{2}}^{x+h}\left(\frac{x_{3}-\alpha}{h}\right)\left(1+\frac{\alpha-x_{3}}{h}\right) d \alpha=\frac{1}{2}\left\{\frac{1}{6}-\left(\frac{x_{3}-x-h}{h}\right)^{2}\left(\frac{1}{2}-\frac{1}{3}\left(\frac{x_{3}-x-h}{h}\right)\right)\right\}
$$

and therefore

$$
\frac{\partial \overline{\phi_{2} \phi_{3}}}{\partial x}=\frac{1}{2 h}\left(\frac{x_{3}-x-h}{h}\right)\left(1-\left(\frac{x_{3}-x-h}{h}\right)\right)
$$

Now if we filter $\partial \phi_{2} \phi_{3} / \partial x$ over $2 h$ for $x_{1} \leq x \leq x_{2}$, we bring in the contribution from $x_{2} \leq x \leq x_{3}$

$$
\begin{aligned}
\frac{\overline{\partial \phi_{2} \phi_{3}}}{\partial x} & =\frac{1}{2 h} \int_{x_{2}}^{x+h} \frac{\partial \phi_{2} \phi_{3}}{\partial \alpha} d \alpha \\
& =\frac{1}{2 h}\left(\frac{x_{3}-x-h}{h}\right)\left(1-\left(\frac{x_{3}-x-h}{h}\right)\right),
\end{aligned}
$$




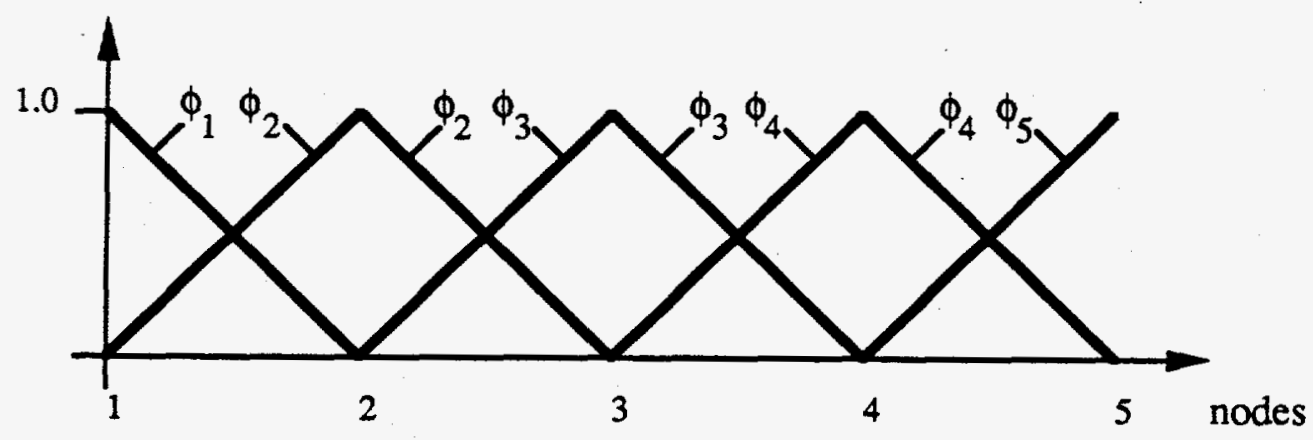

(a)

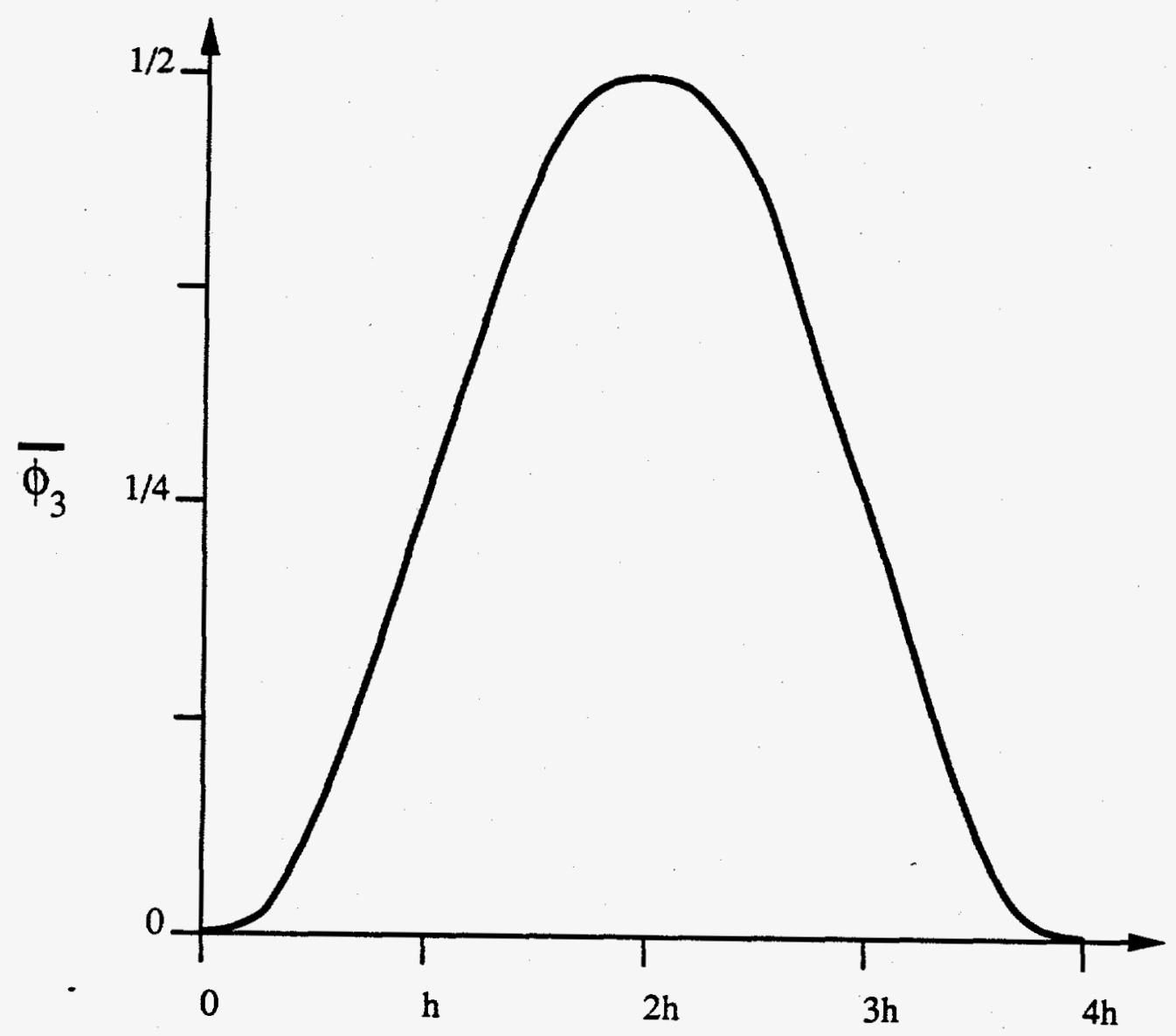

(b)

Figure C1. Plot of the average of the one-dimensional piecewise-linear basis function. 

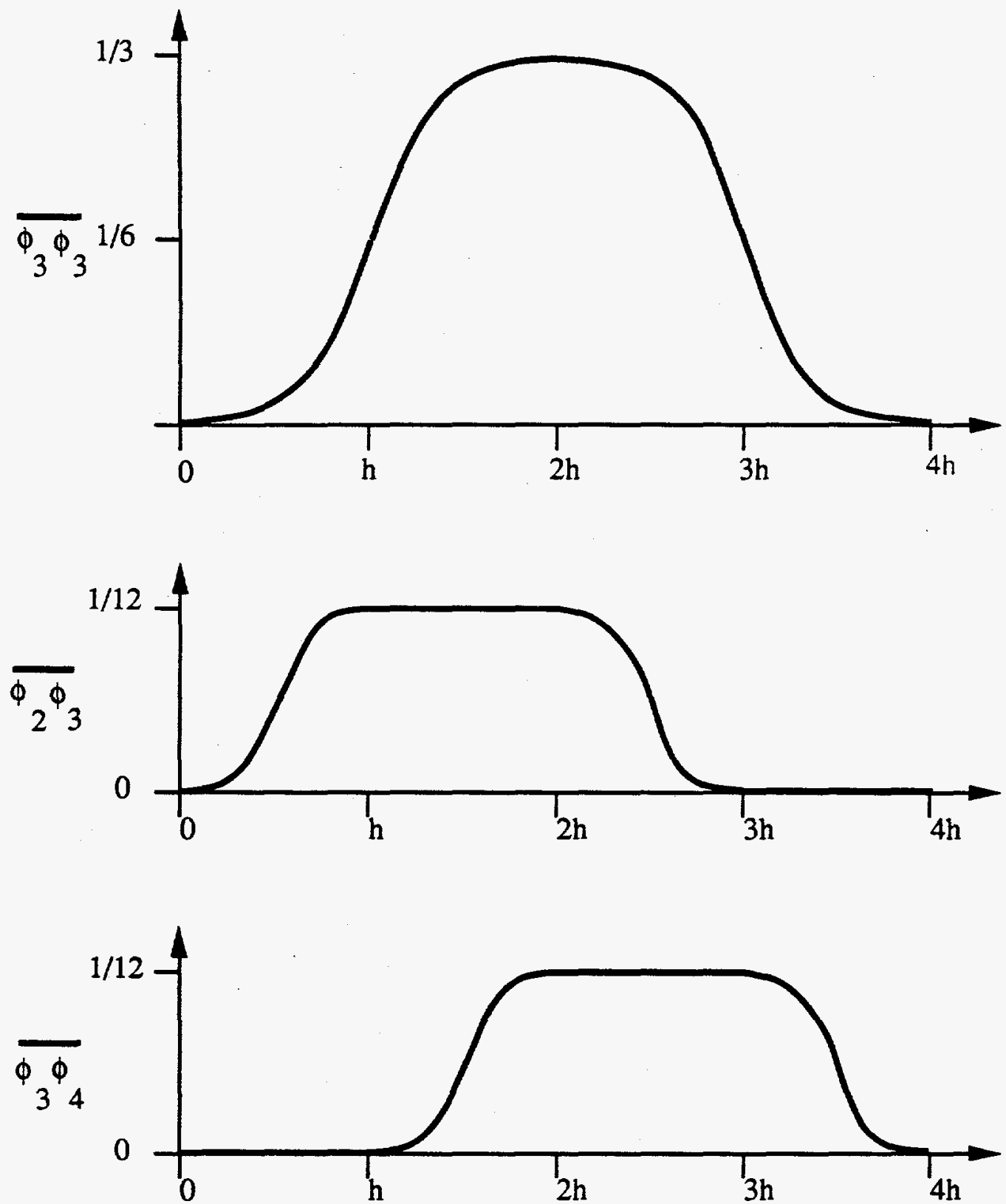

Figure C2. Plot of the average of the product of one-dimensional piecewise-linear basis function. 
and thus,

$$
\frac{\overline{\partial \phi_{2} \phi_{3}}}{\partial x}=\frac{\partial}{\partial x} \overline{\phi_{2} \phi_{3}}
$$

Thus, the averaging operator commutes with differentiation for the product of piecewise-linear basis functions. If the filter width varied with $x$, the error in assuming the averaging operator commuted with differentiation would be

$$
\begin{gathered}
\frac{1}{2 \Delta x_{f}}\left\{\phi_{j} \phi_{k}\left(x+\frac{\Delta x_{f}}{2}\right)+\phi_{j} \phi_{k}\left(x-\frac{\Delta x_{f}}{2}\right)-2 \overline{\phi_{j} \phi_{k}}\right\} \frac{\partial \Delta x_{f}}{\partial x} \\
\leq \frac{1}{\Delta x_{f}} \frac{\partial \Delta x_{f}}{\partial x}
\end{gathered}
$$

(since $\phi_{j} \phi_{k} \leq 1$ ). Thus, for smoothly varying filter widths, the error is small in assuming that the averaging operator commutes with differentiation.

The above also leads to the conclusion that with the GFEM formulation, averaging over more than one grid length is a viable option. Thus, the averaging width, $\Delta_{f}$, can be chosen independent of the grid width $h$.

\section{Eorm of the Averaged Terms in the GFEM Formulation}

In the GFEM formulation, the averaged terms (basis functions) are the

$$
\text { advection terms }\left(\bar{u}_{\beta}^{k} \int_{\Omega} \phi_{i} \frac{\partial}{\partial x_{\beta}} \overline{\phi_{j} \phi_{k}}\right) \bar{u}_{\alpha}^{j}
$$

and the 
model terms $\quad\left(\bar{u}_{\beta}^{k} \int_{\Omega} \bar{\phi}_{j} \bar{\phi}_{k} \frac{\partial \phi_{i}}{\partial x_{\beta}}\right) \bar{\mu}_{\alpha}^{j}$

For each element, at each node $i$, with (local) coordinates $\left(\xi_{i}, \eta_{l}, \zeta_{i}\right)$, we have for each basis function $\hat{\phi}_{j}(\xi, \eta, \zeta)$ for $j=1,2, \ldots, 8$ (each of the 8 corner nodes of a hexahedran)

$$
\overline{\hat{\phi}}_{j}\left(\xi_{i}, \eta_{i}, \zeta_{i}\right)=\frac{1}{\Delta \xi_{f} \Delta \eta_{f} \Delta \zeta_{f}} \int_{-1}^{1} \int_{-1}^{1} \int_{-1}^{1} \hat{\phi}_{j}(\xi, \eta, \zeta) d \xi d \eta d \zeta
$$

where for the tri-linear basis function

$$
\hat{\phi}_{j}(\xi, \eta, \zeta)=\frac{1}{8}\left(1+\xi_{j} \xi\right)\left(1+\eta_{j} \eta\right)\left(1+\zeta_{j} \zeta\right)
$$

Performing the integration we have

$$
\overline{\hat{\phi}}_{j}\left(\xi_{i}, \eta_{i}, \zeta_{i}\right)=\frac{1}{\Delta \xi_{f} \Delta \eta_{f} \Delta \zeta_{f}}
$$

For example, an averaging width of $\Delta \xi_{f} \Delta \eta_{f} \Delta \zeta_{f}=2 \Delta \xi 2 \Delta \eta 2 \Delta \zeta$, with $\Delta \xi=\Delta \eta=\Delta \zeta=$ 2 , results in $\overline{\hat{\phi}}_{j}=1 / 64$. Globally, considering each element contribution, if $i=j$, then

$$
\bar{\phi}_{i}\left(x_{i}, y_{i}, \dot{z_{i}}\right) \cong 8 \cdot \frac{1}{64}=\frac{1}{8}
$$

(contributions from 8 elements) and if $i \neq j$,

$$
\bar{\phi}_{j}\left(x_{i}, y_{i}, z_{i}\right) \cong \frac{1}{64}
$$


(The conversion from local to global coordinates is described in the previous subsection.)

For the product of the basis functions, the transformation from global to local coordinates is

$$
\frac{\partial}{\partial x_{\beta}} \overline{\phi_{j}^{*} \phi_{k}^{*}} \cong \frac{\partial \xi}{\partial x_{\beta}} \frac{\partial}{\partial \xi} \overline{\hat{\phi}_{j} \hat{\phi}_{k}}+\frac{\partial \eta}{\partial x_{\beta}} \frac{\partial}{\partial \eta} \overline{\hat{\phi}_{j} \hat{\phi}_{k}}+\frac{\partial \zeta}{\partial x_{\beta}} \frac{\partial}{\partial \zeta} \overline{\hat{\phi}_{j} \hat{\phi}_{k}}
$$

Integrating from -1 to 1 with the averaging operator, gives

$$
\begin{aligned}
\frac{\partial}{\partial x_{\beta}} \overline{\phi_{j}^{e} \phi_{k}^{e}} & \cong \frac{\partial \xi}{\partial x_{\beta}} \frac{2}{8^{2}}\left(\xi_{j}+\xi_{k}+2 \xi_{j} \xi_{k} \xi\right)\left[1+\left(\eta_{j}+\eta_{k}\right) 2 \eta+2 \eta_{j} \eta_{k}\left(\eta^{2}+\frac{\Delta \eta_{f}^{2}}{3}\right)\right] \\
& \cdot\left[1+\left(\zeta_{j}+\zeta_{k}\right) 2 \zeta+2 \zeta_{j} \zeta_{k}\left(\zeta^{2}+\frac{\Delta \zeta_{f}^{2}}{3}\right)\right] \\
& +\frac{\partial \eta}{\partial x_{\beta}} \ldots+\frac{\partial \zeta}{\partial x_{\beta}} \ldots
\end{aligned}
$$

The leading coefficients are calculated using the determinate of the Jacobian and derivative of the basis functions (Anderson et al. 1984). For example,

$$
\frac{\partial \xi}{\partial x}=\frac{1}{|J|}\left(\frac{\partial y}{\partial \eta} \frac{\partial z}{\partial \zeta}-\frac{\partial y}{\partial \zeta} \frac{\partial z}{\partial \eta}\right)=\frac{1}{|J|}\left(\sum_{j=1}^{N_{e}} y_{j} \frac{\partial \hat{\phi}_{j}}{\partial \eta} \sum_{j=1}^{N_{e}} z_{j} \frac{\partial \hat{\phi}_{j}}{\partial \zeta}-\sum_{j=1}^{N_{e}} y_{j} \frac{\partial \hat{\phi}_{j}}{\partial \zeta} \sum_{j=1}^{N_{e}} z_{j} \frac{\partial \hat{\phi}_{j}}{\partial \eta}\right)
$$

where $N_{e}$ is the number of element nodal points ( 8 for a hexahedran). 


\section{Solution Options for Other Terms in the GFEM Formulation}

The subscript $\beta$ implies summation in the $\alpha$-component of the momentum equation. For example, in $n_{i j}(\bar{u})$,

$$
\bar{u}_{\beta}^{k} \int_{\Omega} \phi_{j} \phi_{k} \frac{\partial \phi_{i}}{\partial x_{\beta}}=\bar{u}_{1}^{k} \int_{\Omega} \phi_{j} \phi_{k} \frac{\partial \phi_{i}}{\partial x_{1}}+\bar{u}_{2}^{k} \int_{\Omega} \phi_{j} \phi_{k} \frac{\partial \phi_{i}}{\partial x_{2}}+\bar{u}_{3}^{k} \int_{\Omega} \phi_{j} \phi_{k} \frac{\partial \phi_{i}}{\partial x_{3}}
$$

In the equation solution, the summation over $k$ can be avoided by averaging $\bar{u}_{\beta}^{k}$ over the element when $n_{i j}{ }^{e}$ (the element coefficient matrix) is formed. For instance,

$$
\begin{gathered}
\bar{u}_{\beta}^{k} \int_{\Omega_{c}} \phi_{j}^{e} \phi_{k}^{e} \frac{\partial \phi_{i}^{e}}{\partial x_{\beta}} \cong \bar{u}_{\beta}^{e} \int_{\Omega_{c}} \phi_{j}^{e} \frac{\partial \phi_{i}^{e}}{\partial x_{\beta}} \text { where } \bar{u}_{\beta_{c}}^{e} \text { is defined at the element centroid, and } \\
\bar{u}_{\beta c}^{e}=\bar{u}_{\beta}^{e}\left(x_{c}, y_{c}, z_{c}\right)=\sum_{k} \phi_{k}^{e}(\underline{x}) \bar{u}_{\beta}^{k}
\end{gathered}
$$

where $\left(x_{c}, y_{c}, z_{c}\right)$ are the coordinates for the element centroid or in local coordinates, we have $\bar{u}_{\beta}^{e}(0,0,0)$. This approximation is already applied in the existing code to be used in this study and is called the 'centroid advection velocity' simplification.

The turbulent eddy viscosity, $v_{T}$, can also be calculated at the element level as the average value evaluated at the centroid of the element. For the term in $k_{i j}{ }^{e}$, we have

$$
\begin{aligned}
& \int_{\Omega_{e}} v_{T}^{k^{*}} \frac{\partial \phi_{j}^{e}}{\partial x_{\beta}} \frac{\partial \phi_{i}^{e}}{\partial x_{\beta}}=v_{T_{e}}^{h^{e}} \int_{Q_{e}} \frac{\partial \phi_{j}^{e}}{\partial x_{\beta}} \frac{\partial \phi_{i}^{e}}{\partial x_{\beta}} \\
& \text { where } v_{T_{c}}^{n^{*}}=(c \Delta)^{2}\left(\left(\bar{u}_{\alpha}^{m} \frac{\partial \phi_{m}^{e}}{\partial x_{\beta}}\right)_{c}\left(\left(\bar{u}_{\alpha}^{k} \frac{\partial \phi_{k}^{*}}{\partial x_{\beta}}\right)_{c}+\left(\bar{u}_{\beta}^{k} \frac{\partial \phi_{k}^{*}}{\partial x_{\alpha}}\right)_{c}\right)\right)^{1 / 2} \text {. }
\end{aligned}
$$


As shown by Gresho et al. (1984), when using 1-point quadrature, if the centroid gradient of the basis function appears in the integrand, it can be efficiently evaluated using the results of the $\mathrm{C}$-matrix. For instance, with the $\mathrm{C}$-matrix we have at the element level

$$
c_{i j_{(\alpha)}}^{e}=\int_{\Omega_{e}} \frac{\partial \phi_{i}}{\partial x_{\alpha}} \psi_{j}=\int_{\Omega_{e}} \frac{\partial \phi_{i}}{\partial x_{\alpha}}=c_{\dot{\psi}_{(\alpha)}}^{e}
$$

since

$$
\sum_{j} \psi_{j}=1
$$

With 1-point quadrature,

$$
c_{i_{(\alpha)}}^{e}=\left(\frac{\partial \phi_{i}}{\partial x_{\alpha}}\right)_{c} \Omega_{e}
$$

and looking at the advection matrix (and using the approximation $\bar{u}_{\beta_{c}}^{e}=\sum_{k} \phi_{k}^{e}(\underline{x}) \bar{u}_{\beta}^{k}$ )

$$
\begin{aligned}
\bar{u}_{\beta_{\varepsilon}}^{e} \int_{\Omega_{c}} \phi_{i} \frac{\partial \phi_{j}}{\partial x_{\beta}}-\ldots & =\bar{u}_{\beta_{\varepsilon}}^{e} \phi_{i_{c}}\left(\frac{\partial \phi_{j}}{\partial x_{\beta}}\right)_{c} \Omega_{c}-\ldots \\
& =\bar{u}_{\beta_{c}}^{e} \phi_{i_{c}} C_{j_{(\beta)}}^{e}-\ldots
\end{aligned}
$$

where the subscript $c$ denotes evaluation at the centroid of the element. 


\section{Appendix D}

\section{Description of The Existing Software}

The finite element planetary boundary layer code (FEM-PBL) was developed at Lawrence Livermore National Laboratory, by the Atmospheric and Geophysical Sciences Division. The code consists of a preprocessor, a main code, and multiple postprocessors. Supplemental reading on details of the various options is available in Gresho et al. (1984). The following is a review of some of the code characteristics that will be exercised or modified for this study.

As outlined in the main body of this report, a modified Galerkin finite element method (GFEM) is used with isoparametric 2D quadrilateral and 3D hexahedral elements for modeling the Navier-Stokes equations. To reduce run time, a lumped mass matrix is used and the coefficient matrices are generated using 1-point quadrature. An hour-glass correction term is included in the diffusion matrices or a higher-order quadrature scheme can be used for the diffusion coefficients. The pressure Poisson equation is solved directly or iteratively and an explicit forward-Euler time integration scheme is used for the velocity solution (the semi-implicit velocity solution scheme is only available for two-dimensional solutions). The option of subcycling on the pressure field solution is also available. These code characteristics are discussed below.

\section{The Equations}

The coded equations are the incompressible, Boussinesq, planetary boundary 
layer equations. The Boussinesq approximation is applicable for small variations in density in the cross-flow direction. The code includes the continuity equation and transport equations of momentum, potential temperature, species, and mixing ratios of water vapor and condensed liquid. Since only the momentum and continuity equations are of interest to this study, they are listed below (note that $y$ is the vertical direction).

$$
\begin{aligned}
& \frac{\partial u}{\partial x}+\frac{\partial v}{\partial y}+\frac{\partial w}{\partial z}=0 \\
& \frac{\partial u}{\partial t}+u \frac{\partial u}{\partial x}+v \frac{\partial u}{\partial y}+w \frac{\partial u}{\partial z}=-\frac{\partial \pi}{\partial x}-f_{2}\left(w-w_{8}\right)+f_{3} v \\
& +\frac{\partial}{\partial x}\left(k_{H} \frac{\partial u}{\partial x}\right)+\frac{\partial}{\partial y}\left(k_{v} \frac{\partial u}{\partial y}\right)+\frac{\partial}{\partial z}\left(k_{H} \frac{\partial u}{\partial z}\right)-\alpha\left(u-u_{g}\right) \\
& \frac{\partial v}{\partial t}+u \frac{\partial v}{\partial x}+v \frac{\partial v}{\partial y}+w \frac{\partial v}{\partial z}=-\frac{\partial \pi}{\partial y}+\frac{\theta-\dot{\theta_{g}}}{\theta_{0}} g \\
& +\frac{\partial}{\partial x}\left(k_{H} \frac{\partial v}{\partial x}\right)+\frac{\partial}{\partial y}\left(k_{v} \frac{\partial v}{\partial y}\right)+\frac{\partial}{\partial z}\left(k_{H} \frac{\partial v}{\partial z}\right)-\alpha v \\
& \frac{\partial w}{\partial t}+u \frac{\partial w}{\partial x}+v \frac{\partial w}{\partial y}+w \frac{\partial w}{\partial z}=-\frac{\partial \pi}{\partial z}+f_{2}\left(u-u_{s}\right) \\
& +\frac{\partial}{\partial x}\left(k_{H} \frac{\partial w}{\partial x}\right)+\frac{\partial}{\partial y}\left(k_{V} \frac{\partial w}{\partial y}\right)+\frac{\partial}{\partial z}\left(k_{H} \frac{\partial w}{\partial z}\right)-\alpha\left(w-w_{z}\right) \\
& \text { where } \Theta=T\left(\frac{P_{0}}{P}\right)^{R / c_{p}}, \theta=\Theta-\theta_{0}, \Pi=\theta_{0}\left[c_{p}\left(\frac{P_{0}}{P}\right)^{R / c_{p}}\right] \\
& \text { and } \pi=\Pi-\pi_{0}(y)-\pi^{\prime}(y) \text { where } \frac{d \pi_{0}}{d y}=-g \text { and } \frac{d \pi^{\prime}}{d y}=\frac{\theta^{\prime}(y)}{\theta_{0}} g \text {, and }
\end{aligned}
$$




$$
\begin{aligned}
& T \quad \text { : temperature } \\
& P \quad: \text { pressure } \\
& P_{0} \quad: \quad \text { reference pressure }\left(10^{5} \mathrm{~Pa}\right) \\
& R \quad \text { : gas constant for dry air } \\
& c_{p} \quad: \quad \text { specific heat at constant pressure for dry air } \\
& \theta: \text { : the potential temperature } \\
& \theta_{0} \quad \text { : constant reference state potential temperature } \\
& \theta^{\circ} \quad \text { : background deviation potential temperature } \\
& \pi_{0} \quad: \quad \text { reference state scaled pressure } \\
& \pi^{\prime} \quad \text { : background deviation scaled pressure } \\
& \pi \quad \text { : the deviation scaled pressure } \\
& u, v, w: \text { velocity components } \\
& f_{2} \quad \text { : horizontal Coriolis parameter } \\
& f_{3} \quad \text { : vertical Coriolic parameter } \\
& u_{g}, w_{g} \quad: \quad x \text { and } z \text { components of the geostrophic wind } \\
& k_{H}, k_{V} \quad: \quad \text { horizontal and vertical diffusivities } \\
& \text { g : acceleration due to gravity } \\
& \alpha \quad \text { : Rayleigh damping coefficient }
\end{aligned}
$$

The above equations can be modified to represent the laminar Navier-Stokes equations by setting the code coefficients as follows:

$$
\begin{aligned}
& f_{2}=0, f_{3}=0, u_{g}=0, w_{g}=0 \\
& k_{H}=v, k_{V}=v, \alpha=0, \text { and }
\end{aligned}
$$

the gravitational term in the $y$-momentum equation will be zero without the temperature solution. We can then consider $\pi=(P+\rho g y) / \rho$. The modeled equations become in 
tensor notation

$$
\begin{gathered}
\frac{\partial u_{\alpha}}{\partial x_{\alpha}}=0 \\
\frac{\partial u_{\alpha}}{\partial t}+u_{\beta} \frac{\partial u_{\alpha}}{\partial x_{\beta}}=-\frac{\partial}{\partial x_{\alpha}}\left(\frac{P}{\rho}\right)+\frac{\partial}{\partial x_{\beta}}\left(v \frac{\partial u_{\alpha}}{\partial x_{\beta}}\right)
\end{gathered}
$$

where $P$ includes the gravitational term.

\section{Mesh Restrictions}

The code uses a logical computational mesh which requires that the number of nodes in each direction be constant and not vary through the field (i.e., mismatching of the mesh is not allowed). The logical mesh requirement is more cost effective for simple geometries, but does pose some difficulties for complicated geometries. For example, the flow around a surface mounted block may require the specification of elements not needed to resolve the problem physics (e.g., excess elements inside body and in the far field).

Choosing the direction with the fewest elements as the $i$-direction (first numbering order in the logical mesh) will minimize the bandwidth of the pressure matrix.

\section{Basis Functions and Spatial Integration (Quadrature)}

The basis functions for the velocity, $\phi_{i}(x)$, are piecewise continuous bilinear in two dimensions and trilinear in three dimensions in the parent element. (They are only 
multilinear in the $x$-system for elements containing straight and planar sides.) The basis function $\phi_{i}(x)$ is unity at node $i$ and zero at all other nodes. For the pressure, the basis function $\psi_{i}(x)$ is piecewise constant; it is unity on element $i$ and zero on all other elements. At the element level in terms of local coordinates $\xi, \eta$, and $\zeta$, we have in two dimensions

$$
\begin{gathered}
\phi_{i}^{*}(\xi, \eta)=\frac{1}{4}\left(1+\xi_{i} \xi\right)\left(1+\eta_{i} \eta\right), i=1,2,3,4 \\
\text { and } \psi^{*}=1
\end{gathered}
$$

and in three dimensions

$$
\begin{gathered}
\phi_{i}^{*}(\xi, \eta, \zeta)=\frac{1}{8}\left(1+\xi_{i} \xi\right)\left(1+\eta_{i} \eta\right)\left(1+\zeta_{i} \zeta\right), i=1,2,3, \ldots, 8 \\
\text { and } \psi^{*}=1
\end{gathered}
$$

where $\xi_{i}, \eta_{i}$, and $\zeta_{i}$ are the values of the local coordinates at node $i(1$ or -1$)$.

The integrals in the coefficient matrices are approximated using 1-point quadrature. The 1-point quadrature technique involves multiplying the element size (area in two dimensions and volume in three dimensions) with the value of the integrand at the element centroid (see Appendix C).

\section{Time Integration}

The method of direct solution of the discrete pressure Poisson equation is that developed by Taylor et al. (1981) (the routine in the code is called FISSILE). The discrete Laplacian $C^{T} M^{-1} C$ is factored into a lower triangular and a diagonal matrix in 
the preprocessor (since it is not time dependent) and stored for use by the main code. (Since it is symmetric, no upper triangular matrix is needed.) At each time step (or at each major time step if subcycling), the pressure field is solved by performing one forward reduction and back substitution (i.e., Gaussian elimination).

The discrete pressure Poisson equation can also be solved via a preconditioned conjugate gradient iterative solver (see Gresho 1986).

\section{Hour-glass Correction}

The hour-glass correction corrects for the lack of diffusion of $2 \Delta x$ and $2 \Delta y$ waves by the 1-point $\mathrm{K}$-matrix.

The present code allows for an hour-glass correction matrix with a choice of scalar multiplier. The specific choice of hour-glass matrix is outlined in Gresho et al. (1984). In summary, for 1-point quadrature, isotropic diffusion, and uniform mesh, in two dimensions the hour-glass matrix is

$$
H=\left[\begin{array}{r}
1 \\
-1 \\
1 \\
-1
\end{array}\right]\left(\begin{array}{llll}
1 & -1 & 1 & -1
\end{array}\right)=\left[\begin{array}{rrrr}
1 & -1 & 1 & -1 \\
-1 & 1 & -1 & 1 \\
1 & -1 & 1 & -1 \\
-1 & 1 & -1 & 1
\end{array}\right]
$$

In three dimensions, a constant multiplier of $h / 2$ is used for the three-dimensional wave and a constant multiplier of $h / 4$ is used for the three two-dimensional waves, where $h$ is a measure of the element size. For anisotropic diffusivity and a distorted mesh in two and three dimensions, the average value of the diagonal diffusivities is used and $h$ is an estimate of the average element length and is used for all elements. 


\section{Subcycling}

With the explicit time integration scheme, there is a stability and an accuracy restriction on $\Delta t$ with, usually, $\Delta t_{\text {stability }}<\Delta t_{\text {accuracy. }}$. With subcycling, the pressure is solved only at the major time step based on the time accuracy requirement and a linear extrapolation of pressure is performed during the minor time steps whose size is based on the stability restriction. Thus, subcycling allows less frequent updates of the pressure field, reducing run time.

\section{Essential Boundary Conditions}

As explained in Section 3.2.1, to set essential boundary conditions, say $\bar{u}_{\alpha}=\bar{u}_{\alpha}^{s}$ on $\partial \Omega^{s}$ (a portion of the boundary), we set

$$
f_{\alpha}=\gamma_{(\alpha)} \bar{u}_{\alpha}^{s}
$$

and let $\gamma_{(\alpha)} \rightarrow \infty$. In defining the coefficient matrices, this term was shown to be in the $K$-matrix (Section 3.2.1). However, in the actual coding a large number $\left(10^{20}\right)$ is placed on the diagonal of the $M$-matrix. The position on the diagonal corresponds to the nodal location with the specified velocity condition. On the right-side of the equation, we have the large number times the specified velocity boundary condition. 


\title{
Appendix E
}

\author{
Details of How the Vorticity Model is Implemented \\ in the Existing Finite Element Code
}

As described in Section 2.5 .3 , the vorticity model for the eddy viscosity coefficient is

$$
v_{T}=\left(c_{\omega} \Delta\right)^{3}|\nabla . \omega|
$$

where $\nabla, \omega$ is defined as the finite difference approximation to the vorticity gradient, $\Delta$ is a measure of the grid size (i.e., $\left.\Delta=(\Delta x \Delta y)^{1 / 2}\right)$, and $c \omega$ is a constant. The form of $|\nabla . \omega|$ to be used here is the FEM approximation to

$$
|\nabla \omega|=\left(\frac{\partial \bar{\omega}}{\partial x_{\beta}} \frac{\partial \bar{\omega}}{\partial x_{\beta}}\right)^{1 / 2}
$$

where $\bar{\omega}$ is the resolved or large-scale vorticity. In two-dimensions,

$$
|\nabla \omega|=\left[\left(\frac{\partial \bar{\omega}}{\partial x_{1}}\right)^{2}+\left(\frac{\partial \bar{\omega}}{\partial x_{2}}\right)^{2}\right]^{1 / 2}
$$

where

$$
\bar{\omega}=\frac{\partial \bar{u}_{2}}{\partial x_{1}}-\frac{\partial \bar{u}_{1}}{\partial x_{2}}
$$


In the finite element code, $v_{T}$ is needed at the element centroid. To calculate the vorticity gradient at the element centroid, we need vorticity values at the nodes. Unfortunately, since the velocities are also nodal quantities, the velocity gradient and thus, the vorticity is inherently a centroid value. To obtain the nodal values for vorticity, the centroid values are moved/transferred to the nodes using the inverse area (finite difference) weighting scheme ${ }^{1}$

$$
\bar{\omega}_{i}=\frac{\sum_{j} \frac{\bar{\omega}_{j}}{A_{j}}}{\sum_{j} \frac{1}{A_{j}}}
$$

where $j$ is the summation over the four elements surrounding node $i$. Corrections can be performed for boundary nodes. If the node is at a boundary, the calculated nodal quantity using the above scheme is a good approximation for the midpoint between the boundary and the first adjacent node. Then using this midpoint value and the value at the first adjacent node, a linear extrapolation can be performed to find the value at the wall. However, this extrapolation was found to often result in a sign change of the values. Therefore, corrections were not performed for boundary nodes because of possible inaccuracies introduced by the linear extrapolation at boundaries.

Using the nodal values of vorticity, the gradients at the element centroid are calculated from the following relations

1 Inverse area weighting will correctly weight the centroid value for a nonuniform mesh, which is not the case for area weighting. 


$$
\frac{\partial \bar{\omega}}{\partial x_{1}}=\sum_{j} \bar{\omega}_{j} \frac{\partial \phi_{j}}{\partial x_{1}}
$$

and

$$
\frac{\partial \bar{\omega}}{\partial x_{2}}=\sum_{j} \bar{\omega}_{j} \frac{\partial \phi_{j}}{\partial x_{2}}
$$

where the summation is over the four nodes of the element. The value of the constant $c_{\infty}^{3}$ was set equivalent to the Smagorinsky constant $c^{2}$ (i.e., $\mathrm{c} \omega=c^{2 / 3}$ ) unless otherwise specified. 


\section{Appendix F}

\section{Estimating a Steady State from the \\ Velocity Time History at a Point Location}

One way to estimate a steady state is to use the velocity values at a point over a period of time and with a simple but reasonable assumption about the time history behavior, extrapolate to the value at steady state. This method was used in this study.

For example, the backward facing step cases for $\mathrm{Re}=800$ with a $41 \times 801$ mesh (discussed in Section 5.1) were checked for time convergence using this method. It was assumed at "long" time wherein asymptotic behavior seemed to prevail that the velocity, $V\left(=\sqrt{u_{1}^{2}+u_{2}^{2}}\right)$, was approaching a steady-state condition according to the relation

$$
V(t)=a+b e^{-c t}
$$

Using times of 80,200 , and 300 with velocities $.998972, .995210$, and .994915 , respectively for the node point location $x=9.975$ and $y=0.45$, the three coefficients were calculated and a projected steady-state condition (i.e., as $t \rightarrow \infty, V \rightarrow a$ ) was predicted, $V(\infty)=0.994873$. The code was then run to $t=400.1$. For $t=400.1$ the exponential relation predicts $V=0.994878$, and the code calculated $V=0.994866$. The relation predicted a value $0.0012 \%$ high. The convergence check could be the absolute difference 


$$
V(400.1)-V(\infty)=0.994866-0.994873=-0.000007
$$

or a relative difference

$$
\frac{V(400.1)-V(\infty)}{V(\infty)} \times 100 \%=-0.0007 \%
$$

If the absolute or relative difference is at or below the desired time convergence tolerance, one can conclude that at this location the solution is time converged. However, several points may need to be checked for convergence. 


\section{Appendix G}

\section{Spatial Filtering of the Direct Simulation}

The filter width $\Delta f$ is defined as

$$
\Delta_{f}=\left(\Delta x_{f} \Delta y_{f} \Delta z_{f}\right)^{1 / 3}
$$

By definition, the filtered velocity field using cell volume-averaging is

$$
\bar{u}_{\alpha}(x, y, z, t)=\frac{1}{\Delta x_{f} \Delta y_{f} \Delta z_{f}} \int_{z-\frac{\Delta z_{f}}{2}}^{2+\frac{\Delta z_{f}}{2}} \int_{y-\frac{\Delta y_{f}}{2}}^{y+\frac{\Delta y_{f}}{2}} \int_{z-\frac{\Delta x_{f}}{2}}^{x+\frac{\Delta x_{f}}{2}} u_{\alpha}\left(x^{\prime}, y^{\prime}, z^{\prime}, t\right) d x^{\prime} d y^{\prime} d z^{\prime} .
$$

Assuming that the integral limits fall on the boundaries of elements (i.e., the limits do not cut through elements), we have

$$
\bar{u}_{\alpha}(x, y, z, t)=\frac{\sum_{n=1}^{N E} \int_{\Omega_{n}^{e}} u_{\alpha} d \Omega_{n}^{e}}{\sum_{n=1}^{N E} \int_{\Omega_{n}^{e}} d \Omega_{n}^{e}}
$$

where $N E$ is the number of elements within the domain $x-\left(\Delta x_{f} / 2\right)$ to $x+\left(\Delta x_{f} / 2\right)$, $y-\left(\Delta y_{f} / 2\right)$ to $y+\left(\Delta y_{f} / 2\right)$, and $z-\left(\Delta z_{f} / 2\right)$ to $z+\left(\Delta z_{f} / 2\right)$. (This assumption is not necessary, but it simplifies implementation of the filtering scheme.) Substituting the expansion of $u_{\alpha}$, which is 


$$
u_{\alpha}(x, y, z, t)=\sum_{i=1}^{N P E} u_{\alpha_{i}}(t) \phi_{i}(x, y, z)
$$

where NPE is the number of points per element, we have

$$
\bar{u}_{\alpha}(x, y, z, t)=\frac{\sum_{n=1}^{N E}\left(\sum_{n=1}^{N P E} u_{\alpha_{i}} \int_{\Omega_{i}^{e}} \phi_{i} d \Omega_{n}^{e}\right)}{\sum_{n=1}^{N E} \int_{\Omega_{i}} d \Omega_{n}^{e}} .
$$

For piecewise linear basis functions

$$
\phi_{i}=\frac{1}{8}\left(1+\xi_{i} \xi\right)\left(1+\eta_{i} \eta\right)\left(1+\zeta_{i} \zeta\right), \mathrm{i}=1,2, \ldots, 8
$$

and

$$
\int_{-1}^{1} \int_{-1}^{1} \int_{-1}^{1} \phi_{i} d \xi d \eta d \zeta=1
$$

Transforming from global $(x, y, z)$ coordinates to local $(\xi, \eta, \zeta)$ coordinates introduces the determinant of the Jacobian:

$$
d \Omega_{\eta}^{e}=\left|J_{n}\right| d \xi d \eta d \zeta
$$

Substituting, we have 


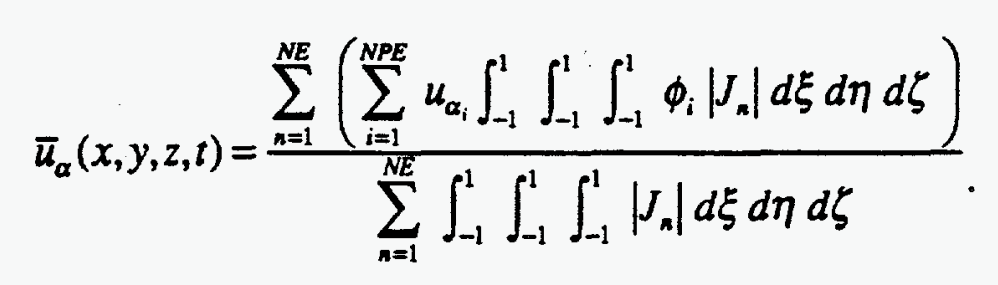

For rectangular elements $\left|J_{n}\right|$ is constant over the element and we have

$$
\begin{aligned}
& =\frac{\sum_{n=1}^{N E}\left|J_{n}\right|\left(\sum_{i=1}^{N P E} u_{\alpha_{i}} \int_{-1}^{1} \int_{-1}^{1} \int_{-1}^{1} \phi_{i} d \xi d \eta d \zeta\right)}{\sum_{n=1}^{N E}\left|J_{n}\right| \int_{-1}^{1} \int_{-1}^{1} \int_{-1}^{1} d \xi d \eta d \zeta} \\
& =\frac{\sum_{n=1}^{N E} \mid J_{n}\left(\left(\sum_{i=1}^{N P E} u_{\alpha_{i}}\right)\right.}{2^{n \operatorname{dim}} \sum_{n=1}^{N E}\left|J_{n}\right|}
\end{aligned}
$$

where ndim is the number of dimensions.

The code stores $A_{n}$ but does not store $\left|J_{n}\right|$. We calculate $\left|J_{n}\right|$ from the element areas according to

$$
A_{n}=2^{\text {ndim }}\left|J_{n}\right|
$$

So for code implementation we have

$$
\bar{u}_{\alpha}(x, y, z, t)=\frac{\sum_{n=1}^{N E} A_{n}\left(\sum_{i=1}^{N P E} u_{\alpha_{i}}\right)}{2^{n \operatorname{dim}} \sum_{n=1}^{N E} A_{n}} .
$$


For a uniform grid, $\left|J_{n}\right|$ (or $A_{n}$ ) is constant so the above expression reduces to

$$
\bar{u}_{\alpha}(x, y, z, t)=\frac{1}{2^{n \operatorname{dim}} \cdot N E} \sum_{n=1}^{N E}\left(\sum_{i=1}^{N P E} u_{\alpha_{i}}\right) .
$$




\section{Appendix H}

\section{Power Spectrum Calculations Using the Fast Fourier Transform}

The following is a detailed description of the method used to calculate the power spectrum for the velocity time histories presented in Section 5 . The coded subroutines used to calculate the Fourier transform and power spectrum were obtained from Numerical Recipes by W. H. Press, B. P. Flannery, S. A. Teukolsky, and W. T. Vetterling, Cambridge Univ. Press (1989) (a book of numerical routines).

The power spectrum is an estimate of the mean squared amplitude of the time history data;

$$
\frac{1}{N} \sum_{j=0}^{N-1}\left|c_{j}\right|^{2} \equiv \text { mean square amplitude }
$$

where $c_{0}, c_{1}, \ldots, c_{N-1}$ are the sampled data. The $N$-point sample at equal intervals $\Delta$ is used to compute the discrete Fourier transform

$$
C_{k}=\sum_{j=0}^{N-1} c_{j} e^{2 \pi j k / N} \quad k=0, \ldots, N-1
$$

using a fast Fourier transform routine. The power spectrum $P(f k)$ for the zero and positive frequencies 


$$
f_{k}=\frac{k}{N \Delta} \cdot \text { for } k=0,1, \ldots, \frac{N}{2}
$$

is calculated using the discrete Fourier transform as follows

$$
\begin{aligned}
P(0) & =P\left(f_{0}\right)=\frac{1}{N^{2}}\left|C_{0}\right|^{2} \\
P\left(f_{k}\right) & =\frac{1}{N^{2}}\left[\left|C_{k}\right|^{2}+\left|C_{N-k}\right|^{2}\right], \quad k=1,2, \ldots,\left(\frac{N}{2}-1\right) \\
P\left(f_{N / 2}\right) & =\frac{1}{N^{2}}\left|C_{n / 2}\right|^{2} .
\end{aligned}
$$

Data windowing is used to avoid data leakage. For a window function $w_{j}$, the equations for the power spectrum are

$$
\begin{gathered}
D_{k}=\sum_{j=0}^{N-1} c_{j} w_{j} e^{2 \pi j k / N}, k=0, \ldots, N-1 \\
P(0)=P\left(f_{0}\right)=\frac{1}{W_{s s}}\left|D_{0}\right|^{2} \\
P\left(f_{k}\right)=\frac{1}{W_{s s}}\left[\left|D_{k}\right|^{2}+\left|D_{N-k}\right|^{2}\right] \quad k=1,2, \ldots,\left(\frac{N}{2}-1\right) \\
P\left(f_{N / 2}\right)=\frac{1}{W_{s s}}\left|D_{n / 2}\right|^{2}
\end{gathered}
$$

where

$$
W_{s s}=N \sum_{j=0}^{N} w_{j}^{2}
$$


The window function used to generate the power spectrums in Section 5 is called the Parzen window

$$
w_{j}=1-\left|\frac{j-\frac{1}{2}(N-1)}{\frac{1}{2}(N+1)}\right| .
$$

To reduce the spectral variance, the data is segmented into overlapping segments. This method reduces the variance so long as the data sample has more points than needed. The total number of data points used to calculate the power spectrum is $(K+1) M$ where $K$ is the number of segments each of length $2 M$ so that the overlap of data between segments is of length $M$. To use the fast Fourier transform routine, $M$ must be equal to an integer power of 2 (i.e., $M=2^{n}$ ). Using the power spectrum calculated for each segment, an average power spectrum is generated.

This method will give the smallest variance from a fixed number of available data points. The larger the number of segments, the lower the variance. However, as mentioned above, it is assumed that there is more data than needed. For example, it was found that if the data has periodicity, it is important that each segment include several full periods. For periodic data, segmenting the data was not as important as the choice of a long segment with many cycles in each segment. 